



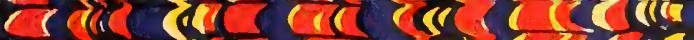

iicrese

acts

iriese

ra eres (anir

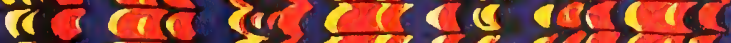

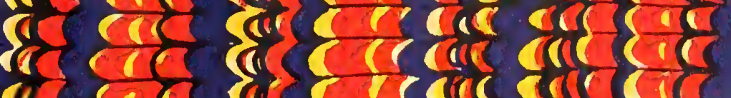

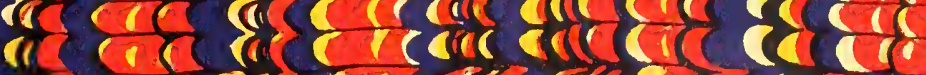

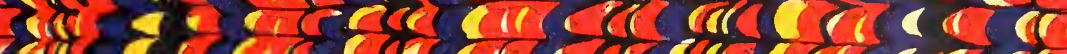

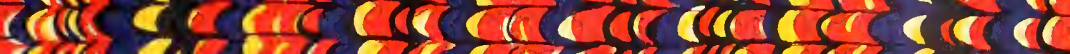

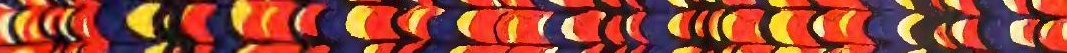

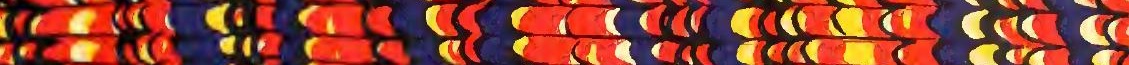
4.

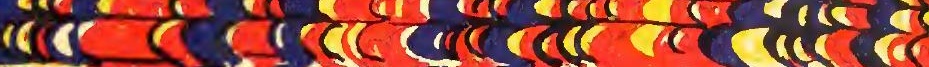

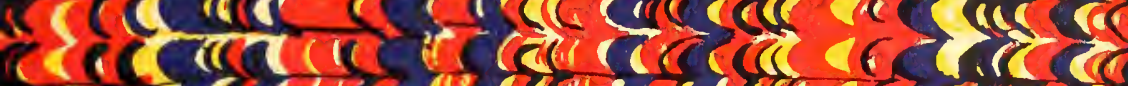
क्षे दो यदिक्र (a) कि हैं। (ii) ti.i

cois $\pi \sqrt{20}$ Crer ace care दोवि $\cos$

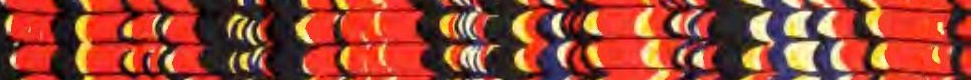

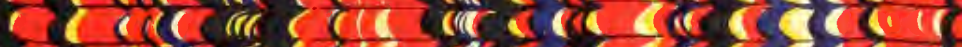

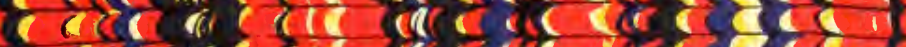
cals icce

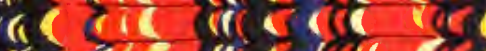

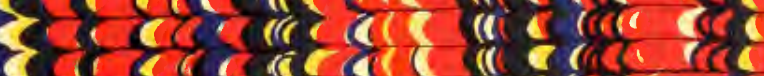

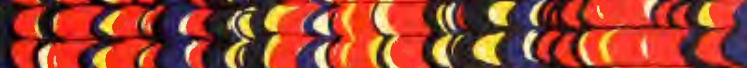
eicer me

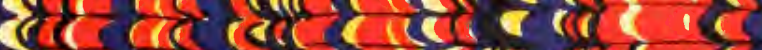

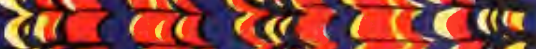

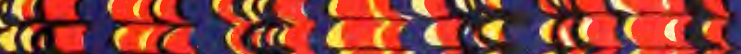

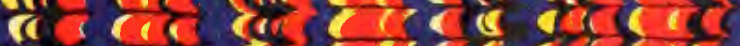

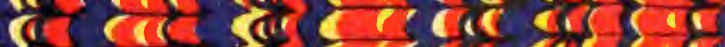

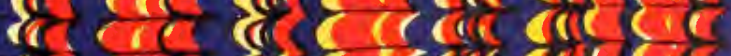
a दित

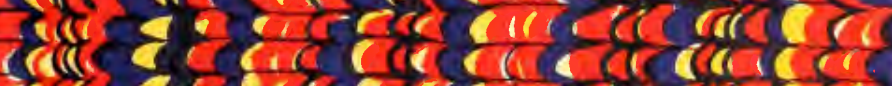

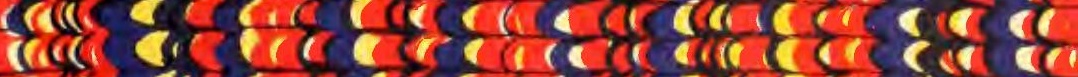

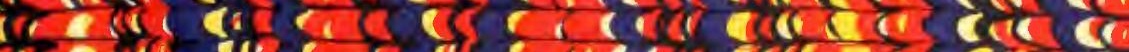

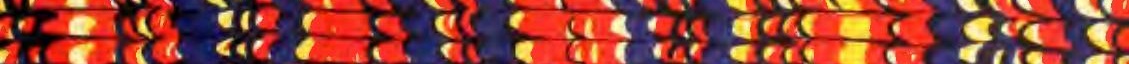

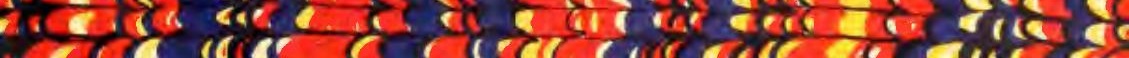

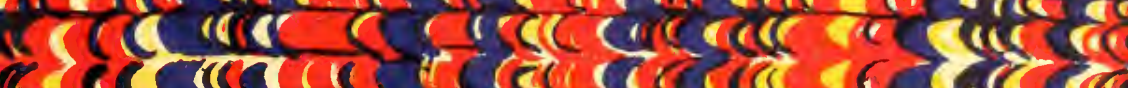









\title{
ÉTUDE MONOGRAPHIQUE
}

DES

\section{Tabanides d'Afrique}

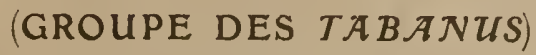

\author{
PAR \\ J. M. R. SURCOUF \\ Avec le concours de \\ Miss G. R I CAR D O \\ Stagiaire au British Museum
}

Chef des travaux de zoologie au Laboratoire colonial du Muséum de Paris

Avec 3 planches en couleurs, 26 figures dans le texte et 22 cartes

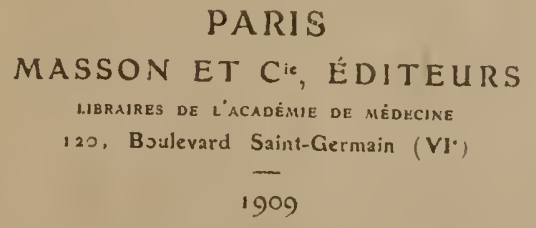




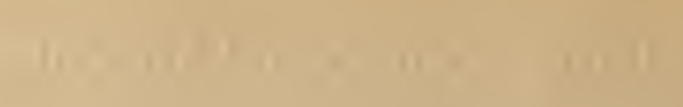

9114 
Tabanides d'Afrique 


\section{FN PRÍPARATION}

1" Etude monographique des Tabanides d'Afrique (suili). Ciroupe des Hemetopotir.

2. Tabanides de Madagascar. 


\section{ÉTUDE MONOGRAPHIQUE}

DES

\section{Tabanides d'Afrique}

(GROUPE DES TABANUS)

PAR

\section{J. M. R. SURCOUF}

Chef des travaux de zoologie au Laboratoire colonial du Muséum de Paris

Avec le concours de

Miss G. R I C A R D O

Stagiaire au British Museum

Avec 3 planches en couleurs, 26 figures dans le texte et 22 cartes

\section{PARIS}

MASSON ET Cie, ÉDITEURS

LIBRAIREs de l'académie de médeciNe

120, Boulevard Saint-Germain (V.')

1909 


\section{A Monseur F. BOUTier,}

Professeur d'Entomologie au Maséum, Mentre de l'Institut.

Cher al Honore' Mitre.

En janviur Iyoj, lorsque je me suis pour la premiere fois présente it volre laboratoire, bous minues aconeilli anec tumt de biemupillance que, grace a vos enconragements, jai pu, malgri de nombreax abstacles, enteeprendre el poursuiure létude des Tabanides.

G"est dans us tonrs et dans votre Laboratoire que j"ui été initié ì ln Wéthode rigourense qui mia permis de mener à bonne fin ce travail, permettez-moi de cous le dédier comme témoinnage de reconnaissance.

JacQUeS SURCOUF. 


\section{INTRODUCTION}

Les Tabanides sont des l)ipteres pui pinpent les Mammiferes et en sucent le sing. Répandus sur to globe entier, an les reneontre sous toutes les latitudes ou les grands mammilères peuvent vive. Les recherches microbiologiques ont prouve an munde savant que les Taons et les antres insectes piquenrs puraient transmettre des microorganismes nuisibles a Thomme ot aux animax.

Létendue des rolonirs africaines, leur importance au point de vur commreial rendent iudispensable l'étude de tous les ètres qui sont susceptilıles d'entraver par leur fréquence on leur nocuité le développement de ces enntrées. Il nous a done semblé que l'examen seientifique des Taons, la comaissane de leurs morurs et de leur habitat était nicessaire à lépoque actuelle. Nos fonctions au Lahoratoire colonial du muséum et les nombreuses domandes de détermination qui nous y ont été adressées nous ont montré l'intript d'actualité ju'aurat cette stude, carmieux on connaitra ces différents ennemis, mioux on saura s'en préserver et ensuite les détruije.

Jusqüiei les travaux exécutés sur les Tabanites d'Ifrique ont été incomplets. Les wures de Laxé ot de Fabricus ne peurent donner que des indications imprócises. En eflet le nombre des especas conmues était si faible güil a sembli inutile aux premiers determinatenrs de scruter lus détails de la morploblugie et, puand les recherches posterientes ont firit découvrir un grand nombre d'espèces dillérentes il a été sunvent presque impossible de les rapporter à relles déji técrites.

Postérieurement, Meuga, Wiedeman, Palsot de Beatrous, ont décrit de nombreux spécinens nouveaux. Plus rérmment, Mcorar, Bgor,

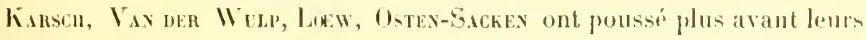
déconvertes et orienté vers une spéciliation plus systimatique l'étnde

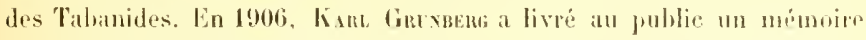
sur les insectes piquents de l'Afripue. La profosseur Mamo Baza, de Turin a étudié les Bipteres de l'Erythrée; M. Andrew Busotn a dirigé et publié les recherehes de l'Ecolede médecine de lihartonm; MI. News reu, 
Dutwor et Tono, celles de l'expridition an Congo de l'Ecole de médecine tropicale de liverjuol. Vers la mème époque le doctenr lientesz a dressé le catalogue synonymipue de tous les Tabanides décrits; ce trarail qui a exigé des recherches bibliographiques considérables facilite l’étude de ce groupe el donne de préciruses indieations.

Mon maitre, M. le prolesseur Bouvier, membre de l'Institut, a bien voulu maceueillir dans son lahoratoire du Muspum wt ma engigé à etudier les Tahanides. Le hasard a voulu qua Miss li. Ricanoo, stagiaire lilore au British Musem of natural Mivtory, vint alors au Musem de I'aris pour y rechercher des doenments sur les mimes insectes, une cooprí ration fut décidée entre nous et le plan de pe prenter ouvare résolu.

Le travail que nous presentons au leeteur comprend deux parties distinctes; la première se compose d un tableau dichotomique divisant les espèces du grenre Tabanus en seize proujes d'après leurs aflinités, puis de la description de toutes les espices qui les constituent. Chacun des seize groupes est prócialé d'un talueau dichoromique. La seconde partie est constituée prar des eartes al' Ifrique indiquant les lienx d'habitat de chatenne des especes décrites. Lin ontre nums avons été amenés à joindre i cos cartes des tahleaux de ripartition geographinge de rhacm de ces Eroupes.

Pour ètre plus chairs, nous avons un preu arbitrairment subdivisé ['Afrique non pakaretique on six pónions. Cette division ne comprend que l'Ifrique tropitale, ast-it-dire la partie siture an-dessous de $20^{\circ}$ latitude Nom; rn effet la rigion sibarienne est rurore peu étudice et en remonlant vers le Nord, on rencontre las Tabanides de l'Nlgúrie, de la Tunisie et du Maroe pui appratiennent a la faune palearetique et se rattachent à celle du hassin rirumméditeranéen (earte 1).

Nous publierons prochainement un revision des Talbunides du bassin de la Néditertanée. Pour une raison analogue nons latissons de côté dans cet ouvage les Talanides de Mardazisear ; les esperess de cette ile sont. it de rares exceptions pris, nettement différentes des formes africaines: la lame malgathe ininsi que sa flore, du reste. n'est plus que le témoin d'un continent disparu. Peut-ètre fuand nous anrons étudiẻ les Tabanides de I'lnde et de la Malaisie, saurous-nous y trouver les passages entre la faune africaine et la liaune indo-malaise.

Les difficultés matírielles de cette fitude sur les Tabanides d'Afrique ont ité aplanies grace an lienveillant appui de $\mathbf{~ l l}$. he docteur Rocx, directeur de l'Institut Pasteur, qui a bien voulu s"intéresser i notre ceure et en entreprendre la publication anx frais de cet établissement. Ciest 


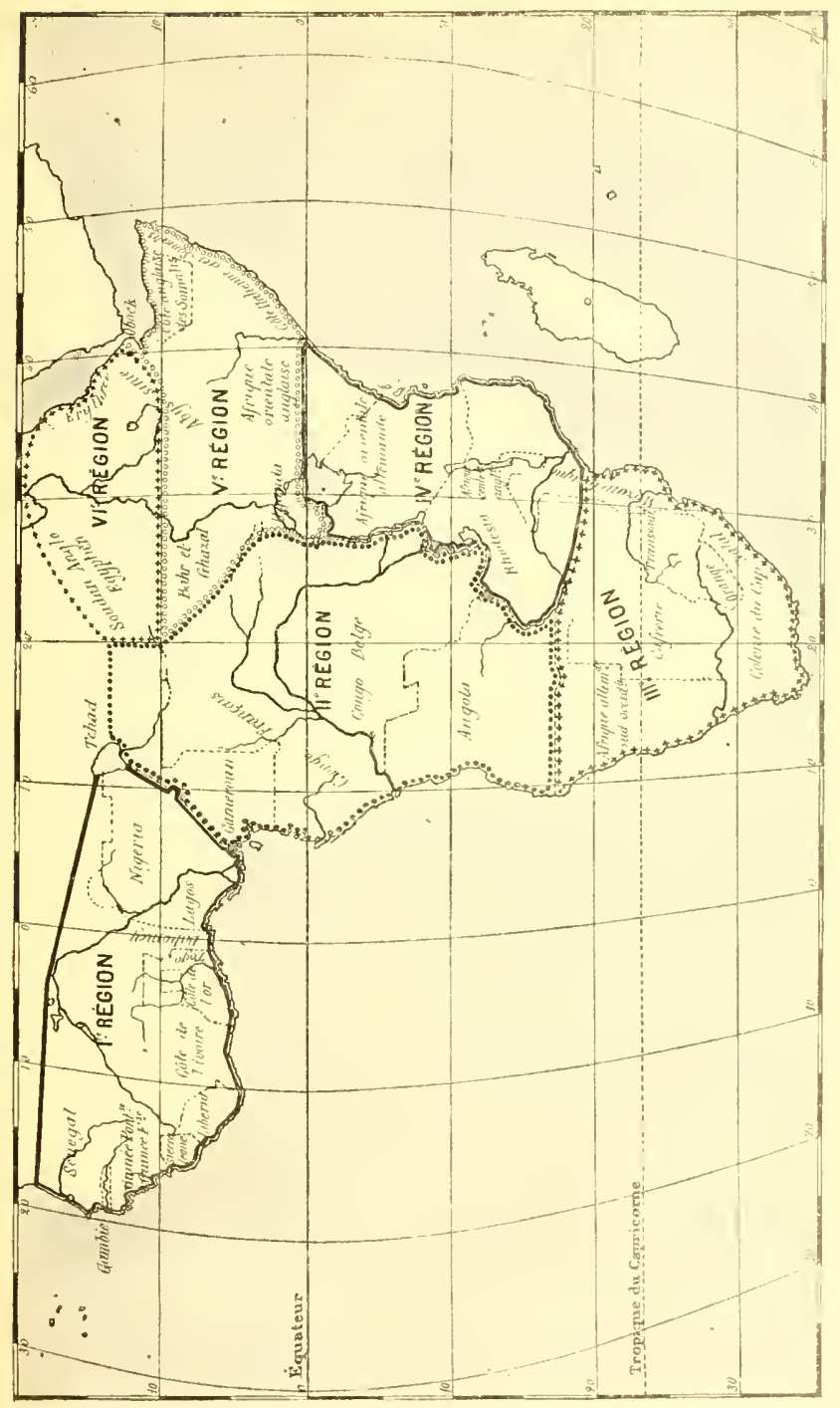


gratce à ce précieus patronage quaura put tre publié ex volume qui est le premier consacré ì la faun complite des Tahanides africains.

Nous adressums at M. le lor Roux ol it ses éminents collaboraleurs I'lommage de notre reconnaissaner.

Par sulte de nos relations at de la lienveillance des diptéristes de l'Europe. nous arons eu a notre tisponstion lit collection des Tabanides du Muséum de Paris, du British Museum, du Musée royal d'llisioire naturelle de Belgique. du Musie de Madrid. du Musée de Lishonne, du Musée de Ilambourg, du Husio de Franclort-sur-Mein, de l'Institut Pasteur, du Laboratoire de fratasitologie de l'Ecole de midecine, du docteur

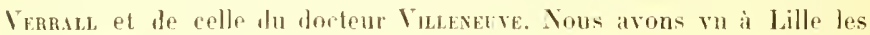
types de Macol art.

l Jans ces diverses collections, sa Irouvent les types de Fabrows, Bosc.

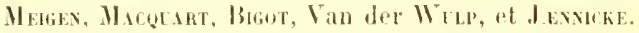

Nous arons pu comprater a ces lypes tous les insectes douteux ou inconnus et ce traval noms a rombluit it établit de nombrenses synonymies.

En effet. le catalogue de Kentex mentionuait 9:3 especes de Taons en

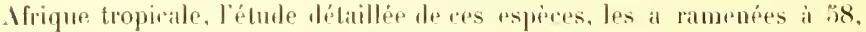

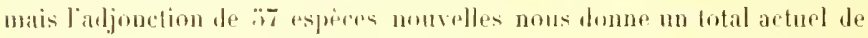
11 ii espires.

Nons ajouterous que le nombre siccroilril sensiblement lorsque le

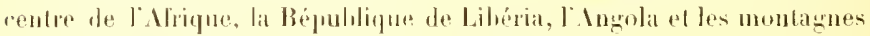
anront été sillonnées par les missions srientidiques. 


\section{NOTIONS GENERALES}

La lamill. des Tabanides conprend plus de 1.600 especes jui se répartissent en plusieurs familles sublivisées en gonres.

Parmi ceux-ci le genre Tatrames est etudie dans cet ouvage. Il importe done d'en rappeler brievenent les caracteress spéciliques.

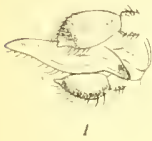

$\xi$

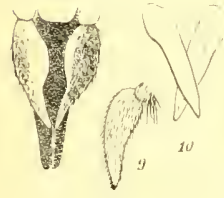

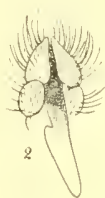

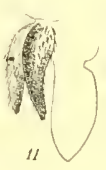

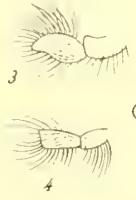
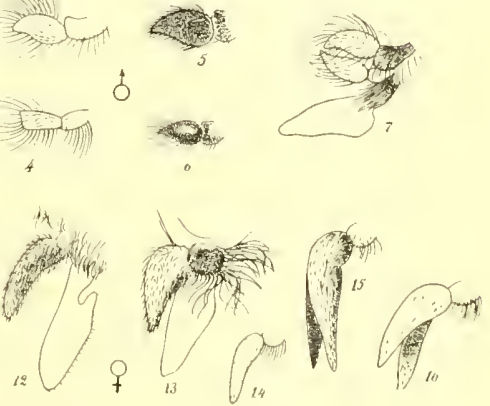

Fis., 1 .

Morphologie. - T'́te courte, allongár Lansversalement, aussi large on flus liage que fo thorix, région antirieure lombée, region posterrienre exavé Antemnes lirigés en avant, presque contigurs a la lase. composies de trois articles, le trobieno est hrusquement élarui a sa base et fre sur lo borl externe une sillif plus ou moins olsolete parfois en forme te croissibut aign Tabamus atre Rossi), parfois présentant une simple expansion (Tabames Rothschildi); la partie terminale de cet artiole porter puatre segumntations. Trompre saillante. non repliée. Palpes (lige. I)

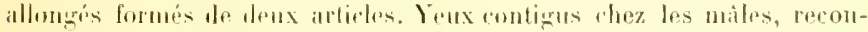
vant le fronten entier et se réunissant le long de la ligne médiano (ID,

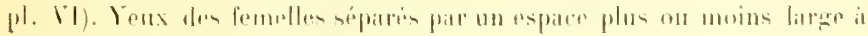




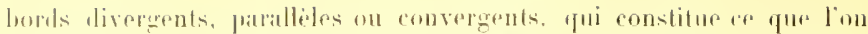

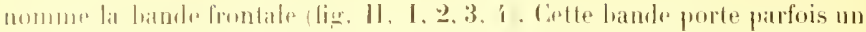

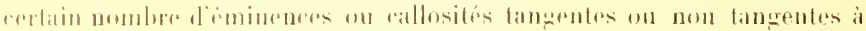

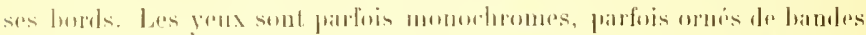

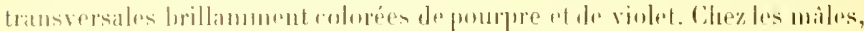

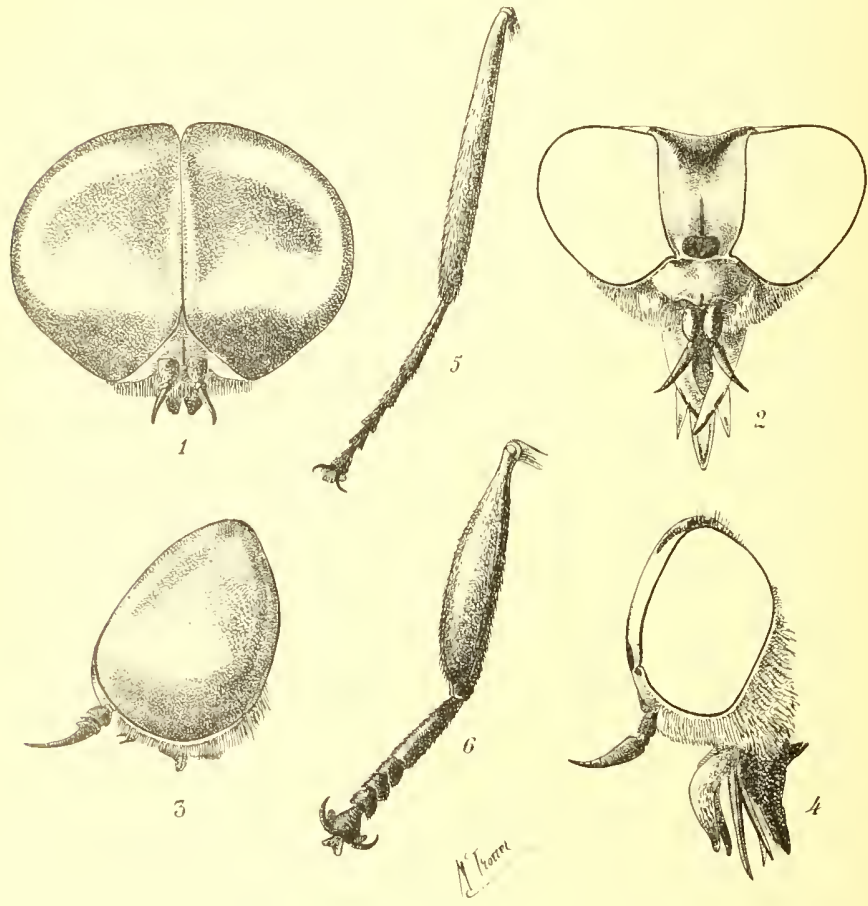

Fis. 11.

las voluminnax yeux composis ss suldivisent fréquemment en zones 11) ou moins nettes de rorneintes plus grosises que celles du reste du hamp. Il existe parlois me villacifi assu dense sur les reux, arei se

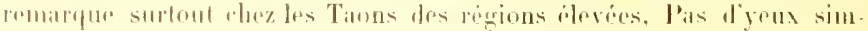

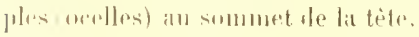

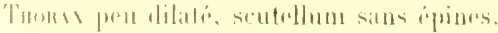


Anosux large et long. parfuis conique, composé de sept segments apparents.

Pattes (lieg. II, $\ddot{j}$ et b) ni longues, ni fortess, sans touffes de poils, plus ou moins pulrescentes sur teur longuenr; lanches postérieures passable-
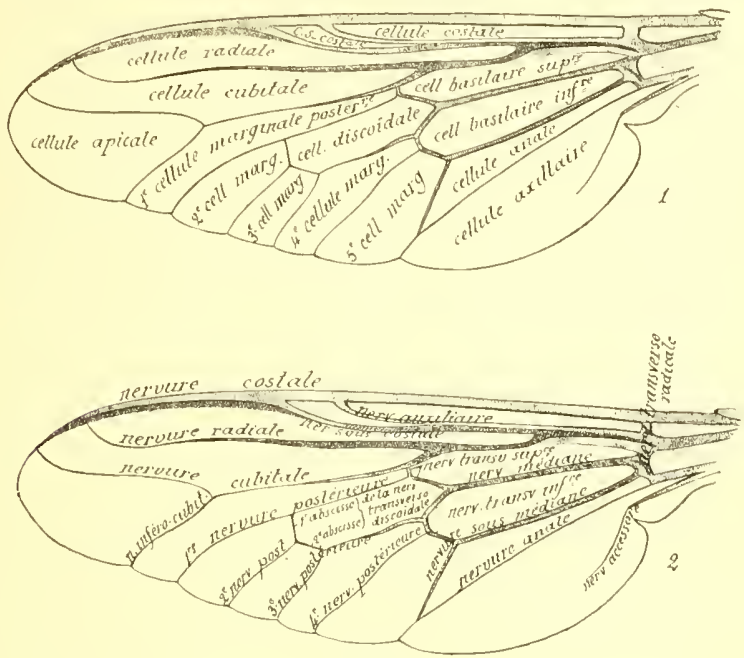

Fig. 111 .

Correstondatice

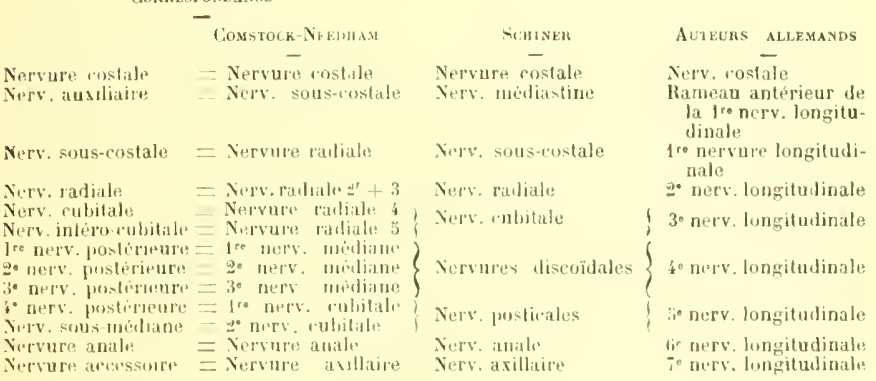

ment longres. Tilias moyens et antiriens armés d'un éperon. Tarses portant truis empolia.

Mlues (lị. III) à cuilterons visibles el assez qrants, nervures réparties tres irrigulierement sur le disque; la nerrure costale borte l'aile en 
entior, la troisième nervore longitudinale est lifide, une de ses branches fournit parfois un hamecon supplémentaire dirigé vers la racine de laale. Cellule discoidale non centrale, mais rapprocluée du hord extérieur de l'aile, entre les quatre cellules marwinales postérieures issues de la cellule discoüdale, il en existe une cinquième, ces cinq cellules sont génóralement ouvertes. Cellule anale ordinairement allongée, fermée an bord de laile ou tris pres de colui-ci.

Les ailes sont un preu beartés en arripre pendant le repos et présentent sourent des taches wu des bandes sombres. Balaneiers non recomverts par les ailes.

Développement. - La vie larvaire des Tabanides est encore pen connue. La première observation fui s lapporte fut fate sur les larves du Tabanms bovimus Laxé et remonte a bf REEn, en 1760. "La larve vit " dans la terre. Elle est allongio, cylindrique, anneie vers la tite qui

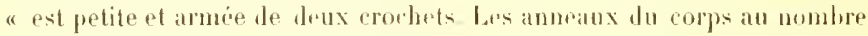
" de douze ont de's cordons relevés. lat nymphe ost nue, presgue eylindri"quc, avec deux tubercules sur le liont, des cils sur le bord des anneaux " ol six pointes à son extremité pustrieure. Elle se rend à la surfice du " sol lursquiplle doit se dépouiltre de sil peau. pour prembe la forme du " taun of sort it moitie de la terre, (ne treen, lusectes, VI, XII, 1(1, 11).

En 1834 Mhcouat rappelle louservation de de liens.

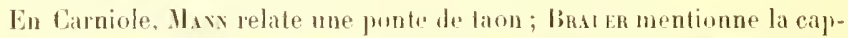
ture de nombreuses pontes sur des lerelves aquatipues. En I90; M. LecanLox (Amuales de la Socirlé Entomologfique de France, vol. IXXIV.

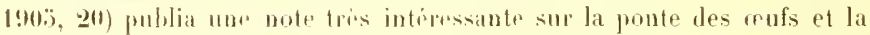

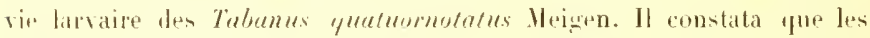

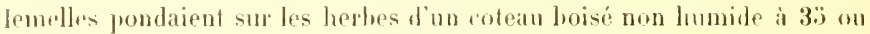
io contimètres du sol; il importr dre remarguner, dit-il, que la femelle en étal de ponte devient indifférentu a ce qui l'entume, se laisse captiver sams y prendre attention et que les puntes peuvent avoir liou dans an. endroit relativement sec.

Les arufs sont pondus en une masse prosque conique forme de cur-

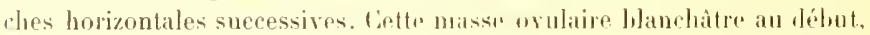
lormil apres quelques jours.

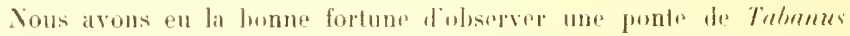

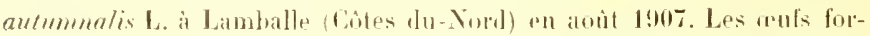
maient une masso tense alhérente a un jont ot la femelle, la tite tomrée rers le sol humide, restirit immolile. noms arons pu la capturer sans qu'ulle fit un elfort pour nous exhapper. Mallurureusement, nous avons 


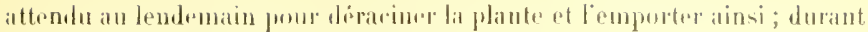
lintervalle un fauchenr avat tort detruit.

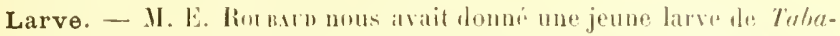

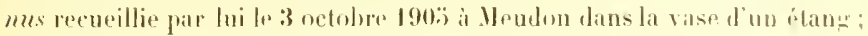

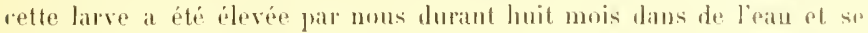
nourrissint non de proirs vivantes mais de matieres arganigues apprortées par une toulle de momsse qui grarnissalit le fond d'un cristallisoir ou elle vivat. Le 13 mat 1907 , cette larve refoula la mousse, de farou a se fire un alni, perdit sa molsilite, devint plus translucide que de eoutume ot le l6 mai elle étit transformée en me nymphe qui prit mallseurensement par suite d'un acrisent. La lawe est allongée. blandatre, trix

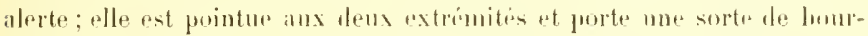

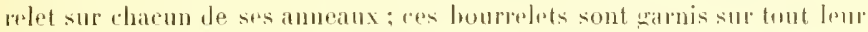
pourtour de tubercules ritratiles qui servent de moyen de progression.

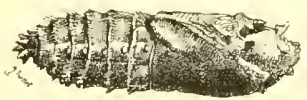

Fig. IV.

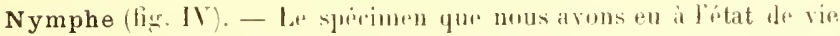

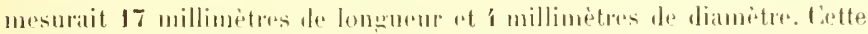

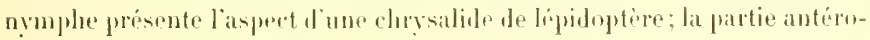

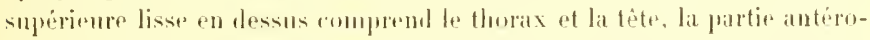

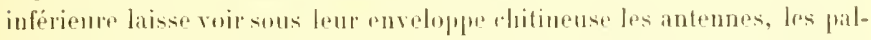
pes, les yeux et les pattes de la premier paire. Les ailes sont contenues dams un fourran yui attrint le sommet de lanneatu scutedlatre. inerme.

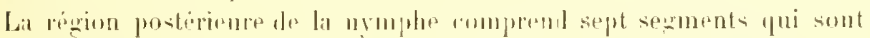

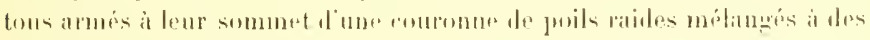

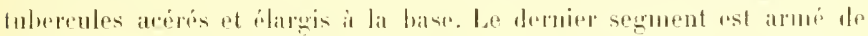

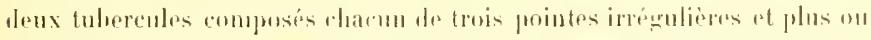

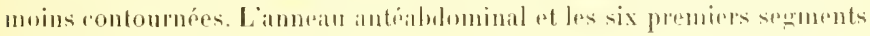

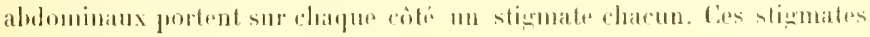
faraissent légirement en moliel.

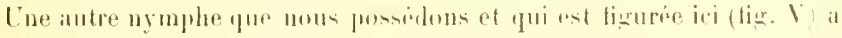

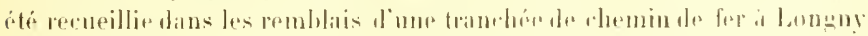

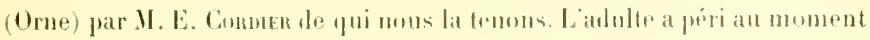

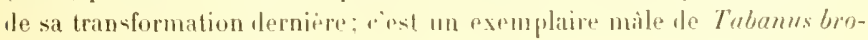


mins L.; il est à Hemi sorti de lenveloppe nymplate dront il n'a pu se débarasser malgré de nombreux alforts, comme le prouve laallongenent innsite de l'enveloppe nymplate. Le morle de libration de l'arlulte est rendu évident par l'examen de celtu rare pièce, la tète fail bélier et défonce le sommet de la coque puis edle-ci sous la pression de l'insecte sourre entierement en long et se dirhire jusqüi la lase da premier segment abdominal qui reste intact ainsi que los segments survants. Laalulte ayant terminé ce stade de son évolutiou s’envole, dès qüil est séché, à la recherche de la nouriture.

Biologie.- Les mảles ne se mourrissent pas du singr des vortébrés, mais du suc des fleurs, ils en sucent les neetaires, ils aspirent aussi la sive des blessures darbres et des fruits tombés. Gertains arhustes a flemrs odorantes attirent les males et les femplles. Cust ainsi qu' it Citrnate (Morbihan) le docteur Lensuz, a recueilli es juillet 1907 , trois spécimens de Tabanus migrifacies Gonert sur los blanches d'un genèt d'Espagne. Les femelles

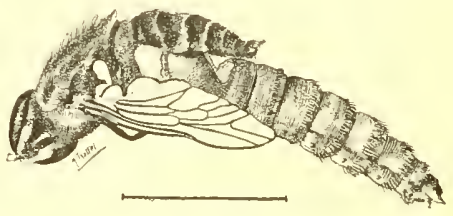

Fig. 1.

assaillent eruellement les chevaus ot les bovilés, elles s'attaruent surtout aux parties nu elles ne peuvent itre atteintes par les jambes, la tè on le eoup de quene. Lorsque les brstiaus sont rentris aux étables, les taons 'pui ne piquent pas dans lobseurité attendent au-dehors. au pied les murs ou sous le chajeron des toits que les animaux de ferme sortent a nouveau. Il arrive assez fréquemment que rertaines especes de taons attaquent l'homme, mais fortquim les saisit entre les doigts ils ne tentent aucum effort pour piquer. En France les Hemalopota et les Chrysops. phus nombreux que les tams du reste, attaquent lihomme avec acharnement. Nous comaissons en Bretanne les prairies au milieu des bois wi en juillet on est immódiatement atsailli par de nombrenx Chrysops. Muoique les tanns agissent le jour uniquement, le grand soleil les gène. ifs se tiennent a l'abri des loris, ot lit all embuscade s'elanent vers les promes qui passent sans jamais les poursuivre longtemps. Sils attaquent un homme qui forte unf omlselle, il est fréquent quau bout diun 
moment on voyant liuutulití de leurs afforts ils viemnent se refugier des ardeurs du soleil à l’intioneur de liombrelle.

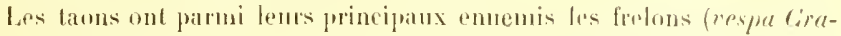

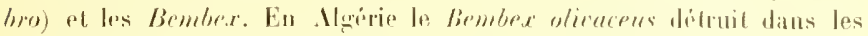
régions sablonneuses de grambles quantitis de Tabamus nemoralis.

Lappareil hucal des femetles a té sprecialement itudié par le profes-

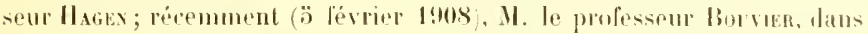
sun cours professé au Museum, a repris les études de Ilagex ot les a fait ronnaitre.

Les pieces de la bouclue sont les suivantes:

$1^{\circ}$ En dessus un voit le labre blarei à sa base, roupant sur les cités ef terminé en une courte pointe aigui a l'extrémité;

-2o Iatéralement, les maxilles qui sont les piores les plus axternes et portent un palpe de deux articles, puis les mantibules; les maxilles et les mandihules sont en forme le lame couprante ef tranchante ;

$3^{\circ}$ Au milieu, Mlypopharym ou langue, piece assez longue et vulnérante, fercée d'un trou pour lo passage de la salive et frour l'aspiration des liquiles nourriciers ;

$4^{\circ}$ En dessons, la live inférieurr. won vulnítante, terminise par deux paraglosses spongirux de forme varialile suivint les especes. Celte live arec ses paraglesises forme un tulw owvert en dessus qui sert de gain aux maxilles, aux mandibules et i l'hypopharynx. I a partie supérieure de re lube est fermée par le lalre.

Cette disposition nous montre que suivant lit nourriture pui lui est offerte, la femelle du taon peut piquer et sucer le sang ou aspirer le suc des fleurs.

Les pieces buccales des màles présentent la môme disposition mais les diverses parties sont extrmement ridnites; les maxilles et les mandihules sont moins chitinises ut moins robustes que chez les femelles.

Yous ajoutons à colle sommaim draription des caractères les plus évidents des bons qu" malgré les opinious admises il y a ell lleperie au moins souvent deux géúrations prir an. C'est ainsi pue tout récemment (mai 1908 il nous a été donno de recueillir sur un cheval plusieurs exemplaires très fratis du Tahamus antummalis 1 ., cetto sopèce se reprend in automne en France et én Ilgípie. 


\section{CLASSIFICATION}

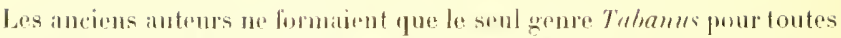

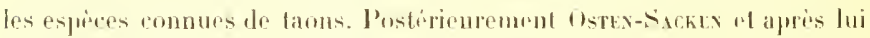
Braver partagerent re genrw en trois genres: Thérioplecters, Alylotus et Tabanus. Ces divisions efaicnt hasies sur les raracteres suivants:

Thérioplectes. - Formes de montagno ou de pays froids, se distingruant par une tense pubrscence sur les grux of la prósence d'un tubercule sur le vertox. 11 y amra lien plus tard l'ítudier la valeur histologique de ce tulvereule qui somble itre une forme te regression des yeux simples (ocelles) que certains groupes roisins (Pangmia, etc.) possèlent sur le vertex.

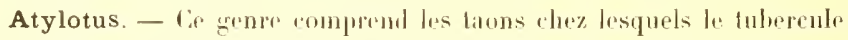
lu vertex est remplace par une callosite de trinte sombre, plus ou moins saillante; les yeux sont puhescents mais la pilositi est moins tense que

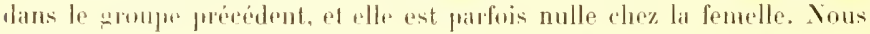
ünsisterns pas sur ce dernier caractere qui indique hien untrment une ¿rolution.

Tabanus. - Sous ce nom se rangunt les taons i hande frontale sams callosité an vertux of à yeux glabres. Ce sont éridemuent les taons les plus ivolués.

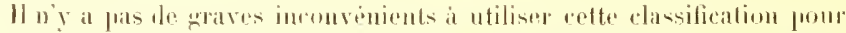

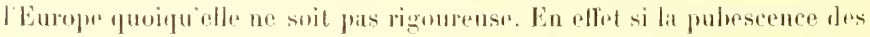

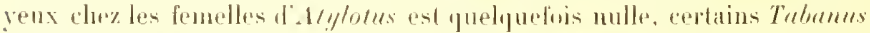

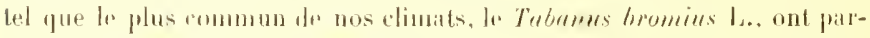

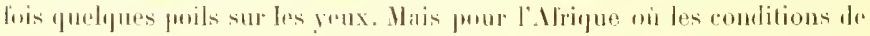

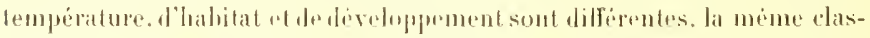

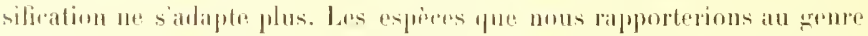

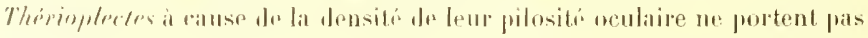

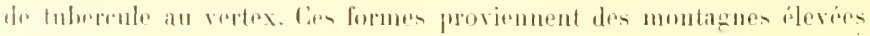




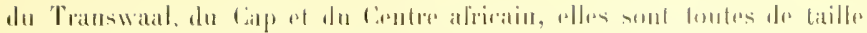

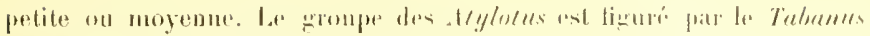

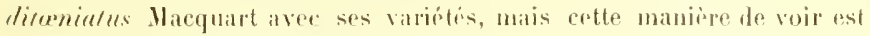

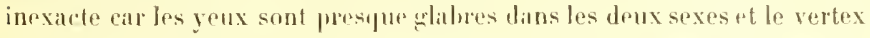

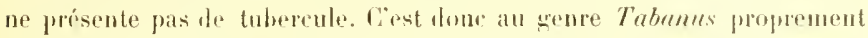
dit que se rapporte lin presplue totalití des repieces artuellenunt commes.

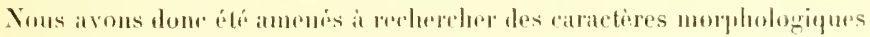

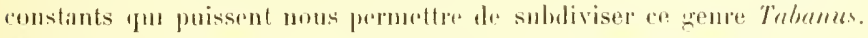

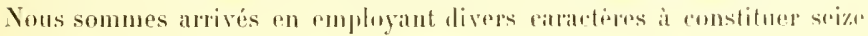
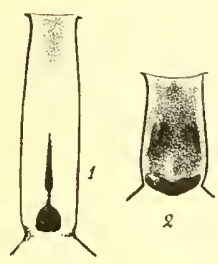

\&

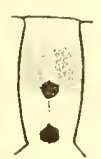

3

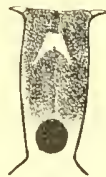

4

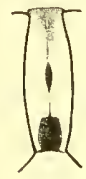

8

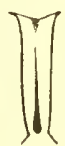

9
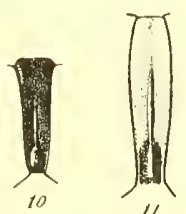

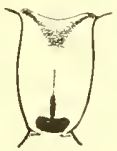

5
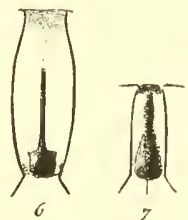

7

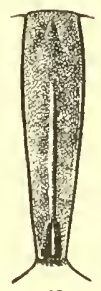

13

Fin. Yi.

groupes dont dharun reunit des esperess aflines et offre une ditrirener evidente aver les grompers roisins.

Les raracteres employés ont été les suivants indiqués en valeur décroissinte : la pubesernce on lit non pubesconce des yenx, le nombre et lat lorme des rallosités de la lande frontale intraoculaire (lier. VI), la forme des tibias el des antenues (fig. II) la nervation dres ailes fig. III). Parfois avous-nons dû faire usage de la nature de la pulescence et des tarartieres de sa coluration, mais seulensent à l’intérienr de chacun des seize sroupes

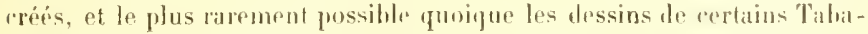
nitles soient aussi constants qur ceux des lépifloptires ot qu il en soit latit nsaze par les lépidoptéristos. 
Plus lard quand nous aurons terminé l'étude des Tabanides du globe pourrons-nous faire des conpures nowrolles, cortaines sont manifestement exactes des maintenant, mais tout essai de nomenclature serait prématuré actuellement et vraisemblablement errome.

P'uris, le fer mai 1909. 


\title{
DIVISIONS DU GENRE TABANLS
}

\author{
Tableau dichotomique des seize groupes \\ qui constituent le genre Tabanus
}

Bande frontale porlint ì la base une callosité ollongue ou enrée, toujours prolonge vers le vertex par une ligne étroile it lorigine, dilitie on non vers le milieu de la bande (is tig. 11).

Bande frontale portint une callusite arrondie ou transverse à la lase of une tarlore ou une calgosité an milien de la bande frontale. Cess deux callosités sont sípurres quamb l'insecte pol frais (2) lic. 1i).

Bame frontile portant deux potites sallusités arrondies, siluies l'un all-ilessus de l'antre, jamais reliés entre elles (3 lig. Vl) . . . .

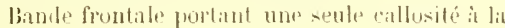
base ( 1 fig. 11 )

T'ibias antérenrs fortement lilatés (ti tị. Il hailes

Bande fronlale trois foris aussi laule que large à lat base, it ligne mádiane courle (.) fig. II). lorps noir, frilten noires, ailes brumes ì extrémites apicale clarire. pis d'appendise

Bande frontalo 3-i fois flus haute que large it la base (fi fig. II). Ailes brunes sans appendice a la $3^{\circ}$ nervure longiturlinale, aldomen purtant une grande tache soufre ou blanchaitre; on ailes hyalines a nerrures rouges, sa nz alppenlice, corps blanchilre it lacles bounes au burt intérieur des seguents abluminamx.

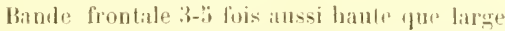

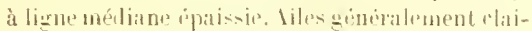
res, thorax salus baudes distimetes. Ablomen rougeatre ou brunatre ì binde méliame itroile, très distincte; pas d'antres lignes ou dessins $7 \mathrm{l}$ lig. 17

Seizime groupe.

3

Treizime groupe.

Quatorziène groupe.

Guinzièue sroupe.

J'remier groupr. i

Troisinne groupe.

Cinquièmegroupe.

seplième gr'oupe. 
linde frontale de q-io his ulus haute que latre is ligur médiane dilitlée en fuspau (S fig. (i), pratjer

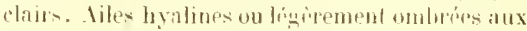

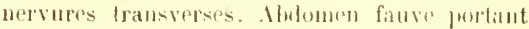

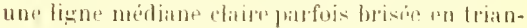
sles of reposint sur une liande plus sombre.

Bander frontale généralenent tris étroite, da 4-7 foris alussi hante que linge. Espreses mon nom ou jomiles. sians ligues ni dessins, jaunes ou rouses. Ailes claires ou lomnatres aver un sans ippendice (9 lim. 11 . . . . . . .

Bandr frontale rimg lois anssi laule pue barge it

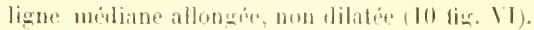

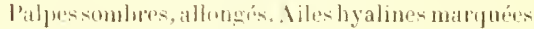
de bandse ou de trate bruns, phosou moinstein-

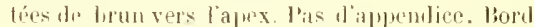
postérieur les soments vatraux toujums matrgriné de hlane. Celluk discoülale tonjours rlaire.

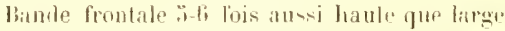

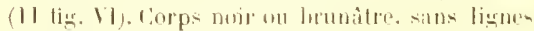

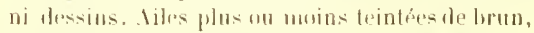

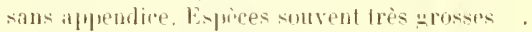

Bambe lrontale : $1 / 2-7$ fois plus laute que large ì ligne módiane non dilatée l:3 lign. V1). Palpes it pubesence noire. Aile plus ou moins branes. Tlmax orné le deux lignes foneriludinales of de denx lignes latérales jaumes. Iblomen variant du fauve rougealle au lom-noiratre, portant une biamle midiane etruite, claim. parfoir visible seu-

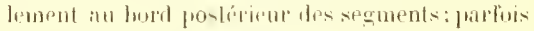
das timbes latérales alydominales peu distinntes.

lamble lrontahe analogue a colle du groupe IX, couleur générate, palpes of ailrs semblables. l'as de handervishles sur te thorix. Modomen fauve on bruntre it ligne meditm olsolite on tris

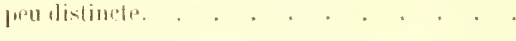

bande lroutale analogne it relle du groupe Ix.

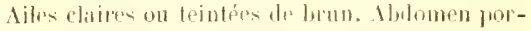
lant sur bi ligne mediane les triangles chairs el des tarhes laterales frix distinetes le plus souvent trianzulaires.

Banle froutale de dimensions diverses. Esprires jolites ou moyennes. Ablomen portant une grambe tarlie sur le servut segment ou deux sur le 3e ol te te segmeats. . . . . .
Ituilième -roups.

sixime grompe.

Deuxisme groupe.

Guatrime groule.

Yenvieme grompe.

Dixièmegroupe.

Onzième groupe.

Douzième groupe. 


\section{PREMIER GROLPE}

\section{Tabieat Demotomone}

Tibias antérieurs fortement dilatés, ailes layalines it bandes de couleur sombre; espreses fauves ou rougeitres, parfois jaunitres.

Antennes entièrement noires, ailes lyyalines arec deux bandes brunes ou noires . . . . . . . . . . . . . . . . 2 2

foneches non enticrement noires, ailes hyalines avec deux bandes peu foncies

Abdomen filuve sans taches, flincs ì tache triangulaires noires, visibles en dessous. Apex des ailes sans finscie noire. . . T. Latipes Macq.

2 Semblable. Ipex des illes avec une fascie noire. T. africanus firey.

Cimate espèce rouge, invers de l'alitomen noir. T. Brucei Ric.

Abromen ì sept taclses noires en dessus . . . T. septempuncta/us Rie.

Abdomen à quatre taclies noires en dessus. . T T. suboitlatus Ric.

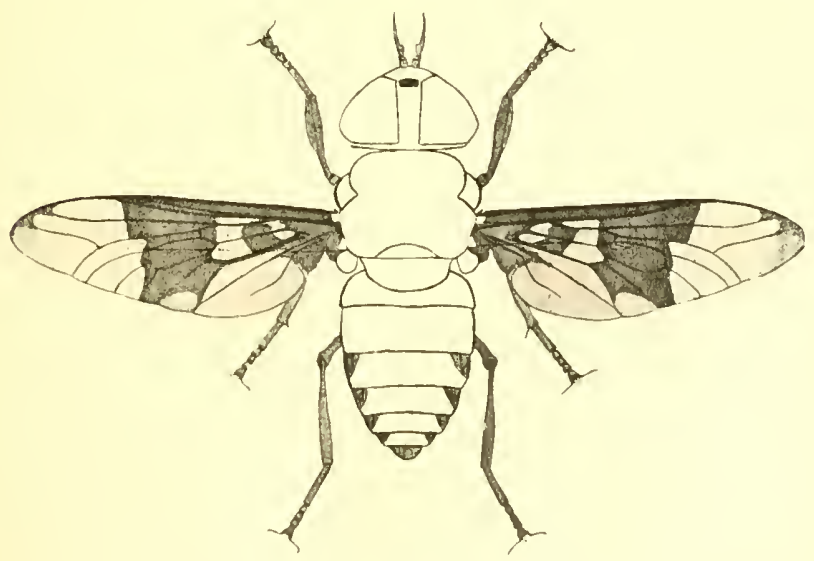

Vize. 7. - Tulames lalipes o Wicor arT 
Tibias antérieurs noirs à poils noirs, les autres libias jaunitres.

T. fasciatus Fab.

3 Tibias antérieur's brun sombro à poils dorés. les

/ autres tibias jaunitres. . . . . . . . T. niloticus -ul. sp. Aust.

Toutes les pattes noires en entier. . . . . T. atripes Van der Wulp.

Tabanus fasciatus Faвpicius ( $\mathrm{pl}$. I, fig. 4 )

Le type $q$ a élé décrit en $177 \%$, de sierta-Leone (collection Drury par Vabricius. Le type ơ a té décrit par Wiedemann en 177t, sur un exemplaire provenant de sierra-Leone. Longuenr 13-16 1 mm.

Tète et lhorax jaune doere en dessus, chamois ou janne cuir en dessous ; bande frontale ocreuse, abdomen jaune chamois rembruni vers l'apex. Ailes claires avec la base brun-sépia ainsi que le bord costal justu'iu sligma et une bande transverse.

Tẻte revêtue en dessous de jaune doré, rallosité frontali: un peu plus claire Ique le front, arronlie ì sil pittie supérieure et prolongée par une ligne étroite sillonoér dans sa partie inférieure et śétendant jusqu'aux deux tiers de la bande frontale. Cette partie linéatre est revêtue de la pubescence jaune ocracé du fond. Intennes brunes, premier et deuxième articles revètus d'une pubescence jaune, dent du troisième article peu saillante. l'alpes assez minces, d'un jaune très pàle, revètus d'une courte et rare pubescence jaune doré fåle, quelques courts poils noirs çà et Jà, plus nombrenx vers l'apex. Yeux glabres à cornéules égales, d'un be'au vert, sins bandes ni taches.

Thorax recouvert en dessus de quelifues courts poils noirs, llancs à plus longue pubescence jaune.

Abdomen rerouvert en dessus de courtes soies lorées, le horl postérieur du troisieme segment et le disque des segments suivantw portent de courts poits noirs augmentiant de nombre vers litpex: dessous jaune it pubescence concolore, hrinches jaune verditre à pubescener jaune, fémurs antérieurs à pubescence noire, fémurs métlians et postérieurs jaune verdàtre à pubescence concolore; tibias antêrieurs très épraissement dilatés, arojués, noirs ì pubescence noire; tibias médians et postérieurs hrun rougeatre ì pabescence noire, ces derniers portent une frange mince, plus fongue et plus dense au hord extérieur. Tarses renflés, brun rougeatre très sombre à jubescence noire, métatarses anterieurs arqués it renllés.

Ailes it bande transverse de 2 m. 12 de largeur entiron, de couleur un peu plus sombre que le bord eostal, cette bande s'efface ver's le bord postérieur de la cinçuiène cellule postírieure; rellule discödale plus ou moins éclaircie au centre Cuillerons brunatres. Balanciers à massue jaune verdàtre et tige jaunâtre.

Les divers spécimens de la collection du nuséum proviennent de liotonou (Dahomey), bords du Rio-Forealo, Onitcha (mission Lexfaxt), N'Goko-Sangha, région d'Onesso (1)r (inavot, 1906), Léopoldville, Congo entre Bomba et Bulobo Dr Bnumpt), Kodok-Nil-Blanc (1)r Balfoun). Un exemplaire de Nil (Sénégral) pris par le $\mathrm{J}^{r}$ Tmroux se montre tris voisin du $T$. niloticus . Iusten. 


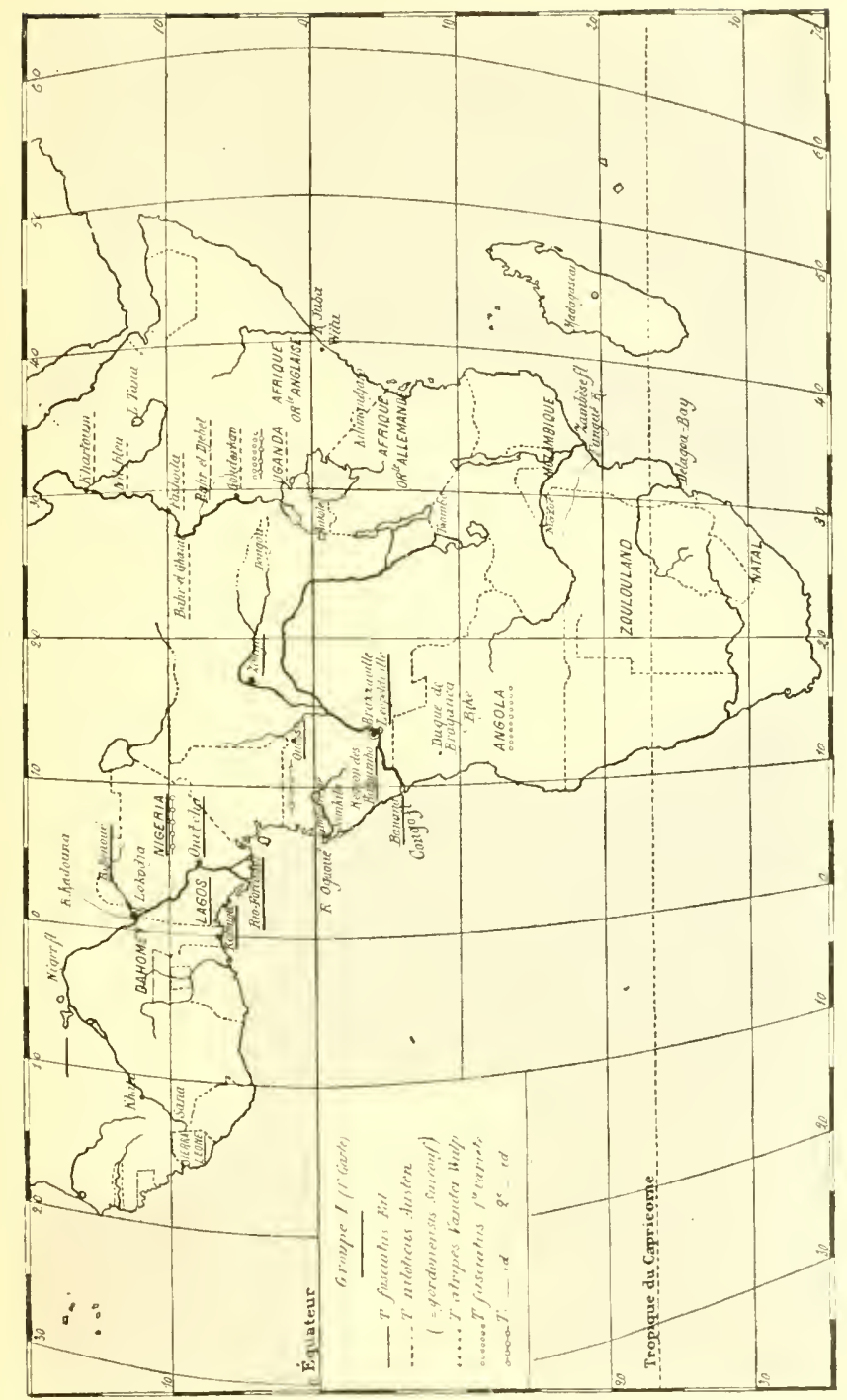


Le British Inseum posside plusipurs formes de $T$. fascintus, forme typique, exemplaires provenant de Sierlib-Leon', Latgos, Leopollwille, Wathen (Xorl die Nigeria), un spécimen de cette derniç rrógion a les fémurs postérieurs chars;

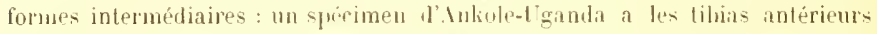
clairs à lat hase mais le's franges les libiak postrieurs sont principalement conposées de poils noirs. The autre forme intrimáliairo est roprésentée par un spécimen du Congo, un de l'l tandit deux du Yorl- Nigerial, ces quatre insectes ont des poils dorés málangés it la puhesence noire sur la moitié basilaire des tibias postérieurs.

Le musílum de Bruxelles posinde plusimus spécimens of provenant de ZongoYokuanghay (Lt TukeNs), Bananitbuma II. T'scholtex 1891, Congo (liasmaert), borils ilu Benoué (Bondo).

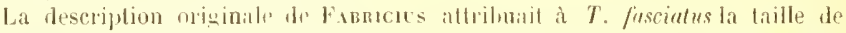
T. bovimas, il semble qu'il y ait eu une erreur de transeription ef ifu'il s'agisse vaisemblablement de $T$. bromius.

\section{BHBLIOGRAPHIE}

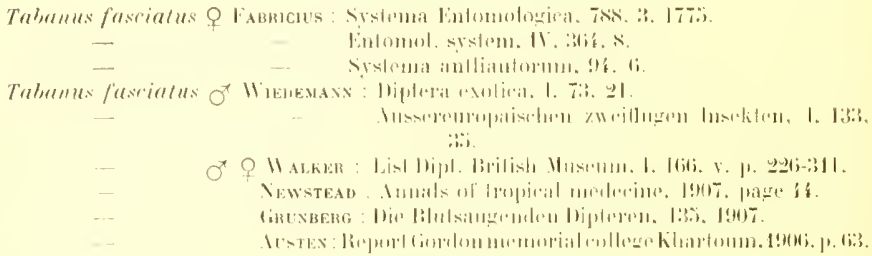

\section{Tabanus niloticus sub. species f Justes}

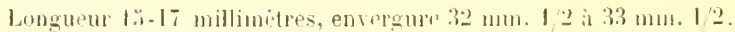

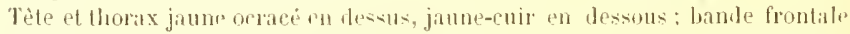

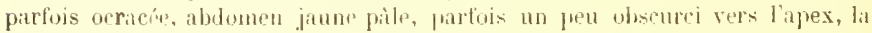
moitié basilaire habitueltement un pen verditre parfois mettement verte. Ailes hyalines, à bord costal brou et portant une hande tranvere lorun sépia.

Celte des ription sommaire ne permet pas de distinguer lo Tubrmus niloticus of Au-ten dn Tubunus fasciutus Q Fabricius matis lat desoription détallée montre des

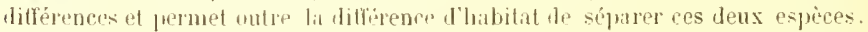

Tète revetue en desous diune jubrocence janne doré, callosité frontale de la coulem du fond comrant la molié de lit lirgeur du front. Intennes jannes

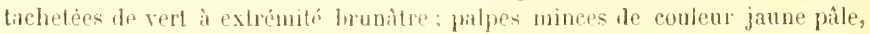
recourerts de poils jume d'or prartois aree yuelques pretits joils noirs ver's l'extrémité.

Thorax recourert en dessus de courts poils noirs répambus éfrarsement sur le fond jaune, llancs à pubescence jaunitre pille plus longue.

Abdomen jaune revètu en dessus de menus poils dorés. lersle bord postérieur 


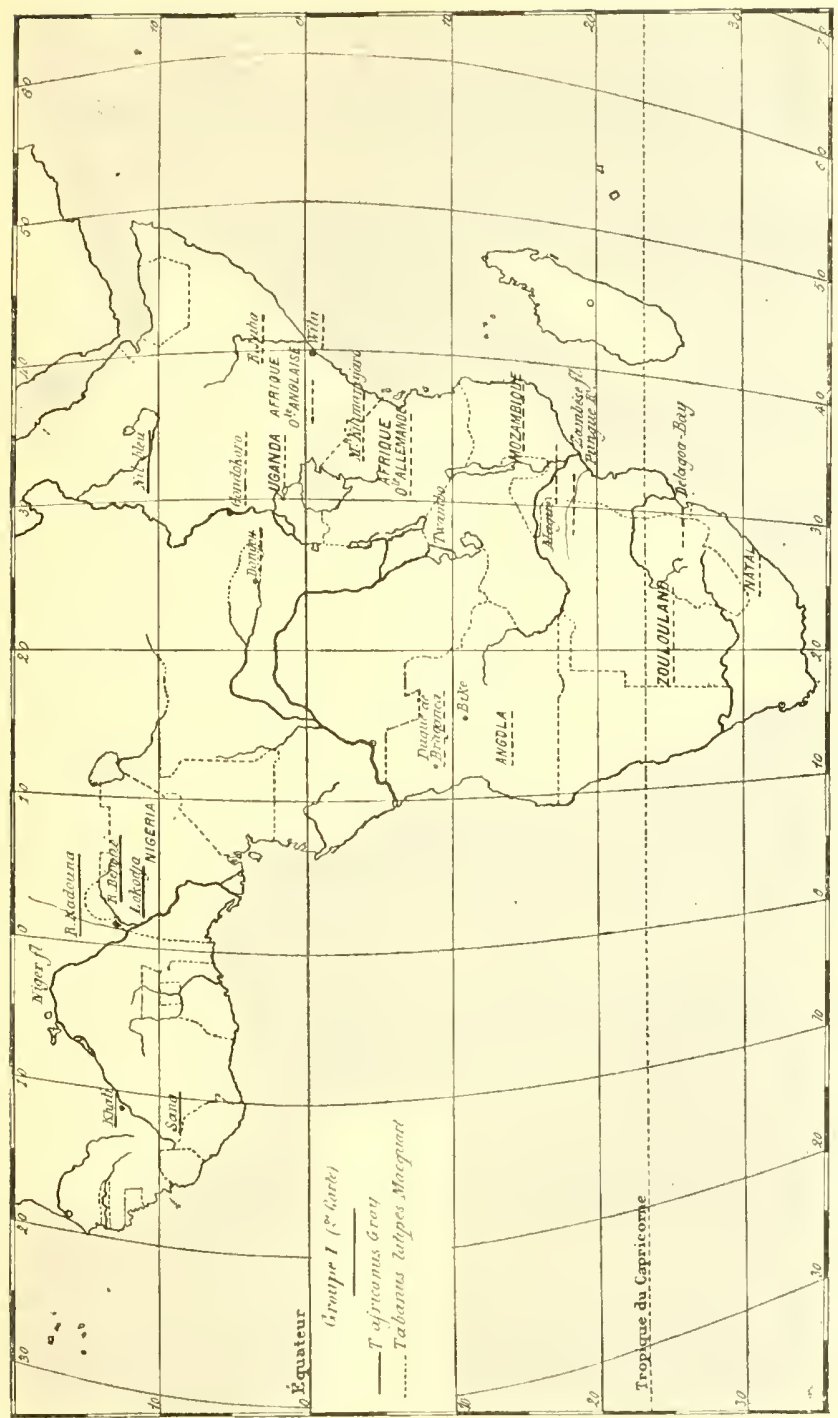


du troisième segment ef sur le milieu de ehacun des segmenls suivants il y a quelques petits poils noirs. Fémmrs jaunes on jaune vertitre, libias antérieurs brun soubre, renftés, à jubesrence noire sauf ì lit base qui est jaunitre en dessus el porte sur une zone trís réduite une pubescence dorée, libias mésians ef gostirieurs junes ou janne verdatm is pubescence concolore, les demiers ont me frange de poils dorés an borl extrrne: leur bord introre est recouvert d'une pubescence courte, jaunatre à la base, noire sur lit moitié apicale.

Tarses antirieurs noirs, tarses intermédiaires el posterieurs d'un lrun rougeâtre plus clair ì la base.

liles lyzilines portant une bande transserse plus sumbre que le bord costal, d'une largeur égale à la longueur di la cellule discoïdale et qui disparaît au bord de fil cinquième rellule postérieure sansalteindre le lord postérieur de l'aile. Cellule discoüdale portant un trail longitudinal, pale, plus ou moins visible. Cuillerons assombris it marge parlois verte. Billuciers in tige jiune et massue vert pâle.

Le lype d"Tubanus niloticus Q est an british Museum, il a eté lécrit par 11. Austex sur un exemplaire recueilli thas le Soulan anglo-égyptien en 190: (br Babfova). Ce Tabanus semble commun sur le Nil Blanc et le Bahr-elfielıel. Il a été pris à Kodok, en décembre 1!000, jar le eapitaine I1. E. Harmes et au commencement de la mème année par le major R. II. Pentox el le colonel f. D. Ilunten ì Abu-Chok, enloe Gondokoro.et Taufikia (29 mai 190:5). Le major Penton l'a repris en nombre au bahr-el-filuazal en février. On l'a recueilli dons les parages du Nil Blanc, I Usanda el la Giambie.

Le Tabamus niloticus lusten dillère de la forme typinge su Tabanus fusciutus Fabricius, espèce commmo du Congo of le sierra-Lone par les caractères snivants :

10 Par la coloration el la pubescence des tibias antrienurs qui dans la forme lype sont intièrement noirs ef recouverts exclusivem de poils noirs;

2 Par la coloration des tibias interuédiaire et postérieurs qui sont jaunes ou jaune vallitre au lieu de noir an de brun rougeitre;

$3^{0}$ Par la frange du lork "xterne des libias postricurs yui est dorie an lien di noire.

En outre, les tibias antérieurs de Tulumes niloticus Insten paraissent un pen plus minees que ceux de Tabmus fascintus Fal., la combure extirieure parait moins accentuée, le métatare antérieur est moins ar qué el moins rentlés.

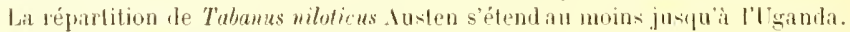
Le Musium possède dos séries frovenant du Jardin hotanique d’Entablse (capilaine (ineag, ts sept. 190\%); ile Bugaya, lar Vicloria, Ankole (colonel Bruce, août 1903). I'n exemplaire d'Inkole (16 mai 1903) apparticnt ì me forme intermédiaire entre $T$. niloticus et $T$. fascintus, ses tibias antérieurs sont pûles ì leur bise, les fringes des tibias poskérieurs sont composées de poits noirs sur lenr moitié distale. Ine forme de trancition se romontre aussi dans le Congo belge, cette forme ne présente pas de soies chorés sur la moitie basilaire des tibias antérieurs, mais il y a des soiss dorées milangérs aux solms noires of plus on moins prédominantes dan- les franges interne el externe du tibia postérieur dans sit moitié basilaire, un exemplitire te cette forme a été recueilli i Fajao, Uganda, par lo capitaine E. D. W. Gremg (novembre 190\%). 


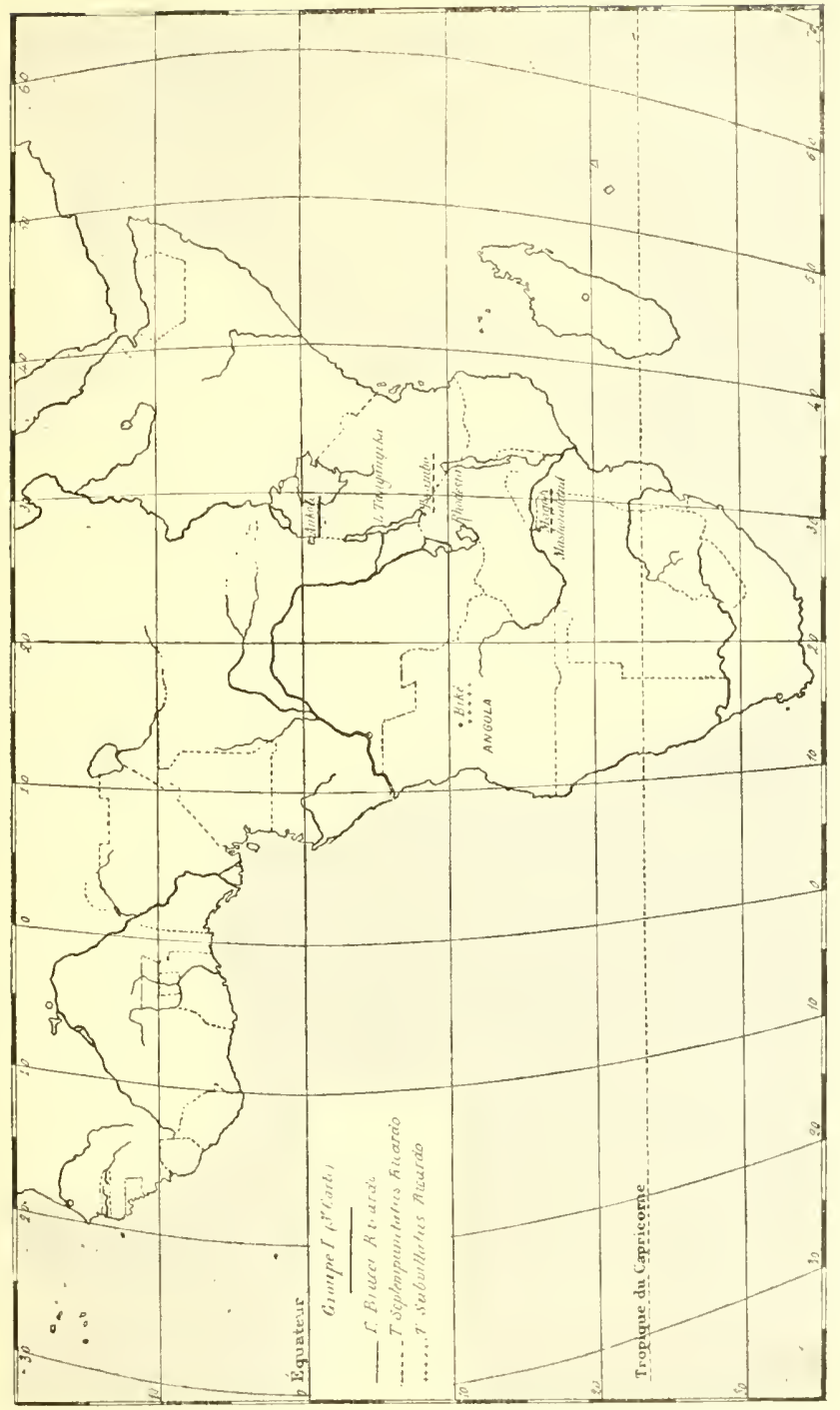




\section{BHBLAGHAMIIE}

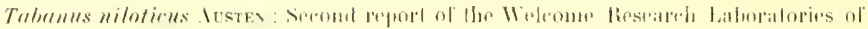

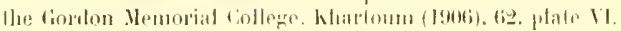

\section{Tabanus atripes ? lan DEB WuLP (pl. I, fig. I)}

Type: une femelle des horlis de l'Ogoune (Afrique urcidentale), oflirte an Mlusée de Leyde, par II. A. Faviel.

Yax ber Wup a donné de cette esjree la diagnose suivante: "Fulvus, ablu"mine testaceo, oculis nudis, intennis pilpisque ochraceis, rostro jedilus alin. "rumque hasi atris, tihiis anticis suldilatatis, alis cinereis, costa-fasciatue a meelia fuscis. Fem. long. 16 millimetres.

" Face, barbe et palpes ocracés, ces derniers peu élargis, pointus à l'extré" mité, front assez large de couleur jaune rougeâtre portant une callusité " petite, testacée et lrillante se prolongeant vers le vertex en mo ligne étroite. leux glabres, apres ramollissement ils deviennent vert smbre are un reflet "violet, mais sans bandes transverses. Les deux articles basilairesles anlennes

" sont jaune rougeâtre, le troisieme article manque, trompe noire: thorax et "scutellum jaune rougeitre, còtés ocracés.

"Ver's la base des ailes se montre une pilosité jaune pàle, courte, mais dense.

" MJdomen d"un testacé uniforme, le dessons plus elair, cendré par endroits "avec les segmentations jaune pitle.

"Jambes noires, tibias légèrement dilités, fonvexes sur le bord externe: " thihias postérieurs frangés des deux còtés de courtes sojes noires; balanciers " vert pâle; ailes grisâtres, Jeur extrème hase noire, le borl antérieur hrun " aussi loin que s'étend la nervure sulwostale et se réanissant ì son extrémité à " une large bande transverse qui passe il travers la cellute discoïdale ef lispri" raît légèremenl vers le bord postírieur".

Celte description de Van den Wubs est exicte et ne nécessite pas une redeseription, il y a à remarquerependant que la partie linéaire de da callosité fronlale s'ítend moins haut que dans les espices précédentes : les antennes n'ont pas la pubescence jaune et claire du Tahmos fasciatus et du Trabanus niloticus et el]es porlent quelques poils noirs sur les deux preniers articles. Les hanches intermédiaires et postérieures portent quelques poils noirs, franges internes et externes des cuisses postérieures d'un jaune doré, quelques poils jaunes sont mélangés aux poils noirs sur les ruissés intermédiaires et postérieures. Balaneiers verdàtres. Le Iluséum posside plusieurs fumelles frovenant de samkila, llaut et Joyen Ogooué, Lambaréné. N"foro sur Je Bas Ogouné.

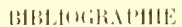

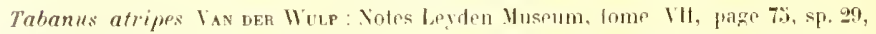
lat. 5i, fig. 1 . 


\section{Tabanus africanus likAl}

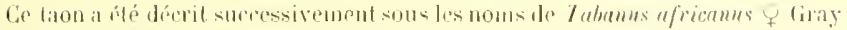

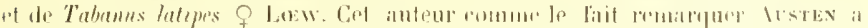

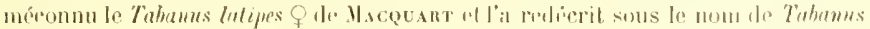

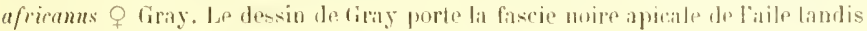

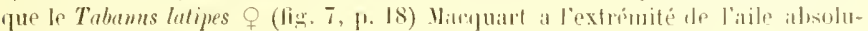
ment hyaline.

longue u $16-18$ millimbes. Envergure dos a iles $32 \cdot 3$ i millimitres.

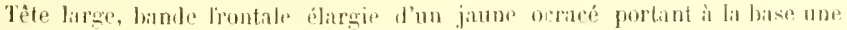

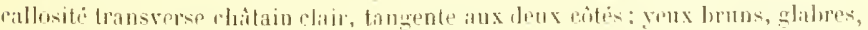

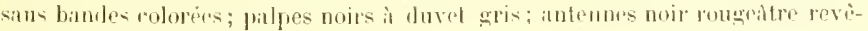

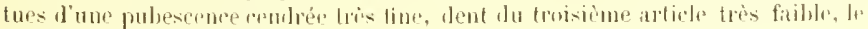
reste le l'artirle tris allongé.

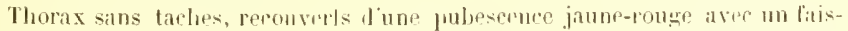
ceau de poils blanes situi it l'insertion dos alps pt une petite tache libuclue un

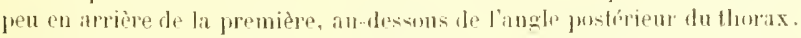

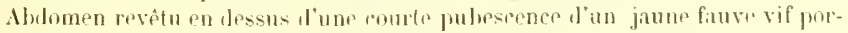

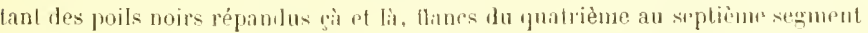

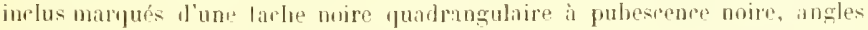

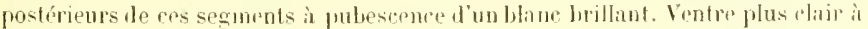
poils dorés lò̀s serrés portinnt une ticlue noire sur les rités du troisième. IgatIrième et cinquitume segments, les trois aloniers sermenls noirs an entier;

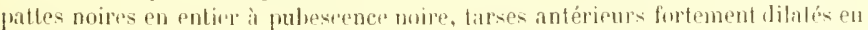
dessus, tilias postrieurs porlant deux rangies depils nuss formant des franges.

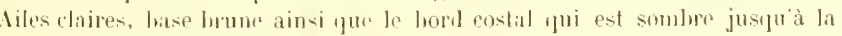

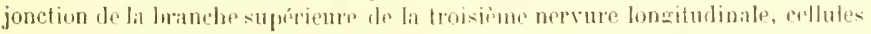

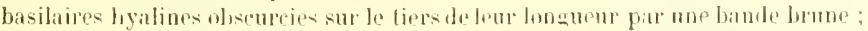

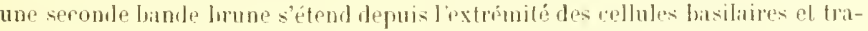

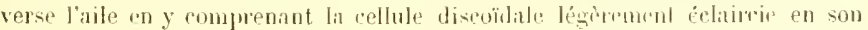

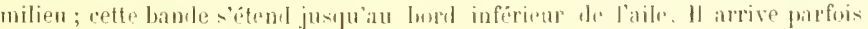

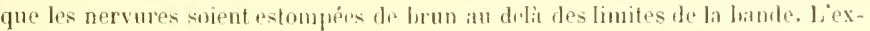

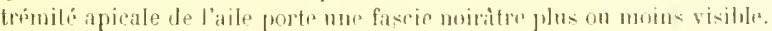

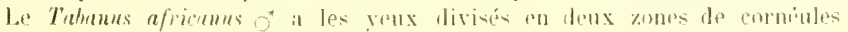

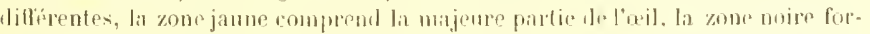

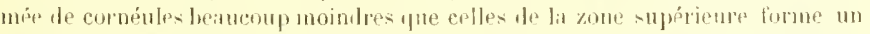

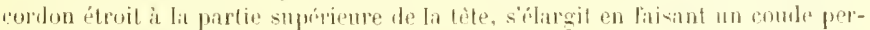

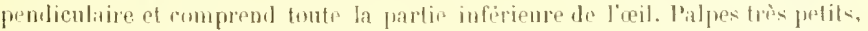

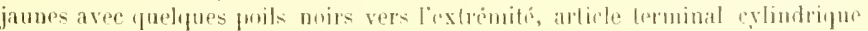
Itrmine en pointe, trompe lomine.

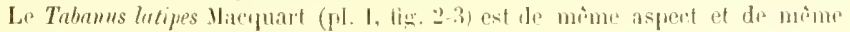
coloration que lo Tubanus a fricomes firay, il en iliflise:

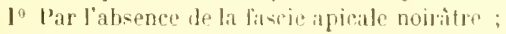

2o Par la Iongueur moindru de la Lande colorin transverso";

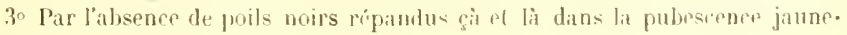
rouge des segments ablominaux. 
Laire de dispersion de ces insectes est très consilérible.

Le Tubemus africumus Giay se rencontre dans loute l'Ifrique meridionale. le Congo el l'Ugamla, il remonte jusifùà linndilioro.

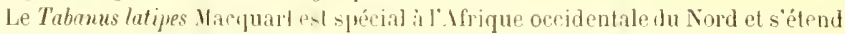
de sierra-leone jusqu"it winar el iu Nil bleu.

La variété femelle décile par Walker sums le nom de Talumus fenestratus o Walker, el prowenaul d'une localite inennnue dait ètre distincte de Tabanus a fricamus Gray el de Tubanus letipes Luew, mais le lype noxiste plus et la description est insuffisantr; il y a done lieu de considérer celle espice comme non avenue et te la rayer de la liste des 'lahanides d'Afrique.

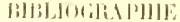

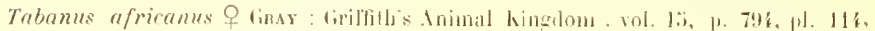

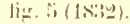

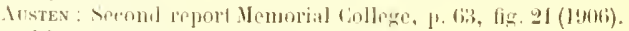

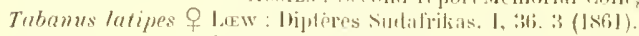

l'eters: livise natelt mozanubinue Zool, 1.2.

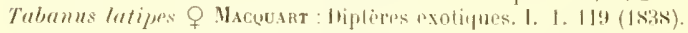

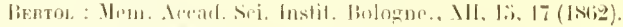

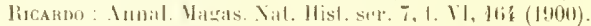

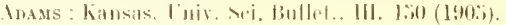

\section{Tabanus Brucei R Ricakdo}

Le type et quatre autres femelles proviennent d'Inkole, Uganda (16.5. 03. $\mathrm{L}^{\prime}$-col. Bruce) 1903. 206 et sont dans la rollection du British Juseum.

Grande espèce arec l'ablomen rougrâlre, ailes à binnles sombres, tibias antérieurs dilatés; elle est voisine de Tabunus septempunctutus Ricardo, mais s'en dislingue far l'absence de laches noires sur l'abdomen.

Longueur 20 à 21 millimitres.

Tẻle grante, toujours plus litrge gue le thorax, face et hande frontale rougeronille; fare, joucs el barlue à pulpescence rougedlres; palpes larges, rougeâtres. noirs il l'apex, plus longs que reux de Tahamas spptempunctatus 오 Ricardu. épitis à la base, aplatis à l'aulre exlremiti, à pulescence rouge : antennes noires porlanl une pubescence noire sur les deux premiers arlicles, premier épais, cylindrique, second très petil, troisième élargi à la base et pourvu d'une dent médiane mediocre; hande frontale environ fuatre fois plus longue que large, a bords pirallìtes, recouverte l'une court pubesinnce rougeâtre. Callosité frontale grande, transrerse, n'atteignant pas les yeux, surmontée d'une tache oblongue brune, non contiguë; yeux clilbres, monochromes.

Tlorax et scutellum hruns, recouverts d'me courte pubscence rougeatre, tris dense. Còtés et poilrine rougeàtres avec une pubescence rouge plus longue, squames noiràtres.

Ibdomen très élargi, courl, d'un rouge brillant uniforme à courte pubescence jatune rougetite, it l'extretue bord marginal des segments 3 , 4 ef 3 on distingue 
quelques poils noirs: ventre noir avee chaque segment fitroitement bordé de jaum ef revetu d'une rourte prubescence jame.

Pattes entierement noires; tibias anlarieurs dilatis, artqués; premirer article tarsal aussi fong que les quatre artirles suivints qui sont courts of ohlongs,

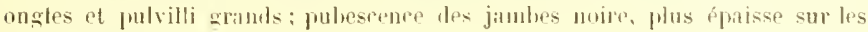
Pémurs postérieurs.

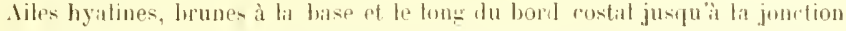

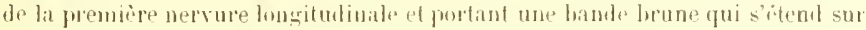
le disque de l'ale justju'ì la ringuième cellule marginale postérieure.

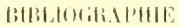

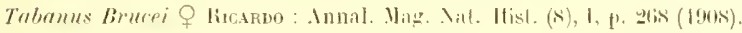

\section{Tabanus septempunctatus $q$ Ricardo}

Le type et trois autres femelles provienent de Fwambo, Nord-Est de la Rhodesia près de l'extrémitésul-Est du lac Tanganyika (W. II. Хutt, 96-83).

Une autre femelle vient de Haze, Mishonaland (G. A. K. Marsiald, déembre 1898). Tous ces insectes appartiennent ì la collection du British Museum.

Cette espèce est roisine de Tubanus africanus Graty par les bandes des aites, mais elle s'en distingue aisément par lis marques noires de l'abdomen. Cirande et belle espice d'un jaune rouneatre, pattes at antennes noires, ablomen portant sept taches ef trois bandes noires.

Longueur 20 millimitres.

Tite grande. aumi large gue te thoritx, tace of bande frontale rouge-rouille avec une longue pubescencr jaune rougutre, pulusersee do la face et des joues de mème coloration; pialpes épais, un preu phos jaunes, terminés par une pointe

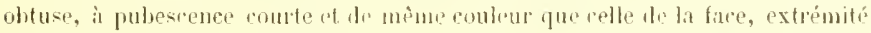
des palpes noine in pulresonce noire: antenms entiprement noires à premier

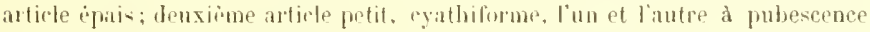
noire; troisiom anticle allonge it dent whtuse: bande froutale large et coute environ trois fois et remie alus longue que large it ba base. portant une eallosite

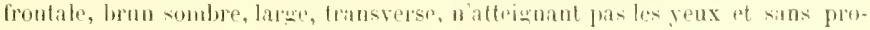

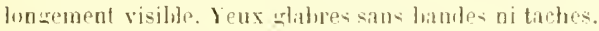

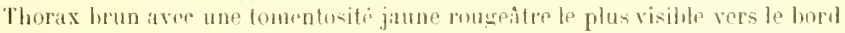

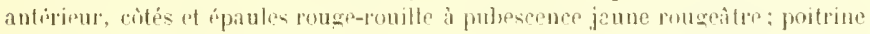
plua brune. Sentellum de meme coluration due to thorix portant des trafes l'une tomentosite dre endeur brillante.

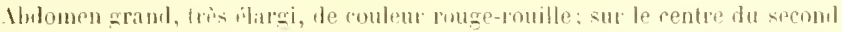

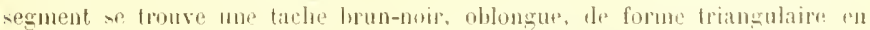

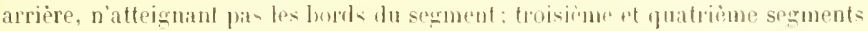

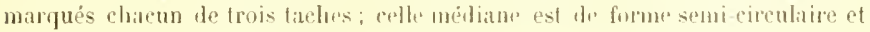
atteinl par sa base fe loril postérieur du segnent, clle s'étend sur la moiliós de sit 
largeur ; les tiches latérales sont plus ohlongues, elles rejoignent le bord antérieur des segments. Cinjuime et sixiom segments ornés au bord antérieur d'une hande noire qui s'tend un pen atu delit de lit mojtié du segment. Septime anneau noir en entier, la pubeseence de tous res segments ent d'un jaune rougeitre et assez chirsemé. Dessous jaunitre itvec du larges bandes noires sur lia moitié antérieure de ebaque segment; i partir du denxieme les borils posté. rieurs de ehacun d'eux sont pecourerts l'une courte pubescence jaune. Fënurs

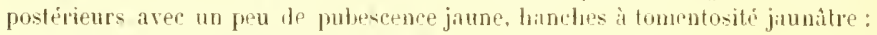
tibias antérieurs largeses, dilitris, itryuén.

Prenier inticle tarsil aussi long que les quatre autres qui sont ilargis of courts. Ongles et pulvilli longs.

Ailes hyalines bran sombre ì l'extrème base et an bord contal jusijüi la réunion de la furemière nervure lonsitudinale, portint en outre une large bande

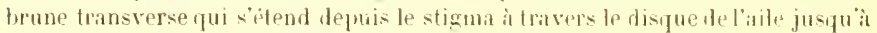
la cinquième cellule marquale pustérieure; nervures brunes, sauf la première longitudinale qui est épaisse et noire.

Le spérimen du Mashonatand a la pubescence de la face et de la bande frontale on pea plus jaune que le tyon el une rourte ligne noire frolonge la callosité frontate, le thorax ef le sfutellum prestue entièrement reeonverts d'une tomentosité jaune rongeitre, par alleurs il est identique aux exemplaires de ha Rhorlesia.

\section{IBH:LOGRUP'IIE}

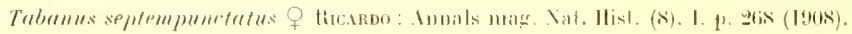

\section{Tabanus subvittatus ? Ricardo}

Le trpe et deux autres femilles provinnent de Biké-Ingola el ont èté recueillis

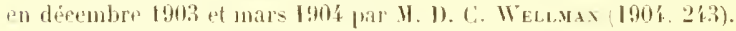

Ces trois exemplitir's apgrartiennent it lit rollection du British Ituseum.

Cette espèce est tròs roisine de Tabums septempunctatus Ric., miis elle en thifrive par les taches de l'ablummon.

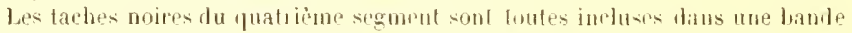

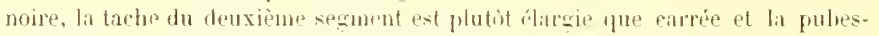
cenee des borls postírieurs des segments ventratux est al'un jaune flus pille. Lat pubescence jaune des fémur postérieurs pst plus visible, elle s'étend anx tibias postérieurs ehez l'un de ces insectes.

Les spécimens sout the tails mointre, 18 millimetres.

Deux d'entre eux portent la mention " rerueillis auture des troupeaux ".

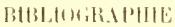

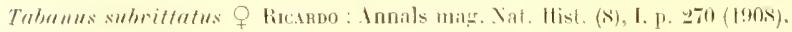




\section{DEUXIENE GROUPE}

\section{TABIEAL DJCHOTHIOE}

I

 segments étroitement marginés de jaune. lbdomen noir à taches médianes triangulaires blancluex. . . . . . . . . . Envers le l'abdomen étroitenent marginé de blane. liles rembrunies à l'apex, sans lrandes. Tibias noirs. . . . . . . Envers de lialdomen marginé de taches blanches. liles it bamle mirliane transverse th apex bruns. Tibias noirs. . . . . Envers de l'abdomen f́troitement marginé de biane, taches dorsales très léduiter. liles enfumées, plus claires à l'intririeur des cellules; tibias antérieurs blanes sur les 34 basilaires. . . . . . .
T. billingloni o Newstead.

\section{2}

T. yuadrigultutus of Ric.

T. marmorosus Q sureuf.

T. obscurefumatus S surcouf.

Ce groupe conprend des Tabaniles de moyenne taille, de conleurs claires et agréables, ils ne sont représentís que frar der spécimens peu nombreux dans les diverses cullections d'Europer.

\section{Tabanus obscurefumatus $Q$ sircour (pl. l, fig. : 氵 $)$}

Le type ast un exemplaire femelle pris par Gunat en 1883 sur les bords du San-Benitu (Congo).

beux autres lemelles de la meme provenince.

Eisèce noire de taille moyenne ì aile- hrumes; abdomen noir marginé du

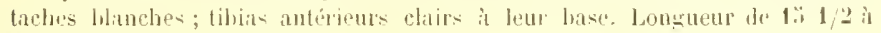
18 millimetres.

Tète plus laroge que bu thorax, ficce noire recouverte d'une tommlonité blam: d'arcent, pubescence et barbe blanches. Palpes llun noir sale aree un peu de lomentosité grise el une pubescence noire, éparse, allongés, mincer et pen dilatés 
¿̀ Jeur base. Intennes noires, le premier article cylindrique avec une tomentusité grise et dies poils noirs, second article tres petit it poils noirs, troisième à dent

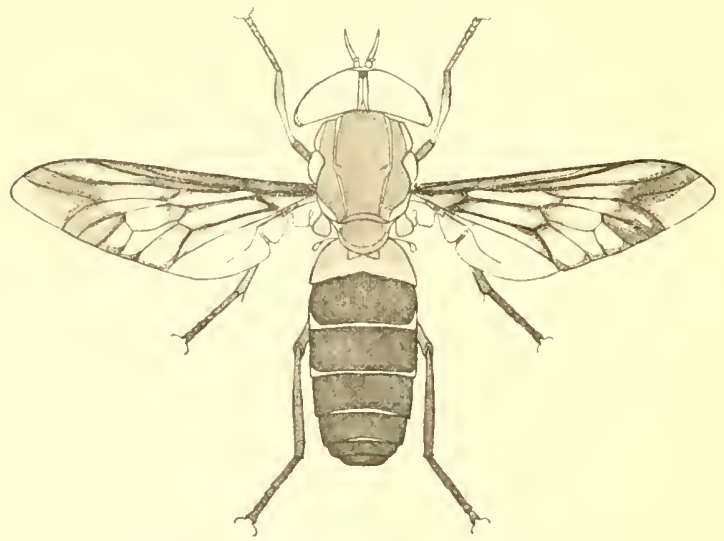

Fig. 8. - Talianus Billingloni O Newstean.

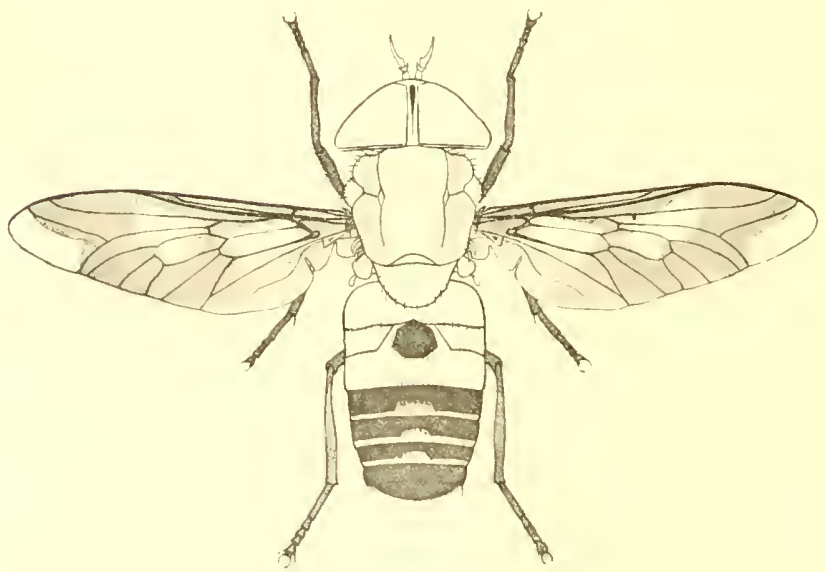

lig. 4. - Tubanus murmorosus $Q$ surcouf.

saillante. Bande frontale étroite, atténuée à la prartie supérieure, a moins six fois aussi longue que large. Callosité frontale allongée, de la largeur de la bande 
DEUXIOME GROLPE

31

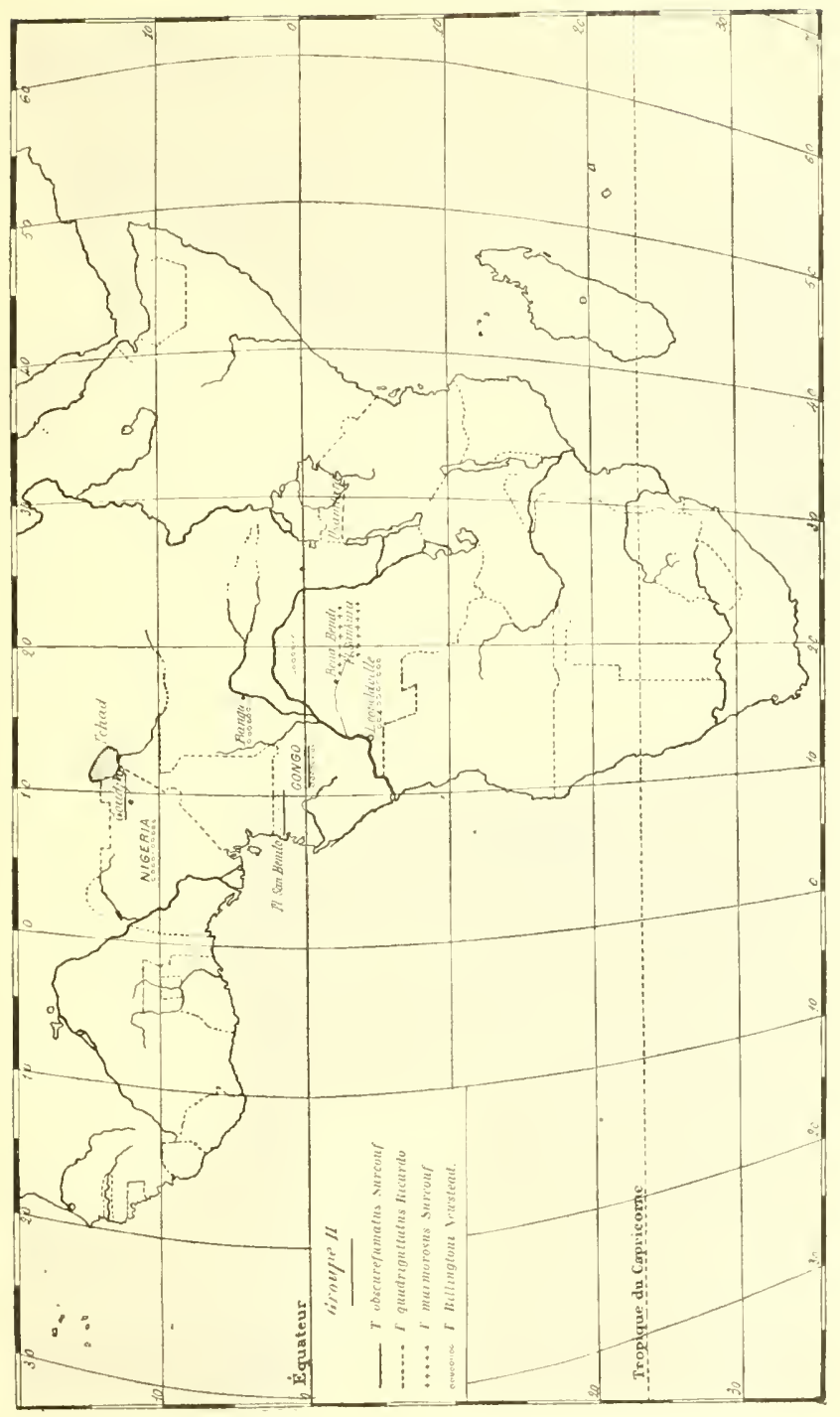


frontale tout entiète à la base, d'un brun lrillant; sp prolongeanl par une ligne fitroite, saillante de mime colorition jusiguan vertex, les parties atroites de lit bitnde frontale jui burdent wettr ligne mire élevée sont noiratres. leux monochromes.

Thorax brun noiratre prortant un peu de tomentosité grise et des traces de bandes longitudinales grises it la juirtin antírieure, flancs avec quelques courts poils noirs, et diantres flus hlines ef plus lonss 'n dessous. Peetus noir, reconvert d'une tomentosite et d'une pubscence grises. Srutellum de mème couleur que le thorax.

Ibtomen noirn̂tre, tris aplati : frmier segment ì tomentosité grise et de la pubesence blanche sur les rôtés; second segment portant médianement une lache subtriangulaire de lomplosite binno et une bordure marginate postérieure de tomentosité, cette bordure itroito s'etarmit sur les cótes et se continue sur tes tlaties en une pointe irrégulière. On distingue des poils blanes sur la tach" médiane "t sur fes cotés : truisiome, quatriome et cinquieme segments semblables au seeond segment mais les marques blanches diminuent de taitle, les tachl's médianes s'amoindrissent jusqu it ne plus former qu'une étroite tache linéaire recourerte de poits blancs ; vixiène el septième segments entiorement noirs avec une pubescence noiratte sur les fotés, sur les flancs des autres segmente la puberence est blanche. lentre de la routeur du dessus. chatue segment postériprement ef étroitement marginá de blane. Ilanches brun rougeàtre

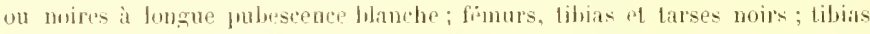
antérieurs arjuris, biancs aver une pubescence blanche sur les $4 / 3$, a partir de leur base, te cinguicm a pieal est noin a prabescence noire. Aites longues ef hrunes avec tes nervures brunes, cothufe discoüdile of eellule basitaire inférieure belaircies sur presigue toute leur surfiter. Cuiflerons sombres. Balinciers noiritres.

Le Iuxín de l'aris poscide ontre le tỵe de nombreux spécimens provenant du Cungo (Missiox Bel et Missiox Cottes).

Le British Husenm avait regu d\%hut, sud-Nigeria, un spécimen of reeneilli pitr. If. lluberox en juin 1906.

Le musie de Portugal a recu de Batetial sim Tomp (12, 1888) de nombreux exemplaires femelles.

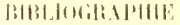

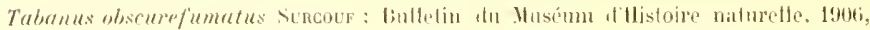

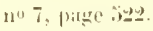

\section{Tabanus quadriguttatus of Ricardo}

Le type est an Brmtsu Museun : if provient d'Usambara, et a été recueilli sur les loords du rivage Sul-Est du liu Nianza aved quelques autres femelles

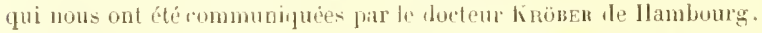

Lisper de taitte moyenne, noiritre; it ailes hyalines, lrunes au bord eostal et à l'apex, pattes, patpes et intennes noires, abdomen noir ì faches grises. 


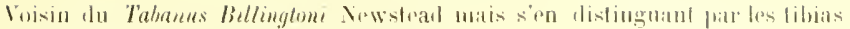
antérienrs noirs el les dessins de liabdomen.

longuenr I!) millimedres.

Tele plus large que le thorax; face rougeatre mas entierement rerouverde

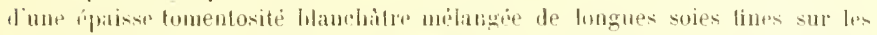

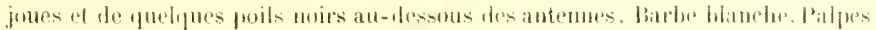

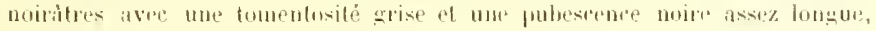

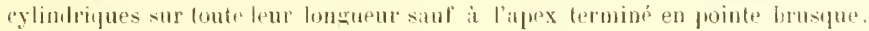

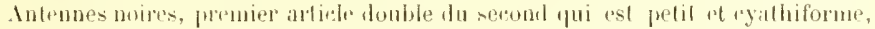

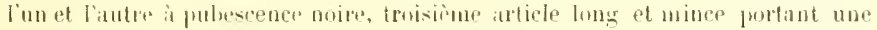

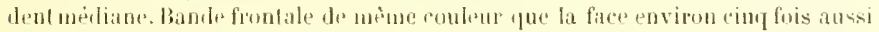

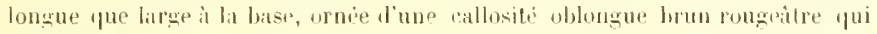

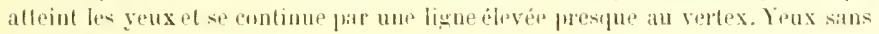
laturles.

Thorax rouge brunitre cunvert de lomentosité grise, sans bandes longitulinales distinites et portant des frikes de pubescence dorée sur les coilés, alu-des-

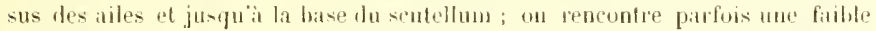

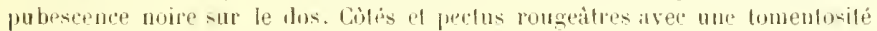
urise, des soies noires dans la partie dortide el des soies grises ver's la régrion ventrale. scutellum semblable au thorax.

Abdomen assez long, d'un noir profoul, desuxième segmen: partiellement if'un rougeatre sombre ave une lomenlosité grise, une larhe triangulaire il la base

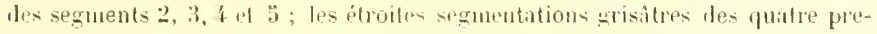

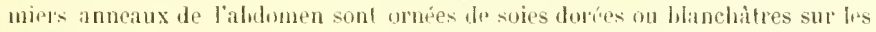
rotés, par ailleurs li pulesenne est noire ainsi yur sur le dessus on la couleur noire parait. Linvers noir ave des lordures etroites de pubesence blanchitre.

Pattes entierement moires; hanches it pubescence et tomentosite srives: fimurs posterieurs aved des régons de pubrecenerblanche; partout ailleurs la

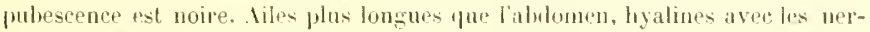
vures et le stigma brun-janne, leintées de hun lo long du bord cosial, at l'extrème hase et a l'apex tont enlier. Celle coloration lume est rependant filibie is la partie supérieure de la seconde rellule sous marginabe, un lait pale est visilhe au milien de la prenière rolluls marginale postérieure, lia burlure postéridure fost prarfois aussi teintée de brun ef laisoe des cellules discale, hasilaires el api-

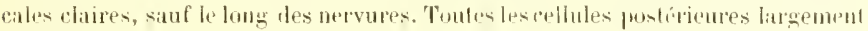
ourertes.

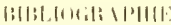

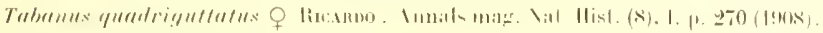

Tabanus marmorosus? Semory (pl. I, lig. $\bar{l}$ )

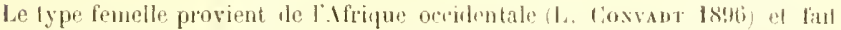

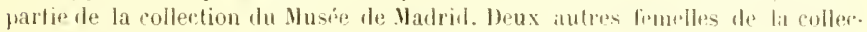
sirioul 
(im thu Vusée Royal d'llistoire aturelle lta Belgique proviennent des BeniBenuli, sankuru, Congo (i. Cloetexs 1.9.3) et Congo (Deleval) (fig. 9, p. 30).

Longueur 21 à 23 millimètres.

Cirande espèce a ailes presque hyalines très voisine du Tabanus quadriguttatus Ricardo.

Tète plus large que le thomx, fare rongeatre pîle entièrement recouverte d'une pulrescence jaunatre mélanúe de longs poils blancs sur les joues. Palpes assez longs, cylinilriłues, brusquement terminés, le couleur noiratre ì pubescence noire. dntennes noires il pubescence noire. Bande frontale environ cing fois auswi longue qu* large ì la base, de couleur brune, ornée d'une callosité allongée lrun rougeatre jui est tangente aux yeux dins sa partie la plus large el se continue par une ligne élevée jusqu'au vertex. Yeux sans landes.

Thorax rouge hrunitre à courte puhescence noire, firse, sans lignes distineles, ]ubencence des cotés mélangée de blanc, origine desiales porlant des touffes de poils blincs. srutellum rouvert dune pubsecence blanche.

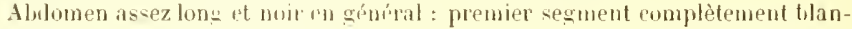

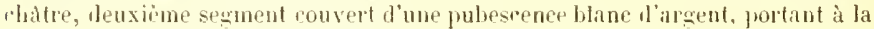
partie suprírieure un tarhe médiane triangulaire d'un hrun rougeàtre, les einy segments suivants sont noirs it jubescence noire; les troisième, quatrième et rinquieme fortent sur feur borl postérieur une tache médiane blanche atteiunant la moitie de la hauteur du segment. Flanes des premier, deuxiène ef yuatrime segments marquís le blan. Ventre noir, le bord postérieur de tous les srgunents largenent bordé de blanr, une bande longitudinale médiane noire parrourt tout labulomen 'n interrompant la bordure blanche et se compose de tarles semi-eirculiares ayaut leur hase au bord antérieur de chaque segment.

Jattes brun noitailre foncé, fómurs ì pulvescener blanche, tibias antérieurs banchitres sur leur flus gramle partie et ayant une pulsescence blanche, les autres tibias et les tarses ont une pulsescence foncée.

diles plus longues que l'iblomen, hyalines, lrunes à ta base et le long du bord costal, portant une petite band transverse hrune it hautrur de lextrémité apicaletes cellutes basilaires et une seeonde bande a l'extrémite apicale de la cellule discö̈dale, ajux chir. Balanciers bruns à tige sombre.

tBISLTHSHASPItE

Espine incidils.

\section{Tabanus Billingtoni ơ REwstead (pl. l, fig. ti)}

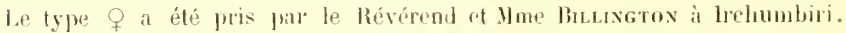
Le trye of a étí eapture par le Révérend 11 . Ave dans les environs de Matadi.

cont insecte anvait ité désigmé in littris jar Miss (i. Ricaroo sous le nom de Tahums splendidissimus qui avait ŕté employé dans une communication faite au

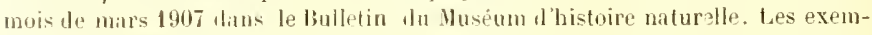
jhaires de Mis. fi lacaroo provenaient de Bolenqui, Congo supérieur (juillet 1903, 


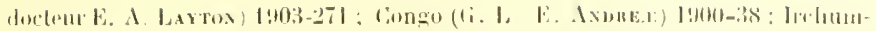

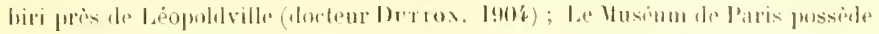

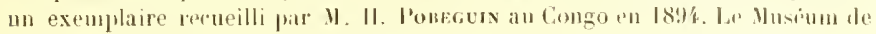

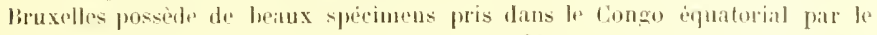

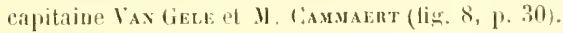

Cetlebejle espier noire et or. de haille moyenne ent voisine de Tabunus fuscinlus. Fabricius of do Tubaus africanus ciray, mais ba disposition de la comleur noire sur les ailes est dillérente, alle allecte plutot laspect de traits sombres yma dre

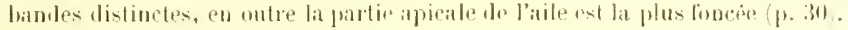

Longueur 18 à 20 millimètres.

Tète de taile moyenne plus large que le thorax, face ef jours grisàlres avee

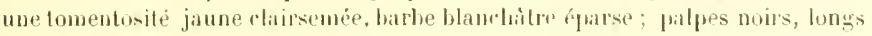

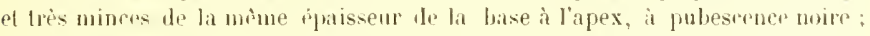
anteunes longues, noires, premier irticle double du second, l'un allaulre it

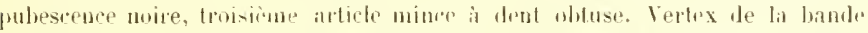
lirontale noir, recouvert d'une éparisce tomentosite rousst, bande frontale lonzunte

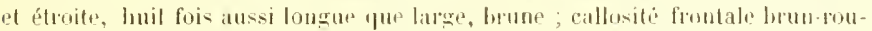

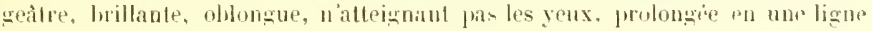
étroite presque jusquau vertex. Tomentositi de la hamle frontile rousse. Teux unicolores.

Thorax brun ave les traces de deux banles jaune rougeatre, rotis avec une pubescence fauve docér qui se continue plus pale autour do lia hase du thurax et du scutellum ; pertus grisitre protant une pulwesenes el une tomentosití jaunàters.

Abdomen noir. premier segment lituvedoré recouvert d'un tomentosité fauve dorée. Segments 2,3 et 4 itroitement bordis postérieurement le fauce ou de

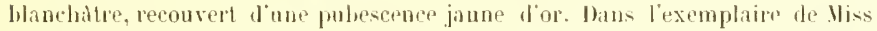
Ricardo cetle coloration est plus paile. Envers sembliblile, mais les bordures des anneaux sont plus larges

l'altes noires, minces ; tibias anterieurs pailes, noirs in l'apex et recouverts

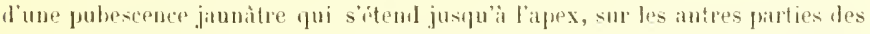
patles, la pubescence est noire sauf ì l'intérieur dres fémurs. Miles jaunitres,

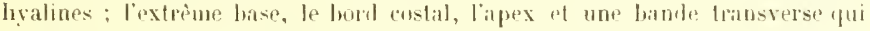

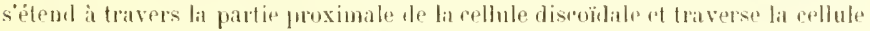
apricale, sont d'un brun sombre. La deuxione collule hasilaire, une fartie de la cellule apicale, le centre do cette dernispe collule, la fulus grande partie de la cellule discoüdale, la moitié basilario de la première collule postírieure ainsi que des cellules suhmarginale et marginale te mème que lextreme apex les l'aile. sont hyalines. Les ailes sont plu- longues yur l'illufomen.

La deseription du màle est donmée par. M. Newstead.

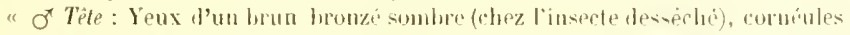
"ocreuses; espace compris mutre les yenx noir lom en dessons: clypens "et jours gris, ces lernieres portint une longur puberencesoyeuse, hanche:

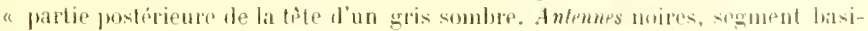

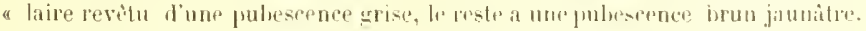
"Trompe et labium woirs. Thorar d'un ehitain somble en avant de la suture a frontale portant une ligne méliane etroite faiblement indingér at denx lignes 
"sulomédianes étroites, qui se terminent en arant romme deux dépression " noires: bords d'un brun grisâtre olsscur ; suture lirun-orange en face des " lignes submédianes el continuée en arrière comme par un court trait étroit; " partie postérieure lu thorax légìrement plus sombre que la partie antérieure; " lo tout est recourrl par une jollinosité grisîlre et une pulsescence noire peu "fournie: scutellum el pleure gris, mais ces derniers sont plus pâles « pue le "premier. Abdomen d'un noir unfume portant d'útroites liandes apieales grises " aux segments, ces bandes dliminuent graduellement vers l’ajes jusqu'à ce " qu'elles disparaissent completement sur le dernier auneau ; ventre de la mème " rouleur ef portant des landes eomme le dessus. Puttes noires: fémurs médians "d'un brun sombre de poix; les denx tiers sujúrieurs des tilias antérieurs

" hlanc sale, le tiers restant noir. Liles ì nervures brun sombre, toutes a vec une " large bande diffuse d'un brun orangé qui les entoure.

"Longueur 16 millinitres. longueur de l'aile 1 :i millinètres. "

\section{BUBLt)GRUPAtE}

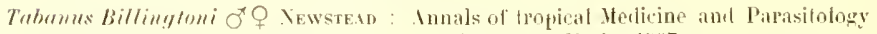
rol. I. ॥० 1. férrier 1907 . 


\section{TROISILME GROUPE}

\section{'T'ABLEAU DICHOTOMIOUE}

dirande esprese noire à pulveserner du thotax ol de: ha face jaune doré on jaune grisitare, parfois des taches médianes arrondies, blan hes, sur tes deuxième, troisième et juatrièmesegmentsabdominaux. Palpes noiràtres.

T. biquttatus Wierdemann. Palpes jaunes (Ifrique occidentale) . . . . . T, croceus Surcoul.

Espèce de taille mointle, fubrecence thu thotax et de la face błanche, palpes hanes (sinégal).

Espèce noire ì ailes noires (description insuftisante d'un type inconnu). . . . . . . . . \% comx Law.

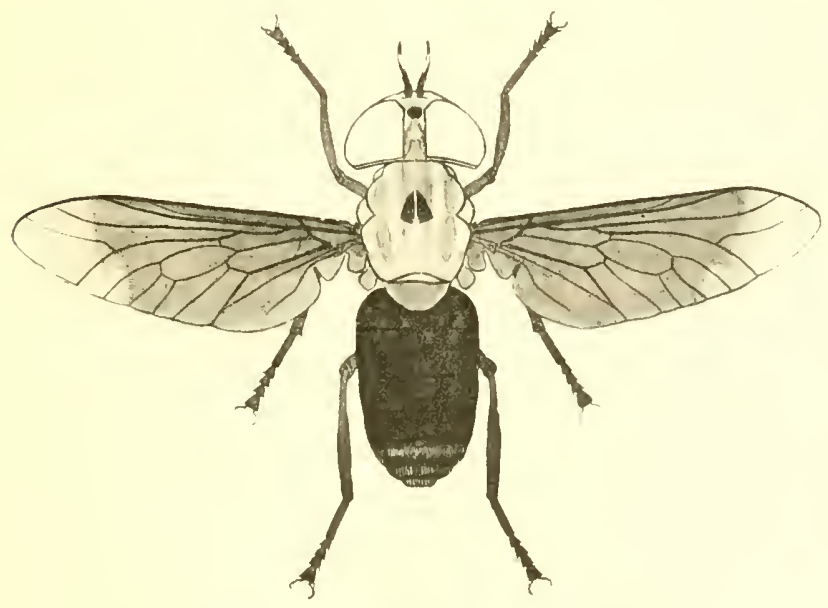

F'ig. 11. - Tabunus bigntlentus ? WiedeMaxx.

Ce groupe comporte une espèce ot ses nombreuses variṕtés, celles-ci parfois tris nettes et luealisées au point de vue géographique sont parfois tollement pen 


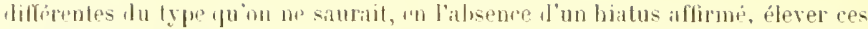

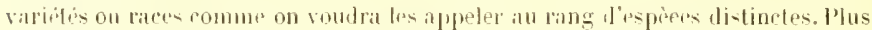

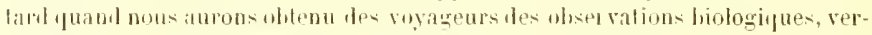
rons-nous appiatraitre des dillirences moselles pour to moment la morphologie de l'insecte sec ne nows permet pas de traneluer lat question.

\section{Tabanus biguttatus or WienEMaxv ( 1,37, fig. 111 ).}

Le type nexiste plus, il at étriecrit pur l'auteur dans les termes suivants :

"Tubumus bigultutus of $q$ noir, ailes concolores, apex cendré hyalin, tibia" "posterieurs filies.

" of : troisième el duatrime segments de lablomen à tache luanche.

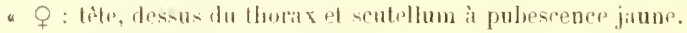

"Longurmr du corjes i 10 lignes, ailes à nervure transversale apicale simple mon appmalieulie. Hu Capp. "

Le rogage soientilipur ate V. DE Rotschu D en Ethiopie at procure au Huseum un rertain nombre d'exemplaires frmelles trè frais qui permuttent de compléter la descriplion de Wienemaxs.

Longueur 17 à 23 millimitres.

Soir it thorax couvert alune puhesence dorer, ailes noires ì axtrémite apicale exlairele.

Thite plus large que le tholiz. yeux à cornéules ingates. Bande frontale plus litrge au vertex qu'à la liase portant contre l'épistome une callosité brume, frans-

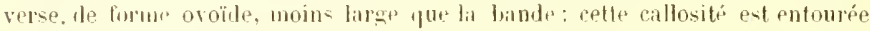

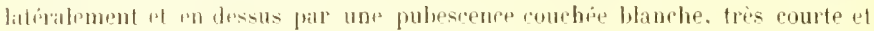
soyense qui, pxaminie sums un ertain angle, laisse paraitre la bande frontale brun sombre. Le vertex est couvert de phos longs poils noiratres avangant en forme de I sur la bande frontale. il y a cu oulre deux monchehres noiritres obliquement -itúes un pera au-dessus du milieu de la bande. Lpistom han-

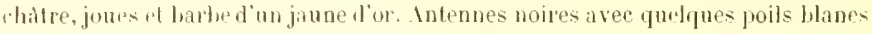

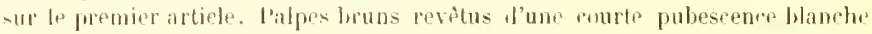
itmatogue comme aspert el comme rellets ì celle de la bande frontale.

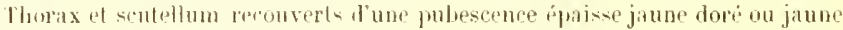

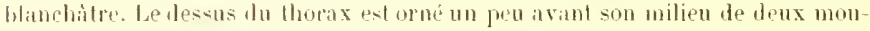
chelures noires, parallidement dispostes et trè voisines. Flanes à poils mar

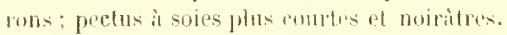

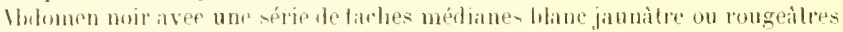

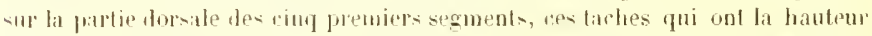
du segment sont tris tugares mais presistent fréfuemment sur les froisime ef

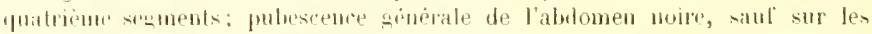
linches oi elle ast jaune ain-i que sur te bond posterieur des ringuime, sixime of septien segments. l'atles noims en entier il pelotes lauves. Cuisses anté-

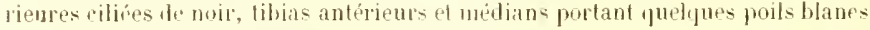
sur la moitié hasilaire, euisses posterieures ciliées axtérieurement de jaunitre. cinillerons sombles ciljo de noir. 


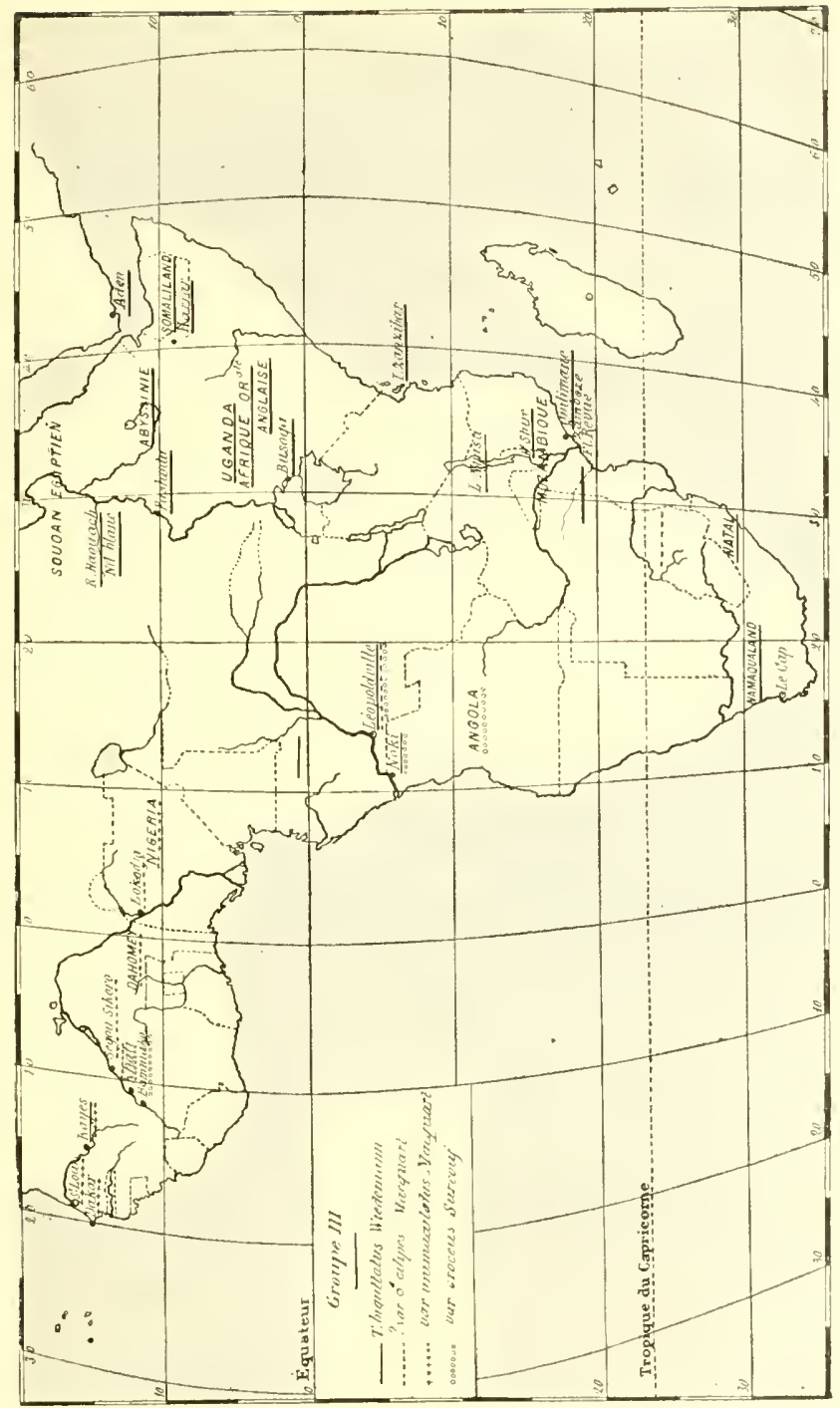


dilen brunes éclaircies à l'extrémité a picale. Balanciers sombres.

L. hahitat extrèmement itendu du Tubamus bigultutus Wiedemann a amence cetidn- auteurs à dicrire sous des noms dilipents plusieurs formes recueillies en

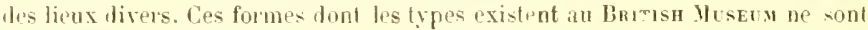
pas séprarables du Tubamus bigultatus. Wied.

lillos ont iti nommées:

\section{Tabauns cerberus O WALAER: \\ Tabaurs moctis of WALEER; \\ Tabanus tripunctifer O WALkER.}

Vous donnons, it titre d'indication et pour établir lo lien, la description origiMitlr de ces insectes:

\section{Tabanus cerberus Walki:k}

Noir; tète et thorax ornis de poils d'un fiuve toré; antennen et jambes " noires: ailes noir cendré.

"Corp noir, large: tite revitue ell desusus of plus épaisement en dessous de "soips dores. Seux, buthe et antennes noirs, thorax couleur de poix, revitu "de soins couleur tan, poitrin recouverte de prils noirs. dldomen brillant. R lamulwe neires, fulsesentes. diles d'un noir grisitre, plus pilles vars l'apex.

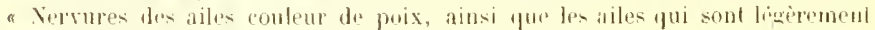
"Polonpres de la mine conleur, halabrins noirs.

"Longurur du corps 8 lignes, des ailes 20 lignes."

Ifrigur moridionale, de la mollection de .I. Artitxo.

\section{Tabanus noctis of Walker}

Corps noir sombre, revouvert de courts poils noirs. Partie de lit tite teintie " de lirun, recouverte de poils noirs courts. Yeuz trés grands, divisés en denx "aires distincles, partie posterieure noire comprenat loute la partie discale à

"facettes très grandes, mais déroissant de taille vers le bord inférieur, partie " antériesre enivreuse heaucoup plus grande qu" celle du Tabauns utratus, sépa" réa de la première par une lighe droite compusce le très petites facettes. "Tromje, jalpes et antennes noirs, angle du troisième article antennaire très pen saillant Jambes noires revetues de courtes soies noires à pelotes noir

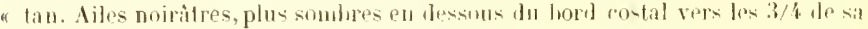

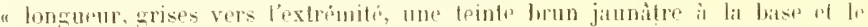

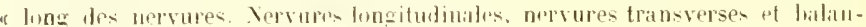

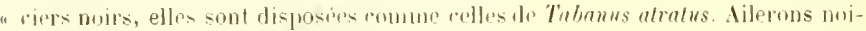
" ritris, franges de courtes soies tan soublue.

" longueur du corps: 8 lignes, des ailes is ligne. " (iap de Runne fi-perance. 


\section{Tabanus tripunctifer $q$ Walken}

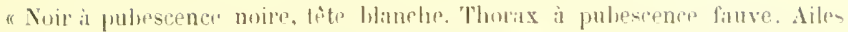

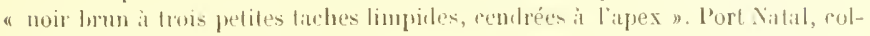
lection Quexzius,

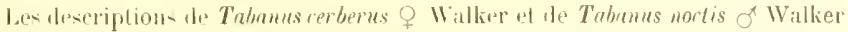

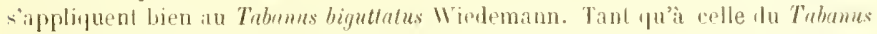
tripunctifer. Walker, elle se rapporle suit à un individu aberrant, soil it un insertu non africain.

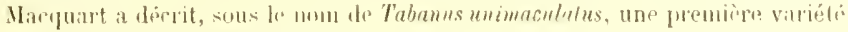
lu Tubunus beiguttatus Wiedemann.

\section{Tabanus biguttatus sub. sp. unimaculatus Macivan'T}

"Longueur : 8 lignes 1/2, an lirun nuiritre, liare et front brunatres, anten" nes noires. Thorax à lignes ham hiatres. 3" et qe segments de l'ablumen, "chacun a tache dorsale prergue contiguï. Piets noirs, ailes noiritues, extré" mili claire chez le mitle. "sénígal.

Matcpuart a décril inglement un Tabmas colipes of, dont il donne la diagnowe. suivanle:

"Viger. Abdomine tortio quartopue spomenlis macula alha. Tilois postiris " riliatis, .lis nigricantibus, apicen grisea ".

"Longueur: 9 l. of.

"Wun noir de poix. Face el partir antéreure du front brunitrm, it poils noirs.

"Antennes noires, menues, il pointe allongre el denl fort petite. Yeux tris "grands; parlie supérieure à zrandes farcettos, l'inférieure à farettes tric petiter.

"Thorax antérieurement d'un lrun noiritre. Irdomen f'un noir lui*ant; troi"siome segurent ì pelite tache allongée d'un blanc jounitre. formép de prits:

" quatriẻme à lache plus grande, rhumboidale, do la mème conteur."

Du Cay, Veseum, collertion de M. Servileze.

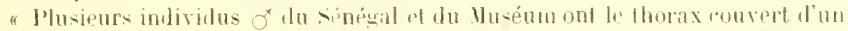
"duvet blanehatre aver des pwils noirs "t qualle lignes banchitres pum dis-

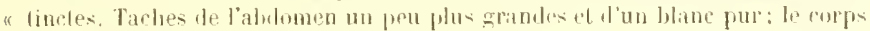
" et les atiles sont d'un noir moins loncé.

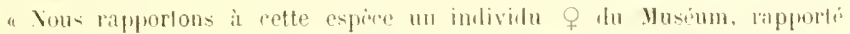

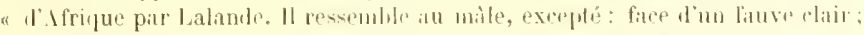

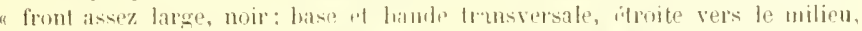

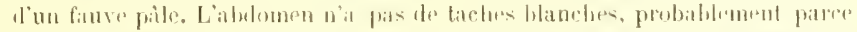

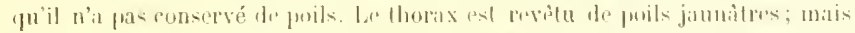

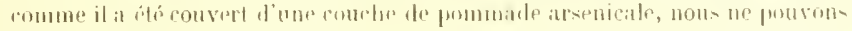

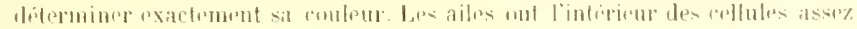
a clair.

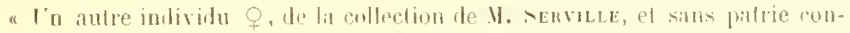

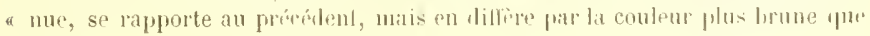


" noire thu corps et des ailes. Les palpes sont blanchaitres. la face et le front "sont d'un gris jannitre; ce dernier it une tache triangulare et le vertex bruns.

"L" thorax est d un brun rougeâtre.

"Celte espece a quelques rapports aver $T$. trimaculutus de Palissot B., qui "est de l'Amérique."

La collection du Iuséum posaide deux sprecimens l'un måle et l'autre femelle itipuetes Tabunus cilipes de la main de thropuart.

Femelle: Thowax blanchatre, une tache le puhescence fauvesur la partie inférieure de la bande frontale, joues et larbe l'un jaune foncé, palpes bruns a puhesience sombre. Antennes brunes, le troisieme article à dent saillante formant un crochet avec l'extrémité antennaire.

Hile: Thorax Hanchatre, abdomen brun avec une tache arrondie sur le deuxime et le troisime segments ablominaux, ces taches atteignont les deux hords de thaque segment, jones et barbe d'un brun chocolat, palpes bruns, petits ef rinflés a pubescence sombre. Les intenues manquent.

Ln résumé la forme ot a pubescence thomcipue brune, provenant du Cap,

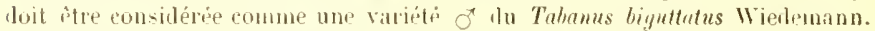
Les males tu sinégal i thorax bhom hitre doivent se rapporter a la sous esprice T. unimaculutus Macquart. Cefte variété unimuculatus the laille un peu moindre que Tabmus bigultutus Wiedemann, daspect phus faible, se distingue en outm lu type par la coloration blanche de la lace et lu thorax et les palpes blancs an lieu àitre noirs. Elle est localisée au cénégal. Je British museum possède un sperimen provenant de lllorin (Norl Nigiria).

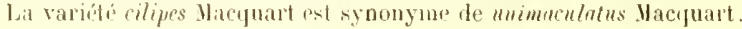

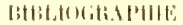

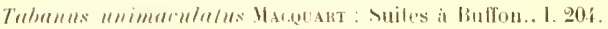

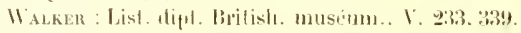

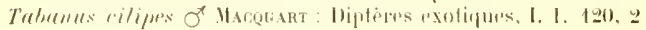

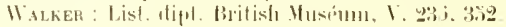

Tabanus biguttatus var. croceus suncolf (pl. I, fig. 8-!i)

Cette variété nouvelle provient de liati (soudan) et a été décrite sur de nonbreux expmplaires communipuén par lt. le professeur Laverax, Memlire de l'hnstifut la varinté crocens prévente la taille of l'aspect général du Tabamus var. unimaculutus Viacquart, elle s'en distingue par une pubescence jaune qui s’étem sur le thorax, le scutellum, la bande frontal". les joues, l'épistome et les palpes. Celte puhescence se rẻpand en outre fiparsenent sur le lord postérieur des deux segments abdominaux et sur le borl lithral des trois derniers. Celte variété a éti: frisis avec unimuculatus mais pfle en beaucoup plus fréfuente qu'elle dans le coudian et elle établit par sis forme et frar son labilal un parsage d'un granl intérèt entre le type $T$. biguttatus $W$. et sa variété fixée unimaculatus Hacquart.

si l'on reporte sur une carte a'Afrique ces différentes formes, on trouve ju'plles eunstituent des froupements bomorènes yui étahlissent ainsi que ces 
différences ollient une certaine constance et impliquent au moins l'exislence dr races giographipues.

liespère Iypique se rencontre le khartoum au Cap le long les flenves of sur le rivage orintal de l'Ifrigue, ou la relrouve au Congo. In exemplaire unipur a comme lieu d'origine hityes. sénégal, c’est vritismblathement une erreur.

La variéte unimaculatus śctend de Dakar au Tohal enlre le $10^{\circ} \mathrm{el} \mathrm{le} 17^{\circ}$ lat. N. et le $20^{\circ}$ long. 1 . et $111^{\circ}$ long. E.

La variété croceus a élé recueillie à Kali (Souhan); Bammako (I)r Boufraro); Luki Mayomba (1!. Exglenent, Collection du Vuse de Rruxelees!; Ingola (Collection lu Ilusée de Lısnoxse). Lukoja, livière Niger; Zungeriit, Nord Nigreria: (iambaga, Cóte de l’or (Colleclion du Batısu lluséun .

\section{HHBLAMARI'HIE}

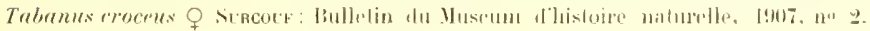
1. I i:S. 


\section{QUATRIEME GROUPE}

TAHLEAN DICHOTOMIQUE

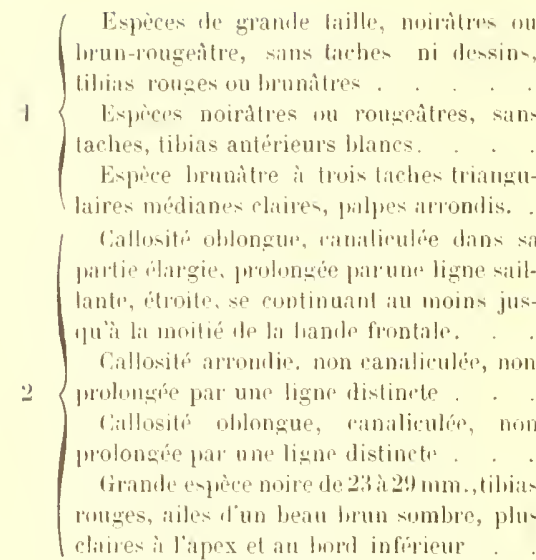

Ibdomen lnum-marron, sans fruinositr. 3

\{ (cotes

Tous Ifs tilobs moge clatir à pubsence Jimwe, ailes claires ì nerrures jaumes (19 à 4

Tilias antérieurs lrun rongeâtre tru noiintres.

diande espèce de 22 à $2 \%$ mon. liles lırnnitles à nervures lirunes ; palpes lom it juberence noire tris dense; fous les libias lnun rougestitre ou noiritres. . . .

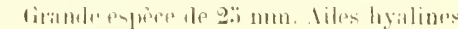
a nervures jaunes, palpes jaune rougratue it pubesience brune non serrée, tous les tibias brun rougêttre on noirattres.
T. enens o sureoul.

T. grandissimus of Rir.

T. fusco-marginatus o licic.

T. Brumpti o Surcouf. ;

T. ruficrus ? Pal. Beanv

T. delutius $q$ Surcouf. 
MIITRIEM: CROUH:

i:

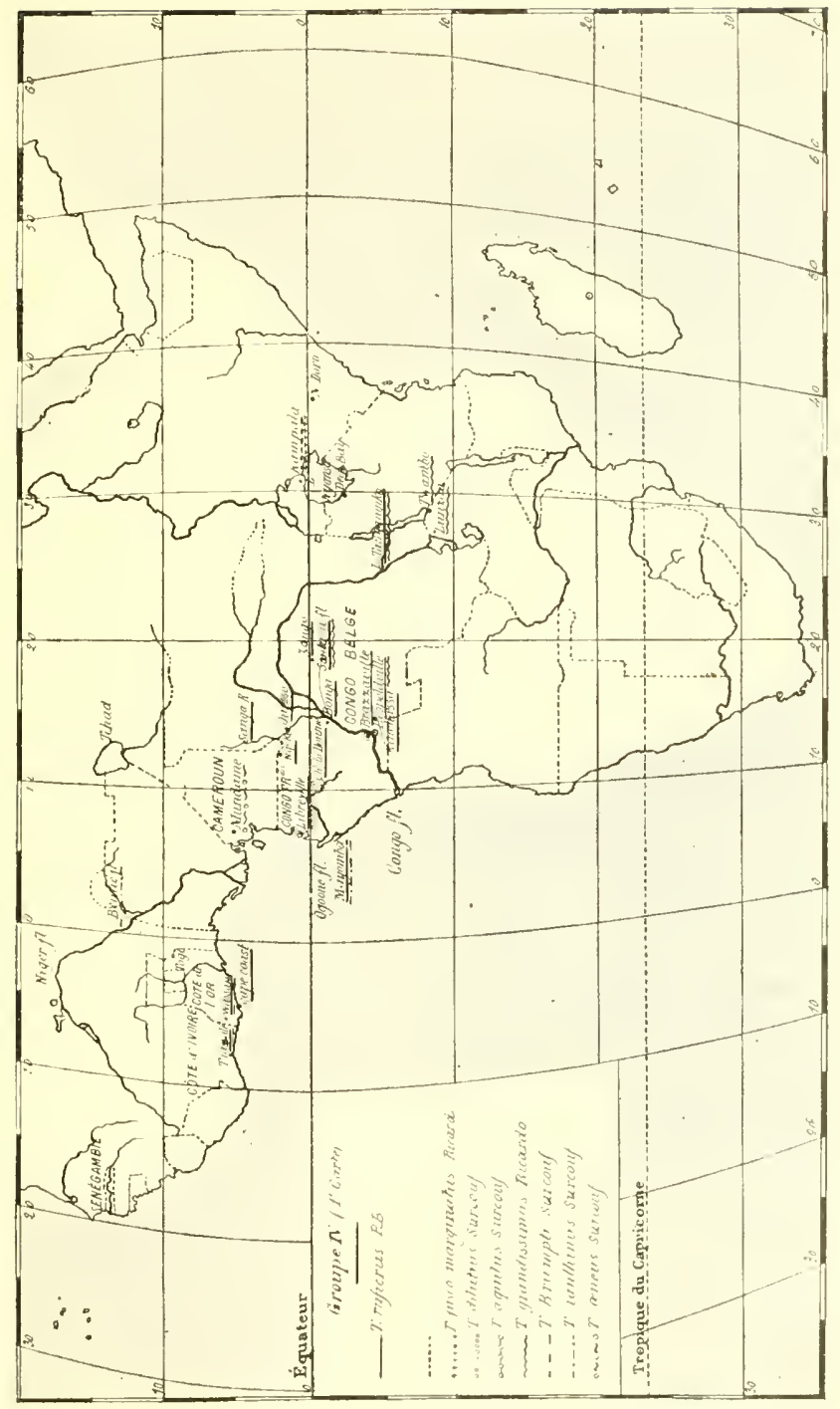




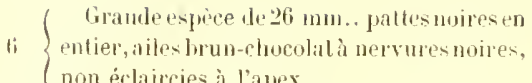
non éclaircies à l'apex. . . . . . .

T. aquilus q surcouf.

Espice de $18 \mathrm{~mm}$. , brun rougeitre à pruinositébleuatre lui donnant un reflet violacé.

Tilvias anterienrs clairs. les autres rouges. . . . . . . .

7 Couleur générale lirun noiratre, tibias

T. ianthimes $q$ surcouf.

antérieu's blanes dans leur moitio Irasilaire,

les autres tibias noirs.

Tons les tihias blancs dans leur moitié

hasilaire

\section{T. Besti q surcouf.}

T. Boneti $q$ surcouf.

Ce groupe "st composé le Tabanides dont les yeux sont wlabres, les tibias antérieurs non dilatés, la batule frontale ring à six fois aussi haule que large; le corps brunatre ou noir sins lignes ni lessins en général : les ailes sont plus ou moins teintes de brun, sans appendice. On y rencontre les plus grands Tabaniles de l'afingue.

Tabanus ruficrus $q$ P. B. (pl. I, fig. If)

Palisot de Beauvois donne de cet insecte une description succincte:

"Fuscus aulennis tibisque tarsis nigris. Femoribus alis palpisque fuscanis.

« Longueur Q 10 limnes. Owar.

P'ar suite d'une erreur nous retrouvons le même Talanus désigné sous le nom de Tubanus rufipes P. B.

En 1821, Wiepemanx redécrivit le Tubanus ruficms l'. B. et en donne la descriplion suivante (traduite le l'allemand) :

a Corps brun en entier, hárissé en dessus et en dessous de l’insertion des ailes-

"Intennes, fémurs, tarses, ajex des tibias, noirs; base des tibias et jalpes

ه roux. Front et espace interoculaire à jubescence jaunàtre; ligne b́levée glabre,

" ovale, oblongue, brillante, canaliculée en dessous, filiforme vers le bas, base

" un peu f́laissie, Ailes brunes ì nervures couleur le poix.

Walken ayant regu le Tubonus rufiorus, P. B. le supposia nouveau et le redicrivit sous le nom de Tahanus permasns.

- Piceus, capite antico fulvo, thoraceque pectoreque rufo-fuscis, illius vittis

* tribus olsscurioribus, abdomine glaucescente, antennis perlibusque nigris,

" tarsis intermediis basi tibiisque ferugineis, alis cinereis, basi costa venarum-

"que marginibus fuscis."

a Corps coulew de poix, tête recourerte d'une pulsescence lrune courte, tan

" sombre et revètue de poils couleur tan antécieurement. Yeux couleur d'airain,

"grands, séparés sur le milieu par un très étruit intervalle, toutes les facefles

"très petites. Trompe noire, palpes couleur de tan somlure; antennes noires,

" premier article trèsolilique à l'apex ; corne du troisièmearticle courte.Thorax et

- poitrine brun rougeatre sombre, thorax arec trois bantles sombres, étroites et 


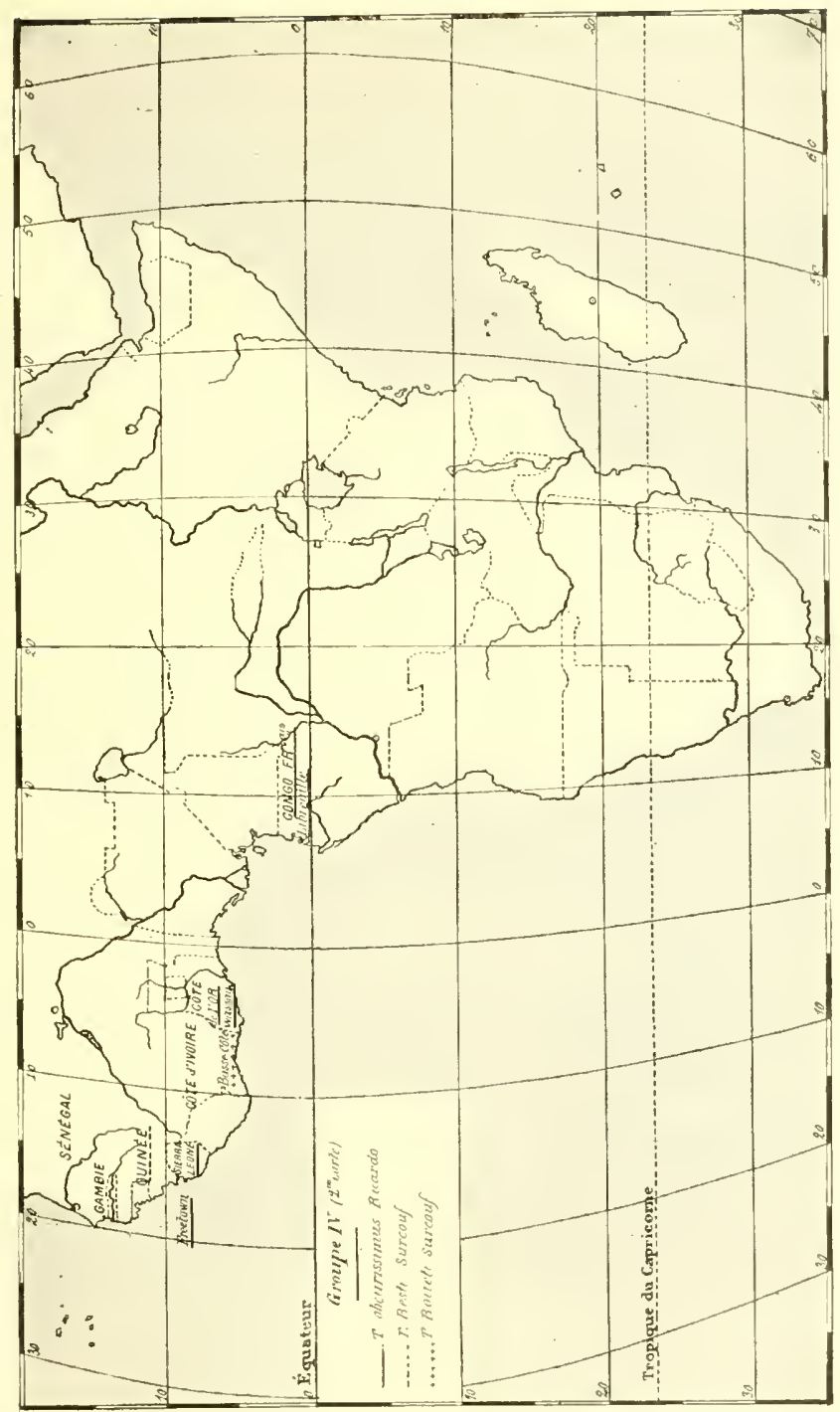




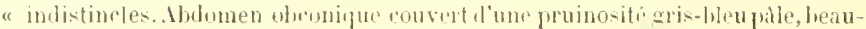
a coup jus loner que le tharax dambes noires, libias ferrugineux avec l'extré" mití apicale noire, fibia jostérieurs frangís de courtes soips noires. Tarses

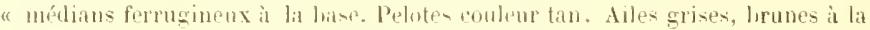

"base of le long de la lordure roslath juspue pres de l'apex et au pourtour des

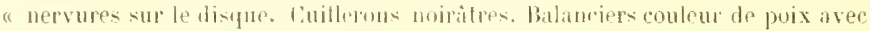
"li" disfup jatune frile. Extrémitri de la nerwe transverse formant un angle "ohlus, sins appendire pros de sil thite. Longueur du ror'ps : neuf lignes; des "ailes vingt-ileux. Afrirpe oceilentille.

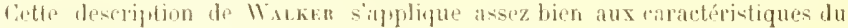

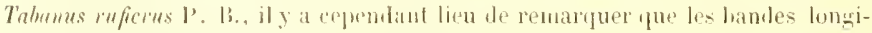
turlinales du throm sont très pen visibles.

Plusirurs espires sont tros roisium du Tabanus ruficrns P. B. main le talieau

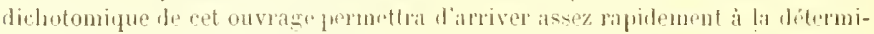
nation exicte de ces formus aftines.

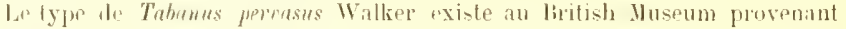

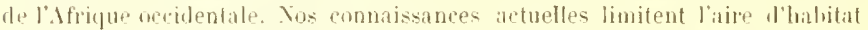

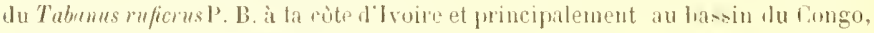
cist done une espece ementiellement tropicale.

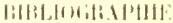

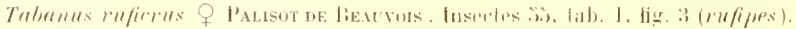

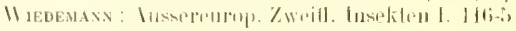

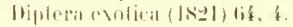

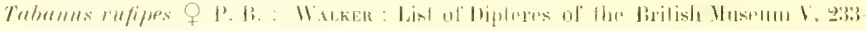
$3: 1$.

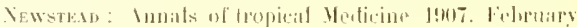
iii. 11. A. (ity. St).

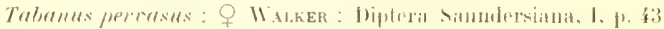

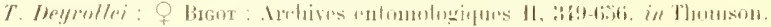

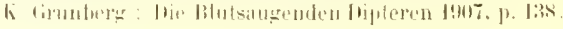

Tabanus Brumpti $q$ sicouf (lil. l, fig. 10)

$=T$. sumpuimipes o Ricaroo (in litteris)

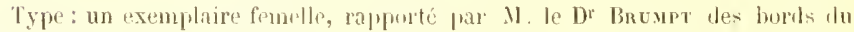
Bénoté, Congo.

Noir, abdomen heuitre, alies lyyalines, tibias rougen.

Longueur $19 \mathrm{~mm}$, envergur $38 \mathrm{~mm}$.

Téte grande, plus large que le thoras. Face lume avec une tomentosité brun jaunâtre et une pulrescene hrum, jlus dense sur les joues. Barbe noirattre.

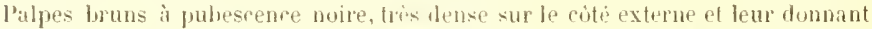
l'apprarence d'ètre noirs, ces organes sont hongs of peu renflés. Antennes lon-

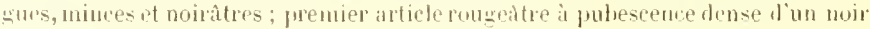
brillint; deuxième article rouge à pulescence noire ćparse; troisième arlicle ì 


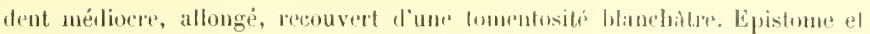
bande frontate de mème couleur que bi fire, millosite frontalle noiritre, itroite, non saillante, non tangente aux cités de fa bande frontale, prolungép par une ligne étroite. Bande frontale environ cinc fois anssi longue que large.

flowax brun rougeattre brillant, dessus glalore, cótés couverts de poils noirs: pectus brun rougeitre i pubesence noiritre. Scutellum de lis couleur du thorix.

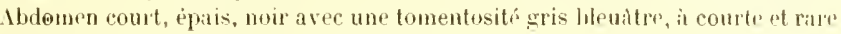

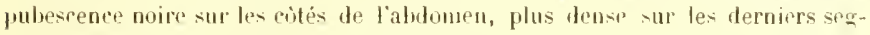
ments. Ventre noir à trè courte pubescence moirs.

t'attes d'un ronge brillant. Ilanches moiritres à longes poils cuncolores, cuisses noiratres it extrémité apicale rouge. pubesence noire sur tes pirtiex sumbren. rouge vers l'apex; tibils rouge clair a pulessence fauve, tarses antérieurs nor rougeitre it puluscener noire, tarses meilians el posterieurs rouges un peu rem. hrunis it liapex par la pubrscence noire, plus deuse it la partie ilpicale externe.

Ailes hyalines, bord costal et stigma jaune lorum. extrine base lorunithe, nervures jaunes. Balanciers ef euillerons noiritres.

\section{Tabanus sanguinipes $q$ lircando}

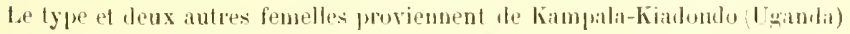
et ont té pris par le lieutenant-columel lincese $(17,8,0.3)$.

Espèce noire it lomentusité d’un lifeuitre indécis sur labdemen, pattes rouge brillant et ailes claires, jaunes au bord custal et noiritres ì leur extrème bise.

Longueur $20 \mathrm{~mm}$.

Le Tabanus sunquinipes R. se rapproche de Tubanus ruficrus P. B. mais s'en distingue aisément par ses ailes ctaires, sps pattes plus lirgement rouges et les palpes dont l'apex est beancouj phas mince.

Tète grande, beaucoul phos large que le thorax. Face lorune aves une tomentosité hrun jaunatre el une puhescence noire, phus dense sur les joues; harbe noirâtre; palpes jaunàtres mais si épaissement couverts de pubescence noire sur le coté externe yu'ils paraissent completement noirs, ils sont tongs avee la partie basilaire midiocrement cpaisse, diminuant ensuite graluellement eu une pointe bongue et mince. Intennes le mème forme que celles le Tabums perrasus Walker, longues, minces, noiratres, les leux premiers articles rouges it pulsescence noire. Epistome et bande frontale de meme conleur que la fice, callositis frontale brun rougeatre, oblongue, prolongée par une ligne étroitr. Bamle fron. tale étroite, environ cinq fois aussi longue que liarge.

Thorax noir hillant, hrun rougeatre sur les cotés el ì la hase, dessus glidre, còtés couverts de courts poits noirs; jectus lrum it pulesenence sombre; stutellum semblable ì pubeseence trun sombre.

Ahdomèn court, épais, noir ivee une tomentosité gris hleuàtre, sans puhescence. Extrémitri des hords latéraux des derniers segments d'un jame transparent, ventre noire ì tomentosité grise.

Pattes d'un ronge briltant, hanches ef tilrias antipleurs noiratres ainsi que les extrémitis apicales des tarses, tilias posterienrs il pubesence faure sur le dessus. 
Ailes hyalines, from costal el stigma jamnitres, l'extrime lonse noiratre, nerrures jaumes, pulesecontes an lord costill. Toutes hes cellules prostériemres largemint onvertes.

\section{litis.106ilitillt:}

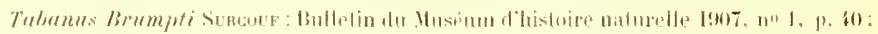

\section{Tabanus fusco-marginatus P Ricardo (j). II, fi心. 1)}

Le type femelle ef deux autres femetles proviennent de Kampala-Kiadondo-

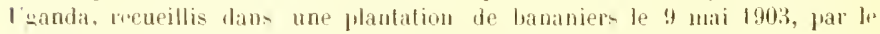
lirutenant-rolond broce $(1903,206)$. Collertion du british Museum.

Espree extrèmement voisine te Tubanus ruficrus 1'. B., mais certainement dis. tincte. cirande et rohste forme a liarge abdomen d'un brun-rhatain, pattes rouweitres, lande frontite étroite et antennes noires. Longueur 23 milliurtres.

Elle se distingue de Tabanus ruficrus P. B. par son abdomen glabre ne portant ancume trace le tomentosite gris blentle, le vintre de mente couleur que le llessus r les palpes un jeu plus allongés aver une pointe plus atténuće.

Bande frontale semblible, environ cin fois anssi longue que large et rétrécie intérieurement ; callosité oblong̨ue, rougeatte, continués par une ligne mines jus-

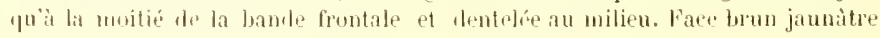

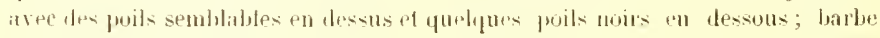

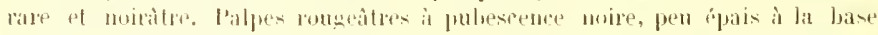
if terumis par une assez longue pointe. Intennes noiràtres, les deux premiers

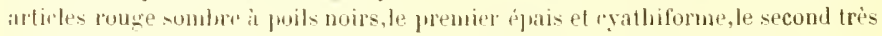
petit, le troisione lonn avee la dent habitum située trìs près de la base.

Tlwrax Inun rougeàtre aree une tomentosité biune el grise et quelques poils noirs à lit partie anterieure, pas de bandes visibles. Scutellum brun-châtain comme l'ahdomen, eouvert d'une pubescence sombre, plus noire i la base.

Ablomen d'un brun-châtian chair, presique completement dépourva de pubescence: il existe sur le promier segment des traces de tomentosité brun jaunìtre; Hinnes ave de courts poils noir's, bords latéraux des quatrixme, rinquième et sixieme segments d'un janne transparent. Yentre de la méme couleur rue la dessus.

Pattes brum rougeatl'p; hanches ì pubescente noire et lomentosité brune ; funurs d'un rongeàtre sile, les antérienrs jlus sombres, tous à pubescence mire; tiblits d'un rouge plus brillant, apex les tibias antérieurs noir, larses anfirieurs noirs, les atutres de la rouleur des tibirs, tous ì pubescence noire plus épaisse sur les tiuses el les libias postérieurs.

diles hyalines, teintées de l,run, plus brunes au bord costal, un peu jaunatres it li hase: nervures lorunatres un peu plus pilles vers l’apex lle l’aile.

\section{BIBLIOGRIPIFL}

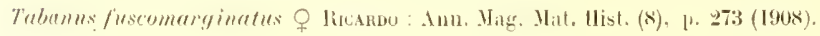




\section{Tabanus aquilus sercous}

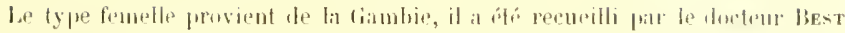

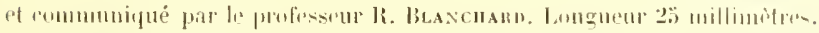

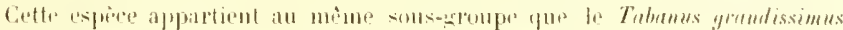

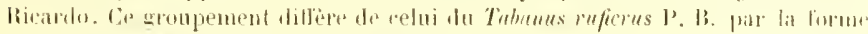
de la rallositis siluese sur lit bande froulale.

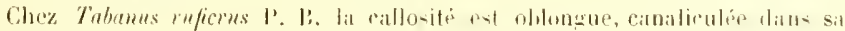

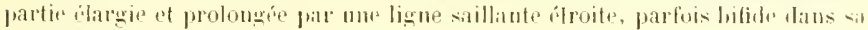

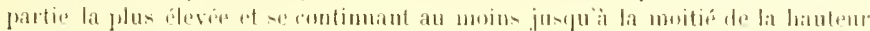
le lit bitnile frontale.

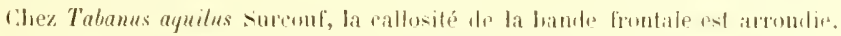

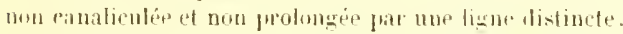

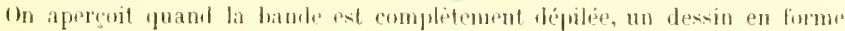

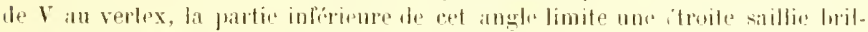

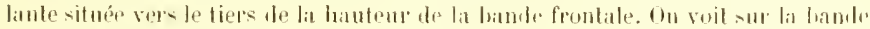

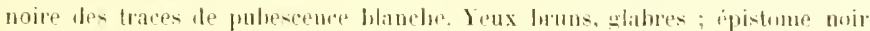

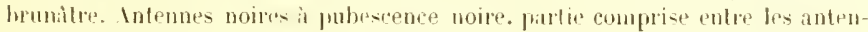

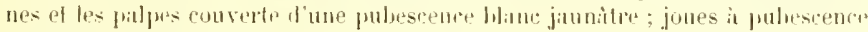

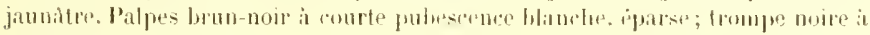
pubescence concolore.

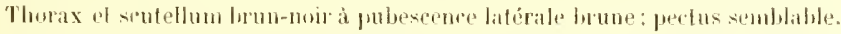

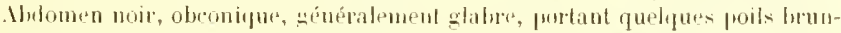

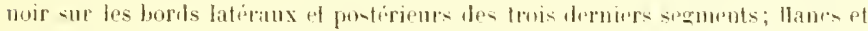

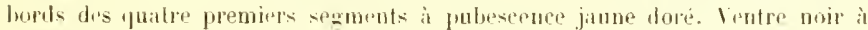

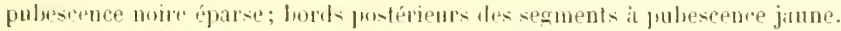

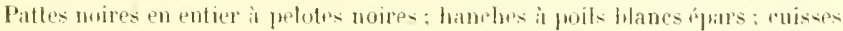

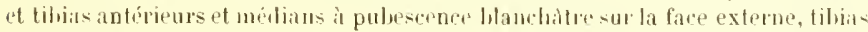

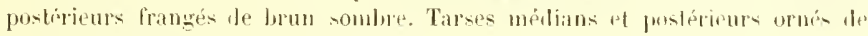
courts poils rouges it teur fice inférieure.

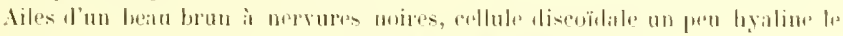

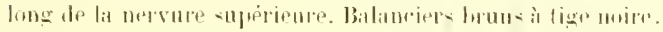

\section{lilliturili Wrill:}

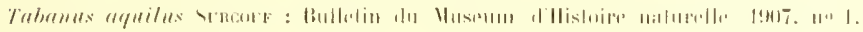
1. : :

Tabanus dilutius o sincove (,H. 1, lig. 13)

Le type et une autre femelle proviennent ale Libreville (Congo frameas) an ant

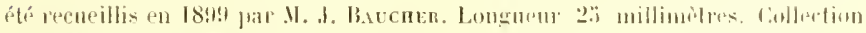
du Muséum de Paris.

f'urt et anpect de Tabames ruficrus P. It. 
Tide grosse, plus large que le thorax, yeux plus gros que claez les antres espires llu groupe. Bande fronlale étroite à tomentosilé jaune-ruir portant une callosití whlongue, non tangente aux cités de la bande et prolongée par une ligne étroite a sallante au milieu qui s'étend justudsux denx tiers de la longueur de la bande. Callosilé et ligne de prolongement d'un brun wair. Jones et barbe it pulescence jaune. Epistome jann bulfle. Intennes testarées, les deux premiers articles à courts poils noirs peu tenses, lent du troisième article normale. Pal. pes jaune rougentle ì pubescence brune non dinse.

Thorax et scutellum rougeatres ne diffèrent pas de Tubunns ruficrus P'. I3.

Mbdomen allongé, derniers segments châtains; dessous et patles comme chez Talumus ruficrus 1'. 13.

Ailes hyalines it nervure jaunes, bord costal et stigma jaunıs de même que le long des rellules lasilaires, pas de teinte brune, uneme à l'extròme basede laile.

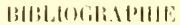

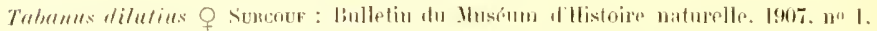
1. 39

\section{Tabanus grandissimus $q$ Ricando (pl. I, fị. 1:i)}

Le type et une aulre fenelle proviennent de Fwambo dans la Rhodesia près de l'extrémité sud-est du Lic Tanganyilia (W. H. Nust).

firand of jobuste espèce noire à ailm lrun sombre, la plus grande de foutes les espreps africanes jusqu'ici décrites. Le lype mesure 29 millimètres de longumr, les antres spreimens varient de 23 i 26 i millimètres. Deux exemplaires pris sur les hords du lac Tanganyika présentent le minimum de taille. Le Tabatmmus grandissimus licardo ressemble a Tabmus bigultutus Wiedemann mais s'en dislingue par sa taille leancoup plus considérable "t ses tilias rouges.

Tètr grande, plus large yne le thorax. Face brune à tomentosité brun jaunâtre el pulvescence noire assw épaisse. bathe noiràtre; palpes jaunes mais très densément recouverts d'une fubesence noire sur le cole externe, le coits interne est jaune ot prespue glabre. Intennes noires, premier article ápaissement revètu de pulsescence noire, deuxième article rougeâtre avee quelques poils noirs, troisime artiele long mince à dent située très près de la base : Iriangle frontal de mème conleur que lit firce, glalıre. Bande frontale cinq fois plus longue que large, rerouverte d'une tomentosité jaunàtre papaise ; callosilé frontale brune, oblongue, n’atteignant pas les yeux, la ligne jui prolonge halituellement la callosité ust ici séparís el indistincte.

Thurax noir brillant avec parfois une leinte rougeàtre, ròtés à poils noirs el

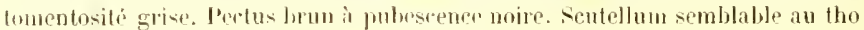
rax, cuillerons brun sombre.

Abdomen long, noir, recouvert d'une tomentositŕ gris bleuattre, saus pubescene; cotés à poils noirs beaucoup plus égais sur le premier segment et ì lapex. Fentre noir il pubescence noire. 


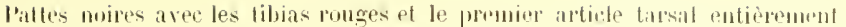

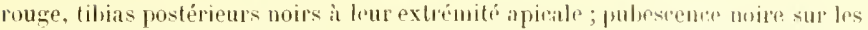
tibias rouges, et plus dense sur les libias postérieur's yni portent une pulseserme rumge en dessous, rette pulnscenes est d'un rouge brillant sur lo alessum des tarses poslúrieurs.

Ailes d'un beau loun sombre, pilissant vers l'apex et au lurd inf'rieur, partio centrale de la deuxiemerellubs basilaire, cellule disfödale of cellule anale hyalines, foutes les cellules postírieures largement ouvertes.

Guelques spécimens de T'abanus grandissimus $q$ licardo ont été pris, sur los

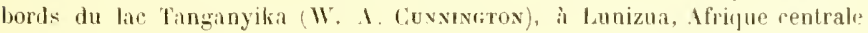
anglaise (A. suanPe); ì l)erp-Bay, cote occidentate du Lac Nyassa à Sito mètres d’altilude (R. Crawsuay, 20 février 1894).

\section{BHBLHOGHAPIIE}

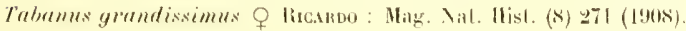

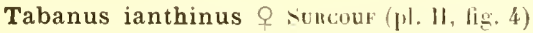

Le type est un spécimen en très hon élat, appartenant au Musér lhoyal el llistoire naturelle de Belginue el provenant lu haut Congo.

Espèce brun rougeitre it tomentosite d'un gris-bleu sm te thorax et l'alubmen; bande frontale tris étroite à callosité fotroite et allongée. prolongre par une ligne qui atteint le rertex. Inlennes don rouge billant. Pattes mougentres.

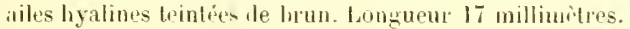

Voisin de Tabamus obs'missimus ot lieardoauruel il ressemble, il sien distingur par la forme le la bande froutale, la callosité, la couleur des antemus, le llos:ax et labulomen rougeatres, la couleur saractéristique de la tomentosilés et la largeur de l'abdomen. Il se dislingue 'n outre de Tabaus ruficrus of P. B. par lat rouleur rouge brillant des antennes et la forme de la cullosité frontale.

Face eouverte d'une tomentosite jume-brun assombrie par quelques juils noirs au milieu, des poils lan jaunilre sur les jouss ef les parties infélinures le la fare, harbe noire. Palpes jutue rougeatre it pulescencr noire, mines, légèremant dilatés a la bise seulement. Intennes d'um rouge brillant. les deux pre-

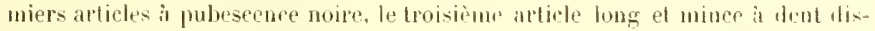
lincte. Bande frontale fitroite, ruviron huit fois plus longue tgue large de ba couleur de la face, la callosilí est brun noiselte, longue, froite, n’attrignant pas

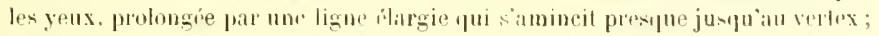

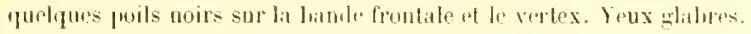

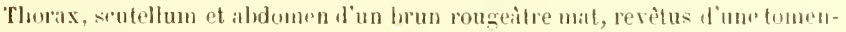

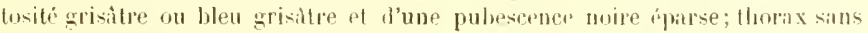
apparence de bandes, envers le lablomen l'un rouge plus sombre aree une

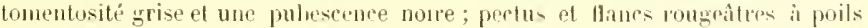
noirs. J'attes d'un roug: brilkat avee une pubesenes noire qui laur donne nne apparence sombre, spécialenent aux fómus at anx tarses; tibias antírienrs 
plus clairs, janditres un mugeilres, mais avee une pubescence entierement noire. Ailes teinlées de lorun-jaune au bord exlerne, nervures brunes, stigma brun jaunâtre. Première pellule marginale non rịlrérie. Balanciers bruns à dis. que jaunatre.

Le nom de innthims a été donnir is cet insecte pour rappeler sa couleur violacée.

\section{Lillulllill'III:}

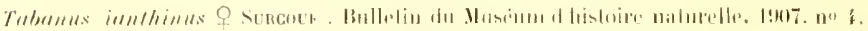

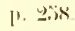

\section{Tabanus Boueti i sincotF}

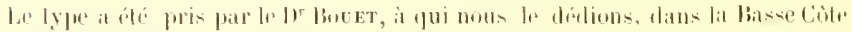
1'lvoire. (Colloction du Musinu de l'aris).

bua noiritre, ailes lowmes, libias hane.

Longuen] : 13 millimilrex.

reux glathes, bronzis, hande frontile froite, rinc fuis aussi haute que large, brune, portint une callosifi alroite prolonye sins étlanglement par une ligne

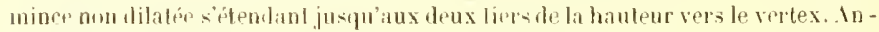

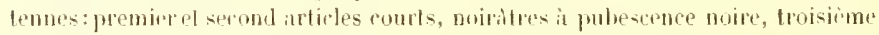
arlicle fris long, rouge, pulant une dent live pris de la lase, segmontsextremes

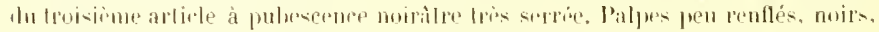

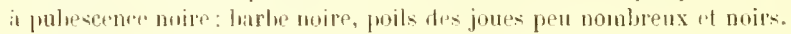

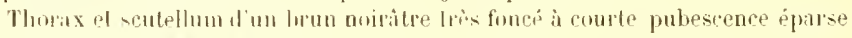
noire, plus dense sur les llincs ol hit partie seutellaire. Pertus semblable.

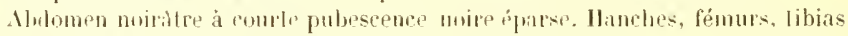

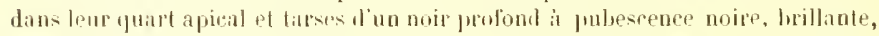

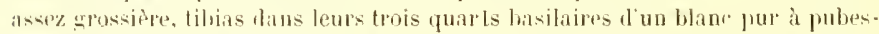
conce blancle. Aites brunes, plus vaires it l'a jux. Balanciers jambilles à disque banc.

\section{HIBLTHESTHIL:}

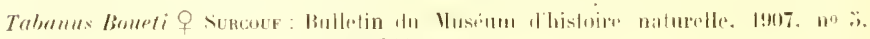
biats $: 3: 3$.

\section{Tabanus æneus $q$ surcule (ןl. I, fic. 12)}

Type q lan-la collection du Muxée l'hisloirc naturelle de Ilanbourg, où il porle eomme indication: Kanerun llukonge Farm hei Jumdane ann YongoFluss. R. Rонde, leqit, ventredi 20. Xl1, 194t:.

Longueur : 2y millimitres.

brun à ailes brunes el palpes jaunes.

leux grands, lronzés; bande frontale it borls légèrement divergents au 


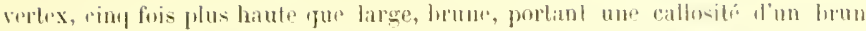

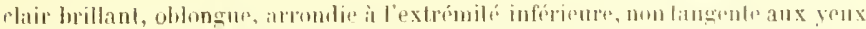

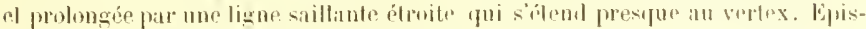

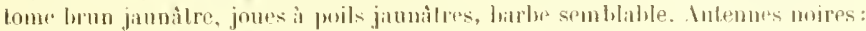

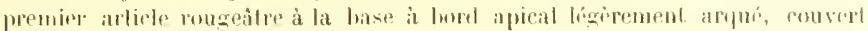

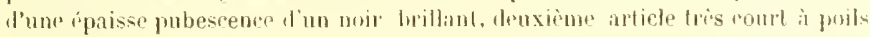

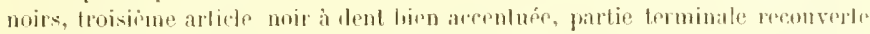

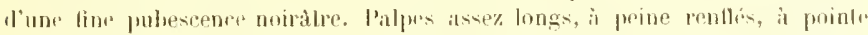

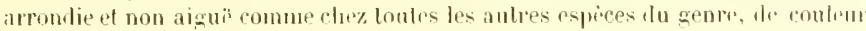

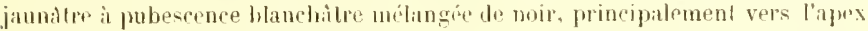
el be cólé externe. Trompe testické.

Thorax roogealre, dinudé, portant des poils blanes sur toul to pourtour "l

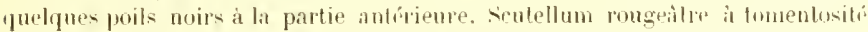
jaunatre ef poils blanchitres atiosi que les rolés du lhorax el lit poitrine.

Abdomen large, arrondi, brun, is rourtr pulsescence noire éparse, portint sml

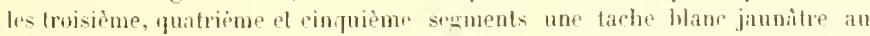
lord postérieur. La tache du hroisime segurent est triangulaire, l'aprex attrignant prespue le bord antérieu du segment, la lache triansulaire du puitric̀ne spomenl es de mime largeur jur lia pridende, les angles de la bise sont ronds; la tache du cinquirme segment at inrondie. Il existe une trace peu proptihle an milieu du horl postérieur des drux premiers segments. Ventre uniformsment brun à pubescenre noire. Cuinses d'un noir rougeatre ì pulesrence noire, les antóriemres ont la pubescence mélangée de joils jamnilres dans la moitié apimale.

Tibias anlérieurs brun rougeatro dans leur moitié basilare, noirs ditns lemr moilic apicale; tibias intermédiaires brun rougeatre flus sombre; thibs pusti. rieurs brun sombre. La pubesence de lit première pritre est mélangín dr janne el de noir, relle des autres libias es nuire; tirses lirun rougealre somber it pubescence noire. liles d'un brun rhmolat ì nervures brunes; cellute basiliam infírieure, collules diseoülale, reflules marginales postérienres légètement éclitr-

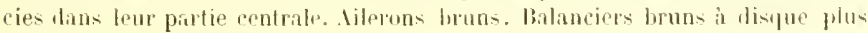
clair.

\section{BILLIOHIIUIIL:}

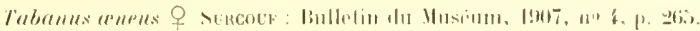

\section{Tabanus Besti o sircouv (pl. I, lim. 11)}

Le type el plusieurs aulres femelles ont de pris par he lorher BEst, mathein des Colonies Britanniques, en Gambie anglatise, dans la région comprise entre

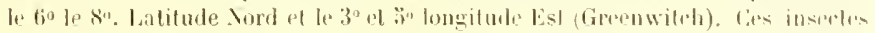

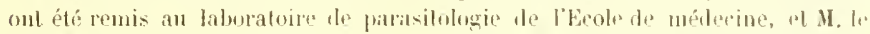

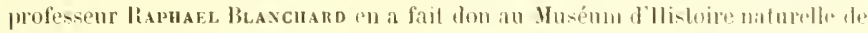

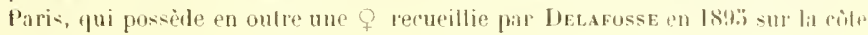
l'Ivoirs'. 
o Noire, longueur du corps : 1.3 millimetres.

lux lronzén, glabres, sans bumles eolurés, à cornéules égales. Bandr intraculaire étroite, brune, [mortant une callosité d'un brun clair brillant, la la largeur le la bande intraculaire is la lase at se prolongeant progresavement en une ligne saillante et étroite qui parrourt la bande en entier. Parties infórieures de la títe jaune chamois. Intennes noir brunatre. Palpes noirs ì pubescence noire.

Thorax d’un noir brunatre reeouvert d'une pubsecence noire, Mdomen noir en entier. Jattes antérieures noires, libias fortement ciliés au bord externe, blancs dans leur moitié basilaire. Paltes intermb́diaires et postérieures noires à tihias brun rougeatre foncé. Ailes enfumées, plus claires au bord externe. Balaneiers noirs.

La collection du musie de Hadrid contient plusieurs exemplaires $q$ recueillis en Afrique occidentale, par L. Coxvant, en 1896.

La comparaison du tyje T. Brsti et du type T. obscurissimus Ricardo étahlit l'identité de ces deux esperes. I titre documentaire nous donnons la description de miss licardo.

\section{Tabanus obscurissimus 오 liacakdo}

Le type a éti recueilli a Lokkoh-Creek - Sierra-Leone, en avril 1904, par le major Sintu. Il fait partir de lat rollection du British Museum. Le docteur liexNET il constate a Lilireville que le Tabanusobscurissimus laicardo sucait le sang de flomme et des animax.

Cetter espice de couleur acajou terme, at ales hrunatres et ahdomen élroit se distingue de Trabums secedens Walker par les soies noires de la face, la barbe noire, fo troisime article de l'anteme diun rouse lurillant à peine assombri a l'apex et jar l'abdomen long et itroit.

Longurur Is millimètres.

Tète phus litrge que le thoras ; fure lorunc aver une tomentosité jaune mate et une pubescence noire, eetle dernière consistant en de rares poils longs et noirs apatississant en dessous des antennes ef sur les joues; harbe brun noiratre. Palpes brun jaunitre avee une pubescence noire qui souvent leur donne l'aspect noiratre, ils sont phus jaunes il Jpur loral interne, légèrement épaissis il leur bise "t trominis en une longue pointe. Intennes rouges, te troisième article rouge brillant, un peu assombri a l'apex, le premier et te secomb a pubesenec noire, le premier ejais, le second ryathiforme. this petil ave un long prolongement recouvirt de soips noires, troisime article is dent médiocre. Bande frontale de mème coulenr pue la fare, brun brillant guand elle est hénudée; plus étroite an vertex, turt fois aussi longue que largo, un peu jus lorune vers la partie inférieure of fortaut une longue callowití d'un lrum-rouge lirilant, tangente aux yeux et continuir par une bitude élevíe atteignant ì peine le vertex et s'amineissant gradueltement en une fine ligne.

Thorax brun mat sans bandes, un peu plus brun sur les còtés, pubescence courte sur le dessus, jaunitre et mélangée de quelques poils noirs ; flancs portant de longues soies fines, qui, en arriere des ailes, sont faures et courtes; poitrine brune it pubescence noire. scutellum comme le thorax 
Abulomen long et élroit, d'une couleur acajou terne, couvert d'une courle pubescence noire: dessous semblable. dlulels bruns. P'ittes brun rougeitre, fémurs brunîlres, tibias rougealtres; tibias antérieurs d'un jaune rougeâtre ou jaunes; noirs à l'apex. larses antérieurs noirs. l'ubescenep des pattes noire, longue el ípaisse sur les fémurs, sur les tibias antérieurs plus clairs la pulvescence est jaunître. Ailes d’un brun pî̀le, plus claires à l'apex, la deuxir̀me cellule marginale et le hord inférieur de l'aile sont parfois complètement hyalins chez quel. ques spécimens. Nervures brunes.

Jusquà présent le Tabrnus obscurissimus Ricardo ne s'est rencontré que dans l'afrique occilentale tropicale, sierra-Leone, Cóte de l'or et Congo frangais.

\section{BHBLOGRAPIIE}

Tabrmus obscurissumus o Ricardo: Inn. mag. nat. lifst. (8) I, p. 372, 1908. 


\section{GINOUILAE GROUPL}

TABLEAU DCHOTOMIQUE

Ailesbrunes à cellules discö̈lales et marginales postérieures clitires at milien. Ibdomen portant une grande tarhe jaune soufre ou hlanchatre, recourrant lo- truis premiers segments abdominaux. l'alpes clairs pattes noires $(22 \mathrm{~mm}$.)

liles hyalines à nerrures rouges, sans appendice. Face, thorax et abdomen couverts diune épaisse pubesence hlanehatre courte. Ahdomen portant an bord antirieur des segments $3,4, ., 6$, duatre petites taches brun rougeatte. Pattes rougeâtres (20 ì 22 num.).

Espece phs petite, pubescence hlanc grisilre. Pattro diveolores (17 i $18 \mathrm{~mm}$.).

T. pluto ơ Walker.

T. camus $q$ Kitrseh.

T. conescens $q$ ivireoul.

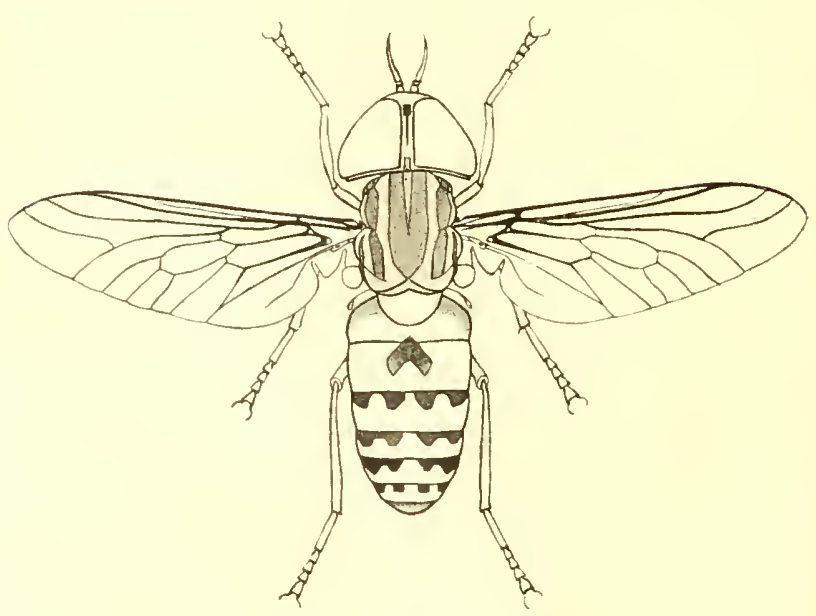

Fig. 12. - Tahrems canus O Karsch. 


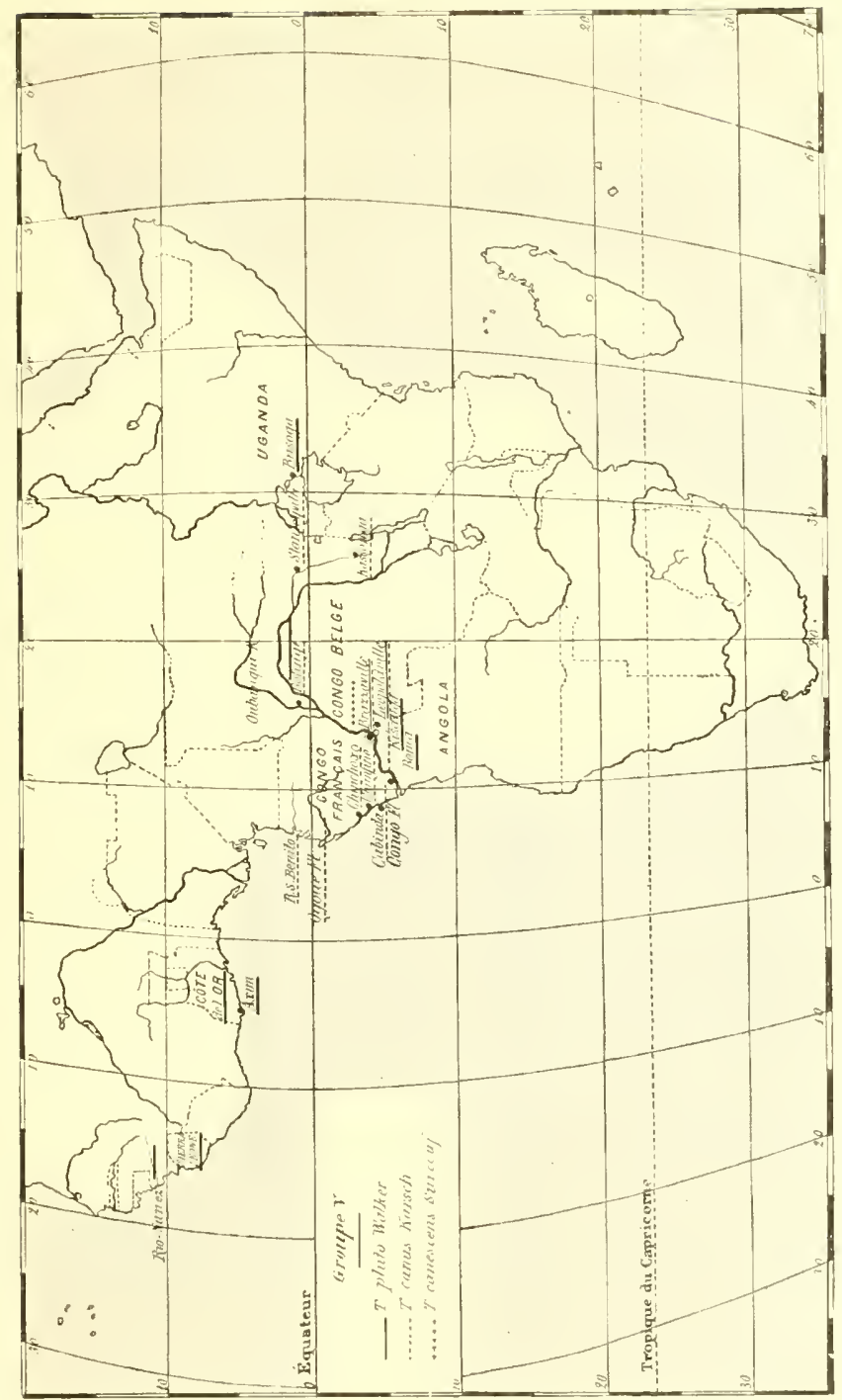


Les caractères des Tabanides de ce groupe sont les suivants:

Youx glabres, hande frontale portant à la hase une eallosité oblongue ou carrée toujours prolongée vers le vertex par une ligne étroite à l'origine, dilatée ou non vers le milieu de la bande; cette bande frontale est trois ou quatre fois aussi hate que large à la lase. Ailes lurunes sans appendice, abdomen portant a la base une grande tache soufrée ou hlan hatre, ou ailes hyalines à nervures rouges, sans appendice, corps blanchàtre à taches brunes an bord antérieur des segments abdominaux.

Tabanus pluto $q$ Walker pl. 11, fig. 6)

Le type o pris par le Révérend D. F. Morgax dans le sierra-Leone a été décrit par Walker qui en donne la diagnose suivante:

"J'iceus, thorax nigro-fusco piceo vix vittato, abelomine fascis duabus angustis " vittisque totidem latissimis aureis, antennis nigris, pedilus nigro-cinereis, a alis subnigris."

a Corps couleur de poix, tète rouverte en dessus d'une pulsescence molle, " blanchàtre et revètue de soies blanches en dessons. Yeux couleur de bronze, "trompe noire; palpes blanc jaunâtre; antennes noires, troisième article avec " une dent peu saillante à la base.

"Thorax brun sombre avec des bandes couleur de poix très indistinctes, et " un léger rellet blanehatre ainsi que sur lit poitrine revètue de poils blancs. "Cotis de l'aldomen recouverts de proils blan's; sur le dessus de l'abdomen " une large bande torée ou jaune occuje les $2 / 3$, de la largeur, de chaqu" côté " et jmesque jusqu'à l'apex. Ces bandes sont reliées entre elles par deux bamles

"étroites de mème couleur ; ailes preșue noires i nerrures noires, pattes "gris sombre voilees de courtes soims hanches. Balanciers couleur de poix. " Longueur du corps 22 mm., des ailes 4 t mm. SierraLeone (Révérend D. l".

" Moligan)."

Cette description de. Walker parait Jasée à la fois sur le màle et sur la femelle qui offrent rependant les caractères morphologiques différents.

ơ Un exemplaire rapporté de Brazzaville par M. Roubaud (Coll. du Musum). longueur $22 \mathrm{~mm}$. environ.

Yeux très gros, bronzés, glabres, ronstituant par leur ensemble lá tète presque entière; cornéules de deux dimensions différentes, celles des parties supérieures, médianes et antérieures sont très grandes, relles de la partie inférieure sont très jetitrs, la réunion de ces deux systèmes de cornéules s'effectue sur une ligne droite situe i hanteur des antennes. Epistome it pubescence courte of brune. Antennes: premier article d'un noir rougeatre à pulescence noire, olliquement tronquí du haut vers Je bas, second article plus court débordant latéralement en leux pointes, troisième article plus lung que les deux premier's ensemble, sinué en dessous, surmonté d'une dent hasilaire peu saillante et arrondie: falpen llancs à pubescence blanche.

Thorax lrun noir à tomentosité cendrée et portant des traces de bandes lonqitulinales au hord antéricur. Pectus à toutfes de poils blance. 
Alulomen hrundre recouvert sur les trois preniers segments, dune large bande dorée éclaircie au milieu et d’une lache médiane, ogivale, blane jaunâtre au hord extérieur du Iroisième et du quatrième segment. Cette tache ne s'álend pas jusqua hord postérieur. Ventre lnun somlre ì tomentosité grisâtr el à pubesence blanche fparse, chaque segment borlé antérieurement de couts poils blancs. Ailes lirunes à nervures hrunes, cellule discoülale éclaircie, première cellule fostérieure légèrement rétrècie à son extrénité. Paltes d'un noir rougeâtre, tibias antérieur's rougeatres, pulsescence des pattes, blanche, rmpodia noirs. Balanciers in lige brune, sommel du disque jaume. Cuillerons bruns.

Q Yeux à cornéules égales sans bandes; hande frontate blanche assez large, plus élroite à la base qu'au vertex, porlant à la parlie inférieure une callosité noire demi-ovö̈de, tangenteaux deux còtés, prolongée par une ligne saillante noire qui s’élend jusqu'au milieu de la bande on elle se dilate un peu ; vertex à partie luisante formant une callosité mal définie, sillonnée de plissements obliques et convergenls. Epistome hanc ainsi que la larbe et les joues. Saillie du troisième article antennaire plus aiguë que chez le mâle, le reste est identique.

Certains exemplaires provenant du Bas liio Nunez (Gambie française) et communiqué par 1 . Ie professeur Laverax en 1904 ont toutes les cellules des ailes éclaircies dans leur partie centrale. Cette variation parait ètre lıahituelle en Gambie.

Deux exemplaires de Uganda (Brilish Ituseum) sont plus éclitircis que le lype.

Le Tabamus pluto Walker a élé redécrit par Yander Wulp sous le nom de Tabanus leucaspis sur un spécimen provenant de la eòte de l'Or. Afrique occilentale.

Cette espèce est nettement tropicale, son point de eapture le plus seplentrional est en Gambie, on l'a reprise depuis dans la rigion de lit côte de l'Or (Axim); au Congo et à Busoga dans l'Uganda.

\section{HIBLIOGIRIPIII}

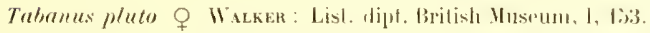

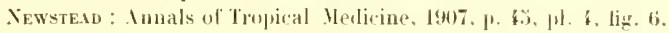

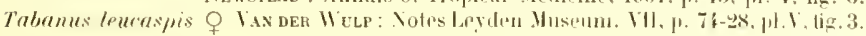

\section{Tabanus canus $q$ Karscu $(\mathrm{pl}, \mathrm{ll}, \mathrm{fig}, 7)$}

Le type ㅇ appartient probablement it la collection de Berlin, il provient des insectes recueillis dans l'ifrique oceidentile par II. stabsartz et le docteur FaLhEXSTEIN,

Lit description originale que nous reproduisons in extenso esl suftisante pour étallir la priorité de sa découverte.

"Cinereus, thoraee cinere-brunneo, lineis tribus mediis longitulinatlibus a cinereis pone conjunctis, lateribus cintrascentilus. Mblomen supra et lateribus 


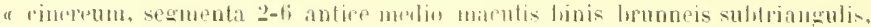
" pune divergentibus et lateribus supra majoro brunneu fuseo ornata, venter " lirumneo-fuscus, pistis lirumei.

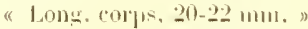

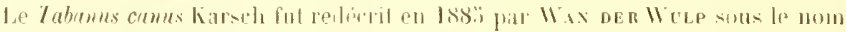

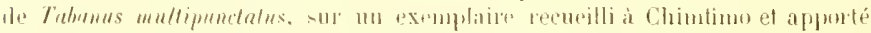

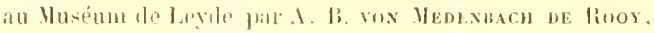

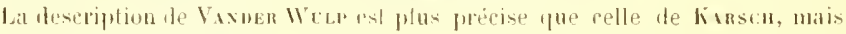
ello est encore incomplete.

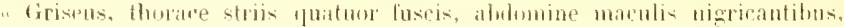

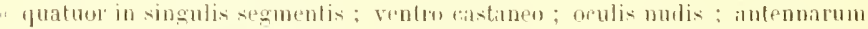
"artirulis bisalibus rulierentibus, artieulo tertis nigro; palpis pallirlis; pedi-

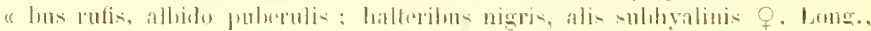
" 21 IIIII.

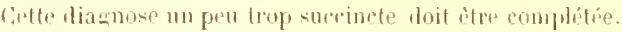

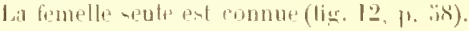

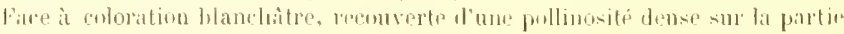

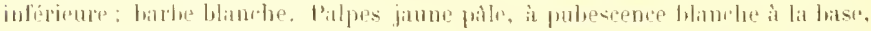

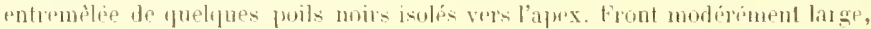
gris chir ; entre les angles les yeux pxiste une callosite subquadrangudire,

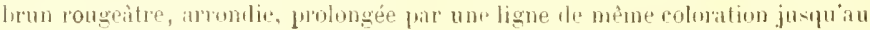

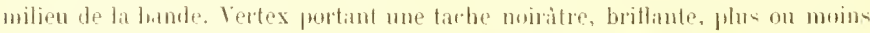

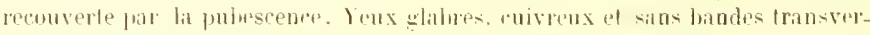

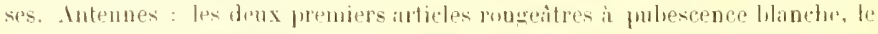

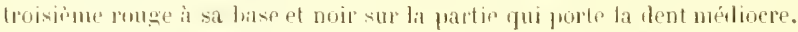

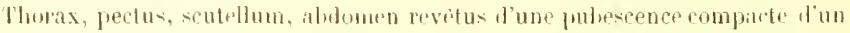

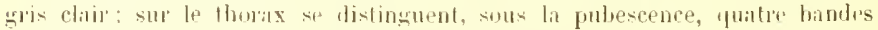
forunes tongiludinales. mal drinim, les deux médianes contluentes en arriore.

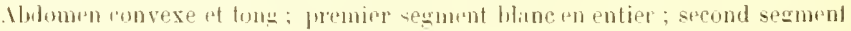

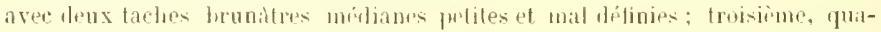
trieme, cinquieme et sixisure segments portant an lord antérient quatre tarhes

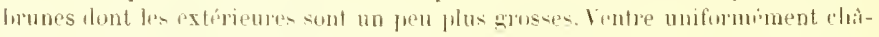

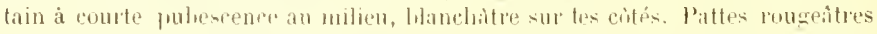

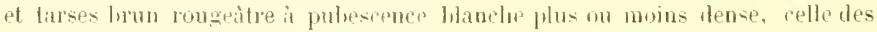

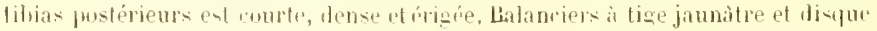

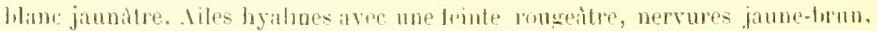

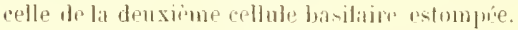

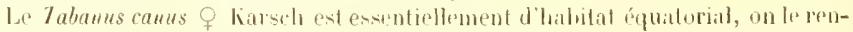
contre jusquici, miquement sur le liltorial entre l'embonchure du Gomo ar le

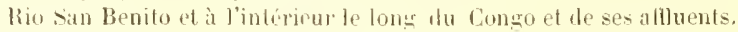

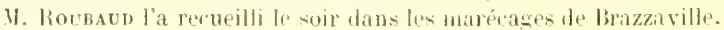

\section{lilliluilill|ll:}

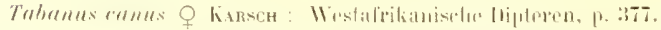

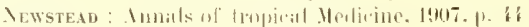

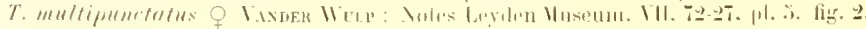


Tabanus canescens $q$ inncoul (11, 11, lig. 8

Le lype $q$ a élé pris dans le Chari ì Beira par le docteur Kéraxoel qui, par l'intermédiaire de l'Institut Pasteur, 'n a fait don au Musium d'Histoire naturelle de Paris. In antre exmplaire en meilleur élat, appartenant au Iusé Royal d'llistoire naturelle de belginue el provenant du Congo belge nous a śtí communiqué.

Longueur 17-18 mm. Blanchatre, offrant l'aspect général du Tabanus caus Karsch, de petile taille. La tomentosité est d'un hane grisatre et est moins dense

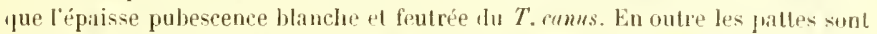
discolores, tes tarses des trois paires de pattes of la moitie apicale des tibias de la première pair sont noirs. 


\section{SIXIEME GROUPE}

\section{Tablead dichotomique}

Pattes entiprement jaunes ou rouges; ailes claires, petite espèce jaune. Callosité frontale petite, oblongue, continuée par une ligne courte qui atteint la moitié de la bauteur du vertex. Troisieme nervure longitudinale appen-

Pattes non entièrement jaunes ou rouges. Ailes teintées de jaune ou de brun . . . .

Pattes noires tous les tibias blancs ou jaunatres. Ajles claires. Thorax noiritre. Bande frontale large à grande callosité carrée

T. par ox $\sigma^{x}$ Walker.

2

T. claritibialis $\&$ Ric.

4

$2\{$ F́murs antérieurs jannes ou rougeâtres.

Pattes jaunâtres, apex des tihias antérieur's et tarses antérieurs noirs. Intennes jaunes en entier; derniers segments abrominaux pirfois rembrunis . . . . . . . . . . Pattes d'un rouge sale, tarses antérieurs noirs. apex des antennes noirâtres, ailes à ner. vure non appendiculée . . . . . . . .

Espèce brun-rouge, tarses antérieurs noirs, ajles portant un long appentice . . . .
(tibias antérieurs blancs à la base, apex et 4 tarses antérieurs noirs . . . . . . . Tibias antérieurs non blanes à la base, apex et tarses antérieurs noirs . . . . . . .

Tihias médians et postérjeur's rougeâtres, fémurs postérieurs frangés de poils noirs. Ailes teintées de brun, pas l'appendice. Espèce fauve, abdomen sans bande . . . . . . leur moitiṕ basilaire, le reste des pattes, noir. Ailes teintées de brun dans la région stigmatique, portant un court appendice. Abdomen avec une bande claire peu distincte. . . .

T. thoracinus ㅇ P. B.

T. combustus $q$ Bigot.

T. impurus 우 Karseh.

T. obscurehirtus @ Ric.

T. licentipes $q$ surcouf. 


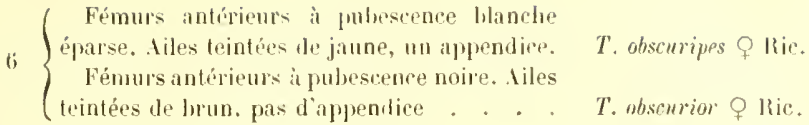

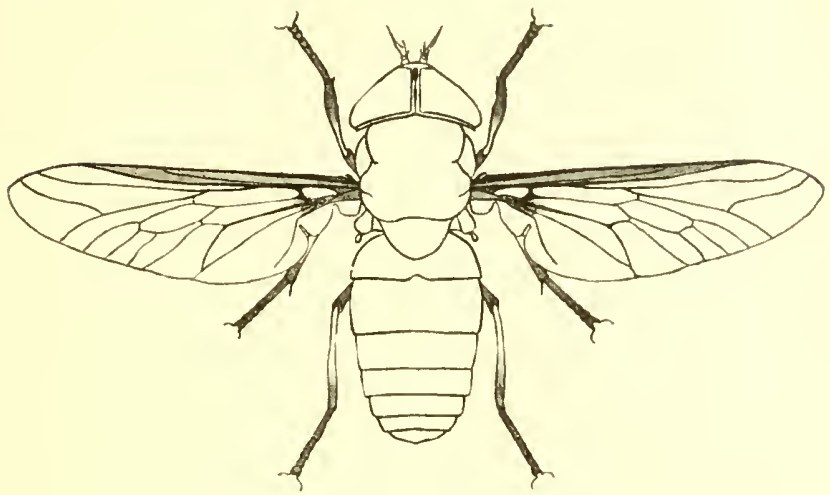

Fig. 13. - Tabanus combustus q Bicol

Ce groupe contient les Talonides qui correspondent aux caractiores suivants:

Yeux glabres ou presque glabres. Bande frontale portant a la lase une rallusité oblongue ou carrée fonjours prolongée vers le vertex par une ligne étroite it l'origine, dilatée ou non ser le milieu de la bande. Bande frontale souvent triow étroite, quatre ì sept fois plus litute que large. Espirces moyennes on pelites, unicolores, fauves ou ronges, orlinairement sins lignes ni dessims. Viles claires ou brunâtres, avec ou sans appendice.

\section{Tabanus par $q$ Walken}

Sous cette lénomination ont été réuniss phusinurs espoces des anteurs, war les

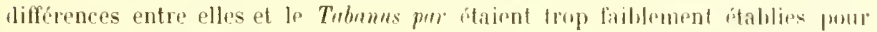
maintenir leur séparation.

Ces aprees sont :

Tabames rufipes o Macouart me Meigen.

Tabanus luteolus o Law,

Tinbremus cereolus o bigot.

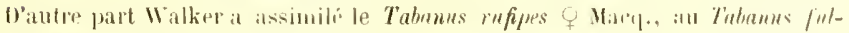
rus o lfgo., er qui ne peul itre armis.

Watker a donné de Tubanus par. Q la despription suivante :

"Fulvus; oculi renei; palpi testacei; antenne rufescentes, pall've, subarcuatit. Surcous 
"simplices, vix dilatata" thorax eervinu tomentosus; pectus cano tomentosum ; " alin subeinera, venis fulvis.

"Penelle couleur tan; lète aver une petite callosité anlérieure; yeux bronzés " composés de trés prtites lacettes. Palpes testacés, antennes rougeâtres, petites, " légèrement rourbes, troisieme artirle à jeine dilaté formant un angle très "ohtus. Thoriax avec une tomentosité fauve. Poitrine avec une tomentosité blan-

"che. Ailes très légìrement grisâtres, nervures rouleur tan; branche supérieur

" hr la nervur cubitale, variant de structure, son angle stant ou lígirement "olvtus nettement dessiné el ayant un ruliment dappendice, ou phus obtus, "quelgne peu arrondi et complètement simple. Vervure subanale rejoignant la " mivure anale à quelque distance du bord. Longueur du corps : ' lignes 12 ;

" des ailes : ! lignes."

P'ort-Natal. Collection Queinzıus.

Le Tabunus par o Wallier est le lype d'un petit groupe distinct par sa taille petite ou moyenne, si couleur unilormément jaunitre ou rougeâtre, la bante frontale étruite, les ailes elaires on légèrement colorées. Les aspèces de ce groupe diffirent entre elles pir la coloration des pattes at de leur pubescenee, et par les atles hyalines ou trintées. Leurs morlifications de couleur dans les paltes sont analugues ì celles du groupe de Tubuns fasciatus fabricius. Toutes les espèces ont des antemnes minces, de rouleur jaune, it dent très faible et une callosité frontale jamaitm ou rougentre sourent petite, parfois indistincte. Les ailes possèlent un apjendire parfois mais re carartère est variable lans ce groupe. le Tubums cluritibialis o Ricurdo compris provisoirement ans le groupe de Tabamus par en differe par la forme de la callosité frontale et la couleur des pattes.

Le Tubums fur $q$ Walker enl une petite espèce jaune, aisément reconnaissable, pattes entierement jaunes ainsi que la bande et les antennes, ailes parfois appendiculées; il varie de taille rutre 4 mm. et $13 \mathrm{~mm}$.

Face ì tomentosité jaune veracé el à pubescence blanchàtre. Pailpes d'un l.lanc jaunitre, épais, terminés par une puinte courte à pulsescence généralement moire. Intennes jaunatres, minces, les denx premiers articles parfois à pubescence blanchatre. troisieme long avec une saillip peu visible ou munie d'une dent siluex normatement. Bande frontale intoite environ einq fois atussi haute gue large, recouverte l'une tomentosité jaune oeracé sombre, callosité frontale petite, jaune, très indistincte, ollongue, n’atteignant pas les yeux, arrondie antérieurement et trianguliare postériemrement, prolongée par une courte ligne jusqu'à la moitié de la bande. leux sans marques mais parfois un peu velus, le plus habiluellement glabres.

Thorax grisâtre, parfois paraissaut plus sombre, couvert d'une tomentosité fance et d’une julhescence jaune jûle áparse, mélangée de quelques poils noirs.

Alulomen unicolorp d'un jaune lerne, assombri ì l'apex, puhescence composée le poils james et noirs entremètés, envers plus pàte it pubescence principalement blanchâtre mélangée de quelques poils noirs. Pattes jaunes on jaune roureatre, fémurs it pulvescence blanche, quelques poils jaunes sur les tibias et le premier article des tarses, partout ailteurs la pubescence est noire. Ailes hyalines, nervures et stigma jaunes.

L'étude de lit inllection des Tabanides dı Iluséum d'Histoire naturelle de 


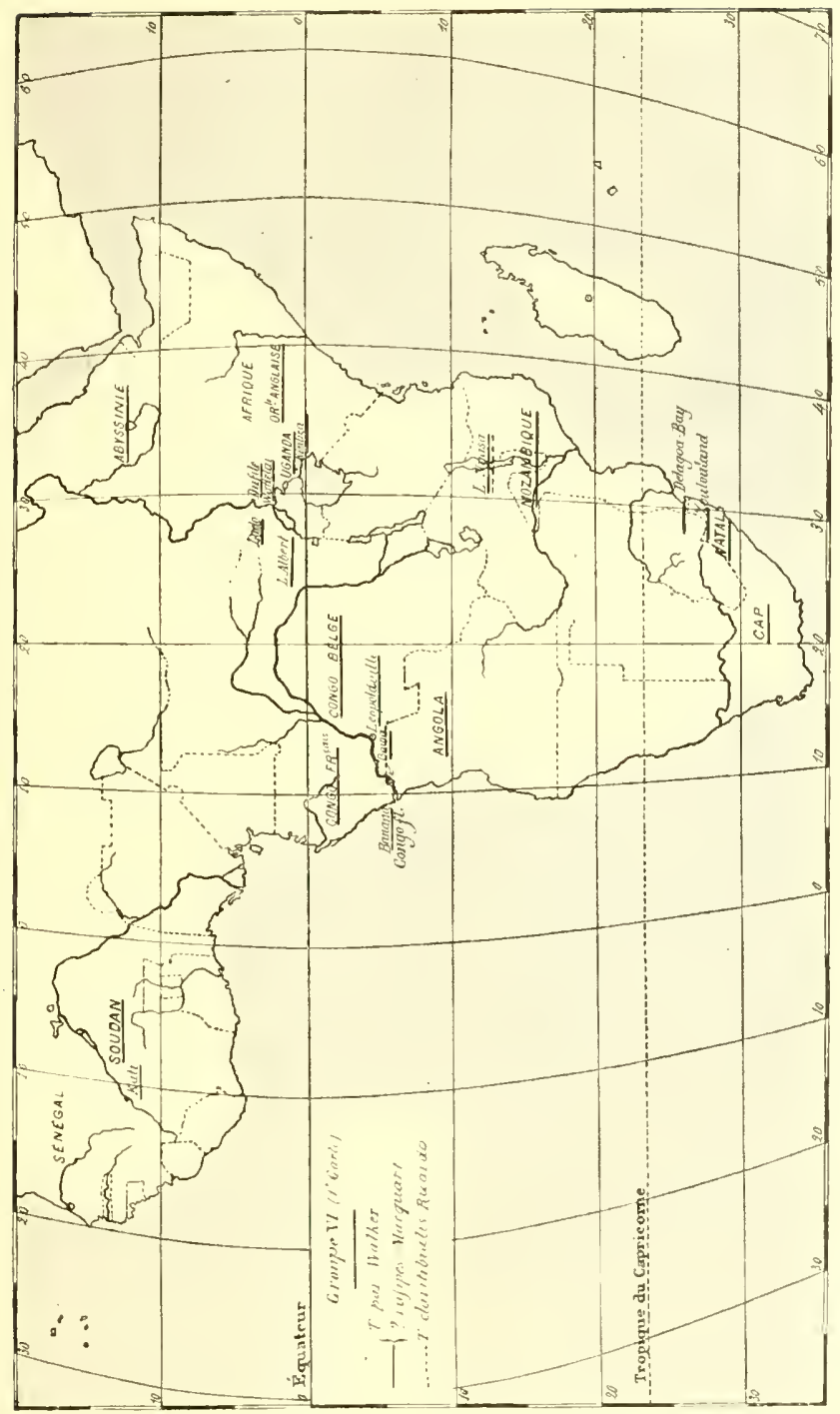


bruxelles nous a promisl'y trouver un tion mile que nous rapportons an Tabumus per.

Il a etr recueilli par. U. Iscriorfex ì Boma (Congo), un second spécimen très usis a éti pris par .11. WaElbrocke it Kinchassa (tongo) en 1899.

Longueur $10 \mathrm{~mm}$. Thte beaucoup plus grosse que le thorax, composie dyeux contluents, glatures. Ces yeux se divisent en diux zones do cornéules, la zone dis plus grosses, de conleur brun-ronge comprem toute la partie médiane w supérieure de J'ail; la zon des petites cornóulas, de conleur foncée, se sépare

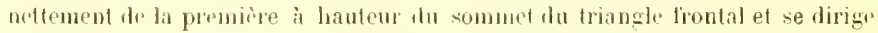
horizontalement jresyue jus ju an hord oi ello se relève pour potourer la premièr zone l'un annean d'autant plus etroit qu'il se rapproche du restex. Triangle frontal lestacé ; antennes rousses; joues et harlue jaunitres; paljes renflés, ollongs, jatune rlair portanl quelques poils lorunitres épars. Pièces buceales testarées. Thorax hrunathe hólissé d'une jubesence et d'une tomentosité jaune un fru verdatre, Hance is poils jaunitres, poitrine ì tomensité rendrée. Scutellum brunâtre it tomentositi jaune.

Ablomen jatune à pubescence fitune el quelques poils noirs éprars, derniers segments abdomina ux légìrement rembrunis; ventre jaune elair i courte pubesrence jaune. Pattes james en entier it fuheseruce jaune. Ailes hyalines, hord costal et stigma jumes, nervores jaunes, gas d'appendice is la troisième mervare longitudinale qui fait an angle obtus. labaneiers jume pâle ì disque un pen ulobuleux.

Pour ètre plus complets nous donnons ci-desious la description originale des expères que nous rémissons an Tahanus pur.

\section{Tabanus rereolus o lirgot}

"Intemnes de nuince orangét, troisiòme segment sans échancrure et briève"ment dentéc en dessus. Paljes et liarbe d'un hane jaunatre. Face el front rou"geatres. Callosité de mème rouleur, étroite en haut, lilatée et arrondie infé" rimement. Tergum, écussons noirs, couverts d'un épais thuvet jaune pâle. "Amlomen d'un fauve rougeâtre. Cuillerons et balanciers janne pàle. Pieds

" fanves : ailes hyalines ftroitement teintás de jamnatre pale val's la base et le

"stigmate. longueur $11 \mathrm{~mm}$."

Afripue orientide. Itu sprécimen.

\section{Tubanus luteolus \& LEW}

"Luteolus, peribus encoloribus, fronts angusta unimarulata, antennis "orluraceis, oculis non fasciatis. Long. corp i lignes 3/4."

Ciafrerie.

Ressemblant an Tabams fulvus mitis en litlírant essentitlement. Face jaune l'oct: à pubescence blanchâtre sous liqquelle se trouvent épars guelques poils noirs. l'alpes blane jaunatre, d'épaisseur moyenne recouverts sur la plus grande 


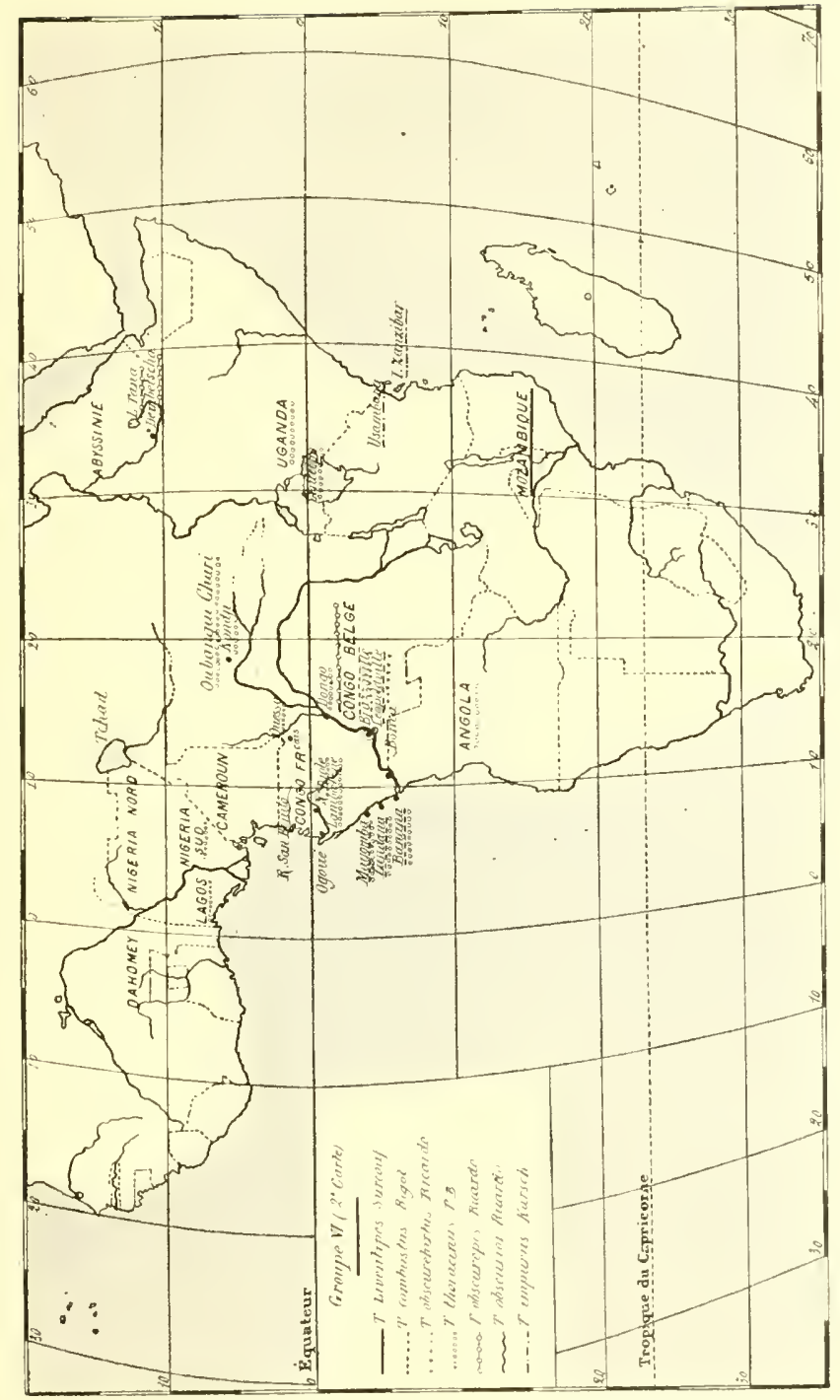


partic de poils noirs. Les deux premiers articles antennaires plus pâles que le troisime, le fremier an forme de capuchon, très éparsement revètu de courts poils noirs, troisime article antenuaire assez mince, it dent peu saillante. Front trim étroit it coloration génelale jaune-orre partois obsore portant une callosité prolonge par une ligne habituellement glabre, l'une of l'autre sont habituellement de tointe bruntre, main penvent etre noirs. Yeux sans bandes transversales, lorsquili sont ramollis ils prennent une coloration verte ou d'un violet chatovant.

Couleur générale thu thorax et de l’écussun uris pour la plus grande partir, dexis d'un jaune liore plus foncé, flanes d'une coloration blanchitre. Ia pulvescencedu dessus est principalement pìle un jaunitre avec quelques poils noirs sur le inilieu tu thorax et sur tes còtés; sur les llanes la pubescence est d'un blane jaunitre.

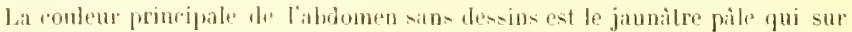
les trois derniers anneaus devient phus sombre. Le ventre est d'un jaunatre pàte plus clair avec des poils claiss assez toulfus et des poils noirs épars non visibles partent et plus abondants sur les derniers anneaux. l'attrs jaune brunitre, cuisses ì pubesence prédominante hlan jaunatre, tibias avee des poils noirs et quelpues poil clairs. Liles hyalines un peu grisatres avec les nervures et le

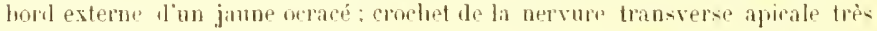

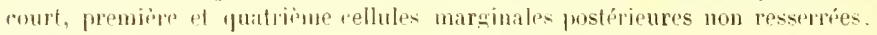
Calterie.

\section{Tabanus rufipes o Macovant}

"Intenuis rutjs. Thorate rinereo. Abdomine pedibusque rutis. Long, corps: " $12 \mathrm{~mm}, \mathrm{I} 2$.

" Palpes, face et front d'un jaune lerrugineax, ce dernier avec une petite cal"Lositi allongée, laisante, de la mème couleur. Intennes d'un fauve rougeâtre, "dent peu saillante. Thorax l'un gris jaunitre. Cuilterons jaunatres. Balanciers "limves. liles claires, base el hom extérieur jaunatres, nervures fauves de

" forme normale."

In Cap, Collection Delalande au Muséum.

Lil collection du Muséum possède deux spécimens étiquetén Táranus rufipes de la main de Macqualit.

Ces deux exemplaires ont respectivement $10 \mathrm{~mm}$. 1 20 $12 \mathrm{~mm}$. de longueur. Face jaune ferrugineux. bande frontale large à callosité jaune n'ayant que la moilié de la largeur de la bande. Cette callosité est triangulaire et se continue par une ligne de mème forme jurquian trois quarti de la bande. Palpes jaunes assè rentlés à pulrescenec blaw jaunàtre assez dense, mélangée de quelque. poils noirs au coitex extetne Intennes jaune rougeâtre en entier (pubescence non appréciable sur les spécimens usés); harbe, joues et épistome d'un jaune plus ou moins ferrugineux.

Thorax ft feuson gris jaunàtre, recouverts d'une fubescence serrée de couleur fauve. 
Ibdomen de forme arrondie, fauve, it pubescence fatue mélangée de praclyaes

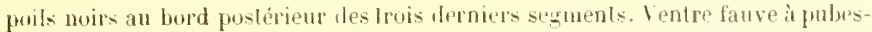
cence fauve. Pattes d'un janne rougeatre elair, tarses jaune ferrugindux clatr. pubescence jaune, en général, composie de poils noirs et jaunes sur les lirtses: tibias posterieurs avec une rourte frange noire an borl exlerne. Diles hyalines it nervares jaune clair, stigma el borl costal jimne, nervure culvitale non appendirulée. Balanciers fauves.

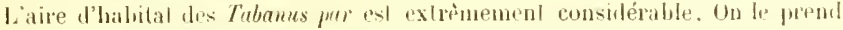
au soudan, Congo frangais, Congo leelge, Ingolin, Calp Mathl, Zoulonland, Mozan-

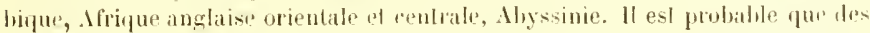

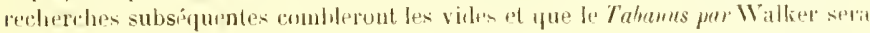

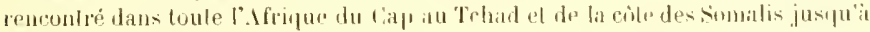
sierraticone.

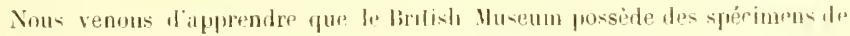
Tubanus pur recueillis dins le Vord Vigeria et be Jyasaland.

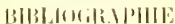

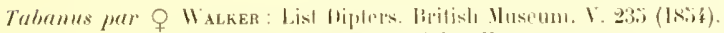

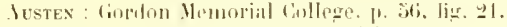

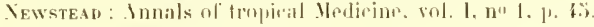

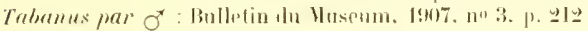

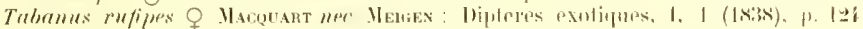
(momen lis lectum).

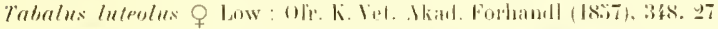

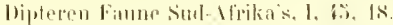

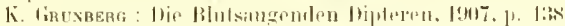

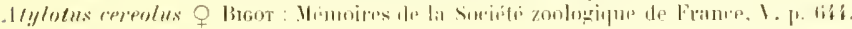

Tabanus thoracinus Q PA.. IBEAEv. (pl. 11, fig. !l)

Ce taon, un les plus anciennement decrils, avail de redecril par bisot som le nom de Tabams notarum, re lernier nom devient donc un synonyme.

Le type provenant de owar of benin (. fripue orislentale) lut figuri el derril brievement par Pal. de Beaur.; sa resrription lut copiée sans une grande "xilr-

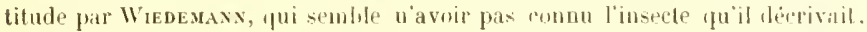

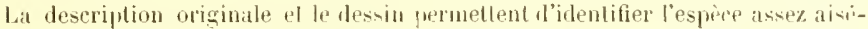
ment. Les types du Tabames notarum Bigot sont pelits et mal conservés, la noluration des ailes est très légìne, à princ pereceptible.

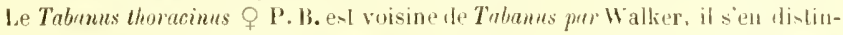
gue par ses ates legiremenl leinlpes de lorun jaunitre, ses tibias antrimms it

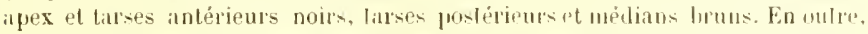

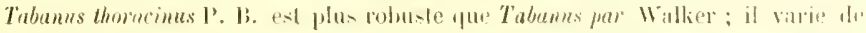

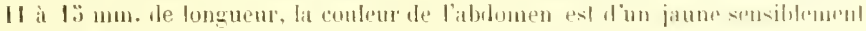
plun rouge et le thorax est plus sombre que rhez Tubanus pur W.

Yeux d'un vert émeraude chez l'animal vivant. Bamde fronlate ittoile, six lios

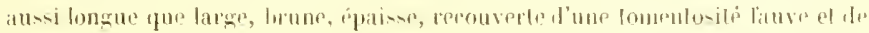


quelques poils noirs. Callosití frontale fiune, fressourent assombrie it peu de distance du hord hes yeux, olilongue, prolonge par une ligne qui atleinl ha moilii dי lat distance de lépistome an rertex. Luisses couvertes d'une tomentusiti wrine, frimurs a pubescence noire sur la prartie dorsile et il longs poils noirs en dessous. l’arfors ces poiks sonl d'une coloration pus claire. Tibias anlérieurs normalement d'un jaune prite a leur base, leur puliescence est pale.

\section{DeschIPTHX OE PaLISOT DE BEAUVOLS}

"Fulvun, thoraen viruli-glauen, antennis luters, far-in fusein.

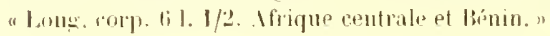

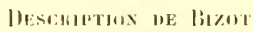

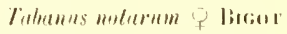

" l.01gueur : 10 is 12 11m.

"Bande fromtale lrès fitroilu sinn dilitation : yeux noirs, antennes aver le troi"sirme segment hrievement donlé, lubles les cellules postérienres de l'aile lar"grment onvertes, l'anale formée en dega du loril, lia bifuration exlerne de lis

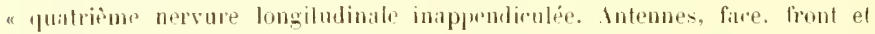

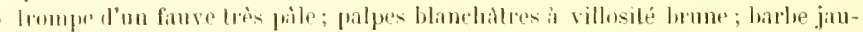
" nitle: Moprix, écusson (démudés) not's aver queldues restiges d'un duvet

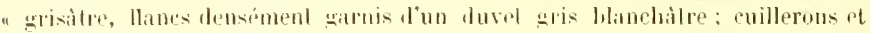

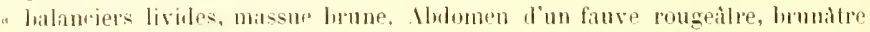

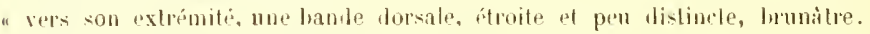

" Ilanches et pieds l'un jaune pille, tarses avee l'extrémilé brunatre, les inté- rieurs noiratres, les tibias antérieurs de teetle mème nuanee avee la lase légè"rment blanchatre. couverl l"un tris fin duret blanr; ailes grises, un peu plus "foncée exhérieurement. Cet insecte, paraît-il, tomrmente cruellement les luale" liers du pays.

"Deux spécimens. Assinie."

Le Tulumus thoracinus P. B. se pencontre dans la région íquatoriale, les dilfé. rentes callections des Musées aEurope qui nous unl ét's communiquées nous donnent les points suirants de dispersion : Lagos, Nigeria sud. Congo frangais, Congo belge, Angoli, région de loubangui-Chari, région de l'Uganda.

\section{RItictolitillite}

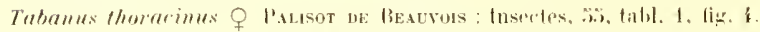
Hienevaxs: Diplera exntisa $(\mid \times 21), 76,27$.

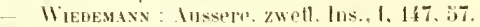

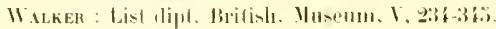

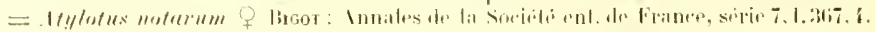


Tabanus combustus 9 Briot (lig. 13, p. 6.

\section{Description de Buot :}

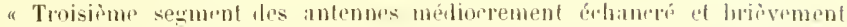
" dente, los yeux sont uus, la bande frontale frès itoite est un peu dilatie en " ovale it son extrimité infériemp", toutes les rellules pustérieures des ailes sont largement onvertes, l'anale lirme en derà du lumbl, la bifureation externe de "La quatriome nervore longitudinale (Ronduni) est dinure d'appendice. Intrunes "fauves, lextrémité du troisiòme regment ainsi que la saillie dorsale du

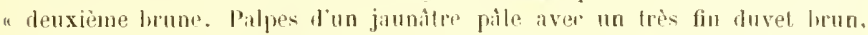
" trompe lorune. Face et barle l'un jaune fauve it front rougeâtpe. Thorax. écusson rougeatres. le premier lópuentment marquis de trois larges bandres "longiturlinales diffuses, noiritress: cullerone of balaneiers rougaturs. Ihdo-

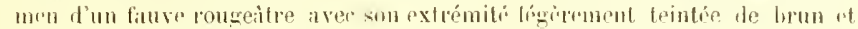

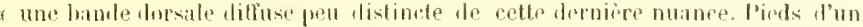
" rhitain obseur, tibias roussatres. Iiles grisitres, brunitresau bord exterue ef "le long te toutes les nervures. longueur: If mm. Issinie. "

Les tyjes sont en la possession de M. Yerralu.

Bigot a en effet disnné le nom de types à quatre spérimens, of il en existe un cinquiome exemplaire non étiquelí.

Les types sont d'un brun rougeatre et mpsurent lis tmm. de longweur. Les ailes sonf teintées do brun-jaunitre, pattes d'un rouge sale, tarses antéricurs noirs. Ces Tabanides se distinguent du Tabaums par Walker par les ailes ombreses et les tarses antrieurs noirs. Ils se differencient du Talomus thoracims: P'. B. par la coloration d'un lorun plus ronge, la bande frontale plus large, la coloration plus sombre des pattes et l'alux noir des antennes. Le Tabanus ohscurehirtus Ricardo en diffère par les tibias antérieurs blancs on jaunes. les fémurs antérieurs frangés, les intennes jaunes en entier et la conteur plus janne de l'aldomen.

Tète phus large que le thorax. Face lrun rongeatre à tomentosite d'un jaunåtre sombre et queleques poils bun Palpes jaune rougutre sombo i pubeseence noire, termines frar une pointes affilie, larges à la lass. Bathe jaune sombre entremèce ar poils bruns. Intennes rougeatres, les quatre berniers divisions du troisième article noires. premier et second article à pubescence nojre dutent obtuse. Bande frontale rougeatre, environ six fois aussi trante que large it tomentosité jaune somlire : callusité frontale olılongur n’atteignant pas les yeux, brune, brillante, portant un sillon mélian, prolongée par une conte ligne saiflante upui eontinue la callosité et qui se dirige vers le vertex.

Thorax loun rougeàtre san- bandes distinctes: sur certains spremens apparaissent trois bautes obseures, partie dorsale refonverte d'une tomentosité jaune wrisitre ef de poils jaunes en arrière des atiles, critus it longs poils buns, pectus rouge à puhescente lırun-jaune. scutellum at iblomen rouge sombre, re dernier sans la bande brunatre perceptilile que signale: Brgor, mais les segmentitions sont un peu plus sombres of l'apex brunitre; la partie dorsale semble

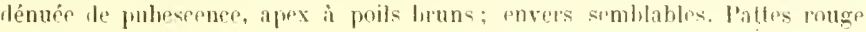


sombre. lémurs antérieurs plus bruns, tarses antérieurs noiràtres ì pubescence noire, un peu de pubescence fauve se rencontre sur les fémurs médians et postérieurs, ainsi que sur les tibias antërieurs. Ailes teintées de brun, plus clares a l'apex et au bord postérieur, nervures licun-jaune, stigma brunatre, pas d'appenlice ì la troisième nervure longitudinale. P'remière cellule postérieure légèrement rétrécie an bord de l'aile.

Les différents spécimens de Tabanus combustus $q$ Bigot actuellement conmus provienment tous des bords du Congo et du rivage de l'Atlantique entre l'embonchure du Congo et la Cóte d'lvoire.

MHLLIMGRIIIIL:

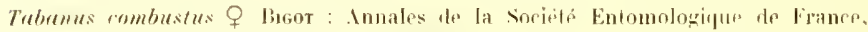

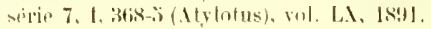

\section{Tabanus impurus $q$ Kiasch}

Descriplion de karscn :

"Tabonus impurus o type recueilli ì Ltambara Ifrique orientale), couleur " lıun rouge avec les yeux et les larses antérieurs noirs, yeux glabres. Branche " supérieure de la troisieme nervure longitulinale avee un long crochel appen" diculaire. Tibias antériemrs non dilatés. Toutes les rellules postérieures mar" sinales largement ouvertes. Callosité frontale antérieure large et arrondie. "l'inférieure se terminant en pointe, d'un lrum-rouge brillant. Ailes nom rlair's " avec une large hande brun-ruuge au bord costal. Cellule anale firmée grant " d'atteindre le bord de l'aile. Longueur du corps : $15 \mathrm{~mm}$."

Il existe une figure jointe à la deseription de Karsch, elle n'est pas utilisable: il est impossible d'identifier le Tabams impurus o Karsch, la descriplion ftant très sommaire. On peut cependant le dilfirencier du Tabanus combustus o Bigot par le long appendice de l'aile. r"est en tout cas une forme du groupe de Tabanus thoracimus $q$ P. B.

\section{BIBLIOGRAPIIL:}

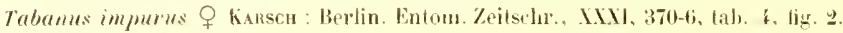

Tabanus obscurehirtus $q$ Ricardo (pl. II, fig. 10)

Le type est une femelle recueillie i Lutete(Congo), le 10 norembre 1903 , communiquée par l'Ecole de yédecise tropicale de Liverpool.

Robuste espèce jaune, aisément reconnaissable de Tabanus par Walker, Tabanus thoracinus $ᄋ$ P. B. et Tabrmus obscuripes Ricardo par ses fémurs à pubescence noire et ses tibias anlérieurs blanes. Le thorax paraît aussi plus rouge que dans les espèces mentionnées ci-dessus. 
Longueur 1 :t $\mathrm{mm}$.

Tite un peu plus litge que le thorax : fiter rougr, ipaissement recouverts d'une tomentosité jaune et l'une pulsescence jaune; barbe jature: prajpes jaunes, minces et longs, légèrement aplittis il l'extrénité, pubestence noim épaisse aver quelıues poils jaunes en dessous. Antennes d'un rouge lrillant a vec l'extrème pointe noire, premier deuxième articles jaune pale i puhescence noire, la dent du troisiome article assez proéminente, rapprochée de la base. Bande frontale étroite, six fois aussi longue que large, jaune, callosité frontale hrun-rouge, hrillante, natteignant pas les yeux, étroite, en pointr en dessus puis prolongér par me ligne átroite qui alteint presipue le vertex.

Thorix fituve arec une courte tomentosité jaune sur le ressus, pechus jaure it épaiss pubescence romposée de longs poils jaunes. Sicutellum of aldomen faures. les segments très étroitement jaune pâle, le dessus à courte pulbescence jaune, hord- latéraux des quatre derniers segments it extrímití jaune transparente, l'envers semblable, portant une courte pubescence noire.

Pattes dinn rougeatre sombre, la moitié basilaire des tibias antérieur's blanchitre ì pubescence blanche; la moitio hasilaire des autres tibias d"un rougeâtre paile arec une pubescence noire sur les tibias médians. Tibias postérieurs avec une pubescence noire et épaisse mélangée de quelques poils fauves yui présnte la plus grande longueur sur les hords externes et internes des tibias médians pt postérieurs. Fémurs antérieurs noir sale, médians et postérieur. rouge sale; tous les fémurs sont recouverts d'une pubescence si épaisse qu'ils paraissent noirs, les médians et antérieurs portent de longues franges de poils noirs. Toutes lrs cuisses sont noiràtres à tomentosité grise. Tarses noirs à pubescence noire.

Ailes teintés de brun, jaunes au bord antirienr, nervures jaunes, ordinairement pas d’appendice; toutes les cellules postérieures largement ouvertes.

Le Muésum de Paris possède plusieurs spécimens provenant de la rive gauch du Congo entre Bomat el Léopoldvills.

\section{BIILIOGRAPIIE}

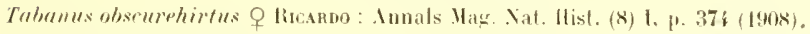

\section{Tabanus obscuripes $q$ Ricindo}

Le type q provient des environs du lac Zegi-Stana en Ibysinie, il it its refueilli par .1. DE.sex an juin 1902, ainsi que doux autres spécimens pris en avril et mai ì Dembrateha-(rodjam, rn Abyssinie. Ces différents spécimens sont an Batisu Museus.

Le Tubanus obsruripes q Ricardo est trẻs étroitemunt voisiu de Tabanus par $q$ Nialker el de Tabanus thoracinus o P’. B., mais il s'en distingue par ses fémurantérieurs noirs.

Longueur $14 \mathrm{~mm}$.

Bande frontale froite, environ six lois aussi longue que large it la hase. Palpes 
il pubescence blanehe, très dense ell dessous, mélangée de quelques poils noirs. Fímurs antérieurs noirs. lrillants, jaune pìle ¿ l'extrémité apieale, à pulbescence noire mélangée de quelques proils blanes sur le bord externe, les antres fémurs unt anssi une pubeseenee blanchatre ; tibias antérieurs à apex et tarses antérieurs noirs, les autres piltes sont d'un jiun rougeatre et les tarses ont une pubeseence noire. Ailes teintées de jaune, un très court appendice existe cliez tous les exemplaires à l'exception d'un sent.

Il est possible que lorsque nons recevons des sujets frais et nombreux nous les identifierons lous an Tabanus par Walkel et que les autres espices ne soient que des variatés distinguées senlement par la couleur des ailes, des pattes ou de la pubeseence. L'halitat actuellement connu du Tubums obscurepes $Q$ Rie. comprend rone l'thyssinie.

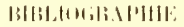

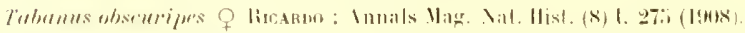

\section{Tabanus obscurior $q$ Ricardo}

L. type $q$ et trois autres spérimens proviennent de Wathen, Congo loetge (If W. II. Bexrley, 190\%). Collertion du tirilıs Museum.

Cette espiere de l’dfrirue oceidentale est très itroitrment alliée à Tabamus obsfuripes o Ricardo d'Dlyssinie, et s'en distingue unipuement par ses ailes plus lirunes sans appendice, par les fémurs antérieurs el les tilias dont la puliescence est noir au lien de blane jaunâtre, la pulseseence des fémurs postérieurs et médians est jaune rougeàtre ot non blanchitri. Palpes légèrement plus sombres aver des poils jaunitres en lesuous. Poils de liı face noirritres, mélangés de poils jaum rougeatre et non lilancs romme rlzez le Tabanus obscuripes q Kic. Longueur $14 \mathrm{~mm}$.

\section{BIBLAOIIRIIIIIE}

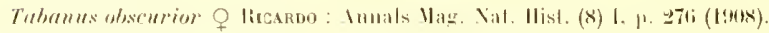

\section{Tabanus claritibialis $Q$ Ricardo}

Le type $q$ a été pris dans la région située entre l'extrémité sud llu Lar Nyaza ut he llaut-shiré (Afrique centrale anglaise) is 500 mètres d'altitude (14, I, 96, E. L. Rhoades); un autre spécimen a été recueilli par E. L. Roades, le 20 jan rier 1906, dans la mème région et it la mème altitude. Collection du British Museum.

Cette pspree ressemble d'une manière générale aux espèces du groupe de Tabums par Walker, mais en dillère par la coloration des pattes et la forme de lit hande frontale. 
Aldomen jamne rougeiler, thorax noir, ailes hyalines, paltes noires avec tous les Iilias lianes.

langueur $14 \mathrm{~mm} .1 / 2$.

Face converte d’une tomantosité gristre et d'une pulesecence blanche. Barbe bianche. Palpes un preu épaissis a ta lase ot se terminant par une pointr obtuse, rougeàtres, couverls l'une épaisse pubescence blanche et dre quelques courts poils noirs sur te bord exlérieur près de l'apex. Antennes rougentres, phus sontres it l'apex; premier article cylindrique plus court ique dans le resle du groupe; second article très petit, l'un et l'autre à pubescence noire; troisisme long avec une petite dent près de la base; la première division de lat partie terminalu est recouverte d'une tomentosilé grise. Triangle lrontal lıun rougeàtre brillant, sechancré au milien, voilé d'une tomentositi gris', jrincipalement autour the la base de l'antenne.

lande frontale plus largo que dans tonies les espices du groupe de Tabums par W. ets., ì lords parallèles, environ quatre fois aussi longue que large, noirître, couverle de tomentosité grise : callosité frontale de même couleur que le triangle frontal, carrée, n’alteignant pas les yeux. la ligne qui la prolonge est courte et épaisse, le vertex porte une petile tache oblongue d'un noir brillant: des poils lilancs existent de chaque cotó de la callosilé fronlate et à sa base. Partie poslérieure de la tête blanchìtre à pubeseence noire.

Thorax noir, quelque peu brillant, avee le commencemenl de trois landes médianes grises el une tomentosité grisn, le reste glabre, còtés rougeâtres à poils noirs. Pectus brun ì lomentosilé grise et pubescence blanche.

Ibdomen uniformément jaune rougeàlre avec les trois derniers segments brunâtres ou noiràtres. pubescence noire, courte.

Ventre semblable à tomentosité hanchitre.

lattes noires à pubescence noire, libias d'un blanc jaunàtre pàle ì pubescence blanchàtre, noirs à leur extrémilé apitale, tibias antérieurs plus largement noirs. Ailes hyalines à nervures lıunes, stigma jaune, pas d'appendice; fromièra cellule postérieure largement ouvirte.

Le Tabanus claritibialis o Rieardo se rencontre dans la région du lar Nyazia et vers la còle orientale d'.lfrique.

\section{BABLIMIRIPIIE}

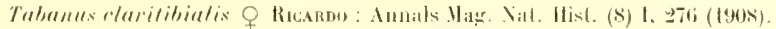

\section{Tabanus liventipes $q$ sucour}

Type: une femelle récollée par M. Guntacme Vasse, en l!06, sur la cóte de Vozambirque; une autre femelle de la meme provenance (wolleclion du Mnsinus).

Espèce voisine de Tubanus claritibiulis o Ricardo.

Abdomen rouge noiratte à bande dorsale chaire; thorax noir: ailes hyalines courtement appendiculées brunes au bord aterme el dans lic région apicale Pattes noires aver tous les tibias blanes. 
Longueur $15 \mathrm{~mm}$.

Tête plus large que le thorax. Yeux verts sans bandes. Face couverte d'une tomentosité jaune cuir à pubescence jaune. Barbe jaunâtre. Palpes blancs à pubescence blanche mélangée de quelques poils noirs isolés sur le còté externe. Antennes: premier article jaune it pubescence noire, deuxième et troisieme articles d'un rouge brillant. Bande frontale jaune-cuir de la couleur de l'épistome, large, portant une callosité noire, épaisse, quadrangulaire un peu moins larque que la bande et prolongée par une ligne de mème couleur, étroite à l'origine juis un peu dilatée. Partie postérieure de la tète étroitement bordée de jaune.

Thorax et scutellum recouverts d'une pulsescence d'une tomentosité jaune verdatre; épaules à pubescence noire, llines à poils grisatres. Poitrine à fontentosité grise et à longs poils mous de couleur grisitre.

Abdomen rouge fauve rembruni sur les còtés depuis le lroisième segment, les trois derniers segments presque complètement noirs sauf sur la partie médiane qui reste rlaire aver une pubescence dorée, eparse sur les six jremiers srgments; parties latérales des segments portant une courte pubescence noire f́parse. Chacun des segments est étroitement bordé de poils jaunes peu visibles. Ventre fause, it pubescence claire, les trois derniers segments noirs à poils noirs, btroitement marơinés de jaunâtre. Hanches noires recouvertes d'une fomentosité grise et jortant des poils blancs, fémurs noirs à pulsescence blanche. Tibias antérieurs blanes dans leurs deux tier's basilaires, tibias médians et postérieurs llancs sur presqne toute leur longueur: pubescence blanchàtre sur les tibias antérieurs el médians, jaune sur lrs tilias postérieurs dans la partie blanche. Tiers apical des libias antérieurs, extrémité apicale des aulres tibias et tarses noirs à pubescence noire. Cuillerons brunâtres, balanciers jaunitres, concolores. Ailes lyalines, brunes le long du bord costal et enfumées dans la région sliginatique et apicale.

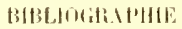

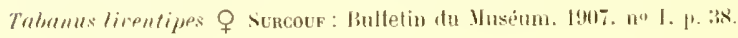




\section{SEPTIEME GROLPE}

\section{Tableav dichotomige}

1 Ibodomen à bancle médiane claire. . . .

Aludomen à bande médiane noire. . . . 7

$=\left\{\begin{array}{l}\text { liles hyalines. . . . . . . . } \\ \text { diles teintées de lirun ou di nerves trans- } \\ \text { verses ombrées. . . . . . . . . }\end{array}\right.$

Callosité frontale ovale, triangulaire au sommel. Antennes d'un rouge brillant à extrémité apicale noire; palpes minces. Abdomen rouge brun à bande médiane blanche, segments très étroitement bordés de blanc, apex noir. Ailes hyalines à stigma jaunàtre, 13 ını. .

Callosité frontale oblongue. Antennes rouge fauve ì exlrémité apicale concolore, palpes blanche, segments non bordés de blanc, apex concolore. Ailes hyalines à sligma jaunàtre, $11 \mathrm{~mm}$.

Callosité frontale oblongue, palpes nettement épaissis. Abdomen brunàtre à ligne médiane blanche. Apex noirâtre. Ailes hyalines un peu cendrées, à lıord externe presque noir, 11 mun

T. unitenialus 9 Ric.

T. Lacerani $\varnothing$ surcouf.

Ailes à troisieme nervure longitudinale non 
Callosite grande, oblongne, moins large que la bande, prolongée par une ligne qui n'atteint pas le vertex. I'ittes et antennes jannes. Iblomell it bande méliane qrise composée de taches triangulaires. Niles hyalines; nervures brunithes estompées de jaune-brun,

Callosité grande, presifue carrée, s'étendant T. conspicun. O Ricisdo. justulaux yeux, prolongée par une ligne clargir en fusean qui atteint presque le vertex. Abdomen dun rouge sitte clair ì bande medime litrge et continue. Ailes byalines teintées m. hun clair. $17 \mathrm{~mm}$.

T. subungustus Q̨ Rirardo.

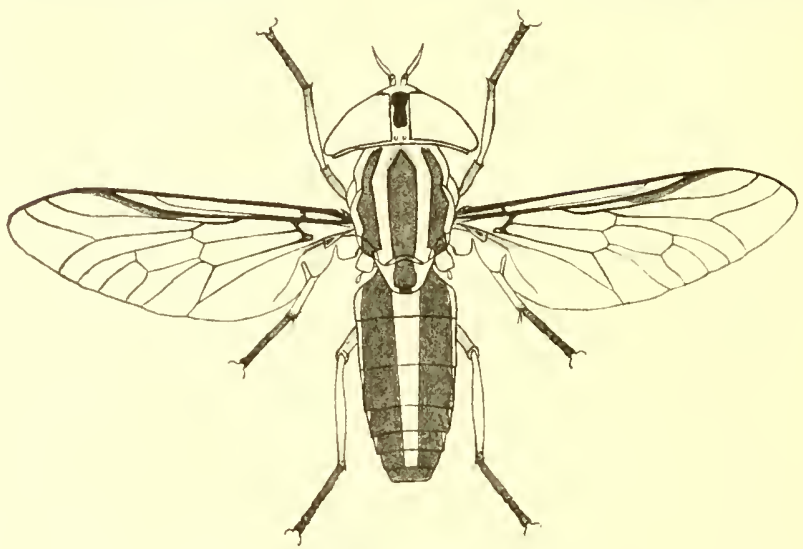

Fig. 14. - Tabanus Larerani o Surcour

( Abdomen uoirâtre ou brun rougeâtre ì bande méliane rontinue et taches latírales. Callosité frontale ef fémurs noirittes. Yenx

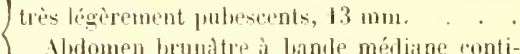
T.ulbustrimas $Q$ Ricatru. nue sans taches latérales distinctes. Callosité lrontale et fémurs rougeîtres. Youx complite ment glabres, $19 \mathrm{~mm}$.

\section{T. albitimen o Walker.}

liles clares, appendice court el épais; pelite espece rougeàtre a bande méliane noire. fémurs liruns it pubescence noiràtre, I2 $\mathrm{mm}$.

T. olssurestrintus o Rir.

Siles hyalines, teintien de brun: abdomen chitain, long et étroit à bante méliane distincte, noire: fémurs lnuns ì pubescene blan(rhâtre, $17 \mathrm{~mm} .1 / 2$

T. nigrostrintus o lice. 


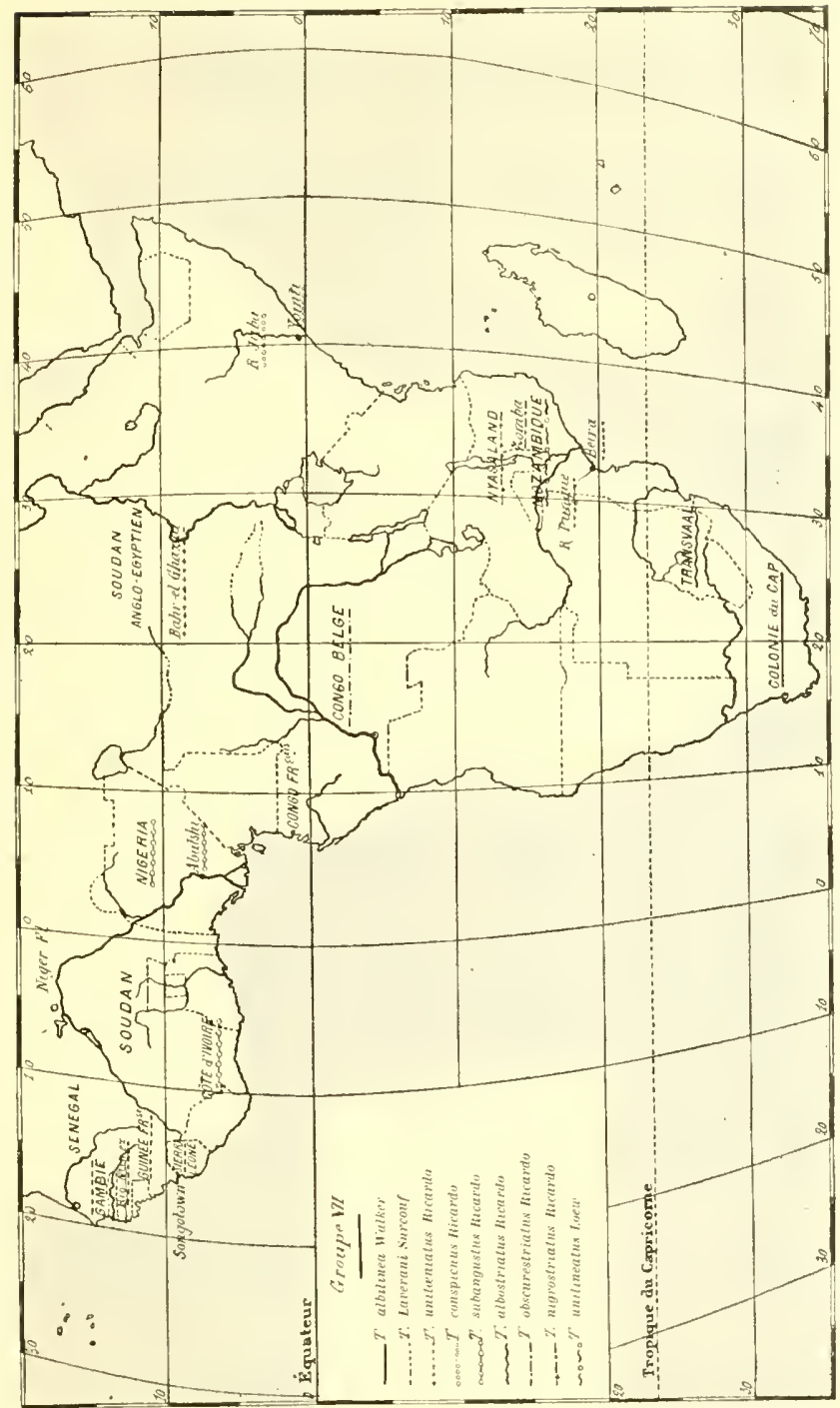

Suriouf 
Ce sroupre comprend les Tabaniles qui ont les aractères génériques suivants: yeux glabres, tibias antérieurs non dilités. Bande frontale de trois à cinq fois aussi haute que large à ligne médiane épaisie. liles génératement claires. abdomen rongeâtre ou brunître ì bande médiane itroite très distincte, pass d'autres lignes ou derins.

\section{Tabanus albilinea $\bigcirc$ WALER $=$ Atylotus niveipalpis o B⿺goт}

Walker a donné de cet insecte de patrie inconnue la diagnose suivante:

"Fuscus, capite pectore thoracisque vittis tribus fulvis, alulomine ferrugineo a allo univittato tuscoque bivittato, antennis pedilusque fulvis, alis fulvis "subeinereis").

Longueur du corps $17 \mathrm{~mm}$. 1/2, amplitude des ailes $33 \mathrm{~mm}$.

Tìte lnome revêtur d'une tomentosité blanchitre en dessus, blanche et surmonté de poils blancs en descous, trompe noire; palpes et antennes bruns. leux cujveux.

Thorax brun à tomentosité blanche, portant trois hamdes brunes indistinctes; cótés et peetus brunatres à tomentositi blanchatre "t une pubescence blanche très fine.

Mylomen ferrugineux, recouvert d'une tomentosité blanchâtre sur chaque còté et desious, portant sur le desus une bande médiane brune marquetée de blanc. P'attes lorunîtres à tomentosité lilanchàtre, tarses et extrémité des tibias felrugineux, tarses antérieurs lrun de poix. Ailes légèrement grises, teintées à la base, au lord externe et le long des nervures.

Le type de lingot est la propriáté de M. Verrale du Cap; un spécimen femelle de lia colonie du Cap (R. Trises, $64,7 i$ ) est jtentique an type cidessus. La redescription ent faite sur ce nouveau spécincen qui est de couleur plus brune que le ty ge de Briot.

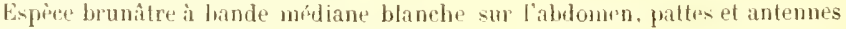
jamâtres, nejvures ombrées, un appendice.

Lonsueur $19 \mathrm{~mm}$.

Thiste erante, plus large que le thorax, fice rougeâtre paraissant blanfhe, à cause d'une tomentowité blanchâtre et de poils blines. ces derniers densément lépandus sur la parti inférieure de la face et les joues. Barlue blanche, pralpes jaunâtres, premier article à longs poils blanes, deuxième article échancré à la hase puis s'attínuant graduellement en une pointe, couvert d'une courte pubescente blanche el de quelques poils noirs vers l'apex. Intennes jaunâtres, troisième article rouge brillant, brun à l'apex ; premier et second articles à pubescence noire, troisieme avec une dint médiocre qui porte quelques courts poils noirs à l'apex. Bande frontale environ quatre fois aussi haute que large de même fouleur qur la face, couverte a'une tomentosité grise; callosité frontale brun rougeâtre, oblongue, à sillon médian, non tangente aux yeux et prolongée par une linne graduellement rétrécie jusqu'au delà de la moitié de la distance du vertex. Yeux glabres sans marques. 
Thorax noir hrunitre it trois bandes grises tres indistinctes, pubescence du notum hlanchatre, critis gris à poils noirs, poitrine grise it prils luanthatres, scutellum brun a bordure rougeatre ef pubescene bianche.

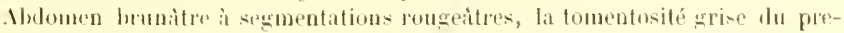

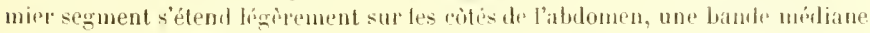
continue composér de tomentosité blambatre alteint le bord fostérieur du sixime segment, la coloration de sa bordure est plus sombre que colle du rest. de l'abdonen, lex lords latraux des sements postipleurs sont d'un jaune transparent; la pulescener du dessus est noire et courte, mais fiaisse of entre-

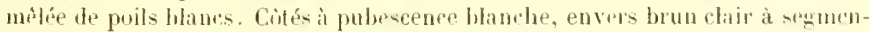
tations jaunatres, tomentositi blanche et pubesene noire. tambes jaune rou-

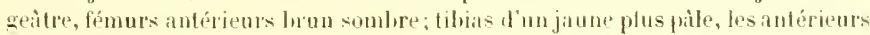

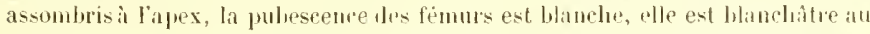
bort externe et it la lase tles libias, partout ailleurs elle ast noire, tarses à puhescence noire. Balinciers jaunes à massu d'un blane brillant. Liles grinder, hyilines à nervures jaunes, presque toutes sont estompérs d'une coulleur janne pille, stigma jauntre, appendiere flistincl.

I'n spécimen de pirie liush. ..-L. de la colonie du Cap (1. Y. Straxixi, $98,191)$, mesure seulement $16 \mathrm{~mm}$.

L'Atylotus nireipalpis + Bigot, du Caj, est identique a l'expice de Wallier. L'auteur me mentionne pas la bande modiane blanche de labdomen qui est cependant clairement visible dans son type quoinu il soit tans un maurais état de conservation, Il mesure $17 \mathrm{~mm} .1 \% 2$.

\section{BHBLACIRUPIIE:}

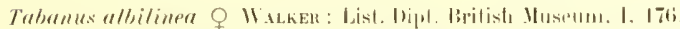

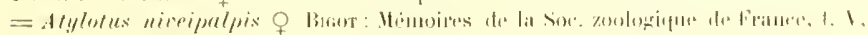
p. lisis.

\section{Tabanus unitæniatus $q$ Ricarno}

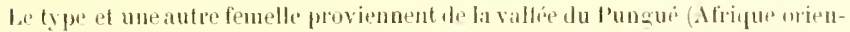
late) par te 20" lat.sud; ces deux spécimens ont ité pris par M.ti.1. K. Marsmat. en 189t (Colfection du British Museum). Cipturés an fuaturzime mille à partir de Beira, sur la voie ferrée. Un exemptaire a éte pris i 1 anu dams le

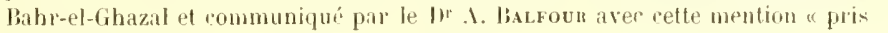
à la lumièren.

Cette espece est trìs voisine du Tabmus unilimpalus of Lrew, le Hozambigut, mais s'en distingue par son abtomen plus rouge, tes tibias antérieurs phos chairs, les palpes plus miners, la callosité frontale triangulatre et lis taille (13 $\mathrm{mm}$.).

P'etite espice à corps rougeàtre aver une hande médiane blandye sur l'abdumen et les ailes presifue hyalines.

Tête petite, plus large que lo thorax; fitce rougeitre couverte l'une tomentu- 
sité grise et diune pubescence hanche, courte ef éparse. laube hlanche. Palpes jaunes, longs et étroits, légèrement épaissis à la base et terminés par une pointe olituse, densement recouverts de poils noirs. Antennes d'un rouge brillant à extrémité apicale noire, premier article court et large, portant quelques poils noirs, second article petit, cyathiforme aver des poils noirs à l'angle supérieur, troisième arlicle à dent obtuse. Bande frontale enriron quatre fois aussi haule que large, ì côtés parallètes, de couleur rougeâtre, portant un recouvrement constitué par un peu de tomentosité grise : callositéd'un rouge brillant, convexe, ovale, rétrécie à sa partie supérieure et continuée par une ligne saillanle et étroite qui s’élargit en forme de fuseau, une étroite ligne part du vertex et se bifurque, ses deux lranches surmontent la callosité en fuseau. Yeux sans bandes.

Thorax lrun rougeâtre avec les traces d'une tomenlosité grise et les rudiments de trois bandes; còlés rougeâtres à tomentosité grise.

Abdomen étroit, d’un rouge brun, portant une bande médiane blanc grisâtre qui atteint le cinquième segment, apex noir, spgmentations très étroitement bordées de blanc, pubescence du lessus de l'abdomen presque complètement noire. Envers testacé avec l’apex phs ou moins largement noir. Pattes rougeâtres, fémurs noirìtres, sprécialement les antirieurs; tibias antérieurs noirâtres a l'apex, tarses antérieurs noirs. Liles hyalines, stigma jaunâtre, nervures brun jaunâtre. Tine des femelles a le bord antérieur légèrement bordé de jaune. Première cellule postérieure non rétrécie.

\section{BIPLTOGRAPHIE}

Tabames unitreniatus of Rirarto: Annals Vitg. Nat, ftist. (s), t. p. 312 (1908).

\section{Tabanus conspicuus $q$ Ricardo}

Le type et deux femelles proviennent de Yoiuti, sur le lleuve Juba, ì vingt milles environ de l'embouchure, sous l'Es fuateur, par 40" long. E. Le major L. II. It. Pope IIexvesser a pris un spécimen tlans lia salle à manger du steaner. (Collection du British Muséum).

Belle espece facile ì reconnaitre ì son aspect particulier, pattes et antennes jaunes, abdomen jaune clair avec une bande médiane grise à peine borlée par une coloration plus sombre, thorax à bandes distinctes.

Longueur $19 \mathrm{~mm}$. ; in autre spécimen ne mesure que $16 \mathrm{~nm}$.

Trte srande, plus large que le thorax. Face rougeâtre recouverte d'une tomentosité blanche si épaisse que la face paraît blanehâtre, de longs poils blanes en dessous, barbe hlanclie. Palpes grands, llanes, épais à la base, prolongés en pointe, sans pubescence noire. Antennes rougeâtres, noires à l'apex; premin article cyathiforme à tomentosité urise et pubescence noire, deuxième article plus petit à pubescence noire, troisiłme long pourvu d'une dent médiocre. Bande frontale modérément large, rinq fois environ aussi longue que large, à bords parallèles, te mème couleur yue la face, mais plus jaune en arrière ou 
mème entièrement jaune. Callosité lnun rougeatre, luisante, gramle, de forme oblongue, moins large yue la bande frontale et prolongée par une ligus parfois interrompue, mais qui n'atteint pas le vertex. Yeux sans bandes ni taches.

Thorax brun avec une tomentosité jatune grisatre formant trois bandes, la médiane plus étroite, les latérales plus grises, pulsescence janne, courte el éparse, mélangée de poils jaunes plus long's sul los còtés, loordẻe de poils noirs en dessus. Pectus gris a pubescence linchatre, scutellum de mème couleur que le thorax.

Ibdomen graml, jaune, portant une hande rentrale de tomentositi blanche composée de faches en forme triangulaire avec un sommet allongé yłui les réunit a lis tache friangulaire voisine. Lil tache du premier segment n'est pas toujours visible; la tache triangulaire du deuxième segment est presique obsolète, la bande a une faible bordure sur chaque còté. La pubescence du desius bst courte et noire, entremèlée de pubescence jaune plus épajsse sur les cótés, les flan's des deux premiers segments ont une tomentosití grise. Envers jaune aver des segmentations très étroitement blanches qui s'assombrissent vers l'apex. P’attes jaune rougeâtre, libias plus jaunes, hanehes rousertes d'une tomentosité grise, tarses antériours et extrème apex des tibias noirs; les autres tarses hrunàtres. La puliescence les hanches el des fémurs antérieurs est hlane dargent, plle est jaune et plus courte sur les autres, noiratre sur les tarses. Les quatre derniers arlicles des tarses antérieurs sont assez élargis. diles plus longues que l'ablomen, hyalines, ner' vures brunatres, estompées de jaune brun, stigma jitunatre.

Première cellule postérieure légèrement rétrécie.

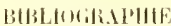

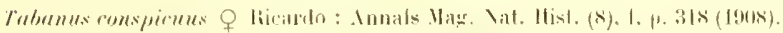

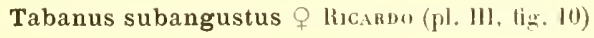

Le type provient de Alutshi sur le Niger Mripue occilentale). Cette enprea se rapproche de Tabomes nigrohimus of Ricarto, mais s'en distingue l'aburt pat' la bande frontale plus litrge, la forme de la callosité frontale, lablomen long et étroil sur lequel "se trouve une hande grise large d'un nillimetre et tris visible. La cellule marginale postérieure rótrérie du Tabuus nigrohirtus o like. ne l'est pas iei. (Collertion alu British Muséunu).

Longueur du rorps $17 \mathrm{~mm}$.

Tète à peine plus large que la thorax. Face rougeatre cunverte d'une tomentosité grise, plus jaun sur les joues oì quelipues soies blanches sont visibles, barbe blanc jaunitre. Palpes jaunes converts de pubescence noire, élirgis légirement ì la base, graluellement effiles en pointe. Intennes rouge brillanl. longues el minces, le premies article oblong el court: le denxième petil avor son angle externe prolonge, l'un et l'antre à pulsescence noire; le troisièm très long et très mince, la dent, petite, est placée près de la base, l'ilpex extréme est noir. Bande frontal brune, assez large, environ trois fois aussi longue que large sur 
toute sa hauteur, à borls parallieses, fallositi frontale brune et brillante, grande

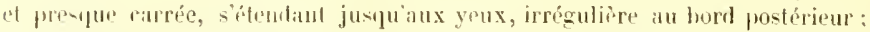
prolongée par une ligne qui s'élargit ru fuseau ef attcint presique lo verlex qui est lrun, tes coltiobordant la rallositi sont reponverts d'une tomentosité jaune. leux sans bandes ni dessins.

Thorax brun rouge it tomentosité grise à Iravers laquelle apparaissent des luandes noiratres ; rités el poitrine rougeâtres, couverts d'une lomentosité grise et de soips jaunes. pulsescenee des rotés du florax noire, scutellum Irunatere.

Andomen Joun rongeatro nun lrillant avec ume bande distincle et conlinue de tomentosité gris dont les eôlés sont droits; puhesence du dessus de l'alodomen noire, sauf la bande qui a une pubesrence blanche et les côlés une pubescence faume. Dessnus rougeitre, plus sombre à l'apex. Pattesbrunes; apex des fémurs jounàtre ; tibias rouge jaunitre, tibits intérieurs assombris à l'apex, tarses antrienrs en entier el extrémiti apicale des autres tarses bruns. Pubescence hanchatre sur les hanches, fì et lì noire et courte. Ailes plus longues que le rorps, lyailines, teintés de lrun jaunitre, nervares jaunes. stigma lorun jaunâtre. Toulde les cellules postrínieures largement ouvertes.

Quelyues sperimens viennent de l'blat de Nigeria of des lords du Niger. Le Tabanus subangustus o Rir.. sembir ètre boralisé dans cette région.

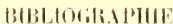

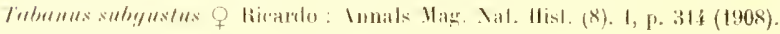

\section{Tabanus albostriatus $q$ Ricando.}

Le lye ct une autre femelle proviennent du Transval (Ross. 97-49) el appartiennent is la collection du British Museum.

Espee noire portant uno bande médiane blanclse sur l'abdomen et des taches latibales grises. Antrnnes noiratres, palpes frès minees, jaune pîle. Patles jaunes, fémurs plus sombres. Yeux très ligèrement pubesrents. Liles teintées de brum portant un appendice.

Longueur $13 \mathrm{~mm}$.

Tète plus large que le thorax: face grise à rare pulvescence blanche, quelques poils noirs au-dessous des antennes. Barbe btanche. Palpes d'un jaune transluciulo pâte, courbes, très minces et se prolongeant en une longue pointe, légèremont ípaissis à la base, il pulesonce composée de courts poils noirs. Intennes minces à dent très obtuse, ne formant qu'un angle obtus au lieu d'une point", premier article jannatre, couvert d'une tomentosité grise ef de poils noirs, it peine ryathiforme; serond, petit, jaunatre à pulescence noire: troisiome noir, rouge it son extrème base. Bande frontale grise, eourte, environ quatre fois aussi haute que large, à crités parallèles, frortanl une petite callosité étroite, triangulaire, de couleur lirun el yui se continue par une lignétroite juspu’au vertex ; la pubescence noire de la bande frontale devient grise au vertex. Yeux sans 


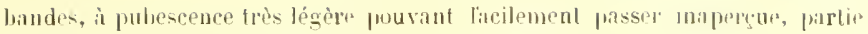
postérieure lle la tèle à poils blines.

Thorax brun noiràtre, recouvert l'une pubescenfe grise, eourle ef modípimenl dense sur laquelle applaraissent trois bandes grises; hanck gris it poils noirs plus longs : peetus brun couvert de poils noirs: une toulte de poils blants atu-dessus de loriwine des ailes. siculellum de même couleur el de môme vestilur. fue le thorax.

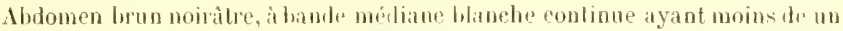
millimetre de largeur et lonjours atroilement limitér. Cellr bande s'álargit fégèrement à la base du deuxième segunent. Sur les rités des seeond, troisième et ¡uitrième segments se voient des laches grises mal définies mais dislincles qui ne dépassent pas le flane mais qui romprennenl toute la bautem de chacun dru segments. l'ubescence du dessus courte, "paisse, noire avee trois bandes de poils gris blanchâtre qui se trouvent sm la bande mádiane et les taches latérales, on peut aisénent s'en rendre compte en regardinat l'inswete tangentiellement. Bords latéraux de l'abdomen transparents aver mo pubescence gris blanchâtre.

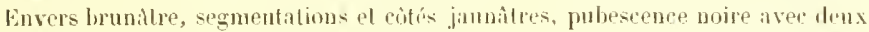
bandes latériles gris blanchàtre. Il tuchus noires cunvertes diune tomenlositit grise et d'une pubescence noire ef llan he, fémurs antérienrs noiritres. fémurs intrimédiaires el postérieurs d'un rougeatre sombre, ayant une fomenlosité grise ef une pubescence blanchître ; tiluas jaunes, les antérieurs rongepilres à lapex et jaunes à la base avec une pulesence blanche ; outre celte coloralion clitire des tibias antérieurs il leur base, las fibias inlerméliaires ont une pubeseence blanche ì leur base et noire à l'apex, libias postérieurs à pubesenep noire. Tarses antérieurs et inlerméliaires lorunàless, tarses postérieurs jaunex: fomportant une pubescence noire. Ailes hyalines, légèrement teintées de hrun sur Jes nervures longitudinales, le bonl rxterne, lextrémité des eellules basilaires or la nervure anale. Appendice distinel, nervores lrunes, stigma petit, brun jiunatre.

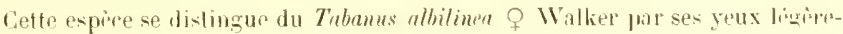
ment pubescents, labdomen étroit of phus sumber la faille moindre, des antennes plus fonfées, la callosité frontale noirithr au lieu de lirun rougeatre, les fémurs plus sombres et les palpes plus minces.

Celle espèce est localisée au Transvial.

\section{pillitorititille}

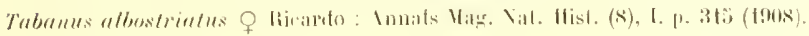

\section{Tabanus obscurestriatus $q$ lícando}

Le type femelle provient du Congo ( 18 novembre 1904) pt fait pirlis de la colleclion de Brilish Muséun.

P'elite expèce rougentre avee une lanule médiane noire ef les ailes claires.

Longuevr $12 \mathrm{~mm}$. 
Têtr moyenue, plus litror que le thorax ; fire recouverte d'une tomentosité grisp et alune fubescence l'un blanc d'argent ; palpes rougeattres à pubescence hlanche, érais à la bane et grarluellemenl attínués en une pointe. Barbeblanche. Antennes minces rouguatres. ì dent trís faible, premier et deuxième artirles ì pulescence noire. Bandr fronlale mviron cind fois aussi haute que large, a cotés fillallibes portant all-dessus tes antennes un triangle frontal, convexe, brillant. finne runcente : caltosite frontale de men couleur, de forme presifue carrée, attrignant los yeux et contimuée par une large bande alteignant presque le vertex, bande frontale recouverte d'une tomentosité jaunâtre, plus sombre sur le velex. leux san handes. Thorax hunitre portant deux taches latéralen faures, hordées de noir, touten inlislincles, dessus à tomentosité grise et courte pubesrence fanve, wités ì poils noirs ef tomentosité jaunâtre, pectus ì pubescence banche. scutillum de mene coulem que le thorax.

Ablomen rongeatre portant une bande noire qui commence au premier seg-

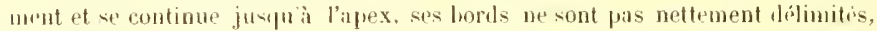
pulnsceme du dessus noire. Wuatrìme, rinquième et sixième segments jaundtr's a poils fauves sur les rités, seplime segment entièrement noir. Envers

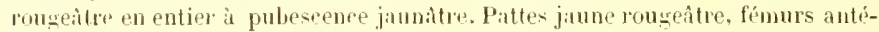
rients bruns ì pulnescence noire, tibias janne paile ì pubescence lilanche, apex des tibias antérieurs hrums. Tarses antériemrs lruns. Ailes claires, jaune pâle ì leur extrémité externe, nervures et stigma jaunatres. Appendice court et épais.

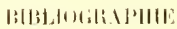

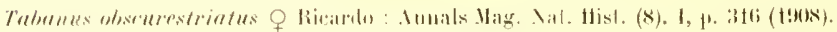

\section{Tabanus nigrostriatus f licakdo.}

Le type femolle provient des plateaux dr Zomba (Nyasaland). Collection du British lluseum.

Espèce complètement distincte de toutes les autres espèces africaines, remarfuable par un long abdomen itroit d couleur chatain portant une bande médiane noire distincte. Ailes hyalines teintées de brun, appendiculées halituellement, mais ce caractère paraît ètre variable.

Longueur $17 \mathrm{~mm}$. I, 2 .

Thte plus large que le thorax, fare recouverte le poils blanes et d'une tomentusitŕ blane grisitre, la coulenr fondamentale est plus apparente sur les joues. Barbe jaune blanchatre, palpes jaun's à pubescence noire, fongs, ì peine plus f́largis a leur hase, terminés par une pointe obtuse. Intennes rouges, parfois plus sombres i lapex (dams un spirimen, la base du troisième article est rouge), dent modérément saillante; premier article oblong, second petit, l'un et lautre à pubescence noire Bande frontale lomnatre, quatre fois aussi haute que large à la base et légirement rétrérim antérieurement, triangle frontal rougeâtre arec une tomentosité gris-jaune autour de la base des antennes. 
Ciallosití frontale brun sombre, lorillante, convexe, atteignant i peine les yeux, prolongre par une ligne stroitr qui s’élargit en forme de fuseau et s'étend jus'juitu vertex, da tomentosité qui le lorde est jaunatre. Veux glabres sans lessins ni landes.

Thorax brun rongeitre aver des trices de bandes noires, formant un bande médiane laroge of deux biludes latírales ; la couleur rouge du fond paraîl entre les bandes en lignes átroites; dessus à tomentosité grise; côtés et peetus lorun rougeatre ic fomentositis grise, seutellum noiràtre.

Abdomen brum rougeatre clair, segmentations tris fablement jamms, loande noire commenģant au second sexment, composée l'une tache noire oblongrue sur "hitpue seguent, res taches offrent l'aspect d'une étroitre bande noirr continue inn tathe ni tomentusiti grise. Premier segment portant une tach' méliane noire indistincte, la bande oflre sit plus grande largeur sur le cinquieme th te sixiòme segments. Septiòme segmont entièrement moir. Bords litéraux du cimquieme et du sixiome segments d'un jaune transparent, pubescence du dessus noire ef rourte, beancoup plus épaisse sur les flanes, parfois montrant iles traces te pubescence jaune sur les segmentations; septieme segment it longs poil noirs. Envers de mème conleur, sans binde, apex noir. Pattes brun noiritre, tibias de mème couleur que l'alodomen, tilias antérirurs et tarses loruns ì l'apex. Pubescence des hanclres et des fémurs blanchìtre, reux-ci à tomentosité blanchitre; quelques proils jatunes sur les tihias antérieurs, partout ailleurs lit pubescence est noire, courte et peu distinetr. Ailes hyalines, teintées de brun, trìs sombres au bord antérieur ; appendice court, épais et peu distinct (mantuant parfois), stigma ef nervures brunes, toutes les cellules largement ouvertes.

Tous les spécimens connus de Tabrnus uigrostriatus: of lic. proviennent du Nyasiland (Afrique orientile).

\section{BIBLWMIIUHI:}

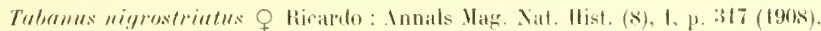

\section{Tabanus unilineatus of Löw}

"Tabanus cinereus abdominis linea longitudinali alba, alarum stigma nigri" cans (Löw)."

"Ce genre appartient au groupe qui contient Tabuus oucidentalis Pab, pt Tabu" mus truiotes Wiedemann, quoijue ces deux espreces soient américaines. Tirille " intermédiaire entre ces deux espuces. La callosité frontale est de mème forme " uque dans les deux ispères précédentes, mais elle est de colorition brun clair "et plus gronse que chez Tabanus tomioles W.

"Il ditfèr en outre de ces deux espices par les derniers segments ablomi" Daux noiratreset par ses ailes hyalines, un peu rendrées et ì hord externe " presque noir. Tibias antérieurs plus minees que dans les deux autres espèces " et brun sombre presque jusqu'is lit base, fantis que le Trabums tomiotes th le "Tabanus occidentulis ont leur tires basilane blanchitre (La faille n'est donnée 
"quapproximativement is ceux du mavais itat de l'exemphares). Longueur

" $11 \mathrm{~mm}$., du Wozanbique."

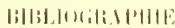

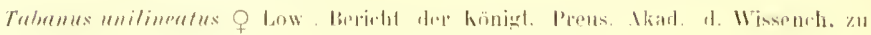
liotin. Isiz. 1. 6is.

Tabanus Laverani o surcove $11.80 \mathrm{fig} .1 /$ )

Type: un spremen fentlle provenant du bis Rio Nunez, Guiné frangaise. en lonk et communique jar y. Je Jor Laverax, Yembre de l'Institut.

Trois antres femelles de la mèm origine. Collection du Museum. Le British Juseum frossète deux spécimens de rette espèce, provenant l'un de sierraLeone, Lingstown (4,04, Yajor F. surtur), le sreoml a été recueilli en Gambie par le 1) Hopkixsox 1). . O. (1906).

Le Tabums Laveruni o surconf existait dins lin collection du Vuséum sous le nom de Tabams unilinpatus Lrew. Ces deux espuces, quoique voisines. sout cependant distinctes. Longueur du corpes: $10 \mathrm{~mm}$. 1/2 i 12 $13 \mathrm{~m}$.

leux sins landes ni drasins, glaloro, bande frontitle six fois ausi haule que large, blanchître; portant une callosité oblongue, rougeâtre, prolongée prar une

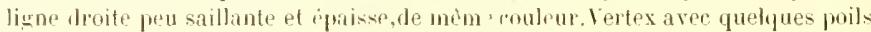
noirs. Bord postériemr de la tète cilie fe courtes soies lilanches, triangle frontal l'un banc jaunâtre. Intennes ronge fauce à extrémité apicale concolore; premier article testacé, à pubescence noire, peu dense à la partie supériure. P.ilpes renllés, d'un blane jaunattre à pulesence lilanche mélangée de poils noirs sur

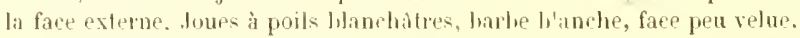

Thorax loun noinhtre portant deux landes médian's et deux landes latérales l.lane jaunàtre, entières et atteignant le sommet du thorax, à puluescence ratre et toneolore. scutellum bordé par une tumentositi grise; llanes i proils épats. pectur cendré à pulvescence blanchâtre et nolle.

1Mlomen ronge Jrun dans toute sa partie supérieure, portant sur les six premiers sements une bande blanche étroitr de largeur constante. La puloscence de l'aldomen est noire sur les partir. hrunes et augmente de lensité vor's l'apex, elle est blanclie sur la bande, les poils noirs s'étendent ceprendant sur cette lande lans les deux derniers segments; les llancs portent de chaque cité une lande blanche semblable. La puhescen's des deux derniers segments ventraux est banche et mélangée de nombreux poils noirs. Pattes d'un rougeitre pâle ì pulescence blanche, tarses bruns it pulsescence concolore. Ailes hyalines ì stigma jaune; disque des balanciers jaune.

Cette espèce est intermédiaire entre Tabrmus unitreniatus o Ricardo et Trbanus unilineatus o Lrew, Elle ditlère de Tabanus unitrmiatus $\mathrm{R}$. par ta forme de la 
callosite frontale, les patpes renflés, les antemes à exlrímilf́ concotore, les seg. ments ablominaux non bordés de blane, l'apex non rembruni.

Le Tabunus Laverani $Q$ surcouf so distingue du Tabunns unilineatus lem par l'apex noirître et les ailes hyalines il stigma jaunatre, landis que te Tubunus unilineatus Low a le hord externe des aites presque noir.

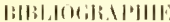

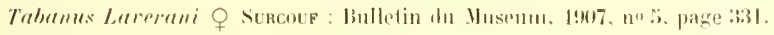




\section{HUITILNE GROLPE}

Tableav DIChotomige

Siles hyalines . . . . . . . . . .

I $\left\{\begin{array}{l}\text { liles teintées de brun ou pstompées aux } \\ \text { nervures transverses }\end{array}\right.$

Abdomen à bande médiane et taches latérilles distinctes. . . . . . . . . ches latérales indistinctes.

Bandr médiane de l’abdomen droite.continue, formée de taches rectangulaires l,anchâtres. Intennes testacées, 1 : 1 2-18 $\mathrm{mm}$. Antennes noires, $15 \mathrm{l} / \mathbf{4} \mathrm{mm}$.

Bande médiane composée de taches trapézoülales. Intennes testacées, $17-19 \mathrm{~mm}$.

Rande composée du $2^{2}$ an $6^{\circ}$ sezment de taches triangulaires, atteignant le bord antérieur de chaque segnent, à bases étroites, còtés dn triangle un peu échancrés vers le ventre. Cuisses noires à apex rougeatres, tarses antérieur noirs. Antennes sombres, $161 / 2-18 \mathrm{~mm}$. . . . . . . . . .

diles hyalines à hord costal lorun noirâtre, abdomen brun à hande médiane composée de triangles blanchatres, palpes hunitre pâte, $9 \mathrm{~mm}$.

Espèce brun rougeâtre, très déliée, à aludomen pointu à l'apex: taches médianes triangulaires visibles en regardant l'inzecte jpar derrière, taches latérales indistincles. ronge, $14 \mathrm{~mm}$. .

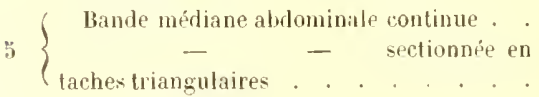

T. styitturius o Macq.

T. timiolu o P. B.

T. sugens of Wied.

T. socius of Walker.

T. namus o Wied.

T. coniformis o Ric. 


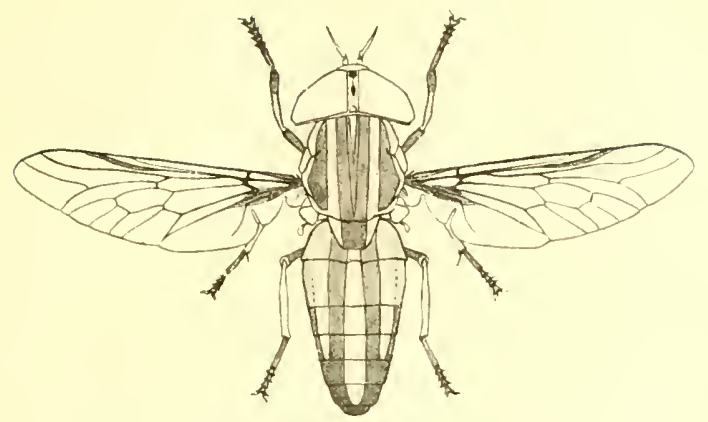

Fig. 15. - Tabanus tomiolı O Palisot de Beauvols

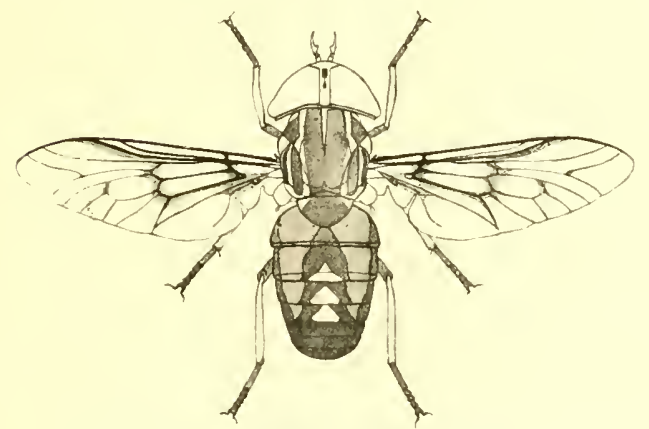

Fin. 16. - Tabanus frulerms $O$ Macevart

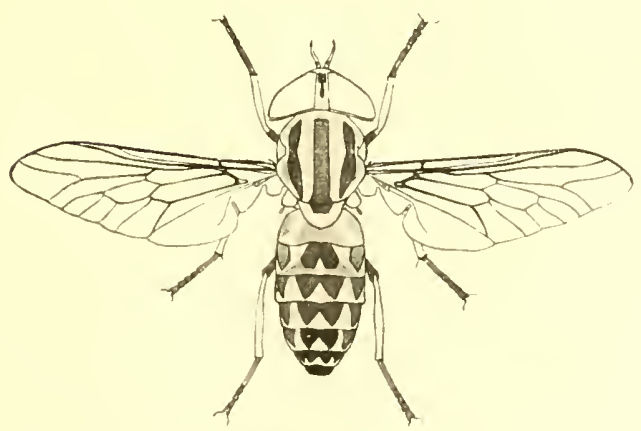

Fig. 17. - Tubanus quadrisignalus O Ricardo 
Ailes hyalines, toutes les nervures estompées de brun; aldomen d'aspect soyeux à bande médiane étroite continuediun jaune grisatre, les trois derniers sezments abulominaux noiratres, 15 is 16 mm. . . . . diles teinties de brun vers le lroril costal, aldomen chitain clair à lande médiane étroite jaune, flerniers segments non remlorunix, 1:-1:3 $\mathrm{mm}$.

T. spriciventrix $\bigcirc \mathrm{La}+\mathrm{w}$.

T. Ricardoe $q$ surcouf.
8

T. qualrisigmutus Q Ricardo.

T. distinch $Q$ Iticardo.

T. sticticolis $q$ Surcouf.

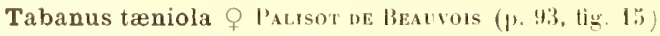

Le Tabumus tomiolı O t'. B. est un des taons les plus communs de l'Ifrique tropicale, il a été suecessivement décrit sous les noms de:

Tabanus taniola o P. B.

Tabumus grumensis $Q$ Wiedenaxs.

Tubruns subelongatus $Q$ llaceuart.

Tabanus dorsivitta O WALKEn.

Tubumus macrops OT WaLken.

Trbornus lomgitndinalis o Lew.

Tabanus proximns $Q$ CORTI.

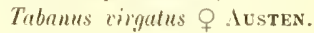




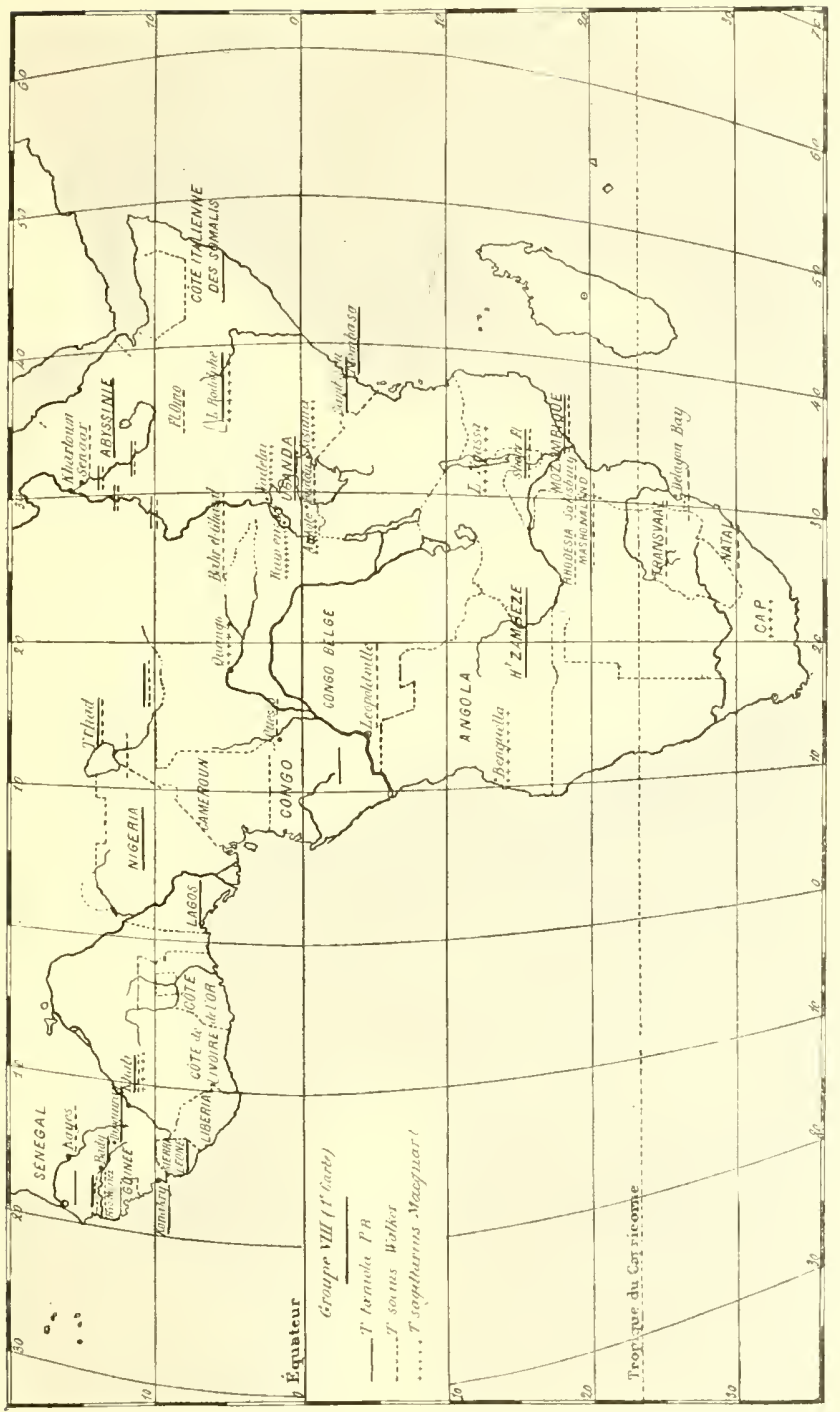


Le Itusín de I'iris possède un exemplaire femelle de Tabanus tomiola q P. B.

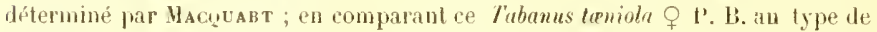
Tabams dorsiritta $q$ Watker on se rend compte qu'ils sont identiques. Le dessin de Palisot de lobaurois pst tris inexact, l'abdomen est trop noir et les bandes latérales sont trop distinctement définies, tandis que d’après sa deseription l'abdomen est brun au milien, lo corps d'un gris brun et les bandes latérales grises. WiEdemanx yui na certainement pas connu le type, copie simplement la description dr P. B. Le Tabunus dorsirillu o Walken a été renommé Tabanus

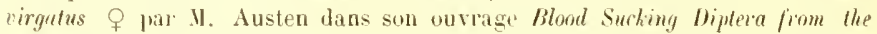
Soudau, dans le second mémoire du Gordon College de khartoum en 1!06, parep que Walkeb arait déji employé le nom de Tabunus dorsivitt pour une espèce de patrie incertaine, probablement originaire de l'Amériłue du sud. tiespice de la Cafrerie décrite par Macipuart sous be nom de Tabmus plonyutus 0 est sans aucun doute l'espèce lécrite par Palısot de liearvors et plus tard par Watker, le type de ce dernier concorde dans toutes ses particularités avec la drseription de Macyuart à l'exeption de ta callosité frontale qui est plutỏt brun-rouge que noire. Le Tubanus guimeensis $q$ le Guinée, décrit par Wiederaxs peutêtre identique au Tabanus Lwniola o P. B.; quant au Tuhanus ynimpensis or Wiedemann, i] roit appartenir à une autre espèce. En effet Wiedenann dit que les yeux ont deux bandes rougeatres qui se rejoignent ver le hord extérieur, ceci peut s'ajpliquer an mâte seulement et non à la femelle, car aucune femelle de ce groupe ne porte de bandes, mais il arrive pu' des mâles possèdent des bandes oculaires lorsque les femelles en sont privées.

Wiedemann nous en donne un exemple dans to cas de Tahame occidentalis Wied., espèce du Brésil (roir Aussereurop., Zweifl, Inseliten, I, 1). 171), et HacQuar (in Diptern exolicn, 1, p. 117), remarque que des landes existent parfoik sur les yeux des deux sexes et parfois seulement chez les matles. Nons pourons citer comme exemple de ce dernier cas : Tabanus fulvus Meigen, Tabums alexandrinus Wiedmann, Tabanus lineole Fabricius et Tabamus carolinensis Macquart. Irrauer en décrivant Tabanus paradocns Joennicke et Tabums miki Brauer remarqu" que les lrandes se rencontrent chez ces mites seulement(Denkr. Acak. Wien. XLH, I). 179-196 (1888).

Le Tabamus froximus Corti, de l'Abyssinie, que les anteurs rapportent it une variété de Tabamus subelongalus Macquart ne nous est pas connu, si on le maintient comme sous-espèce, ce nom doil ètre changé. car il al iti prérédemment employé par Walker pour uncespice du Mexique.

Description de Tabanus tæniola P. B. d'après l'exemplaire de Macevant (eollertion du Muséum)

Longueul $15 \mathrm{~mm} .1 / 2$.

Cette femelle prise par lteudelot au Sénégal en 1830 porte à son épingle le n0 249 bis et la mention: Tabamus lmiola de la main de Hacquart.

Tête plus large que le thorax, yeux glabres, bronzés, sans bandes; face ef bande frontale recouverte d'une tomentosité blanchâtre, pubescence des joues et 


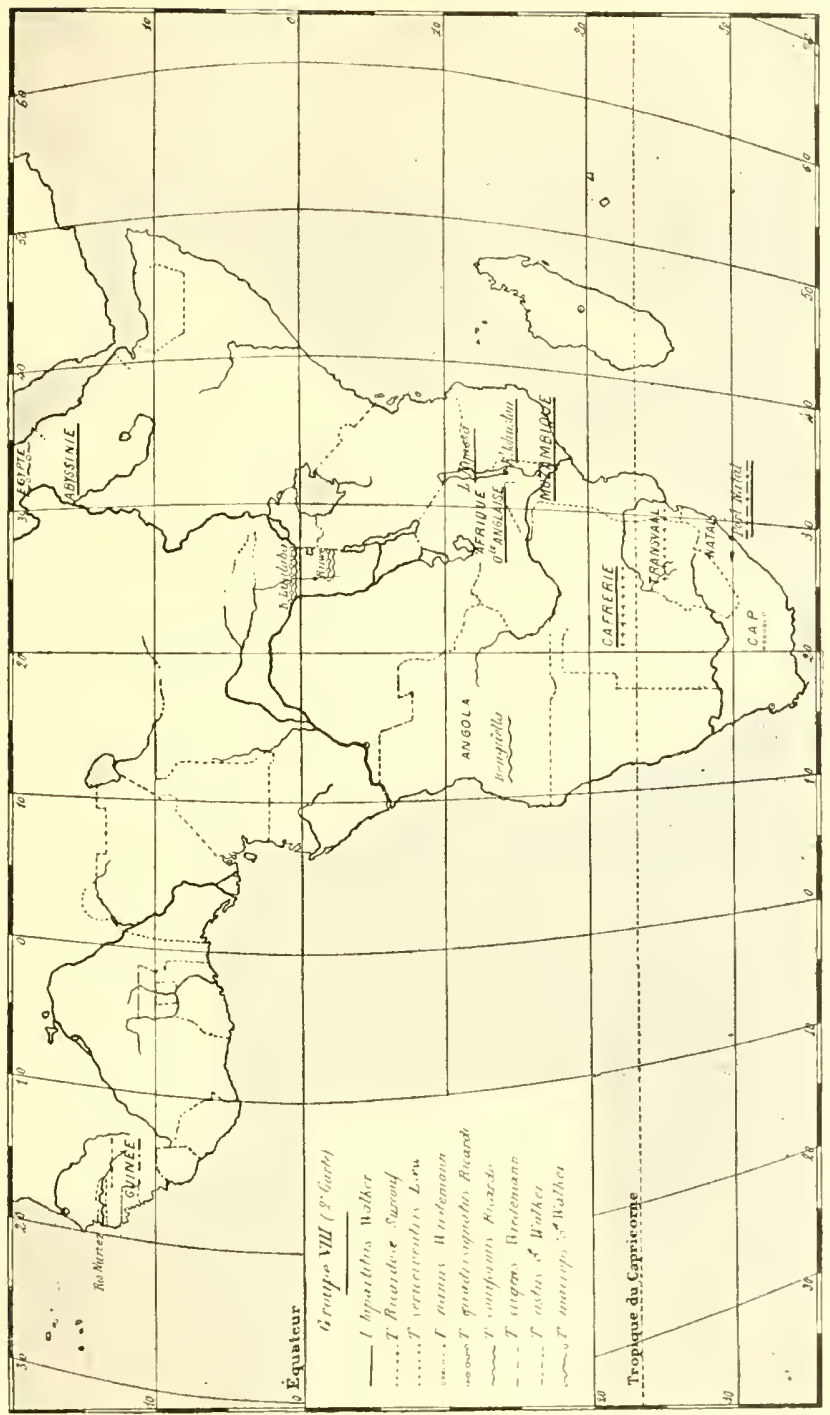


barlw banche. lande frontale assez large, environ quatre fois auss haute que large, couverte d'une tomentosité gris jaunatre, cottes do la lande ligimement

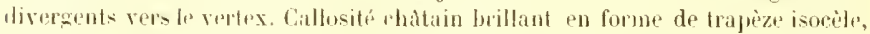
lit base n’attrignant jas pactement le borl des yeux, base supérieure occupant

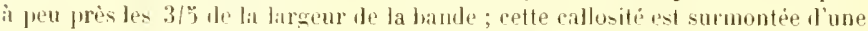
ligne élroite de mème coulemr qui se dilate ten l'usean 1 t se termine un peu an

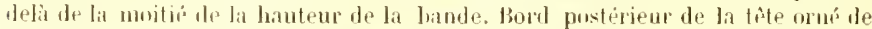
courts joils blanes dresses. dutenne- premier article testacé à pubescenere blanche, yuelques poils noirs it l'angle apical externe, bord apical formant une ligne droite: secont article testacé ì pulvescenre luanche of une couronne de poils noirs à l'extrémité apicale: troisiome artich testacé ì l'extrème base (te reste de

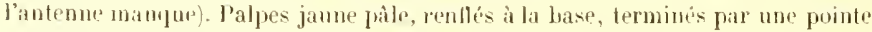
allongér à poils noirs et quelques poils hanis a la base. Trompe lrune, stylets mandibulaires testacés.

Thorax (démulé) rougeatre arec des bandes longitulinales très sombres et des tranes de tommotoxite blandre. l'ectur rougeitre i tomentosite blanche et de Jongs froils mous, blanchitres, cer poils sont noiratres sur les flanrs: une toulfe de poils blanes au-dessus de l'origine des ailes. Scutellum brun rougeâtre ¿̀ tomerblosité blanrlie.

Aldomen lanu-rougeatre portant en son milien une bande fongitudinale d'un

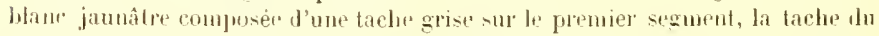

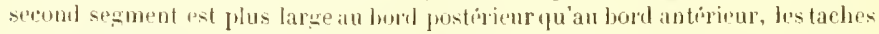
lu troisime, Ifuatrième et cinjuieme segments font suits aux taches des deux jremiers secoments it ronservent une mène largeur, le bord externe dre chaque tache est ligèrment convexr: sixième segment à tache trìs rédnite. La pubesene dr

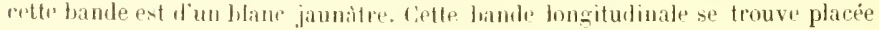

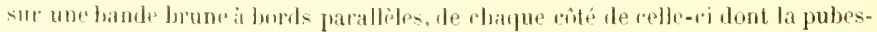
ernce est noine existe une hande blane jaunitre qui se tromine an sixiems segment et alteint preque le flanc brunatre de l'abdrmen. Cette zone tai se termine en pointe an sixirme segment recouve toute la région latcrale de la base

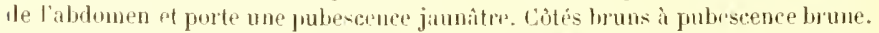
llernir segment brun ì puhesienre sombro. lessous rougeâtre ì tomensité hanche et quedques poils blanes au hord postirieur drs segments, les deux derniers ont une pubescence noire, on y listingue une bande médiane rongeàtre. Jlinches et cuisses reourres d'une tomensité blanchitre, denne, leur donnant un alspect rendre: tibias rougeatre rlair a tomensité of pubeseenet blanches; extremité apicate dres tilias antérieurs noire, tarses brun noiratre à pubescence noire. les larses postéricurs plus clairs. Ailes hyalines, bord externe el deuxieme mervore longitudinale bruns, le stigma et les autres nervures jaunatres. Balanr.jers it disclue pougestle.

Lia collection du Muséum compreml te nomhenx spécimens provenant de diverses localités.

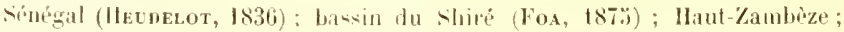

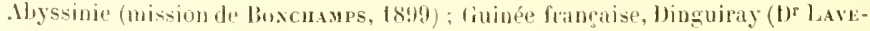
Rax); llatut-Niger et Bénour (Wand, 189.i); Guinép frantgaise, Benty (1)r Laverax, 1!904); Gambie, Sainte-Yarie de lathurst (E. Brovrt, 1906); Congu (1)yowski, 1894); lit du Dakoto, lladen, Pays des somalis a sud de llarrar 


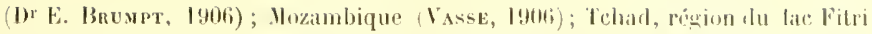
(L' Lebas, 1904); Guinie lramģaise, Konatkry (1) Tautas, lanï); région d'ouesso (1) (inavot); lac liodolphe (1)r Brosur, t:202).

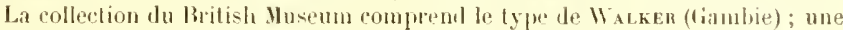
longue série de femelles du Laqus; Sierra-Leone; Nond Nigeria ; dambir ; Ionbasa (Afripue orientale), Vyasaland, Zambize, Nil Bleu Simnaar) et Nil liane. Une note épinglíe ì un spécimen provenant du Vord le Nigeria porte celte mention : "l'ris aux limpes la nuit en essaims " W. F. li.

The autre note de sennar dit: "Cette espeee aussi hien que le Tahanus luthps "Matuart suce le sans. Dilles sont tres nuisibles et ginent considérablempnten "laissant une cettaine irritation is lendroit de leurs pipures ». Cilptain II. II. W. Duxx. Bezzi sighale rette espèce, soun lt nom de Tubamus subelongatus Nacquart, du Somalilanel (.1nnal. mus. cie. Genore sirie 2, tome XXXII, p. 18.3.

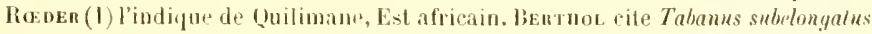
de Inhambane (Est africain) (2). Le capilane t'Lowen remarque que le Tabunus dorsicitta $q$ Walker est appeté "serool Fy " par les naturels et qu'on te rencontre depuis Ondiuman jusqu'it Faschoda sur le Nil Blane, le Bahr el Gihazal et le Zeraf. 11 rapporte aussi qu'il pique (3).

Cette espèce a donc une large distribution en lfripue ef cela rend d'autant plus surprenant l'absence de tout mile dans les ditlérentes collections d'Europe.

Les exemplaires de l'lganda ainsi que ceux du Kilimandjaro et du Zambèze oul des poils noirs sur les tibiats postérieurs, mélitugés aux poils lilanes de la pilusite normalement pilte, il y a auns de nombreux poils noirs aux palpes.

Nous donnons les descriptions originales des expèces que nous réunissons atu Tabamus tenioh \& 1. 13. Ces descriptions ont ité traduites exactentent.

\section{Tubunus subelongutus o Hacevar?}

" Mlongre, noiratre. Thorax à lignes blanches. Mblomen à bande dowale

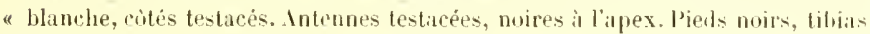
roux

"Longueur 7 l. Q. Palpes d'un jaune pàte. Fitce et devant du liont bin" chattres; le reste de ce dernior d'un gris rousuitre, à callosite noire. Intennes: " les deux premiers articles of la bise du froisime lestarés; te pate noir. Yux " nus. Thorax noiratre à lignes de duvet banchitre. Abdomen it bamde dorsalas "hlanchatre, bordée d'ume band" noiritre; rités textreés, ventr" faure; les " Irois derniers segments noiritres. Cuisses neires ; jamles fauves : antrirures

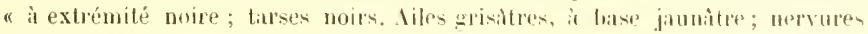
"normales."

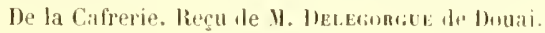

(1) Roeder : Jahrb. Hambury. Wissenssh. Instaltt. A, p. 2 (1893),

(2) Berthol: .yem. Acart. Sciene. Bologne. XII, p. 16 (186i-2).

(3) C. Flower: Proceed. Zool. Sor, Lindon. 1900, p, anI. 


\section{Tubranes dorsivilla O WALKEIt}

"Niger, sulutus albido tomentosus; caput ferruginenm; oculi cenei; palpi " albidi; antenna ferrugine. parver, arcuatie, vix dilatitæ, apice nigre, thoracis " latera fulva; abdonen fulvum, vitta testacea ferroginea marginata basi cine" reat, apice piceum, tibia fulva; alic cinerea.

"Noir, tète ferrugineuse aree une pubescence blanche courte an avant et en "dessous. Jeux Lronzís, glabres, composés de trèsuetites faceltes. lalpesblan.

- châtres. Antennes ferrugineuses, pretites, courbes, noires vers l'extrómité;

" troisieme article à peine dilité mais formant un petit angle légèrement aigu.

"Thorax conleur de tan de chaque cité. Abdomen couleur de tan avec une

" large hande ferrugineuse qui enferme une bande testacée pàle, cette dernière

- rendrúe à la base; extrémité couleur de poix liles grisîtres, nervures noires.

- Nervure cubitale simple formant un angle obtus près de sat hase; nervure

"sub-anale rejoignant lia nale à quelque distance du burd. " Longueur du corps: fi lignes : des ailes: Il lignes.

fiambie.

\section{Tabanus quinerensis or W WEDEMANx}

" Birun, thorax blanchâtre, abdomen à trois landes clistinctes.

Veux it deux bandes.

Longueur li lignes 1/2. - Civinér.

Illié à Tabanus striatus Fabricius.

"Intennes terrugineuses, apex du troisime article largement brun. Epistome " blane jamnattre avee un duvet jaunâtre. Barbe lianche. Palpes jaunc-rhanois "clair. Front nu-dessus des antemnes largement jamne ocre et brunatre "au-dessus vers l'angle des yeux, avee une ligne longitudinale ratuse. Yeux "bruns aver deux bandes rongeitres convermant vers l'extérienr. Thorax brun " is pulsesence blanche dans une dirertion constante. I travers la pubescence "s'entrevoient cing taches en forme de bandes rougeitres. Triangle antérieur de " l'aile jaunâtre. Ex́usson brun rongeitre. cilié de blanchâtre. Còtés du thorax "en entior gris cendré à jubeseence blinche. Iblomen lorun avee trois handes "et le bord des côtés lianc. La bande médiant va l'un bout ì l'autre sans être "interrompue. A la sixiome regmentation soudainement elle se rétrócit et ne "s'étend pas justgüi l'atpex. Bindes littérales preague pas interrompues deve"nant coupres conrt au bord externe. La couleur foncière entre les bandes est "ferrugineuse. Ventre brun-romse, cilié de blan sur les bords, noiritte il l'apex. "Dins la jartie endauque bianchitre se trouse de chaque coté sur chaque segment une ligne diagunale d" jwint brillants. Incisions ciliées de thlanchitre. diles entierment hyalines aver les nervares brunes et le bord rustal jau" nâtre. Cuisses brunes à pubescence blanche, tibias jaune lorunàtre, les antérieurs avec des poils hlancs, les jostérieurs ciliés de brun extŕrieurement. Base " des tarses brune ou en grande partie noir brunatre. 
" Femelle brun plus clair ou lrunâtre. Thorax tacheté de Bhanchâtre. Bamle " míliane de l'ablomen non raceourcie, s’étrulent jusqüa l'apex du sixime "segment, Bandes latirals's au contraire sarrènt avant le milieu et se confon"dant avec la bordure latirale. Ventre ferrugineux, symétrique. Jambes plus " claires. Tjbias posterinur's non ciliés de hrun. Muséum Iupérial. "

\section{Tahame longatudinalis o Law}

Cet inserte a été probablement dícrit par Lew sur un spécimen uniqur on 18.2.2, arant lit publieation de sis nouvelles isprices du sul de l'Ifrique (1860). Law remarque expressement que Tabanus lonyitudinalis est distinet li" Tabrmus subelongatus Vacquart, Tubanus sugens Wiedmann et Tabanus quimensis Wiedemann. Sa deseription semble se rapporter exactement an Tabanns tanioln I'. B. Löw mentionne quatre bandes brun-noir sur l'abromen et les bandes latróraldé moins distinetes et quelque peu plus pàles en avant; or ces bandes existent sur des spécimens de Tabanus teniola P'. B. "Iu British Musem de mème tue la coulfur foncière de l'alutomen est plus ou moins limitée par les bandes latírales jaunes. Dans un spócimen de l'Africjue oriontale ces handes atteignent. le premier segment, habitupllenent elles s'artêtent au fuatrième segment, lat seule autre dilférence a relever dans la description est lis coloration de la bass les articles antennatres, law les décrit gris-noir, tandis que dans los spécimens de Pariset de londres ees articles sont ou testacés on d'un testacé noiratre.

lour le moment nous puuvous considérer Tabamus lonyitudinalis Law roum

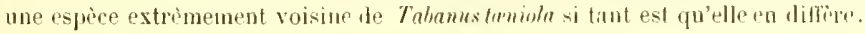

DESEMPTION WE LAR

"Thbanus cinerens. Ibdomen subrufeseens striis quatuor longitudinabus " obsrure brunneis, frons femina callo ollongo linearue fusiformi signati. "

Partie inférieure de la face à pubesene blanchatre. Front d'un jaune blanchatre jusqu"i l'angle des yeux, où coumence une callositŕ allongée, revtanculaire en dessus de laquelle il y a une ligne plus sumbre en forme de fuseau. La couleur de lit partie parallele du front est gris jaunatre. l'renier article antennaire gris noiratre de meme que le secoml (troisime manque); te tholix ad gris rendré clair avere trois latgess lignes longitudinales gris bountre dont la médiane est partagée à sal partie antérieure par une ligne lougitudinale eris clair, pendant que lans lit partie postérieure de chaque bande latérale il y a une ligne longitudinale prespue gris rougeàtre. Flancs gris cemdré clitir aver um pubescence blanche. Aldomen rougeatre avec quatre lignes longitudinales bron

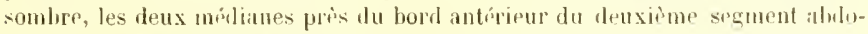

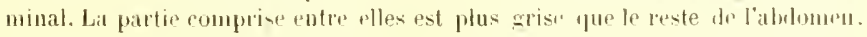
Les bandes laterales w' sunt pas si nettement ditimites et sont un peu convergentes dans la partie antérieure. Elles se ríunissent aux bandes médianes sur le sixième segment et déji sur le cinquìme elles ne sont séparrée que par 
un ligne longitudinate. Le dernier artirte de labdomen pst brun-noir de chaque rite $e^{2}$ sous le ventre les deux derniers soments sont fortement coloris an brun.

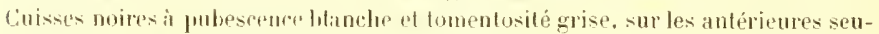
les il y a des prils mirs. G'axtrumité apicale pst jaune rougeátre. Tibias jaune rongeâth" lextrémité dẹ libias antérirurs est noire sur un asse\% grand espace.

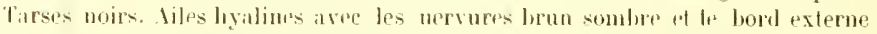

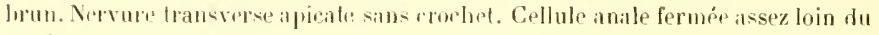
borl.

\section{Trabanus virgatus o lostex}

C.e symomyme a the donné par ll. Austex à cause du donble amploi du mom de Tabanus dorsicitha. Le Tubanus dorsiritta o Walker synonyme de Tabanus titniola of P. B. n'est pats le mème que le Tahamus dorsivitta o Walker décrit lians son ouvrage Diptera Stumdersiana, patt. I (18:30), p. 35.

M. Austex en donne lit diagnose suivante:

"Thorax grisitre avec des bitules plus sombers, abdomen rougeàtre, bande " médiane longitudinate gris latir, jambes jaunâtres ì tarses bruns. Assez " commun dins lesoudan anglo-bgyptien, sur le Nil Blanc (1900, major H. W. " louxs), région de sénatar sur le Nil Bleu (septembre 1902). Lire d"habilat "romprenant lin Nigeria, los antres régions de l'A frique occilentale at s'étena dant itu protertorit Est africain at il l'Afrique centrale anglaise ".

\section{Tabremes macropls or WALKEI}

Dewx mâtes ont dé ain-i nommés par. Walker, dont un taon sans lète provenant d'Egypte, communiqué par sir li. Wickssos, l'autre mile d'une localité

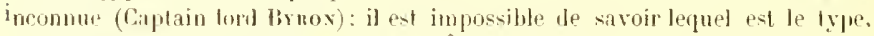
l'un ef l'autre sont probablement des cotypes, ils sont du reste illentipues.

Espire lirun rougeitre de taille moyenne. Abdomen it leux bandes distinctes brunes qui occupent toute la fongueur do l'abdomen of des bandes latérates frunes indistinctes. difes hyalines. lattes rougeatres, longueur du rorps $17 \mathrm{~mm}$.

Ifile. - Face "ouvert"d'unt tomentosité lrun rongeàtre d'une puhescence jitune palle. Pirlpes jatunes, plus fâles ì l'ajex, à pubescence blanche. Intennes: les deux promiers ardicles rougeitres a pubescence blanche, troisieme article manyuant. Wallee le dicrit romme lirrugineux. Triangle de la bande frontale

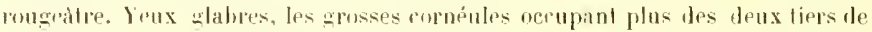

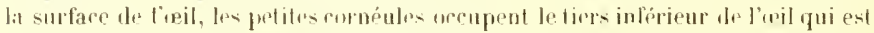
traversé parr une litrge bande jaunatre allant de la latse des yeux vers le virtex, elle est hordiu par une bande noire ef an deli par une autre bande jaunitre qui form. le borl externe des grandes corneules. Thorax brunatre à tomentosite fauve el d'indistinctes bandes nuires. Scutellum rougeâtre.

Aldomen jaune rougeatre ì hantes médianes, brunes, atteignant la longueur 


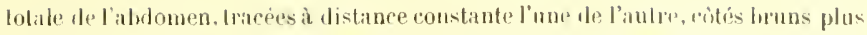
latuves vers la base de l'alubmen, sixième segment inlièrement noir ; segurnta-

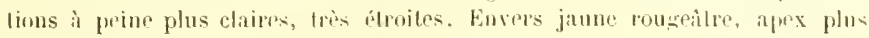
somlur, segmentalions ì prils blanes. Le dessus de l'alulomen prrlr une lomentusili grise yui formedes laches lalérales distinetes romure relles du groupe de Tubanns truioln P. B., la pubescence semble avoir ati principalement noiler. l'ittes jaune rongeâlre, fómus plus bruns, tarses bruns, puhescence des fímurs blanche. partout ailleurs noire, sligmil el nervures brun jaundre.

On pent rajporter avee probabilite le Tabaus mucrops of an Tabunus temiola dont il serail le male. Il mol imporsible de le sivoir al'une faron cortaine puistgue le nombre des miles al partout extrimement ríluit, main celle assimilation est hantement probable.

\section{IBILLIOGHAIIIH:}

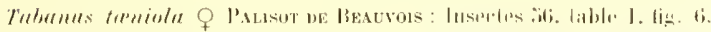

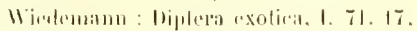

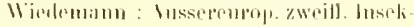
ton, İ?. 8 .

Malker : Jisl Miph. Mrilish Musemu. I. ב2:3:-3:1\}.

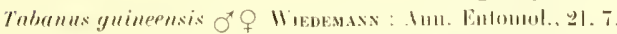

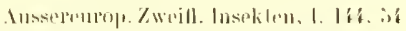

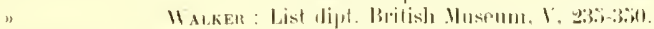

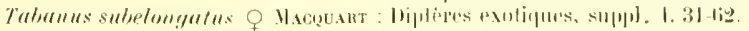

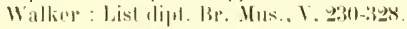

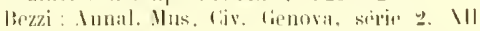
(XIIII), IS:3. \&.

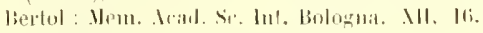

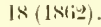

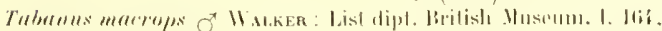

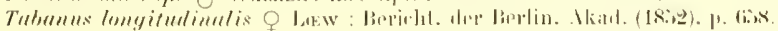

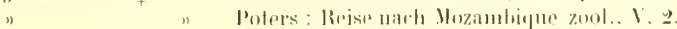

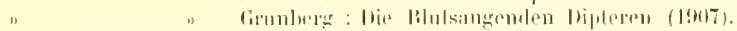
1. 1:36, liz. 10!3.

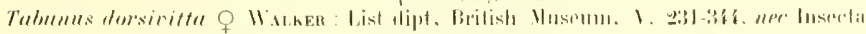
Simmolexsiatur.

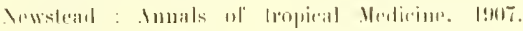
Feliruary, 11. it.

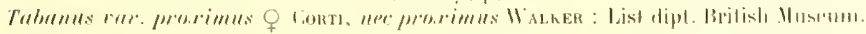
I. 137 .

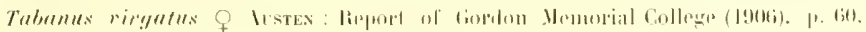
tier. 9

\section{Tabanus sugens $†$ WiEnEMAN}

"Thurace rubilo, abulonine fuseo, albu-vittalis antennic nigris."

Long : 7 lignes. - lininie.

Miedemann a tonné lit lescriplion suivante:

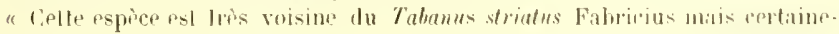


" ment disturte. La dittérences les plus importantes sont les suivantes:

" intennes noires, landes de lablomen flus menues, cuisses tris sombres

" (brun morritre). La lrande qui desend de la callosité frontale est large en

" torme dr fuseau ef dre chacun de ses cités s'étend un ligne fine qui vient con-

" verger avec elle aut sommet de lit cillosité."

Collection du Prisce Cirnstian ì Coperuhague.

Wiedemann dablissant que le Tabamus sugens o Wiedemann est extrèmement voisin de Fubunus striatus o Fabricius, esperee orientale qui a Je méme mode de dessins abrominaux que le groupr de Tabmus trmiola P'. B., on peut le considérer comm très voisin de colui-ci. Il semble dilfërer de Tabanus guineensis

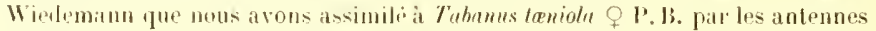
fntièrement mores, mais Wienemave n fotablit pas ba diflipence qui existe entre

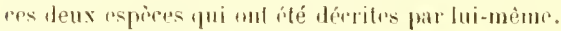

\section{lilil,IIIIIIIIF:}

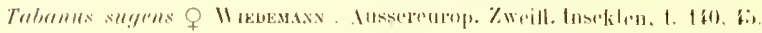

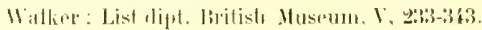

\section{Tabanus socius $f$ WALKEl $(\mathrm{pl}, \mathrm{Il}, \mathrm{tig}, 13$ )}

"Fuscus, eapite llavo sublus allo, thorace cinepeo quinquevitlato; pectore "fulvo nigroque valio, abdomisn nigro, fulvo trivittato; basi ventrique ferru" gineis, perlibus nigris, femorilus fulvis, tarsis piceis, atis subcinereis."

The jaune, partie suyérieure blanche à poils blancs. Yeux couleur de poix, pieces bucendes jaune paile, Irompe noire, thomax brun sombre avec cinq lignes grises; pectus varté de lurun et an noir; abdomen noir, fermgineux vers la bise a trois handis couleur tan. Envirs ferrugineux, brun pảte de claque còté, now it l'extréunitó: pattes noires, cuisses jaume lirunitre, tarses couleur tre poix. liles légèrement grises, nervures brunes, balanciers jaune frile. Longeur dı corps "l lignes, envergurt des ailes lti lignes. sud de l'Afrique. liecueilli par le J) I. SMITII.

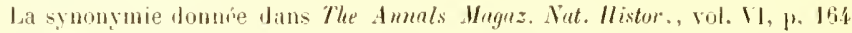
(1990) is incorrecte, car en comparant le type de Tabanus socius of Walker it Tubanus sugitharius o Macquart dont le type existe au Muséum de Paris, on reeonnait qüil y a leux especes distinctes. La Taluams serratus Low quon a voulu assimiler it Tubanms socius of Walker un est également ditferent.

d.e ilusém de l'aris posside de nombreux exemplaires des provenances suivantes: Ifrique occillentale (Dr Laverax, I906); Fleuve Omo-Butei (D) E. Brumpt, 1901i); Khati-soulan (1)r Lavehas, 1:107); Tehad, rives inondées

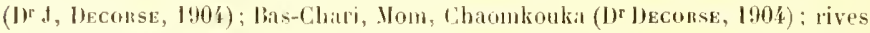

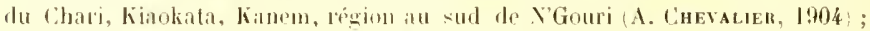

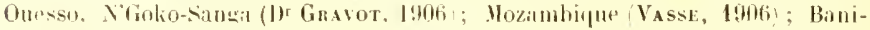
Soudan (1)r Laveran, 1904); t'chald oriental, archipel houri (1. Chevalien, 1906); Senegat-Sélibuby (1)r Lavenan, lan6); région da lac Fittri (lieutenant Lebas, I904): 1byssinie (mission de Boxcuanp's, 1899). 
Le tritish Yuseum possède le type de Trabanus socius of Walker provenanl du

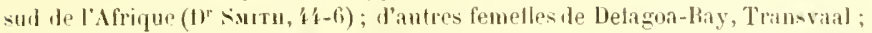
Tufuli River, Natal; Salishury, Masbonalind ; Bahr-el-Ghazal; Sennatar, Nil

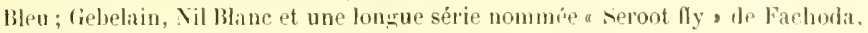
Nil Bline, 6-xu, l!mo (II. E. Ilarues); l'aulres exemplaires proviennent de l'soga-l'gandi et un vient de Léopoloviche ( 7 décembre 1903), alressé par l'Ecole de médecine tropicale de Liverpool.

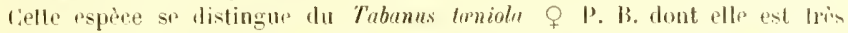
roisine par la forme de la hande médiane grise de l'abdomen, cette bande n'esl fits etroite et continue mais se compose d"etroites laches triangulaires a colis presque droits, leurs bases repusent sur lo bord postérienr du sigment nel se terminent par un court inex ill lomil antirieur n. Longurur du rorps, in a $1 ! \mathrm{m} \mathrm{mm}$.

Iyant pu comparer entre eux les spécimens de presque tous les Yusées "Weurope il résulle que le Tabamus socius ressemble en tous foints atu Tabanus turniola P. B. sauf par la bande abdominale médiane; les triangles de tomen losité grise yui composent la bande médiane rommencent par une tachr tomenteuse grise, do forme irrégulière sur le premier segment, la tache du deuxième segment est grande, triangulaire el porte un long apex qui atteint le bord antirieur tlu segment, còtés droils, parfois la tache est complètement oblongme, sa hase est généralement plus étroite que cello de la tache du troisième segment, celle-ci et la tache du quatrièmo segment ont une base plus large, les còtris tígèrement concaves et les apex inrondis. sur le cinquième siggment la linche est très petite ì base lrìs atroite, sur le sixième seguent on voit parfois une très petile lithe oblongue.

Laire de répartition du Tabamus socius o Walker, comprend actuellement le Sénégit, Soudan, Congo franģais, Tehat, Congo helge, Angola, Natal, Transvaal, Jhumlésil, Mozambique, tegandit et la vallée du Nil jusquauprès de Kartoun, qui ainsi que liayes (Śnégal) semble marquer la limite seplentrionale de celte espice.

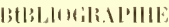

Tahamus sorius of Watker: List lipt. Briljsh Wusenun. I, llio.

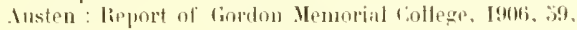
fiz. 2 í

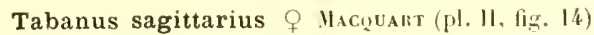

1 celte espèce se rapportent les discriptions des esperes suivantes :
Tubrumes varintus o Walker.
Trhanus rubicundus o WaLkel.
Tabomus serratus of law.
Tabranes arclemuationis q limarn.

qui sonl des synonymes de Tibanus sayittarius o ltirquarl.

Le lype appartient à la collertion du muséum de Paris. Il porle oulre l'étiquette mamuserite de Vaequart la mention : Afrique, Delabande, 306. 
Maequart en donne lit courte diagnose suivinte:

" Intennis nigris. Indomine lateratibus testaceis, singulo segmento macubir "dorsali nigricante. Pethihus rutis. Longueur fignes $q$.

" t'ilpes, face et front a'un gris jamitre pâle, ce dernier is callosití carrée, " hrune, prolongé d'unp ligne, à l'extrénití de laguelte il y a une tache brune, "wrale, imitant l'extrémités d'une limhe, dechingue coté. Vertex brun. Antennes " norim; premier article rongeatte. Thoras noir ì léger duvet blanchatre, at " quatre ba ndes d’un rougeâtre pàle, latges pustériemroment, linéaires at presifue " nultes antérieurement; chables ruugritres; côtés gris: écusson noir, Abdomen "d’un fauve testacé sur les còtés, une grande tache lorsale noirâtre sur charue "segment, sur laquelle it y a um tache triangulairede duvel grisitre : une autre "lialhe semblable sur le laure descités ; incisions jamitres; ventre d'un gris

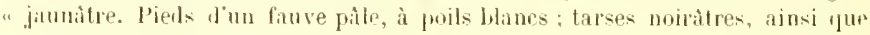
" lextrémité dex jambes antérieures. Batancier" brunâtres. liles rlaires ; bord "extérieur un peu jaunatre".

Đu cap. Melaraxde. Muséum.

La longueur indiquée ent exact' mais l'état d'u-ure du type ne permet que hirn alifticilentent de l'identifier.

Tite plus large que le thorax, yeux glabres. lande frontale it hords sensibtement parallèles, trois fois plus haute gue large it la bise et couverte d'une tomentosité gris jaunàtre paile. La hadale frontisle porte à sa partie inférieure une callosilé chàtian clair tangente aux yeux, prestue carrée et prolongie par une ligne courte saillante of de mème couleur qui s'etend presque jusigu'ì la moitió du vertex on elle se dilate in une tigure atlectaut lit forme d'un fer de llèche, une antre callosite tris petite ef arrondie, de meme rouleur existe an rertex. Intennes noires a premier article rougeatre (d'apres Marquart). Le type ne posserde que les deux premicrs artiols's dui sont rougratres à pubescence noire. Patpes renflés d'un grio jitunitte pîte ì pubestenor noire éparse.

Thorax noir ì léger duvet blinmhiater et quatre bandes d'un rougeattre pate, filargies postériemrement, linétires ot presque nulles intérienrement, épaules rougeatres, thanes gris, dessous it pubescence diun blanc rongeatre. scutellum noir.

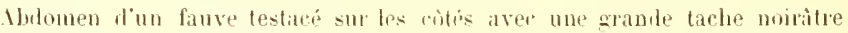
ditfuse sur le milieu de chaque segment, élargie an hord postérieur ; sur cethr tache il y a une lache lriangulaire de duvet gris jaunitre bien visible depuis le troisieme semment. Ine serie de taches triangulines semblahles se trouve sur tous lás segments dans la partir faure. Incisions juunâtres : ventre d'un gris jitunàtre. Pattes d'un fauve palle à pubesence blanche, larses rembrunis ainsi que l'extrémité des tibias antóripurs. liles hyatines ì nervures brunes. boril costal et stigma jaunatre. Balanciers brunitres.

Catte expece très voisine du Tabanus socius. O Walker en diffère surtout parl lo drsin dex triangles qui composent ha ligne médiane de l'ablomen. Cette bande atroite est composée dle triangles comme dans Tabrmus sacius o Walker, mais ils ont tous une lase plus large pt leurs rotés sont tres coneaves de telle sorte que ces triangles forment une véritable liğne en zig-zagr, le froisième ot le quatrième ont les plus larges bases. Lit bande commente par une tache indétinie sur le premier sament sur un fond moir; le deuxime triangle for me un tache allongrs 


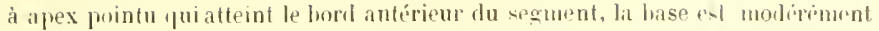

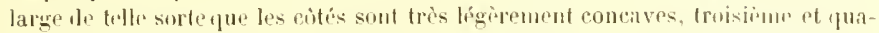
trième semblables mais leurs bases sont plus larges of leur's cotés plus conraven, les apex sont enclés sous le spament qui des prócide; cimpuiène triangh plus court et litge, le sixime forme une tache allongée. Lex apex du sermul ot trojsiène triangle sont pirlois tronqués. Longueur du corps. 11 it 18 mu.

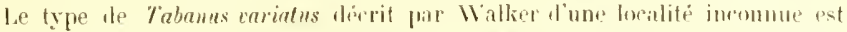
identiqur au type de Tabams rubicundes provenant de l'intílieur de l'Afrique, ainsi que l'a constaté II. Iusten. Le type de Tahuns vuriatus il été acritlentellement détruit mais ce nom prend lis priorité sur relui de Tabumas rubicundus, dejjà emplové par llacerant pour un tion do l'Inde ot de Java. Tabanus rubirundus Walker n'est pas la méme espèce que Tabamas secedens Walker conme l'imliquent les catalogues de liertesz el de Bezza ; eette confusion est due i une erreur commis par low dans sa note sur les Diptères du Sul de l'A frique (page 33) au sujet d'un changement de nom fait par Walker pui le premier dérrivit Tahamus secerlens sous le nom de Tabiamas tibialis et qui posterieurement retrouva l'incien nom alors employé (roir Dipteres of the British lluseum. supplement, I, p. 224 (1834).

\section{Tolmnus sermatus o Lew}

"Brunneo cinereus, thorace albido vittato; abdomen brunneo rafum seg"mentis ultimis nigricantibus: vitta mestia sequalis brunnea in singulis seg"mentix triangulum album includet; rallus frontalis unicus inferus rufesecus. "Long. corp. $8: i / 6$ lin $"$.

Expere tres s'mblable at Tahans trisignatus milis cependint listincte. Llow les exemplaires les phs chairs la couleur du fond lu thorax of du scutellum est rouge brunâtre, assez noire sur les parties inférieures du thorax seulement et brun-moir entre les bords sombres du dessus. Jans les exmplaires plus sombres cette coloration ent plus ètentue, principalement au delit de la maijemre partie du sentellum de sorte que les bords latéram du thorax, les intervalles entre les landes thoracinues sombres of las bords du scutellum restent roug lirunitre. La couleur fondamentale de la face est habituellesent noiritre et rougo brunitre au-tessons mais ta tomentostio blan he est si denso que la couleur du fond nest visible que par rénudation; la pubesence time de la fice est blanchatre. l'alpes blane jaunate a pubecence blawhe pris de la base ef noire par ailleurs. Intennes noires, le premier, le deuxieme et parlois aussi la base du troisìme article sont brun sombre; fremier article fortement en forme de capurhon, couvert d'une touentositó blanchatremélangie de poils noirs principalement donser vers

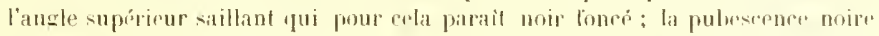
du sprond article antennaire forme presque un toupt it l'extrémiti dr l'inglu

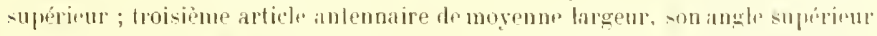
fortsment sidlant al consert t'un assez grand nombe de très courte poils. Front à pubescenec et tomentositi romme relle de la fare jusqu'i l'angle antirieur des yeux, il porte une callosití quadrangulaire l'un lom luisant qui

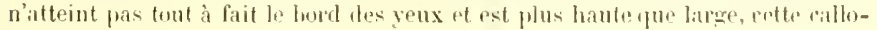


sité est rejointe par une ligne brune qui remonte et semble produite seulement par le frottement car parfois elle mancue fout it fait ou presque tout à fait. Dans la région ocellaire et au vertex il se forme par le frottement des tiches irrégulières où la couleur brune du foml apparait. Après le ramollissement les yeux restenl très sombres et ne présentent aucuue trace de bandes. Coloration et desus du thorax romme chez Tabams trisignatus, Lew. seutellum la plupart du temps noir seulement ì la base. Le dessin de labdomen est très earactíristique chez cette espece, on le voit t'autant plus clairement en regardant l'insecte par l'arriere, on apergoit alors une tris large bande médiane brun-noir dont les lords latéraux sont droits el qui devient plus noire el plus étroite vers la partie postérieure. Du heuxième au siximme segment lablomen porte une tache trianguharo qui atteint be borl antérieur dr chatgue annatu et qui a une fomentosité gris Winnhatre ou gris rougeitre, les cotés du triangle tournés vers le vertex sont un peu échancrés de sort pue les ingres postérieurs sont les plus nettement distincts. Il se forme ainsi par la réunion de tous res triangles une ligne médiane dentée. Sur les anneaux postériours l'apex antérieur de la tache triangulaire est carhé sous le bord postérieur les anneilux prócédents; le septième segment abdominal est noir en entiur. Sur le sixiòme segment commence une bande qui va vers le haut près du bold latéral, elle consistr en des taches qui ocenpent toute lit hauteur des amneaux et qui sunt plus lirges, plus claires et moins nettement distinctes sur chanur anneau suecessif, on peut aisément les distinguer habituellement jusqu'iu troisieme annean. La bimde formée de ces taches est noins nettrment Jimitée que la hande médiane, elle est dentée au cóté interne et droitr au còté externe qui est très facilement limitr. Sur le siximme anneau ces bandes comvergent souvent aver lit bands médiane, le reste de l'ablomen plus ou moins brun rougeatre est recouvert d'une tomontosile gris blan hatre. La pulescence de l'abdomen est surtout noire an dessus, elle est blanchatre sur la majeure

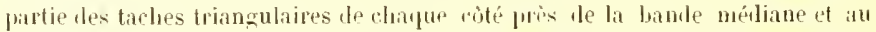
lord latéral externe. Ventre muge lorique it pubescence blanchatre longue sur les còtés, elle est noire sur la ligne médiane en grande partie et sur les anneaux postérieurs en entier. C'est pourquoi il peut arriver que la ligne médiane vue sous un certain angle semble plus brillante quoique lorsiguon la regarile plus de còté eille prenne la coloration de la ligne latérile, les derniers segments lu vantre sont noirs. Cuisses noires a apex rougeatre, tibias jaune rougeatre ì fomentosité et pubescence blanchatre, le tier's apical des antérjeurs noir, les autres ont lapex un peu noir. Tarses antérieurs noirs, lia couleur des autres passe du brun au noir. Balanciers a pédoneule brunatre et a bouton blanc jaunatre. Siles grisitres, hyalines ì nervures brun-noir, lyord marginal très peu distinct, d'un jaune lorunâtre elair. Prenière cellule marginale rétrécie, la quatrième ourerle.

Chez quelques exemplaires la tachr médiane du sixirme segment abdominal est très nette.

Cafrerie et llozambique. 


\section{Tabanus mblicundus o WaLkER}

"Rufus, thorace fuseo quinque sittato alvlomine nigro quadriviltato predibus " nigris, tibiis rufis, alis limpirdis.

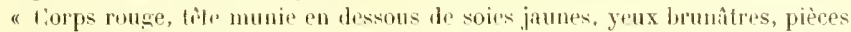
" burrales jame pâle, trompe et antennes noires. Thorax avee rinq bandes bru" nes, poitrine marjuce aussi de brun. Ibdomen a vee quatro bandes noires dont " les deux mílianes se réunissent sur les bords antérieurs d" plusieurs seg" ments. Ventre noir ì f'exlrémit́. Jambes noires, tibias rouges recouverts " A'une pulsescence blanche et courte. liles sans coloration, nervurescouleur

"de prix. Balanciers fauve pale brillant. Longueur du corps 8 I. 1/2.

"Ifripue du sud. Rapportí par l. Comte de Derny."

Cette description très iusulfisantr pour diff́rencier une espèce dans ce groupe très aftine est heureusement complétés patr la bésence du type au British Iuseum. Liexamen du Tabauns rubicundus $q$ Walks! le rattache avec certitude au Tabonus sngiltarius o Macquart.

Le Tabanus exclamationis o Giraml a été reeueilli entre $10^{\circ} \mathrm{lat}$. S. et $16^{\circ} \mathrm{l}$ ! $\mathrm{j}^{\circ}$ long. E. (Greenwith). Girard dit qu'il se rapproche de Tabanus longitudimulis Lew, il doit d'apris cette lescription être très voisin de Tulbams suppllarius Macq. et probablement appartenir à la mème espìce.

L'auteur dit en outre que la callosité frontale a la formed'un point al'exclamation, ce qui peut s'entendr" de toule callosilé dilatér au milieu.

Le Trbamus sugittarius O llacquart est rrprésenté au Muséum par le type el des exemplaires provenant de Khati-Soudan (1) Lavenax) : Kisoumou, lictoria Nyanza; ellvirons de Khartoum; Mbyssinie.

Le musée de Lishonue posside deux sprérimens de Quango.

Le British Museum a le Lype d Tabunus rubicumes $O$ Walker, provenant do l'intérieur de l'Afrique, collection du comte de Derby ; II. Waterhouse du British Museum nous fat savoir que cette collertion provient principalement drs environs du Lae Vgami; d'antres femelles proviennent de Biké et Benguela, Ingola : Ifrique orientale; Budlu; Walelai; Ankole; tulamwezi, Nord dl'soga ; Kisembara: Jundit, Igandiı (s.C. Tountxs, 19013); quelıues-uns ont éti rerueillis dans les plantations de lananiers pus de lil riviere Seziva; d'autles dans la forit de la riviore tukoge; un eximplitire de Gambie, et un autre des monls Ruwellzori à 3.500 pieds d'allitudr.

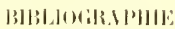

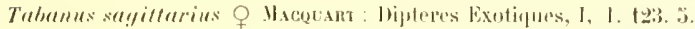

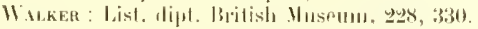

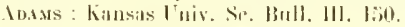

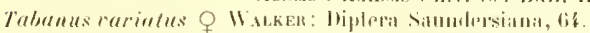

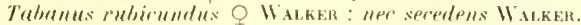

Miphares of lintishl Musinam. 1. p. Itil

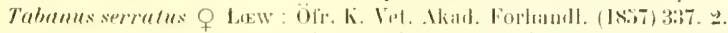

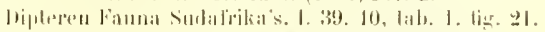

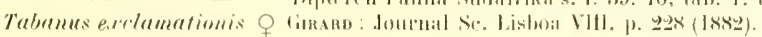
It. 11. $3677(1881)$. 


\title{
Tabanus fraternus VacouarT
}

II faut rapporter an Tabuns fraternus $q$ Macipuart les insectes suivants qui sont den synonymes:

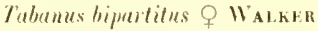 \\ Tubanus trisignatus o Law
}

Le type le llacquart provien! de la Cafrerie est en mavais état, il fait partip de la colfection du musée de Lille.

Maceuart en donne la description suivante:

\section{Tabanus fraternus 9 Macevart (p. $93 \mathrm{fig} .16$ )}

"Thorace nigro, vittis rubris. Abdomine fusi'o, maculis dorsalibus albis; vittis " Tateralibus rutic. Antennis nigris. Pulilus nigris; tibiis testaceis.

"Longueur 8 I. Q, voisin du T. autummulis. l'alpes d'un jaune pảle. Face et " barbe blanches. Front blanchìtre à callosité lorune. Antennes noires. Yeux " nus. Thorax ì quatre handes pougedtres et ì duvet blanc; dessous it duvet "blanc. Abdomen à taches dorsales, triangulaires, le duret blane, sur les pre" mier, deuxiène, troisième et quatrième segments; une bande fauve de chaque " còté des mèmes segments; cinquieme, sixieme et septième noirs, avec un pen " de faure sur les bords; ventre fauve, à líger duvet blanchatre sur les cótés. "Cuisses noiratres à duvet blanchitre; jostérieures testacées en dessus; jambes " linuves; antérieures blanches, à extrémité noire; tarsés noirs. Ailes assez " claires; base et bord extérieur jaunâtres; nervures normales.

" De lit Cafrerie, reçu de M. Melegorgue de Douti.

Lin examinant le type de Macoquart on remarque que les taches triangulaires dérrites par l'auteur sur les quatre premiers segments sont en réalité situées sur les second, troisième el quatrième avec une faible tarhe oblongue sur le cinquieme segment. Walker et law ont redecrit Fuburs frulermus of Jacquart en 1830 ti et 18.57 . Lew l'a recueilli de Cafrerie et le Yozambique. Le type de WALKER, qui existe au British I useum provient du Natal (68-4). Collection Saunders; d'autres spécimens de la meme collection proviennent du sud de l'Afrique; nne femelle vient le la rivipre Lunigina, llenga, Guest du lac Nyasa i 1.000 mètres d'altitude (R. Crawsmay); une lemelle du sud de l'Ifrique recueillie pendant l'expédition du lar Lavingstoxe et apportée par Lord liussech (63. 86); une femelle de l'Est africain prise par le Dr SPeke dans son expédition aux sources du Nil, donnie pir Lord liussell (1864).

Karsch rapporte que les spécimens males et femelles de Bondeï (Est-Africain) ont les yeux higarrés. Bezzı remarque que les femelles d'Ali-t gri, Erythrée, sont un peu plus petitrs que le type de Macovan et que le thorax est rougeatre avec des lambes noires. les exemplaires du Hustum de laris provionnent de lit cỏte de Mozambique et ont été juris par H. Ci. Vasse.

E-pèce rougeatre qui se distingue aisément par les trois taches triangulaires de son abdomen et par ses ailes estompés. Longueur du type 16 millimètres. 
Celui de Walker a la mime lonenfur, mais entains spécimens atteignent 20 millimities.

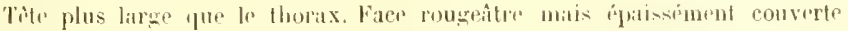
d'une tomentositi hlanehitre et de nombremx poils blanes. Barbe blamehe.

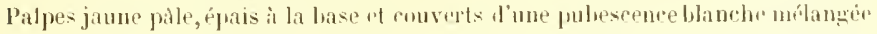
Ar quelipues poils noirs ì l'ipex. Antennes hron rougeatre, premier article jaune

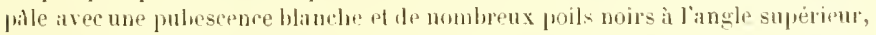
re qui lui donne un aspert sombere, il est découpé en forme de capurlon; serond tres petit a poils noirs; troisieme article hrun sombre. rougentre seubment is lis base, orné d'une dent distincte couverts de poils noirs. Bande frontale rnviron tuatre fois aussi longue que large, Jéginment ritricie antérieurement, mongitre, couverte l'une tomentosití grise. Callosite frontale rouge, srande, pristue carrée, atteignant les gemx, ha ligne qui la prolonge est en forme dr fuseau th atteint plus de la moitié de la bande frontale: de charpue póté de cettr ligne il y a une épaisse pubescence noire leux sans binules ni tarhes. Le thorax viric do couleur du noir au rougeitre, il est hildituellement noir sur sa partir antérieure et plus rougeatre postérieurment, lit couleur noire apparait souvent en bandes; il porte einy bandes distinctes de lonentosité grise, la médiane est itroite (elle nexiste pas dans le type de Wallior). les autres sont plus larges et ont min pulescene grisatre ; rotés rougeatres it pulescence grise, sur le descus elle ast principalement jaunitre. l'ectus rougeatre ì tommosité grise of pubescence blanchìtre. scotellum d'un noir rougeitre.

Alulomen janne rougentro. las trois druiers segments noirs, les trois taches triangulaires sont égales de dimensions et ont une large bise, l'apex du premier triangle est court, lorsque ces taches ne sont pis dénuléps, elles sont recouvertes de poils blancluatres; sur le premier sengment on apergoit une toulfe de poils blanes dans les spécimens très frais. sur le cimpuime segment il y a une lache ollongue grisatre, les triangles gris sont hatbitudlement entourés d'une bordure noire formant une bande irrigulicre de ehinge rote. La pubescence du desous est rourte et jaune, noire sur les parties sombres.

Envers jaune pale arec une tomentosité et une pubescence blanches. lattes janne rougeatte; fémurs noirs; tibias antírieurs noiratres a l'alpex; tous les tarses noiratres. P'ubescence des pattes luanche sur les parties claires, noirt sur les parties sombres. Ailes hyalines it nervures brunes, stigma jamnitre, horl externe et nervures transverses ligèrement ombrees de brun, promière rellule postérieure rétrécie.

A titre de documentation nous joismons a notre description celles des anteurs rux-menes.

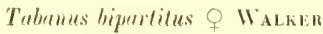

"Fulva; caput allum, vertice ferrugineo callo nigro; palpi albi intenuts " nigre; thorax supra niger, cano tuadrivittatus, diseo postice latrribuspur "fulvis; abdomen è marulis alhis univittatum, hasi macula nigra, apirem ver"sus nigrum; femora tarsique nigra, illa apire fulva: abu subeimera", venis " honnullis nigro marginatis; halters alli. 
Couleur tan. Tête blanche; vertex ferrugineux; callosité noire. Yeux couleur l'airain, toutes les facettes très pretites. Palpes blancs. Antennes noires: premier article formant un angle aigu en lessus et en avant; troisième mince, légèment courbe, sa corne tris courte et petite formant un angle presiue droit. Thorax noir en dessus, aver quatre bandes blanchâtres, de couleur faure de chaque còté et sur le disque en arriere. Ibdomen noir vers l'apex avec une tache noire is la base et une tache blanche triangulaire sur chacun des segments intermédiaires. Fémurs el tarses noirs, ceux-ci avec l'extrémité fauve. Ailes grisatres it nervures noires, nervures transverses et quehjues-unes des nervures longitudinales ombrées de noir. Branche antérieure de la nervure cubitale simple formant près de si base un angle très obtus et un peu arrondi. Balanciers blancs. Longueur du corps 6 lignes, des ailes: 13 lignes.

Port Natal.

\section{Tabanus trisignatus O LEW}

"Brunneo-cinerascens thorace albilo-vittato, abdomine lrunneo-rufo, postice " brunneo triangulis tribus albidis segmentorum secundo, tertii et quarti " signato. Alce cinereo-hyalina, venis transversalibus fusco-cinctis: callus fron" talis inferior et linea frontis longiludinalis brunnescentes.

"Long. corp. $8-8$ lin. 1/6.

Couleur du corps trìs variable mais l'inserte est aisément reconnaissable. Chez les exemplaires les plus clairs, la couleur fonciere du thorax et du scutellum est d'un rouge brunitre, pumant que chez les exemplaires les plus sombres, ha majeure partie inférieure des llanes et du thorax est noiraitre, recouverte d'une tomentosité et d'une pubescence lihnchatre. Les bandes sombres de la frartie supérieure prennent alors une coloration brun noirâtre sombre de sorte jue le fond reste d'un brun-chatain. La couleur fondamentale de la lace est toujours jaune rougeàtre pàle recouverte d'une pubescence blanchâtre et l'une dense tomentosité blanche. Palpes blanc jaunâtre à pubescunce blanche entremèlé de quelques poils noirs ripars. Antennes noires avec une coloration rouge brunatre foncée depuis la racine jusqu' ia la base du troisieme article. Premier articte en forme de capuchon, ì pubescence et tomentosité blanchâtres, l'angle supérieur saillant porte une dense pubescence rouchée qui lui donne un reflet noir foncé, les petits poils noirs de l’angle supérieur du deuxième article antennaire sont en forme de toupet; troisieme article d'une largeur moyenne, angle supérieur fortement saillant, recouvert d'un nombre assez grand de petits poils noirs tout à fitit courts. Front ayant jusqu'ì l'angle antérieur des yeux, la mème tomentosité et la mème coloration que la partie inférieure, et portant une grande callosité quadrangulaire brune qui va d'un ril à l'autre; alu-dessus se trouve une autre callosité linéaire qui semlle due au frottement parce que chez les divers exemplaires elle montre un aspect diflérent, parfois la région orellaire est pourvue d'un petit dessin qui commence nu point ou serait placée l'iselle antérieur et va par deux branches embrasser l'emplacement ocellaire. I'rís de la bande longitudinale dénudée du front se trouve sur le bord de chaque cil un 


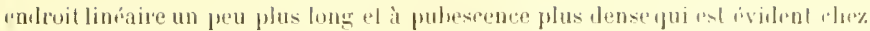
les exemplaires les plus sombres. Les grux restrul très sombres prolant le ramollissement et ne montrent aueune trace de bandes. Thorax it tjualre bandes

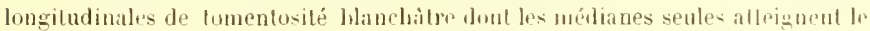
bord postírieur. scutellum noir drapuis sal base sur une aire variable.

Coloration et dessin de l'abdomen très caraptóristipues. Couleur du lomd brun rougeatre, au milieu du bord postórieur du premicr anneau se trouve un pelit"

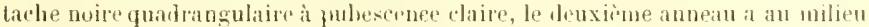
du bord postérieur une petite larhe triangulaire à tomentosile blanehâtre ôt it pubescence d'un jaune pàle clair, troisieme anneau à tache semblible qui linil en pointe vers le bord antérieur, une troisime tafhe se tronve sur le quatrimme anneau el sil prointe antérirure est recouverte par lo bord pos érieur de l'innein

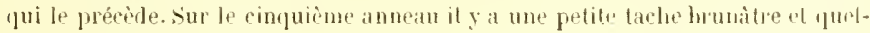
ques petils poils clairs gui montrent sur le lord postérieur un dessin semblable non défini. Les cinpricme el sixirme anneaux ont une coloration brun foncé et whe pubescence noiralre landis que le loord laleral reste de coloration alare it pubescence claire. La couleur lorun des dernicrs anneaux se continur commer une bande longitudinale prísulu bord latéral an-4lessus des anneaux anlérjeurs at plus ou moins loin selon que les spteimens sont plus on moins coloris. lentre plus clair que le còté supérieur de l'alulumen. les deux derniers anneaux sunt plus ou moins bruns ou noirs. Cuisses noiràtres en général, ehez lés exemplaires les plus clairs elles sont lurunatres sauf au cotb exlerne des anterieurs, les cuisses ont une tomentosité et une pubesence blanchattre. Tibias jaune rougeàtre, In tiers apical antérieur noir, les autres tibias un pen noirs à l'apex. Balanciers à tige brunâtre elaire et lìte bianchître. Liles grisitres, hyalines, à nervures brunnoir: Base et hord antérieur's un peu colorés en brun. Les nervures I rancversales et la base de la deuxième cellule submarginale onl une bordure estomprée de brun sombre; première eelhule marginale postérieure plus étroite, lit puatrirme cellule élarsie.

Les différents exemplaires des rolleclions d'lurope froviennent du Natil, Cafrerie, Mozamhique, Afrigne rentrale anglaise el Myssinje.

\section{l3|B|,|1MilithIII:}

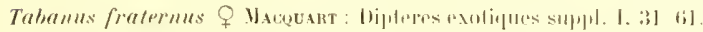

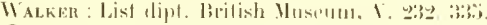

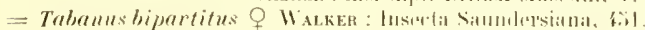

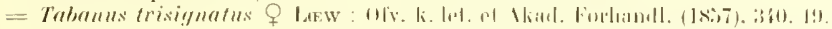

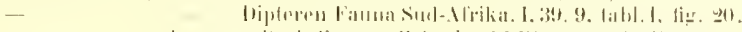

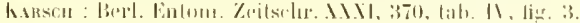

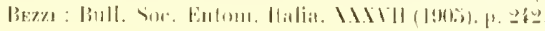

\section{Tabanus quadrisignatus liıCA}

Le type famelle et un autre exemplaire proviennent de kuwe, rwièré luat-

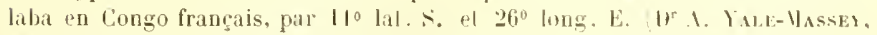
février 1906). Collection du Britıslı Muserum. 
Espice bien marquare, voisine de Tabuns fraternus q Macpuart, plus romnu sous le nom de Thinams trisignntus $q$ Lipw, qui lu est synonyme. Le Tuhanus quatrisigualus o licartus'en distingur par ses tathes midianes triangulaires au nomlire de quatre a lieu de trois, son thomx i larges handes et ses antennes d'un jounr rouge brillant. Les bandes thoracidues le rapprochent de Tabmus lingsleyi q lairardo dont il sóloigne par les taches de rabdouen.

We taille moyenne, thorax noir it deux larges bandes grisitres, distinctes. Abromen lirun pougedtre aver des taches latérales gris jamnitre en outre des taches médianes. l'attes noiratres, tibiar jaune-rouge et ailes claires trós légèrement teintées de brun.

Longueur 1312 it $15 \mathrm{~mm}$.

Tète plus large que le thorax. Face rougeatre (démulée), dans l'autre spérimen elle est rouverte l'une tomentosite jaum urisath et d'une pubescence blanche. Barle blanche. Pillpes d'un jaune très pâle ì pubrocence blanche, légìrement épaisis à la basp, terminés far une pounte médiocre. Intennes jaunerouge brillant; premier article cylindripue, couvert d'une tomentosité grise ef d'une pubencenee noire mólangre de quelınes poils blaurs; deuxime article très petit, ì julpescence noire; troisiome article long, plus sombre à l'ajex et portant une dent bien développée. Bande frontile pougeitre (denudée), couverle d'une tomentusilé gris jaunitre environ ciny fois aussi longue que large, rétrórie antérieurement, portant un callosité frontalo lrun-rouge, ohlongue, n'atteignant pas les yeux, la ligne gui la prolonge ex comrte thaisse avec des poils noins au vertex.

Thórax brun nuiratre, ses deux larges bandes composées de tomentosité qrise it pubescence jaunatre se contiment jusqu ì sa base. Côtris à tomentosité grise ; la pubescence du dessus, noire avec des poils blanchatres autour de la base des ailes se continur antum lu thrax. Pectus et flanes rougeatles, couverts de tomentosité grise et de pulescence hinche avec des poils noirs en descus. Srutellum jaune rougeatre it la base, portant sur la partie rentrale une pubesence noire et quelques poils noir jaunitre.

Ablomen brun romgeàtre purtant un péu de tomentosité grise sur le premier segment, et sur les quatre suivants une tarhe triangulaire mécliane le tomentosité grise: tuntes ces titches ont une bite ditrgie, la premìre est plus allongée et toutes ont une pubercence Hanchatre. Les taches latirales sont, chez le type, plus granden sur le second segment, et moindres sur te troisième ef le quatripme; elles sont tont's de rouleur jaunàtre, revètues d'une tomentosité grise et de quel'jues froils han's. leur forme est irrégulirie. Cinquième segment noir sur les còtés, sixième noir an entier, l'un el l'autre aver les angles latraux transiucietes. sur l'autre femelle il y a des trates de laches latérales sur le cinquième segment. lat pubescence du dessus mot nure d'un jatune noiritre sur les taches claires;

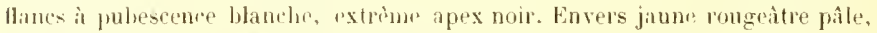
noirate ì l'apex, puleseruce gfiniralement banchitre. l'attes lorun noiratre à tibias jaune-rouge; elles sont semblahles à celles de Tabanus comiformis 0

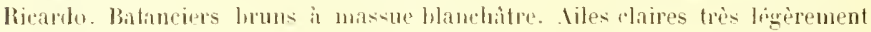

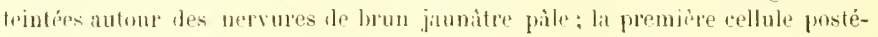
ricure un peu rétrécie au bord, nerrures brunes, stigma jaunatre. 


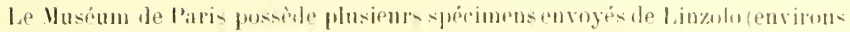
de Brazzaville) (1)г ILcaix, 1907).

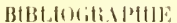

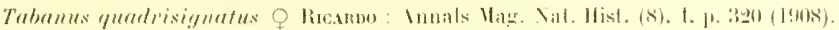

\section{Tabanus distinctus $O$ llicardo ( 11. II, lig. I., )}

Le typeqprovient le Benguela Ingola, il it etir perueilli par 1. Yale. Massey al rommuniqué par le colonel $G$. firsis; une autre lemelle a fle prises sur les berds

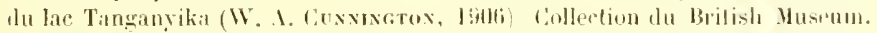

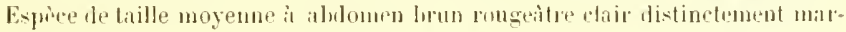
qui de trois series de taches triangularen grises, thorax it bandes gris's pen diatinctes.

Longueur lo mu. $1 / 2$.

Tète plus large que le thorax. Far jaune rugeilre converte d'une tomentositi blane grisitre et d'une courte pulpecente blanche, epaisse sur les joues of sur la partie inférieure de la face. Barle lianche, longue. Palpes jaune paile. épais ì la base, légèrement arquís of trminés par une pointe allongié pubrscence blane jaunatre mélangée de quelipues poils noirs. Intennes rouges, jurmier article très légèrement en capurlun, portant les poils noirs sur l'angla extérieur el quelques poils hlanes atu lord "xterne; second article très petit à puhescence noire; troisième article rouge sile ì dent obtuse, noir à l'extrímitie apicale. Bande frontale couverte de tumentosité grise, callosité frontale d'un lorun rougeitre brillant, atteignant ì peine les yeux, allongée, prolongée par une

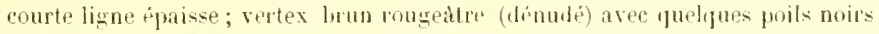

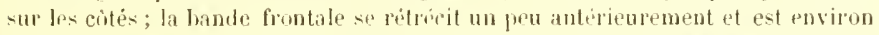
cinq fois plus large que haute; partie pustérieure de la tète blanchatre ì poils blanes.

Thorax rougeitre avec cinı bandes grises indistinctes et quatre hamles noirea, les bandes grises sont composies de tommtositi grise, la bande médiane mat

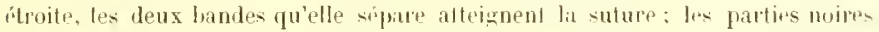
jaraissent flre des bandes fat conlraste avec lil couleur foulimentale rouge du thorax. Dans les spécimens du lac Tanganyika, ces bandes ne sont pain appa-

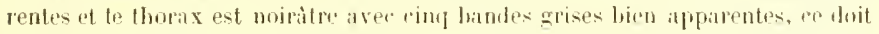

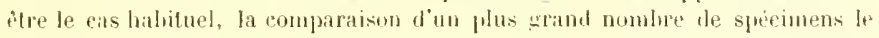
prouvera. Pubesence du dessus du thorix noire, ellr est jaune bianchitre sur les bandes grises; flanes d̀ poils noil's, des poils hanes priss de la raciur des

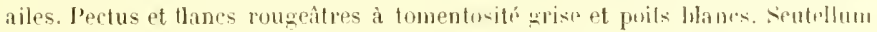

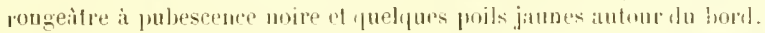

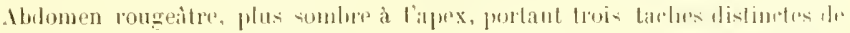

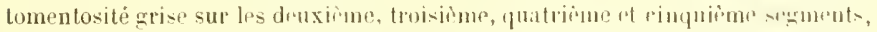
des tracessur le sixième; lernipr spgument entièrement noir. Les tarlues malianes

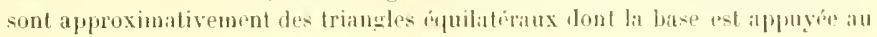


bord postrírieur de rlatue segment, chinque apex atteint la base de la tache prórédente, toutes ces taches sont le taille parale sauf celle lu sixième segment qui est plus petite. Jes tir hes latérales sont des triangles isocèles. le còtéle plus rapproché des taches mérlianes at la base qui repose sur le bord postérieur du segmont forment un angle Iroit. La pubesence ost noire sur les parties sombres du dessus, jaunattre ou blinche sur les taches grises. Envers rouge jaunitre pâle à tomentosité et puluscence grises. daubes rouge jaunatre, aprex des cuisses et fémurs antérieurs lırun rougeitre; tarses antérieurs et apex des tilias antérieurs noiritres; les autres tarses brunàlres. Hanches à longue pubescence blanche; fénur's antírieurs et médians à pubescence noire en dessus et blanche en dessous, fémul's postérieurs ì pubescence entièrement blanche: tibias antérieurs ì poils hanchitres sur la base jaunitre; los autres tibias ont une pubescence principalement blanchâtre, elle est noire par ailleurs. Ailes claires, à nervures brunes. stigma lınn jaunatıe: première rellule postèrieure distinctement rétrécie au lorit de l'aile. l'as d'appendice.

Le spécimen du lac Tanganyika est de conteur plus lorune.

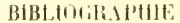

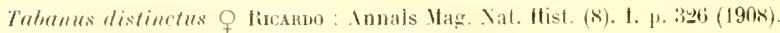

Tabanus sticticolis q ivrcouf ( $\mathrm{pl}$. II, fig. Iti)

Type récolté par W. Chevalier dane le Fouta-Djalon en b!nt, deux autre femellé de la mème prosenance.

Brunattre tair, abdomen portant une hande médianede triangles clairs et deux lignes latérales de taches imprécises. Ailes rendrées. brunàtres vers le borl costal. Longueur 14 à 1 : millimètres.

Tette plus large que le thorax ; yeux bruns sans handes, glabres. Bande frontale étroite à borks sensiblement parallèles, couverte d'une courte pubescence jaune, portant une callosité brun rougedtre de la larzeur de la bande à la base de formespmi-ovoïde th surmontée d'une ligne brone saillante qui s'étend un féu au delit du milieu du vertex. Antennes d’un testacé brunatre, premier article à soies jaundtres, lent lu troisieme article arronlie. I'alpes clairs avee quelques rares poils uoirs courts a lit patite externe. Epistome jaune, borl postérieur de la trite blanchàtre.

thorax ef scutellum bruns recouverts d'une pruinosité cendrée à pubescence rare ef courtes composis de poils noirs, flanes ciliés de noir, poitrine blanchatres avec quelques molles sojes blan hatres.

Aldomen brun maron portint sur rhaque segment un triangle méelian blanchitte ayant sil base sur le borl postérieur du segment. En outre deux bandes latrinles blanchàtres, mal délimitées sétendent sur chapue còté de l'ablomen, formant une tache pui so relie sur chaque segment a la base du triangle. Pubesconce claire ou noire sclon la teinte des zones colorées. Flancs à pubescence jaunitre. Ventre uniformóment rougeàtra à rourte pubescence blanclue, noire à 


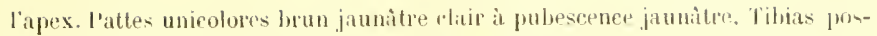
térieurs frangés, tarses rembrunis. liles centrées plus brunes vers le hord costil, sans appendice. Balanciers jaune pâle à tige rougeitro.

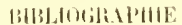

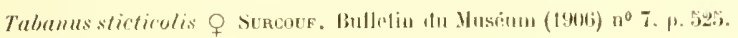

\section{Tabanus sericiventris $q$ LaE}

Le British Museum renferme dans sit collertion de Tabanides six femelles provenant de Potehefstroom, Transvaal (189:3).

Le type vient de dia Cafrerie. Ainsi que law le remarque, celte esprice est

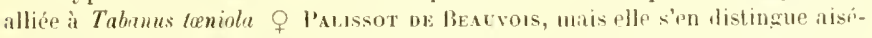
ment par plusieurs caractives et entre antres reux des atiles.

lispiee jaune rougeatre ì thurax noir reconvert d'une tomentosité grise. Ibumen rougeatre avee un reflet gris soyeux ut une droite bande mérliane grisu bordée de noir. Apex noir. Ailes teintiás de hun. Longueur 1 inm.

Tête plus large que le thorax. Face converte de tomentosité grise el d'um pubescence blanche fyarse. Babe blanche. Palpes minces d'un blanc jaunitre pàte ì pulsescence blanche. Intennes jaune rougeàtre, troisicme article noir, jaune ì la base; le premier article est en forme de eajuchon et it puhescence noire, second tres petit it poils noirs, troisieme mince it dent distincte. Bande frontale de mème couleur que la fice, environ trois fois anssi haute que large, d'ígale largeur sur toute sonétendue; callucití frontale, grande, de forme trianqulaire, n'atteignant pas les yeux et prolungée par une ligne courte un peu plulongue que la callusité elle-mème. Yeux sans maryues ni dessins.

Thorax couvert d'une tomentosité grise et d'une pubescence jiunitre pitle. Lew dit quil ny a pas de handes visibles mas dans certains spécimens il $y$ at les traces de trois bandes grises indistinctes yui probablement n'appitraissent

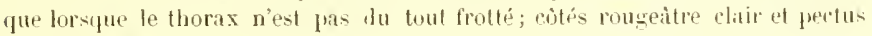
gris, tous tes deux revètus dune pubescene blanchatre. Scutellum semblible an thorax.

Ibdomen jaune rougeatre sur bes coris des quatre premiers segments, lestmis: derniers noirs, la bande médiane arise continue whorde de phaque còte frar une hande moire; tout le dessus de liatromen revètu d'une tomentosité juune prisatre ou grise offre une apparence soyeuse ; la pubescence est jaune sur les fratties rlaires el noire sur les parties sombres: envers d'un jaunâtre prìe ì pubes-

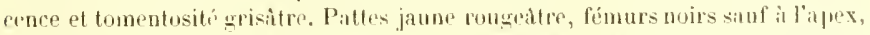
tilias anteripurs noirs it l'apex; puhporenes lilanchitre; sur les parties sumber des tiluas ct les tarses efle est noire. Siles hyalines, toutrs les nervures teintere

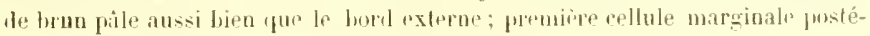
rieure non rítrécie; nervures lmancerses et stigma brun jaunâtre.

Deseription de LeW :

"Flavido-cinereus, thorace non striatu; abdonten rufescens, cintero-stria ceum, stria-longitudinali medià-pallidialuabus yue hane includentibus nigri- 
" eantilum pretum; alde cinerece, venis ommibus hrunneo-einctis. Long. corp.

$71 / 3$ lignes.

Assez mine, trompe noire, palpo lbancs il pubescence blanche. Tête revâtue l'une tomentosité blanchithe qui passe au jaunatre sur le front assoz large. l'remier article antennaire jamitre, un peu en forme de capuchon, orne de courts poils noils, troisiome article rouge à l'extrême base, le reste de l'article est noir et assez litre, angle supérieur arsez aigu. Yeux sans bandes, d'un beau vert apres ramothissement, les parties lacisses dans l'ombre sont d'un bleu-violet. Callosité de la bande frontile jetite, d’un brun brillant, n’atteignant pas le bord des yeux. prolongée dins une ligne étroite. Couleur foncière du thorax noiritre sur le dessus et rouge brunitre sur les còtés, main rette coloration est misquée par une tomentosité dense d'un gris cendré jaunâtre qui ne forme ni ligne ni lande longitudinale nette. Pubescence du thorax d'un jaunàtre pâle. Sicutellum semblable at thorax.

Abdomen rougeatte sur les cotés, noiràtre sur les trois derniers anneaux, tomentosití de labomen d'un gris jamnitre otfrant un aspect soyeux ru de biais. La partie dorsale de l'abdomen porte ume hande médiane droite d'un jaune zrisitre, formíe par une tomentositè of une pubescence claire qui s'arrète au Jernicr segment. Ceste zonr ent entourée de chaque còté far une bande noire, itruite, de mème largeur, de tomentosité elaire st de pubeseence noire, lien vi-ible en regardant l'incerte tangentioltement par derriere. L'ensemble de ces Iruis landes verupe le tiers de la largeur do labdomen. Ventre sans dessins, la lomentosité qui ent de mème conleur que relle du dessus masque presque complìtement hit coulem foncière. Cuises it tomentosité gris clair ol it pulsescence jaune grisitre claire. Tilias juune rougritre, les antérieurs à moitié apicale noire et il pubescenere noire sur cette partie. La pubescence des tibias est blanchitre, les tihias postérieurs ont vers l'itpex quelgues poils noirs. Balanciers brun clair, massue jaunitre it l'apex. Ailes teinteses de gris, tontes les nervures estompées de brun, lord antérieur brun. Premìre st quatrème cellules postérieures margrinates non rétrécies. - Ciafrerie.

\section{HIt:LHMILIPIII:}

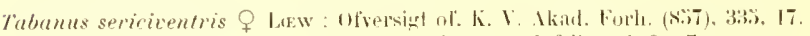

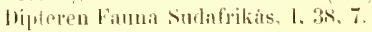

\section{Tabanus nanus $q$ WIEDEMAN}

"Nigellus; iblomine fusco trifariam maculato, naculis mediis imsurinque " albilis; Tibis tarsisque onlariteris.

"Longuene 4 lignes, du Ciap."

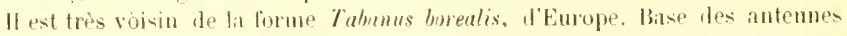
brune, atrtive terminal fermgineux vif aver laapex noir. Epistome gris glampue. Barbe blanchitre. liatpes brunatre palle. Front parfois aver une puliescence grise, portant une bandolete unie sitargissint gratuellement en ltssous. Tho- 
ras noiritre ayant au milien une tacle linéaire hrune ef des tarkes latérales blanchatres et délieates. Borl des coités brunitre, cotés du thorax gris ardoise, it pubescence blanchitre. Ibdumen brun. Bande mediane composie de triangles Wanchatres, bantes laterales composées de taches quadrangutaires il pulpesence erise. Bord des segments brun jaunatre clair. lites hyalines avee le loril costal Jrun noiratre. Tige des halaneiers jaume, disc[ue brun, banchatte en dessus. Cuisses anterieures lrun noiritre. Collection Westermans.

Xous ne connaissons pas ce Tabumes qui appartient probablement au wroupes de Tabunus turniola of P. li.

\section{BabLGuikiptit:}

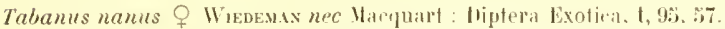

lussereeuror : Zweill. tnsetiten, 1, 187, 11'

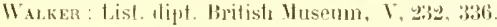

\section{Tabanus Ricardoæ $q$ Suncour}

Le type provient du has fio Nunez en Ciuinée francaise et a étri communiqué par $\mathrm{H}$. le D Laveran en 190\%. Une autro femelle de mème provenance.

Je dédie cotte esprece ì Miss G. Ricardo, ma distingués collaboratrire.

Longueur 1:3-14 mul.

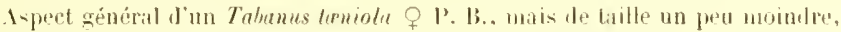
d'apjarence plus légère. Aites teintées de linun au bord costat.

Tite plus large que le thorax, yeux bruns ì cornéules ígales, glalnes. Bande Irontale plus targe atu vertex yu'it la bane, jaume soufre, portant une callosite brune, Je forme trapiézö̈lale, légrement sillonnic au unilieu, surmontée llun prolongement peu sitilitnt, fitroit, s'dtendant jusque vers be vertex. Antennes testacées, premier artirle portant de courtes soies noires, troisieme irticle it dent oltuse, noir danstoute la partir apicale. l'alpes minces, longs, blauchitres, avee

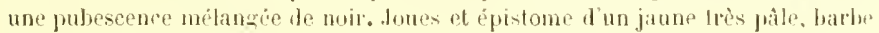
blanche, partir postéricure de la tète à pubescence très courte.

Thorax brundtre, orne de quatre lianes longitulinales de pollimositio blanchatre et d'une cinquième ligne médiane abrégée. siculellum bron, recurvert d'un pollinosité jatunitre. Flanes el jertus d'un cendré jaunitre it pulwsecence blanche.

Mblomen chitain, orne d'une bande mialiane étroite el le denx bandes latirales plus larges, se terminant au cinquirme soment inclus. Pubecence dr

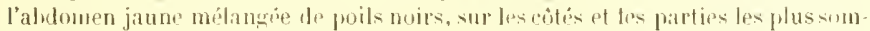

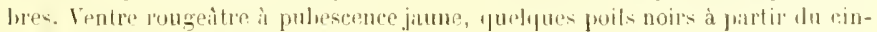
quimesezment. segment anal horde dr soies moires. Pattes mnirolores alun

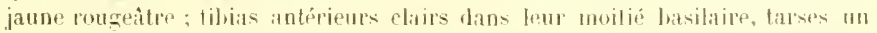

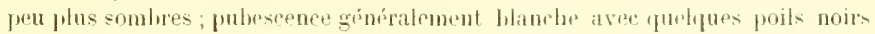

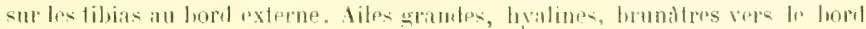


costal et lat rellute strmalmue. Cuillerons lorunàtres. Balaneiers jaune elair it lige soncoloper.

BHLGHitidphte

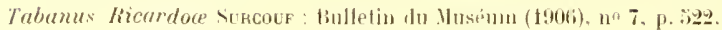

\section{Tabanus coniformis $q$ Ricardo}

Le lype et un autre exemplaire femelle proviennent de Ruwe, riviere Lualaba (Congo Belge) par $11^{\circ}$ latitude sud et $26^{\circ}$ Jongitude Est, ils onf été reeueillis par le or I. Vale Masser (1906), "dans une toile d'araignée près du campement." (Coltection du British Vuseum). Le muxén de Lishonne possède deux spécimens provenant de Benguella-Angola .

Expèce brun rougeatre, très líliére, aver l'alulomen très pointu à l'apex. Taches médianes et tarhes łatrirales indistinctes, apex noir. Longueur 1 i mm.

Tête plus large que le thorix. Face converte d'une tomentosité grise qui devient lorun jaunatre sur le triangle frontal et le bord extrème des joues, pubescence hlanche. Barbe blan he. l'alpes l'un ronge jantâtre à courte pulsescence blanche. modérément épais, apex court. Intennes rougeàtres, premier article en forme Ju eajuchon, ì pubescence blanehe et des poils noirs sur l'angle supérieur; second article petit ‘̀ fubesernce blanchâtre, troisième long à dent noyenne, Je yuatre dernieres divisions de cet article presque aussi longues que ba premirire. l'extrémite apicale noire. Bande frontale rouge, converte de la mème tomrotositís rolorée que le triangle fronlal: callosité frontale brun rongeàtre, atteignant ì peine les yenx, uvile, prolongée prar une ligne epaisse, canaliculée in son milieu et atteignant presque le vertex. La bande frontale est environ quatre fois aussi longue que larqe, très lig̀̀rement rétrécie anlérieurement. Youx sans taches.

Thorax brun rougedtre avec des bantes indistinctes, les unes sombres, les autres crises, dessus it tomentosité srisitre et pubescence noire, il y a des traces de puleseence blanchitre antour d" la racine de l'aila et à la base du thorax. Citís et pectus recouverts l'une fomentosité arisitre à foils blancs. Scutellum rougeatre, noir vers la base, couvert d'une tomentosité grisàtre mélangée de yuelques poils blancs ou noirs.

Nydomen brun rougeitre portant trois élroites taches médianes, triangutaires, grises, risibles seulemenl en regartant par derrière, sur les deuxiòme, troisiome of quatrième segments; citł́s avec des laches oblongues irrégulières, jiune rougeatre, couverts d'une tomentosite grisittre sur les quatre premiers segments, les trois dernirs sont noirs en entier. Le einquième ot le sixième seguments ont bes incisions plus rlaires. Puhesence du dessus noire, les bords poxifipurs du cinquième et du sixime segments ont des poils mélangés blanes

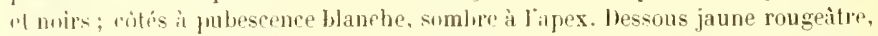
il apex noir, les incisions très etroitement blanches, pubescence błanche, noirs à liapex. Pattes brun noirître, tibias antérieurs jaune-rouge sur la moitié 
banitaire, les atres tibias entirement jaune-rouse, tarses médians ot postérieurs do mime conleur mais plus sombres it lapex. Pubeseence des hanches el tes fémurs llanche aree une tomentosité grise, lianche sur les tilias antérieurs dans leur partie hasilaire, noire par ailteurs. Batanciers it tige sombre et massue jinnatre. Iiles claires, nervures brunes, stigma jaunitre, première vellule postérieure non rétrécie.

Dans les exemplaires de l'Angola, les taches de l'abdomen sont distinctes ot il $y$ a des marques mélianes noiritles sur le second, Iroisième el quatrième segments L'espèce sera toujours aisément reconnue it son abdomen conique, noir it l'apex, en outre le thorax et le scutellum sont un peu plus sombres que chez le Tabanus taniola 아. P. B.

\section{BHBLHMIRARHIE:}

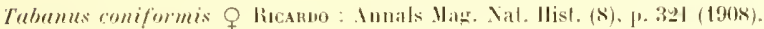




\title{
NEUYIFAE GROUPE
}

\author{
Tableat Dichotomoue
}

1 Fémurs antíriems brun noiratre. . . 2

f Fémurs antérieurs ronges. . . . a baude médiane grise, peu distincte, habitwellement rontimue, parfois réduite a de petits triangles grisitres au lord postériem

2 Irs segments et des taches tatriales mal

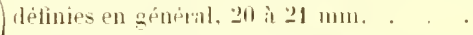
Petite espere lixun rougeatre à bandes laterales rontinues sistendant jusqüau fuatrime segment, 12 it $16 \mathrm{~mm}$. . . Tingseyi o livardo.

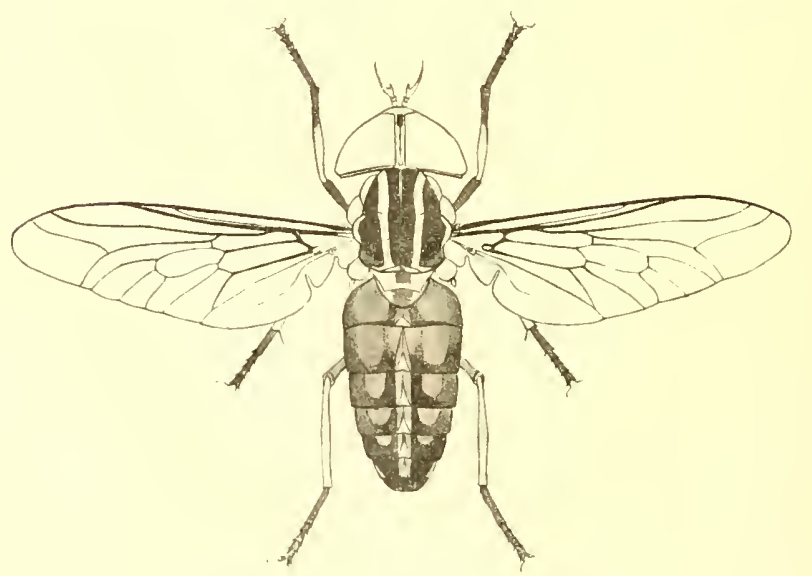

Fig. 18. - Tahamus claripes O Ricardo 


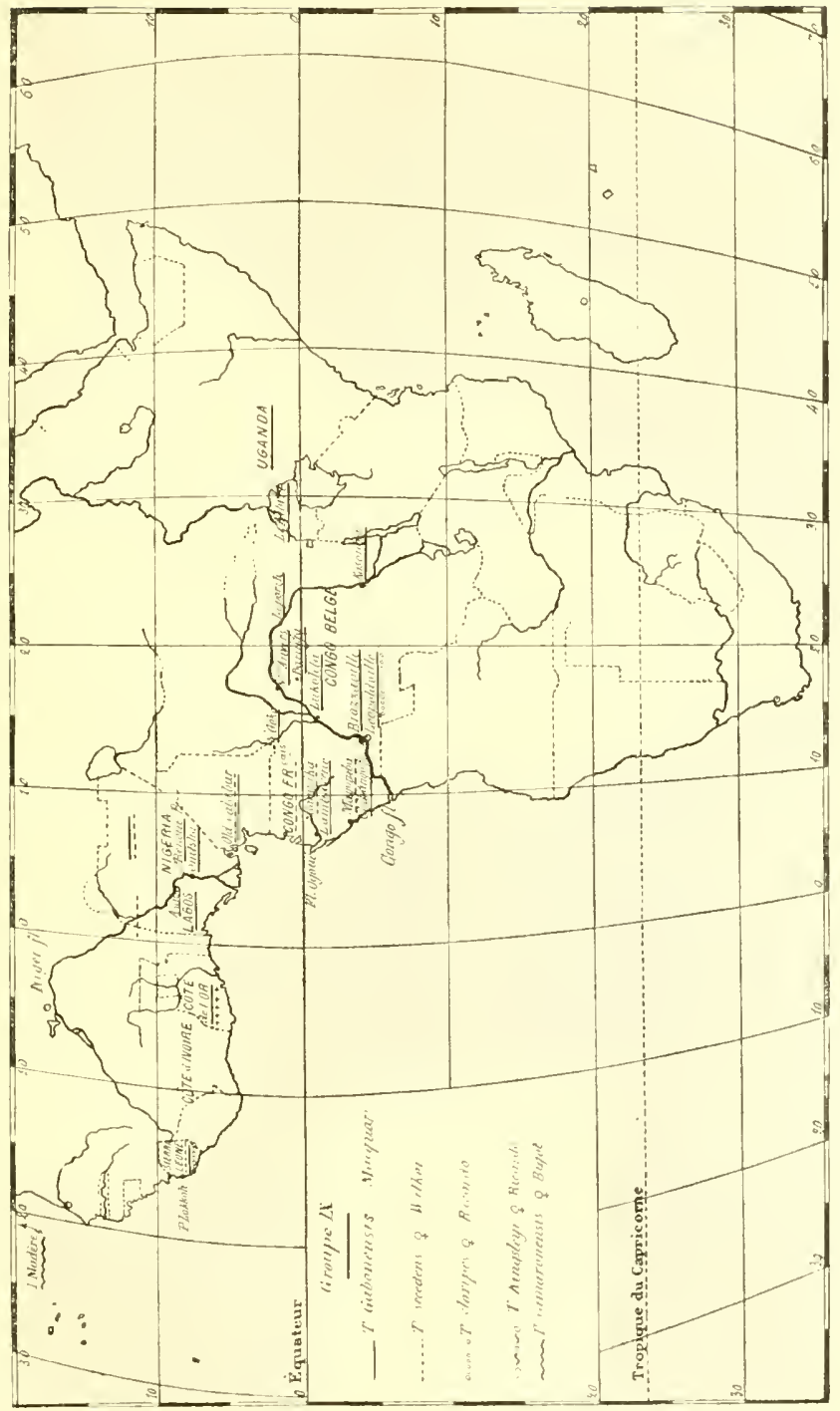


Espèce indécise de l'île Madtre, près de T. secedens.
T. claripes $q$ Ricardo.

T. camaronensis $Q$ Bigot.

\section{Tabanus secedens $q$ IVALker (pl. III, tig. 2) \\ = Tabamis gabonmsis o Macquart \\ - Tabume Blandeardi Q Suncovf \\ = Tabamus brunuescens O licardo}

Le Tabanus secedens est un des insectes les plus repandus sur la còte oeei-

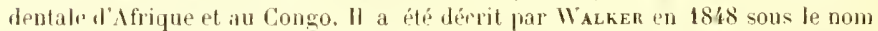
de Tabamus tibialis, mais es nom ivait déji rité employé par Macguart en $\mathbf{1 8 4 6 .}$ Wacker changea le nom de son insectr en $18: 44 \mathrm{ot} \mathrm{tr}$ nomma Tabamus secedens. Pendant ce temps, Hacount lui avitit donne le nom de Tabams gabonensis en 185\%. Hes diffierences qui avairnt été ronstatérs entre le Tabamus gabonensis $q$ Macpuart "t lo Tabmus secedens $q$ Walker ne somblent pas suffisintes pour maintenir les deux esprem distinetes. to nom de \%abams gabonensis Q Macquart devient alor's synonyme de Tubamus secedens $q$ Walker.

Le Tabunus rubicundus $O$ Walker n'est pas l'ailleurs te synonyme de Tabanus secedens. $Q$ Walker, mais lo Tabunus sagittarius $q$ Macquart.

la femelle du Tabamus seredens Walker tait seude connue borsque te Musémm le Paris refut en 1906, deux spreimens males quion peut ripporter aves certi-

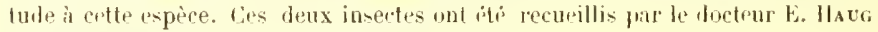
en 1906 ì X'Gomo, sur les hords de l'Ozooué.

Longueur 171 \& $199 \mathrm{~mm}$

or Thit grande, yix confluents sur tout leur bord interne et atteignant $4 \mathrm{~mm} .1 / 2$ de longueur, composés de denx champs de cornénles nettement dislincts dont une zone médiane de groses cornéules yui comprent toute la partie médiane et interne et yui est entourée prar un anneau étroit le petitescornéules: au verlex cet anneau est très étroit ot il augmente trìs peu de largeur jusque vers lo bord externe de l'sal ou il s'élargit pour former toute lit partie inférieure de l'oil, la ligne de séparation est courle et atteint presque le sommet du triangle frontal, elle est nettr el brusque juntout. Triangle frontal marron vers l'apex sur los $2 / 5$ de sil hauleur, le reste est ilun jaune dore brillant; joues d'un banc jaunàtre à longue pubescenee jaune. Barbe blanchàtre. Intennes petites, rouges; premier article noir, en capurlon, portant de nombreux poils noirs, courts et plus denses vers l'angle smpérieur; denxieme article a pointesiblante, cilié de courts poils noirs ; troisieme article à dent basilaire externe de taille normate, ornée de quelıues poils noirs, bord infúrieur sinué ; extrémité annelée couverte d'une courte pubescence serre donnant une coloration noire à cette partie de l'antenne. Palpes petils, vésiculeux; dernier article oblong d’un jaune grisitre pâle à longs poils noirs et blanchatres. 


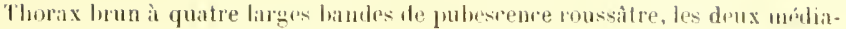
nes se prolongent sur le s'utellum du'etles contourat; dresous du thorax cendré it longue jubescence jaunâtro.

Ablomen long, brum rougeatre, à rare pubesence noire, portant une ligne médiane frstacée, contime, commengant an premicr segment et se terminant au

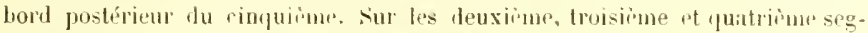
ments il y a rn outre une tir he latirale frès jeu visible.

Dessous de l'aludomen brun à courte pulpessencr d'un jaun doré, lord des segments frangé de poils jaunes. Hanchescendrées, fémurs brun rougêtur foner à pubescence noire en dessus rt quelques poils blines sur la partie inférieure. Tibias et tarses comme la femelle. Ailes hyalines, tointées a bord costal, stigma hrun formant une tache nébuleuse étendue, nervures brunes très tígìrement estompées de jaunitre. Balanciers à tige rougeàtre, hase du bouton noiraltre, partie terminale testacér jaunître.

Cette espèce varie considérablement de coloration et passe du rougeâtre atu brun.

Q Tète plus large que le thorax, face gris jaundtre dinsément couverte d'une toutentosité grise, qui est plus jaunatre sur la partie supérieure des joues et sur le triangle frontal; pobescence d'un blanc d argent assez longue et mélangée de quelques poils noirs sur le milieu de la bande frontile. Palpes jaunes couverts de courts poils noirs, grands et nettement épaissis à la base, arqués et terminés en une pointe allongée. Antemes „l'un brun rougeâtr", premier article rougeâtre it épaisse puhescence noire, en forme de cajuchon; second articte très petit, rougr avec quelques poils noirs ; troisiime article d'un lirun noiratre, parfors rougeattre à la base, la juemiore division de cet article porte une tomentoxité grisitre ou jaunitre, les dernières sont romplètement noires ot glabres, la tent siture près de la tase est nettement marrjure. Bande frontatr brune, couverte d'une tomentosité jaunàtre ou fauve, étroite, environ huit fois aussi haute yue large, rétrécie antírieurement; callosité frontale lrillantá, atlongée, d’un brun noisette, atteignant presufue les yeux et surmontée vers le vertex par une ligne saillant jusqu'au delà thu milieu de lit binde frontalr. Youx glithres, sans margu's ni dessins.

Thorax brunâtre portant quatre larges bandes éfuidistantes, formées dr tomentosité jaunatre, parfois les bandes sont plus wrisen que jaunes: la pubescence du pronotum dans fos exemplaires bien conservés est courte, noire atver quelifues poils jaunes antérieurement et sur les cintés; poitrine et danes d'un brun grisâtre ì pulsescence noire sur les cótés avere quelques poils gris épars. Scutellum brun rongeàtre, plus sumbre au centre.

Abdomen brun rougeitre, noiratre sur les segmentitions et rers l'apex; la bande métian très fitroite, continue dans la forme typique se compose souvent dreptites taches arronties sur te premier segment et d'une tache triingulaire allongée sur clacun des segments suivants, l'apex de chacume de ces tarbes atteint le bord du sement prícédent ; it $y$ a seuloment une trace di: tache sur le sixiẻme segment. Lorsque les wérimens sont fritis, des taches sont recouvertes

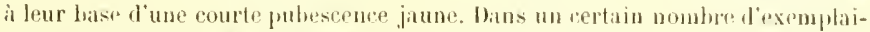
res il existedes laches latérithes arrondies parlois tris nettes, lat prósenere de ees taches avait servi de base pour sipares Tubanus secedens Wallier de Tubanus 


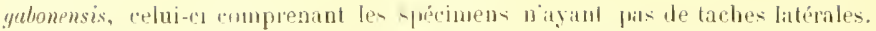
lhans quelques exmplities du Vieux Cababar ol de Niseria, lat bande médiane est formúe des mèmes tarhes mais elles sont plus élargies à la base, rourtes et de forme triangulaire. Ins apex de ces triangles ne dépassent pas généralement la

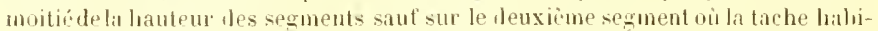
tuellement longue atteint le borel du pranier serment. Euvers jandite ou rouge jaunatre. brun sombre au uilieu ; seguentations très étroilement jaunatres vu llanchàtıs. Pattes rougeâtrẹ, fémurs antérirurs lorun noiràtre, les autres lémurs sont lirun rougeatro ; les fémurs ont une tomentosité grisatre en lessous et des poils noirs en dessus. Cliez guelques sprimens de l'Uganda, tous les fémurs sont rougeatres. Tibias lougeatres; antirienrs rougeatres ou d'un jauna tre pale, noirs a l'apex; la pubesener est subent blandhatre à la bilse ef revèt parfois les libias antirieurs en leur donnant une appareure blanchitre quoique sa coloralion suit plus jaunatre yute blaneles, lat pubmeence des autres tilias est noiratre. Tarses antérixurs noiratres, les autres lorun nuiratre, tous à pubes. cence noire en dessur, mélangfe de quelyues puils rouge brillant en dessus. plus ́pais aux tirses postérisurs et médians. Ailes grisitres teintés de lorun pille, jaunes it la bake ef au bord costal, uervures brunes, stigma jaunàtre, cuillerons bruns.

Le Maséun d bondres pussèle de nombreax exmplaires qui ont dé pris en

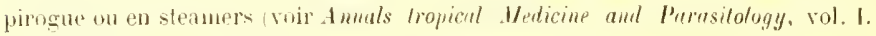
1. 4 is $(1: 107)$.

Laire d"extension dn Tabmus secettens Walker romprend sierra Leone,

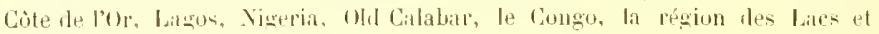
1'lomanla.

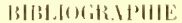

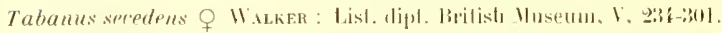

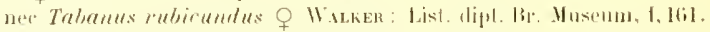

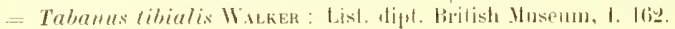

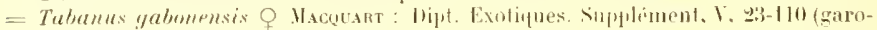
nensis).

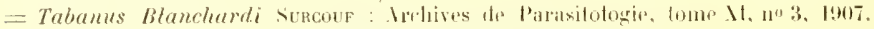

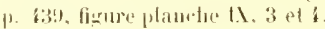

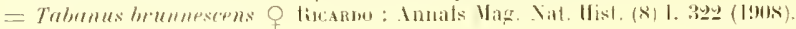

Tabanus claripes o Ricardo (r. fig p. 12.2

Le typ provient le Lépolkville (Congo indépendant), reeneilli le Is janviej 190 f par les docteurs butrox, Town et Comstr et communique prar l'école de médecine tropicale de Liverpool 190\%. (Collection du Britinh Mu-éum).

Celte grande espre brun ruggeatre it ailes brunatres et bandes thoraciques distinctes se distingue atisement par ses hanches d'un jaune rougratre clair do Tabumus secedens Willier ainsi que par si plus grande taille, les pattes plas 


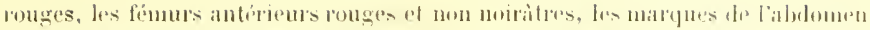
qui ronsintent rn taches médianes fritugulaires. grises. nou continues ot les taches laterales d un joume rougeitre imblistimet.

longueur $24 \mathrm{~mm}$.

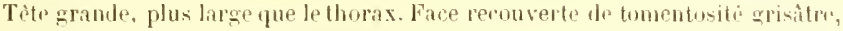
elle ent jaunatre suy les rités des joues et su lo triangle frontal au-ilessus des antennes: les parties infirieures de la face ont une pubescenfe blanehitue el plus longue.

Barbe blanchàtre. Intennes brun louguatro sombre, noiratres it l'apex; pre. mier artich grand, en form de cipuchon, it pubesence noir extrementmit épaissie à langle supérieur; deuxièm" artich potit il pubescence now ; troisième article phus grand, muni doue petite dent très aiguë, premire division du troisiène article eourerte l'une tomensité brunitre. Bamle frontale longue,

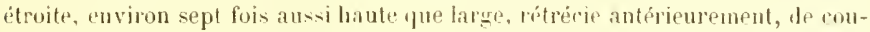
leur jaune comme le triangle frontal; callosité frontale brun rougedlre hrillant, longue, atteignant les yeux, sillonnée au urilien et prolongée par uni ligne éparisse au delì du milieu de la hande frontale. La pubescence de colle bande frontale consiste en quelques courts prils noirs vers ba base et pueligues poils jaunatres au delia. Palpes jaunes, longr et arojućs, pas très épais i la base ef teruninés en une pointe allongée. rrourerte d'une courte pulsescence noire.

Thorax brun portant deux larges bandes et deux plus étroites. composés dis tomentosité jaunàtre pàle. Pubescence de drssus courte et noire, jaunitre sur les bandes; des touffes de longs poils blanes it la litse des ailes; cotés el poitrine couverts d'une fomentosite srisitre at de poils blanes. Scutellum brum it tomentosité jaunàtre, plus rouge à l'apex, portant une pubescence route ri noile.

Aldomen long, épais, brun, la couleur rougchitre apparait sur les deux premiers segments et sur les côtés, sur le premier segment il y a une trace tle tache grise, sur le second segment cette tache est oblongue et a poine plus large a la base, sur le troisième la tache triangulaire est fongue et atteint le bord antrieur, le quatrieme segment porte une litro tache triangulaire qui natteint pas le horl antérieur, te sixième segment portr une comrle tacle triangulaire ; toutes ces tavhes sont d'un jame grisitre avec un pubesenes de memr couleur. La rouleur foncière rougeatrts de l'abulomen parait sous form de titches indistinctement irrégulières sur les cotis. I'nbescence dre dessus courte, noire rt éfraisse, ell' est blanche sur les hanes. Les hords latéraux des derniers segurenti sont d'un jaune transparent. Envers d'un rougeatre fuble ì large bande métiane plus sombre recourute dune courte pubescence blanches. l’attes f'un rouge brillant. Hanches antérieures et postínieures i leur extrímité apicale et hanches mélianes noires en intier; genoux, apex des tibias antérieurs et tarses antérieurs noiràtré : tibias antérieurs d'un jaunâtre pâte sur lemr surface externe. Pubescence dos hanches antrieures longue bt blanche. sur les autres lianches ello est éparse al courto; blanche sur les lïuurs, julu-

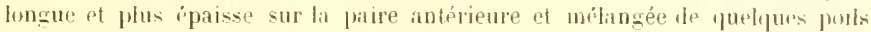

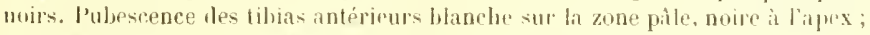
noire sul les autres tibias et sur les tarsos. liles brundte futh ; borl extéricur brun jaunatre avee quelques espaces clairs au milieu des collules; ner- 
vures et stigma hruns, première cellule postérieure un peu rétrécie. - Pas d'appendice.

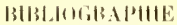

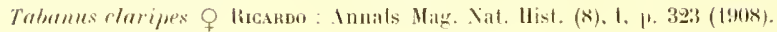

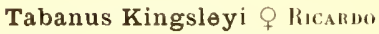

Le lype et plusieurs femelles proviennent de Jort Lokkoh, Sierra-Leon (190\%)

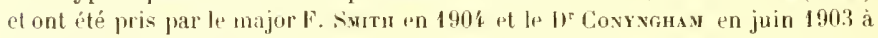
Baiwalla (sierra-Leone).

Cette espèce diffère de Tabmus secedens Walker par ha présence de bandes latérales composécs de taches contiguïs les unes aux autres et formant une bande distincte tandis que chez Tabamus secedens Walker, les taches latérales sont isolées et l'abdomen est en entier d'une teinte brun rougeadte, les quelques dégradations de teinte qui apparaissent les unes près des autres ne forment pas de bandes noires bien mariguées comme dans le groupe de Tabamus tieniola o P. B. Le Tabanus Kingsleyi O Ricardo se distingue encore de Tubanus limiola par son thorax à bandes épaisses semblables ì celles de Tabrmus secedens Walker, et ses ailes teintés de lrun, estompées sur la plus grande partie des nervures.

Longueur de 13 à $16 \mathrm{~mm}$.

Face couverte d'une tomentosité grisatre qui devient brun jaunàtre au dessus des antenres, et revêtue l'une courte pulsescence blanche. Barbe blanehe. Palpes peu épais à la base et obtusément terminés, jaune palle à pubescence blanche à lit luase puis courte et noire partout ailleurs.

Antennes rougeatres, premier article heaucoup plus court que d'habitude, couvert d'une pubescence noire; seeond article prtit, à pubescence noire, troisième article long avec une jetite lent près de la base, brun rougeàtre au delì de la base, les quatre dernières divisions complètement noires. Bande frontale environ six fois aussi laute que large, légèrement atténuée antérieurement couverte d'une tomentosité brun jaune Callasité frontale brun sombre, oblongue, canaliculée au milieu, atteignant complètement ou ì peine les yeux, prolongée par une longue ligne mince.

Thorax noiràtre, portant deux larges bantles le tomentosilé grise rerourertes d'une pubescence jaune, eòtés grisâtres avec un peu de pubescence jaune qui se' continue autour de la bas? du thorax, noire partout ailleurs. Flanes et pectus couverts d'une tomentosité grisatre el d'une pubescence blancle. Scutellum noi. lâtre ì tomentosité grisittre, seuf sur le centre.

Aldomen brun rougedtre, noir sur les trois derniers segments, portant une bande médiane, continur et distinete de tomentosité grise jusiqu au sixième segment, bortée de bandes lirun sombre ou noiratres, auxijuels sont contiguis des taches latérales jaunatres qui forment une bande aisément distincte qui s'étend jusqu'au quatrième segnent, liı pulsesernce de la bande médiane et des taches 
latérales est principalemont jaunatre, olle est noire far ailleurs. Envers jamitres avec une farge bande médlane noire, recourerte le pulescence lilanche. l'attex noiratres; tilnas jaundtres à apex noir; fímurs mólians et postérieurs souvent largement rouge jaunàtre, revitus d’une tomentosité grise et d'une pubsescuce blanche; tihias à puloscence blanchatre, pillout aillemrs elfe est noire. Ailes hyalines, teintées de brun, coloration plus intense au bord antérieur, stigma brun rougeatre, nervures biunes.

\section{BIBLIMIR.IPIIE}

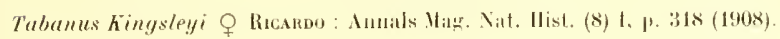

\section{Tabanus camaronensis $\sigma^{x}$ Bigot}

Le type de Brgot provient de Madire et il est en très maurais état. Il parait identique au T'nbamus secedens Walker, mais les fémurs antérienrs sont ronges et non noiritres, le reste des pattes est complètement noiratre a l'exception des tarses qui sont bruns. Pour le moment nous maintenons Trbamus comuronewsis of Bigot comme espèce distincte, la capture de nouveaux spécimens dans celte celle région pourra seule trancher la question.

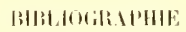

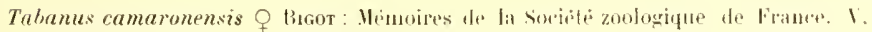
p. bit (afylotus). 


\title{
DIXIEME: GROLPE,
}

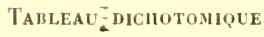

Bande abdominale étroite, indistincte. Abdomen brun rougedtre. Fémurs antérieurs jaunes ou hrun-rougeitre. Ailes lingerment teintées de brun, 16 it $20 \mathrm{~mm}$. . . . . . . .

Callosité frontale pointue, noiràtre, vers le vertex. Abdomen jaunâtre, parfois ì bande médiane indistincte. Ailes presque rlaires 18 ì $19 \mathrm{~mm}$. . . . . . . . . . . .

Bande aldominale olssolite. Aldomen rougeâtre à segmentations noirâtres ; fémurs antérieurs noirs, 18 il $21 \mathrm{~mm}$. . . . . T. testaceirentris O Macquart

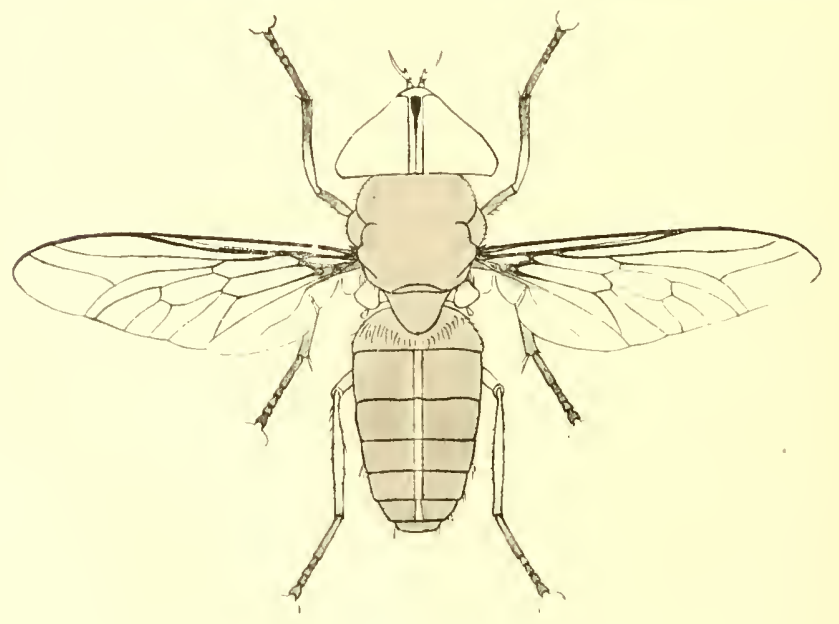

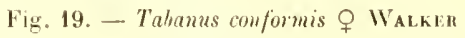

Ce groupe comprend les insectes qui réunissent les caractères suivants :

Yeux glabres, tibias antérieur's non dilatés, bande frontale de cing à sept fois plus 
bIxII.M G.HoUPE

131

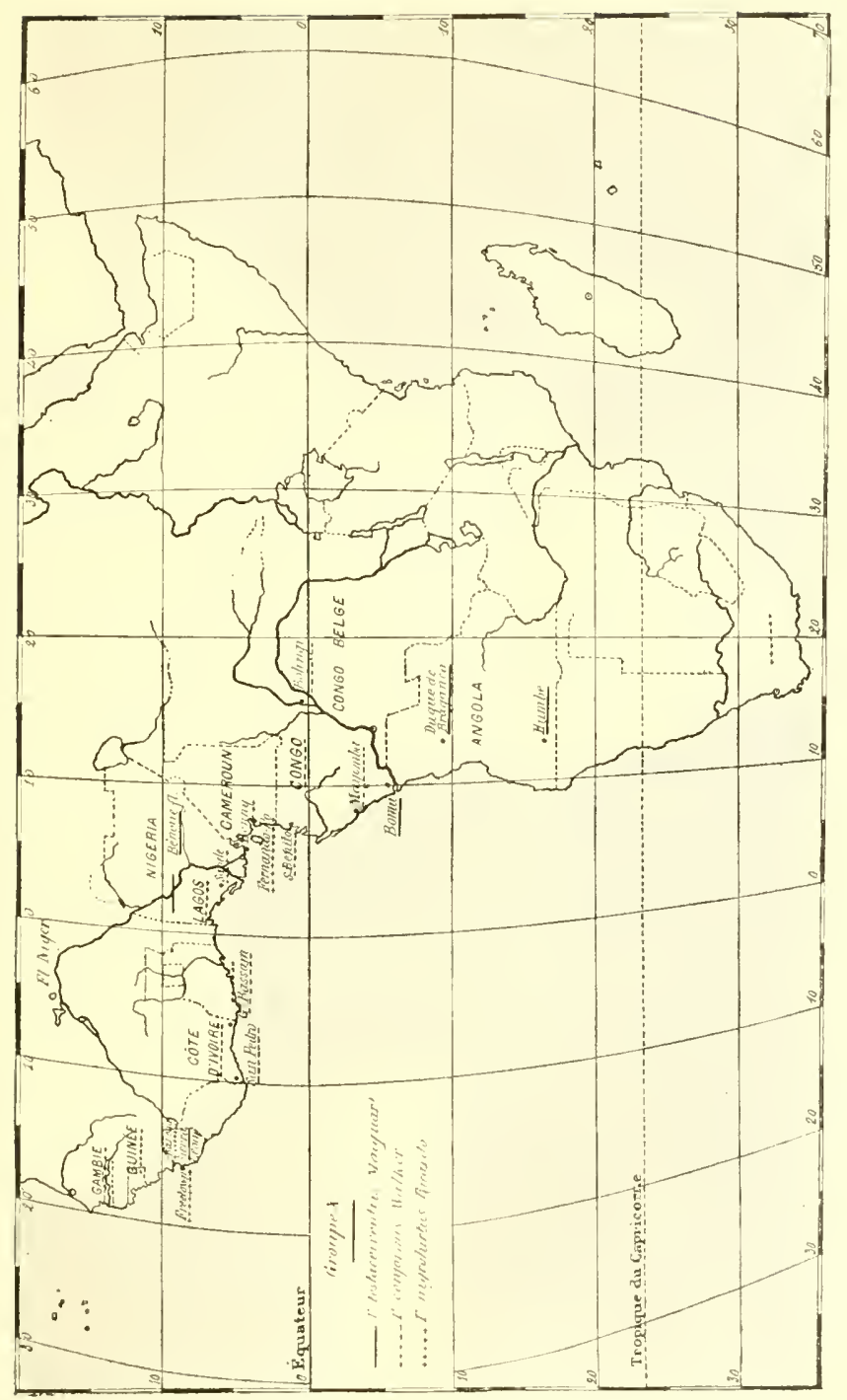


Laute que large, callosité longue el étroite, ligne médiane non dilatée, palpes clairs à pubescenen noire, ailes phus ou moins trunes. Pas de bandes visibles sur le thorax. Ablomen fauve ou brunatre à ligne médiane obsolète ou très peu distincte.

\section{Tabanus testaceiventris $Q$ Macquart}

Le type de Macquart ne porte pas d'indications de localité.

\section{T. testaceiventris o MacQuant}

"Thorax noir bleuâtre. Aldomen testacé. Intennes noires, testacées à la " base. l'ieds noirs, tilvias testacés.

" Longueur 9 lignes $=20 \mathrm{~mm} .1 / 4$.

"Palpes jaunâtres. Face d'un gris un peu jaunâtre. Front assez etroit, " lauve, à callosité allongée; vertex testacé.

"Intennes noires, premier article testacé, lent ordinaire. Thorax noir à "reflets bleus; des vestiges de duvet jaunâtre; ppaules d'un testacé lrunàtre. " Cìtés noirs. à tiaclies testilées et duret gris. Ibdomen d'un testacé un peu " brunitre, sans duvet; ventre de mème. Cuisses pt tarses d'un noir lrunàtre. " Ailes brunatres, assez elair's au bord intérieur, nervures normales.

Cous ne connaissons plas cette espèce (Afrique).

\section{BHBLIOGRIPHHE}

Tubanus testacpinentrix o Hacouart : Diptires exotipues suppl.. 2. 16-92.

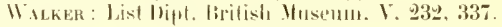

\section{Tabanus socialis o WALKER \\ $=$ Alylotus hypolencus o Bigot \\ $=$ Tabamus leomum O B1GoT \\ $=$ Tabamus nigralivtus o Ricakdo}

Le tyje de Biriot porte: "Atylotusalbotomentusus del'Issinie», nous pensons que cest en réalité le type de Atylotus lypoleucus $O$ Bigot. Y. Coluss a attiré notre ittention sir ce que l'Atylotus hypolencus o Bigot identifié par nous à Atylotus ulbotomentosus porte seulement un nom manuserit et il remarque que fréquemment Brgot donnait un nom à se's typers et le modlfiait ensuite en oubliant la première etiquette, nous ne doutons pas de la valeur de cette explication. Le type de Atylotus leonum bigot est un exemplaire mal conservé provenant de Leone. Nous trouvons que le Tabrnus nigrohirtus Ricario est le mème que Tabamus socualis. Cette expèce est assez viriahle et difficile à identifier. La couleur des pattes semble variabli cloz Tahanus socialis o Walker; les fémurs antérieurs du type de nigrohirtus of Ricarlo, el de quelques autres spécimens 
sont d'un jaune rougeâtre, chez d'autres exemplaires ils sont brun rougeâtre ou brunâtres, cliez les femelles de Kariua ils sont complètement noirs et les antemnes sont tres sombres, fo troisièm article st entirentent d'un noir lorunåtre.

\section{DBSCRIPTION DU TYPE DE nigrolirtus RICANDO}

\section{I.ongueur 16 ì 20 millimètres.}

Brun-rouge. Face rougeatre, recouverte d'une tomentositri blanche qui devient graduellement hrun jannitre sur les cótes supriripurs les jones et au-dessus dos antennes, pulesence blanche rlairsemée. Barlw londre. Palpes jaunes it pubescence noire, minces et un peu lilatés à la lase, légèrement ar'fuf́s to trminés par une pointe allongée. Bande frontale grise, couverte d'une tomentosité brun jaunatre et d'une rourte pubescence noire, la forme de la bamia es étroite, beaucoup rétrécis antérieurement, envirun huit fois aussi haute que large. Callosité frontale lrun-rouge, atroite et longue, atteignant les yeux is sir partie inferiemre, puis samincissant graduellement, s'icartant du bort des yeux et se terminant en un ligne minre; che\% quelupes spécimens la callosite niatteint pas le bord des yeux, mame it lit bilse. Intennes rouges, apex du troisiome article noir, le premier article un peu en rapuchon, second très petit, l'un et l'autre à pubescence noire: troisième article long. mince et muni d'une petite dent.

Thorax brun rongeatre portant deux lignes étroitrs, grises, très indistinctes, le dessus du thrax est revètu l'une tomentosité glauque et d'une courte puhesrence noire; pectus rougeàtre à pubescence et tomentosité grises. Sirutellum rougeatre à tomentosité glauquee.

Aldomen brun rougeattre, devenant un peu plus sombre a l'apex, portant une étroite bande médiane grise, continue, s'itendant s n fremier au cinquiènte segment; dessus de l'aldomen converte d'une rourt" pubescente noire, segmentitinns de l'abdomen parfois trís étroitement lulanchìtres; chez quelıues spécimens la bande méliane se divise en courtes taches triangulaires du second an sixỉmp segments qui sont couverts le poils d'un j:une grisitre, la lande médiane, mème dans ce cas, paraît continue, en regardant l'insecte par derrière ; parfois il ya a à peine une trace de bande. Cottés de ladudomen it poils noirs et quelinges poils blanesvers lapex. Envers d'un rouge brillant, luisant, avec moins de puhescence noire et un p’u de pulvescence l, la nche sur les ròtés, scomentations ì tourntositéblanche distionete, il y a lit trace d'une large hande noiratre longitudinale, les deux dernitrs segments sont litrement noirs. Pattes jaune rougeattre, hanches it pulvescence grise, fimurs antérieurs parfois brun rongedtre, apex des tibias antérieurs et tarses antérieurs noirs, tibias antérieurs d'un jaunatre clair sur lat moitié hasilaire, tarse's médians et postérieurs hrunitres. P'ulıscence les fémurs noire mélange de poils blines en dessous ot in bord exterue, noire partont ailleurs. Diles grises à hord rostal jaunatre, tris lingimenent teintés de brun. Yervures brun-janne, stigma jaunitre, plits d'appendice. t'reniage rellule marcinals postéricure tris lémirement rétrérir.

Nous donnons lis descriptions de Tabanus hypoloncus of Bigot et de Inhanus: 


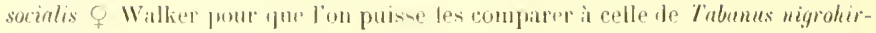
lus. f licicarilo.

\section{Tiebumes heypotemes of Bigot}

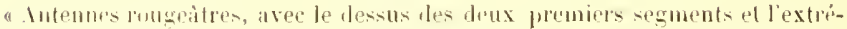

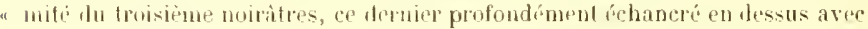

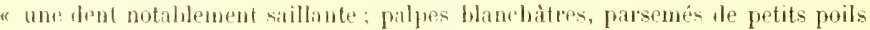
" noirs ; front et fare rougeatres. Callo-ité frontale l'un claitain rougeàtre, " allongép of fort itroite en haut, fígirement of graduellement dilatée en bas;

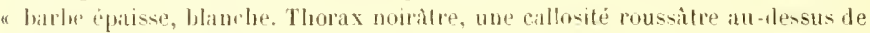
"la base des ailes: flanes et poitrine couverts de duret blanc. beusson noiritre. "Coillrons et balanciers d'un chàtain pàle. Mbdomen brun foncé, avee unt "band" diffust, élroite, at le bord des segments teinté de rougeâtre obscur ; "ventre rougeatre. diles grisitres aved le bord extrme froitement teint de * roussiltre pale. l'ieds rougeatres; lase des fimurs, extrémite des tibias, les "postérieurs plus largement ef tous les tarses miratres. Hanches eouvertes " d'une villosité blatuche. Les nervures chares ef tout le reste comme chez les "leux précédentex espèces ( $T$. combustus). 1 spécimen ".

Asinie.

\section{Tabanus socialis ? WALKER}

" Cinerens, pectore cano, alwlomine ferrugine, antennis basi pedibustue "fulvis, alis subrinreis basi et atd costam fulvis ".

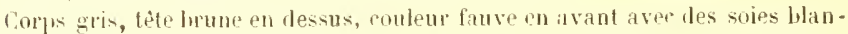
cles rn dessous. Youx ruges séparís en dessus pir un intervalle étroit. Toutes les firentris tris petites. Trompe noire, stylet ferrugineux; palpes jaunes; antennes hun-noir à la base. The trinte brunitre sur chaque còté du thorax. Pectus banchâtre. Mblomen ferruxineux pîle, obconique à pubesence courte coulenr tan, beacoup plus long que le thorax. dambes fauves, tarses noirs, pelotes lrun pàle. Tillias postérieurs avec une très eourte pubescence noire. diles forgèrment grises, fauves it leur base et sur frs trois quarts de la longueur tu bord costal. Neriurs ferrugineuses, une courbe pres de la base de la nervure transverse du sommol, mais ni angle ni appendice. Cuillems fauve sombre. Balanciers ferrugineux pàle.

Longueur du corps: 8 lignes, des niles: 16 lignes (Congo).

Les exemplaires de Tabanus sociulis q Walker se trouvent répartis sur la Góte ocridentale d’.tfrique et proviennent des rẻgions suivantes: Gambie, sierraLeone, Lagos, Nigeria lu surl, Cameroun, Fernamlo-Po, Congo betge (région équatoriate). 


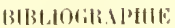

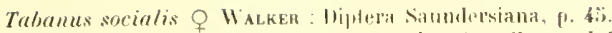

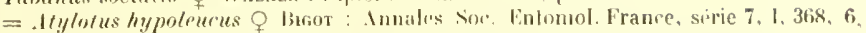
101. I. (t $8:) 1)$.

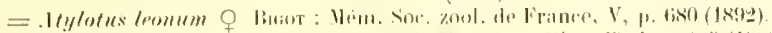

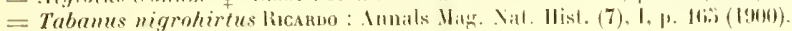

\section{Tabanus conformis $Q$ Walker ( p. 130)}

Le type femelle vient du Conso (1868). Colleclion Saunders, quoique vieux et en matuvais étal il ollre unt grande ressemblance avec le Tabanus socialis Walker (que nous identifions au Tahrmus uiyrohivtus o Ricardo), mais il paraì cependant distinct par la forme de la callosité frontale qui se lemine postérieurement en une pointe prolongér par une courte ligne étroite. Ailes presque Iransparentes, à peine aussi teintees que celles de Tabanus socialis $q$ Walker, seuls le bord externe et les nervures transvers's de la hase sont jaunâtres; fémurs antérieurs brun rougeître. Ihdomen élroil, jaunitre, apparemment recouvert d'une tomentosité grise ì lil base et sur les còtés; pubescence du dessus noire.

Longueur $18 \mathrm{~mm}$.

"Ferrugineus, capite subtus albo, lhoracts disco fuseo ferrugineoque livit" talo, abdominis lateribus fasriis gue fulvis, apiee fuseo, pedibus fulvis, lar" sis piceis, illis subcinereis fulvis ".

"Tète ferrugineuse blanche en dessous. Yeux couleur de bronze; palpes et "antennes faures (ces dernires manjuent, sauf leur base). Trompe brone. Tho" rax ferrugineux, le disque avec une itroite bande ferrugineuse sur rhaqu" " coté. I'oitrine plus palle ave" une prunosite blanchàtre et une vestitur" " de soies jaunes. Mbdomen ferruginux, brun il l'extrémité. Bordures posli "rieures el cotés des segments lauves, jaulues fauves, hanches plus philes " a vee une pruinosité blanchàtre, tarses bruns. Ailes avee une légère teinte " fauve grisatte. Nervures brunes plus sombres vers le bopl antérienr, fanves "vers la base. Balinciers jaunes. Longueur 19 inm. Envergure 38 mm.

Le Tabanus torminatus Willker indique de I'Imérique du sud et le Trubums Janus $q$ Walker de localití inconnue spmblent tous les deux the dr mimp espece que Tubanus conformis o Walker. Le Tubanns terminatus a une handr médiane grise assez distincte qui ne si voit pas sur les autres, probablement à cause de lenr atat de dénudalion, tous ont une tomentosití grise sur l'alulomen jannitre avec une pubescence nuire el la callosilé de la bande frontale est proinlue.

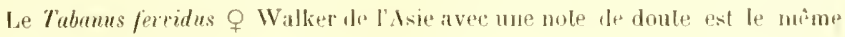
que Tabanus conformis. 


\section{BIBLIOTRAPHIE}

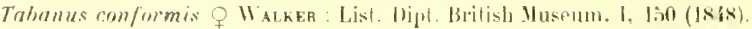

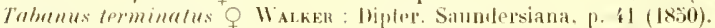

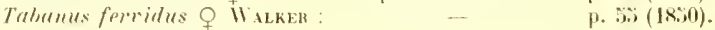

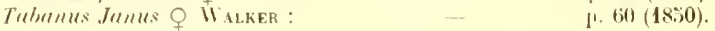




\title{
ONZILME GROUPE
}

\author{
TableaU DiChOTWMIQUE
}

$1\}$

Aites claires ou rembrunies seulement 1

au bord costal.
liles teintées de brun ou brun jaunàtre

Tarhes latérales grisitres, distinctes, triangulaires: taches médianes non relicé entre elles, taches latérales non reliées it la tache médiane. Espece brune,20 mm. Tirches latérales blaurbes. très listinctes, taches médianes des segments 2 et:3 n'atteignant que la moitié de la hauteur du segment. Taches latérates du secomi 2 segment non reliées it lia tache médiane. Espèce noire ì taches blanches, $14 \mathrm{~mm}$. Premier segment à tache blanche arrondie, leuxième ì triangle médian de lit hautewr du segment, troisieme, yua. triìme, cinquieme à trois rourts triangles. Taches entourées d'une coloration plus sombre. Espree noiritre de Hozambique. $18 \mathrm{~mm}$.

Callosité frontale longur, rétrécie : l'apex el prolongée par une ligne étroite. Caltosité frontale large et courte, prolongéc par ume ligne ditalée en fuseau. 3 Triangles mélians formant une band irrégulièr Taches latérales, oblongues, indistinefes. Face it longs poils blanrs epais; pattes brun rongeatre sombrr. 20 à 22 inu.

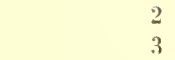

1. disjunctus o Rirardo.

T. Martini $q$ surcouf.

$T$. Inhambanensis o Bertol. 4

T. Nyesser o Ricardo. 
Girande espèce puissinte; triangles mélians de l'abiomen ayint toute la hauteur des segments, larges it lat base; taches laterales distinctes, grandes, I!t it $23 \mathrm{~mm}$.

Exprep plus tine, moins robusto: bise du it second triangle médian étroile, haches latérates plus petites; face ì courte puluescence blanche éparse; pattes rougeâtrés, 16 à $18 \mathrm{~mm}$.

Triangles medians reliés aux taches latérales. arrondies, de la hatuliu du segment: pattes lounatres à pubescence b) lanehitre; $18 \mathrm{11m}$.
T. ustus or $q$ Walker.

T. congoiensis $q$ Ricardo.

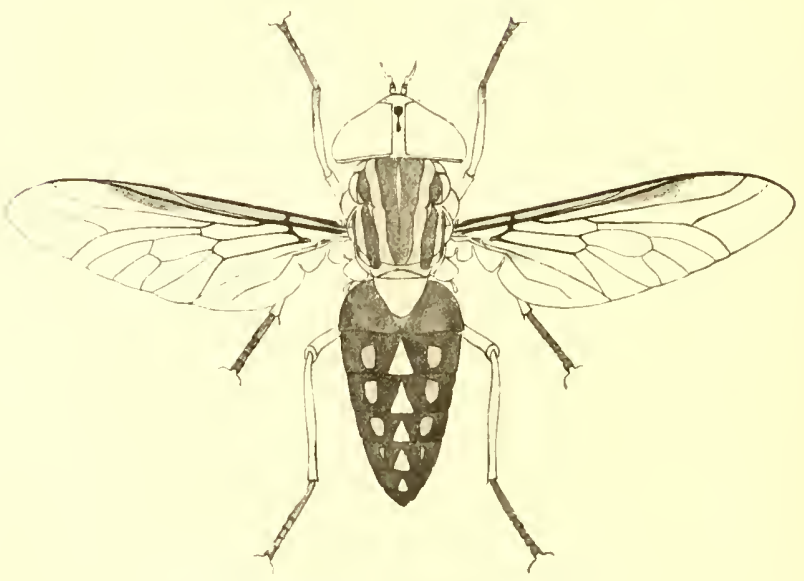

Fig. 20. - Tabumes disjmuctus Q licarbo

Ce groupe contient les insectes réunissant les caracteres suivants: Yeux glahres: tibias antérieurs non dilatés: lande frontale de cing à sppt fois plus haute que large à ligne médiane non dilatée, prafpes clairs à puhescence noire. Niles plus nu moins teintées de brum, sans aljundice. fhorax à handes plus ou moins visibles. Aldomen portant des triangrles clairs sur la région médiane et des taches latérales très distinetes, généralement triangulaires. 


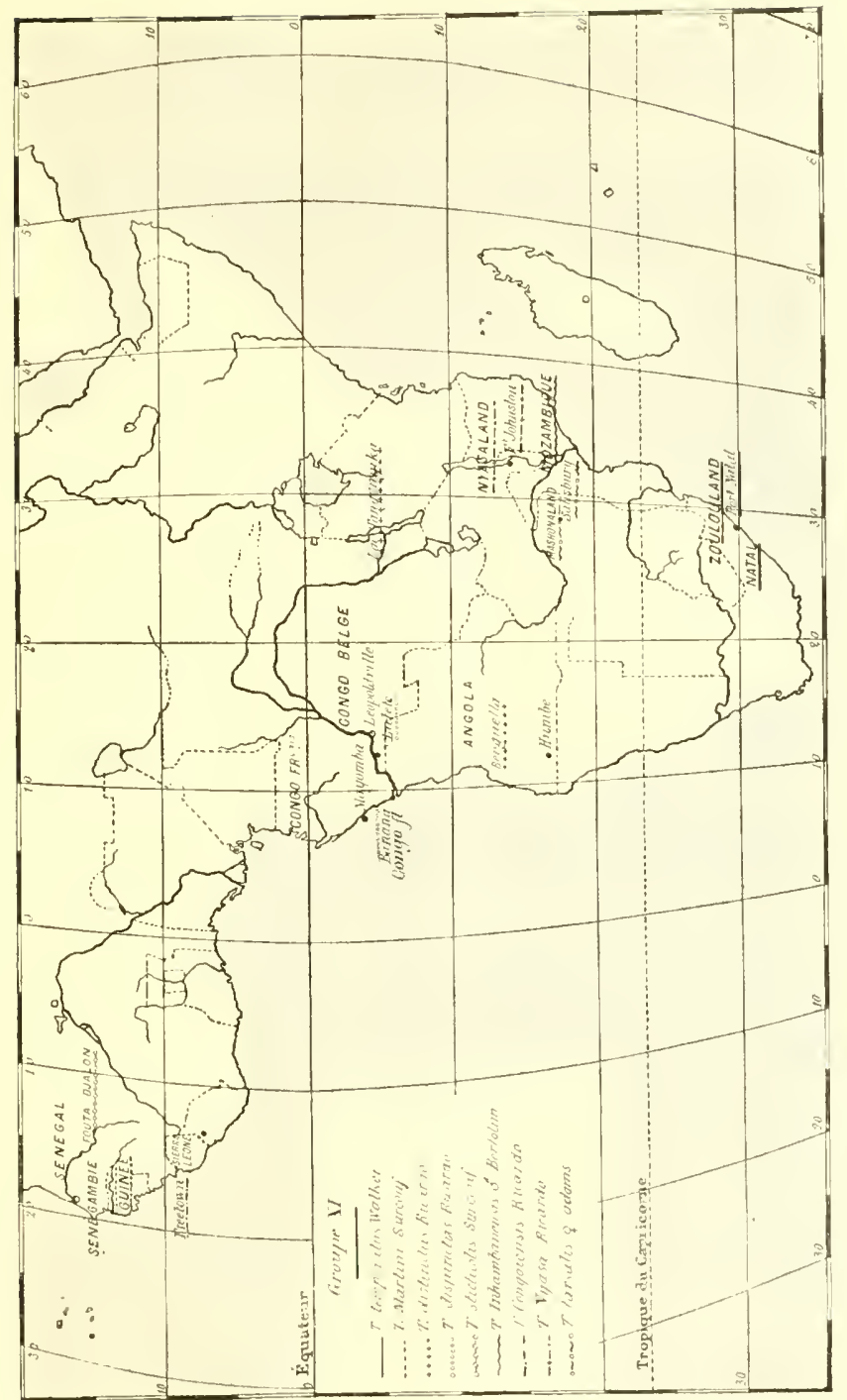




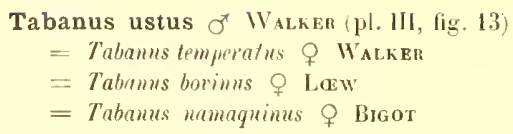

Le type provenant de Port-Natal (49. 227). Collection Quienzius, fait partie de la collection du British Juseum.

"Piceus; caput llarmm; antennar lufe, basi nigre; thorax fulvoruinquevit" tattus; abdomen ferrugineum, apice piceum, maculis trigonis fulvis orna" tum ; pedes ferruginei, tarsis piceis; alit riners, all costam fuscie, ventrum " marginibus subfuse is."

Couleur de poix: tète jaune, antennes rousses, noires à leur base. Thorax it eing bandes fauves. Alulomen lerrugineux, apex couleur de poix, orné de taches triangulaires fauves; pieds ferrugineux, tarses couleur de poix, ailes centrées, lorunes ì la rỏe: bord des nerrures presigue lirun.

Port-Natal.

Ce taon est évidemment le màle de l'espèce dont la femelle a été nomuér piur Walker T. temperatus.

11 a de grands yeux, les cornéules des denx tiers de la partie supérieure des yeux sont grandes, lit face portr les poils jamnes. Il a l'abdomen d'un rouge un pren plus brillant que les femelles, aver des titches médianes moindres, les taches latérales sont indistinctes; labulomen est aussi plus court, les palpes sont james avec ces proils noirs.

Longueur : $20 \mathrm{~mm}$.

Le type femelle provient du Xatal (collection SAunoers) et apprartient au Britislı II (févier et mars 1906), recneillis sur les troupeaux (F. Toppra) at communiquies par E. Warrex en 1!307. Le type de Tabamus namaquimus $q$ Bigot provient du Xatal. Le muséum de Bruxelles possède un spécimen détermine Tabams boximus par VAx DER Wup ef iue l'un doit rapporter an Tabanus temperatus o Walker. Le type de Walken est sans conteste l'espèce que Lew range sous le nom de Tabamis borimus quoiquil remarque que les spécimens qu'il possède diffirent un peu de la forme européenne et qu'il en spérifie les différences. Yous conserverons le nom de Wakken pareo quon pent considérer cetle forme sud-africaine do Tabanus hovimus comme ansez dissemblible du type pour rn faire une espèce distincte. Le type de Wallier mesure $23 \mathrm{~mm}$., les autres spécimens varient de 20 à $23 \mathrm{~mm}$.

Callosité frontale plus longue "plos étroite que chez Tubanus bocinus; abdomen de coloration plus sombre surtout les còtés du second et du troisième segment dont la couleur jaune est moins pridominante, mais les grandes taches latérilles grishtres ou jaune rougeitre sont beaucoup plus distinctes, de forme irrégulipre et toujours visibte sur rhinge segnent quoiqu'elles décroissent gratduellement de taille. Intennes d'un rouge brillant; dans le type de Walker, noires seulement à l'aprx: dans quelques-uns des specimens, cepenlant, la couleur rouge du troisième article est continné à la base où elle est obscurcie par 
une tomentosité sombre; le troisieme article ast plus étroit al lat base que relui de Tabanus bocimus L. Thorax a handes grises plus distinctes.

Alylomen brun sombre noiratre, les taches grises triangulaires tourlent par leur apex le bord antérieur de claque segment, les plus longs triangles sont ceux des deux preniers segments. Taches latérales grisitres, grandes et de forme irrégulìre, atteignant leur maximum sur le second segment et décroissint gral Juellement de laille. Côtés ì poils lllanes; enters à bande médiane noire; segmentations étroitement brillantes. P'altes rougeàtres, férnurs antérieurs noiràtres, médians et postérieurs parfois brunàtres; tous il pubescence blanche of tomentosité grise. Apex des tibias antérieurs noirs, tous les tarses d'un brun noirätre. P'ubescence des tibias blanche, entremèlér te poils noirs, par ailleur's elle est noire. Ailes teintées de brun. Tous les exemplaires connus proviennent du sud africain.

\section{Description de Walken : Trubumus temperatus q Walker}

«Piceus, cinereo tomentosus ; caput supra ferrugineum, apud oculos luteum, " callo piceo; antenna rufie, hasi nigre; thorax ferrugineo quinque vittalus, "scapulis testaceis. Abdomen vittis tribus nacularibus testaceis, literibus basi " ferrugineus, segmentorum marginibus posticis subtus testaceis: pedes fulvi, " tarsis, tibiis anticis antice femoribusque anticis nigris. Ha cincreæ, hasi " rosta et apud venas discales fusca, halteres fulvi, apice albidi. "

De couleur brune avec me pulsescence cendrée. T’̀te tomenteuse, ferrugineuse en dessus, jaune vers les yeux, testacre en arric̀e et en dessous; callosité lrune. Veux brillants, toutes les facettes trìs petites. Palpes cendrés. Antennes noires, premier article prolongé it sa lase en un angle aigu ell dessus, second article rouge, formant en dessus une mince corne aigui"; troisième article rouge, courlhe. sa corne est petite, courte et forme un angl" presque droit. Thorax aver cinq bandes ferrugineuses indistinctes, épaules testacées.

Abdomen hrun, ferrugineux de chaque fòté vers la hase et portant trois petites taches en forme de bande testací pale, des taches latérales diffuses et des taches triangulaires dorsales, décroissant successivement de taille. Bord postérieur des segments testacé en dessous, lambes fauves: tarses, fémurs antérieurs sauf l'apex et apex des tilias antérieurs noirs. Ailes grises, hrunes a la base, le long du bord rostal et vers les nervures discales. Nervures noires, lranche antérieure de la nervure cubitale simple, formant un angle obtus, arrondi près de sa base. Balancier's fauves ave l'extrémitŕ blanchàtre. Longueur du corps 9 lignes, des ailes 18 lignes.

l'ort-Natal.

\section{Trbanus botimus o LaW}

Note de Lew

" On ne peut pras le séparer de notre Tubums bovinus L. quoiju il ditfìre un peu "de tous les exemplaires que je peux lui comprer. Il se rapproche des spóci- 
" mens chez lespuels la coloration noritre est largement étenlue sur les denx

- fares de l'abdouren. Les petites lillirences sont les suirantes:

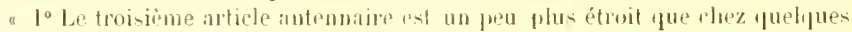

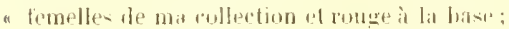

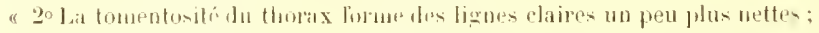

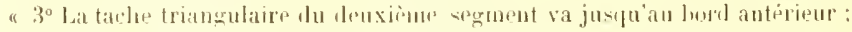

\& $4^{\circ}$ La pubescence noire s'blargit sur l'ahdonen, principalemont sur le der-

\& uier anneau et sur les còtés plus que dins les exemplaires européens;

" "o ha tomentosité forme des teux potés de chapue anneau une tache plus "nette it plus wrise";

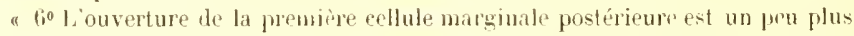

" birge quechez quelques femelles d"Europe, quoigues res dernieres varient asse\%

" fortensent entre elles;

a $7^{0}$ Les cuisses médianes et puslérieures ont sur leurs côtés inférieurs et pos-

" terieurs la mème conteur ronge brunalte que les libias el ne sont colorés en

"Irunâtre que sur lemrs còtés antérinurs et supérieur's.

"La seule différence un yreu évidente riside dan- la forme des antennes, in

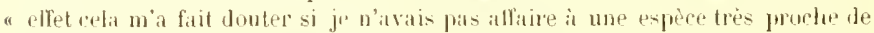

"Tuburus bovinus L. La rrsiemblancedes autres prolies ne permet pas de faire

- de lunique spéciuren femplle recueilli par Walılberg une variété de Tabamus

" hovimus L. Seul un plus grand numlu" d"expmplatres concordants justifierait

" peot itre la création dune nouvelde esprece. Les reax ue montrent auruardif-

"Pérence après le ramolli-iement."

lafrerie (Wahlberg).

Tabanus momnuimas o B1iot

- the femelle, longueur $20 \mathrm{~mm}$.

"Intenn's noires, troisime segment a hase rougratre, notablement concave

" "t denté en dessux. Palpes d'un fanvo pile. Fare, front et barbe jannatres; le

" wilieu du front teinté de hrunilr". Callosité hronatre, flargie el presque car-

"rée interieurement, teminè en dessus par une ligne fort étroite. Thorax d'un

" brun rougeitre avec deux bandra plus pales et peu distincles. Ecuscon ron-

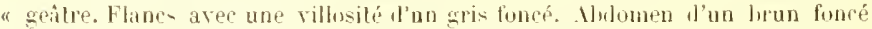

" avec une rangée dorsale de macules trizonates, grisaltres, ef de rhacque côté

" une large lande dilfuse, rougedilre, interrompm aux sutures. Cuilleron.

" Houns. Bahanciers jaunatres avec la massue hlanchatre. Pieds d"un fauve rou-

- geâlre aver l'extremité den tibias et des tarses antérieurs brunatre. Ailes snriser,

a la base et le bord externe jusqu'auprés de l'extrémité et les nervures transver-

- sales bordíes de brun paile.

Port-Natal, un spécimen. 


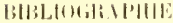

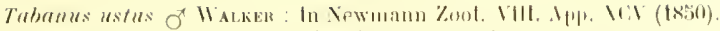

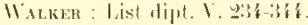

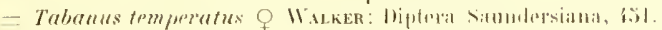

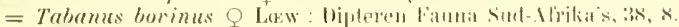

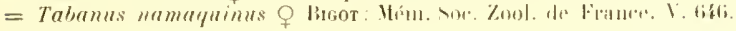

\section{Tabanus disjunctus $q$ liıcardo (pl. III, tig. 6)}

Le type ot une antre femelle proviennent de Lulete, Congo (19 novembre 1903 et ont été communiyuís en 1!104 par l'Ecole de métecine tropicale de Liverpool.

Espèce à trois síries de taches alulominales grises semliables à celles de Tabanus distinctus q kicurdo, mais les lirhes métlianes ne se relient pas entre elles et les pattes sont plus somplètentent rougeàtres, fémurs antérieurs brunìtres, taille plus grande et rouleur flus brune. Le Tabams disjunctus q Ricarilo se distingue du Tubrmus sticticolis surcouf par sa taille plus grande et ses tarhes latérales plus distinclement définies. Le Tabamus disjunctus of R. (p. t38) diffère Tabanus distinctus o Ricardo par les particularités suivantes:

Face couverle d'une tomentosité brun-jaune et de courts poils épais el blanes. Palpes jaune clair plus grands et plus épaix. Anlennes plus sombres, letroisiène) arliçe noirâtre, rouge seulement à la hase. Bande frontale à tomentosité jaunàtre, vertex non rougeatre mais avec une tomentosité sembiable ì celle de la bande qui est environ six fois aussi haute que large, rétrécie légèrement vers le vertex.

Thorax lirunitre avec cinq landes grises indistincles, scutellum de mème couleur.

Abdomen larun, lache médiane du second segment avec un long apex qui cependant n'atteint pas le bord postérjeur du premier segrment; la seconde tache siluée sur le troisième segment est triingulaire et atleint à peine la moitié de la hauteur du segment, troisième tarlse avee un apex un peu plus long; la quatrième tache placée sur le cinquième segment est prestue triangulaire et arrive près du bord postérieur du segment prérident. las de taches sur le sixième el et le septième segments. Les taches latériles sont an nombre de quatre sur chaque còté et complètemeut semblables in relles le Tabanus distinctus mais clles sont moins hien définies et irréxulirrs. Envers rougeâtre, segmenta tions à poils hlancs. Pittes rongeàtres: extrínités apicale des cuisses anlérienres, apex des tibias antérieurs et tarses noiritres. Ilanches it longue pubescenre blanche; fémurs antórieurs à courte puhescence noire vers te haut el une longue pulescence blanche vers le has; il $y$ a aussi ume pubesence llanche moins longue sur le hord externe des autres fimurs. Tibias antérieurs rouverts d'une épaisse puhescence blanehe sur leur moilié hasilaire, les autres tilias ont une pubescence blanche moins dense, elle est noire sur les tarses. Lilps claires, jaunâtres au hord antérieur, stigna jaunàtre; nervures brunes, première rellule marcinale jostérieure légèremenl rétrécie. 
Longueur 20 min.

Le Tabunus disjumtus of Ricardo est connu du Congo, de lit Rhortesia et the I'Toganda.

De nouveaux matériaux de Thbunus ustus Walker et de Tabunus disjunctus Ricardo, rerus par le Brilish Museum depuis la descriplion at Tabomus disjunctus permettent de voir de nouvelles relations entre ces deux espices, et il ent possible que plus tard il faille les réunir.

Dans Tabanus disjunctus les triangles médians sont súparés les uns des autres, les taches lalérales triangulaires dans le tyje de disjunctus sont parfois plus irrẻgulières et plus rondes. comme celtes de $T$ temperatus.

Les ailes sont halituellement claires.

\section{BIBLJOGKAPHE}

Tabames disjunctus Q Ricarbo: Innals Vag. Nat. Hlist. (8) 1, 32.; (190k).

\section{Tabanus Inhambanensis of Bertolisi}

Type de Inhambane, Mozambique. Cetle espece doit appartenir au groupe de Tabrmus bovimus L. el étre voisine de Tabanus disjunctus o licardu mais son sexe ne permet pas de l'identifier avec certilude. Vapric la figure de l'auteur, l’abdomen porte les taches suivantes : sur le premier segment une lache blanche arrondie; le second segmenl porte un triangle médian large à la hase dont l'apex atteint le bord anterieur du segment, chacun des trois segments suivants porte trois couts triangles, sur le sixiome segment se lronvela lache médiane oblongue habituelle. La description ne donne abeun detial sur ces taches mais elle indique qu'elles sont entourées d'une coloration plus sombre. L'auteur décrit son insecte comme une espẹce noiråtre, allongée, le thorax a trois handes blanches de mème que les côtés: fémurs et tilias rouges, tarses lruns. Intennes d'un jaune rougeâtre, sombres ì la base et noires à l'apex. Face courerte d'me épaisse pubescence blanche. Palpes rougeatres à pubescence noire. Alulomen thorax d'un marron noirâtre, lahdomen est plus rouge. Ailes complitement claires. Longueur $18 \mathrm{~mm}$. envirun.

\section{BIBLIOGKAI'IJE}

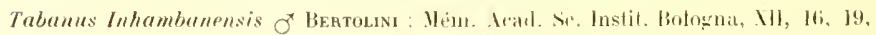
tab. 1. lig. 7 (1862).

\section{Tabanus congoiensis o Ricardo}

Le type femelle provient de Wahthen, Congo francais (Rev. W.-H. Bentenx 1904). Deux autres femelles de Wathen, une de Tumbo (Congo).

Espèce lıun sombre à laches alulominales mèdianes et lalèrales grises bien 


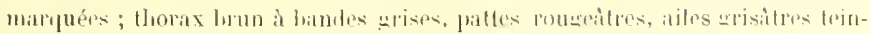

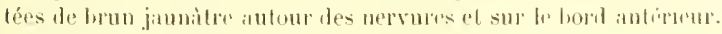

Longueur lli

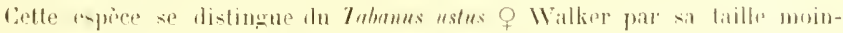

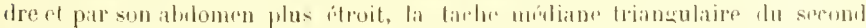
segment est plus itmite a sil base of de rontour plus oblong; la crmber de

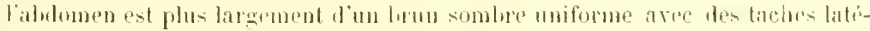
rales petites mais bien distinctes.

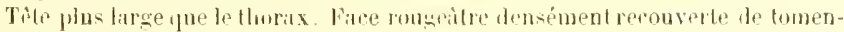
fosilé grisitte ; condeur du fond visible a lit partie supérieure de la liace et vers la

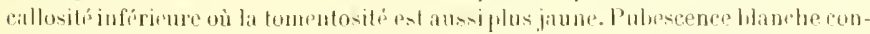

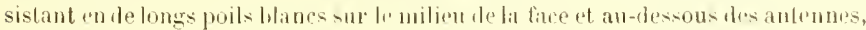

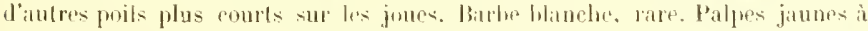

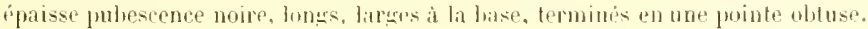

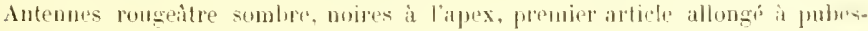
cence noire; second arlicle court, muni le poils noirs vers le prolongement

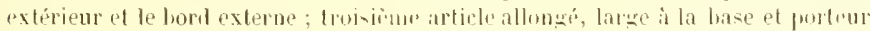

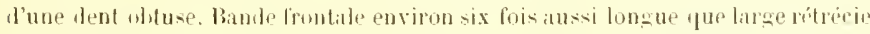
vers le haut, rouge ave une tomentositi lombe at jaube ; rallositi forntale lom rongeatre. ollongue, convese, alleignant romplitement les renx ef protongep par une ligne rpaisse juspu'a la moitié di la hauteur de la hande frontalı. Yeux sans marques ni dessins.

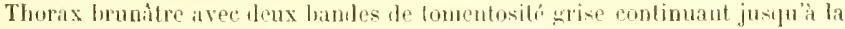
hase, crités et hordure antérieure aris, pubesernce formée de long pails noirs

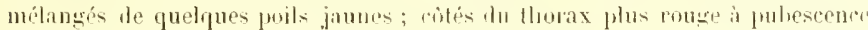

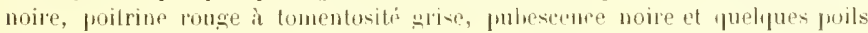

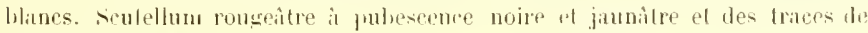
tomentosili grise.

Ildomen brunidre, passant au lorun rougeatre sur le scomd spment ; promier segment recouvert d'une tomentusití "zrise au hom antéricur, sul le lurt postírieur se trouve une petitu tache métian jammitre à poils faurts, division deux par un trail ou un sillon. Sur le deuxieme segment lit liche grise triangulaire rol longue, atteignant le horid antirieur, étroile a sa lase ; str les trois segments suivante les taches sont plus darger, plus eoutes et n'alteiznent pits lo

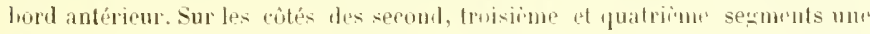
tache grise, arrondie et isolée est visible. Mitns une ales femelles do Wathen ces

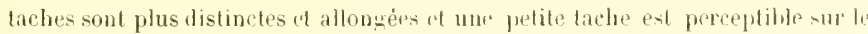

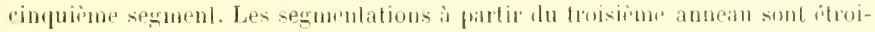

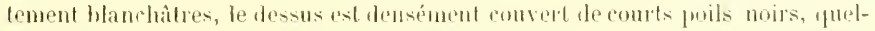

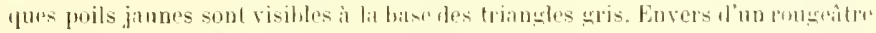

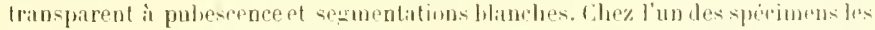
tacheslatérales sont presque indislincles, to triangledu serond sugment n'attrint

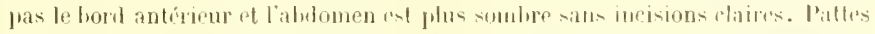

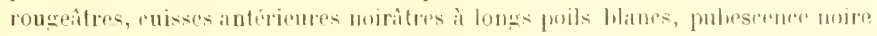

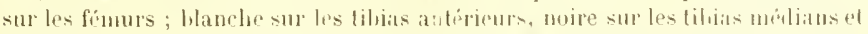

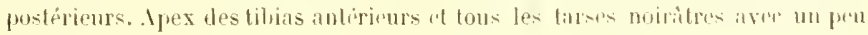
de tomentosité grise sur les fémurs. Ailes gricitres trinties de brun jiunilre Surcout 
sur toules les nervures et sur le hord intérieur, stigma de meme couleur, nervures transverses lirunes.

In autre sperimen de. Wathen a les taches latérales indistinctes, le triangle du second secment natteint pas le loril antérieur et lablomen est plus Jarger sans segmentations claires.

Le Tahanus congoipusts O licarlo se rencontre sur ta còte occidentale d'A frique, phusirurs exemplaires proviennent à notre connaissance de Sierra-Leone, Congo français, et Iluilla-Ingola (CA.yp «xa, 1886).

\section{BHBt(MRRAPHIE}

Tabames comgriensis O Ricardo: Aunats Mag. Nat. Hist. (8). 1, f. 32N (1908).

\section{Tabanus nyassæ $q$ Ricardo (1900) \\ $=$ Tabquus tarsalis o ADAMs (1905)}

Le type fenselle appartient à la collection de M. Imstant et provient du fort Johnston, Nyassaland (Rexpala). The femell, de salisbury, Mashonaland (R. Il. Thomas).

Espice itun brun rougedtre olscur; abdomen it tarkes medianes triangulaires formant une banle et it taches latérales indistinctes. Thorax à handes bongitudinales indistinctes. Ailes lrìs légìrement rembrunies, Longueur 20 millimètres.

Face recouverte d'une tomentosité jaune grisitre et d'une épaisse pubescence hlanch sur la partie inférieure. Barbe blanche. Palpes jaunitres à pubeseence noire, morlirément epais à leur hase. Antennes d'un noir brunâtre, premier article rouge, angle supérienr nuirilre recouvert d'une pubescence noire ; second article rouge it pulsescente noire sur l'angle supritieur ; troisirme article rouge à son extrine hase portant une dent listincte. Bande frontale couverte d'une tomentosité uris jaunitre, environ ciny fois anssi haute que large à la base, lígerement rátrécie antrieurement. Callosití frontale, brillante, brun rougeàtre, n’atteignant pas les yeux "t prolungée par une lịne longue é épaisse qui s'élargrit en fusean; parfois un peu de pubescence noire sur la lande frontale.

Thorix noiritre portant ciny landes grises tris indistinctes et une tomentosité urise; soutellum lougeatle à tomentosité grise.

Abdomen drun lnun rougentre olisem ì taches médianes triangulaires grises

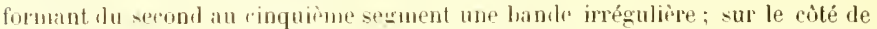

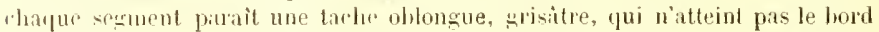
antífirur ; trorl postérieur des segments est étroitement gris ; angles postérieurs du finquième et du sixime sogments fauves; dessus de l'ahdomen couvert d'une courte juhescence noire ef d'une tomentusité grise. Envers rougeatre portant une batute médiane indistincte noire. Pattes rongeàtres; larses antérieurs noirs, les atries lirun lougreatre; puliescence dles fémurs ot des tibias blanche, aver des proits noirs mélangés sur les tibils postérieurs et l'apex des autres tilbias. f'ulrescence des tares noire. Ales livalines faihlement teintées de lrun jaunâtre, au bord contil et intour les nervores hasilaires, stigma loun jaunàtre, ner- 


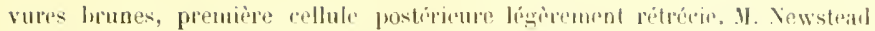

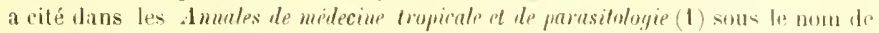

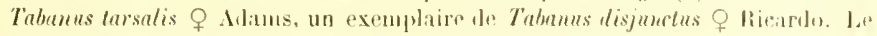

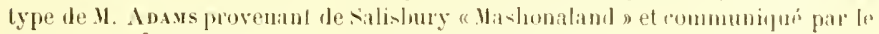
Jr sxow, esi semblable au type de T. Nyeser o licarlo.

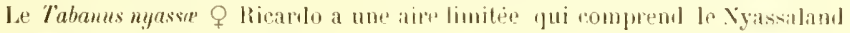
et le Mashoniland (Est aftirian).

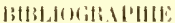

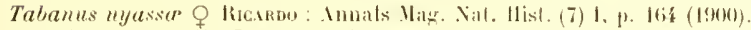

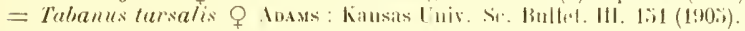

\section{Tabanus Martini o suncouf ( $\beta l .111$, tig. 4)}

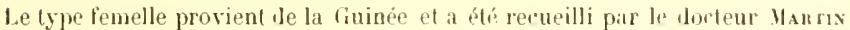
a jui nous le dédions (1907). Cing autres femelles de la mème jrovenance (colleetion de Vusém de laris). Noir is taches médianes et latérales triangulaires, blanches.

Longueur 1't millimetres.

Yeux sans bandes ni dessins, bande frontale cing fois plus haute que large, recouverte par une courte tomentosité blanchitre el portant une callosití onivile non tangente aux yeux et prolongée par une ligne droite un peu épaisicie jusqu'au delì du milieu de la bande. Antrnnes noires, premier article à pulbescence blanche; jalpes allongés d’un blan: jaunàtre il pulescence blanche et portint sur toute la partie externe des poils noirs rares et régulierement distribués, triangle frontal d'un blane junatle brillant, larbe blanche, joues hérissées d'une pubescence blanche.

Thorax noir à cing bandes blanches, lat mediane druite et courte, deux laterales et deux externes qui se rejoimment au smmet du fhorax au-dessus du scutellum; pubescence du thorax rare et concolore. Flanes a poils noirs, prectu a il pubescence cendrée et à quelgues toutfes de poils jaunes scutcllum it fornentosité blanchitre, bordé de jroils blanehatres.

Ibulomen lorun noiratre sombre putant un triangle médian hlanr sur les segments deux a six inclus, des triangles latritux blanes nettement visibles

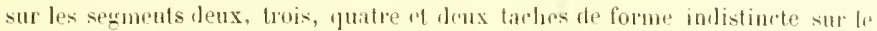
cinquieme segment. Le triangle médian injuilaténal du secomd segment atleint la moitie de la bauteur du segment, at n'est pas relio anx triangles latíraux; to triangle mélian du troisième segment nalleint que le tiers de la hautar du segment; sa lase est plus large que celle du triangle pricédent et ser relir ans

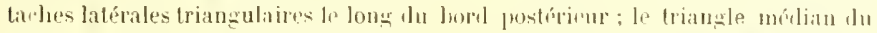
quatrieme segment ocene la hauteur totale ef est refies an taches latínles:

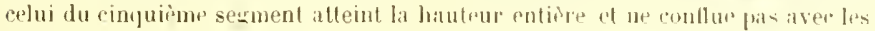

(1) I (1), p. 45 (1907). 
taches latírales de forme indislincle. Pubescence rare delacouleur des diffírentes régions colorées, ventre cendré ì pulescence blanchâtre. Pattes d'un rougeâtre pîle à pulescence blanche, tarses rembunis à pulescence noire. Ailes hyalines un peu grises, stigma jaunâtre, cinquième nervure longitudinale lérèrement estompre Je hrun. Balanciers à disque jaunatre.

\section{BIILIOGRMPIIIE}

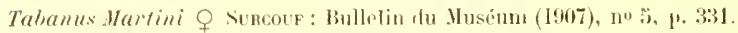

\section{Tabanus Lemairei $q$ Surcour}

Type : un exemplaire femelle provenant de Katanga, Congo, recueilli par la mission Lemarre et appartenant au Musée royal d'hisloire naturelle di Belgique.

In second exemplaire frmelle porte la mention Heyns, Bas-Congo (musée Treveren).

Nous dítlions à M. Llemalne ce nouveau Tabanus.

11 appartient au groupe de T. temperatus WaLher et est très voisin du T. Martini 9 Surcovf (Bulletiu du Musium, n" 5, p. .332, 1907).

Longurur, 18 millimètres.

Noiratre are une hande abdominale composée de triangles gris et dr chaque cóté unc hande composie de taches grises, arrondies el non reliées entre elles.

T'ète srande, plus large que le lhorax; yeux sans bandes colorées; bande frontale ciny fois aussi baute que large à la base, d'un brun jaundtre; portanl à la base une eallosilé hrun noirâtre, étroite, allongée, prolongée par une étroite ligne noiritre, saillanle, sélendint jusque vers le milieu le lit hande. Triangle fronlal d'un blane grisiltre. Joues ornées d'un poil blanchatre, partie supérieure le la tète peu velue. Intennes brunes, promier article obliquement tronqué du dessus vers lir dessous, rouvert de poils blancs; deuxième article court, glabrescent; troisième article de mème coloration que lo précédent, portant une dent peu saillanle mais neltement visible. Palpes allongés, d'un gris brunâtre, portant quelques courts poils noirs.

Thorax noiratre portant les fraces de cinq handes grises au bord antéricur. Scultellum noir, recouvert d’une jollinosité grisittre. Abdomen lırun noirâtre, portant sur le milieu de charjue segment. le premier compris, un triangle de pollinosilé grisitre, dont la base it partir du deuxième segment limite le bord de l'anneau et se relie aux taches litterales. Celles ci existenl sur chaque segment sons la forme d'une tache blanchitle atrondie, occupant foute la hateur du segment. Dessous du corps grisàtre.

Pattes brunes recouvertes d'une lomentosilé blanchâtre et d'une pubescence de mème couleur, tarses d'un noir rougeître ì pubescence noire. liles rembrunies à nervures brunes, bord costal el stigma d'un brun foncé.

\section{BILUMRTIPIIE}

Tabanus Lemairei O Surcovf: Bulletin du Mhséum, 1908, 10 2. 11. 122. 


\section{DOUZLEME ( IROUPE}

TABLEAT: Dichotomove

(Espèces portant une tache mèdiane sur les seuls segments abdominaux 3 rt 4 ; tarses antérieurs dilatés . . . . . . . . . .

Especes à dessins abifominaux differents, tarses antérieurs non dilatés. . . . . .

(Tarhes du deuxième el ln quatrième sig. ment, transverses, argentées; ailes teinlécs. Taches du deuxième et du iquatrime seg\%. argentens 9 surcoul.

Ailes hyalines, à stigma jrestue invisible: taches du troisième du quatrieme segment 3 grises ou argenties; bandes thoraciqnes bien visibles.

diles teintées à stigma tròs visible

T. Sharpei $q$ Iusten. it

Taches du troisième et du quatrième segment grises ou argentées, bandes thoraciques gris prile, visibles seulement par róllexion;

Des triangles et de grandes laches latérales

$5\left\{\begin{array}{l}\text { sur chaque segment, ventre jaume d'or . . } \\ \text { Dessins differents, ventre jimais jamm }\end{array}\right.$ 7. sulcipalpus o lacw. dior.

$6\{$ Siles hyalines. . . . , . .

t diles teintées, au moins au bord costal. 
. Ddomen portant six tirhes blanchessur le second segment. . . . . . . . . . Atrdomen portint denx ficlios blanches sur le second spgment. Jydomen noir. $11 \mathrm{~mm}$. - Abromin à tarhes latérales blanches sur le

T. insignis o lum.

T. diversus o Ricardo. seenol segment at une tacho triangulaire blanche sur cliague siguent; the rangée latérale de points blanes sur lis swments 3.

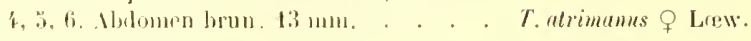

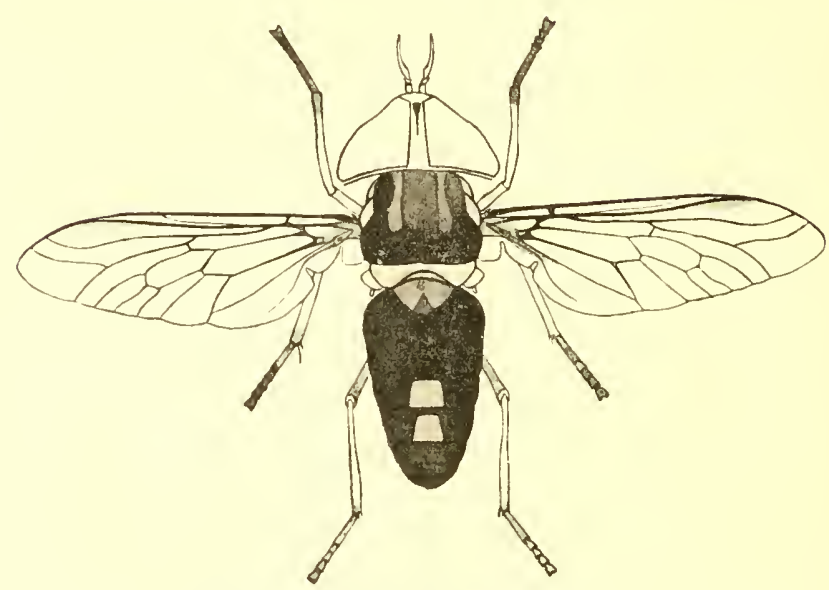

Fig. 21. - Trebanus Roubandi o suncour.

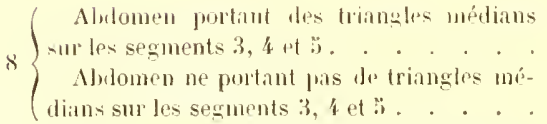

( Mulonen rougeatre portant sur le dellxime stzment deux taches orbiculaires tangentes au bord antérieur et une tache médians triangulaire sur le- anneaux $3,4,5$. . .

Alromen brun portant une tirche médiane triaugulaire sur los segments 3 , 4, 与, rea triangles atteignent da moitié de la hauteur du segment

\section{T. Sererimi o surcouf.}




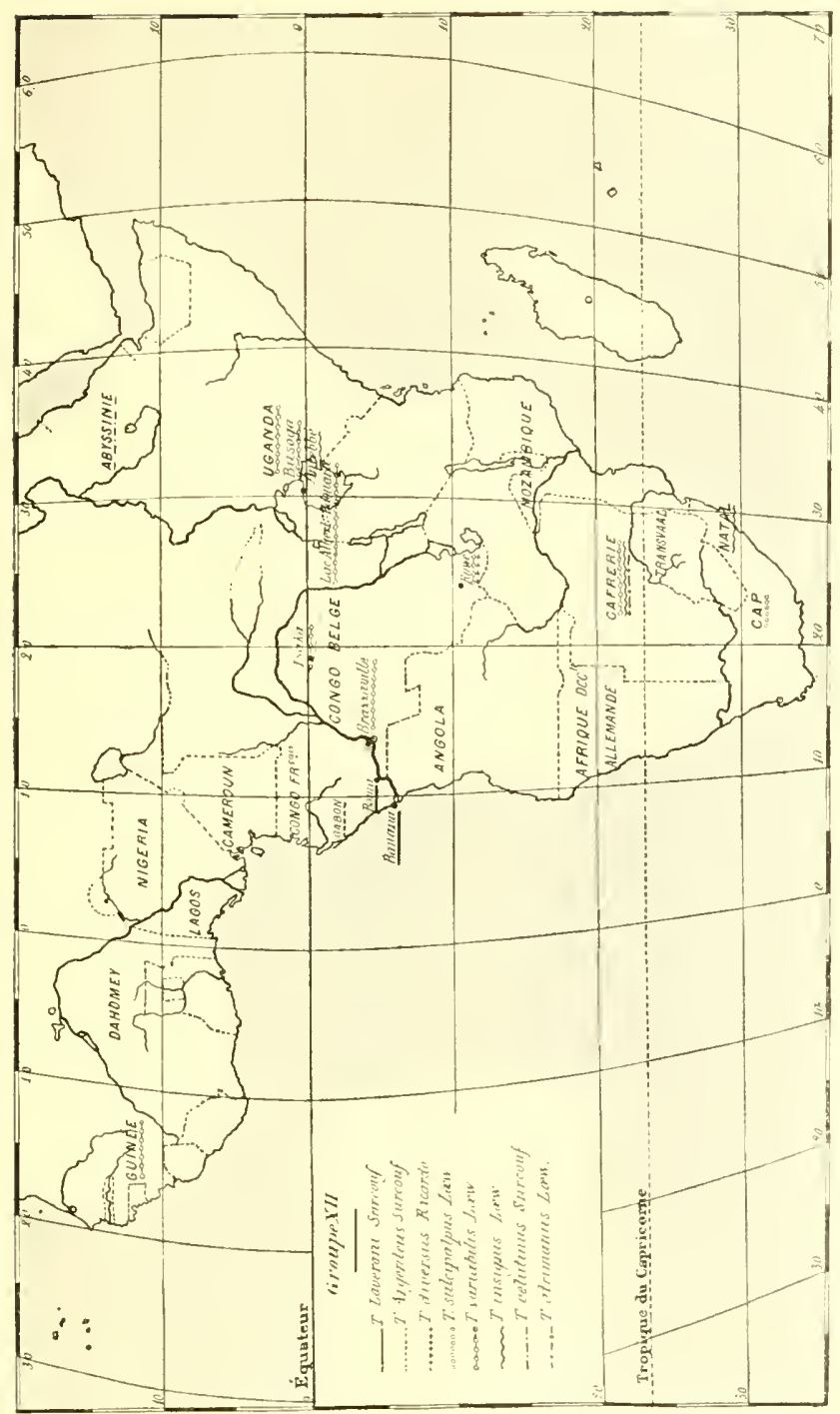


(1)

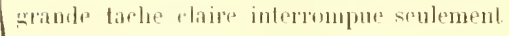 atu milien par mo tir.lue du fond, palpes
1 benxieme segmenl abdominal portant une blancladres . . . . . . . . . . .
Deuxime segmenl ablominal fortant une gramle tache claire interrumpue par la tache

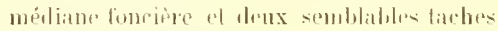 lithinals's ; pitpes noirs.

Cie roupe comprend des insoctes te laille moyenne ou pelite répratis dans

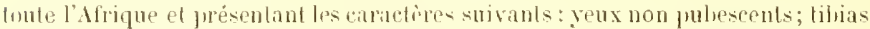
antirieurs non dilates; handes frontales de dimensions diverses. Ibdomen por-

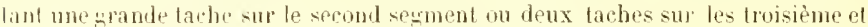
qualrieme segments. Liles hyalines ou limirment rembrunits.

\section{Tabanus argenteus $q$ Sivcou (pl. 111, fig. i)}

Le trpe femelle est dans lis colleclion du musie de llambours, recucilli par 11. Sortux en IS8I an Vialwn, il portail lin mention: Tubunus guttatus WiedeIIIำ11.

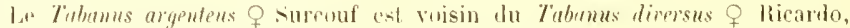
matis il sin distingue par l'illsence de foutes taches biterales et le lrandes mar le thurax. Les antennes ef lit hamale lrumbles sunt par aillewrs rompletement dilfépentes.

Lonuluend 11 millimelres "j.

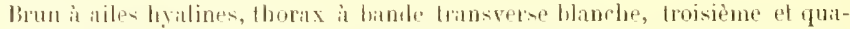

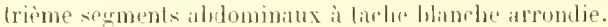

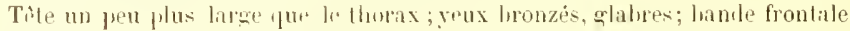

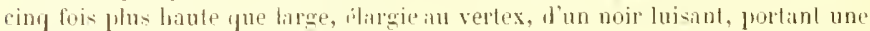

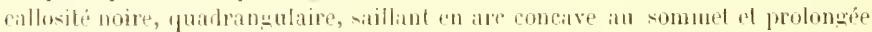

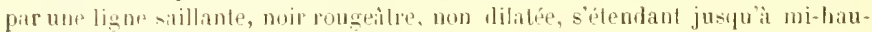

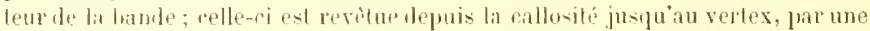

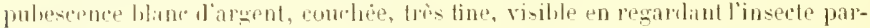
dessus. Triangle fronlal noir it pulescence blanche analogue à celle dr la londe.

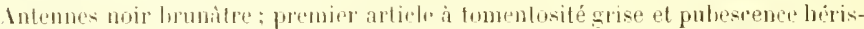

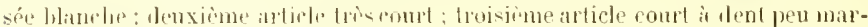

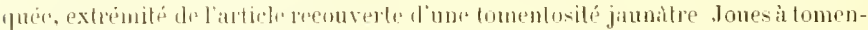

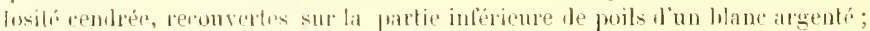

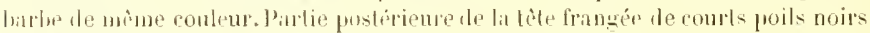

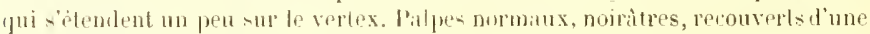
line pulupsence arsentér.

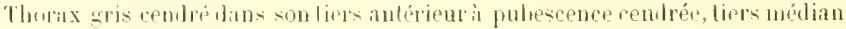

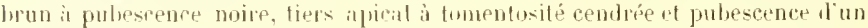
Hanc argenté yui së́lend sur lit moitié hasilate du seutellum. Moitié apieale du

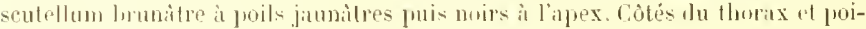
trim it tomentusite cendrie gulesence blanc d'arment. 
Ilulomen brun ì pulescence noire, troisiimes et quatrième segrments étroite-

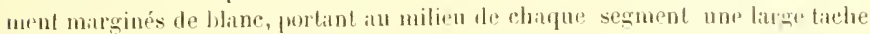
semi-lunaire d'un blan argenté à pulwsience hlinche. Ventre lrun, promipr segment it tomentosité cendrée ot pulescence hlanche; les quatre segments suiVituts marginés de lilane, dernier segment ì poils noirs. Hinches à tomentosité cendrée; cuisses noires à pubeseence noire en dessus, argentie en dessous. Tiltiis

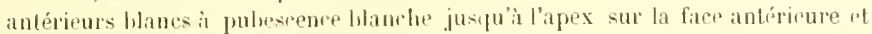
jusquat la moitis sur la face interne; tilias médians el postérieurs lobnes à apex rembruni; tarses loruns is pubesence noire sur la face externe et jaunitre sur lit

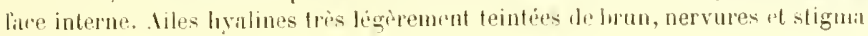
bruns.

Lat collection du Yuséum le l'allis postible un "xemplaire femelle. donné par l'Institut Pasteur et recueilli par le docteur Bovet, en liasse cồte d'I voire (1907).

\section{Btbtoritidrlte:}

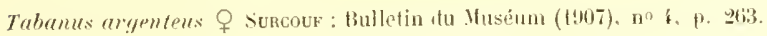

\section{Tabanus Sharpei $q$ IUstex}

O (spécimen), $11 \mathrm{~mm} .5$; largeur de la lìtr. $4 \mathrm{~mm}$., largeur du front au vertex, $0 \mathrm{~mm} .6$; longueur de l'aile, $9 \mathrm{~mm} .25$.

Ressemblant étroitement à Tibumus Wellmani f Austen mais distiuct par ses marques alulominales et sa face plus large. Brun, hande frontale grir-prerle; thorax orni l'une paire de bindesétroites, gris-perle, lien délimilérs, terminées

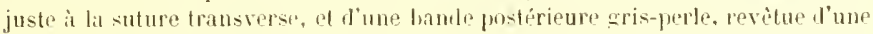

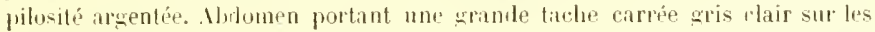
angles postérieurs du setond sexment el me double bande transverse émarginie an lord posterieur des troisieme ef quatrieme segments, cette bandr est plus épaisse au centre ; les cótés llu premier segment sunt gris clair.

Tite a follinosite grise, fafe of joues reviture de poils blancs; bankle fronlate rétrécie antérieurement; calbosité frontale un pru allongèe, langente anx yeux is sil partie inférieure, plus étroite en dessus; palpes fortement élargis a la lasp, fuis s'ilplatissint rapidement, jannitres, revetus de poils blinchitres. Les deux premiers articles des antennes sont grisitese leurs angles superieurs sont noiritres, premier article revitu de poils blanchitres sur la plus samle partie de sa surface, troisième article brun, sil portion batsilate est élargie.

Thorax : quedques proils jannatres pris du lood antérieur du desosus, entre les

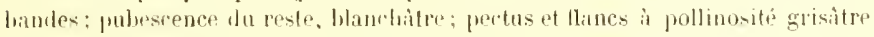
et ì poils lianchitres. sicutellum lorun, phus pille ì son extrème base.

dbromen : les maryues arises sont writues de poils blanchilres, couleur fonrire surmonte d'une pilositis noiratre on brun sombre. Ventre gris avee une bande Iransvers brune it lit base d. chatue segment, siuf le premier el le serond, qui sont entirrement aris et le dermier segment qui est entierement brun; une longur pubescence noire, friqée el dense se montre sur le còté infórieur tlu ternier segment. 
Ailes hyalines, stigma invisible. Alulie l,runâtres. Balanciers brun-noisette. I'attes: fémurs d'un lrun grisitre sombre: tibias, sauf l'exlrémité, jaunâtres: tarses antérieurs et extrémité les tilias antriemrs noirs : larses mérlians et postérieurs, exlrémité des tibias mérlians et poslérieurs bruns. Tarses antérieurs dilatés; fémurs el tilias couverts d'une pilositi jaunitre ou jaune blanchatre ; on rencontre aussi des poils blanchiatres sur le còtí externe des trois premiers articles tarsaux.

Protectoral lu Nyasaland (Ifripue Centrale anglaise): un seul spécimen de Katumhe, Nyasa Noril. 6. 12. 1906 (1) J.-E.-S. ()LD).

Le carnet de notes du Wr old porte la mention suivante : "Contrée marécisgeuse avec de très grands roseaux et une forèt d'arbrissaux.

Le Tabanus Sharpei O Austen a reçu son nou de sir Alfred Sharpe, k. C. II. A.; C. B., gouverneur et commandant m chef du protectorat de Yyasaland, dont les efforts pour la couservation du gros gibier dans le territoire de son adninistration ont mírité la gratitude de tous ceux qui ont au coeur le sonci du a meilleur devenir " de la faune africaine.

Ontre les différences jei mentionnées entre le Tubanus IVellmani $q$ Austen et lo Tabamus Sharpei q Austen, ce dernier peut ètre dislingué du mrenier par ses ailes liyalines, l'absence presque totale de stigma, les tarses antérieurs plus étroits dont les angles des parties saillantes sont plus arrondis el beaucoup moins dilatés.

Il se sépare du Tabamus aryenteus o Surcouf entre antres choses par la bande frontale plus élargie, la région basilaire du troisième article de l'antenne plus courle et plus large, les palpes plus courts, les bandes grises visibles it la parlie anterieure du dessus du thorax, le contour des dessins abdominaux et les ailes hyalines.

\section{BHBt.tORR.IPIlte}

Tabamus Sharpei 9 IUsten: Immals and Magazine of Natural History, sẻr. 8, vol. 1, mar's 1908, page 226.

\section{Tabanus Wellmani 9 . IUsten}

Longueur (6 specimens), $11 \mathrm{~mm} .1 / 2$ à $12 \mathrm{~mm}$. 1/3: largeur de la lìle, $4 \mathrm{~mm}$. is $4 \mathrm{~mm} .1 / 3$; du front au verlex, $0 \mathrm{~mm}$. 3 ; longueur de l'aile $10 \mathrm{~mm}$. à $11 \mathrm{~mm} .3$.

Front étroit, gris-perle en dessus : thorax brun-girofle, avec une paire de bandes gris-perle sétendant à peine au delà de la suture iransverse, et une étroite bande de pilosilé argentée sur le bord postérieur; abdomen d'un lrun de eire, troisième el quatrième segmentsayant chacun une tache médiane d'un blane grisâtre en triangle arrondi ou en demi-ovale et revèlue d'une pilosité brillante et blane de crème.

Tète lrune: bande frontale, face, joues et occiput à pollinosité grise; face et joues couverles d'une loison blanche; callosilé fromlale et subcallosité brun som- 
bre dénmlíe? , la première en forme de rectangle, s'étendant d'un oril ì l'autre; falpes bru sombro minces, revatus l'une puliesfence blanehître; premier et second irticles des antennos gris ardoisé, converts vers le haut do tins poils noirs, le premier article a anssi lles points blanchitres en dessons, troisième article stroit, allongé, brun, légèrement ferrugineux ì sun extrème base.

Thorax : fos bandes dorsales grises sont visibles en regardant par derridre, l’intervalle est aussi grisitre et divisé par une étroite ligne médiane brun clair : la couleur foncière te la bande transverse postérienre (rontenant une grande portion des callus post-alaires) est gris-perle; pectus et llines à pollinosité grisàtre, surmontíe a'une pitosité blanchâtre; scutellum ì pollinosité gris bleuitre, partie supurieure du bord postérieur brunàtre.

Abdomen : taches médianes reposant par leurs hases sur le hord postérieur Jes segments, tache du troisieme segment n'atteignant pas le bord antérieur; còtés du premier sexment, angrles postérieurs du scoond, troisième et quatrième segrments gris, revètus de poils blanchitres: bords postérieurs des troisième et quatrième serments étroitement marginés de gris sur chaque cóté de la tache médiane. Ventre à pollinosité grisitre, portion havilaire des segments habituellement lirunitre, les deux derniers segments jevêtus de poils noirs érigés, le resle porte une puleseence blanchître, courte, plus on moins appliquée. Ailes teintées de brunàtre, stigma lien indiqué, brun. Alulir brun sombre. Balanciers bruns à extrémités un peu plus claires. l'attes lrunes; tilias, sauf l'apex, de couleur crème el revêtus de poil l, lanchâtres; second, troisième el quatrième article des tarser antérieurs forlement dilatés.

Anzola : le type et cinq autres spéemens proviennent du district de Chiyaka,

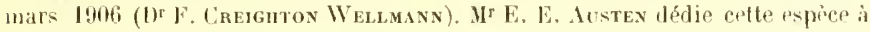
son inventeur, qui rapporte que ce taon mord avec acharnement et qu'il l'a rencontré dans une jongle épaisse.

te Tabamus Wellmani o Iusten est allié au Tabanus insiynis Lœw, mais otlre une ressemblance encore plus étroite avec le Tabanus argenteus $q$ surcouf $\mathrm{d} u$ Gabon.

Il en dilfere par la présence de bandes gris-perle sur le thorax, la bande de poils argentés du bord postérieur "st beancoup moins épaisse, le scutellum est pour la plus grande part gri bleutre au lieu de noir, sauf à la base, les taches aldominales ne sont pas semi-circulaires et leur diamètre transverse est leaufoup moindre; la largeur de chaque tache est moindre que l'espace compris entre elle et le bord du segment.

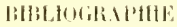

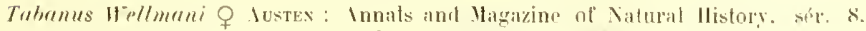

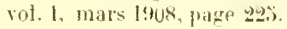

\section{Tabanus Roubaudi $Q$ sircote}

q Trois spécimens. $11 \mathrm{~mm}, 1 / 2$ à $12 \mathrm{~mm}$. 1 2. Collection du Muséum de Paris. 
Caractères généraux du groupe : Liches dorées, ailes un peu enfumées.

Títe hrune, bande frontale portant une callosité noirâtre, rectangulaire, tangentr aux yeux et prolongée par une courte saillie de mème couleur; la bande frontale est revètue d'une pilosilé lulane argenté jusqu'i l'extrémité du prolongement de la callusitŕ frontale, in-dessus elle est couverte d'une pilosité brune mélangée de quelques poils noirs: région située au-dessous de la callosilé, sáillante et noirille : fice, joues et orciput a pollinosité grise, joues et face eouvertes d'une toixon blanche. Palpes jaunityes, lairs, peu renflés, amincis à l'exlrémité. revitus d'une pulnescence blanche, mélangée de poils noirs vers l'extrómité et sur le còt externe. Premier et second articles les antennes brun somlore, le premier article est revetu de poils gris, troisième brun-noir, recouvert d'un très fin duvet argenté jusqu'ì lia partie apirale.

'Thorax brun portant quatre laudes dorsale's de pilosité jaune d'or, les deux médianes se terminent brusquement ì la suture transverse, les deux extérieures aboulissent it la racine de l'aile; l'espace compris entre les bandes est couvert d'une pilosité noire mélangée de tuelques poils dorés, le reste du thorax a une pubescence noirâtre. La bande postérieure transverse qui borde le thorax et qui comprend les callus sous-alaires est d'un lılanc d’argent mélangée de poils dorés. scutellum en entier recouvert de poils dorís. Flanes et pectus ì pollinosité cendrée et pubescence d'un lilane jaunâtre.

Ahdomen lorun sombre sur lit partir dorsale et couvert d'une pubescence noirître, angles postérieurs de chicun des quatre premiers segments ćclaireis et portant des poils dorés, troisirme et yuatrirme sesments fortant chacun sur lenr région mésliane un triangle étroit, arronti au sommet, de pilosité dorée; le triangle du troisième segment n'atteint pas la hauteur du segment.

Vintre phus clair, les cinq premiers segments sont étroitement marginés de hlane jaundre et ont une pilosité lulane juunitre, le dernier segment est hérissé de longes puils noirs.

Ailes teintées de brunatre. stimma triss atlongé, brun. Alula bruns. Balanciers lorun-marron à massue renllée. concolore.

Pattes noiritres; cuisses revètues d'une longue pilosité arcentée; tilias elairs à pubescence blanche et dorée, les tibias anlérieurs sont noiritres à leur extrémité apicale; tarses brun noiritre it pulvescence sombre, les tarses antérieurs sont fortement dilatés.

Le type et les deux autres exemplaires femelle proviennent du poste de Pangita (Boulé N'tangou) au nord de lBrizzitville. Ils ont été envoyés par M. Baudon administrateur, sur lavis de notre ami M. Roubaud, agrégé des sciences, à qui nous sommes heureux de dídier refte nonvelle esprèce en souvenir des nombreusws dicourertes dont il a enrichi la collection de liptires du Muséun.

Ces tions ont été recueillis à la fin de la saison sìchre en ortohre 1907.

Le Tabanus Roubaudi o Surcouf so distingue à première rue du Tabamus Wellmani anquel il ressemble itroitement, par les handes thorariques visibles normalement, les taches dorées, tes antennes claires, les fémurs clairs à puleseence blanche. 


\section{Tabanus sulcipalpus $O$ Lew}

Le classement de Tabanus sulcipalpus of dans le groupe do Tabamns ruriubilis $O$ Loew laisse à désirer; fet insecte étant cependant plus voisin te ce sroupe quw des autres nous l'y taissons provisoirement.

Description de Lin :

"Nigro-cinereus, thurace cinereo lineato, ablomine subtus flavesente, supea rius serie triplici macutarum tlavido-rinerasentium, vix distinclorum - signato; antomnis rufis, apice atro; palpis llivescentilus angustis, longitudi" naliter sulcatis; alis rinereo-hyalinis, stiguate ohseure lorumneo. Iongiludo " $\operatorname{cor}^{2} 51 / 6$ lignes 5 .

"Espèce distincte pir des traits si spéciaux que juse la dérire d"a prè̀ un " seul exemplaire, malheureusement pas battatement conservé ».

Face à tomentosité gris-bleu, recouverte au-dessous de poils b]anchitres et sur le dessus et les côtés de poils noirîtres. Palpes trìs étroits, jaune lrunâtre, recouverts de poils noirs et ayant sur le cité supérieur un sillon longitulinal qui va jusqu“il l'apex. Antennes d'un rouge-rouille saje, l’apex du troisìme article est noir; le premier inticle est un peu en forme de eapuchon et est recourert de poils noirs de mime que le deuxiène; le troisime article est etroit, l'angle supérieur peu saillant. Front à tomentositi gristre portant vers laangle antérieur des yeux une trìs petite callosité, non nettement limitée, qui peut provenir d'une dénudation; de cette callosité part la trace d'une ligne fongitudinate nue, dirigée vers le haut. Les yeux sont hrun-noir après ramollissement, sans bandes.

Thorax gris-noir, la tomentosité grise forme sur le côté supérieur les lignes plus ou moins claires, pas très évidentes; la pulvesence des côtés du thorax de mẻme que la partie sujurieure est d'un blane jaunitre ; the est noire entre les épaules et les racines des ailes, sur les handes haterales sombres et a milieu du scutelkm. sur l'abdomen se montre une raugée de petits triangles ì tomentosité jaune-gris cendré łui se trouvent au lord jrostérieur des segments, enoulre, de chaque cìté existe une rangée longitudinale de grandes taches indistinctement. limitées, recouverter d'une mème tomentositó, enlin les hords postérieurs de's anneaux ont une lorilure claire. Partout ou il y a celte coloration plus claire, la couleur foncière se transforme en un rougic brunstre sale. La puhescence de l'ablomen est daire sur les taches triangulaires, an lood postérieur de lous les segments et itu hort des segments antirieurs, elle est noire partout ailleurs. Ventre jaune t'or it pubescence claire, saufl les derniers segments qui ont une pubescence noire. Jatmbes brunes; cuisses, apex des tibias et tarses lirun foncé. Les cuisses ont une tomentosité jaune grisitre of une pulsescence claire tandis que les tibias ont depuis leurs bases une pulbescence absolument noire. Ailes gris hyalin, nervures lrun noir, hord externe brun foned; premiere of puatricme cellules postérieures non rétrícies.

La petitesse des antennes, l'étroitrse des jalpes, leur sillon, la pubescenee noire des còtés et de la moitie supírieure de lit facor, la prédominance de la pulrescence noire aux tihias sont tous des caractires d'apris lesquels rette "sper se laisse facilement reconnailtere, quoique les carartires du dessin du thorax et de 
l'abdomen, il cause de l'ótat de l'exemplaire décrit, ne sont pas aussi précis qu'il serait lésiralie.

\section{BHBLtUGRIPItE}

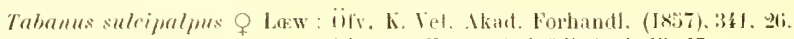
dipteren Fauna Sulafrika's, 1. 4.. I7.

\section{Tabanus insignis $q$ L.EW}

Lrew donne la description suivante:

- Yigricans, thoracis lineis duabus postice albrerialis fasciaque apicali albis ; " abdominis segmentum secundum maculis sex albis, sequentia ternis signata; " alæ hyalinæ, stigmate lı'unneo." Long. corp. 6 1/12 lignes.

Cafrerie.

Espèce dislincle par son dessin extraordinaire, brun-noir, un peu lirillante avec des dessins lılanes. Face à dense tomentosité blanehe et pulsescence semblable.Palpes assez minces, blanchitres, à pulrescence blanehe, surl'apex des palpes i] se trouve quelques petits poils noirs. La bande frontale n'est pas large et porte une tomentosité blanche jusqu'à l'angle antérieur des yeux; puis une petite callosité brun noir, au dessus le front est d'un gris-brun avec une tache noir mat au milieu et le vertex noir, le front dans ses autres parties est blanc. Antenues noires, seule la lase du trisième article est rouge; le premier article en forme de eapuhon a une tomentosité blanc te neige et une pubescence blanche, langle supérienr, fortement saillant et recouvert de petits poils noirs est d'un noir profond ; te troisieme article des antennes est assez étroit aver son angle supérieur un peu saillant. Les yeux restent sombres après le ramollissement ef ne montrent aucune trace de bande.

Le còté supérieur du thorax est brun-noir, les bords latéraux et postérieurs onl une tomenlosité et une pubescence hlanches; en outre il y a deux bandes longitudinales courtes et litrges qui ont une tomentosité et une puleseence lilanches mais gui sp terminent an milieu du thorax; sur les landes noires du thorax la pubescence est principalement noiràtre. Cótés du thorax noiratres, recouverts d'une tomentosité très dense et d'une puhescence hlanche. Scutellum d'un brun-noir.

Alwomen brun-noir, tomentosité lu premier anneau presque complètement blanclu, elle est noire seulement an milira; leuxième anneau portant quatre taches de tomentosité blanche, les deux postérieures sont situées vers le bord postérieur et se rejoignent presifue, tandis que les deux autres se trouvent prìs du borl antérieur et sont jlus éloignées l'une de l'autre; en outre, de chaque côtr du horl postérieur se voit une tache transversale à tomentosití blanche qui se dirigre en avant, près du bord latéral. Le troisième anneau de l'abdomen porte au milien de son lord postérieur une grande tache de tommosité blanche en forme tle demi-cercle et le chaque còté de celle-ci des taches transversales conme celles du deuxième anneau, mais elles ne se prolongent pas aussi avant et sont unies à la tache médiane par une délicate bordure blanchàtre. Le dessin du quatrième annean ressemble à celui du troisième, mais la tache médiane est 
un peu plus grande et les taches latérales sont plus petites; sur le cinquièm, anneau le dessin se répite encore une fois, mais la tache médiane et les tarhes latérales sont plus petites et d'une conleur gris cendré. Sur le sixième anmaril il y a encore de petites taches latérates et une bordure postérieure claire clont les traces subsistent encore sur le lernier anneau. La pulsescence de l'abdomen est noire sur fond noir et claire sur les parties hlanches. Ventre brun noir; la moitié postélieure des anneanx, à l'exception tes derniers, a une tomentosité litanche, lit courte pubescence du ventre est blanche sur les anneaux postérieurs avec un mélange de quelques poils noirs, elle est complètement noire sur le dernier anneau. Cuisses noires avec une tomentosité el une pulsescence blanches. Tihias jaune blanchatre, vers l'apex ils sont noirs sur un assez grand espace, pubescence concolore. Tarses noirs. Balaneiers it pédicelle brun clair et à bouton brun. Ailes hyalines avec les nervures brun-noir, borl externe brun assez foncé. Le rameau vers la nervure transversale de lapex est très court. Prenière et 'juatrième cellules marginales postérieures non rétrécies.

La collection du British IIuseum renferme des spécimens provenant du Natal (collection Sauxders) et une femelle prise dans le Jarlin botanique d'Enteble, Uganda (capitaine S. D. W. Greig-I. M. S. 190\%).

\section{BIBLWORALPIH}

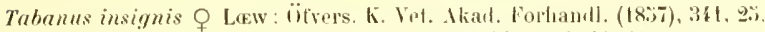
bipteren Famnar Sulativia's, 1, if, 16.

\section{Tabanus diversus + Ricando}

Le type femelle et une autre femelle proviennent de Ruwe, rivière Lualaba, Congo, entre $11^{\circ}$ lat. S. et $26^{\circ}$ long. E. (Greenwich), recueillis en février 1:106 par le docteur A. Yale Massey (Collection du British Museum).

Cette petite espèce noire a l'ahdomen maryué de taches grises très distinctes et le thorax orné de bandes et de taches grises:ailes claires; antennes courtes; paftes brunes à tibias jaunatres. Très voisine le Tabmus insignis $q$ Lœw de l'Ifriłue orientale, mais s'en tistinguant aisément par sa couleur noire et non lirune; frar l'absence des quatre taches blanches sur le second segment de l'alsdomen; par' les grandes taches du troisieme et du puatrieme segments de forme plus oblongue; les antennes plus coutes; la callositó frontate prolongée en une trís courte ligne épaissie et par le thorax "jui a deux taches lle pilosité grisł' à la lase au lieu du hord à tomentosité blanche de Tabanus insignis $q$ lanew.

Longueur 11 millimètres $1 / 4$.

Tìte phus large que le thorax, fice couverte de tomentosité grise et de pulsescence blanehe. Barbe blanche, palpes jaunes ì longue pulvescence blanche, peu ́pais à la base et terminés par une pointe aiguỉ. Antennes courtes, rougeitres; premier article jaunatre is pulsescence noire sur l'angle supérieur el binche partout ailleurs, eylindrique, non arqué au sommet; deuxiène article petit, rouge, à pubescence noire; troisicme article rouge à apex noir ; première divi- 
sion courte el large portant une lent maryuée, les quatre dernires divisions très petites. Bande frontale large, tris légìment rétrécie antólicurement, environ quatre fois aussi haute que large, brune; couverte d'une tomentosité srise avec quelques poils gris et noirs. Callosite frontale lorun rougeìtre, presifue carrée, atteignant les yeux, lit ligne qui les prolonge ent très courte el cpaisse - dans un autre spécimen elle est encore flus large - presijue autant que la callosité elle-mème: le charur crití de rette ligne la couleur brune du fond parait comme une longue tache mal détinie, vertex lrunâtre. Partie postérieure de la tîte blanchâtre ì poils blancs.

Thorax noir brillant présentant denx bandes médianes tomenteuses grises qui ne dépitsient pas le milieu et portent deux tarhes de poils glis à la base, côtés sris a pubescence blanche, clle ext hlanchatre sur los landes, noire par ailleurs, sanf à la lase des ailes où il y a une toulfe blanche épaisse. Cótés et poitrine it tomentositégrise, puluesence llanche. Scutellum d'un rougeàtre sale à tomenlosité grise et pulescence hlanche.

Ablomen noir; sur le premier segment il y a la trace d'une petile tache médiane grise et lescrités sont largement lomenteux; deuxième segment à tar he de tomentusité lianche sur chaque côté, l'angle supérieur te cette tache est dirigé vers l'intérieur, il atteint les trois puarts de la largeur du segment et se rontinue sur tout le lord externe. Les taches latérales des yuatre segments suivants sont semblables mais de laille décruissante; sur le troisième segment existe une grande tache médiane carrée comprenant toute la lauteur du segment; le qualrième segment porte une tache un pur plus petite, toutes ces taches sont convertes d'une tomentositi arise; pulescence hlanche sur le dessus, noire ef blanche sur les colés. Envers d'un jame rougentre sale, noiritre sur les cités et vers l'apex, par aillours couvert d'une courte puhescence hlinche. lattes brun noirâtre; thlias jaunâtres, antérieur's noirs sur leur moitié apicale; fómurs a tomentosité grise et puhescence lianche: tihias il puhesence liane jaunàtre, elle est noire sur l'ipex sombre des tilias et çà et là. Ailes claires, nervures lrunes, stigma loun jaunatre: première cellule marginale postérieure non rétrécie. Pas d'appendice.

lilsthutititutte

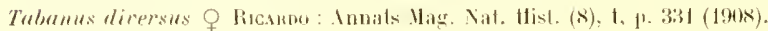

\section{Tabanus atrimanus क Law (pl. III, fig. Iㄹ}

Description de Lew :

"Nigro-cinereus, thorace allidlo- lineato ; aldominis maculis trigonis, incisu-

“ ris, maculisque magnis segmentorm lateralibus allodocinereis ; rentris

" allito pollinosi vitta longitudinali merlia lata nigrieante; antennir nigrap, cal-

" lus frontalis unicus inferus et linea frontis longitudinalis nigricantes ; vertex

" nigropilosus. Long n. corp. 6 1/4 lin.

Apparenté au Tabanus variabilis Q Law, mais rependant distinct. La couleur 
foncière de la face est recouverte l'une tomentosité blanche et l'une dense

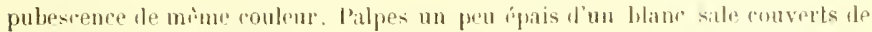

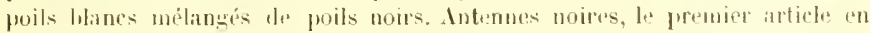

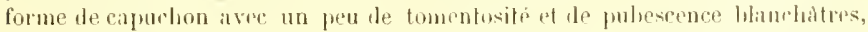

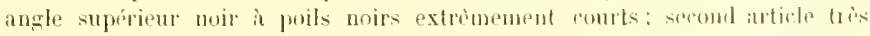

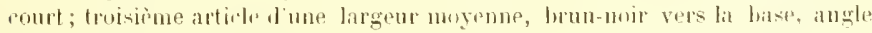

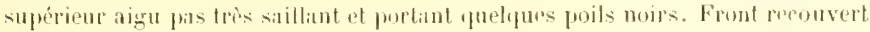
l'une tomentositi blanchatre jusqu'aux angles antérirurs des yeux, et portant une callosité quadrangulaire pas très birn limité d'un hrum-noir, prolongie

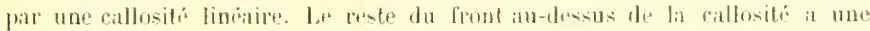

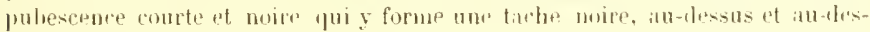

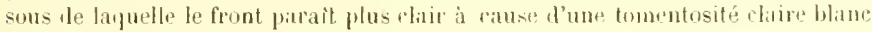
quisitre et de poils blancs.

La région du vertex est noire, un pen brillant ot porte une pulresenene noire, ivilente. quoique his courte les yeus, almis lanollisisement, restent sombres et ne montrent aucune trace de lignes transwersales. La couleur lu foml th thorax est noiritre, mais chle passe au rouge brunilre sur les cótés et entre less

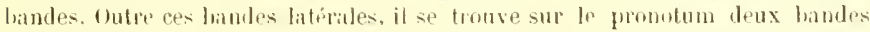

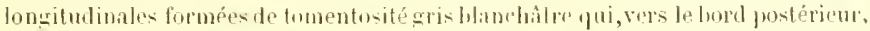

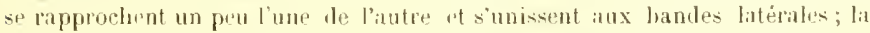
puhescence des robtís du thorix, qui ont um tomentosité hanchitre, est auswi blanchitre. La puhesonce du promotum semble de mime lobaschatre prur la plus grande partie ut nest noiratto que seulement sur une prartie des handes sombres. scutellum noiratre à pubescence principalmment noire.

Le dessus do l'aludomen est d'un hrun-noir mat, le premias segment est gris liknchâtre sur le ròté. Sur le deuxième segment se trouvent deux grandes

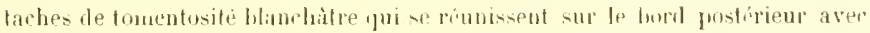
un likge triangle de minr couleur, de sorte puil ne reste que trois itroitrs taches noims; lit srande tarhe méliane noire commene an loord anteriaur, s'élargit vers le lord postérieur of śaromblil vers l'extrémité. Les tarhes litha. rales sont subtriangulaires et sont situéc dins l'anste extérieur du segment et

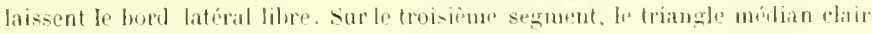
"st plus aigu, la protnde macule latérale quadrangulaite it tomentosite gris hlan-

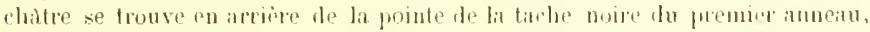

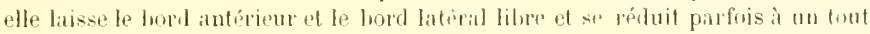
petit point qui se tromva au milieu du segrnent, sur les sesments suivints, hi

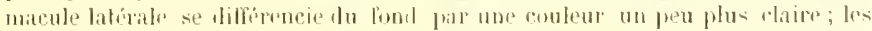
tarhes dorsales triangulaires sont encore tris nettos sur le quatriome ef lan anquième segments, quwique moin nettement cireunserites qu' sur les prictidents.

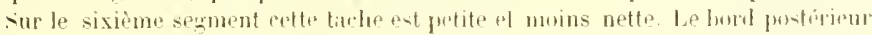

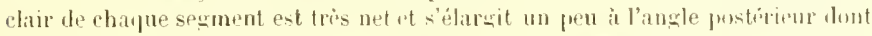

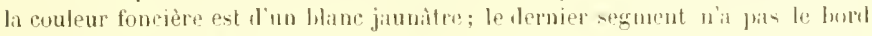

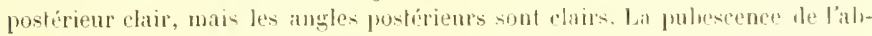
domen a partout la couleur du fond sur bequel ellese trouve. Lat rouleur du ventre est gris noir, la tom 'ntosité hlanche est plus rare sur la lign' mésliane qui semble ainsi plus noirp; les segmentations ont une dílicate lordure hlanche, la pulosence est hanchitre, mais phis noire sur los dernirs seguents, surcouf 
in insi qu'au milieu des segments précélents. Cuisses noires, l'apex externe rouge jaunitre, elles ont une tomentosité blanche ef une pubescence clitire; tilias

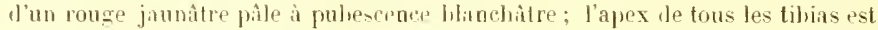
noiratre it pulsescence noire. Tarses boirs. lablancier brun noiritre. Ailes hyalines un peu grisitres ì nervures hrun-noir. Hord externe d'un brun pâle. Iremirre et quatrième rellules marginales postérieures non rétrécies.

Cafrerie (Wanlberi).

La collertion du Mnséum de Paris poside un exemplaire femelle provenant t'Isika, bords du soukourou (1)r Brodex, l904).

\section{BHBLIOT.R.IPHE}

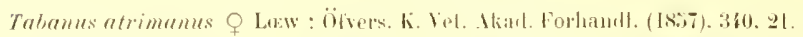

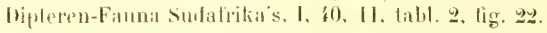

\section{Tabanus velutinus $q$ Sincove}

Type: une lemelle de l'Aliyssinie, prise par W. L. Mubien en 1903 ; trois autres femelles de la mène jrovenance (Collection du Aluséum de Paris).

larun-rouge aver les derniers segments de balulomen noiràtres et une tarhe binche sur le troisiène ef le quatriènt segment. liles cendrées. Longueur : $11 \mathrm{~mm}$, मे 12 $1 \mathrm{~mm}, \mathrm{I} 2$.

fite plus lirge que le thorax; yeux transverses, sombres, à cornéules petites ct égaless, glalures. Bande frontale hrune, normale; callesité lrunâtre, brillante, saillante vue de profil; arrondie vue de face et tres pral visille, sumonte d'une ligne lirunatre étroite et courte. Intennes: fremier article lum rougeâtre d̀ pulsescence jaunatre, forcl pxtrème te la saillie apicale noir : troisième article brunåtre it dent preu saillante. Palpes renflés, clairs, ì pubrsene jaune clair, dense. Bord postérieur de la tète glalıre.

Thorix et écussons lrunatres, hordes de lolane, coblés du thorax ef peclus a longue pulescence jaune.

dhomen hrun-rouge sur les trois premiers segments, s'assombrisant progressirement depuis le truisieme segment justu’a l'apex : second segment portant deux tachrs lilanchàtres, arromlies et mal délimitées, tangentes au bord intérieur, hord postérieur du segment luanehatre. Troisième et quatrième segments portint une tache triangulaire linche plus ou moins arrondie, sur le hord postérieur de chacun d'enx, cette bande se continue en une bordure étroite qui s’élargit sur les còtís. linnuìme segment a tache semblablr plus petite, jeu distincte, parfois nulb. berniers segments abdominaux bruns en entier à pulsescence usée paraissant claire. Ventre rougeàtre recouvert d'une pruinosité cendrée à julsescence composie de poils clairs. Pattes discolores, cuisses brunes it julsescence blanche ; tihias plus cliairs, ascombris à l'apex; tarses noirâtres à fubsecnce somlure. Ailes cendríes, translucides, presque transparentes; nerration normale. Balanciers à bouton marron brillant, tige plus claire.

\section{BELLOHRAPItE}

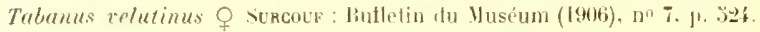


Tabanus Severini o surcove (pl. 111, lig. 14)

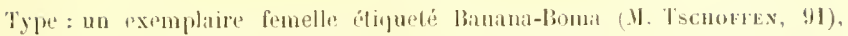

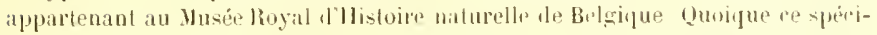
men ne soit pas en hon état, il est cepeniant si net gue nows arons aru devoir 10 décrire et didier celle espice nouvelle a M. le docteur siverin, du Musé lioyal d'llisture naturelle de belgigue, jui a lien voulu mettre at notre disposition les nombreux documents quil possediat.

Longueur 13 millimètres.

Tèle plus large que le lhorax; yeux lronzés, glalores; I tande frontale cinu fuis plus haute que large, a coté légèrement divergents an vertex, de couleur jumm

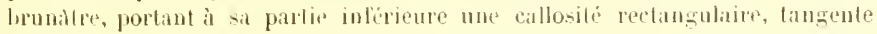
aux yeux, f'un hrun lrrillant el prolongée par une ligne étroite légirement diatlée, yui satrète au milieu de lat hateur de la hamke. Epistone jaune brunilre; larbe et pubescence blanches. Intennes: fremier article trongue obliquencut, brun à épaisse pubescence nore, hase loune; denxieme article lrun rougeatre, revèlu sur son bord apicial de denses soies noites; troisieme article noir à tomentosilé noire, rougeatre à lit biree. Palpes aswaz allongés, peu renllis, blances in pubescence noire, courte et régulìre.

Thorax lirun à quatre handen de tomentosité blanchàtre, pubescence du fessus concolore; llanes à poils noirs, pectus à tomentosité cendrée et proils blanes. scutellum de la couleur du thorax.

Nulomen brun portant un triangle blanr sur les troisiome, quatricme ce cinguirme segments; tés triangles ont hur batse sur le bord postérieur wr leur segment et ualteignent que la moitié de sal hateur; deux firscles hanchitres litérales sur les deuxième, troisiome th puatrirme segments ; les deux derniers segnents à pubescener noire. Ventre rougeatre ì pubesene noire éparse, le bord posterieur de chacun des negments est blanrhatre.

Hanches anterieures brunitres is lomentositi cendrée fubescence blanche; fémurs antérieurs lorun rouseilre à pulrescence noire; tibias antérieurs testacé, plus sombres ì lapex, à pubercence blanche dans la partie claire; larses anti-

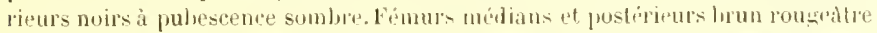

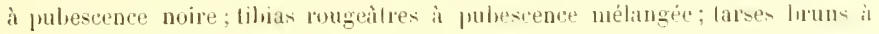
pubescence concolore.

Ailes leintées de brun au hord costal et it la partie inférieure de latile; stimmat Inunatre; premièrecellule marginte fostérienre fortement rétecic halincierloruns à disque jaune.

\section{libi.|WGRIPIII:}




\section{Tabanus varians $q$ Surcour}

7 Unspéciman. $13 \mathrm{~mm}$. Collection hu Wuséum de Paris.

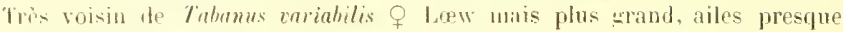
lyalines, serond sument almounial hassint roir en son milieu la teinte fon-

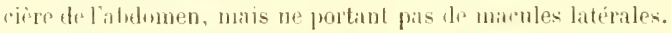

tète hrum, hamle fromtale portant ane callusite noirâtre, rectangulaire, tangente aux yeux ef folongíe par me ligne átroile, nou dilatée jusqu'aux deux tias's de la hautem th vertex; ha hande frumble est hlanchitre et porte une macule hum vers sun milieu ainsi qu'au vertex, les poils occipitaux sont noirs.

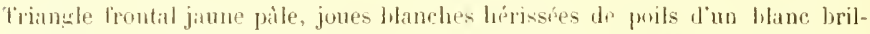

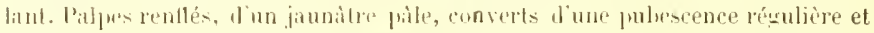

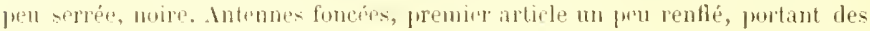
prits noirs gros et peu nomberix ; second articte portant un pinesa de poils

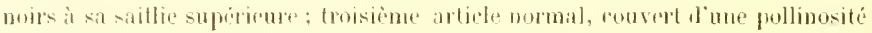

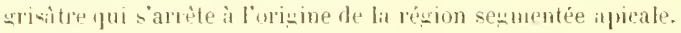

Thorax hrun noiritre un peu lorillant, retouvert d'une tomentusití jaune grisitre formant ciny liznes pu marypées; la pulestence du crite superieur du thorax semble, pur lit plus grande part, ètre blanc jaunitre, mais on y ren-

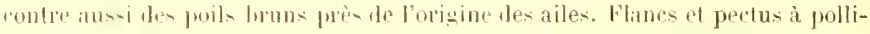

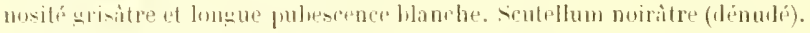

Alutumen : premier sesment brunitre, maremo de blane, pertant une toufle de poils fimmes it lit bise du sruteflum; seeond segment envahi par une tache

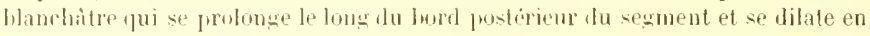
on triangle himchiter au milien de l'arcen, lit roulem du fond napparait

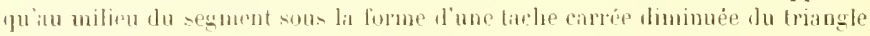

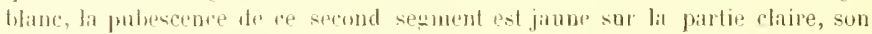

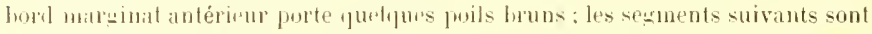
noirs et lo bord postirieur de halcun deux ast hane urisitre, charm des segments 3, fot is prort ma minucule tiche méliane et deux laterates formées de juils durion.

Ventre grimitre à pubestence hlamehe portant une bande médiane noire à poils noirs. sement and lériese de poils mojrs.

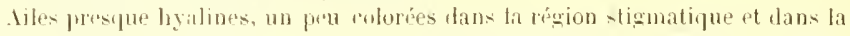

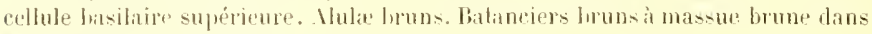
sat region lit phus extrime.

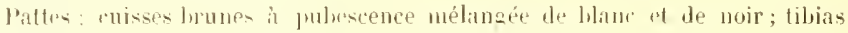

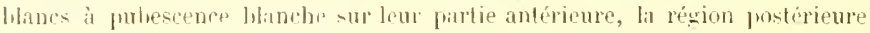

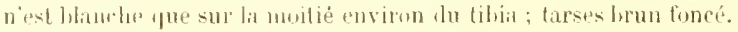

l'exumplite qui nems sert le type a éti recueilli aux "nvirons de Brazzaville Conso) par Y. Routiaul. 
Tabanus variabilis o LEW fl. III, fig. \&

Cette espice assez variable a une aire de dispersion considéralle. Iu typu est décrit de Cafrerie. mais liı figure donnée par luew dians son ouvrage Dipteren

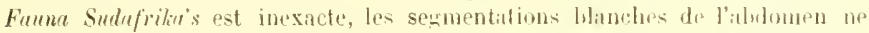

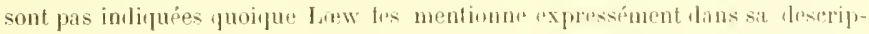
tion; les taches latérales sont aussi beameoup plus arandes dans ledesin quion réalité. Quoique les descriptions de Lrew soient lisujours il relenir, ses dessins ne sont pas toujours fideles.

bescription de Lew :

"Cinereo-nigricans, thorave lineis duatus ohsoletis allidis picto: aldominis

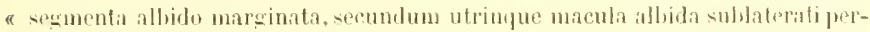
" magna, sequmtia marulis ublongis parvis, ounilus allicantibus signata ; "venter ruluro-nigrieans nitulus, sesmentis omnilus allomarginalis. Antennaz " frontis callus micans inferus lineaque longiluslinilis integra, ef vertex atla.

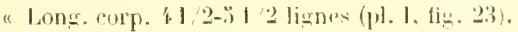

Espreve très jolie mais semblant un peru varialste. Lat couleur foncière de la face semble ètre noiràtre mais alle wh combletemunt recouverte pitl une tomentosite blane grisatre et une pulsescence lianche. Palpes d'un lorun sale aver des poils btanes ef noirs. Antenues d'un noir protund, vers l, lase du troisiome intiut il y a une trace de roloration hrun-noir; le premier article a la borme d'un

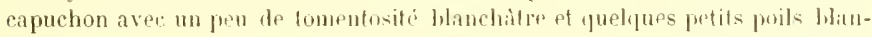
chatres sur to rite inférieur, par ailleure le promier article est recouvert de poils courts et noirs; deuxirme article tres count; troisime article d'un lirseur moyenne, angte superipur tris peu saillant, portint questques puils tris rourts. Le front jusifuax angles intérieurs des yeux jurte un tomentositi gris blane sur un fronl noir, et au-dessus nne caflosité quarlrangulaire briflante non fangente aux yeux et jui sounit au vertex noir frar un callosité uniforme. liniatire et noire. La tomentosité du front assez itarei est atu brun gris foné. Les yeux

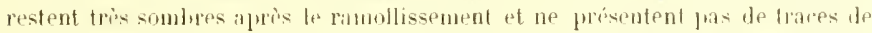
bandes transversales. La conleur foncière du thomx et du scutellum est movitre

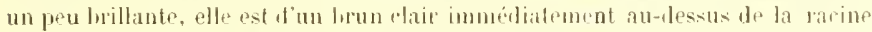

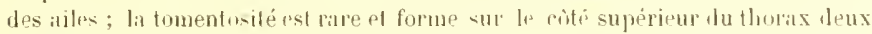

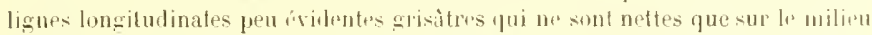

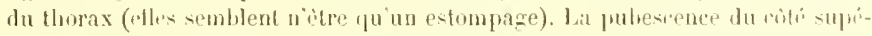

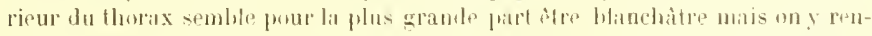
contre ansi des poils noirs, priaripalement pros delorigine des ables. Les ritris

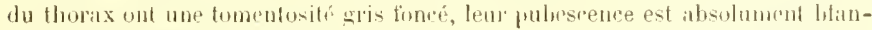

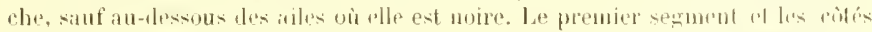

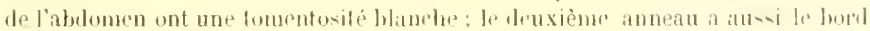

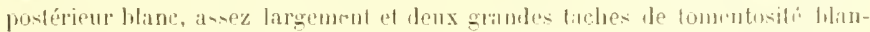

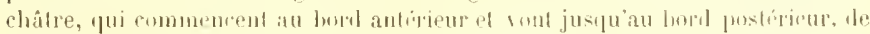

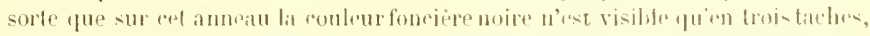

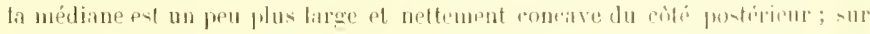
tous les anneaux suivants les bordures pustrifires blancluilres sont answi 
Indles; les laches latirales yui ont une fommontosite blanchâtre deviennent sur chapue segment suivant jolus étroites of moins mottes, pt comme leurs extrémités postirieurs sont dirighes un peu plusextéricurement sur daque anneau successif, pllp forment une bande latérale, itroite of dentele qui disparait lientot. La puluseence lle l'abdomen est noire sur un fond noir, elle est blanche sur les partips rlaires. Li: rentre est assoz brillant, génèralement brun-noir mais il préssente vers la base el au milieu un passige uet au rouge. Cuisses noires à poils noirs; tilias d'un rouge jaunitre, les tibias antérieurs sont romplètement noirs sur leur fare interne et sur le rité externo depuis le milien, curtins spécimens ont semlement lo tiers apical noir; la pubsence de la partie claire des tibias antérieurs est prestue exclusivement hlanihe elle est noire sur les parties noires. fiurses noirs. Balanciers loruns, le houton blane jaunatre à lapex. Ailes hyalines it peine grisatres; les nervur's des ailes sont d'un brun-moir. Bord externe lrunitre, premicre el quatrième cellules marginales postérieures non rétréries.

Le Iluseum de Paris possede des spécimens de toealités très diverses. IlanteSangha (T'. 1. Fenmère, 1900); Brazzarille (liovbacd, 1!167) : simba, Afrique

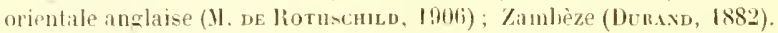

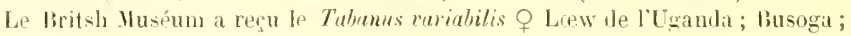
extrémité X. E. du lac Allort-Edward; linwe (Conso belge).

\section{liltLLIMiRAI'ItEE}

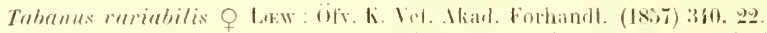

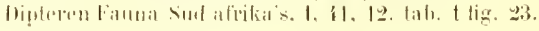




\section{TREI\%IEME (iROUPE}

\section{Tableav dichotomiQue}

$1\left\{\begin{array}{l}\text { Yeux sans bamdes. } \\ \text { Yeux portant des handes }\end{array}\right.$

Esprecs ressemblant it iles Homutopotu.

Andomen birun ì tarlaes latériles grisitIres.

E Espres ne ressemblant pas it des Hemulopota. Abiomen hrun on noir it bande médiane et liches latríales. .

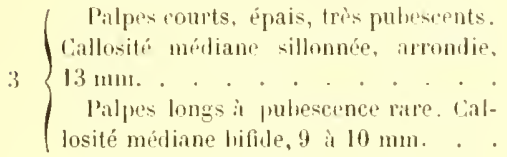

T. morsitums o Ricinto.

T. Rothschildi o surcout.

Triangle frontal d'un noir lrillant. . bidomen noir ì tarbes grises. Rande frontale trois fois anssi haute que large.

T. obliquemacutatus Q Dacquatt men brun it taches grisitres. Binde frontale environ cinq fois ansis hiute que large . . . . . . .

T. pallidifucies o surcoul.

Yeux portant trois landes colorées. Nudomen it trois series de talles grises. fes latrírales sont obliques. Liles ì nervures transverses noires, 8 is 9 mm.

Esperess a trois bandes louritudinales sur l'ablomen. . . . . .

T. sufis o Jirnnitelir.

$\left\{\begin{array}{l}\text { leux purtant une himle transverse } \\ \text { colorée, } 10 \text { mon. . . . . . . . . . . . . . }\end{array}\right.$ 6

T. yralus go lapw.

T. Iritmiatus o Rirarelo. 
Ce groupe contient les insertes prísentunt l'ensemble des caractires suivants : veux gladres : tibias mu dilatés, bande frontale portant une callosite arrondir ou transverse it la hase el une tache, une fillositi au milieu de la bande. Les drux rallositén sont séparés lorsqu" l'insercte est frais.

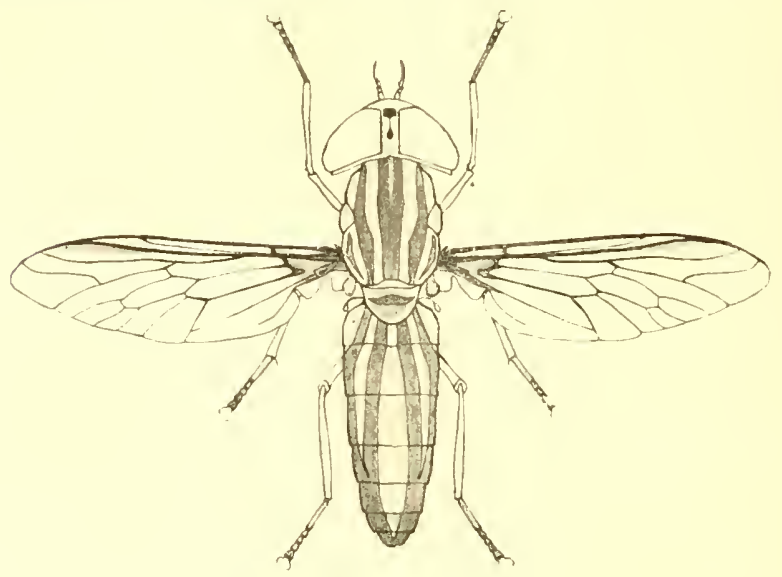

lig. 22. - Talumus qratus $q$ Law

\section{Tabanus morsitans Ricarvo}

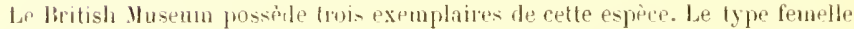

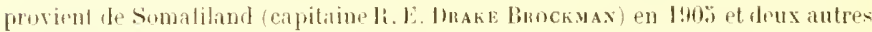
femelles du mème- pays recuellin's par le caphitain swaxa 94-201, une d'elles est trim endommagée.

Le? aptaine Bnock max croil que ce tann propage une maladie fatale anx che-

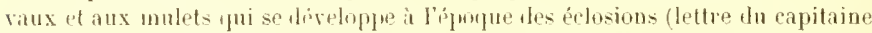

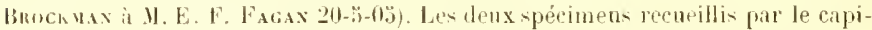
tainu sivare sont mentionnis dans une note de la Monographie des mouches Letsi de M. L. E. Iuster, p. 3tiz et y tiquent sous le nom somali de Baland.

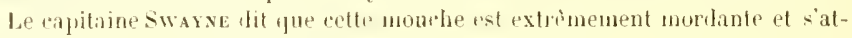
titque aux chevaux et aux chammax.

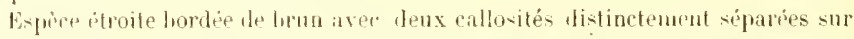
un larce lande frontale et purtant tes taches srises arrondies sur channe cote

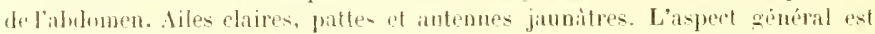

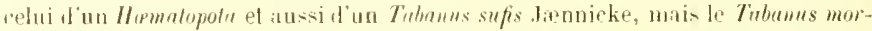

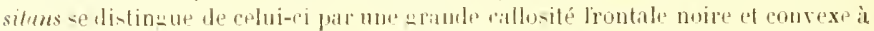

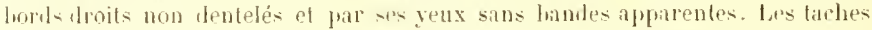

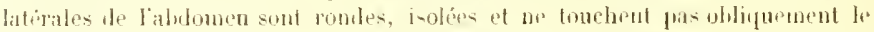
bord postérieur de chaque segment comme dans Tibanus sufis. 
TREIZILME GROUPF

169

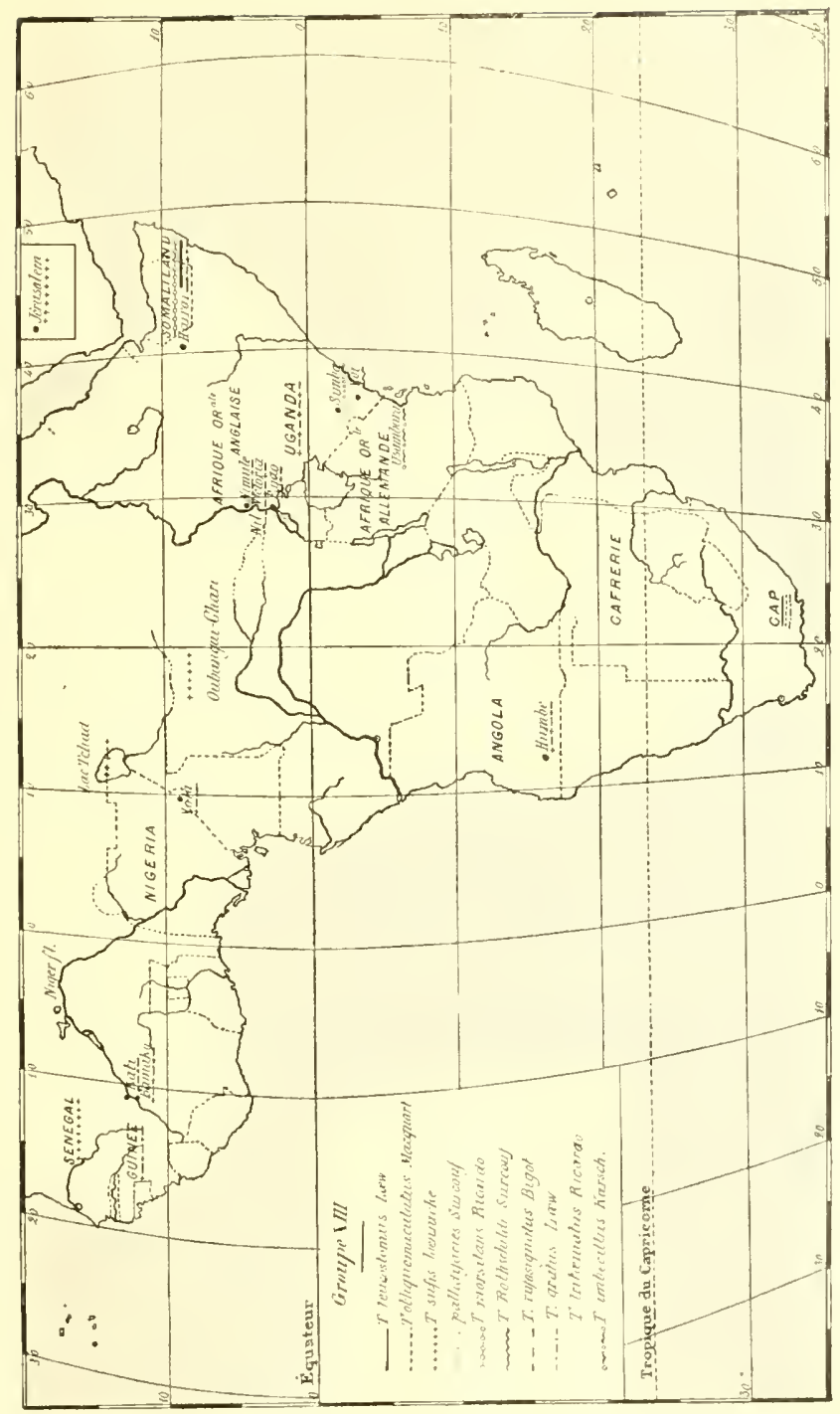


Longueur $13 \mathrm{~nm}$.

Tète plus large que le thorax. Face grise à longs poils lilanes. Barlve blanche, l'alpes hlan's, courts, cpais à la hase, terminés lorièvement en pointe revètus l'une courte pubsecencener nuire mélangée le quelyues poils llanes argentés.

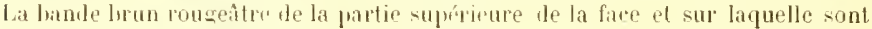
inséries les antennes est rouverte de lomentosité grise. Premier ct deuximme attielrs des antennes, jaunes a pulvescence noire; le troisième article manque. Bande frontale large ì lords parallèles, tout au julus deux fois anssi haute que large, couverte l'an tumentosité brun jaunitre; callosité frontale large, noire, Lrillante, tris convexe, transverse, alloignal les yeux; at-dessus et distinctement separée, s'étont une tarhe noire de forme très irrégulirre, légèrement sillonnée au milieu et une autre liche lrune plus petite au vertex. I cux glabres sans bandes apprarentes.

Thorax brun noirître ì tomentosité qrise et deux bandes grisittres indistinctes: cités grisitres; dencus it pubescene noire áparse; pectus ef lannes rougeitres rouverts d'une tomentusitŕ grise of d'une pubescence blinche; scutellum lirun

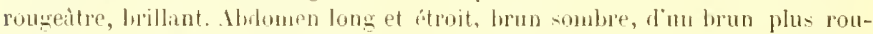
geatre sur les segments basilaires; premier segment portant une tache midiante qrise el deux taches de tomentusité urise sur lus rôtés: tous les autres segments, sauf le dernier, ont une tache grise, ronde, isolée sur chaque cóté et une tache midiane grise, distincte sur le deuxième et le troisième.

t'artie dorsale glalıre, lis segmentations atroitement grisitres, Ventre lurunàtre aver les sequents fitroit, janne grisitre. Pattes jaune rougeitue, tarses antérieurs et apex des tibias antéripurs plus sombres. Ailes liyalines, nervures et stigma jatuatres: les deux spécimens recueillis par le eapitaine Brockisas ont les ailes appendiculées; première cellule postéricure largement ouverte.

\section{BELLOGRIPItE}

Tabamus morsitans of Rusado: Ann. Has. Nat. Hist. (A), 1, 1, 277 (1908).

\section{Tabanus Rothschildi q Surcour (p. 171, fig. 23)}

Type: I q provenant de Voi Ifrique orientale anglaise), prise par .I. Maumice de Rotuscinld en 1906,2 autres $q$ de mème provinance.

Etroit et long, brun "lair avee doux taches rondes, hanches sur chaque segment ablominal. Liles hyalines.

Longueur du corps, 10 millimètres; de l'aile, 9 millimètres; de l'ahdomen, fi millinètres.

Tile large et d'apparence liangulaire voe do face: yeux hrons, à cornéules exales ef glabres. Bande frontale tris large, d'un gris jaunitre, frensie au vertex, fortant à la hake une callosité transverse. réniforme, d'un hrun marron lorillaut, au-rlessus de laquelle se trouve une autre callosité peu distincte, lifigde.

Antennes aluassirs, testaries, ì lfent thès peu saillante. Palpes allongis, 
jaunitres, à pubescence rare, asse\% longue of hlanehitre; quelıues froils moirs éprars.

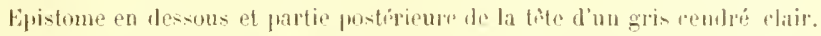

Therax bron rougeatres avee trois lignes longitudinates blanches, la mérliann

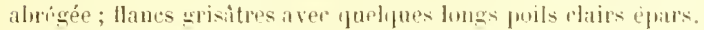

dulumen lirun clatr, allongé, glabresernt.

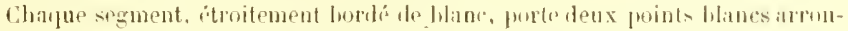
dis; le premier et te deuxième segment ont une fiscip méliane bianchatre.

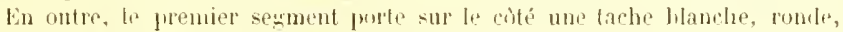

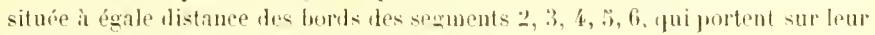
llane une tache semi-lunaire, hlanche, langente an hord antérieur.

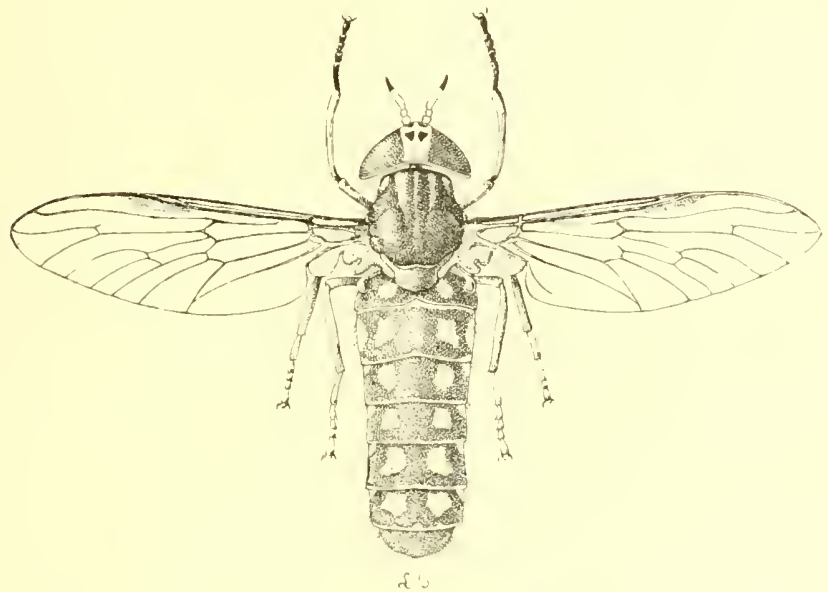

Fig. 23. - Tubanus Rothschildi O Suncour

Ventre noir à premier segment lecouvert diune très courte pubescence cenIrée, les autres segments sont martués de litur et portent rhacun deux laches latérales blanchàtres.

Pubscence ventrale composée dro tfuelutu's proils hlancs répandus sur toute lis surface.

Pattes testacées a pubesience blanibe, rembrunies aux tarses.

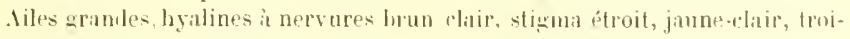
sième nervure longitulinale courtwm ilppenticulise. Cuillerons hyalins.

Balanciers rougeatres.

Cette espice nouvelle est très roisine dn Tubames morsitans Ritarto

Elle en diffère:

$1^{0}$ Par ses palges allongés non rentlés, jeur velus: 
$2^{\circ}$ tar la forme de la callosití dont le hord supérieur est concave chez $T$. Rothschildi et convexe chez $T$. Morsitans ;

$3^{0}$ f'ar lat callosité médiane, qui est bifide au liẹ d'être ronde et non caniculée.

\section{BIBL,IOGIIAP'IIIE}

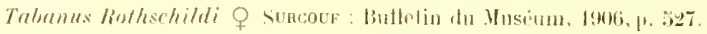

\section{Tabanus obliquemaculatus $q$ Haçuant}

Le type de Macouart existe dans la collection le M. Verrall. Description de Vlacovalz :

"Niger. Intennis nigris. Mblomine manulis lateralibus ablis, obliquiis, lasi " laterilus rutis. l'edihus nigris ; thliis llavis. Alis cellulà submarginali appen-

"dieulata."

" Longueur $61 / 2 \mathrm{l}$. 우.

Palpes et face d'un lilane jaunatre. Front ì quatre eallosités sur un fond d. " duret blanchâtre: première à la base Jpe antennes, saillante, brune, transver"sale; dpuxième contiguï à la première, testacée, transverkale, en forme d’ócus"son: troisième trstarce, presque matr, large, transwursale; quatrième eontigüp " it lat truisieme of nemugant le vertex, mate, testacép, un pu blanchâtre. Antennes " t'un noirgrisatre. Yeux bruns. Thorix noir i lignes blanchitres; lach rouged-

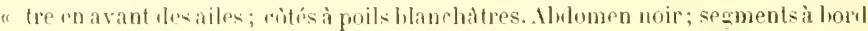
"postórieur fauve étroit. el taches dorsalestriangulaires, petites, de poils faures; "premier et deuxième à cités d'un fiuve testacé; les quatre premiers à tache "hanchatre, ovate, oblique, de chaque cots; ventre noiritre; lord postérieur des a segments faure. l’inds noirs; jambes d'un jaune btanchatre in extrémité noire.

"Cuillerons hlanchitres diles assez claires; nervures lígèrement bordées de " rousittre pitle; tarhe stigmatique hrune; deuxiome cellule sous-marginale "appendiculée is la lase ".

In Cap. Collection dle M. Serville.

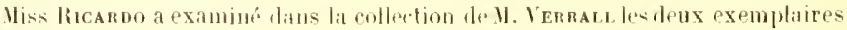

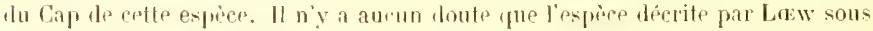
le nom de Tubamus lencostomns et par J.xxvicke comme Tabamus psusemnis ne soit identicue à l'espèce do Macovart. Cepttr expèce se reconnait aisément it la face couverto de poils blancs, les palpes éprais el courts, la fare très développée à peine ausci haute que large. mesurant $2 \mathrm{~m}$. 1/2 à la base. ot $1 \mathrm{~m}$. I 2 au sommet dans les exemplaires de M. Icocart pui ont $19 \mathrm{~mm}$. de largenr. Il y a quelques poil- moirs sur les patpes et les tilitias postérieurs portent aussi une pubesenese noire, milis les types sont an tres maturais itat de conservation.

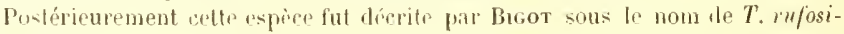
gnatus. 


\section{Tubunus lencostomus o Lew (1860) \\ $=$ Tabunus psusennis of Jäsicke (1867).}

Deseription de Law :

"Nigro-rinereus, farie candida antriore frontis parte atri, antennis alpis, "alodomine bifariam allo-maculato, perlibus nigricantilus, tibiarium antica"rum dimislio biasali albe ; alarum cinprascentiun nervis et stigmate nigro. " lrunneis, nervis transversis lorunneo-limbatis ". -- Long. corp. 7 lignes.

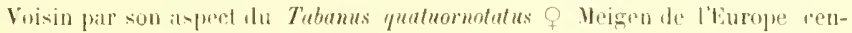
trale. - Longueur l't-I:i millimèlres

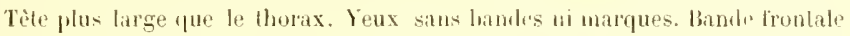
diugie, de eouleur lorune, prortant à la base une large callosité carrée, tangente

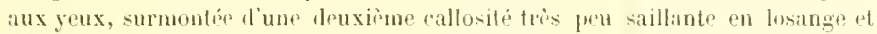
canaliculie an milieu. Une pollinositi blanche et trie deuse ocempe toute la largeur de la hande an-dessus de la callusite carrée el encatre les rótes du losinge puis recourre tout le vertex et les praties laterales de la hande foutale de chatque fóté de la réscion ranaliculée. Epistone d’un hrun-noir luisant. Intemnes moires: premier article dilaté recouvant le deuxième en eapuchon: deuxis̀me

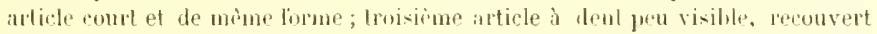
d'une fine pubescence jaune-bun : la pubeseme des deux premirl's articles

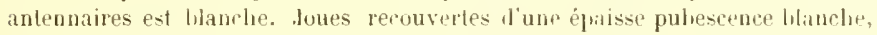
brillante. Palpes épais, renllés, hlanes it pulvescence hlamche.

Thorax brunatre portant quelques lignes bianchitres peu distinctes; érusson de mime; pectus cendré à pubescence lilanchatre.

Malomen à fond noiratre portant un ligne módiane jaunàtre, indecis", se dilatant en triangles peu distincls, jaundres sur claque segment et des taches blanches de taille décroissante disposées de chaque cóté de la ligne móliaue, en oulre, les llanes de chaque segment porlent une cpaisse pulrescence blamelà lre gui

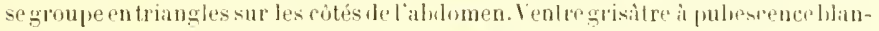
chatrr, chacun des seguenls étroilement marginé de blanc. IJanehes cendres à pulescence blanche; fémurs noir brunàtre ì pubescenceblanche. Tibias anlirieurs launtres dans leur partie apicale avec une pubescence mélangée, et blaues it pulescence blanche dins leur moitió hasilatre: les autres tibias sont d'un hrun

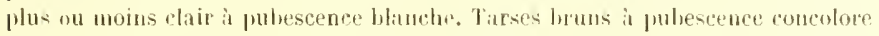
ef quelques poils rouges au cité interne de la paire postrírieure. Ailes cendrées, nervures longitudinales el sligma bruns, nervures lransverses estompures de lrun-jiune; un appendice. Cuillerons incolores. Ballaneiers jaune-brun it minsue livisle.

l'exemplaire type de law provient de la cifrerie. Le Muséum de l'itris possède trois spécimens le Tubanus tencostomus q luew recuejllis par le doeteur 1.. Jinumpt, it Ggaden, pays des somalis, an sul de llarrar, en juin 1901.

Postérieurement ì la description de Tabanas lencostomas o Law, darnicke dérivit sous le nom de Tabranus psusennis un spérimen male de Tabmus tencostomus. Le musée de Francfort-sur-Mein a bien roulu nous contipr l'xemplatire male de Tabrnus psusennis recueilli en Aliyssinie pirr le docteur liupreld. 
f.exniche en donne la desiription suivante:

"E eicereo niges" thorate albilo lineato, abdomine serietus duabus e macu"lis albis; oculis nulis, prmagnis; foto marsine prismatibus minimis rinctis; "alis layalinis, nervis fusco limbatis; fura nervi tertii appendiculata o.

Longueur 15 mm, Patria : Alỵsinia (Ruppez).

\section{Alylotus ruficeps of Bigot \\ = rufescens \& Bigot}

Lat description de Biggot établie sur un senl exemplaire femelle est notoirement insuftisante, nous la reproduisons en entier.

" Une femelle, $1 / 4 \mathrm{~mm}$. Intennes noires. troisième segment médiocrement - échancré el hrivement denté. Ailes : hifurcation externe de la quatrième "nervure longitudinale (Rondani) ì peine appendiculée. Palpes, face et barlie " hancs. Front rougeitre avec un peu de duvet gris (eallosité?), transversale" ment maryoe do deux sillons peu profonds. Thorax noir avee cind lignes grises, écusson moir horlé de gris. Cuillerons blancs, lalanciers loruns à mas"sue blanche. Flancs gris it poils gristres. Alubmen gris avee quatre rangées " de macules noires allongér. Pierls noirs in duret blane, latse des tibias rougea* tre. Ailes hyalines, stigma étroit, allongé, doun poussatre pale. " Capude Bonne-Espúrance.

Celte espice decrite sur un seul spécimen at très voisine de T. Tenrostomus Lerw et the $T$, obliquemaculutus liter., elle se distingue de ces deux espèces par sps alibs complètement claires et sécarte de l'esuce de Marquart par les martues et la muleur de lablomen, de celle de Law par sa bande frontile ronge-hun et ses callosités qui sont noires et brun noir dams Tubamus lencostomus lewe et

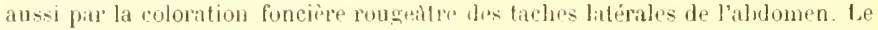
Tubrums rufosignatus Lew, appartient a petit groupe ì hande frontale élargie portant deux callosités séparées. Les palpes très courts et la face recouverte de proils blanes séparent de facon curactéristique ce groupe de celui du Tabunus trmiola P. B. dont les taches de l'ahlomen offrent une certaine ressemblance. liratce à un spécimen retrouvé dans li collection du British lluseum nous avons purefaire la description de Tabanus rufosignatus $q$ Bigot.

E-piee de taille nuyenne it largr bande fronlale, epistome d'un lıun brillint; ailes hyalines; alubmen étroit, aoir aree trois séries de taches disposées en handes irrégulières. Longueur 1 ' $\mathrm{mm}$.

Tète plus large que le thorax, face rougeitre couverte d'une tomentosité grise et à taches noires irrúgulières appariaisant à traver's la pulsescence eomposée de longs poils blanes. Barbe blanche. Palpes courts, epais, jaunes, terninés en une pointe nince, converts d'une pilosité d'un hlanc argenté relevée de quelupes poils noirs a rapex. Epistone linu sombre, lorillant. Intenmes doun lorun olsseur ; prenier article petit, ollong ; second très petit en forme de capuchon; tous deux à pubescence noire; troisième article ì dent très peu aceentuée, les trois dernières divisions noires à tomentosité grise sur la première division. Bante fruntale large, t'un hrun rougeatre, environ deux fois aussi haute tpue 
large s'ánoouissant vers le vertex ou elle acquiert an moins le tiers de lit largenr de la tâte; callosité frontale lorun rougedtre, lranswerse, altoignant it peine les yeux, moins colorée que l'épistome ; la callosilá médiane est irrégulirrmont eordiforme, et séparée de la prentiòre pror une hande de pulvescence gris jau-

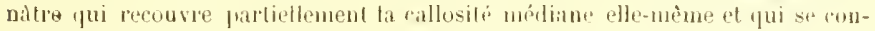
tinue vers le vertex qui est orné d'une courta pubesence noire. l'artir posterieures de la lète portant des puils jaunatres. Yeux sins marpues.

Thurax noir, rougratre sur les coités, is trois haudes grises indistincles; pulsescence gris jaubitre, grisiltre sur les còtén aver: tes proils lolanes en dessus et noir's en dessous. Pectus noir à tomentosité grise et pubescence blanche.

scutellum noir i pulsescence grise. Abdomen long et étroit, noir portant des tachesmédianes triangulaires et étroites, de conlenr qrise, sur les deuxirme, troisièmeet quitrièn" segments, et des track d'une tache semblable sur le cinquième segment. Sur les còtés sont des handes irrégulières, bien marquées, composées de longues taches triangulatres rougeitres qui atteignent les bords antérieur et postériem de chaque segment, charune de ces taches a la base et le

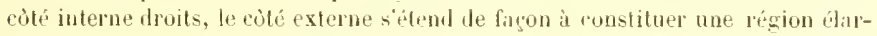
gie et un apex ohtus. Ces titches ont leur maximm de dimension ef de nettete sur les deuxième, troisìne et quatrième segments, sur le cinquiène dles sonl moinrouges et plus pelites. La coloration rusce est probablement recouverts d'une pubsecence blanchatre quand l'insecte es frais, ce qui explipuerail punrquoi Bigot $n$ "a pas mentionné cette couleur rongoitre.

La puluescence est grise el épaisse sur toutes les taches, sur le bord postérieur des segments et sur les cotés de labulomen; elle ast noire sur les régions noires et à l'apex. Envers noir avec un peu de pouge, courert d'une tomentosité grise, segmentations jaunalles a pulyescence blanchitre.

Pattes noires; fëmurs à tomentosité grine el pubescence blanche; tibias jatune rougeatre sur leur moitie hasilaire, libias antínieur's frès brillants, pulesence llanche sur la moitié basilaire des Liblas, noire sur les apex et les tarses.

Ailes hyalines, à nervures brun-jaune, sligma jaune, appendice distinct, mais court; toutes les cellules postérieures largentent ouvertes, l'angle de la troisième nervure est lésirement umbrè.

\section{BHBLIOCRIPIIL:}

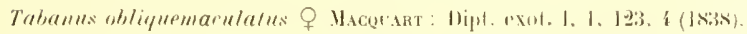

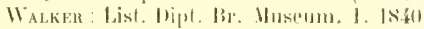

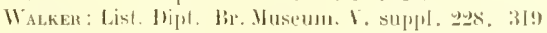
(1):4).

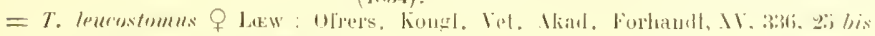
(1nis).

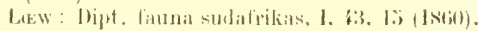

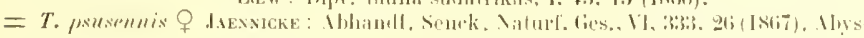
sinit.

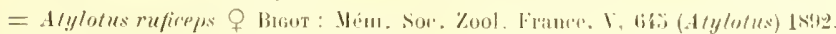

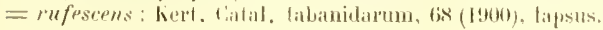




\section{Tabanus pallidifacies Surcotf (pl. HI, tig. Ili)}

Typ': un exemplaire q recueilli is simba (Afripue orientale anglaise), par

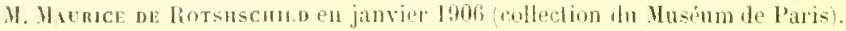

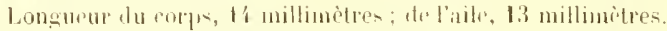

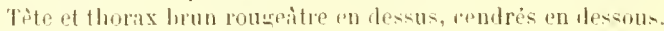

Bande frontate large, elaitain.

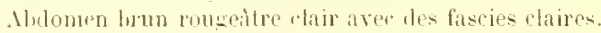

Aites hyalines ì stigma hrum et étroit.

Tètr : veux luruns it cornéules rigales, petites, ghalores. Bande frontale plus large au vertex, romurepte doune putsesenes jame hrunitre portant deux callosités. La première est siluée entre les angths tos yeux, grande, chitain clair.

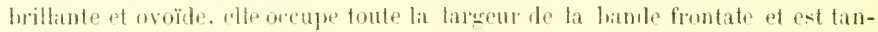
gente aux yeux. Lit callosité superieuse situe vers te milieu de la lande frontale est de meme coloration, oroulde et peu satlanle. Chez les pxemplaires tris frais, elle doit ètre presqur recouverte pirl ta pulbescence de la binde frontale.

Antennes. I'remier article testacé ì fubsecence blanche' aver unc tache trianyoulaire noire sur l'extrémitr distale suprériene.

Deuxiène article trè rourt. Wordi de soles noires rades.

Troisine article it dent thase rougeitres, la reste noir.

Pilpes renlles, courts, hlaneluthes, il pulwsence lianche mólangér de qual-

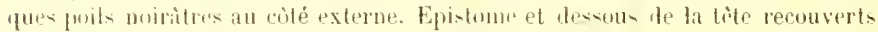
d'une atondante viltusite hanchatre.

borl prostrienr de la tile presque slabre.

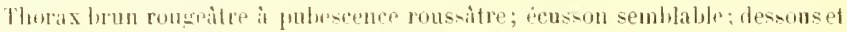
Hanes it longue puleseners hanchitre et hérinsée.

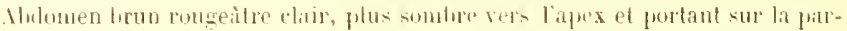

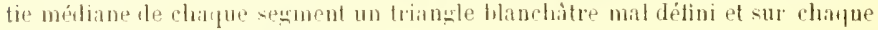
cois um tache armolie rougeitre.

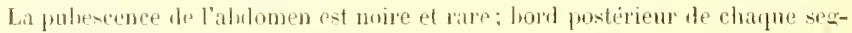
ment aludouninal mareini de blam hitte.

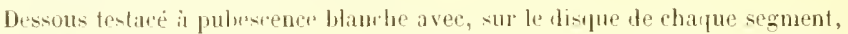
'puetques poils noir ì partir du quatrime segment. ils deviennent plus nombreux rern lapex.

Jambes, hanelips, ruisses, tilias d'un brun rougcitre rbair à pubescence ban-

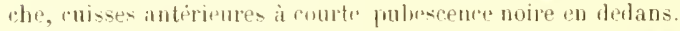

Tibias postérieurs ave une courte frange noire, tarses lorun rougeitre a pulesence sombre.

diles transarentes, grandes ef rlaires a stigntil átroil; hord rostal cliar.

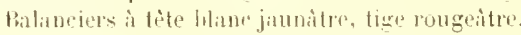

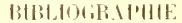

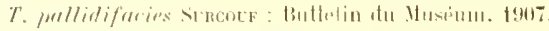


Tabanus sufis of Jxxike $(1867)$ ( [1, III, tig. I

$=$ Tabanus alboventralis T TeWSTEAD (1907)

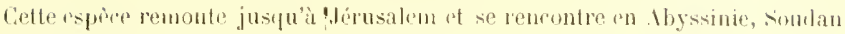

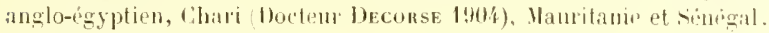

Longuem 8 à 9 mm.

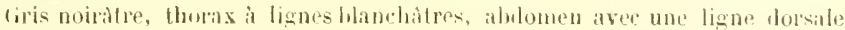

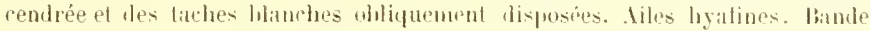

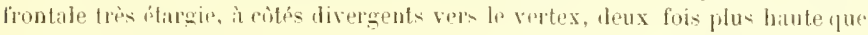
harge au milieu, thinchitre, portant it ha hase une callusité transverse arrondie. presque tangente aux cótés, vers la prirlir maliane sont situres deux taches

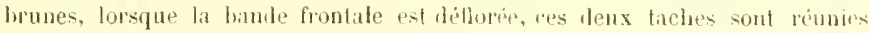

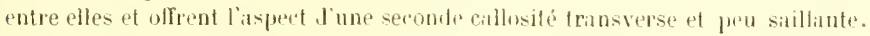

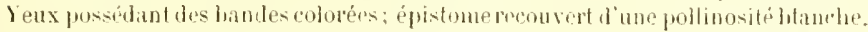
Antennes brunes a purescence banchitre sur te promier artiele moins abondante sur le troisieme article ; celui-ri ne porte pis une dent distinete maic

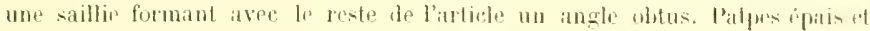

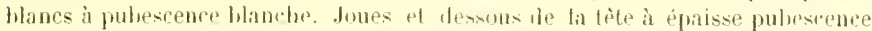

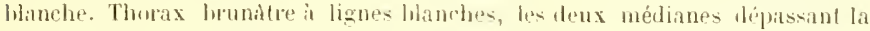

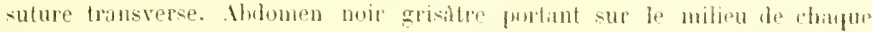

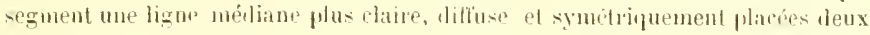

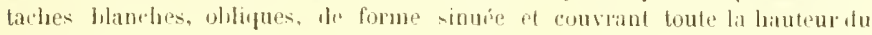
segment.

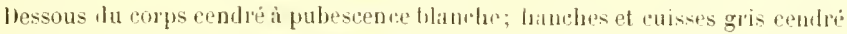

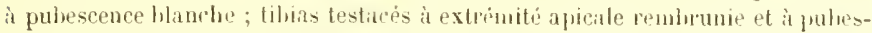
cence claire sur la partie trstacée. Tarses antérieurs sombres a pubescence concolore, tarses médians et postérieurs it métiltarse presque aussi long que be

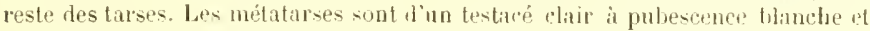
sont rembrunis ì l'extrémité appicale: les articles tarsilux sont sombres.

Ailes hyalines, un peu cendres, appendirulées; nerves hongitudinales of stigma jaunatre; nervures transverses ombrées de lrun. Cuablurons blanchitres. Balanciers blanes.

Récemment M. R. Newsteab a décit sour le nom de Tahomus allocentralis

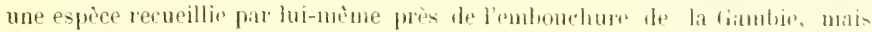

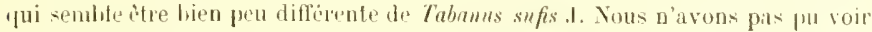
le type de M. R. Newstad et nous donnons la dracription de l'auteur.

Thorax brun noir portant trois lignes etroites; abromen d'un lmon flus sombre portant une sirie hilatérale de tarhes lolanches oblipues. Toutp lidendue dir

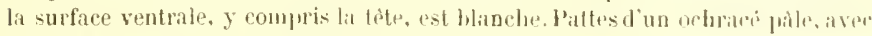
les tarses et les apex des tibias flun lirun somlure.

Femelle, - Tète: apace entre les yeux d'un gris ochreux aree drux larges bandes transverses brunes, la parlie inférieure de lit tite de man due la région postérieure est revètue d’une deuse pulescenre hlanche. Intennes hrun piontr. Palpes d'un blanc jur l'aspect eirenx.

Thorax d'un grislrunatre, sombre, aver une ligne mediane el dexis sousmédianes grises, hords gris aussi bien que fo lwol postériour du s'utellum. 
Ilidomen hrun sombre, it handes apirales itroiles ot palles. Du premier au cinequiemo segunent existe sul charun d'ux une hache grisitre allongép sous-

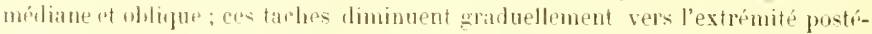
rienre, de telle sorle que da dernise pst difficilement visilde tandis que sur de suond segment, elle stemd ì travers toul le segmenl et se termine it son bord apiral. Il existe anssi une faible ligne mérliane forme de tarlies de poils jaune horé ventre d'un lirunitre pilte revítu d'une pollinosité et d'une pulrescence dilnchitres. Bord apical de tors los semments arant une étroite bordure ochreuse. Jiltes ivere les cuisses, les trochanters el les limurs blines; tithias d'un ochracé pite arec le tiers apical brun; tous les tarses lorun sombre. Ailes transparentes sans densins.

Longweur $10 \mathrm{~mm}$. fongueur de l'aile: 8 mm.

" liceneilli en septembre octolue 1902. an voisinage de lyster Creek, près de l'embouchure de la ciambie. Très étroitement allié au $T$. obliquemaculatus " Maepuart, mais M. E. E. Arstex ì qui ces pximplaires ont été somuis pense " "fu'il s'agit d'un esprée non dérite (Newsteal)".

\section{BAtBLGGRAPIIE}

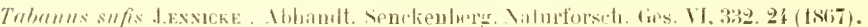

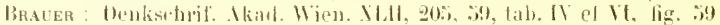
$(1 \mathrm{~s}+(0)$

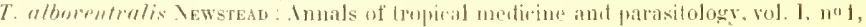
tiovitrition. 19. it;

Tabanus gratus Low or Karsen (1. 1li8, fig. 22)

Cimeraseens, thotace pillide vittato ; abdomen superius nigrum, pallide trivittatum, vitti intermedia integerimat, vittislaterilibus undulatoserratis; antenne flave frruginir, seutellum el perles pallide rufescentes; frontis callus inferior brunneus, superior ater; oculi fasciatı. - tongurur corp. ò 1/2, lignes.

Lil coulem du fond de la face a l'asped noiritre mais elle est extrêmement

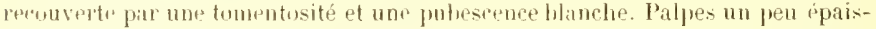
sis d'un lolane sale avec une pulescence blanehe et quetques poils noirs entre-

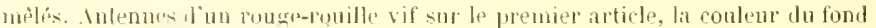

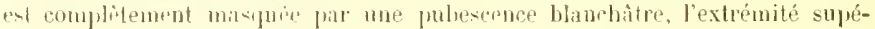
rienre de lit corne qui est trè saillante est si completement recouverte de poils noirs yrille sembl noire; deuxime article antennaire tris petit; troisieme article un peu plus large, la saillie pxterne tric arentuée est comverte de poils noirs,

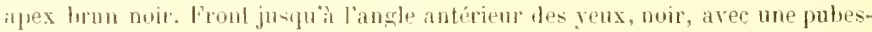
cence hlinehithe il se trumeen or prinl une caltesité quadrangulaire d'un brunnoir brillant gui vit diun lurd des yeux it l'autre. Sur le milien du front se trunvencore une aulare callositi moins uctement tracée, noire et brillanle, on apereoit auni sur le vertex un petit rudroit noir. l'ar ailleurs la lomentosité du front st urisitre, fuoinue julus brunatre entre les callosités. Youx d'un beau rert, langhe inferieur et langle supérieur sunt d'un rouge pourpre de mème 
qu'une bande transwersale droite et peu large tui s'átond ì la hauleur de la callosité inférieure lu front.

couleur fourie du thorax gris noiritle el lrunatre en avint do la racine des

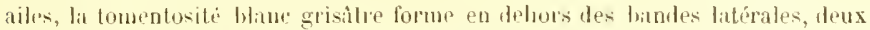
bates longitudinales nettes au milieu du còti supérieur el une ligne longiludinalo nette au moins an còté antérieur ; la pubescence du dessus du thorax est génératement hanchatre; sur lis handes sombres entre lestuelles la couleur du lond devient gà et là rouge brunilre, elle est hmon sombre ainsi qu'une parlie du sculellum. En avant de lia racine de l'aile il y a answi des poils moirs.

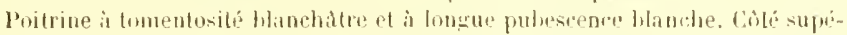
rieur de labdomen noir arec trois bandes gris hanchatre et le loril lateral de mème couleur; la baude uédiane rommence en pointe au horl antérirur tu deuxième segment où elle se joint ì une petite tache le ha mème couleur qui se trouse sur le premixr segment, la bamle s'dargit progressivement jusidua fuatrieme segment bi efle commence it se rétreir plus vite ot se termine au hord poslérifur du sixième segment. Lis burdures latérales sonl conlinues ; un peu distante lu horl de lablomen se trouve de chatue coté une bamle en zigzag gris blanchatre, forme de flusieurs trits gris-blanes, qui orcuge toute la longeur de chaque segment et s'conte flus en dehor's a l'extrémilí postérieure quà l'extrémité antérieure, sur chaque segmmot successif ces traits sont plus étroits, de sorte que les bandes fu'ils forment commencent id disparintre sur le dernier segment. Lit coulemr du lord justícieur des spgments ahdominaux est jaune isabelle, la pubescence est aussi flus blinche que celle du restr de labubuen. Au hord postérieur de chaque segment on voil la traced une borlure claire. La pubescence des parlies claires st him hatre, eftr est moiratre sur les parties noires.

Tentre rouge jaunilre. plus gris sur les colles et sur les derniers segments. recouvert d'une petite lomentusilé de poils banchàtres très courts.

lattes louge jaunàlre à pubescence hanchitre. Apex des tibias antrieurs et

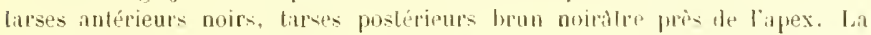
puleseene des larses est completrment hanehe maix il s'y mple des poits noirs, les plus évidents se tromvent sur les ruisses antélieur's, it lextrémite des artirles tarsaux el sur le crité exlerne des tibits postérieurs. Balanciers hancs. diles

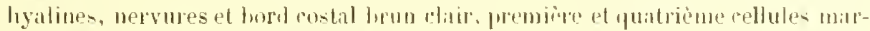
ginales poslérieures non ritréries. - Caltrerie.

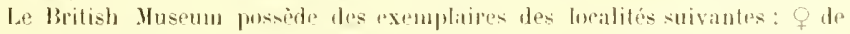
Vola, nord de l'Etal de Nigeria (14, II, 11.5, W. T. Ciowers); I f dr Fajo-Yil-

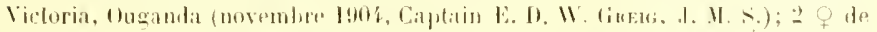

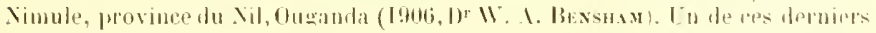
spreimens porte la note suivante: a lu pusiemrs fois it Nimule; recueilli sur des

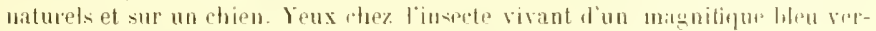
datre avec des bande- horizonlates en zig-zag de pourpere violicín".

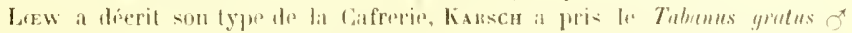

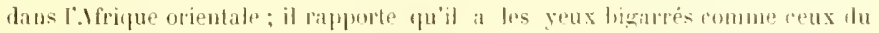

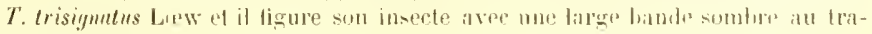
rers des yeux.

l'etite espèce noiràtre à jambes et antenufs jaundles. Thurax à bandes grises, 
alulomen marqué de trois bandes grises continues. Yeux ornés d'une bande colorée. Lotgneur 11 1нm.

Tete ligerement plus large que le thorax ; lace noiratre courerte de tomentosité cot le pubescence tranches. J'alpes jaune pale épais à la hase, terminús en une longue pointe, pulbesence blanche mélangée dle quelques poils noirs parfois très visibles. Parbe blim lue. Antenues d'un rouge-rouille brillant, premier article couvert d'une tomentosití et d'une pubescence blanchàtres, angle supérieur à iprisse pulurence noire; second article très petil; troisième porlant une courte dent listincte dont l'apex est brunàtre. Bande frontale noirålre, environ quatre tois aussi haute que large, couverte d'une tomentosité jaune grisatre, rétrécie à la partie supérieure el portant deux callosités. Celle du front est large. presque carrée, le couleur brun jaunatre, atteignant les yeux, à bord postérieur irrégulier; la callusitri mediane est oblongue-itrondie, de forme irrégulière, brun noirâtre lrillant, parfois réunie ì la callositi frontale par une liggne fine.

Luw décrit la callosité frontalı noir-brun, la callosité médiane noire el signale une pretite tache noire sur le vertex. Cette tache re se présente pas dans les exemplaires cités. Veux aver une hande qui s'itend ì hauteur de lis callosité frontale it travers les yeux jusquan bord postétirur.

Thorax noiratre à ring lrandes distinctes de tomensité grise, lat médiane est la plus étroite; lrandes latírales rounceitres à tomeutosité urise et pubescenre blanthe; sur ledessus lit pubescence est noirattre entre les bandes et hanchatre sur celles-ri. scutellum hirun rongeatre à honentosité grise et quelunes poils noirs. Ibdomen noiràtre à laind médiane s'étendant du premier au sixième segment. plus large sur le truisiome et le quatrième; bandes latérales quelque peu en ziggaz, irregulieres sur leur tord externe, efles atteignent seulement le rinquième segment où elles sont imlistinctes at prtites; pulrescence noire sur les prarties sombres, blinche sur les handes de tomensité grise. Cötés à poils hlanes, hords lintéraux transparents vers l’apex: segmentations très éfroitement et indistinctement plus claires. Envers flus rlair recouvert d'une fomentositŕ grise et d'une pulvescenee noire, jambes jaunhtres aver un peu te tomensité blanche, pubesrence principalement hanche, apex des tibias et les tarses brunatres.

Ailes claires, nervures jaumes, pas dappendice.

La collertion du Musinn de Paris possède un exemplaire femelle recueilli entre le Ounbi Chebrti et l'Oneb (pays Somalis septemlure 1901, Ir Bromer beux autre: fenelfes proviennent de Kati, soudan, et ont été commnniquées par M. le yr Laverax, La rollection du Muséum de lishonne contient une femelle provenant de llumbe.

\section{BIBLIOGRAP'tIE}

Tabanus gratus o Lew : ïls. K. Yet. Nkatl. Fortandl, 18:5, 340, 23.

Law: Dipteren-Fauna, sud-Atrikis, 1, 42. 1\%.

- ol harsca : Berlin. Ent. Zeilsch., AXX1, 379, 4. 


\section{Tabanus tritæniatus $\bigcirc$ licanmo}

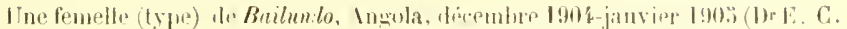

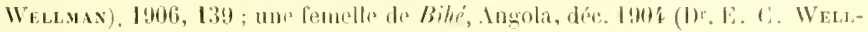
3ax), $1906,139$.

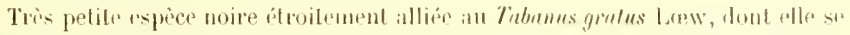

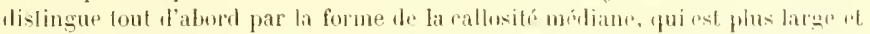
occupe prespue tout l'intervalle le la hande frontale, carró, lourts anlérieur nt

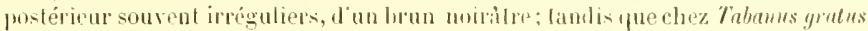

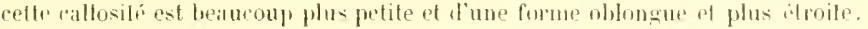

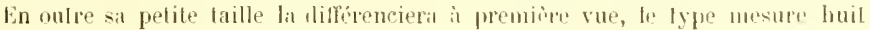
millimilres, et l'autre femelle seulement six millimitres demi.

Abdomen avec trois handes grises, lat médiane attemt trixirme segment, et les latérales la quatrìme seguent, parfois mème alles se terminenl auparating. Inlennes rougeilfes ; preunir articto grisilfe, à proits noirs sur l'angtr supérieur ; troisì̀me artiele noirîlre ou brun ì l'extrémilí. Yeux avó au moins deux bandes colores. Thorax à bitndes grises. Callositio frontale beune, brillante, lingirement próminente, veeupant toule la largeur de la tèle el roliéc par une courte ligne au callus médian.

Bord occipilal gris, portant une pubescence noire au vertex. l'attes rongedtres. liles completement hyatines.

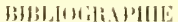

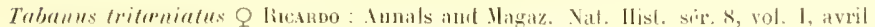
$1910,11.311$. 


\section{QUATORZIEME GROUPE}

T'ABLEAU DICHOTOMIQUE

C reux portant une bande, parfois rérluite

1 à un trait horizontal. . . . . . .

Venx ne portant pas de bantes. . .

2

3

Whlomen noirattre aver dux banden Intérales de pulvestence grise, jambes noires, tibias jaunes, $10 \mathrm{~mm}$. . . . . T. albipalpus $q$ Walker.

2 Alulomen noiritre itres deux bandes laterites jounatros, lanches ol fémurs jinnes, 11 iो $12 \mathrm{~mm}$. . . . . . T. dihmentus or $q$ Mfarquart.

llanches pt fémurs boirilles. . . . T. pusciptes o Ricarlo.

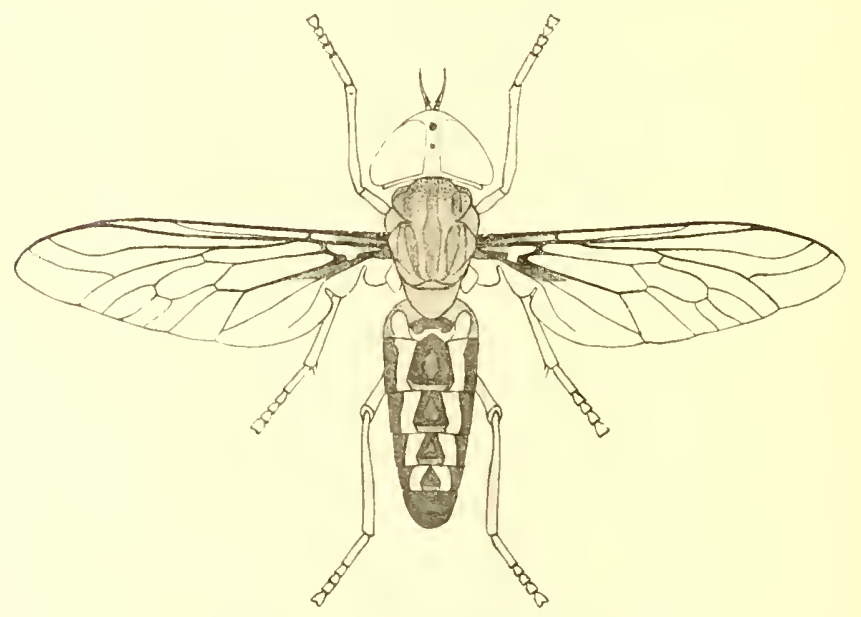

Fig. 24. - Tubmus ditumiatus o Macevant 


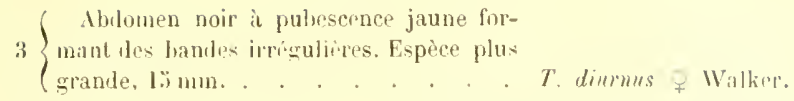

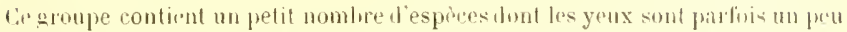

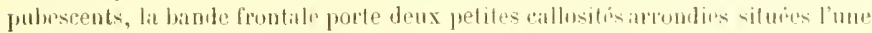
au-tessus dp lautre on non relibes entre elles, fare glalure.

\section{Tabanus albipalpus $q$ WALKEH}

Type femelle provenaut de la liambar. Collertion du British Museum. Ce Iype pol un spérimen en mauvais ítal.

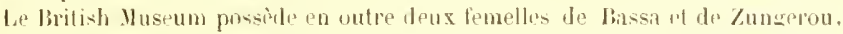

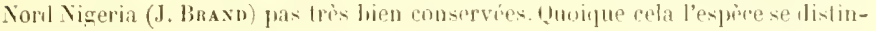

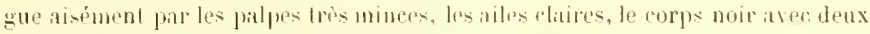
bandiss tirterales formpes d pubescenre grise.

Longueur $10 \mathrm{~mm}$.

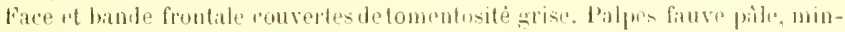

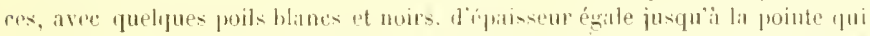
est rourte. Intennes fitures it dent pretite. Biande frontale environ trois foris aussi haute que large, un peu ritrieje antérieurement, portant une maltosité noire, Iransverse, irregulire, n'atteignant pas les youx, et une seconde callosite noire oblongue, siluér au milisu de bis bande frontale. leux avec une bande étroite.

Thorax noiritre, sans bantes visibles. Iludomen noiritre, un pru jaume sum

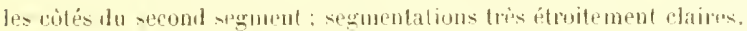

dambes noires, tibias jaunes, les anterieurs moirs it l'apex ainsi que les tarses antérieurs. Niles claires portinul parfois une nervore appendiculate.

Desrription de WaLker:

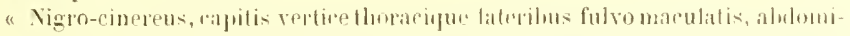

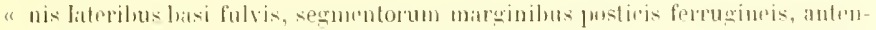

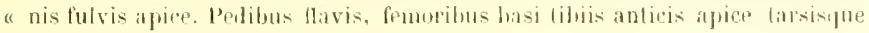
" anticis nigris, alis limpiclis."

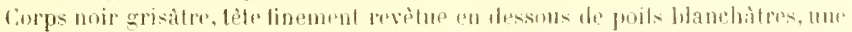

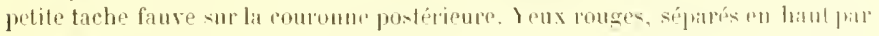

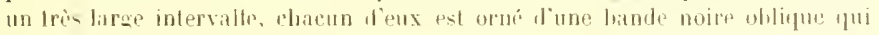

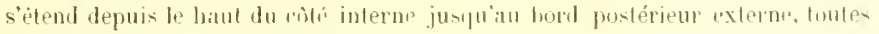

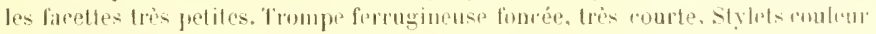

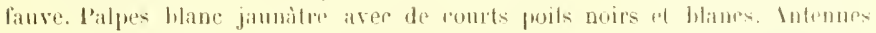

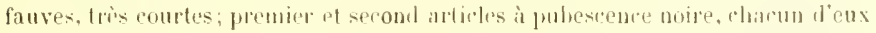

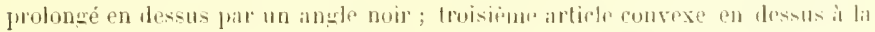

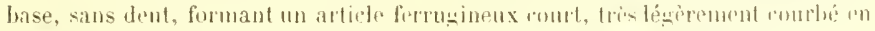

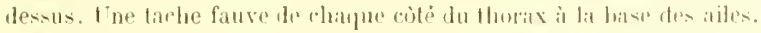

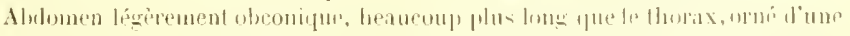

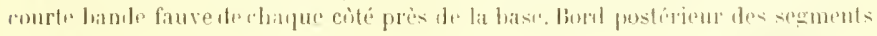

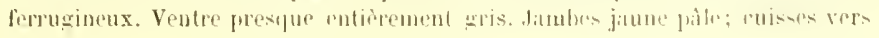


leur hase et hanches noires: extrimiti des tares brune. Ongles, tihias anté-

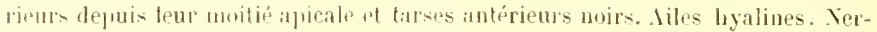

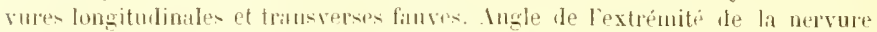

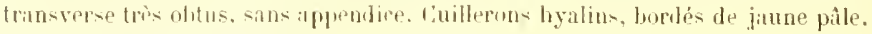
Balinciers jaune hanchittre.

Longueur du corps thenes $1 / 2$, des ailes ! lignes.

fiambie.

\section{lilliLlurili|PIII:}

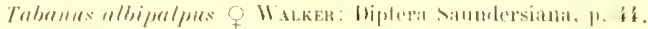

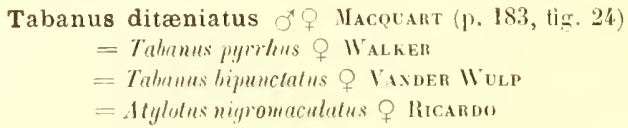

Le Trebanus detemintus Macquart forme un petit sous-groupe est voisin du Tabanus insticus Linné, d'Europes.

L. Tabums ditemiatus Marquart et les especes roisines se distingnent du groupe de Tahamus sufis diennive, par lit très lígère pulsescence des yeux qui est souvent difficile à voir chez les femelles "t part l'ablomen it bandes plus régnières. Le Tubunus bipunctutus Vander Wulp, ext tris voisin du Tubanus ditaniatusMacil., suivant le descriptenr, il a il pas de hande transversale aux yeux. De l'examen du type et des cotypes nue le directeur du Musée lingal d'histoire naturille de belginge a hien voulu mettre à notre disposition, il semble résultro l'ilentité des deux espreses. Le Tuhams ayricolu o Wiedemann qui provient de

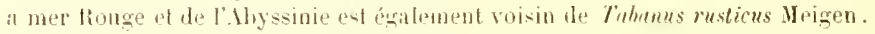

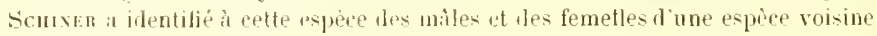
provenant de Hong-kong.

Certains expmplaires du Muréum de Paris ont été déterminés par Bıscnof sous le nom de Trhanus flarmereis II ied. cotype, ces spécimeus se rapportent au Tabanus duanialus Vaey. sins nul doute. Le type de T. pyrrhusW. vient del'Inde.

Le tyju de Mariquat e-t au Musém de haris ainsi que trois cotypes, il est presifue completement détruit. It existe un antre cotype à Lille, provenant de l'ile Maurice. Deux des eotypes de Paris ont un ajpendice it la troisième nervure lunsiludinale, celui de Lille en a seulement ha trace. Les types de Mitcquart pro-

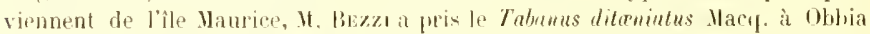
\&ul la côte des rumalis (Aurales cin. Genova, 2-X11, p. 184, 1892). L'extension de cette espece est ronsiderable. olle comprem l'Ifrique entière an-dessous d'une ligne réunissant le sénégal, le Trchal et le pays Somali. ll y a une note du major 11. R. Pope 11exiessey (19 novembre 190,i) qui indique que les somalis du pays de Juba ajpellent ce faon: Bual ad ou Bunl at. D'après It. Austex il est probat-

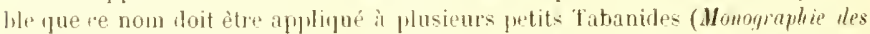
mouches tsitsí, [. 307, 1903). Le Britısh Yuseum possède des spécimens provenant le Wei-llai-Mei. Chine; de l'Inde. Le Huse de Calcutta a des exemplaires du Bingale, Marlras, Coite N.-E. de l'Inde, Ceylan, et de la frontic̀re du Belout- 


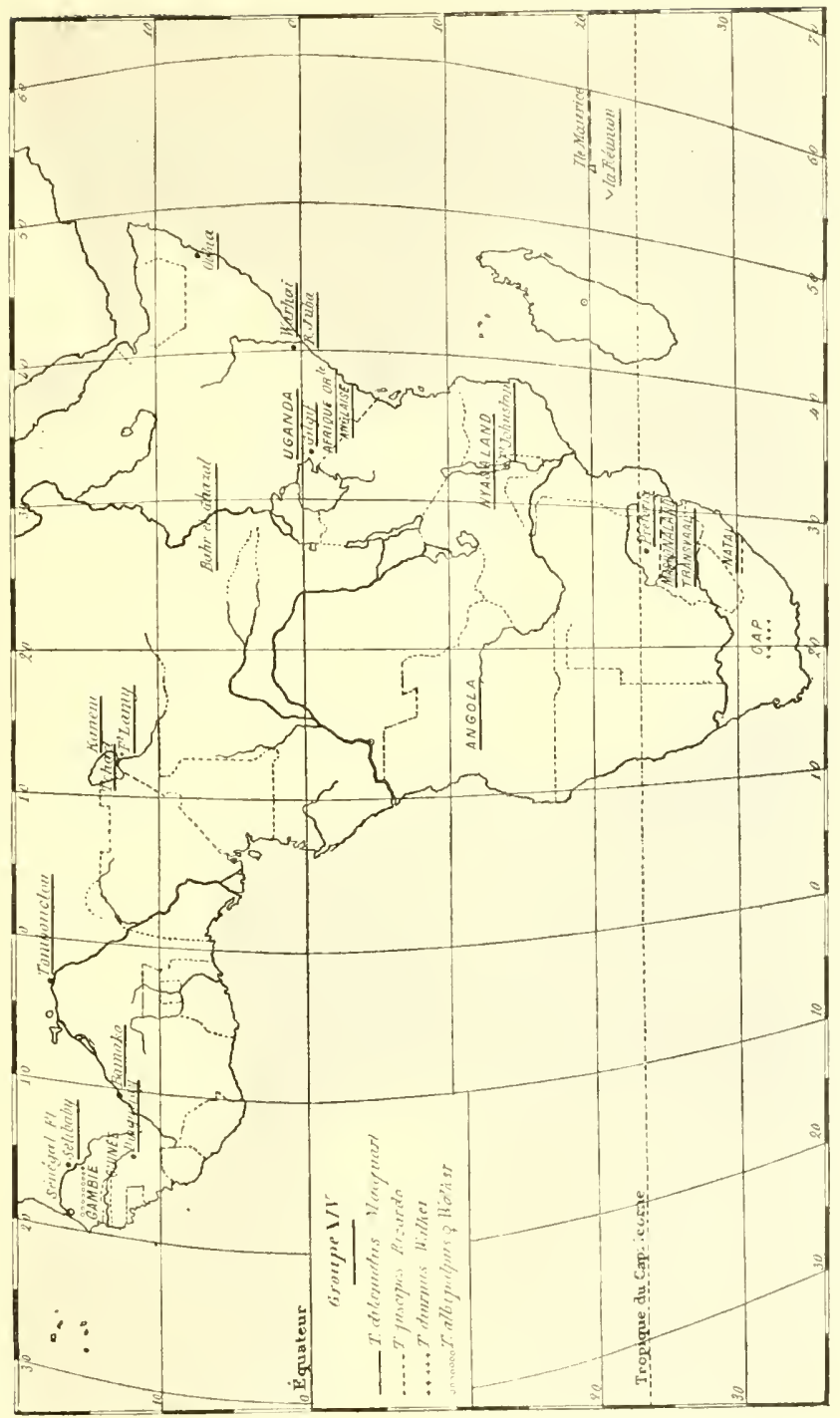


chistin el de la Perse. I. Coquillet lu signale du Japon sous le nom de $T$. pyrrhus Wallier.

Cette espee posside un appendire in lit troisieme nervure longitudinale, mais

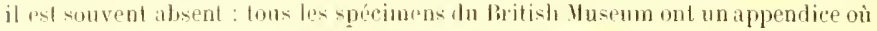
bien l'angle de la neprure est aigu satul' chez un spécinen de Preloria, qui n'a ni appentipe ni angle aigu. Mackuart dil qu'il y a parfois un appendice. Labdomen varie somvent l'aplitrence it cause de lit dénudation de la tomentosité mais la conleur fondamentale jaune de liatutomen parait toujours ontre les bandes mídianes et litérales ipoiqu'elles varienl souvent d'itendur et de longueur.

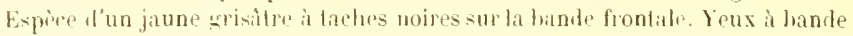
étroite, ablumen jaunitre à larges banden dun hrun mirître. Patles et antennes jaunes. Longueur 11 à 1 ' $\mathrm{mm}$.

Tistr grande, plus large pue fe thorax; face couverte dume tomento-ité grise ef

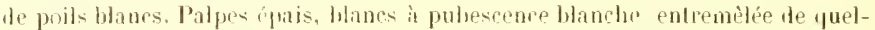
ques poils noirs. Antennes l'un jame roughâtre brillant : presuier el second ardirles plus palos avee puelpum poils moirs à leur apex, dent du troisime articte peu prominente. Barlue banke. Bambe frombale environ fuatre fois ansi

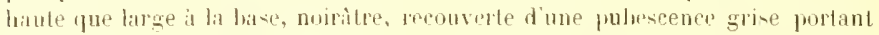

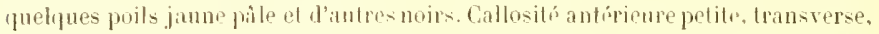

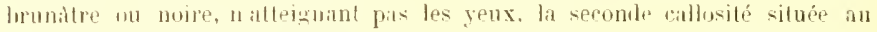
milien de lin bance frontale en arrondie, moire, luisante el porte parfuis un fai-

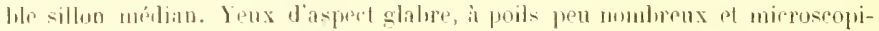
ques, les yeux sont traversís par un trait ì hateur de la fallosité antérieure. Thorax et scuteltum noirs, eumerts d'une tomentusité aris jaunitre ef dune pubscence jitunitre pille on fauve.

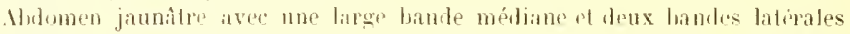
miratres ou brunatres. Sur lia bandr médiane la tomentosití jaune grisitre forme une handecomprose dre triangles a apex tronfué sur ehaipue segment; les

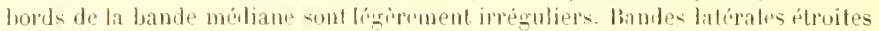
natleignatul pas les citŕs do l'ablumen, elles se fusionnent aver la hande médian vers th sixime serment, te telle sorte que lapex de liablomen est noir : la pubescence du dessus de lablomen es principalement jaune grisitre el sétent en forme de bande sur tes deux segments terminax, il y an peu de

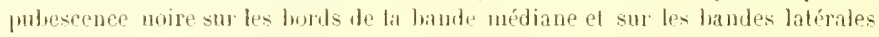
noires, lat prartie dorsale de l'abubmen est couverte en outse l'une tomentosité

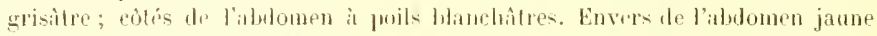

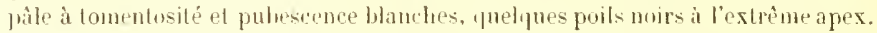

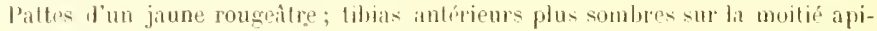

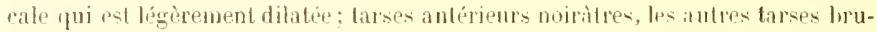

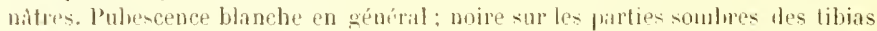
at dro latres antérieurs, quelques poils noirs sur lis autres tilvias ol tarses. Ailes claires, nervores el stigma jaune pats, troisieme nervors longitudinale portant lablitueldement un court appendice balanciers blanchitres.

Write semblible à la femelde, li zone d's petiles cornúules qui entoure la zone les grosses cornéules en est trís nettement séparée.

Lit collection du lluséun de l'aris comprent de nombreux specimens of lrois $0^{\circ}$. 


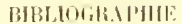

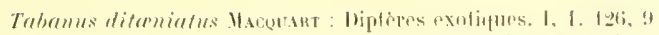

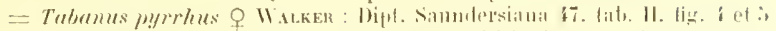

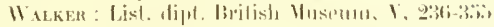

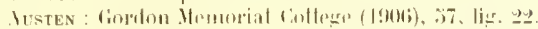

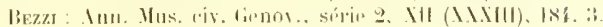

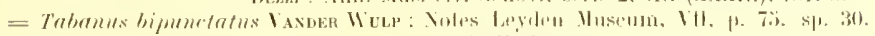
latb. Vi, lige. is.

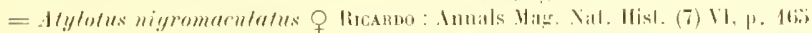

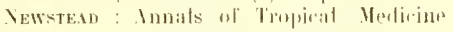

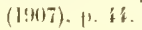

\section{Tabanus fuscipes $\sigma^{x} q$ lírcardo}

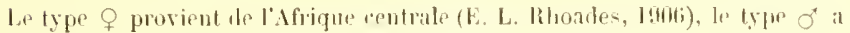
été pris à Estcourt-Natal en janvier $18 ! 17$, A. . I. K. MarsuabL).

Longueur 12 à 1 't $\mathrm{mm}$.

Collection du british Museum.

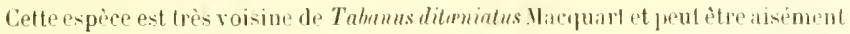
confondue are elle à fremire vur, ma is plle en ditlere par les hanches noires, les fémurs noiràlres presque en entier of l'alsence de loute tomentosité grise sur la partie méliane de la lande noire de l'ablumm. Intennes légìrement assom-

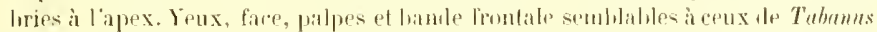

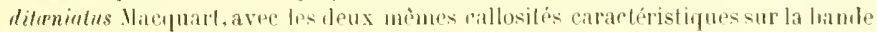
frontale. Il n'y a pas de triangles aris sur les bandes noires de l'alulomen des femelles. lattes jaunes phus sombres, frmurs noirs sur les deux liev de lour longuenr, le tiers apical seul en jaune. Jiles daires it court appendire.

Le male a les yrux jaunitres, lorums en descous, il facetles inégales, sans bande apparente. lables claviformes, jaunes, transparents. Lalulomen a lapex plus largement noir que les femelles of une bante tomenteuse grise est visilhe sur la hande médiane noire; paltes semblibles à celle des femelles, mais les fómur sont entiorement noirs, seml l'extreme apex est jaume.

La collection du British Musemm iontient en outre un specimen $q$ de Salis-

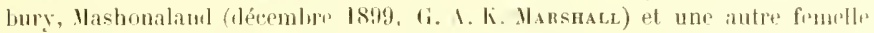
frovenant de Gadzima, Mashonaland (demembre lsili, G. 1. K. Marshalo.).

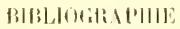

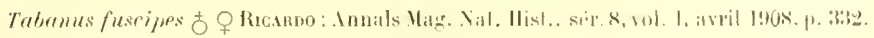

\section{Tabanus diurnus $O \mathrm{~W}_{\mathrm{Al} \text { KKER }}$}

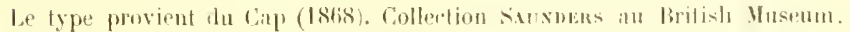

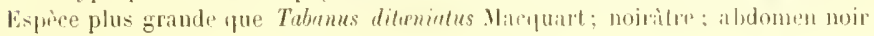


rerouvert l'une courte pubescence jaunàtre qui forme trois larges bandes. Yenx sans bandes. Ailes portant un long appendice à la troisième nervure longitudinale.

Longueur 1:i $\mathrm{mm}$.

Tète plus large que to thorax. Face grise à poils jaunitres entremilés de quet. ques poils noirs. Barbe jaune. Palpes jaune pale ì pubescence hlanchàtre et quelques poils noirs. Intennes d'un ferrugineux pile brillant; dent du troisiome article î courts poils noirs à sa hase; premier et second articles à pulsesrence noire et longs poils jaunes sur lin partie externe du premier article (d'après Walker). Bande trontale gris jaunatre environ cind fois anssi haute que large; au-dessus des antemnes sont drux courtes stries sur chaque còté; bande frontale ì courte pubscence mélangée, jamne st noire; première ratlosité noire, rorliforme, petite; la seromle plus petite et plus ovile, Yeux très légrèrement pubescents sins apparence de hamle.

Thorax noiritre it tomentexite grise, sims hamles visibles; flanes et pectus gris à poils jaunes : traces d'une courte pubesrence jaune sur le dessus du thorax. Abdomen noiritre, recourert d'une courte pubescenre jaunatre donnant l'aspect de trois larges bandes jaunes avee une bande noire de charge eité bordant la bande médiane jaune; ta couleur foudamentale noire apparaît plus largement à l'apex. Extrémité des bords latéraux de l'alıdomen fauve. Ventre noiritre avec une courte pubescence joum pale; segmentations paraissant fauves. l'attes jaunàtres: hanches à tomentosité grise; fúmurs noiràtres à tomentosité grise et blanchâtre, fare interne des fémurs antérienrs glabre et d'un hrun, plus on moins brillant. Apex des tibias antérieurs et tous les tarses hruns sauf le premier article des tarses postérieurs: puhescener des tibias noire. Ailes hyalines it nervures jaunâtres, appendice assez long.

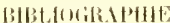

Tabonus diurnus Q WaLken: Diptera Simndersiaua, p. 43. 


\section{QUINZIEME GROUPE}

\section{T'ablead uchotoniQue}

Idomen: cinquirme, sixirme, septième segurnts à hord postérieur fauve rougeìtre bordé de poils jaune doré; trois derniers segments rentraux fauve vif (du Cap). Apex de l'aile clair portant deux petites taches . . . . . . . . . .

Abdomen: cinquième, sixiłne, septième segments à bord postérieur noir rougeittre bordé de poils noirs; trois derniers segments ventrax rougeitres, ciliés de noir (du Congo). Ipex de l'aile presque completement noir . . . . . . . T. irrorutus Q surcout.

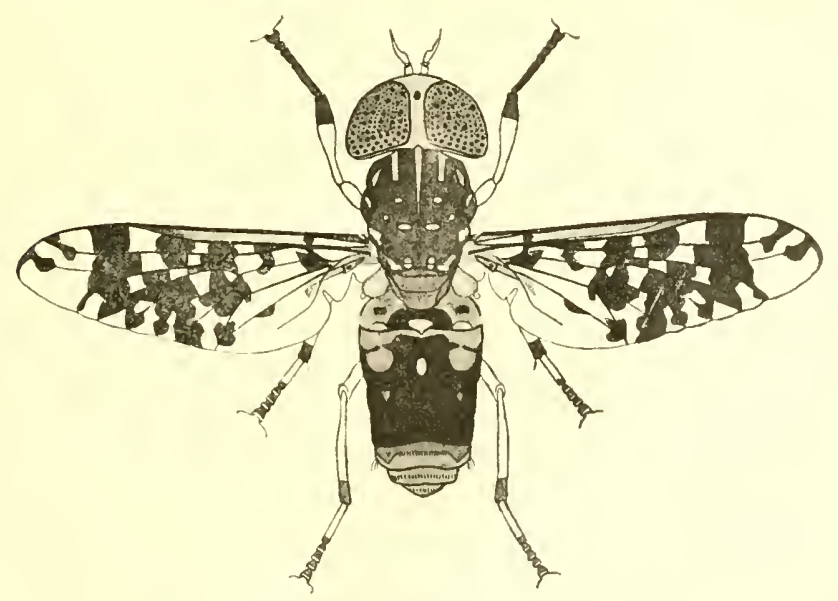

Fig. 29. - Tabanus maculatissimus o Macquart. 
Cie groupe composiatuellement de deux especes comprend les insectes réunissant tes caractères suivant :

reux glabres, tibias antérieurs non dibtés, bande frontale portant une seule catiosité, ailes tachetées.

\section{Tabanus maculatissimus o Mageart (1. 189, lig. 28)}

te type femelle a éti resueilli an cap de bonne-Espérance (Huseum de Paris). Lil deseription de Macouant est très exalcte, nous la donnons en entier:

"Yiger, antennis rutis, apice fusco, oculis maculatis. Thorare abounculato, " abdomine ablo maculato, apice rufo. Pedibus abbirantibus. Mlis nigricautibus " alho matukatis, batsi apice que nigro maculatis.

"Trompe brune. 'alpes, fare ef front l'un blanc jaunatre, ce lepnier ì petite "tache prestue ronde d'un noir luisant. Hoitio superieure du front d'un brun a uarron mat avee wne petite tache de duvet blane au milien. Antennes d'un " liuve clair: premier article blanchàtre, troisieme à dent très courte; les quatre " dernières divisions d un Irun noiritre. Yenx bruns mallqués d’un grand nom" bre de petites taches d'un brun plus foncé.

"Thorax noir, is petites tarbes de luvet blanc, quatre au hord antérieur, " Iuatre plariés en carré sur le disque, ifuatre au borl postérieuret une te cha"que côté en avant de la base des ailes; côtés fiuves à poils jaunâtres un peu "phs pàles. Ecusion marrou, huisant; une tache de duvet blane de chargue côté " du bord postérieur.

"Abdomen l'un noir mat (te foml est diun noir vert brillant quand if est

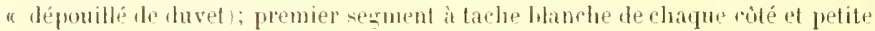
" tack dorsaled'un blan jaunitre: druxime hord antérieur blanc, interrompu " an milieu, une tarhe blanche de chaque coté n’atleignant ni le bori antérieur

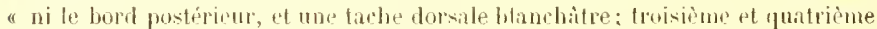

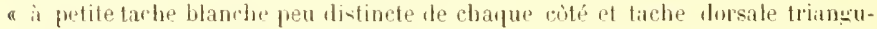

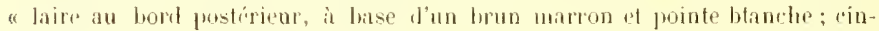
" quième. sixime et septìne d'un fauve vif; cinquime et sixiène ì tache " hamchitre le charjur coté. l'ieds d"un hlane juunatre; hanches intermédiares "ut postérirures noires; denximue anticle des an térieures noir; jambes à anneau " noir it l'extrémité; anterieures un peu éprisses; postérieures à petit annealu ì " lit base: tarses noirs: premier article des intermédiaires et postérieurs blinc, " it extrémité noire. Cuillerons obscur's. diles hyalines it la base, jusque vers le " mitien, larhetes de noir; ensuite une large lande transversate noire, it nom" hrenses petites laches hyations; entin l'extremite hyalins ì trois petites taches " noires fleuxime celtule sous-marginate it long appendice it la base.

"Du Ciap. Coltection de II. Serville ef de Vt. Ciucrin."

Le British Nuseum possè te une femelle de shire, Ifrique tentrate anglaise

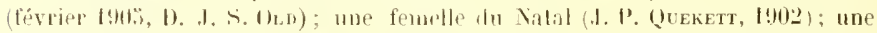

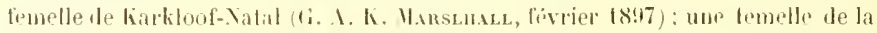
Cafrerie (F. 1'. Mavse Weale, IX78); deux femeltes de l'Afrique du sud (1) INDREW :MITH, 1849. 


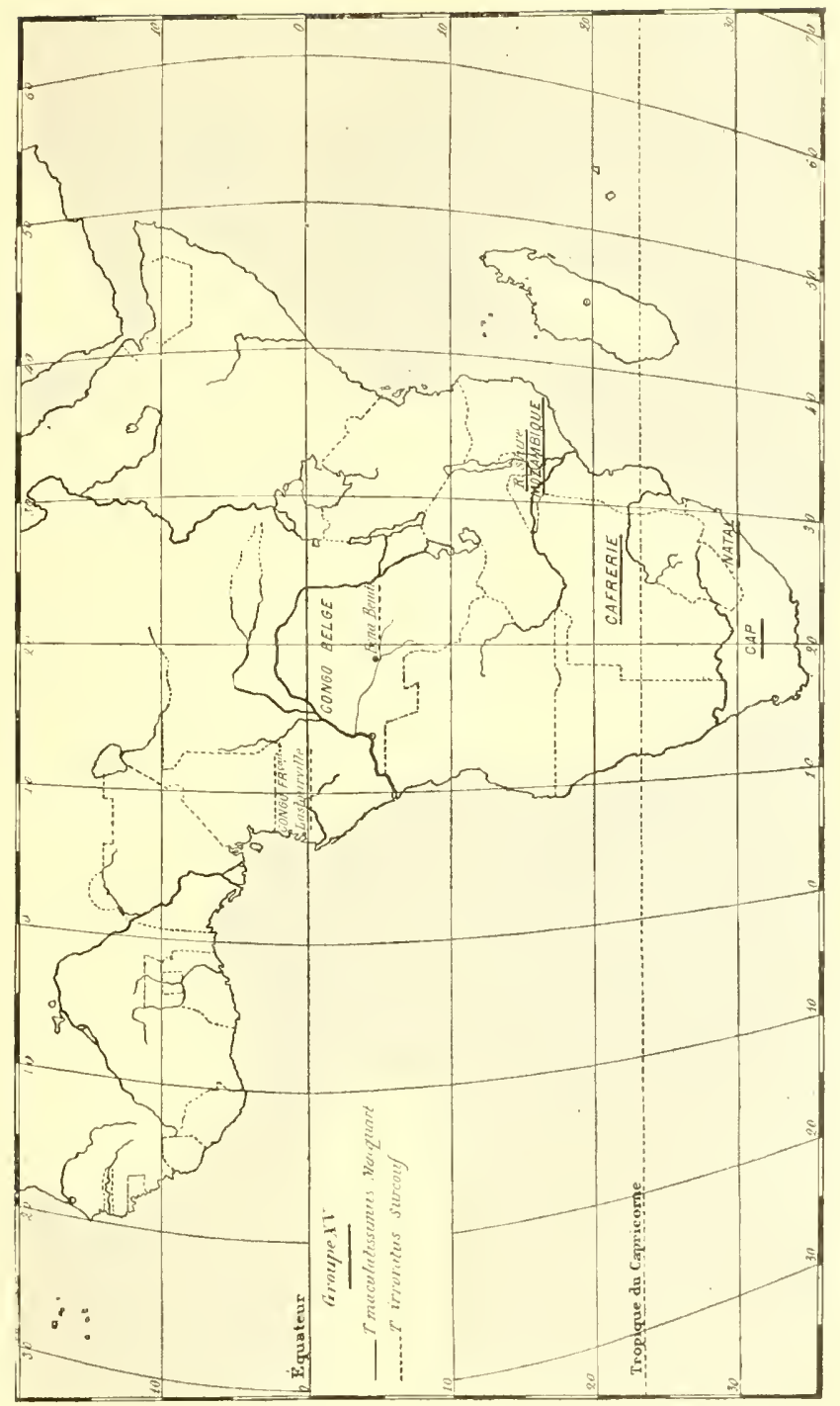




\section{BIBLIOGiRIPIIIE}

Tabanus marulatisimus

Macquart : Miptimes exoliques, 1, 1. 121, 3 (1836), lable W11, ti... 2.

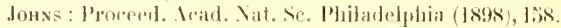

Law :

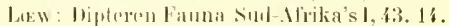

Walkele : List. dipt. Britisth Museum. I, 1titi.

Tabanus irroratus + Surcouf (pl. 111, fig. 20)

Colle espèce est très roisine de Tubunus maculatissimus O Macquart ; le trye provienl de Lastourville (Congo) et a dé recueilli prar. Machal en 1903.

lande frontale el triangle frontal marron en enlier, callosité frontale brunes, quadrangulaire. Yeux uoirs. I'reutier arlicte antennaire lestacé. Les trois derniers stoments alulominaux à borl postérieur noir rougeatre, horlé de poits noirs. Venlre à derniers segments rougeìtres, ciliés de noir. Niles à extrémité apicale noire.

Le reste comme chez Tahums maculntissimus o Wacquart.

Le Musée Royal d'llstuire naturelte de Belgique possède deux exemplaires de Talmas irroratus: its provipmenl de Beni-liendi. Sankurn, Congo (lieuteninl l:Loftens, 1 . (1:3). 


\section{SEIZILME GROUPE}

\section{Tamleav dichotomige}

liande frontile portant deux petites taches noires. Cafrerie, $13 \mathrm{~mm}$. . .

Bande fronlale large da callositús non distincteruent séparies . . . . . . Bandefrontile non alargie a callosités nettement distinctes. . . . . .

Biznde frontale large, callositp lrasilaire transverse prolongée vers le vertex par une tache ovale. Ibelomen srisitre it bande mésliane ditfus et taches latéales aromolies blanchitres. Funta. Djalon, y à 10 mm.

Ablomen à bandes transverses jaunes ou blanches. . . . . . . . .

Mblomen it hindes longitulinales.

Bandes abdominales transverses it pubescence blanche, pattes jannes en

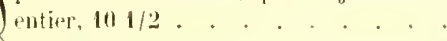
bantes alodominiles transverses it pubescence jiune, pattes non entièrerement jaunes, 10 ì $13 \mathrm{~mm}$.

Aldomen noir portant leux handes 4 fongitmlinaleshlauches atteignanllapex $11 \mathrm{~mm}$. . . . . . . . Aldomen gris jamitr portant deux comrtes handes brunes, 10 num. . .

Abdomen noiritre avec le dewieme segment largement rougeatre; thorax a bandes longitulinales grises. l'itlpes blane grisitre. Ju Cap, 13 1mm . . Thorax sans bandes, palpes noirs. Ilonts Ruwenzorj, 16 mm. Surcouf
T. fulriums o Loew.

\section{$\underline{2}$}

:

$T$. Chenalieri o surroul.

i

$T$. tomicormis o Mact].

T. capensis o Macy.

T. temiatus of Maciunirl.

T. laprifons o law.

T. verme q lism.

T. Runenzorii \& lice. 
Ce groupe comprent quelques espices de taille petite dont les yeux sont nettement pubescents. La plupart des insectes do ce groupe appartiennent à la légion sul africaine.

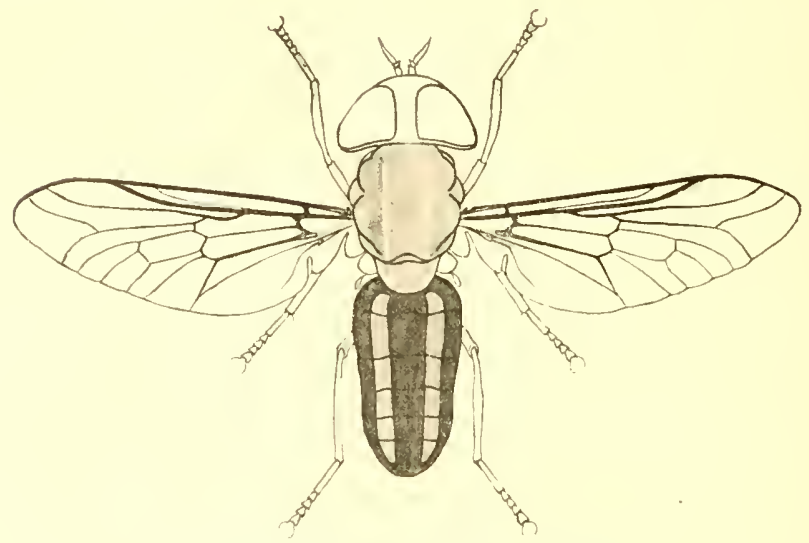

Fig. 2ti. - Tubernus truintus O Macerant

\section{Tabanus fulvianus $q$ Law}

Cipt insedo nous est inronnu, noms reproduisons la diagnose latine et nous donnons la traduction de la description.

Le type o provient de la Cafrerie.

"Cinerascens, dense orhrareo tomentosus, Talrano fulvo simillimus, sed " angustior, capit" thoracrepe magis depressis frons distincte latiore, abdomi" nis vittis lateralibus ochraceo-frellucilis in fuartum usque segmentum pro" lluctis."

Longueur corp. 6 lignes.

Yeux fortement puluescents. Troisime nervure longitudinate portant un appendice.

Il ressimble tant au Tabams fulous Meigen dins toutes ses parties quion pent

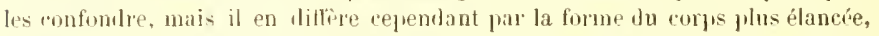
la baude frontale didemment plus large, portant ontre les yeux me toute petite callosité ot en son milieu un très petit joint noir. Ces deux callosités se rencontrent ausvi chez Tabanus fulms mais manyuent souvent. Les yeux présentent aussi aprés la mort une tine ligne transverse. Les landes latérales jaune d'orre et demi-transparentes de l’aldomen sont plus rlégagées sur le còté du troisième segment et sétemlent jusqu'au deli du quatrieme segment, pendant que chez Tubaums fulous 9 , ces landes se terminent toujours an hord postérieur du troisime segment. 


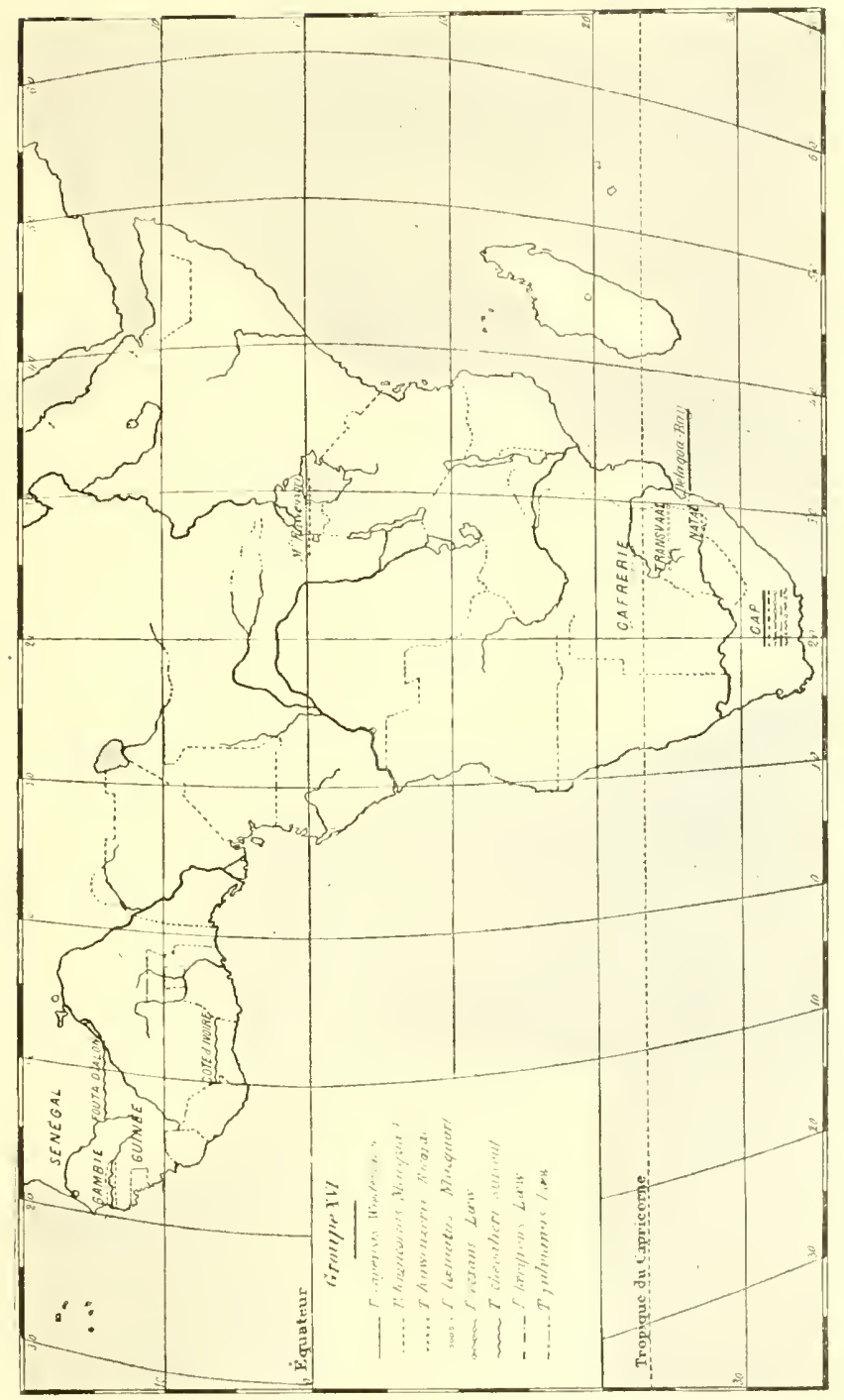




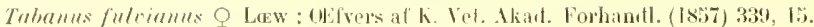

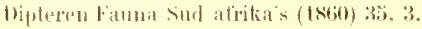

\section{Tabanus Chevalieri q icrcour (pl. III, lig. 18)}

Tyje : un exemplaire o recueilli llans le Fonta-bjalon par ll. Chevalien en 1904: une temelle envoyée de la hasse Cóle d’lvoire par le docteur Bovat (2n 1907 .

Collection du Muś́um de Paris.

Nous lédions celte espuce ì .I. Chevaluen, à qui nous sommes redevables de la dérouverte de plusirurs espèces intéressiates.

l.ongueur !) à 10 millimètres.

firis à teintes neulres.

Tite plus large que le thorax. Yeux puhescents, lirun verditre, sans bande ni traits. Liande frontale larese, recouverte d'une courte pulnescence rendrée. Callosité hasilaire large, brune, convexe au bord postérieur, bisinué au hord antérieur tit prolongée par une tache ovale rejoignant le vertex qui est dénudé. Antennes à premier anticle renllé, rerouver d'une pubescence cendrée très courtre de quelques puils noirs assez longs ; deuxième arlicle très court; Iroisime article noir dans la prartie apicale. l'alpes assez longs, cendrés avec quel jues juils noirs jaune-gris an cóté externe. Epistome, parties inférieure et postérieure de la lète de couleur cendrée, glahres.

Thorax brunatre ì pulfescence jaune sur le disque. Pectus rougeâtre. Sculellum brunàtre à pubrscence noire.

Ablumen court, bilargi, grisatre, portant une large bande médiane dilfuse plus fonce циi séplaircit an milieu du hord pusterieur de chapue segment et une Larhe triangulaire claire sur chanue cotr. Partie médiane munie d'une longue pubsernce noire. Fintio latérales a pubescence jaundtre. Yentre grisàtre à pubescence jaune.

l'attes rougutres ì cuisses roncolores recouvertes d'une pubescence jaunâtre peu dense. Tarses ohecurcis.

diles transparentes, grises. Nervures jaune-brun, troisième nervure longitudinale non a plpendiculée. Stigma hmu clair, étroit. Cuillerons brunåtres, balanriers rougeatlprs.

\section{BHBtUGRAPIIE}

Tabamus Chevalieri Q surcouf : Bullelin dn Muséum (1906), no 7. p. 52\%. 


\section{Tabanus tenuicornis ? Macouart $=$ Tabenus stigma O WALKER}

Le type de Macquart, dams la collection de $\mathbf{W}$. Verrill, provenail du Cap de Bonne-Espélance. Macquart en donne la description suivante:

" Yiger, antennis tenuilus, rutis apice nigro. Meulis hirsutis aldomine inei"suris albis. Pedibus rufis. Mlis relluli submatginali secundi inappendieulatit. "Longueur $10 \mathrm{~mm}$. \&.

"Palpes jaune piile, un peu plus longs, plus grriles et plus pointus qu'it l'or-

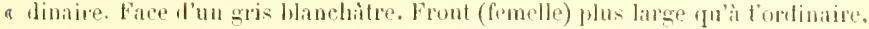
" noiritte, nu, luisint; une lacle jaune transersale, un peu sidilante it la bas"

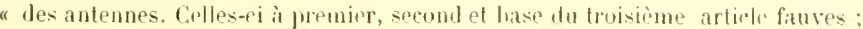

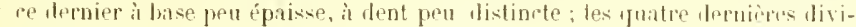
"sions noires. leux relus, bruns. Thorax d'un noir luisant a proils d'un gris "Wanchitre, rares. Cistés à poits gris. Ibdomen d'un noir luisint, boul posté"rieur des segments l'un blane grisitre assez large; une petite tache fauve de " chaque coté du secomol, au lorid postirient. l'ierls entidement fitures. dites " assez claires ; tache stigmatique brune, deuxicme cellule sous-marginale non "appendiculéen.

Du Cap.

Macquart commentant sa description remarque jur Tabams temuirornis $q$ Maequart ressemble au Tabums capensis o Wieslemann mais n'a point de poils jaunatres sur le thorax, ni ne fauves sur les incisions de l'abdomen th que les cuisses ne sont pas noires.

Posterieurement is Macecant, Walken dferivit le Tabumus stigma $q$ :

"Fuscus capite sultus atlo, alobonine fascis fulvis, sultus ba-i fulvo, anten" nis pedibus que fulvis, tarsis obsembribus, alis limpidis.

"Tête lorune, fauve en avant. lanche en lessous où elle est épaissement "recouverte de poils blanc jaunitre. Yeux bruns. Bourhe et antennes fauves, " trompe brunitre. Thorar et poitrine brun sombre revêtus lo puils loline

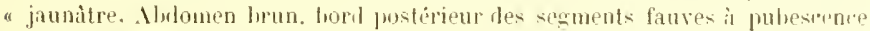
" jaune. Descous faure sur plus de la unoitié à partir de la base. damber faures. " tarses plus sombres. Ailes sans cuuleur. Xervures condeur de poix, fiuves vers " la base et le long du hord costal. Cuillewons lirres. lunns. Balineiers fitures. "Longueur du eorps 4 //2 limnes, les atiles, 8 lignes. In exempliare q du sul de

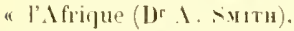

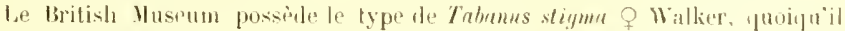
soit en mauvitis état, on peut cependant l'identifier ava certitude an Tabomas temicomis o Maejuirt.

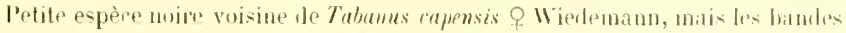

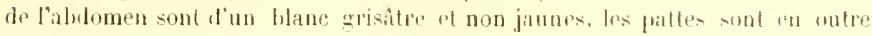
jaunes en entier. Longueur : 9 millimitres.

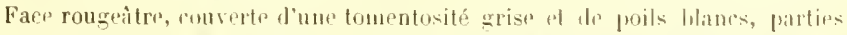

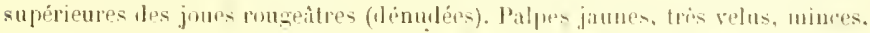

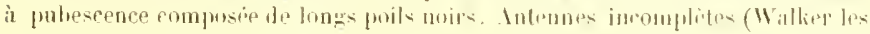




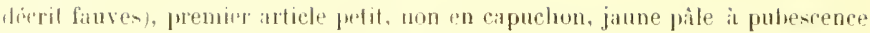
noire: deuxime artick semblable, mats plus petit. Triangle frontal d un jaunatre lrillant aver une tomentosité arise antour de la lase des antennis. Bandro fron-

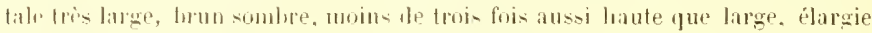
antirieurement, de sorte que lit rigion la phos etroite se rencontre au vertex; la callusití trontale est d'un brun rumgatre, latre. transverse, atteignant les yeux; au delà, lil tramle frontale est marunée do lignes métlanes et latérales álevies

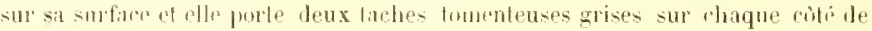

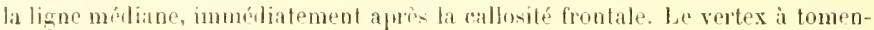
losite srise porte une petite protnhérance. Jeux velus.

Thorax ef stutellum noirs à pubescence l'un lilane jaunatre.

Alwomen d'un poir lrunatre à segmentations largement velues de gris; deuxième spgment un peu rouge sur les còtés, puhescence principalement grise sur lo dessus. Envers plus pate, jaune rougeatre, à segmentations banches. lfex noiràtre. Pattes jatunes, fémurs à pubescence hanche, partout ailleurs dhe est noire. diles hyalines. stigma of nervures hruns, pas dapyendice.

Mxcovart se contredit dans sil Jeseription au sujet de lanpendice des ailes.

Le type porte un appendice.

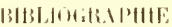

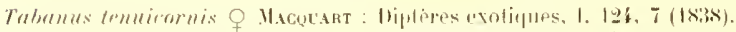

IIalker: lisil tiph. thit. Musenul. 1. 229, 326.

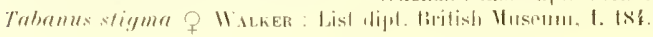

\section{Tabanus capensis o Wiedenaxs}

Ciette mproce lécrite par Wioldomn est repuésentée an Muséum de Paris par drux exemplatires detriminés par Macipart.

meseription de Wifdemaxx.

" Viger, tlavion hirtus, adolominix basi utrinyue ferruginose, incisuris

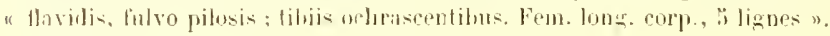

l)u liap.

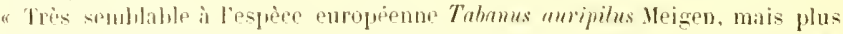

" pelit. Antendes noires, base du troisième alticle brun ocreux. Palpres jaunat-

"tres, harlw jaum dorép. Face srisitre. Yenx à pubesence grise avec

" Jeux bandes romrergant exteriemrment. Thorax noir lorillant aver une

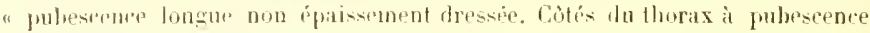

"jamnitrm pltas épaisise.

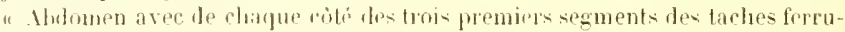

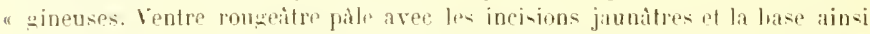

" pue to treis derniris segments noiritms. Ailes hyalines, nervures tranverses

" it burture jaunitres. Bonl custal brun noiritre. Balaneiers hruns avec un

" disque blanchatre. Cuinses noires, tilias brun orrenx . Apex des tarses noirs

"lase brun orreux".

Collertion WestenMax. 


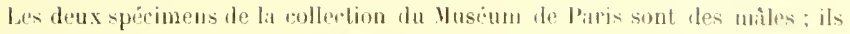

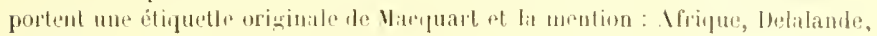
$n^{0}: 309$.

Nous en donnons la descriplion :

Longueur $10 \mathrm{~mm}$. :

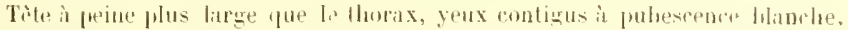

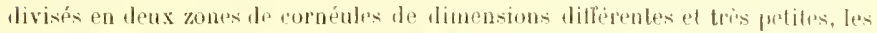

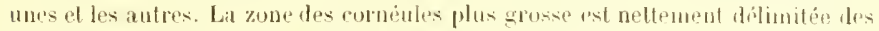

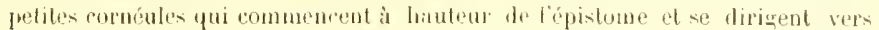

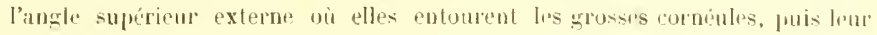
anneau se réduit et we constitue plus qu une ligne an horl postérieur des yeux, ceux-ci portent une bande transverse horizontale vers leur partic mirliane, borl postérieur de la lète hérissé de soies dressies jaunàtres, infléchies vers les

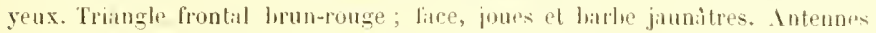

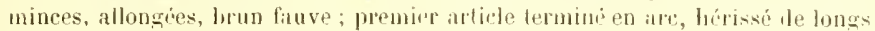
poils jaunitres ; deuxiène atrlicle molndre mais non très petit; troisiòme articlu

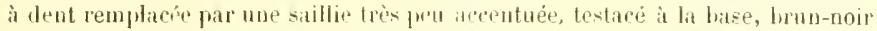
dans la région apicale. Prilpes blanthitres it dernier article vésinulenx terminé en pointe, hérisiés de longs prils jamms milis de quelques longs poils noirs. Trompe brunàtre.

Thorax (dénudé) frorlant une hongu pulnsence éprarse, jaune. Sirntellum noir (dénulé). Còtés du thorax el pectus recouverts d'une épaisse pubescrnce jaune doré.

Ahdomen noir sans tandes ni trits longitudinaux, lo loord postérieur de chacun des segments est jaune rougeitre et repoissement recouvert l'me pubes-

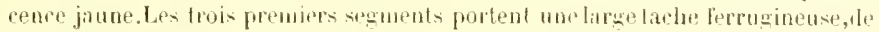

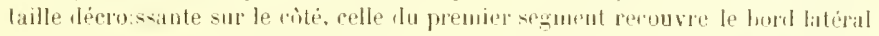

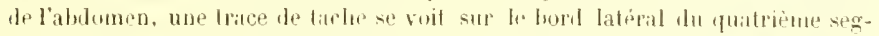
ment. Ventre rougeatre fible aver les incisions phus claires; la biate ot les deux Jeruiers segmentis sont légèrement insoubris. Le ventre prorte une dense pulsescencé jaune. Fémurs d’un hun rougentle frès sombre à puliescence jaune : tibias

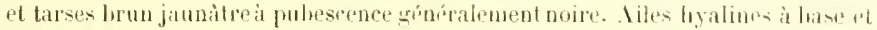

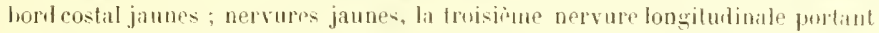
un appendire. Cuillerons jaunitres. Balinnciers it lige jaune et distue lirun.

Lit collestion du british Museum conlipat une femelle du cap. Collection SAUNDERS, ISTRS).

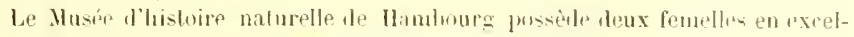
tent état, provenant de Deligoil-bey (Ir ll. linarss).

\section{Ballatorili MIItE}

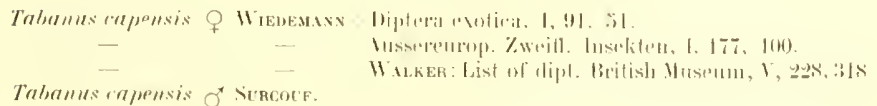


Tabanus tæniatus M MçUant (p. 194, fig. 26)

- Tabauss strigientris o LaE

Le type femelle a di recupilli au cilp de Bonne-Espérance (collection GuéraxVéverate el fait partie de lil collertion du Yuséum de Paris.

Longueur: 11 millimitres.

Noirattre. Aldomen portant deux bindes lilanches.

Téte plus large que le thorax; roux i pulvescence blanchitre, dense. Bande lrontale large, a lorids parallèles, deux fois of demie plus haute que large, de coulpur testacie, sans callositis apparentes, quelques poils noirs au vertex. Face et joues i épaisse pulieserner lihnclue, lailse lianche. Triangle frontal couvert l'une tomentosité hanchitre. Anteunes festáes, premier article non en capuchon, à poils noirs disposés en rouronne it l'apex; deuxime article moindre, it poils noirs à l'apex, hr troisiome article manque. Palpes blanes, renflés, à pulescence hanche; pières luccales testacées.

Thorax noiratre (dénudé) portant, d'iprè Jacquart, fleux lignes d'un blanc bleuitre, peu visibles sur le type usé; Hanes et pectus à tomentosité grise et puluscence blanche. scutellum noiritre portint des traces te tomentosité banche.

Aldomen non brillint portant deux bandes blanches, qui prennent naissince lle claque cobs du soluthom et se prolongent jusqu'au dernier segment inclus ou etles se rilpprochent saus se rúuir (contribement ì la deseription de Maciuart). Les hords latrinux du serond segmeut portent une tache brun-rougeatrede la bauteur du segment; angles postérieurs des denx dernier's segments jaunitres. Pubescence de la couleur du fond sur lequel elle se trouve. Ventre recouvert l'une tomentosité liane jiunâtre.

Pattes testacíes: tirses un peu plus sombres. P'ulesenenre des fémurs longue,

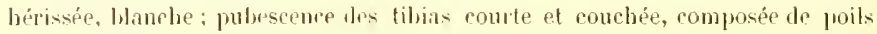
noirs et litanes, les premiers an majorití, larses a poits brundres. liles hyalines, nerrures ef stigma jaunithes Cuilkerons jaunatres. Balanciers à tige testacée et houton châtitin.

Le British Museum possèle un spérimen mảle le l'iet-Retief, Transvaal (1R. Cuawshay, ler décemlore 1!103); une fomelle de la mème provonance; une femelle du sud de l'Afripue (1)r simtu en 1844); une femelle provenant le simons Town (février 189t, P'. de la Garde).

Le llasée de: Hamlourg possède un expmplaire femelle provenant d'Algoa-13ay (1) linauss, 12 novemilre 18!)

J)ans certains des spécimens mentionnés ri-lessus, les fémurs sont noiritres it la base, comme le dicrit Lrew, non complètement jaunes comme lans le trpe do. Macquant. Le troisime article antenuaire est noir; dans le cotype de Nar'juart, yui se trouve à Lilte, il est rouge.

Le màte non décrit jusqu’i prísent, est simblable à la femelle, mais plus petit et plus pulpescent. Patpes jaunes à longue pubescence hlanche et quelques poils noirs sur les joues: le triangle frontat est boiràtre. La partie postérieure to la tite porte des poits blanes et une toutle sur to vertex. 
Ilorax noir, sans apparence de bandes, couvert de longs poils blanes, ainsi que le seutellum.

Aldomen petit, pointu, couvert de tongs poils blanes it travers lespuels les bamles blanches paraissent distinctement, mais elles sont plus atroites yor chez la femeltr. Vers lapex lia pulescence est boiritre. Envers un peu flus pâle it pubescence blanche et tomentosití grise.

Tous les fómurs noiritres; libias antéricurs jaunes, noirs it l'apex ; tarses noirs; tibias médians of postérieurs complitement jaunes ; tarkes linuat tres.

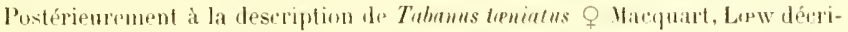
vit, en 18:.7. la mime espece sous le nom de Tubams strigirentris $Q$ : quoiqu la similitule des deux esperes ne puisse ètre mise en doute, nous donnons la

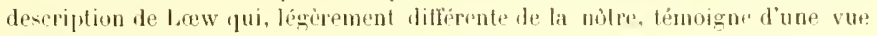
un pru dillérente.

leseription de Lew.

"Cinereo-nigricans; abdomen superius lrunneo-nigrum, vithis duabus lon"gitudinalibus aryualibus integris lhaviulo-rinereis, inferius flavo rulescens; alaz alimpidan.

longueur corp.: 6 1/6i lignes.

"Voisin de Tabanus viltulus Fabricius. Face el front ì fomentosité dense, claire, gris jambitre jusqu'it l'angle anterieur des yenx, la pulescenre, assez longue de la fare, est blanc jaunitre. lalpes blane jamnatre avee des poils blane jaunatre et quelquessuns noirs. Treisieme article antennaire noir, assez large. rouge à la base externe; l'angle de cet article pren aigu porte quelifurs poils tris rourts. les deux premiers artirles antennares sont bruns i tomentositi grisitre el puliescence noire; le premier non nettement en forme de capulion. Front large, portant it la partie inférieure une petite callosité brum, un peu brillante, qui n'occupe pas tonte la largeur ; un peu au-dessus de rette callosité, de chaque róti de l'angle des venx, existe une tache de tomento-ite gris faunatre, plus audessus le front est brun. La callositis porte un prolongement atroit el brun : trois petits points lorms au vertex indiquent les ocellss. Yeux a pulescence courte el dense I'un hruntitre clatir, satns landes transverses; apres ramollissement, trs yeux ont une couteur d'un lueu violet merveilleux ef d'un vert dori miroitant. L' borl poskérieur des yeux porte une tomentosité hlanche.

"Thorax noiritre; sur le dessus, la tomentusité forme qualre lignes longitulinales d'un janne gris cendré qui sont convergentes en avant, deux di deux; les latórales se biturquent au-tessus de l'origine des ailes. Peotus convert d'une tomentosite gris jaunitre. Pabescener du thom assez longur, jaum clair, moiritre sur la partie longitudinate sombre de la bande et en avant de lat racine des ailes. seuteflum noir, còtés ì tomentosité grisiltre el ì pubesfonce presugue partout jaune elair.

"Abdomen orné d'une bande médiane d'un brun noir mat, ì lork très droits qui se rétrérissent rers le dernier segment, cette baute est limiter de chatue cóté jar une hande droite d'un gris jaunatre clitir sur latyelle est une courte pubeseence jaunitre, puis au dwà existe une hatndr longitulinalo lomn-noir ì pulescence noire qui attrint presifue le hord latéral et qui it son hord externe ast moins limite ef moins aigniz. Les còtés eux-mèmes ont un aspect jaune dorpe 
et une pubescence jaunatre. Ventre de mème couleur, mais cette coloration sẹnhle provenir de la tomentosité el atre plus sombre, cela se voit principalement sur les derniers segments. P'nbescence jaunitre mélangée de poils noirs sur les derniers segments.

Cuisses noiràtres avee l'apex jaune-lrun, une lomentosite jaune grisitre el une pubesence jauntre qui devient noire pros de lapex. Tibias jaume-brun a puliescence composée de poils noirs el jitunatres; tarses bruns à poils noirs. balanciers it massue jannâtre. liles hyalines in nervures brunes, en asant de la premicre nervure longitudinate se voit un espace brun clair. Bord marginal átroit, couleur eafé. Yervure transverse apicale sans appendice l'remière th ıuatrième cellules marginales postérieures non rétrécies.

Cafrerie (Wahtberg).

\title{
BHBLWHRARIIE
}

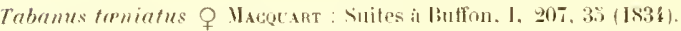

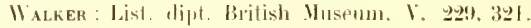 \\ 1. 184,3 .

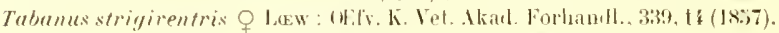 \\ Dipteren Fauna sul drika"s. I. 333
}

\section{Tabanus lævifrons 9 LEw}

Yous ne connaissons que la description re l'auteur que nous traduisons, le type provient de Cafrerie, il nous est resté inconnu.

"Thorax nigricans, polline llavo cinerascens; abdomenex cinero llavescens, " viltis dualuus brunneis postice value abbreviatis; antenne anguslip, tlave, "apice nigro: frons latissima, tota ulseure brunnea, levis, ala hyaline, stig" mate pallide brunneo. "

Longueur corp. $41 / 2$ lignes.

Face et partie antérieure du front ì pubescence el lomentosité jaune pàle. Palpes jaunes à pubescence jaune clair pritiellement noire. Antennes jaunes; second article non en forme de capuchon; troisième très étroit avec un angle très peu maryué, la deuxième segmentation de cet articleet les suivantes sont noir foncé. La puluesrence des deux jremiers articles est liane jaunitre et extrèmement rourte, plle contient quelques poils noirs épars. Front extrimement large partout, d'un brun foncé brillant sauf au milieu on se? roit une fine tomentosilé jaune blanchitre. Youx d’un beau vert sans traces de lisne transverse. Partie jostérieure de la tèle à tomentosité blanche sur un fond sombre, surtout visible au bord des yeux. La couleur foncière du thorax est noire; sur un spécimen frais elle est d'un gris mat, ce qui provient d'une lomenlosite jaunattre peu brillante: le Ihorax porte trois lignes lonsiludinales indistinctes grisatres. Sculellum gris comme le dessus du thorax.

Dessus de l'abdomen d'une couleur oris de lin, il porte deux larges bandes brunes qui se prolongenl jusqu'au hord postérieur du quatrième segment ou à 
la partie antírienre du einquième. Entre ces bandes il y a une bande de la mèmo largeur, plus chaire qui, vue obliquement, prent une coloratton lilane jaumâtre. Pattes jaune hrunatre, farsis brun sombre vers l'extrimite. P'ulwsence des cuisses pustrienres jaune blanchatre, cellos des cuisses antéricures pat principalement noire. Tarses et tibias antérieurs ì pulescenre moire; tibiats intermédiaires pontélieurs à puleseence généralement éclaireie vers la hase. Ientro jaune sate i pubescence hlane jannàtre. Ailes hyalines aver un teinte grise peu évilente, hord antérieur hrun jaune ansi ugue la phus garande parlie des l misième et einquième nervure. Les autres nervures sont hrun somllae.

lhu Cap. (rollection lictoris).

\section{BtBLtogti WHAE}

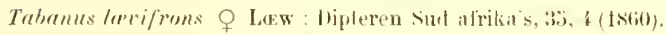

iff k. Vet. Mkial. Forh., 3ati. 2 bis.

\section{Tabanus vexans $O$ LEW}

Le type femelle de cette espèce provient du Cap; comme elle nous est inconnure, nous donnons la diagnose latine et la traduction de la deseription de Lew.

"Nigricans, thoracis lineis einerasentibus modice distinctis; ablominis seg" unentis secundo ad latera late rufo ef tertio utrinque maculi alhidopollinosit "signatis ; yuatuor ultimis hrunneo-nigris, margine apicali flavescente; pedum " nigricantium tibiis posteriorilus totis et anticarum dimidin basali hrunneo " testaceis : atarum distincte cinerearum nervis et stigmate obscure brunneis ". Long. corp. 6 lin.

Palpes hlane sale it pulesience noire. La couleur du fond de la fice est presque miratre, elle est a'un rougeitar pale de chayue cóte de l'onverture de la bonche et en dessous des antennes, sampoudrée partout de hlane: la pulescence de la face est blanchatre, flle est noinàtre sur la partic supérieure. Intennes noires; deuxime article cyathiforme: troisième it angle supirieur trìs peu saillant; la pubescenes courte des deux premiers artirles antennaires est parfaitement noire. t'artie antóricuredu front, dejuis les autenues justu'aux angles anterieurs des yeux, d'une roukur rouge loripue aver un tomentosité blanche. front relativement étroil, portant une callosite quidrangulatre d'un lorun-chitain sombre yui va d'un angle des yeux ì l'autre sous forme de saillie, une ligne lrun-noir, "n furme de callosité, surmonte la callosité alle-mrime ef est enveloppise at milieu du front par une tache noir mat. Sur le vertex il y a aussi une petite callositis loruo-chitain, mais complètement grlabre; le lexte du front porth une tomentositi jame blanchatre. Les youx après lo ramollinement prennent. une couleur lrum-noir sombre of ne montrent aurne lande. l'artie postérienre de la tite noire avec une tomprotosite hlanche; une tache rougeatre sur le vertex.

Thorax noir, les environs de la racine de liaile sont rolores fu brun-sunge ainsi que les flanes, ils sout revitus d'ume tomentositio gris blanchatte plus grise? 
sur le côté supérieur qui présente trois lignes blanchâtres, longiludinates, peu évidentes. La pubescence llane jaunatre du thorax se mólange de poils noirs. srutellum comme le thorax.

Sur l'arlomen prélomine la coloration brun noir, mais le deuxième segment esi, de chaque còté, coloré sur un grand espace en rouge brique sale, sur cette coloration se montre une tache ronde de tomentosité blanchâtre peu nettement dómitie. une tache semblable se retrouve au mème endroit sur fo troisième segment. Le bord postérieur de chaque segment, ainsi que l’angle postérieur et l'angle latéral sont jaune-ronge; une tache dorsale triangulaire brunitre est évidente sur le troisième segment, mais elli n’est pas nettement limitée déjà sur le segment précédent romme sur le suivant, il n'y a que de failles traces de cette lache. La puhesence de liahdomen est hlane jaunatre sur les parties claires pt noire sur le reste. Ventre gris-brun sur les hordures postérieures claires ì tine tomentosité gris llanchâtre. Il n'y a quiune trace non claire d'une lande méliane large et ascombrie.

Pattes noires: l'apex externe des ruisses, la moitié basilaire des tibias antérieurs et les autres tibias sunt lirun-jaune, saul' i l'apex externe. Côté interne des cuisses postérieures et médianes blanc jaunâtre, il s'y mèlt quelıues poils noirs; cuisses anterienres noires au còté interne. Pulescence des larses et des tilias noire, mais vers la lase des tibias se mélangent des poils qui s'éclaircissent vers l'apex. Ailes nettement assombries de gris; hord externe hun-noir, sans que les nervures transverses soient comprises dans une zone plus somlre, aucune des cellules marginales postírieures n'est rétrécie.

lou Cap (collection Victorin).

Bit3itugti.tPHE

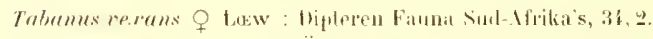

iili. K. Vit. Mkat. Forluanll. 336, 1 bis.

Tabanus Ruwenzorii $q$ limcardo

Le type femelle provient des monts Ruwenzori, vers 2000 metres daltitude, repneilli par llon. fr. Legce et I F. R. Wooldastox en 1906, wne autre femblle a été prise par les mémes rapleurs à 400 mètres d'altitude le 7 férrier 1906. Collection du british Museum. Roluste extree poilue ì yeux velus. Indomen noir, cotris du seeond segment rougeatres; thorax d'un noir hrillant. Intennes, face et palpes noirs. Pattes noires, tilias jaunes. Ailes tris légèrement teintées do hrun jaunàtre. Longueur: 16 millimètres.

lace noipe à tomentosité brun sombre et pubescence lirun jaunatre, quelques poils noirs sur la parlie supérienre drs joues el sous les antennes. Barbe lirun jaunatre. Palpes d'un noir lisunitre sombre à pubescence noire, épais et teminéx en une courte pointe. Intennes noires; troisième article long et mince à dent à peine indiquée: les deux premiers articles it longue puhescence noire. Triangle frontal de mème couleur que la face. Bande frontale environ trois fois 
anssi haule que large, à bords parallèles, d'un brun noiratre sombre à tomentosité brun jaunatre et Jongue pulsescencr noire. Callosité frontale petite, brun rougeâtre, ovale; la ligne rui la prolonge est indistincte. Vertex noir portant. une protubérance.

Thorax d'un noir lurilant, sans bandes, dessus presque glabre, taches humorales rougeitres avec ijuelques poils gris jannitres qui se continuent autour de la base du thorax. Cốtés à pubescence noire; poitrine noire it pubescence d'un jaune grisitre et Ifuelques poils noirs. Scutellum d'un noir Inisant.

Alıdomen noir un peu brillant; premier segment étroitement rougeâtre sur les còtés, second largement ronge sur lus flancs; les segments suivants ont les incisions à pubescence grise très étroitement limitée; pattes noires, tibias jaurîtres à apex noir, fémurs à puhescenee urisatre; tilias à pubescence blanche, elle est noire sur l'apex et les tarses. Iifes byalines, la coloration brun-jaune est extrèmement faible, un pen plus apparente autour des nervures longitudinales du bord antérieur de l'aile. Nervures et stimma lornos.

\section{BHBLIOGIIAT'III}

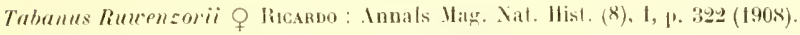

\section{Tabanus imbecillis O Kanscr $^{2}$}

Deseription de Karsch.

Brum aris it pubescence courte gris-jaune. Alubomen hrun-rouge ared le bord postérieur des segments à pubesenée jaume. Antrones brun-rouge, troisieme article aree l'apex plus noir. Jamhes noiratres, tibias lirun rouge Yenx glabres. Brancle antérieure de hi troisime nerve longitulinale avec un court appendice formant crochet. Tibias anterieurs non épaissis. Toutes les cellules marginales postérieures largement ouvertes. Callosité frontale arrondie, se continuant en dessus eu forme de ligne brun-rouge. Ailes hyalines. Cellule anale st frmant cout au hord de l'aile.

Longueur de eorps $10 \mathrm{~mm}$.

Usambara (Afrique orientale allemande) deux femelles.

\section{BIBLIOGRIN'IIE}

Tubanus imbecillus o Kansch: Berlin. Lntom. Zeitsch., XXXI, 370, 7. 
Aux espèces lécrites dans les piges qui précèdent, on doit ajouter quelques autres, qui nous élaient restées inconnues.

Tubunus infars Walker, Dipt. Saundersiana, 1, 45. Le lype n'existe plus, il parait appartenir an groupe de $T$. ditcuiulus et provient du hap.

Tubremus seguens Walker, de l'ile de liodriguez, type perdu.

Tabunus brumeus T'нuxвеrs, Cap de Bonne-Espérance. N. Acta. reg. Soc. upsaliensis. IX, 53-62, 1827.

Tuhams sudanirus Cazalbou (1904), tu Soudan, inséré par le professeur Bezzr dans ses Diptères de l'Erythrèe, p. 220 (1900), jamais decrit, peut ère le Tabamus croceus surcour.

Tabams macrodonta Vacevalit, du Cap de Bonne-Espérance, ne provient pas de cette région s'il n'est un Rlinomyza.

Tubamus fuscinerris Macevart = Tabumus diluniatus Macovart.

Thbunus abicans Macquant, de lidrabie et dusénégal, doit provenir d'Arabie, le type dans la collection du Huseum le l'aris le montre proche de Tubrums agricola Wiederans, ce ne doit pas ètre une espèce africaine tropicale.

Tabanus fallax Iacouart, de Cafrerie, est un siltius. 


\section{APPENDICE}

Depuis le commencement de l'impression de retouvrage, nous avonsen l'occasion de recevoir de nombreux Tabiniles d'Afrique, plusieurs espèces ont atis décrites far N. E. E. Iustex, dont denx dans le douzième groupe; en comparant ses descriptions aux nôtres et ì nos insectes, nuus atrons trouré une espèce trìs voisine de Tabanus wellmanni Iustex ot en outre une forme d'Afrique occidentale, Tabanus varions, confondue avec le Tabanus variabilis loew du sud de l'. Ifrique. Pour mettre au point notre ouvragr, nous avons refait completement le douzième groupe. De plus. les nombreux matériaux qui nous ont été communiqués comprenaient de nombreux mâles.

Nous avons ainsi été amenés à faire cet appendice qui comprend deux parties distinctes.

La première partie donne la description de deux Tabanides nouveaux : Tabanus Denshami 오 E. E. Austen ot Tabanus Cortieri $q$ Suncouf.

En outre, cette premiere partie donne des inclications sur les espèces que nous n'avons pu classer'; elles sont réunies sous la mention: "Specirs incertá sedis ".

La seconde partie comprend la description de tous les taons màles ronnus actuellement dans la faun tropicale africaine et quelques indications de classification. 


\title{
PREMIERE, PARTIE
}

\author{
ONZIEUE GROIPE
}

\section{Tabanus Denshamii Austex}

Longueur (4 spécimens) 17 is $21 \mathrm{~mm}$. : tête de 6 is $7 \mathrm{~mm}$. de largeur; front au vertex $0 \mathrm{~mm}$. fi, et 1 c. atu-dessous; aile 14 a $17 \mathrm{~mm}$. Collection du Brilish Museum.

Brun brillant, les bandes longitulinales du dessus du thorax grises ou gris enfumé ainsi qu'une serie midiane do tris larges triangles sur les segments abdominaux; ailes à leur extrème has", bordure costale jusqu à la tin de la première nervure longitudinale et base de la rellule anale d'un brun sombre; nervures dans les deux tiers froximaux de l'aile distinctement estompées de brun.

tète: front, face et joues recouverts d'une pullinosité grise; face et joues recouvertes d'une pilosité blanchatre; còtés de la bande frontale légèrement ronvergents vers le bits; callosité frontale al son prolongement linéaire (quand il est visible) châtains, la callosité pst de fornı rectimgruliare arrondie en dessus, de la largeur de bande frontale ou tangente à peine aux yeux; région ocellaire légèrement brunatre. Yeux glabres; palpes aplatis vers l'extrémité, revêtus de poils d'un jatunitre pale, parfois melanges de nombreux poils noirs an lood externe.

Premier arlicle antennaire l'un gris pullineux roussâtre, avec l'angle supérieur d'un brun noiratre; deuxième article lorun sombre; troisième partie noire ou lorun nuiratre.

Thorax : pectus. tlanes gris; une large bande grise mal délimitée au-dessus de la base des ailes, ainsi que trois bandes dorsales, métlianes, nettement marquées. la médiane est très étroite el s'itend à peine jusqu’au milieu; un trait noir ì la base de chaque aile; callus post-alaire portant une tache bien visible de poils blanchatres. Les bandes dorsales grises sont revètues de poils jaunatres. la rérion unédiane porte un peu partont des poils brun sombre ou noiritres; le pectus el les flancs ont des poils blanchitres.

scutellum ì pollinosité grise, li couleur foncicres ferrugineuse, brunitre à la base, il poite sur le disque et à la partir inlérieure des poils brun noiratre; le tour du scutellum est frangé de poils blanchâtres.

Abdomen à série dor sale médiane de très larges triangles tronqués, d'un gris enfumé, très visibles tl nettement délimités, formant une série continue soétendant du deuxième au cinquieme segment inclus. 


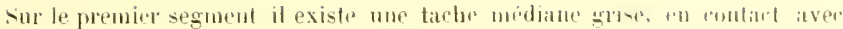

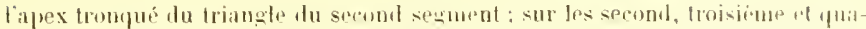

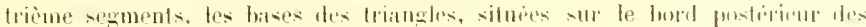

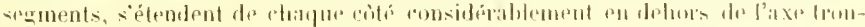

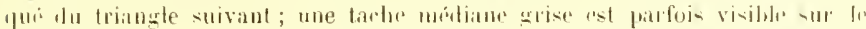

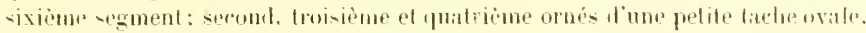

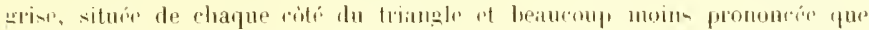

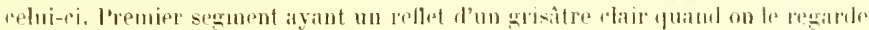

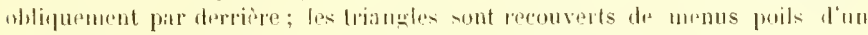

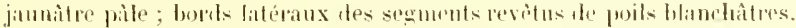

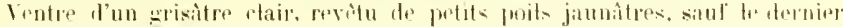

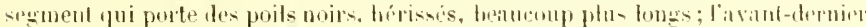

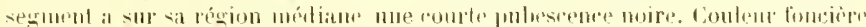

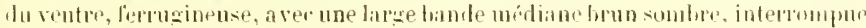

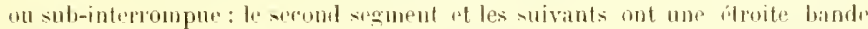

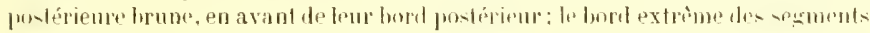
wi jaunaitre.

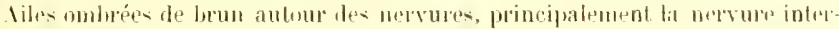
rataire antírieure et la transverse postéricure (qui entourent ensemble fextré-

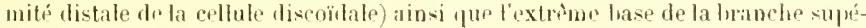
rieure de ha troisime nervure longitudinate. Iluta el hase de fingle anat brums. pratie centrale do's alukp plus pâtes; squmm (écailtes) d'un brun noiràtre. Batanrins bruns à extrémités habituellement jauntres.

Pabtes d'un ferrugineux assonbri, fómurs antirieurs, extrémitís des tifials

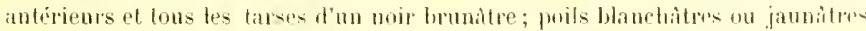
pour lit phupart, une frange vixihl an bord externe des tibias posterieurs.

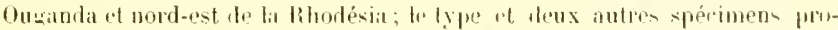

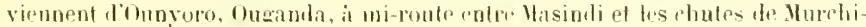

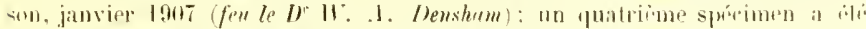

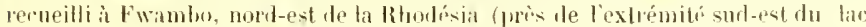
Tanganyika), entre ortobre 1892 ot février $18 ! 3$ ( $1 \mathrm{H}$. H. Nutt).

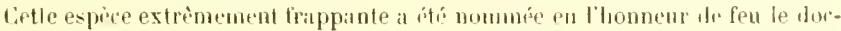

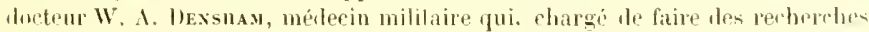
sur lextension de la maladie du somm il dans fouganda. a jerdu la vir it la tin

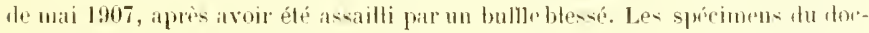
teur Dexshas, que le Museum de lontres venat de recevoir, deux mois avint

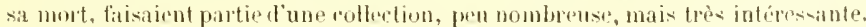
de biptires suceurs de sang, de l'ouganda, tris soigneusenent piquée, en excetlent état et aceompangée de notes. I'ne autro eolteetion avail déjálé énvoyée par le docteur Denshas et it n'y a aucun doute que, si n'était survenue eette mort prématurée, nos conuaissances des mourhes suceuses de sang d'une des plus

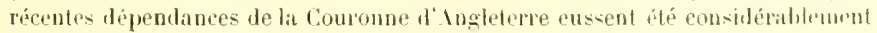
angmentées par les etforts dece chercheur intrépile.

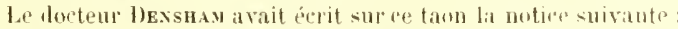

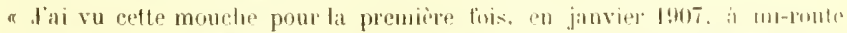

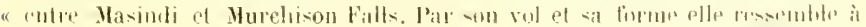

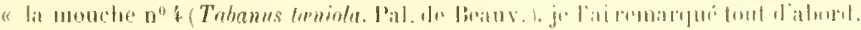




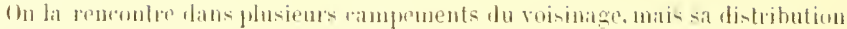

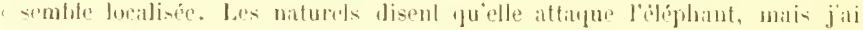

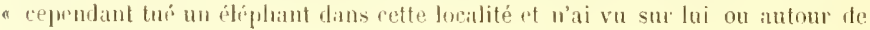
"lui anrune mon'lue".

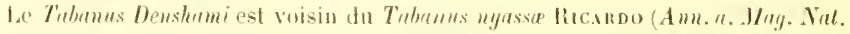

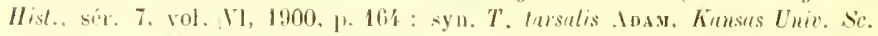
Bull. vol. Ill (XII) 190:, f. I:il) Iont le tye provient de l'Afrique eentrale anglaise. l.e T. nyossa, rependint s'en distingue par ses ailes plus hyalines, fos nervures noins fortement oubrées, la fordure contide de l'extrémité de lat premirị mervure, jaunâtre an lieu de brun foncé, l'alssenfe d'obsourcissement ì Ja hase de la branche suprírieure de la troinime nervure. las taches aludominates latériles baucoup flus grandes. les triangles des segments abdominaux plus étroits, et les tibias ct les fémurs plus pilles.

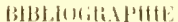

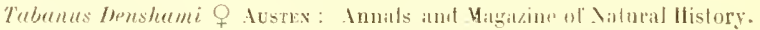
Sér. s, rol. 1. Mills 190s, p. 222.

\section{Tabanus Cordieri S Sucour.}

\section{Longueur 17 millimitres 12.}

lirum, abdomen portint une lignr longitudinale midiane de triangles blanes ef des points latérax très petits. Thorax portant plusieurs handes longitudinales blanches. Ailes brumes le long du lord costal. leutre sans bande longitudiniale.

Coftection dn Iluséun de l'aris.

'Tite: tring ghe frontal, face et jours rerouverts d'une proflinosité cendrée, face et jour revètues d'une pilusité blanche : cités de la banle frontale légèrement ronvergents vers te has. Bande frontale d'un liane jaunitre avec quelupes courls prils jaunes, fortant une callosité rectangulaire chatain, tangente aux yeux et surmonté d'un jrolongenent linéaire, clutain, peu dilati; re prolongement est sripar de la rallısité frontale par une partie un peu plus élarrice fjue la ligne siallante mair revòtue de la pullino-itó grise de fa hande frontale ; région ocellitire tris peu visible, chatain.

Yeux glibres à rormules égales. l'aljes un peu renflés à la courbure, allongés, pointus, blanes, revouverts d'une pubsecence courte et rare, noire. Antennes: premine article jaune rougeitre in poils rourts, jaune rougeitre, apex supurieur le eet article couvert d'ume pitnsite dinse et noire; denxieme articte de mìm. coulen que le premier, cilir for courts poils noirs; troisiene article brunitre freouvert d'une trís tine pollinosité urise, qui disparail aux dernirres segmentations ajucilos.

Thoritx lrun ì puhesence roncolore portant une ligne médiane très fine silJomne dans sa longueur par un mincr trait brum qui sélatrgit en une tache brune au-dessus du scutellum, outre cette ligne médiane grise, il y a deux bandes late- 


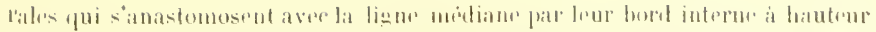

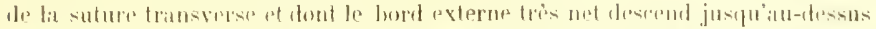

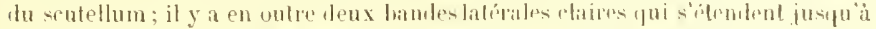

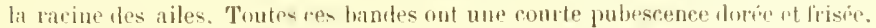

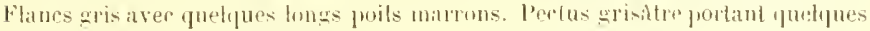
poils blanes el blane grisillw.

scutellum marron chas portant une puhescene jatune hiandultre. Callus alairésurmontés d'un pinceatu dre proils hanes.

Whomen portant une série dorsate médiam do très larges triangles un peu

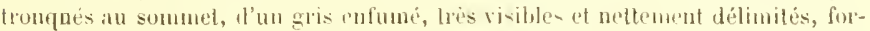
mant une série continue s'ètendant tu somel an rinpuirme sergment inclus. Sur

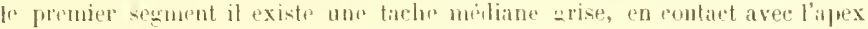
tronque du secoml serment : sur les sismol, troisieme et quatriome sumenls les

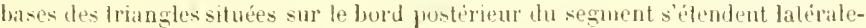
uncht tris en dehors de lapex tronque du lriangle suivint; une tache médiane grise, uhtongue se voil sur le sixieme segmenl. la pubescence de l'aldomen est concolure avec le fond et les dessins.

Ventre rougeàtre chat couvert d'une hes time pollinosile un jreu plus claire, l'avant-dernier el le ternier negment furlent une pilosilé noire.

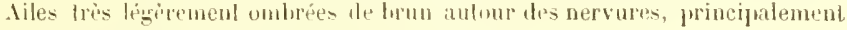
antour de la cellute diseoüdale et de la cellule anite.

Iluk d'un lrun noiratre, balanciers a tige lrume et massue claire.

l'altes l'un ferrugineux chir, fémurs anterieurs, exlrinité des tibias antérieurs et tarses ferrugineux; poils blanchitres on jaunitres pour la pluyart ; la trange externe tes tibias postririnurs est mélangée de poils noirs vers l'apex.

Le Tahmus Cordieri o Surcouf se distingue tu Tabanus Denshami o Austen par ses palyes blancs, le ventre sans bande, les jambes julus elatres.

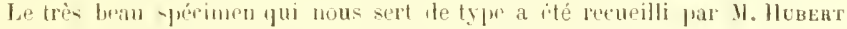

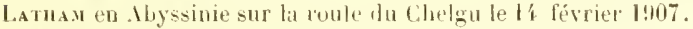

Nons dediuns cette esprece a M. L. Corbarer yui, depuis de longs mois, nous it aidé dian nus recherches et dont les uhervations prersonmelles et l'amitió nous ont toujours ite thun grint recours. 


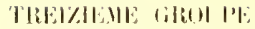

Tabanus Mesnili o rucuel (fl. III, life 1:9).

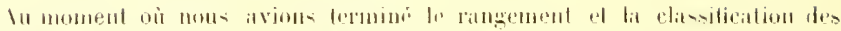

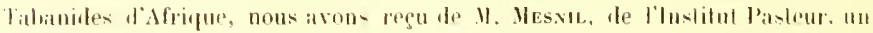

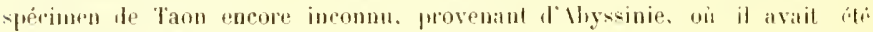
rerueilli par le lor loneav.

liefle expece alprartient dans notre dissilicalion all groupe qui comprend entre autres Tabanus lencostomns Low, Tatumus qullidificies o sureonf at Tobunus gralus $q$ loew.

longneur : 12 millim. "5.

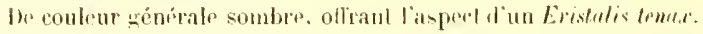

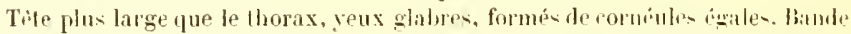

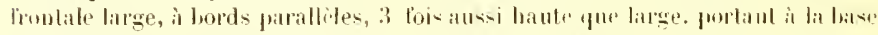
ture eallositi tran-verse, brune, surmonter dou espare couvert de poils jaunes ¿qui te separe d'une scconde eallositi módiane, transwrse, d'un noir brillant.

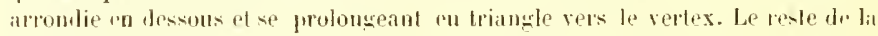

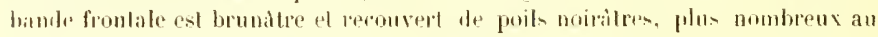
vertex. Le triangle frontil forme un sillie evileole, visilate de profil, dun

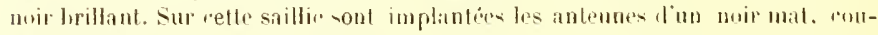

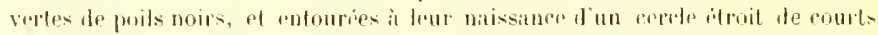

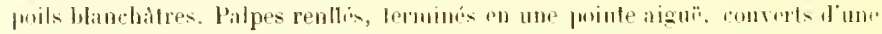
fipaisse pribsité blanche qui se mélange de quelque poils noiro sur te cribrexterne

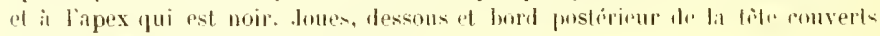
liune éprisse pilositiblane jiunitre.

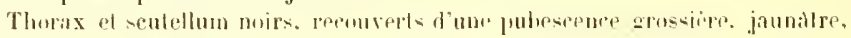
lanoce pectus simblithtex.

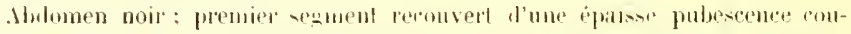

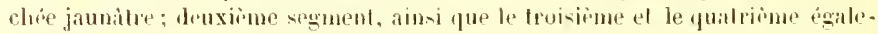

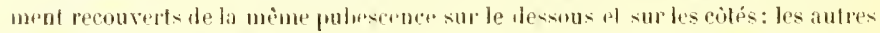
sements ont une longue pulesence l'un moir mal. mélange de puelques rilles

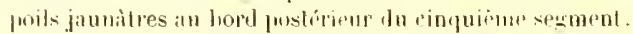

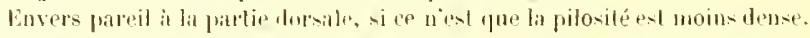

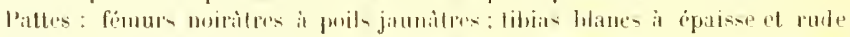

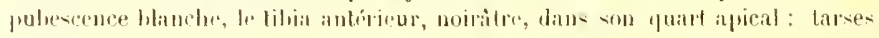
birulls.

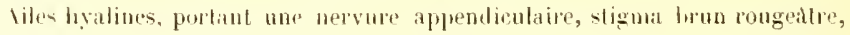

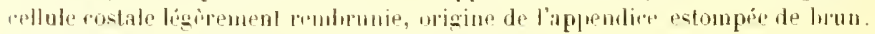

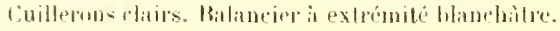

\section{HabLumilidelle:}

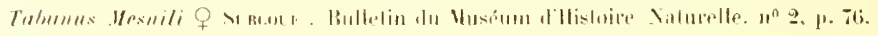
1!m: 


\section{SPECIES INCERT $Æ$ SEDIS}

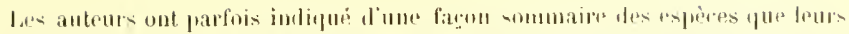

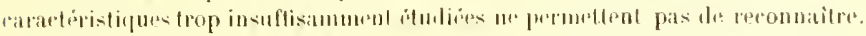

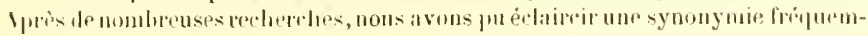

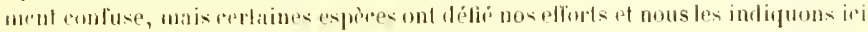

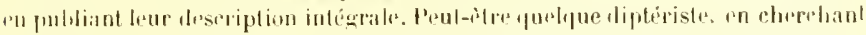

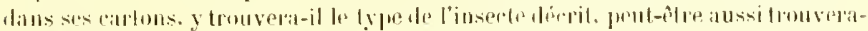

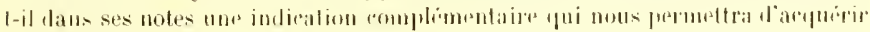
uncertitude.

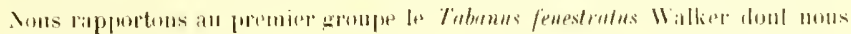
domnons lit deseription.

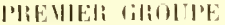

\section{Tabanus fenestratus $\mathrm{WALkF}$}

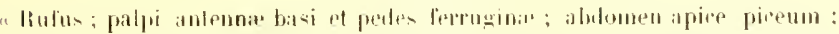

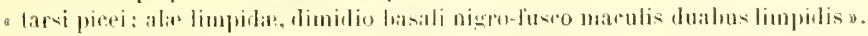

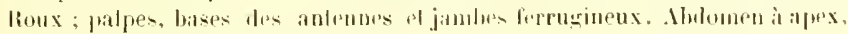

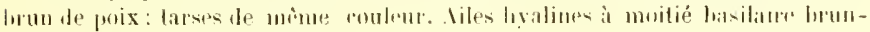
noir portant deux taches daires.

l'atrir inconnur.

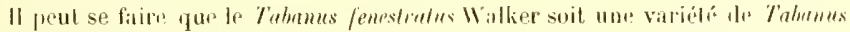

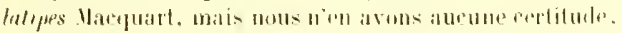

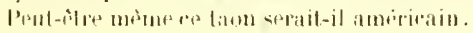

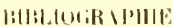

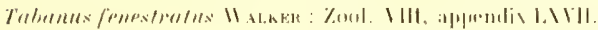

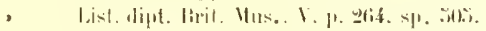

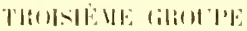

\section{Tabanus corax L,10.W}

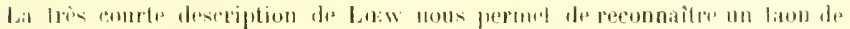

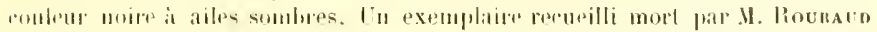

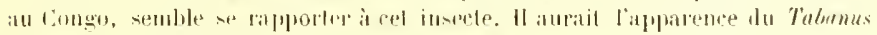

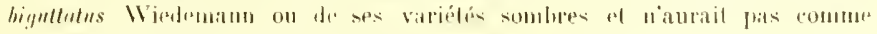

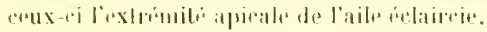

\section{Iillilolili Illll:}

\%. rormes o law:

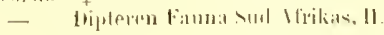




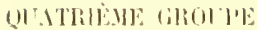

\section{Tabanus Deyrollei ligot}

Ce taon de tress grande taille appartient sans aucun doute au grougu te Tabamus rufrus $Q$ Walker mais les caractères non derrits de la bande frontale ne permettent pas d'intiquer ì quelle espèce ronnue se rapporte le Tabanus De?rollei Bigot.

liécenment. It, Karl Grüneng, dans son unvage: Die Firlsangomlem Dipleren, a décrit ce taon parmi ceux qui hathitent l'ouest africain.

La deseription de M. Karl Gicïnlserg se rapuorte au Tabmus ruficrus Walker avec insez d'exartitule, mais witre le Fulumus Heypollei?

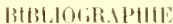

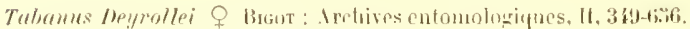

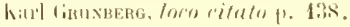

$\therefore$

Les deux espices suivantes Tubrenus hehes o Willier et Tahamus zouloupnsis kigot nous sont inconnues. Ceprendant te Tabamus hebes Watker de grande taille devrail ftre connu, mais est-il africain?

\section{Tabanus hebes? WALKEK}

"Cinereus, thorare, puadrivitlato, abdomine, ferrugineo fulvo cincto macu

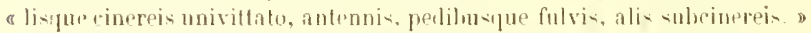

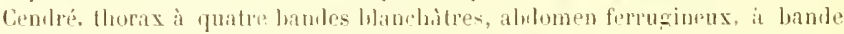

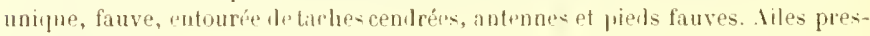
y jur rendries.

Tite brune en dessus, converte d'une pubescence rare, jaunitre ef légèrement revètur de soirs blanches en dessous. Thuche noire; palpes couleur tan ; intennes de méme, ylus ou moins noiles ver l'apex.

Thorax eriv avec quatre landes blanchatres. Cólés et pectus jaunitres, le peetus avec une courte pitwití blanche. Ilwomen ferrugineux, rharpue segment aver une large bande ferrugineuse le long de la bordure pustriemre qui ent

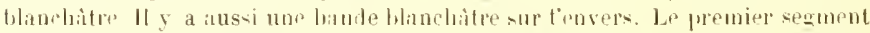
it une grante tache grise qui entwure le lord pustérieur de l'ócusson ; les segments suivants ont des tiches grises fourchups qui dícroisunt sureesavement te laille justu'au cinguième segment oì la tiche pat presige olsolite, mais sur le siximasegment ello et plus large et presque semi-circulaire; sur le septième ella arape presque lout te lessus.

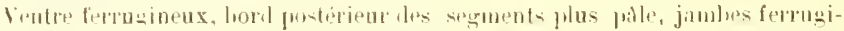

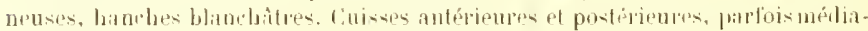

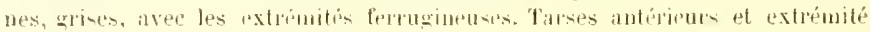
des tibias antérieurs coulenr de proix. 


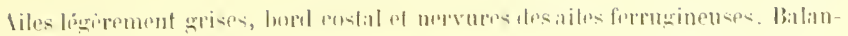
diers lirunitres.

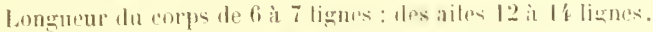

Ju tial? - Collection de .l. Combonex.

\section{bitideritillit:}

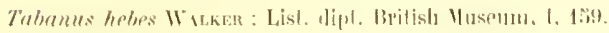

\section{Tabanus (sulgenur dtylolus) zoulouensis $q$ Bbots}

Ithe femelle, longurur 11 millimèlres 1 '2.

"Intennes (incomplites), les leux premiers segments fanres: palpes llun faure pàte : fice et harbe hlanchatpes. Front pruineux, ronsedtre, fallosité de "mine couleur, fort stroite mais hrusquement dilatée inférienrement. Tergum, "rousson et llancs fauves aver une courte villosité jaunitre et clairsemée, fo "premier marqué de einq larges hamles noires n'atteignant pas le bort jus-

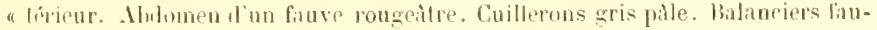
"ves. Pémurs noirs; genoux, tihias el tarses fauves. Tibias antérieurs noirs à a lexprimite avec leurs tarses entiorement noirs. Ailes d'un jaunatre fort pailo «à slignate roussittre.

l'u spécimen: Cap de Bonne-Lisérance.

L.* Tahanus ayricola Wiedemann qui, dans fo calaloguede U. Kentecz, est inuli-

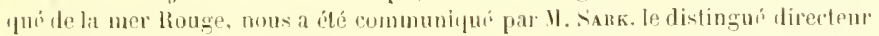

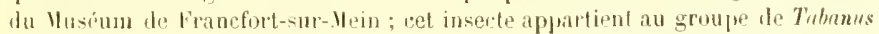
fulcus veigen et ne doil pas ètre compris diuns celte faune tropie:alir a fricaine. 


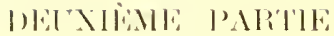

Les taon mites sonl toujours braucoup plus rares que les femelles, wh pitres

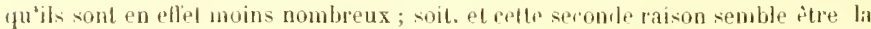
plus exarle, parce que l'habilat al la faron de virre des trons miles sont différents de reux de l'autre sexp.

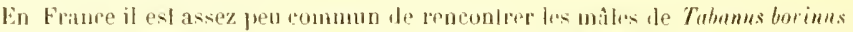
et de Tolmuns aummnalis, ce pui provient de reque les màtes la res mpices ainsi

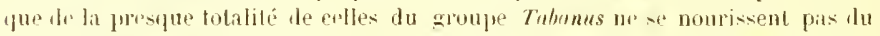

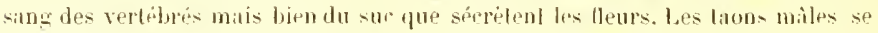
rencontrent amszangent au hord des ruisseaux où viennent pondre les femelles. Personnellement it l'aulomne de 19017, nous avoum pu recueillir le long d'uu

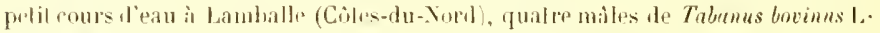

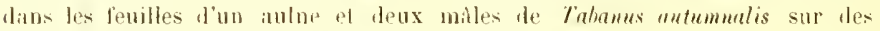
poires fombreses, for matin, vers la mème éfqurue.

If y a une exception it firire pour les undes da grupe atylotus - comme nous l’avons va dans la préface - "e groupe nest pas pxaclement représenté en Afrique Iropicale, mais dans F. Ifrique du Nord, en Algripe, en Tuaisie el au Maroe nous arons en livecassion de prendre parfois autant de milrs que de femelles de Itylotus tomentosus llacquart.

I)une faz̧on générale les taons màles oll'rent it l'observaleur les mèmes dessin-

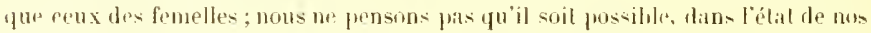
comnaissances andueldes de dresser un lableau dicholomique ulilisible; nous

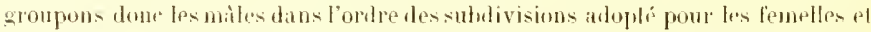
nous estimons que le tableau thohotomique gentiral, en faisant abstraction de

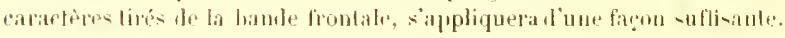

\section{I'RENHER RIIOIPE:}

\section{Tabanus fasciatus or Wigdemaxx (page 1 18 ).}

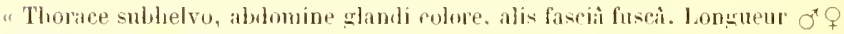
" $Z$ lignes $1 / 2$. Sielra-Leme.

Wienemaxx dat quedane l'exemplaire mitledu Muséum de Vienne, la conleur do l'abdomen esl ferrugineuse, lapex noiratre el que sur chatue segment se

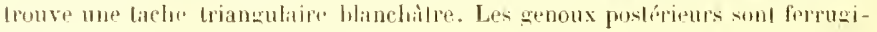
He'ux.

BHBH(MiRIJHI:

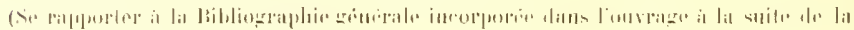

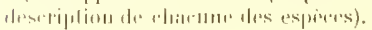


Tabanus latipes or Vaceuarr pl. 1, tig. 2).

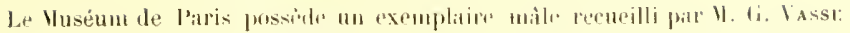

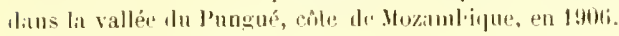

Longurur 15 millimittres.

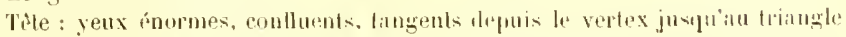

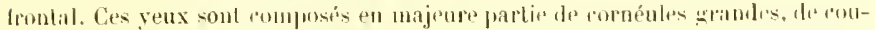

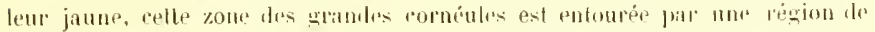

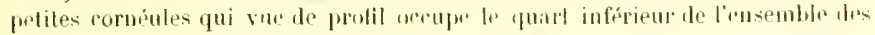

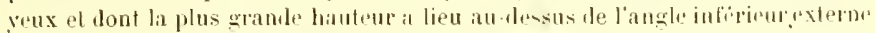

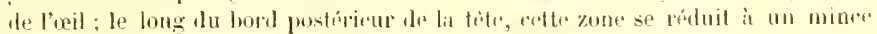
rordon qui fait toul le tour des yeux et recoupe to triangle fruntal sans le lra-

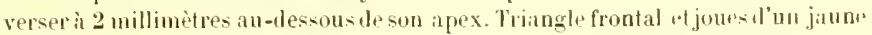
orangé intense portant quelıues poils coneolnces. Intennes semblialiles it celles

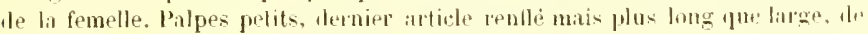

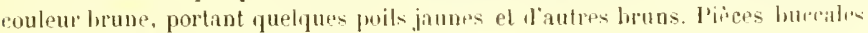
illongées, mesurant 4 millimitres.

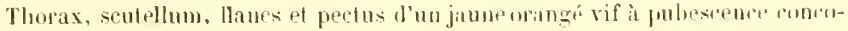

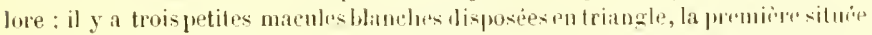
en avant contre l'inserlion anterieure le l'aile, les deux antres sont plactirs,

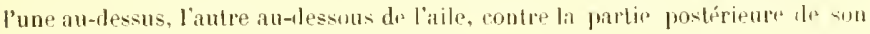
insertion.

Ablomen d'un rouge plus brun que la fhorax it pubescenre concolore: llane

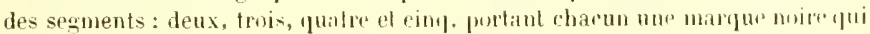
rst marginée de poils blanes it sun lwot posteriente.

Ventre de la couleur du dessus, miris depuis le serond somenl, dhann dis

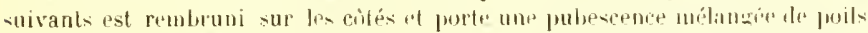

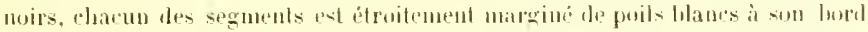
postérieur. Siles brunes jusqu'i hateur du quatrieme segment de l'ablomen,

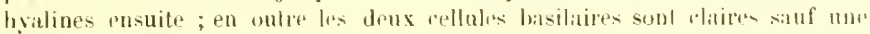

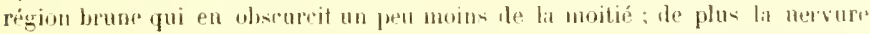

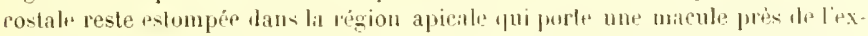

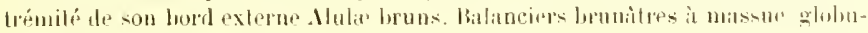

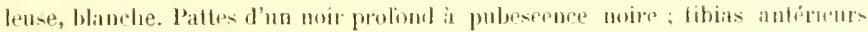
furtement rentlés.

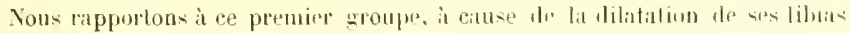

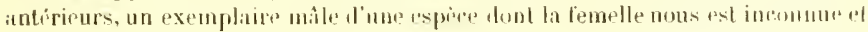

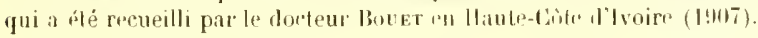

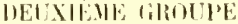

\section{Tabanus Billingtoni $O_{*}^{4}$ \EWSIEAl}

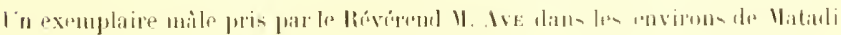

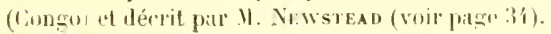




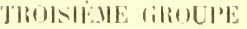

Tabanus biguttatus or WIEnEMANx

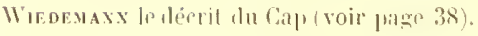

Tabanus unimaculatus Vacovant

lécril par l'anteur, du Cap (p. A

\section{Tabanus cilipes Yacǫran}

Dír ril par l'auteur, du Sénégal (p. 42).

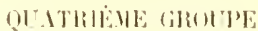

Tabanus ruficrus $\sigma^{7}$ Palissot de lieavors (pl. II, fig. 17).

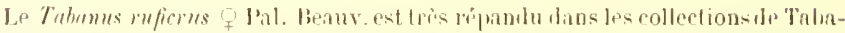

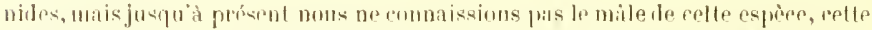

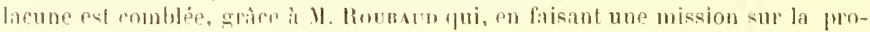

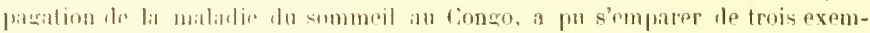

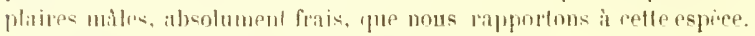

longurur 1 ! millimbles à 19 millimitres $1 / 2$.

Type of Iongurur l! millimètres. Refueilli is limzzaville (Congo francais), le

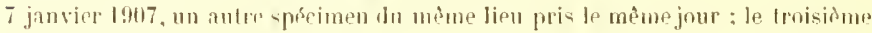

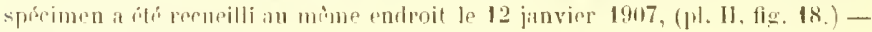
Collertion da Vusium de laris.

Voir, atles unfumros, ilulomen portant trois tarbes mádianes de pilosite jamne.

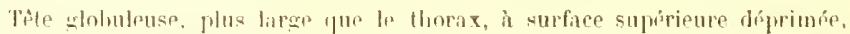

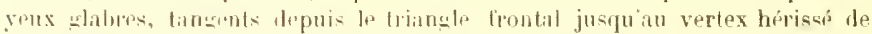
rourts poils noirs: les rormbles des youx se divisent en denx zones: l'une

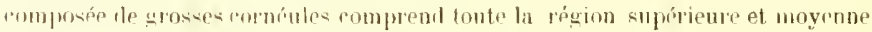

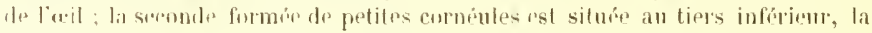

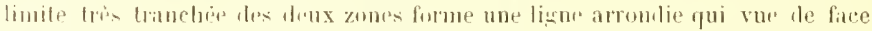

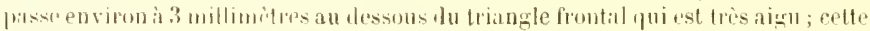

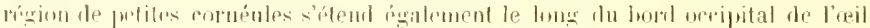

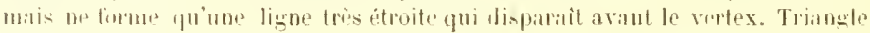

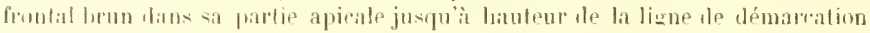
rutre les drux zones de liril ; partie in lirrieure du triangle frontal et joms d'un hrun jaunatre portant des poils marron plus on moins assouturis vers le déssous. 


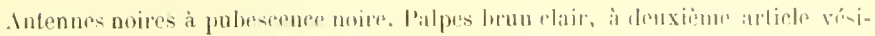
culeus plus long que large, hérissés de prits marron.

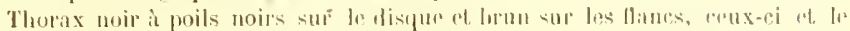

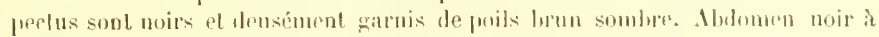

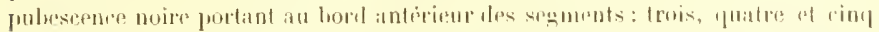

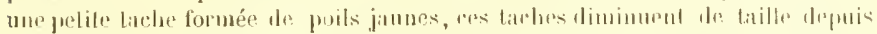

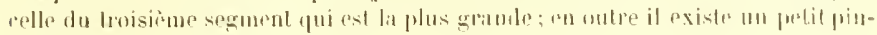
reau de proils jaunes an milieu dlu bonl pustiriem du segment anal.

Ventre noir it pubesenene noire.

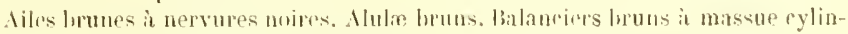
dritpue, plus claire dans sa partie apricalir.

l'alles noires à pubescence noire, prelolles clatires.

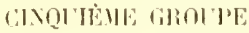

\section{Tabanus pluto WALER}

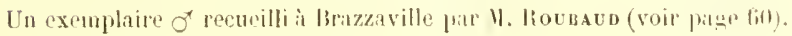

SIXIEVIE RATOLPE

Tabanus par WALKER

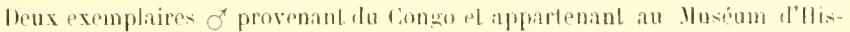
loiru naturlle de liruxelles (voir pitge lis).

\section{SHTHEVE GIROLPE}

\section{Tabanus Laverani $\vartheta^{*}$ surcorf , pl. 11, lig. $2 \vec{\imath}$.}

Le Laloratoire d'entomologie du Muséum de Paric a recu par l'inturmáliatre

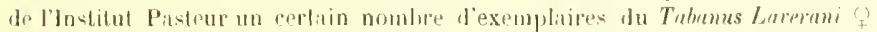

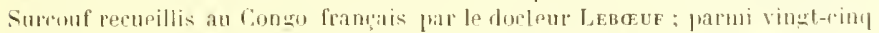

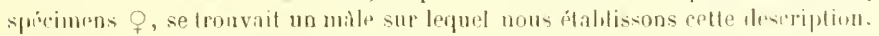

longurur: 11 millimilles. - Colleelion lu Vusémm de Paris.

lyout general du Trebums lavereni o sumouf.

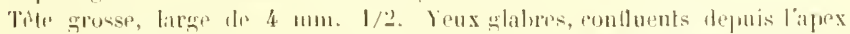

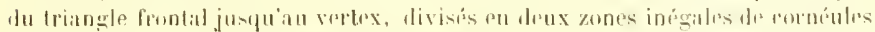

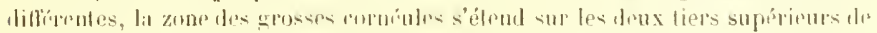

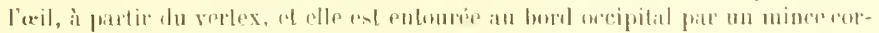

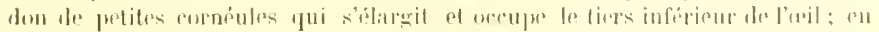

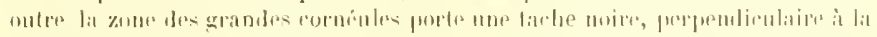

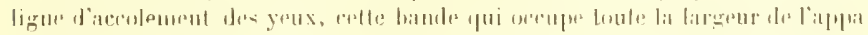

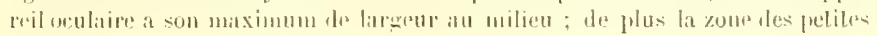




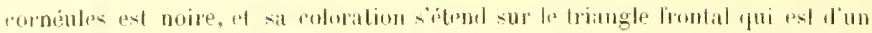

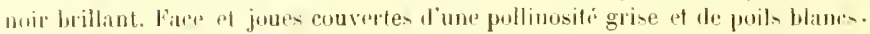

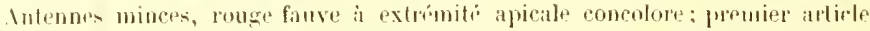

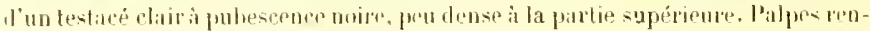
llos, d'un blane jaunitre, à pubesernor blanche.

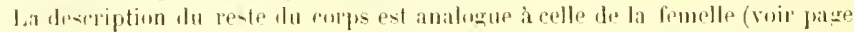
(191).

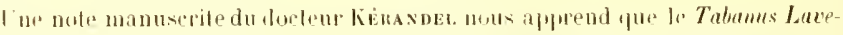

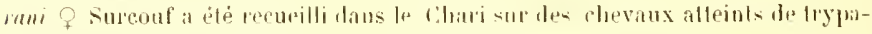
musomiane ( 11 juin 1907).

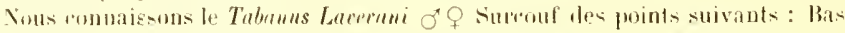

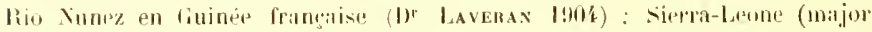

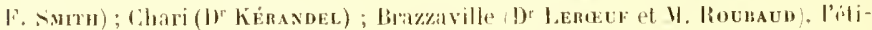
quette de eaphre en ce dernier fminl porta la menlion suivante : Brazaville.

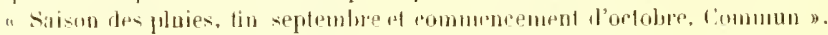

Vous lapportons an septirint grompe un exemplaire mile de taon, recueilli

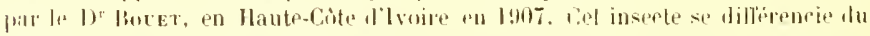
Tulunus Latermi sureouf par les bandes noires nelles du llurax, les antenues

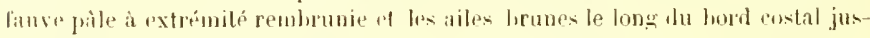

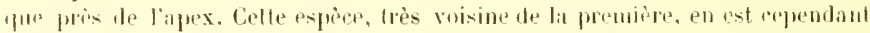

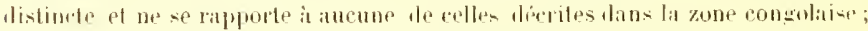
nous la mentionnons four y attirer l'intention, sans lui imperser un nom.

Tabanus subangustus $\sigma^{\prime}$ licando (jl, III, tig. II).

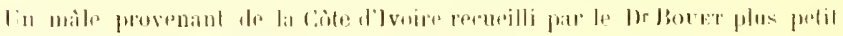

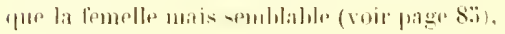

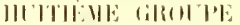

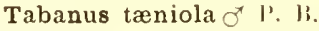

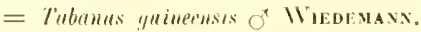

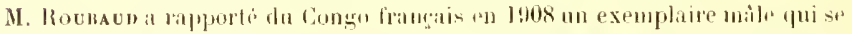

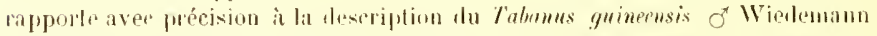

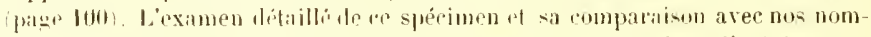

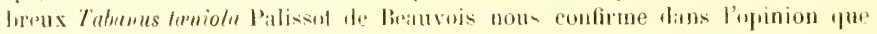

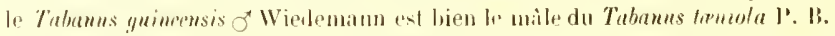

La soulr dibrirence que nous constations est dans la couleur des palpes pui

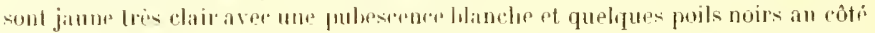

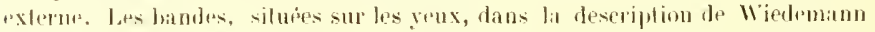

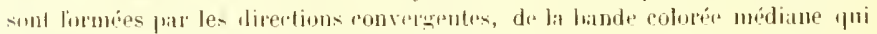

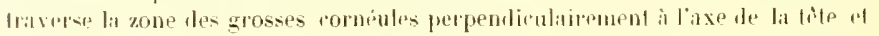
de relle de la zone oblique de peliles ionénes. 


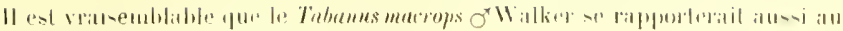
Tielunus deniole I'. B.

Lat synonymie doit doue itre ainsi etablir:

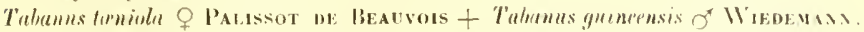

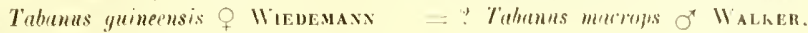

= Tubanus subdomyulns o llacuUart.

ete.

\section{Tabanus quadrisignatus of linian}

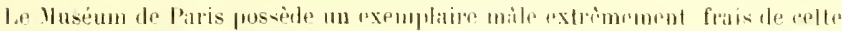

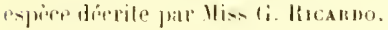

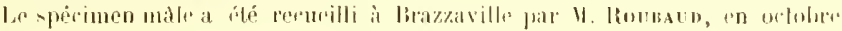
$1: 10 T$.

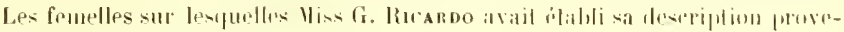
nilient du Congo lianuir.

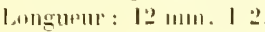

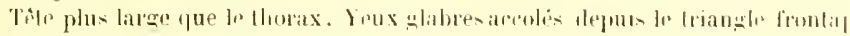

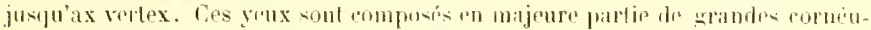

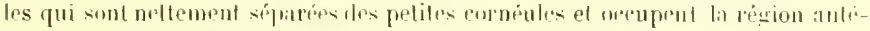

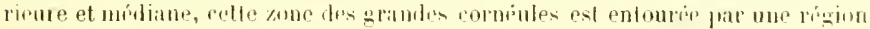

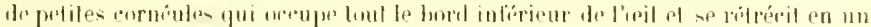

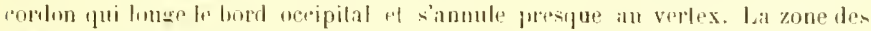

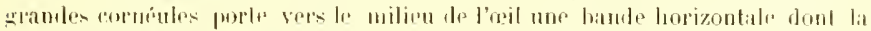

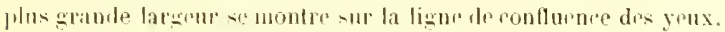

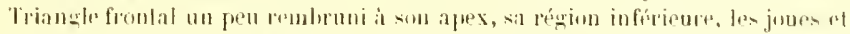

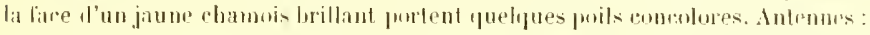

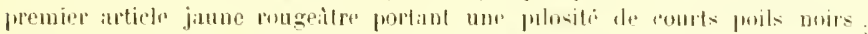

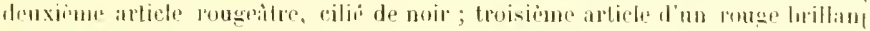

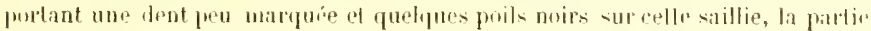

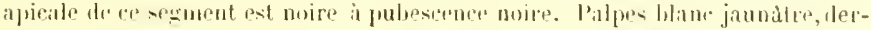

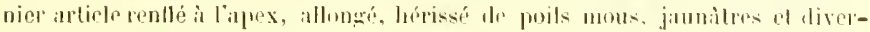
sents.

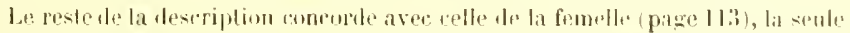

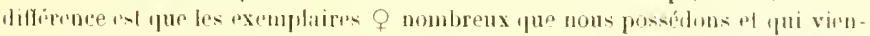

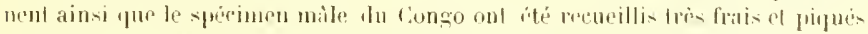

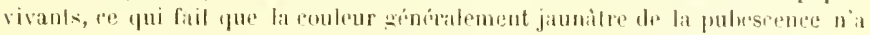

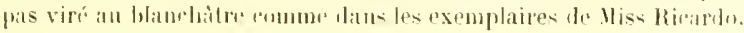

\section{NEI TIENE TIBOI IL}

\section{Tabanus secedens OT WALLKR}

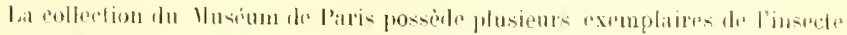

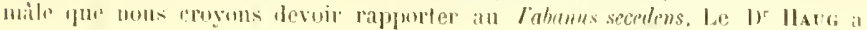




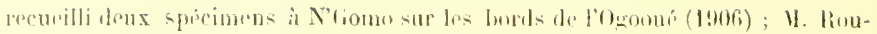

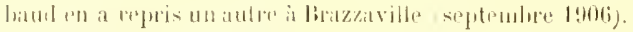

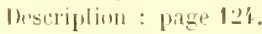

\section{Tabanus claripes or licando (pl. III, lig. 3)}

Xon- mpportons ì celte esprece, deux tris boax specimens illsolument frais

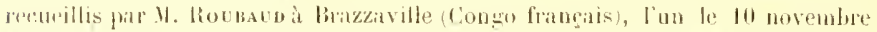
1:07, l'autre au commencement des pluses. Ces deux spécimens sont dans la collection du Muséun d. Paris.

Longueur: : 2.2 mm. $1,2$.

Tète grande, plu- liage que le thoma. Yeux conlluents sur toul leur borl mbrne et atteignant sept millimeitres de hargeur, cumposés de deux champs te curmónles, nettement distincts dont une zune míliane le grosses cornéules qui

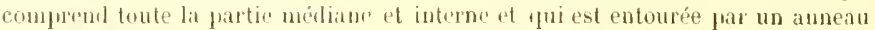
de petites corncules; au rertex cet anneau est trís itroit et il augmente très jeru de largeur justue vers le hord externe de lieil ou il s"elargit pour former toute la partie inlibieure de l'ueil, la ligne de séparation fait une courbe dont la convexite trés accentúe est tomrnée vers le hat, rette ligne arrive au triangle frontal quelle semble couper à I millimitre $1 / 2$ au-dessous de son apex; la séfraration est nette et hruspue fartout. La zom des grossos cornéules porte une tache noire triangulite sur chaque wil et une autre tache bacillaire le long de lit ligue méllane. Triangle frontil maron vers liapex sur les $2 / 5$ de sa hatuteur. le reste est d'un jaune doré brillant; jones jaunes à pwils jaunes. Barbe jaune. Intennes petites, rouges; freurier article rougeatie, à poils noirs, obliquement tronque; , leuxirme article un fren phs clair, cilié de noir; troisione article rouge ferrugneux, étroit, assumbri vers la région moyenne, rembruni à la partie apirale, qui partait noire. J’alpes petits, renllés à l'extrémite apicale, couverts d'une pollinosité jeu dense et noire.

Thorax brun portant juatre larges bandes longiludinales recouvertes d'une

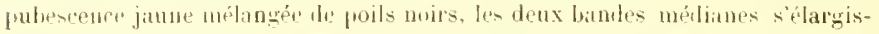
sent un peu a hauteur de la muture transverse puis se rítrécissent et se prolongent sur le scutellum quielles entourent. Flanes et pectus cendrés à longue pubeacene jaunâtre.

Ibdomen long, conique; de couleur rougeitre, is rare pubescence noire, portant une ligne méliane continus, très peu visible au bord antérieur de chaque seg-

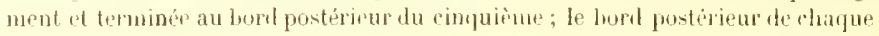
sesmmot ext cilié de proils jauner rlans la région occupée par la ligne longitudinale fus ou moins obsolite. l'as de taches latírales visibles.

Itessoms de labdomen rougeitre, it stoments itroitement bordés et ciliés de blate is lexur lord postéricur, une large bande longitudinale, noiritre, à pubescenew noiràtroserupe la région módiane du ventre.

llanches rougeritres; fémurs rougcàtres à pulsescence noire sur la face supúrieure et blanche sur les autres rógions des fémurs; tibias antérieurs rembrunis dans leur moitić apicile, la pubescence jumbitre devient noire dans la région 


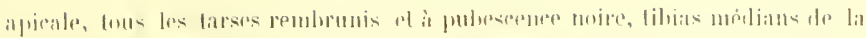

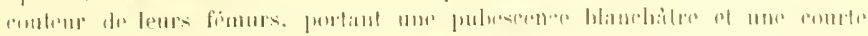
lrange noire à l'angle externe, thias postérienrs rougeitres comme les fémurs

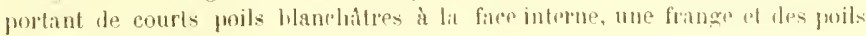
noirs au cilé exlerne.

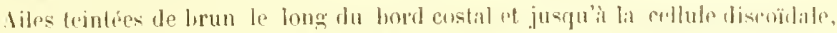

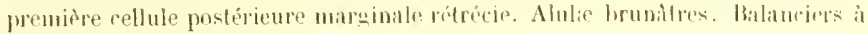
tige brunitre al massue jaunâtre.

\section{Tabanus camaronensis or Bu:ot}

In exemplaire détirioré provenant ar Nislite.

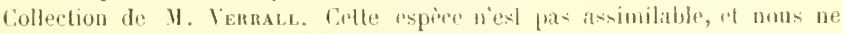
savons rien des Tabanides de Mndire (paige Iay).

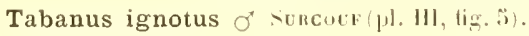

Nous rapportons ì ce groupe un exemplaire mile, recueilli par 11. Roubaun it

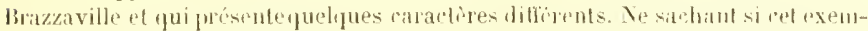

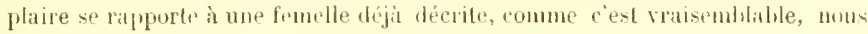
lui donnerons te nom de Trabamus ignolus or.

Type : un exemplaire or.

Longueur : 19 millimitres.

Tite grande, plus large que le thorix ; yeux ronllurnts sur tout leur lwal

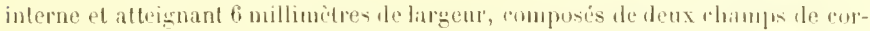

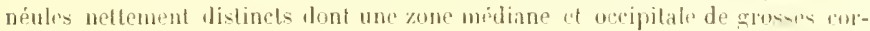
néules yui comprend lat partie rentrale of yui est enturée par un anmean de

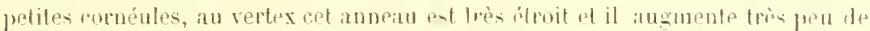

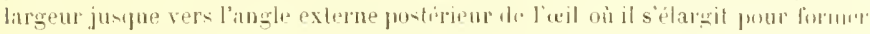
tout la partie inferieure, la ligne de separation fait ume rourde lont la rourexiti

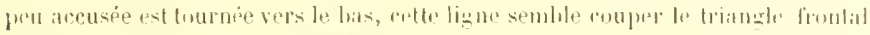
à un millimètre environ all-dessous de sin apex ; la séplitration entre les deux

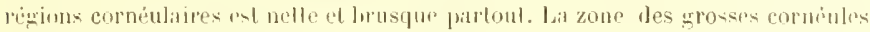
porte me latre lrande meliane, transverse.

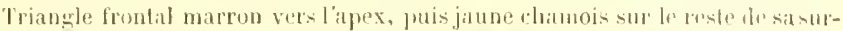

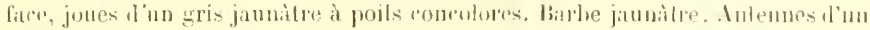

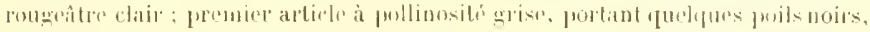
obliquement trongué; second article plus elitir ; troisiome antiele rongathe,

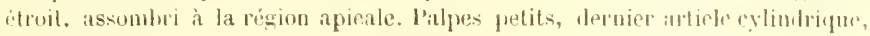
jamnitres à poils slivergents jaume grisitro.

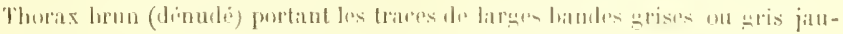

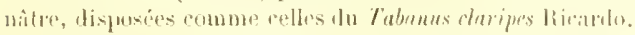

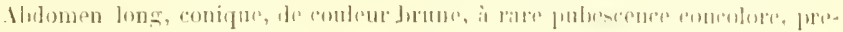

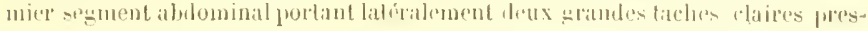




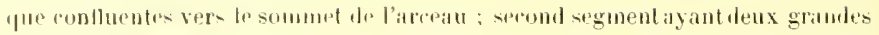

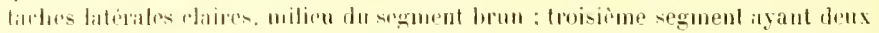

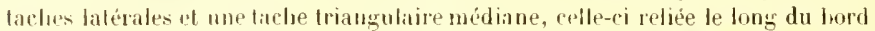
fostrieur du segment aux marpons litérales, ce triangle flus large que haut

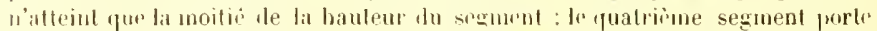

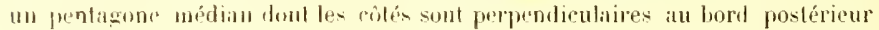

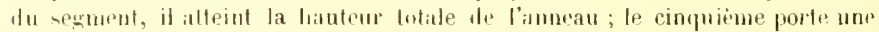

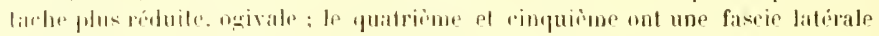

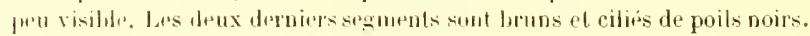

lintre limu ferruginenx à prulescence noire. llanches rougeâtres, couvertes d'une pullinosilé grise el le longs proils hlaneluilres; firmurs rouges à poils blawe

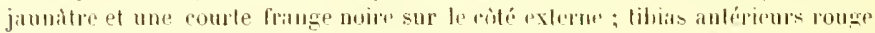
flus sombre ì prubesence melangée de proils noirs on de poils blanes, libias

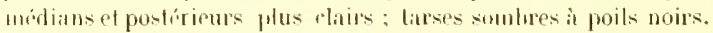

Siles daires, un preu jaunes le long du lomil costal, sligna jaune, extrumités

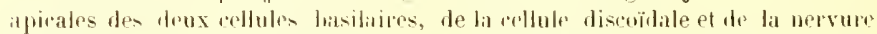

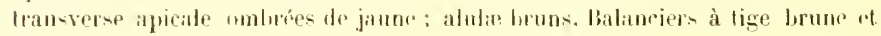
missu jauthlor.

\section{MXILYE (iRG)TE}

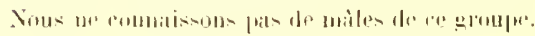

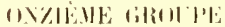

Tabanus inhambanensis lienulox।

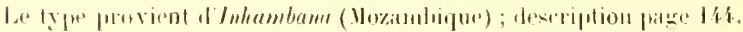

\section{Tabanus ustus O WALKEN}

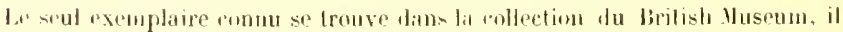

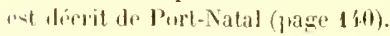

\section{IWI 'ZIEYE GIRUCH':}

1.e Muséum de Patrin at reçu de Simba (Afrique orienlalde anglaise), un exemplaire màle de Tabanus carinlilis larw, "apturé frar la mission de M. Miturice de

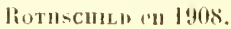




\section{Tabanus variabilis}

Longurur 13 millimètres.

Tite un peu plus large ine fe thorax. Yenx velus, contluens sur loul leur ford interme et alteignant 3 millimitres $1 / 2$ de largeur, composés de deux rhamps de cornéules à séparation indisturte, lit zone médiane ef orcipitale composíe dr.

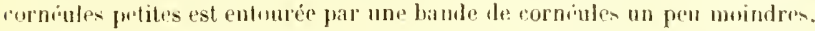

Triangle frontal brun dans sa partie apicale, freste est flun gris jaunitle is courte pilosité noiràtre. Jours et fare grivilres à poils mṕlangés bruns et blatres sur les joues blanes en dessous.

Intennes: fremier article gris rougeatre portant une pilosti nowe assez

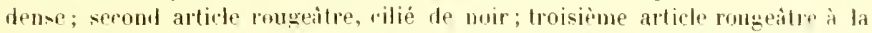
base et noir dans la partie midiane et apicale. Palpes : dernier article en forme de citron, jaune brunitre diar, birisé le proils lanehitres.

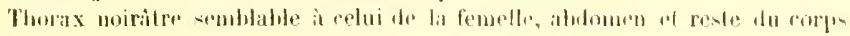
somblibles a ceux de la femelde (voir page tfiit).

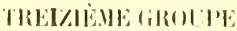

\section{Tabanus gratus f liascil}

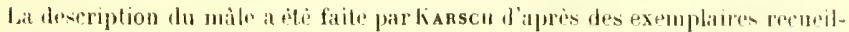
lis en . Ifriqur orientale.

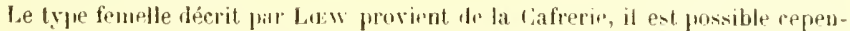
daut qu'il $y$ ait eu confusion entro deux especes voisines (voir lat descriplion fiage 178).

\section{Tabanus obliquemaculatus or VACQ̨an Tubunus psesemenis of J.ENNICKE}

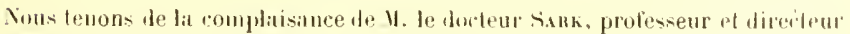

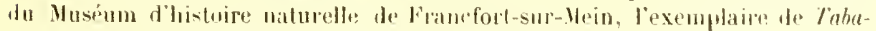
uns psusennis or daennicle recueilli en Mhyesinie pill le br liüppet.

L'étude de re type nous conlirme dans l'opinion yue cet insecte est le mitu deTabanus lpucostomus Low qui est le synonyme de T. obliquemacululus Hacquary.

Puur obvier à la brièveté de la description de lexsiche, nous redécrivons ret exemplaire.

Longueur : 15 millimitres.

Tite grande, plus large que le horax. Jeux glatres, conlluents sur toul four

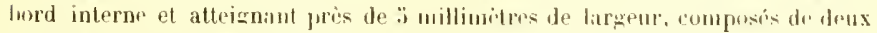

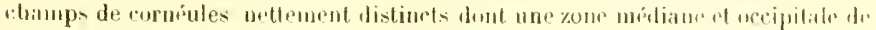

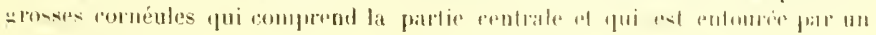
Surrouf 


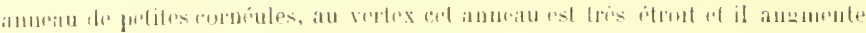

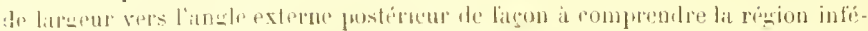

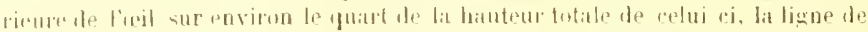

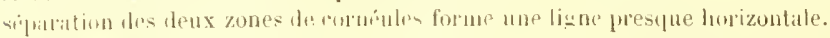

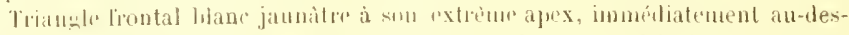

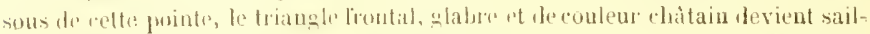
lant, fanaliculé au milieu et se prolonge sums be burd inferieus de chacun des rax. domén couvertes de proils liburs, Barlue hauche.

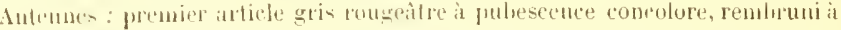
sat saillin apicale: deuxième artich rougrảtre; troisième article manquant.

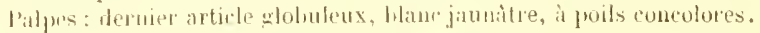

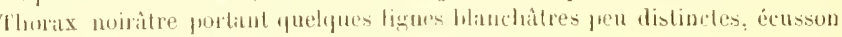

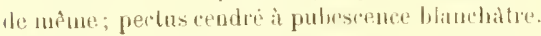

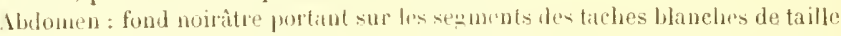

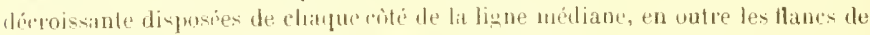

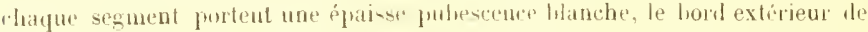

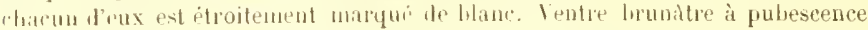

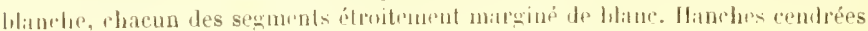

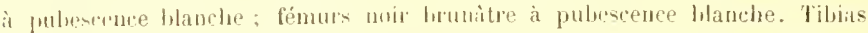

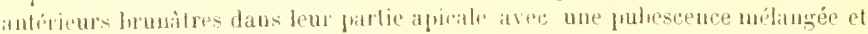

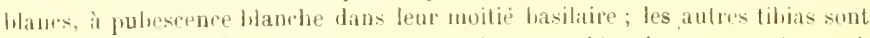
lou rougeitre plus ou moins "lair i frubrence banche; tarses luruss it jullesrence conculore.

liles cendrées, stıma brun, les nervures longitudinales, la réginn stigmalifue, et les nervures qui entoureut la cellule discoüdale estompées de brun-jaune, ainsi que l'appendice. Cuillerons incolores. Balinciers jitune brunàtre à massue clitile.

(1ITTOHZIIVE GIROLPL

\section{Tabanus ditæniatus or Maçueant}

1, Museum de Paris possède l'exemplaire màle type de Macouart. La colleclion comprend de nombreux spécimens (voir pase 18 i).

\section{Tabanus fuscipes o liıcardo}

Le type mâle (colleclion du British Mluscum) a été recueilli dans le Natal en $18: 7$ ( (., A. K. MarshaLl) (deseription page 152).

\section{QUTKLEVE (iROUI'}

Nous ne connaissons ancun mitle de re groupe, constitué jusqu'it présent par teux expeces, l'une du Cap et la seconde du Congo. 
SEIZIEIE, GRQIPL

\section{Tabanus capensis}

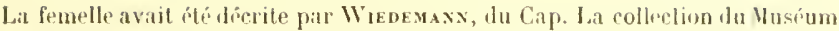
de Paris possède deux exemplaires miles, ils ont ité diterminés par Vacorant et proviennent do la mission Delalande dans le sud de l'Alrique (description lage 198).

\section{Tabanus tæniatus $\sigma^{7}$}

Ypus a vons trouvé dans la collection Ju Ylusíum de l'aris un exemplaire male provenant de la Cafrerie (deseription page 200 ). 


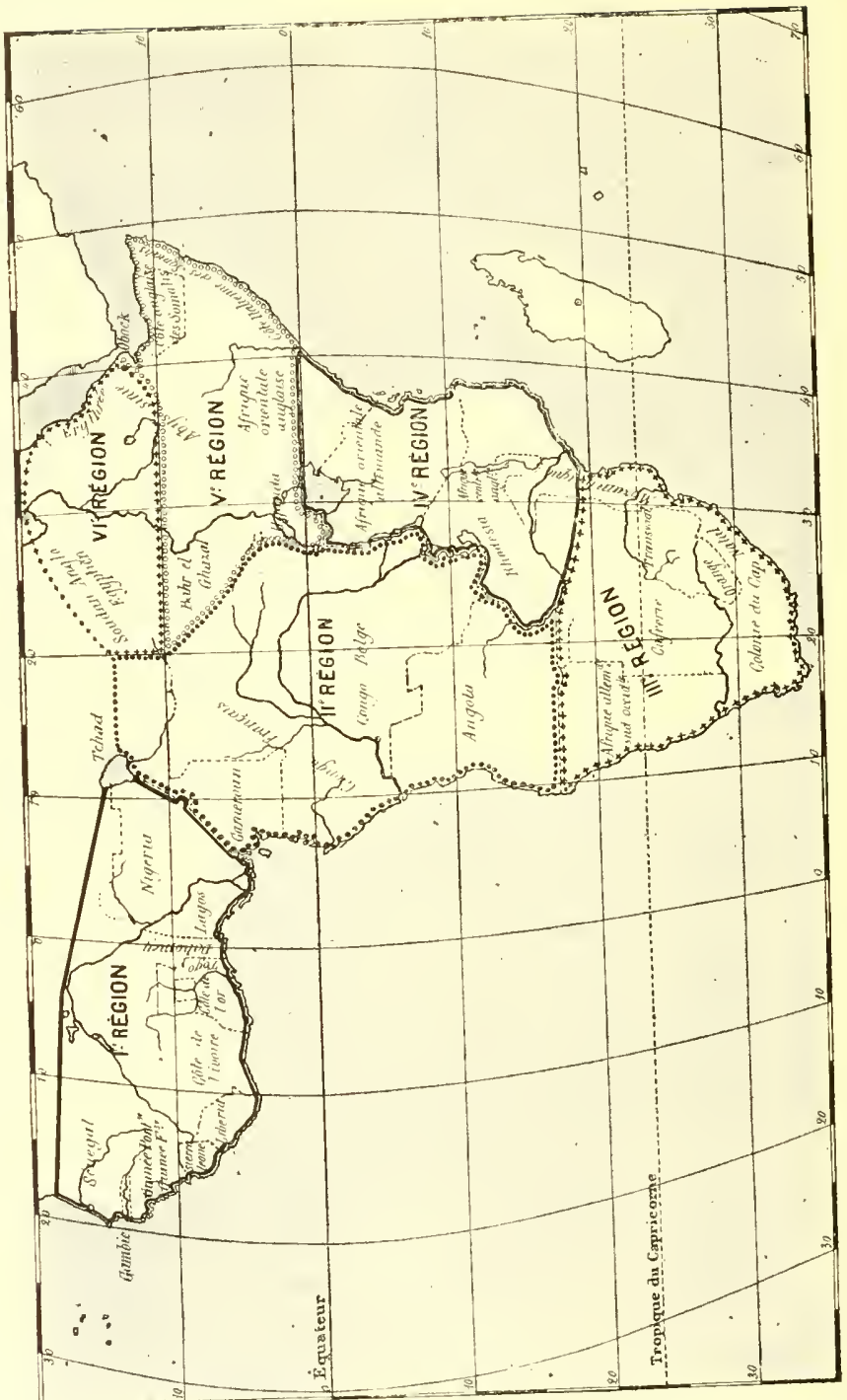




\title{
RIPARTTTON GROARPHQLE
}

\section{Première région}

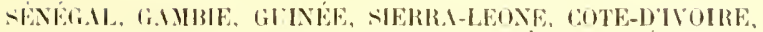
COTE IEE LOR, TOGH, DAHOMEY, NGGRIA, IIBERLA

\author{
PKEMIER GROLPE
}

Tabanus fascintus Fabricius.

Thamas afrioums Gray.

$$
\text { DEVTIETE GROUPE }
$$

T'ubanus olsererefumatus surcouf.

Tabamis billimylomi Newstearl.

\section{TRUSU:UE A:ROHPE}

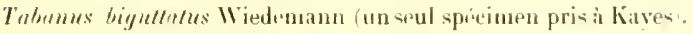

Trebermus unimaculates Dlitequart.

Trabmes rerecens Surroul'.

QVATRIEME GHOUPE

Tabarms armilus surcoul.

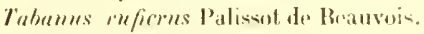

Tubaneses lirnmpti surrouf.

Tabamus besti Surcoul.

Tabanus Boneti Sureoul.

$$
\text { CINQUUV GE GRUPE }
$$

Tishemus glum Walker.

\section{SIXIENE GROUPE}

Tabamus par Wralker.

Tabanus thoracinus I'. 13. 
7abanus Lacerani Surcouf.

Tubanus subanyustus Ricardo.

IIUTIEME GROUPE

Tabanus tieniola P. B.

Tahamus sorius Walker.

Tabams sayitlarius Macquart

Trbunus Rirardos sureouf.

Tabanus sugens Wiedemann.

7 abames sticticolis Surcouf.

NEUVIENE GROUPE

Tabanns seredens Walker.

Tubunus Linysleyi Ricardo.

Tabanas ramaromensis Bigot.

DIXILME GROUPE

Tabanus testaceicentris Marquart.

Tabunus conformis Walker.

Tubanus nigrohirtus Ricardo.

ONZIEME GROUPE

Tabanus Martini Surcouf.

Tabanus congoieusis Ricardo.

DOUZIÈME GROUPB

Tabamus argenteus surcouf.

THEI\%IFME GROUPE

Tabumus sufis Jiennicke.

Tabanus gratus Lew.

QUATORZIEME GROUPE

Tabmus diteniahus Macquart. Tabnnus albipalpus Walker. 
SFITIBME GROTH

Tabumus Cheratirri surcouf.

Fous les groupes sont reprisentis (sauf fe quinzième) et forment 37 esporees.

\section{Deuxième région}

C.MMEROLN, GONGO FRINCAIS, GONGO BELOE, INGOLA

PREMIER (;ROUPI:

Tabumus fostialus Fabricius.

Varititis de Tulanus fascialus.

Taluanus alripes Vian der Wulp.

Tabomus lalipes Marquart.

Tubunus sulviunhus Ricardo.

DEUXIIME GRUUPE

Tabamus obsturefumatus Sureouf.

Tabarus Billingioni Newstead.

Thbanus marmoratus Surrouf.

THOHIINR GBOUPE

Tabamus biyuthatus Windemanu.

Tabamus croceus Surcouf.

QUATRIEME GROUPE

Tabamus Brumpti Surcouf.

Tabamus anens Surcouf.

7 ahanus ruforms l'. B.

Tabanus ianthimus surcouf.

Tabunus desti simenul:

7 ubamus dihutius sinronf.

CINQUIEME GROVPE

Talumus I P/ulo Walker.

Tubumus comus karsell.

Tabmus conesorns surouf. 
Tinbarnes pur Wallies.

Tabumes romblustus Bigot.

Tabaums obscupphirhs Ricardo.

Tubames thoracinns P. B.

Taburus otserurion Ricardo.

SEPTIENE GIRULPE

Tebamus olssermestriatus Rinitrdo. Tabaums Laterami Surcoul.

HUTTIEME GROIPE

Taham taminla P. B.

Tabamus sorins Walker.

Tatumms sagintorins Macquart.

Tolianus quatrisignatus Ricardo. Tabunus roniformis Ricardo.

Tulames distiurfus Ricardo.

NEUVIENE GROUPE

Tahonus secedens Walkur.

Tabanus claripes Ricardo.

Tabanus Kingsleyi Ricardo.

Tabautus ignotus surcouf.

DIXITAE GROVPF

Tabunus lestaceiventris Marquart. Tabamus conformis Walker.

Tutumus migrohirms Ricardo.

ONZIÈME GROUPE

Tab anus congoiensis Ricardo.

Tabanus disjunctus Ricardo.

Tulames Lemeirmi surroul.

DOUZEME GROUPE

Tabumus diressus licirardu.

Tabanus creriatilts LolW. 
Tabuuns atrimanus l,frw.

Tabamns severini surcouf.

Tabaums argenteus Sureouf.

Tabanus rarians Surcouf.

Tahanus Wellmani Iusten.

Tubanus Rontundi Surcouf.

TREIZIÈME: GROUPE

Tabuuns sufis Jiennicke (région Nord.

Tabanus gratus LarW.

Tabarmes trilirnimlus Ricardo.

Q̨ATURT.JËE GHOUPE

Tabanns ditrniutus Macquart.

QระIXZTHE GROUPE

Tabames irroratus Surtoul.

Le seizième groupe n'est pas représenti. La deuxième région réunit cinquanteainq espèces.

La région d'Angola est encore très peu connne pt il existe trics pert d'inserdes du Cameroun.

\section{Troisième région}

AFRIQUE ALLEMANDE SLU-OCGIDENTALE, COLONE DL CAL', NITAL,

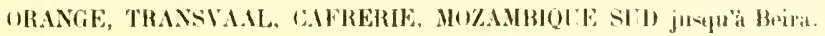

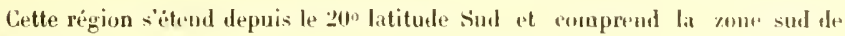
l'Mrique.

PREMIEN GHULPE.

Tubanus hulipes Nacpmart.

THOISIRY AROUPB

Tubunus higultutus Wiedemann.

Tuburres e. cilipes Maequart. 
Tabams por Walker.

SEPTIINE T:RIUPE

Tulumus alhilimea Walker.

Teluaures albostrintus Ricarilo.

Tahmus muitmniatus Ricardo.

HLITIEบE GROLPE

Tabanus socius Walker.

Tabauks stypitfarius. Ilarquart.

Thbames fiethemss Macquart.

Tubanus sericiventris Law.

Tubanus atmus Wiedemann.

ONZIEHE GROUPE

Teluunes ustus Walkir.

Tubunus inhumbanensis Bertolini.

\section{DOVZIIME GROUPE}

Tabumus cariabilis Law.

Tabanus insignis Lew.

Tahames atrimuntes Laew.

Tatmanus sulcipalpus larw.

TRBIZIĖMB GROUPE

Tabanus obliquemaculatus Macquart.

Tabanus rufosignatus Bigot.

Tahanus gratus Luw.

OUATORZIENE GROUPE

Tabunus ditmialus Maequart.

Tubanus fuscipes Ricardo.

Tubanus diurnus Walker.

QLINZITME GROUPE

Tubanus muculatissînus Macquuart. 
SEIZSYYM GNOUPE

Tabames zoulouensis Bigot.

Tolumus limnintus Marquart.

Tobumus fulcimmes low.

Tabamus capensis Wiedemaun.

Thbemms tennicrormis Ilacquart.

Tabumus rerans lirw.

Tubanus lievifrens Law.

Tubumus hebes Walker.

Tabums infans Walker.

La troisione région ne comprenel pas de spicimen des groupes: : 2, 4, : $, 9,10$ et réunit $3: 3$ ispèces.

L.Afrique sud-nceidentale allemande of la Cafrerie ne donnenl presque aucunes indieations actuellement.

\section{Quatrième région}

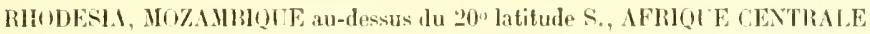

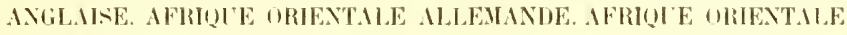
ANGLIISE jusqu it l'Equateur.

\section{PREMIER GROUPE}

Tahames latipes Macquart.

Talianus Brucei Ricardo.

Tabanus septrmpmnctutus Ricardo.

DEUTHEYE GROUPE

Taluans quarligututus Ricardo.

TROISITHE GROUPE

Tabumus biguttatus Wiedemann.

QUATRIEMF GIIOUPE

Talmues mufirens P. R.

Tuhtmms yrumlissimus Ricardo. 
Fabunus pur Walker.

Tabanus clurilibialis Ricardo.

Tabunus: licrutipes Surcouf.

Tabanus thotacinus 1'. B.

Tubumus impuress Karsed.

SEPTIEME GHUIPE

Tubanus nigrostriatns Ricardo.

Tubanes milineatus Low.

Tabrums unitirniatus l.m.

UUITIENE GIUUPE

Tabanus lieniola P', B.

Tabanus sneins, Walker.

Trubauns sayillarius Macquart.

Tubumes firaterums: Macyuart.

Tabamus quadrisignalus Riraralo. Tubunus roniformes Ricardu.

Trabumes distiuetus Rirardu.

WXZIENE GROUPE

Tabanus Myassio Ricardo.

Tabunus inhambanensis Bert.

Tabemus Denshromi Iusten.

DUUZIEME GHOLPE

Tabunus rariabilis Lew.

Tabunas insignis Lew.

Tulumns shonpei Austun.

TREI\%IEME RROUPE

Jabamus lhothechildi surrouf.

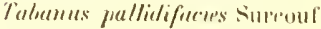

QUA TORZIENE GROUPE

Tiulumes ditereiulus Matequalt.

taluuus fuscipes Ricardu. 
(IUIXIEME I:ILUPF;

Tubunus maculutissimus Mawpint.

SEIZIENE GROUPL:

Talumes imbecithes Kiarsels

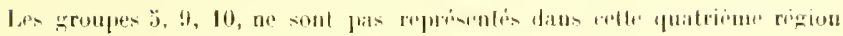
qui couprend 34 expiress.

\section{Cinquième région}

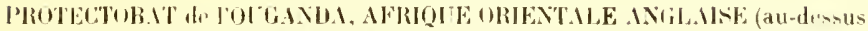

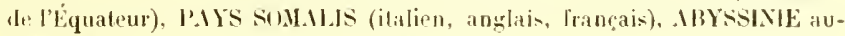

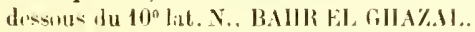

PREMIER REATPE.

Tabanus niloticus Austen.

Variétionde Tulnaus fasfialus.

Tabonus lalipess Macquart.

Tabanus seplempunchulus Ririarilu.

Tulunus Brucei Ricardo.

TMUISHME GIIHPE

Tabanns bignllalns Wiedemann.

QUATRIENE, GROENE

Tubames brumpli surrouf.

Tubanus fusco-marginatus Ricardo.

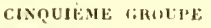

7 uhanes pheto Witker.

SIXIEME GiRUUPE

Tabunus pme 11 alker.

Tabunus thorucinus I'. IS.

SEPTILYE GROUPE

Tubauns conspiruns Ricarde.

Tabanus olscuripes Rieardo. 


\section{HUITIENE IFROUPE}

Tubanus lieniola P. B.

Tubauns socius Walker.

7 abanus sayittarius Macquart.

NELYISM GHUUPE

Tabumus secetens Wallir.

)(UZILNE GROUPE

Tabanus variabilis Lew.

TREIZIENE GROUPE

Tabanus gratus Lew.

Tahanus obliquernaculatus Micquart.

Tabanus morsitans Ricardo.

Tabenes Rothschildi surcouf.

Tabanus pallidifacies surcouf.

QUATORZIEME GROLPE

Tabamus ditimiates Macquart.

SEIZIEMNE GRUUPE

Tubanus runenzorii Ricardo.

La cinquieme région ne posside pas d'insectes des groupes 2, 10, 11, 15, elle comprend 2 sespeces.

\section{Sixième région}

ABXSSLNE, ERYTHREE, SUUD.LN ANGLO-EGYPTIEN

PREMIEK GROUPE

Tabanus miloticus Austen.

Tabamus africanus Grey.

TROISIEAIE GROUPE

Tabamus bigullatus Wiedemann. 
SIXII:ME Ritho?

Tahanus par Walker.

Tubanus obscurepess Ricardo.

HUITIEME GHOUNE

Tabanns irniola 1'. B.

Tabernus socius Walker.

Tabenus fratermes Macyuart.

ONZIEME G:KOUPE.

Tabanns Cordierisurconf

DOUZIEME GROUPE

Tabanus velutims surcout.

TREIZIEYE GROUPE

Tabanus obhquemuculutus Macquart.

Tabumus su/is Jaennicke.

Tabanus Mesnili Surcouf.

Cette région peu connue ne donne que 13 expices dilférentes.

La région s'étendant entre le 180 latitute Nord et la Méditerrannée fera l'objet d'un mémoire à part ainsi que celle de Narlagascar. 


\section{LISTE ALPHABETTIQUE}

\begin{tabular}{|c|c|c|c|c|c|c|c|c|c|c|c|c|c|c|c|}
\hline \multicolumn{2}{|c|}{ Tabmums aturess surcouf } & & & & & & & & & & & & & & 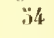 \\
\hline$"$ & "fricanus Giray. & - & . & - & & ${ }^{\circ}$ & . & · & - & . & . & . & . & & 23 \\
\hline$"$ & nlbicans Nacquart. & . & - & · & . & . & ${ }^{\circ}$ & . & " & . & 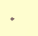 & . & . & & $2: 2$ \\
\hline$"$ & allitined Vacquart. & . & . & & & . & . & . & & . & . & . & . & & 82 \\
\hline v & ulbipalpus Walker. & . & • & 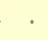 & & . & . & . & . & . & - & $\cdot$ & 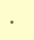 & & 181 \\
\hline$n$ & alhostriutus Ricard" & . & - & - & 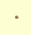 & . & $\cdot$ & . & . & . & . & · & $\cdot$ & & 86 \\
\hline$n$ & alboreutrulis Newsteac & & . & · & & . & . & . & & . & . & . & . & & $17 \pi$ \\
\hline$"$ & aquilus surcouf. . & 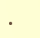 & . . & . . & i & . & . & . & . & . & . & . & . & & 沙 \\
\hline$"$ & "rigrnteus surcoul. & . & .. & . . & . & . & . & . & . & . & . & . & . & . & 152 \\
\hline " & alrimanus Low. & . & - & & 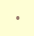 & & . & . & & . & - & . & . & & 160 \\
\hline & roripes Vander Wup & . & . . & - & 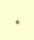 & . & 。 & . & 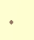 & . & . & . & . & & 24 \\
\hline$"$ & Besti Surroul . & . & . . & . & . & . & . & . & . & . & . & . & . & & ;i) \\
\hline D & bigullatus Wiedeman & & - & . & ${ }^{\circ}$ & - & . & . & 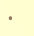 & - & . & $\cdot$ & - & & 38 \\
\hline$"$ & Billinytomi Newstead & . & . & & - & 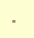 & . & . & $\cdot$ & . & 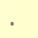 & . & . & & 31 \\
\hline$"$ & lipartitus Walker. & . & . . & & & . & 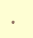 & . & . & . & . & . & . & 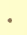 & 111 \\
\hline " & bipunctatus Wan der & Wu & & . . & . & . & - & . & . & . & . & . & . & 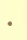 & 84 \\
\hline$n$ & Blanchardi Surcouf. & & 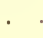 & . . & . & 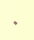 & . & . & . & . & . & . & . & & 124 \\
\hline " & Bonetisureoul. . & $\cdot$ & . . & . & . & . & . & . & . & . & . & . & $\cdot$ & . & 54 \\
\hline$"$ & bovinus LaיW . & . & . . & . & . & . & . & . & . & . & . & . & . & . & 141 \\
\hline 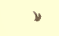 & Brucei Ricardu. . & . & . & - & & $\cdot$ & . & . & $\cdot$ & . & $\cdot$ & $\cdot$ & $\cdot$ & • & 86 \\
\hline$"$ & lrumescens Ricarto & . & . & . . & $\cdot$ & . & & . & . & . & . & . & . & . & 124 \\
\hline$"$ & Irunneus Thunberg & . & . & . . & 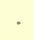 & . & . & . & . & . & . & . & . & 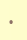 & 252 \\
\hline , & Brumpti surcoul . & . & . & • & & $\cdot$ & $\cdot$ & . & $\cdot$ & $\cdot$ & $\cdot$ & $\cdot$ & $\cdot$ & . & \\
\hline v & rumaronensis Bigol. & . & . & . & " & . & $\cdot$ & . & . & . & . & & . & 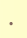 & 129 \\
\hline$"$ & ranescens Surcouf. & . & . & . . & . & . & . & . & . & . & . & . & . & . & (i3 \\
\hline$"$ & camis Karsch. . & . & . & . & . & . & . & . & . & . & . & . & . & . & \\
\hline$"$ & rapensis Wiedemann & . & . & . & . & . & . & . & . & . & . & - & . & . & $1 ! 18$ \\
\hline$"$ & cerberus Walker. & . & . & . . & . & . & $\cdot$ & . & . & . & . & . & . & • & \\
\hline$"$ & cerrolus Bigot. . & . & . & . . & . & . & . & . & . & . & . & . & . & • & \\
\hline . & Chevalieri surcoul. & . & . & . & • & $\cdot$ & $\cdot$ & . & . & $\cdot$ & $\cdot$ & $\cdot$ & $\cdot$ & . & $! 16$ \\
\hline$"$ & rilipes Nacquart. . & . & . & . & . & . & $\cdot$ & . & . & . & . & . & . & . & 位 \\
\hline 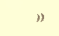 & raripes Ricardo. & . & . & . & . & . & $\cdot$ & . & . & . & . & . & . & $\cdot$ & 126 \\
\hline 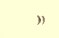 & eluritibiulis Ricardo & . & . & . & . & . & . & . & . & . & . & . & . & . & \\
\hline " & combustus Bigot. & . & . & - & - & . & . & - & . & . & $\cdot$ & - & . & - & \\
\hline "n & conformis Walker. & . & . & . & & . & • & . & . & . & . & - & . & . & 135 \\
\hline$"$ & congoiensis Ricardo & . & . & . & . & . & . & . & . & : & . & . & . & . & 144 \\
\hline " & coniformis Ricardo. & . & - & . & & - & . & . & & - & $\cdot$ & . & . & . & 120 \\
\hline & cumspicums Rieardo & & & & & & & & & & 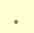 & 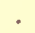 & 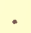 & 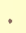 & \\
\hline
\end{tabular}


Tabumus cotm.t lenw . . . . . . . . . . . . . . . . 21?

Cortieri surroul. . . . . . . . . . . . . 210

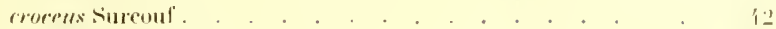

Denshami Justen + . . . . . . . . . . . . 2018

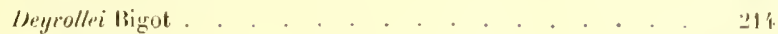

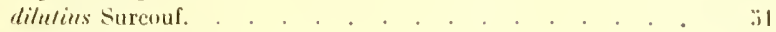

elisjumetus Ricarlo. . . . . . . . . . . . . . 143

distinelus Bicardo. . . . . . . . . . . . . . . . . . 11 ;

diterniatns Macquart. . . . . . . . . . . . . . 18'

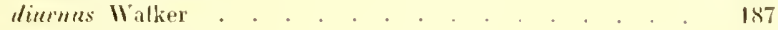

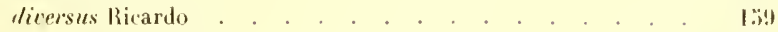

rlorsivitta Walker. . . . . . . . . . . . . . . . 1010

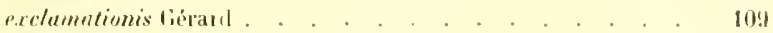

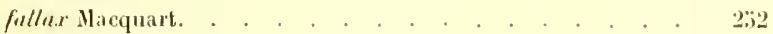

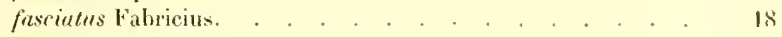

ferestratus Walker. . . . . . . . . . . . . . . . 21:3

fervidus llatker . . . . . . . . . . . . . . 13.;

frmmons Macquart. . . . . . . . . . . . . . . III

filviums Lew. . . . . . . . . . . . . 1!4.

fuscimevis Macquarl . . . . . . . . . . . . . 184.

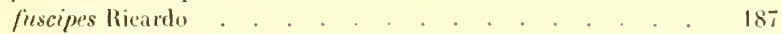

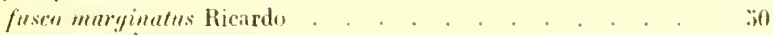

gubonensis M̉arquart . . . . . . . . . . . . . . 121

grandissimus Bicarto. . . . . . . . . . . . . . . i2

gratus Latw. . . . . . . . . . . . . . . TI8

ymineensis Wiedeman . . . . . . . . . . . . 1161

tebes Walker. . . . . . . . . . . . . . . 21'

thypoleucus Bigot. . . . . . . . . . . . . . . 134

ianthims Sureoul . . . . . . . . . . . . . . . . .3

imbecillus kiarsch . . . . . . . . . . . . . . 20.;

ignotus Surcouf . . . . . . . . . . . . . . . . 2.23

impurus Karsch. . . . . . . . . . . . . . 74

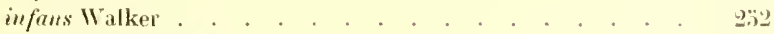

inhamburensis lierth. . . . . . . . . . . . . 144

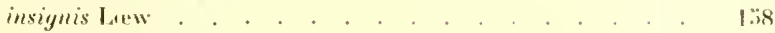

imorntus sureoul . . . . . . . . . . . . . 1!12

Jamus Walker. . . . . . . . . . . . . 1:3

kingsleyi Ricardı. . . . . . . . . . . . . . 128

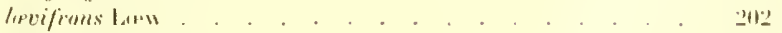

lulipes Misequart . . . . . . . . . . . . . . . 201i

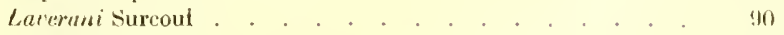

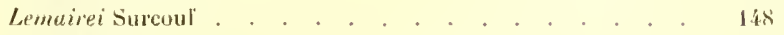

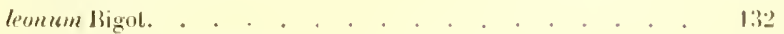

lencaspris Vander Wulp . . . . . . . . . . . . 61

lencostomus Lom . . . . . . . . . . . . . . 17:3

liventipes Surconf . . . . . . . . . . . . . . . . 7 . . .

suroul 


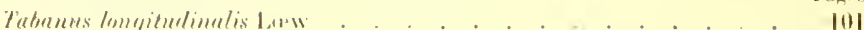

lutenlus low . . . . . . . . . . . . . . . lis

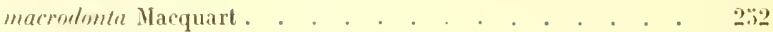

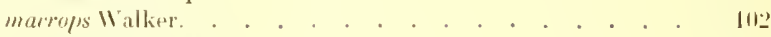

marulatissimus $1 \mathrm{~V}$ alker. . . . . . . . . . . . . 1!n

marmmalus Surcoul . . . . . . . . . . . . . . 83

Murtinisureoul. . . . . . . . . . . . . 1/7

Itesnili surrouf. . . . . . . . . . . . . . 212

morsiluns Ricarlo. . . . . . . . . . . . . . . Ilis

mulfimmatalus Van der $\|$ ulp . . . . . , . . . li2

namaquinus Bigrot. . . . . . . . . . . . . 1往

muns lliedemann. . . . . . . . . . . . . . . 118

niygohirhss Ricardo. . . . . . . . . . . . . . . 1333

nigromacnlutus Ricardo . . . . . . . . . . . . . 18.

migrostrintus Ricardo . . . . . . . . . . . . . . . . א

nilolicus Austen . . . . . . . . . . . . . . . 20

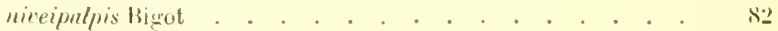

moctis 11 alker. . . . . . . . . . . . . 11)

moturum Bigut. . . . . . . . . . . . . . 72

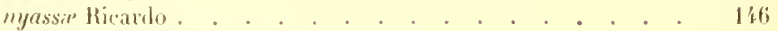

oblignemaculutus Matequarl . . . . . . . . . . . . . 172

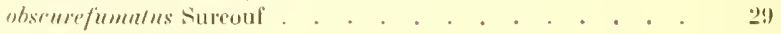

ubscuphirms Ricardo. . . . . . . . . . . . . . 7t

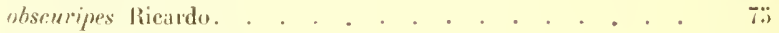

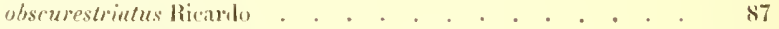

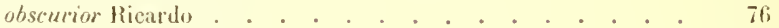

ohscurissimus Ricardo. . . . . . . . . . . . . . \$6

pallidifucies Surcouf . . . . . , . . . . , . . 176

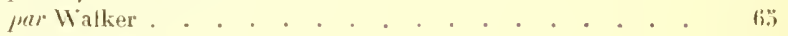

pervasus Walker . . . . . . . . . . . . . . 16

I'uı Walker. . . . . . . . . . . . . . . 60 60

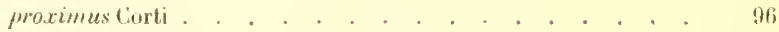

psusenms Jiennicke . . . . . . . . . . . 17,

Pyrohms Walker. . . . . . . . . . . . . 184

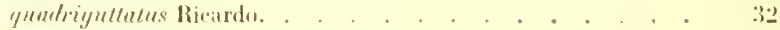

quadrisignatus Ricardo . . . . . . . . . . . . 113

ticardoir sureoul' . . . . . . . . . . . . . 11!1

Mothschildisureoul . . . . . . . . . . . . Tïl

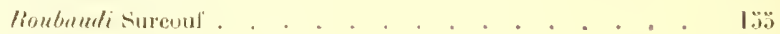

rulicumius Wilker. . . . . . . . . . . . . 109

rufescens Higot. . . . . . . . . . . . . . 17

ruficeps Bigol. . . . . . . . . . . . . . . . . 17.

mificress l'alimsut de Reauroí. . . . . . . . . . . . 46 46

mplipes IVimlentann . . . . . . . . . . . . 4 46

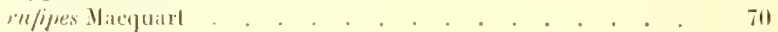

rnfosignulms ligul. . . . . . . . . . . . . . 17t 
Tabumus Romprnamii licardo

Pagise

$211 \%$

sngiltarius Mlatequat. . . . . . . . . . . . . . 111;3

specedens Walker. . . . . . . . . . . . . 126

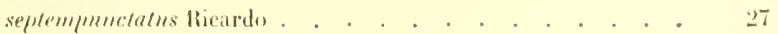

sequms Witker. . . . . . . . . . . . . . . . . . . 2

sericientris Lm. . . . . . . . . . 117

serratus laew. . . . . . . . . . . . 107

Sexerini surconf . . . . . . . . . . . . 1613

Sherpuei Auntran. . . . . . . . . . . . . . . 153

sorialis Willker. . . . . . . . . . . . . . . . 132

smous Wilker. . . . . . . . . . . 104

splendidissimus Ricardo . . . . . . . . . . . . . 34

sticticolis sureouf . . . . . . . . . . . 116

stigma Walker. . . . . . . . . . . . . . . 197

strigiventris Lew . . . . . . . . . . . . 201

subangustus licardo . . . . . . . . . . . . . . 83

subelongalus Macquart . . . . . . . . . . . . .

sutwillatus Ricardo. . . . . . . . . . . . . . . 28

sulunicus Cazalbou (i.l.). . . . . . . . . . . . . 2.82

sufis dirnnicke. . . . . . . . . . . . 177

sugens Wiedemann . . . . . . . . . . 103

snlcipalpes Lew . . . . . . . . . . . . 1.77

timialus llacquart. . . . . . . . . . . . 200

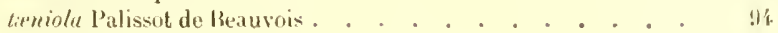

tursalis Alams. . . . . . . . . . . . . . . 190

temperatus Walkitr. . . . . . . . . . . . 1 1

tennicomis Mtitquart . . . . . . . . . . . . 1:17

terminutus Wialker. . . . . . . . . . . . . 1:i:i

Ifstucpiventris Marquart . . . . . . . . . . . . . 132

thorucinus Pallissot de lieauvois. . . . . . . . . 71

lithalis Walker. . . . . . . . . . . . . 12k

tripunclifer Walker . . . . . . . . . . . . 4

trisignalus lew. . . . . . . . . . . . 112

tritieniatus Ricardo. . . . . . . . . . . . . 181

milineatus Low. . . . . . . . . . . . . 89

unimaculatus Macquart . . . . . . . . . . . 41

umitieniatus Ricirdo . . . . . . . . . . . . . . 83

"stus Walker. . . . . . . . . . . . l佃

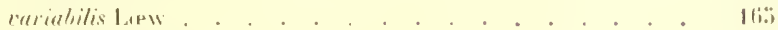

carians suroul. . . . . . . . . . . . . llik

Mariatus Wilkere. . . . . . . . . . . . . 1117

celutinus Sureoul . . . . . . . . . . . . . lit

rexhes law. . . . . . . . . . . , . . . 2018

virgatus Auten. . . . . . . . . . . . . 102

It ellmami Justion . . . . . . . . . . . . 1:84

zoulournsis Bigot . . . . . . . . . . . . 213 


\section{TABLE SISTEMATTQIE L'T BIBLIOGRAPHIOUL:}

INEFMIER R,HII PE:

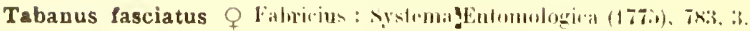

Entomml. sist., 11. 364.8 .

Sistema Aulliantorum. 94. 6.

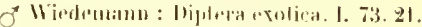

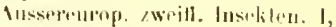
I:3i, :3:

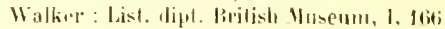

Tewstead: Innals of lropical Vealicint (1947), ii. pl. 4. Jig. 1 i.

h. Crmoluerer : lite Blatsaligenden bipleren $(11107) 13 \%$.

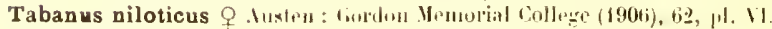

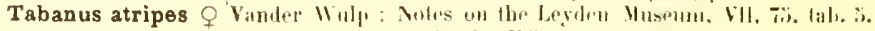
lig. \& (18:i5).

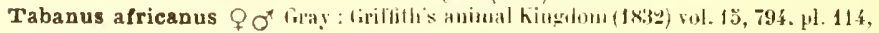
tim.r. $\because$.

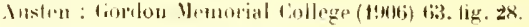

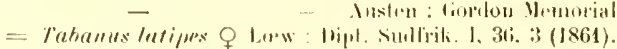

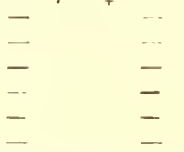

Peoters, Reise Vach Mozambinue \%ool. 1.2.

Matiquat : Iripteres exotiques I, 1. 119 (18:38).

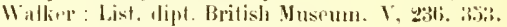

- Mhedulid, tome V.p. 328.

K. Limulerg : Vie Blutsang. Iipteren, p. 234 (J907). ormoul :

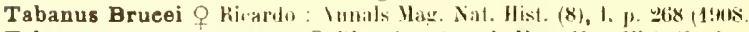

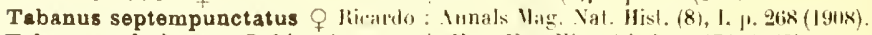
Tabsnus subvittatus $q$ livillitn: Imuls Milg. Nal. Hisl. (N). I, p. 270 (1!108).

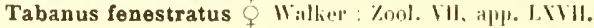

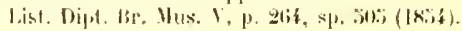

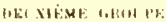

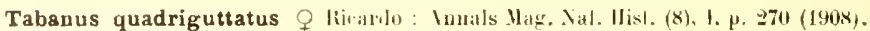
Tabanus marmoratus $q$ surout $=$ Tabames marmomosus surcoul : Bull. du Mus. - $\quad$ - $\quad(1909), n^{\circ} 6$.

Tabanus obscurefumatus $Q$ sirroul : Bullelin (lu Yusium (1906), 107,322 .

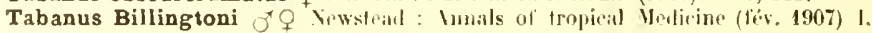
II" 1

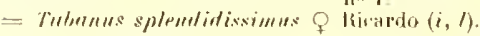




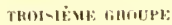

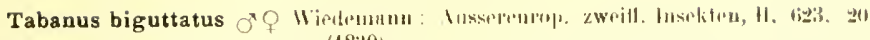
(18:30).

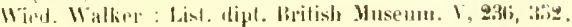

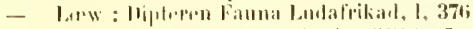

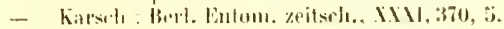

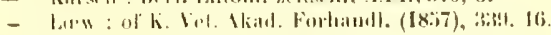

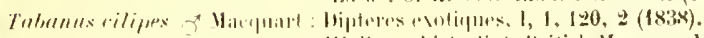

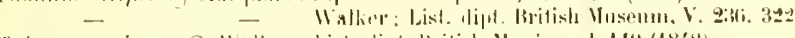

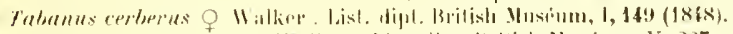

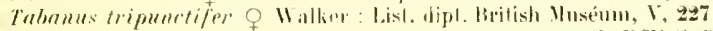

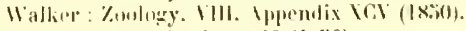

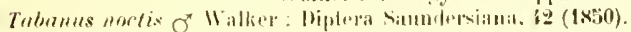

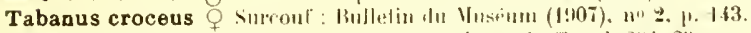

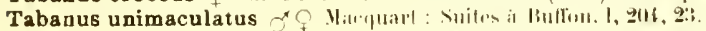

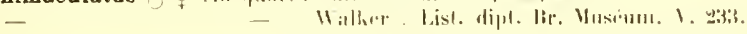
:3:3y.

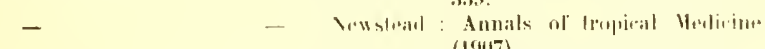
(I9ii)

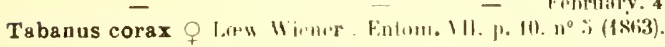

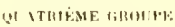

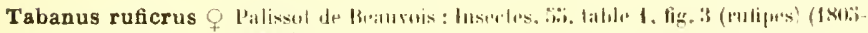
$1 \times 21)$.

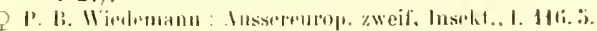

Hiptera "solica (1821). (il. 4 .

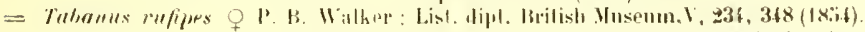

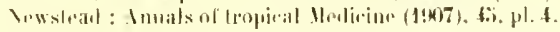
lis. $S$.

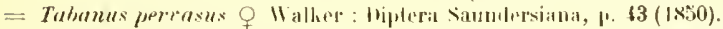

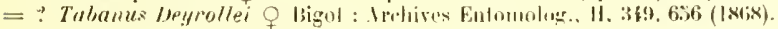

$=$ Tabunus miferus of vinouf.

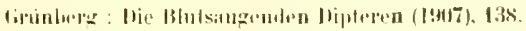

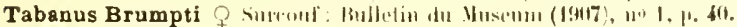

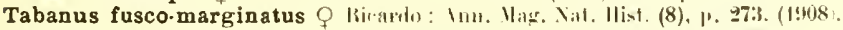

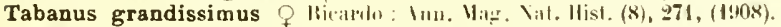

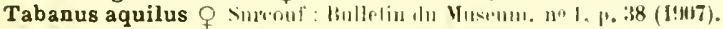

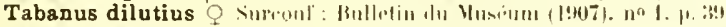

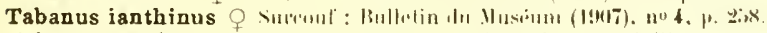

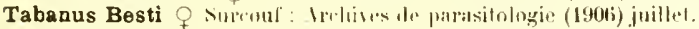

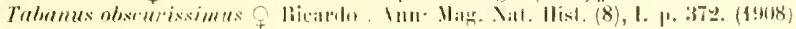

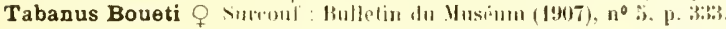

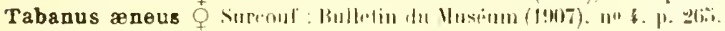

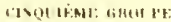

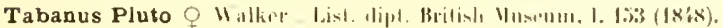

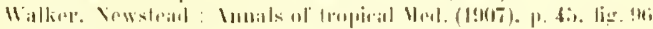

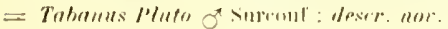




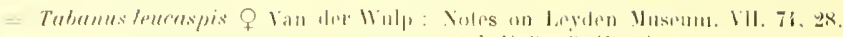
(p). 1. lig 3) (IN

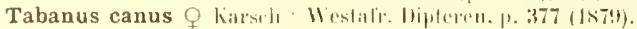

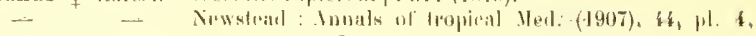
fi:. 1.

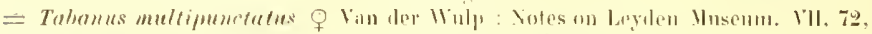

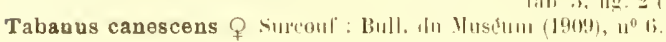

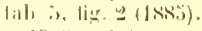

SIXIEME MHOLPE

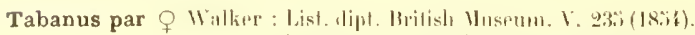

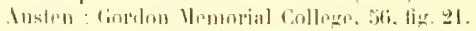

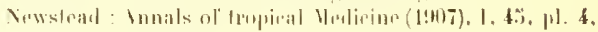
lit. 6.

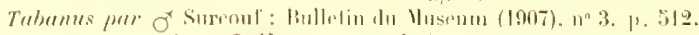

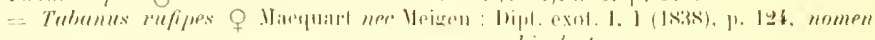
bis lectum.

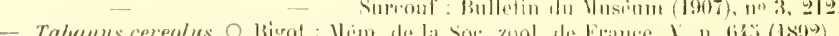

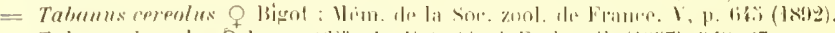

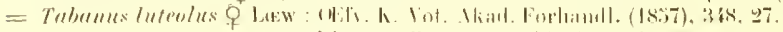

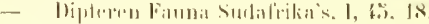

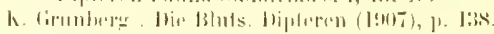

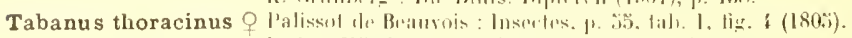

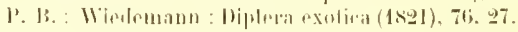

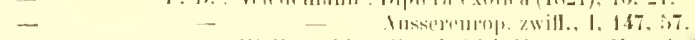

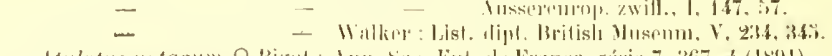

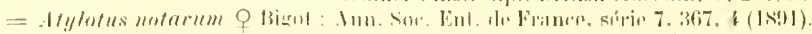

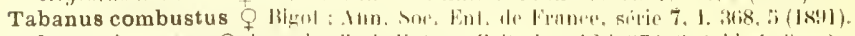

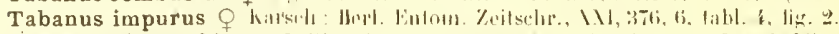

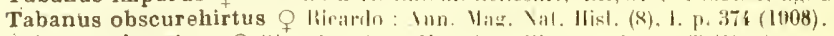

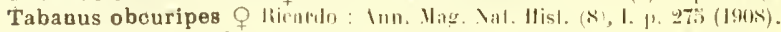

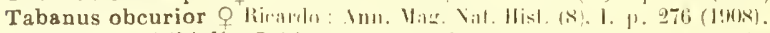

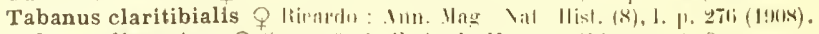

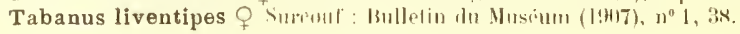

SEPTIÈME GROUPE

Tabanus albilinea $q$ Walker: I. ist dipt. British Wusemm, 1. 176 (1848).

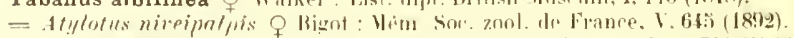

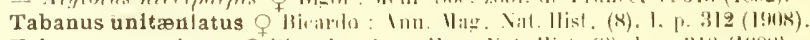

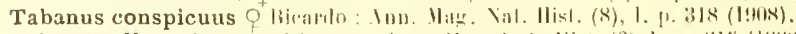

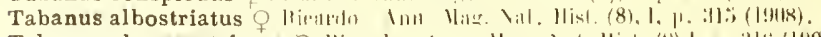

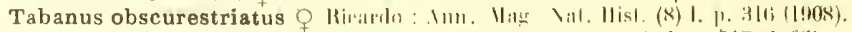

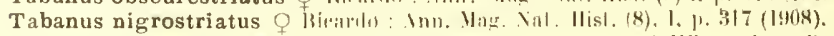

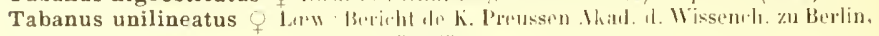
1xit. 6is.

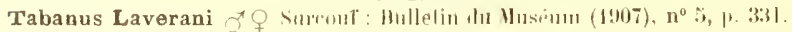

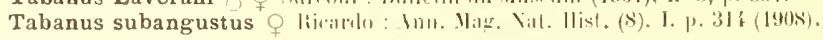




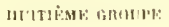

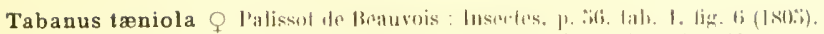

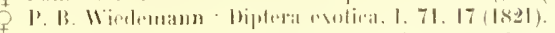

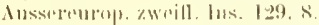

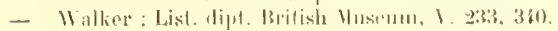

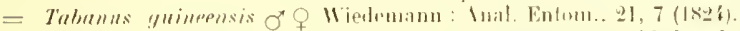

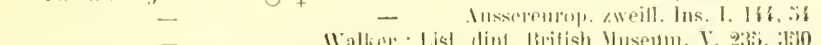

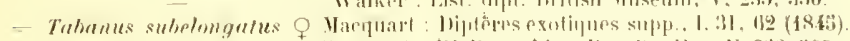

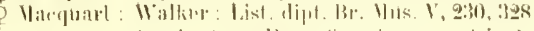

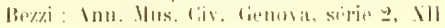
(11111), 1:3, 2.

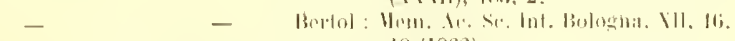
$18(1869)$.

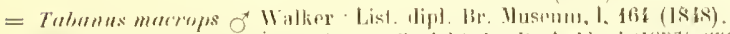

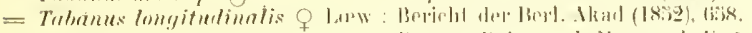

\begin{tabular}{|c|c|c|}
\hline - & - & 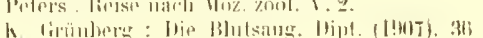 \\
\hline
\end{tabular}
lier. $10 \%$.

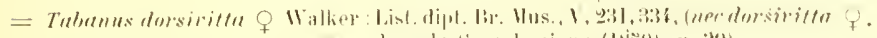

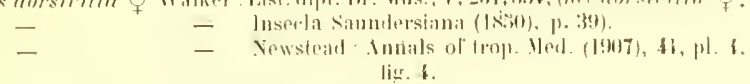

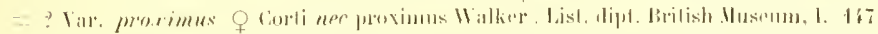
(1)!ij).

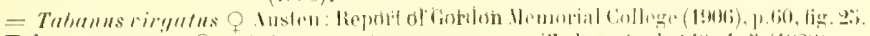

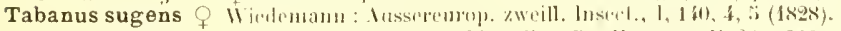

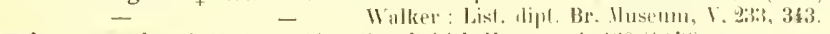

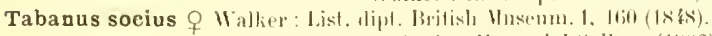

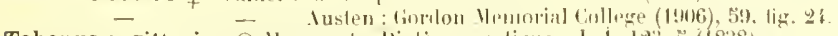

Tabanus sagittarius o Marquat : liptems nutipjues, 1. 1. 123.

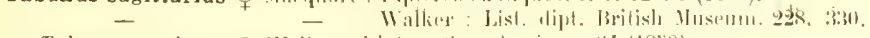

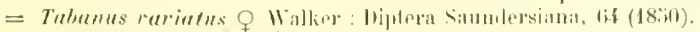

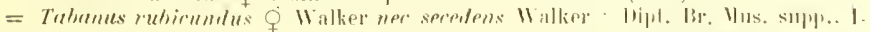
226 (1):ii).

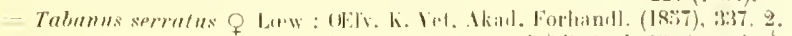

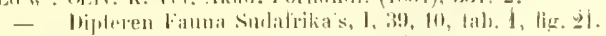

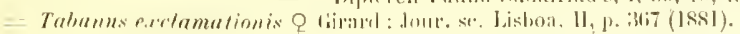

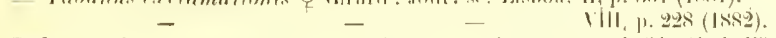

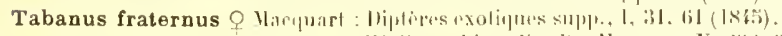

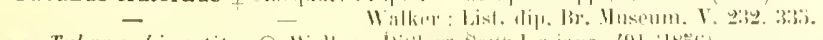

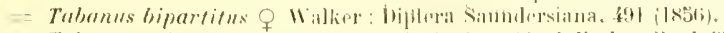

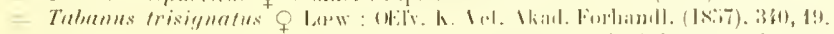

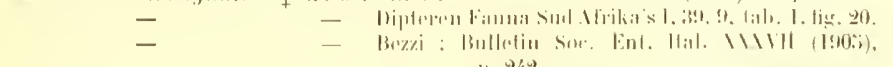

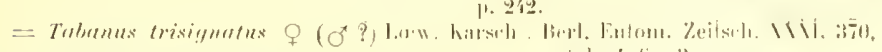
lil., i. lier. : .

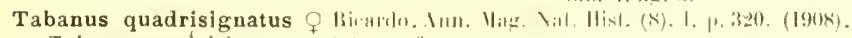

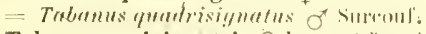

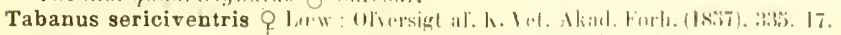

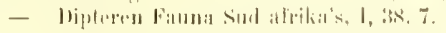

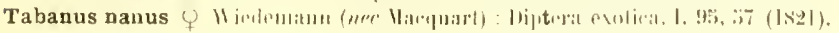

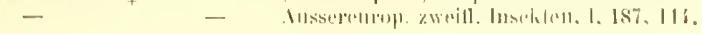




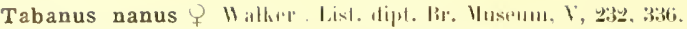

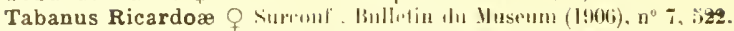

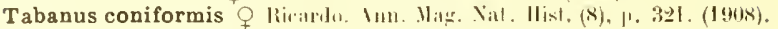

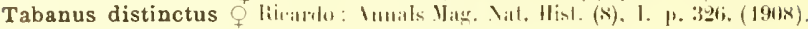

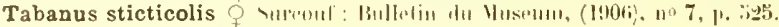

DETIFME GBUUPI.

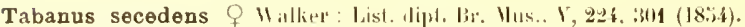

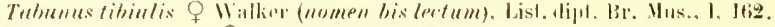

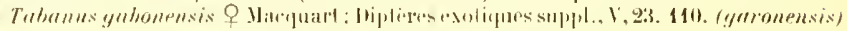
(15:ii).

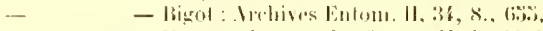

= Tubumus gabmensis ox surrout.

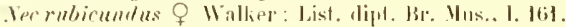

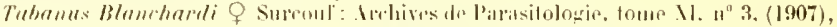
tim. 439. |jir. 3 at + pl. II.

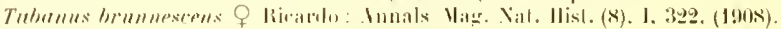

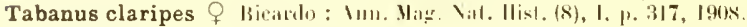

$=$ Tubutus rlaripes of Surroul.

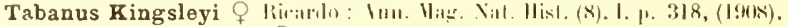

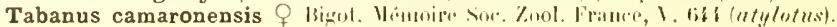

Tabanus ignotus $\sigma^{*}$ sistrunl nom.

IIXIEMt: diROL'PE

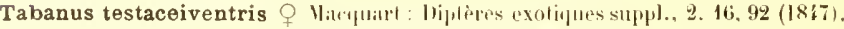

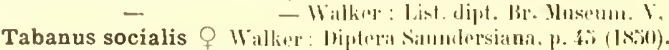

$?=$ Itylotus hypulenens o lsigot: Inu. Sor. Int. France, serie 7. I. 168. t. Nol. L.T. (1891).

- Ityletus leonum ? ligot. Mint. Nor. Zoologique de lirante. 1. 680, (1892).

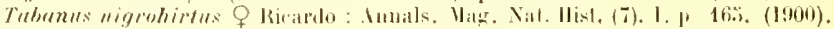

Tabanus conformis 9 Wallier: List. Ilipl. British Musmun. I, 150. (I848).

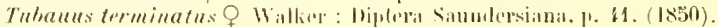

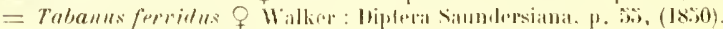

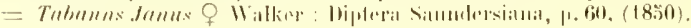

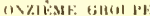

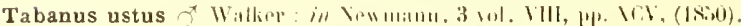

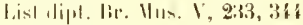

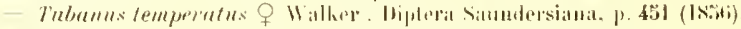

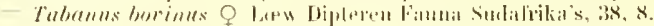

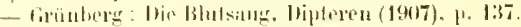

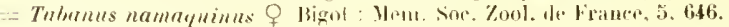

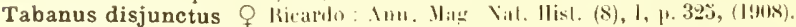

Tabanus inhambanensis of Butoloni : Viril. Si. Inst. Balogna. MII. tti. 19, 1ah, I,

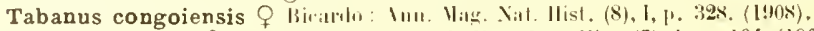

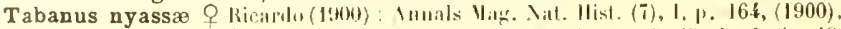

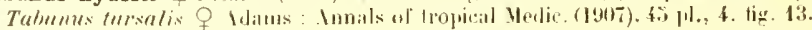




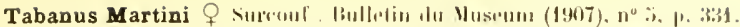

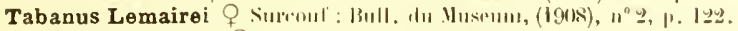

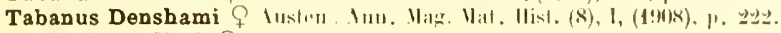

Tabanus Cordieri $q$ sureonl mox. sj.

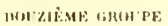

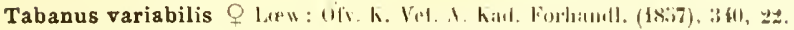

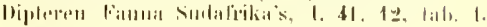
lig. $\mathbf{2}: 3$.

Tabanus insignis $\oint^{\circ}$ latwoul. - Itiptrrent litma Sulatrika's, I. H. 16.

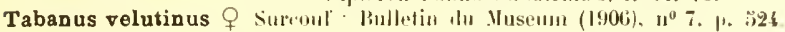

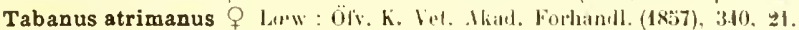

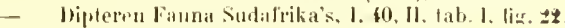

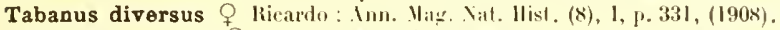

Tabanus argenteus? Sureorl': Bulletin du Museun (1907), $\mathrm{n}^{0}$ f. 263.

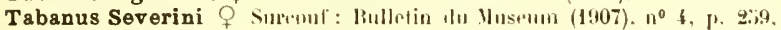

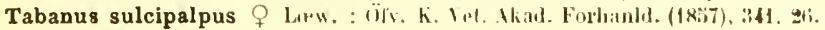

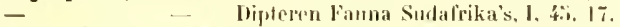

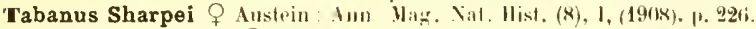

Tabanus Wellmani Q Iusten: Inn. Mas. Yill. Ilisl. (k), I, (I!108), |, 22:i.

Tabanus Roubaudi $q$ Surroul': Bull. du Hus'um, (190)!), di.

Tabanus varians $O$ Surrouf: : Bull, lu Museum. (1909), 1i.

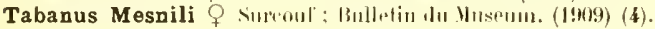

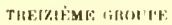

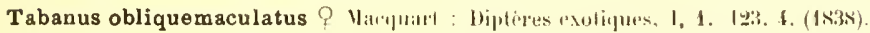
- Mallere : List. Miph. Br. Mus.. 1. 184. IJ. Wilker : list. dipit. Br. Mus.. 1. 22k:319. (18isi).

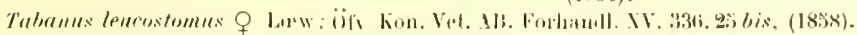

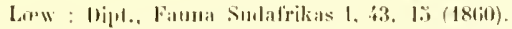

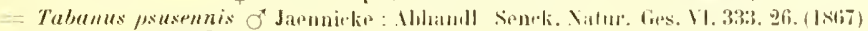

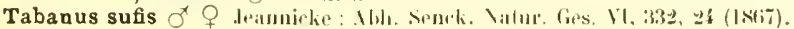

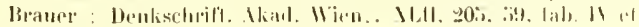
liz.

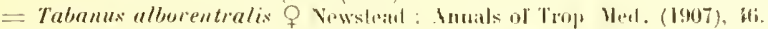

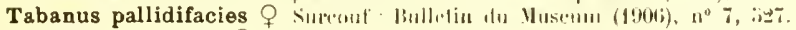

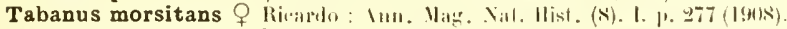

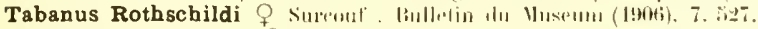

Tabasus rufosignatus o lijoul.

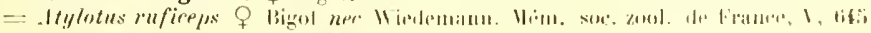
(1S!)

$=$ Tabamls rufescrens

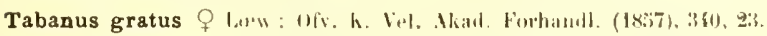

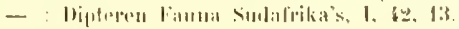

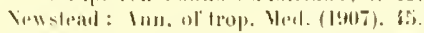

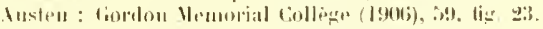

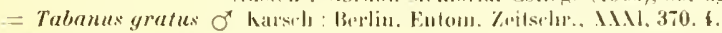

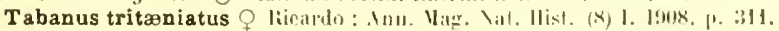


QU.ITORZIÈMF: GROLPE

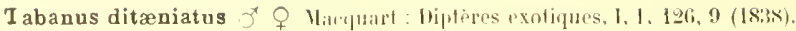

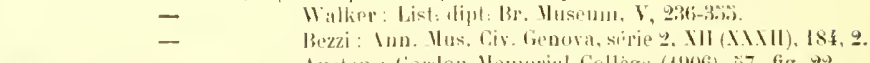

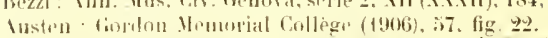

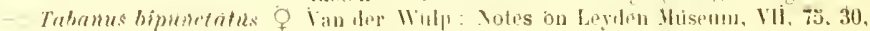
|al1. . . lis. is.

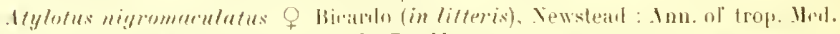
(1!)7), if.

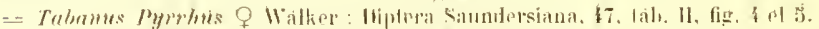

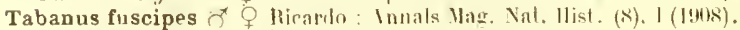

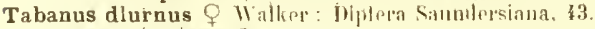

Tabanus albipalpus $q$ Walkre : Hiphera Samblersiana, p. If.

U) INZIĖ.HE IFROTPE.

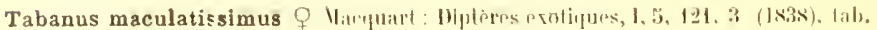
Xill, fig. 2.

1, 1) : 1) K. Vel. Ikail. Forh. (18:i7), 348, 27.

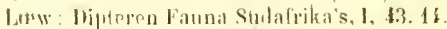

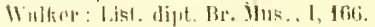

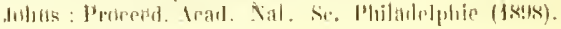
lis.

Tabanus irroratus $q$ Sum

SEIZITHSE GHOHPE

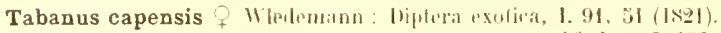
Inssupurop. zweil]. Ins.. I. 177, 100.

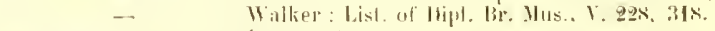

= Traburs inpensis of surroul.

Tabanus tenuicorsis $q$ Varqual : Hiplires pxotigues, I, 124, 7 (1838).

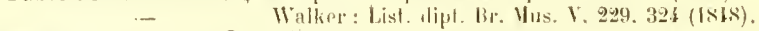

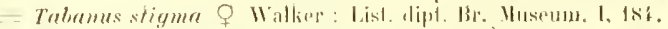

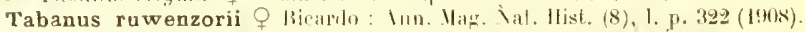

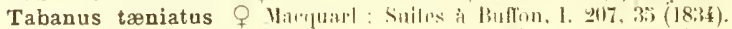

- Wallier : List. dipt. Br. Musemus. Y. 2290,331.

- Wallier: N $\quad$ - I84.5.

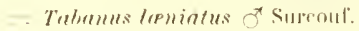

- Thlunus strigitentris $\mathrm{O}$ Lew : Ofr. K. Vel. Mliad. Forlantl. (18iti), 339, If.

- $\quad$ : Hipteren Fauta Sudafrilsits, 1, 3:3, 11.

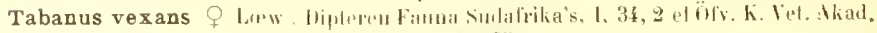
Furt. 336i, his (1850).

Tabanus Chevalieri $Q$ Shroul: Billplin lu Iluseurik. (I906), no 7, p. 523.

Tabanus lævifrons $Q$ Lew : Dipleren Filutin Sulafrika (IN(i)). 3\%, i.

- _ - iifs. k. Vet. Ikar. Forh. 336. a his.

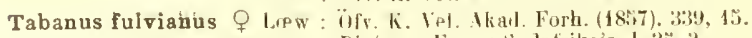

- $\quad$ - Dipleren Fuma Sidafrika's, 1, 33, 3.

Tabanus Imbecillus $q$ Katrse :Het. Ent. Keilsthr, AXII, 370, 7 (1887). 


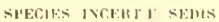

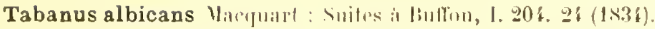

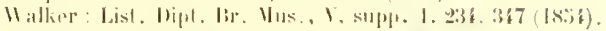

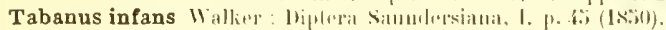

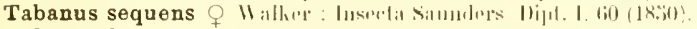

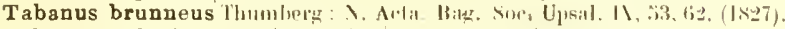

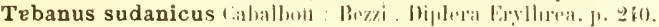

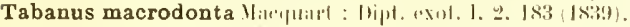

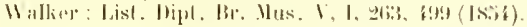

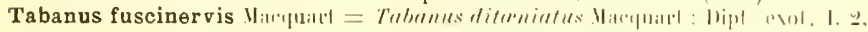
15it $(1 \times 3)$.

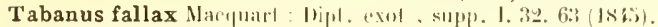

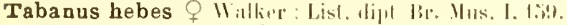

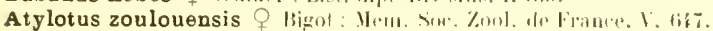




\section{TMBLF INAITIOUE}

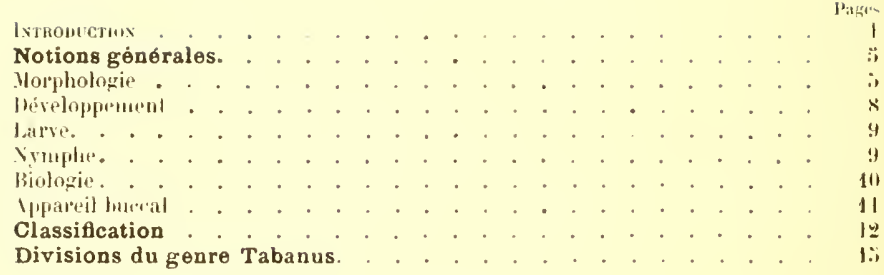

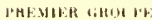

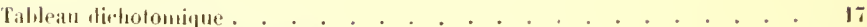

Trbanus fuscialus O Fibricius. . . . . . . . . . . . . . . . . Is

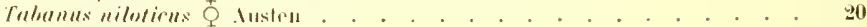

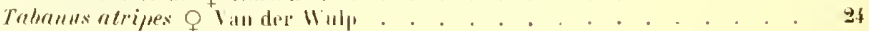

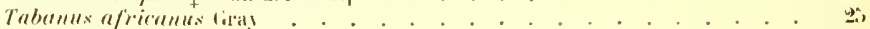

Tabanus latipes Harquitil . . . . . . . . . . . . . . . . . . 20

Taburus Bruceri o liealilo. . . . . . . . . . . . . . . . . . 20

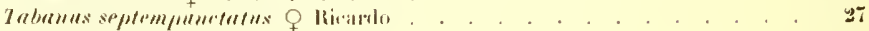

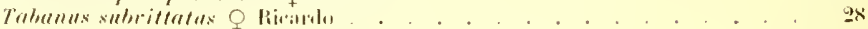

[1] XI: 4:111: 1.

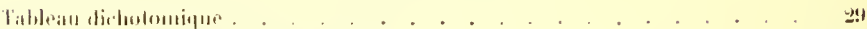

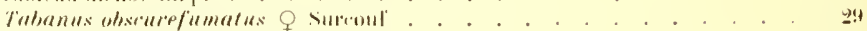

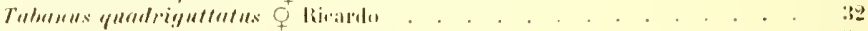

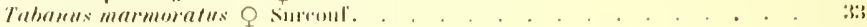

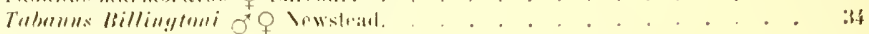

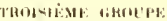

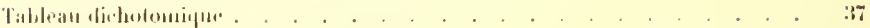

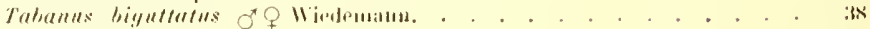

Tubatus cerberus o llilliul. . . . . . . . . . . . . . . 41 


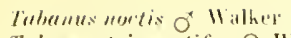

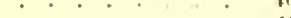

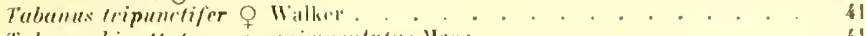

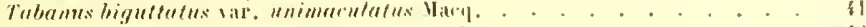

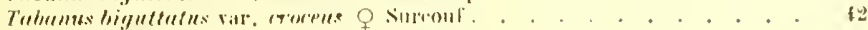

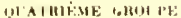

Pithean didhotomingu" . . . . . . . . . . . . . . . it

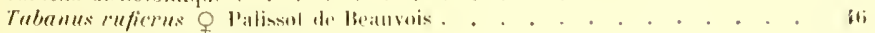

Tubanas brumpti to surenil. . . . . . . . . . . . . . . . . is

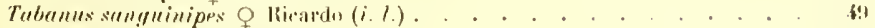

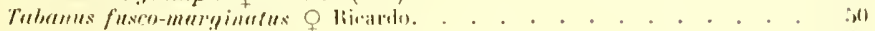

Trabures utpritus o surront . . . . . . . . . . . . . . . . . if

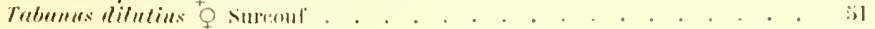

Tubanus gretudissimus $Q$ Rientrito . . . . . . . . . . . . . 52

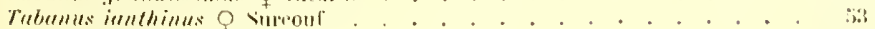

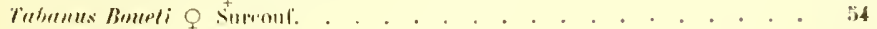

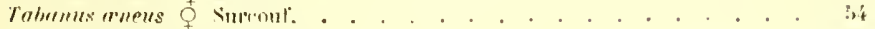

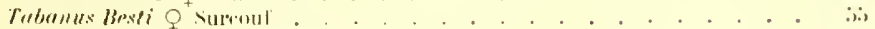

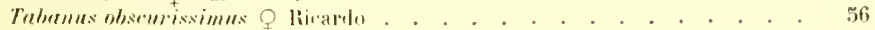

CIVITHE GKOLE

Tableau dicholontique . . . . . . . . . . . . . . on

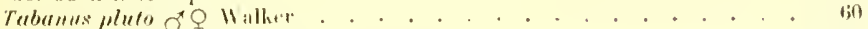

Telbanus cumus o hit'silt . . . . . . . . . . . . . . . . til

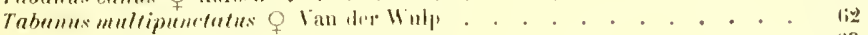

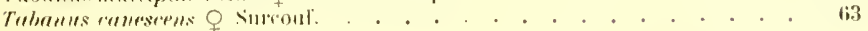

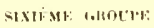

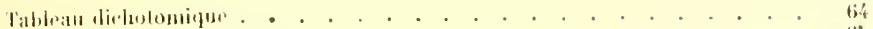

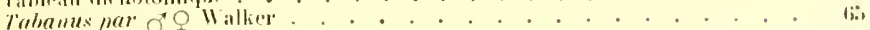

Fabumus cerpolus 0 ligol . . . . . . . . . . . . . . . . dis

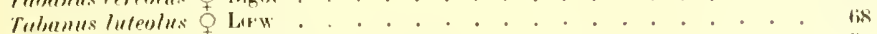

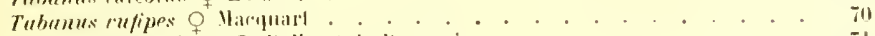

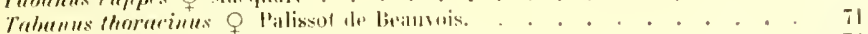

Tehmus noturum o lijent. . . . . . . . . . . . . . . . . . . 72

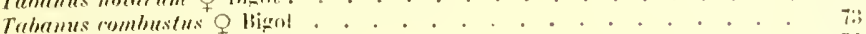

Tuluames impures: $Q^{+}$hirsill . . . . . . . . . . . . . . . it

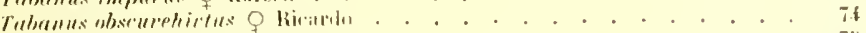

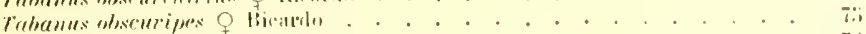

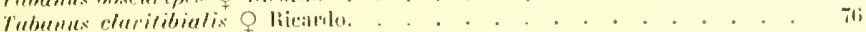

Tubanus absenrior o Rirardo. . . . . . . . . . . . , . . . . 70

Fulunus liventipes o silmont. . . . . . . . . . . . . . . . Ti

SFTTËMF: ARELTP:

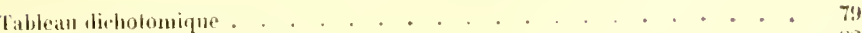

Tabamus albilinea 0 Walker. . . . . . . . . . . . . . . . . 82

Tabalus unitarniatus o kirarto. . . . . . . . . . . . . . . . 83

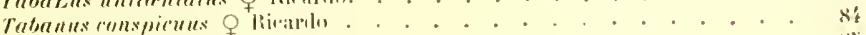

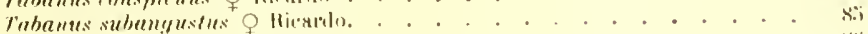

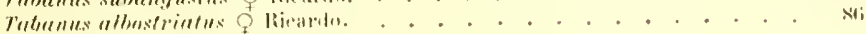




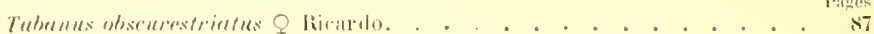

Tubanus nigrostrialus o livianto . . . . . . . . . . . . . 8s

Tabanus unilimentus Q larw . . . . . . . . . . . . . . . 89

Tabanus baremai o surroul. . . . . . . . . . . . . . . 90

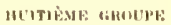

Tahlean diclutomiefuc. . . . . . . . . . . . . . . 92

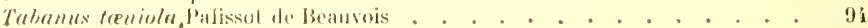

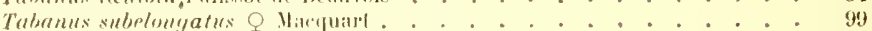

Tahames dorsivitha 0 II allier. . . . . . . . . . . . . . . . 100

Tabraus ynimpensis of IIiedenann . . . . . . . . . . . . . . 100

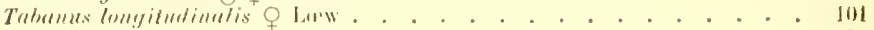

Tabarus virgatus of lusten . . . . . . . . . . . . . . 102

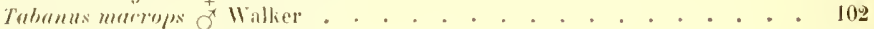

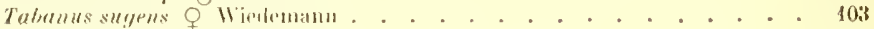

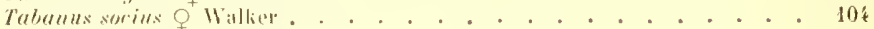

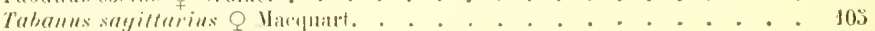

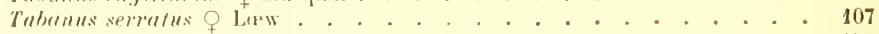

Tabatms rubirmadus 011 allial. . . . . . . . . . . . . . 109

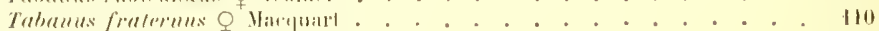

Tuhames hiparlitus 0 Wilker. . . . . . . . . . . . . . . 11 I

Tabumas trixignalus o l,w . . . . . . . . . . . . . . . 112

Tabomus quadrisignatus of Rirando . . . . . . . . . . . . . . 113

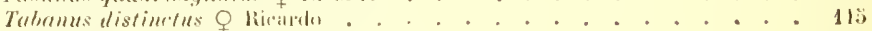

Trabanus sticticulis of surrum . . . . . . . . . . . . . . . 116

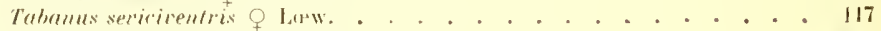

Tubanus nanus o lViedomann . . . . . . . . . . . . . . 118

Trebame dieardou o surroul. . . . . . . . . . . . . . . . 11!

Tubanus roniformis o livirido . . . . . . . . . . . . . 120

NEUTha: tikoc'te

Tathran didolomitre . . . . . . . . . . . . . . . . 129

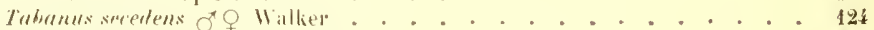

Tabanus elaripms o livimin. . . . . . . . . . . . . . . 126

Tubunus hiugsleyi o livimlo . . . . . . . . . . . . . . . . 12N

Thbumus comeromemsis ot ligot . . . . . . . . . . . . . . . . 129

WXILNE AHUE'TE

Tableau dirlobonique. . . . . . . . . . . . . . 420

Tabantes testureirentris $\bigcirc$ Vitequart . . . . . . . . . . . . 132

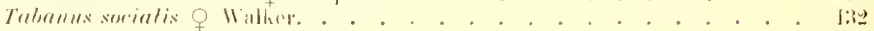

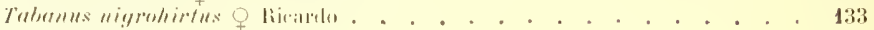

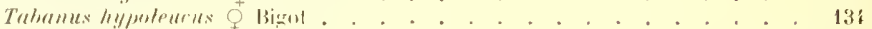

Talumus socialis 011 alher. . . . . . . . . . . . . . . 134

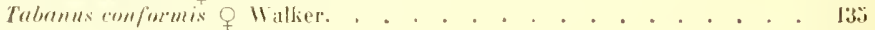


uxatrate: hatpe:

Tablemal dicholominge. . . . . . . . . . . 137

Tubumes ustus of 11 alker . . . . . . . . . . . . . . . . . 1810

Tubanks temperatus o llallier. . . . . . . . . . . . . . . . 14l

Tabauss burinus o laxw . . . . . . . . . . . . . . . . . . 141

Tulumus mamatuinus o Bigel. . . . . . . . . . . . . . . 142

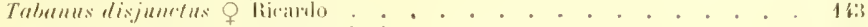

Tahames inhambamensis o lintoloni . . . . . . . . . . . . . 16:

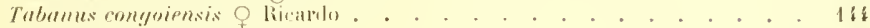

Taluanus nyasser o livalrlo. . . . . . . . . . . . . . . . . . 146

Telumes Martini o smmont . . . . . . . . . . . . . . . . . 147

Trbums Lemuirei

EotzILAF: AROLPE

Talıleall diwholomique . . . . . . . . . . . . . . . . . . 149

Talumus argenteus o surouf. . . . . . . . . . . . . . . . 18ie

T'ehanus sharpei q Inslen. . . . . . . . . . . . . . . . . 1;i3

Taluams. Hethmani o Iuslen. . . . . . . . . . . . . . . . 1:ic

Tulumes Renbendi o simpoul. . . . . . . . . . . . . . . . . 1:ici

Tabrmus sulcipalpus o Low . . . . . . . . . . . . . . . . 1:17

Fubames insignis o Law . . . . . . . . . . . . . . . Lis

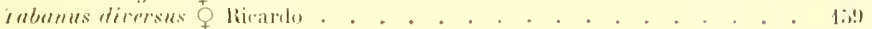

Tabamus atrimmmus o law . . . . . . . . . . . . . . . . 160

Tabame colutims o surouf. . . . . . . . . . . . . . . . . 162

Tabanus sererini f surroul . . . . . . . . . . . . . . . Itii

Tabamus iemints of surcoul . . . . . . . . . . . . . . . . 1tiq

Tubams ratiabilis o Law . . . . . . . . . . . . . . Mii,

TREIZIENE GRULPE

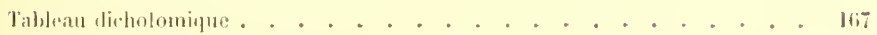

Trabmus morsitans o kicardo. . . . . . . . . . . . . . . . ltiv

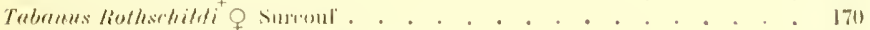

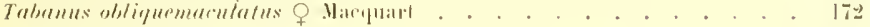

Tabarmus leurestomus o Low . . . . . . . . . . . . . . . . . 17:3

Tabrmus psusemuis of Jirnniclie. . . . . . . . . . . . . . . . 17i

Tabauns ruficeps fligol . . . . . . . . . . . . . . . . 17i

Tabanus pallirlifacies o sumoul. . . . . . . . . . . . . . . 176

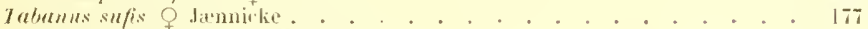

Tuhamus albomentralis o Vowsteal. . . . . . . . . . . . . . 177

Tabmus gratus o laww. . . . . . . . . . . . . . . . 17s

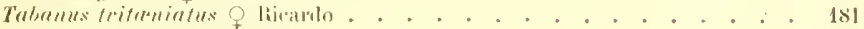

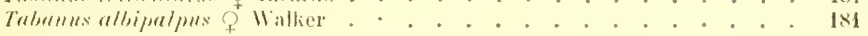

gUTTOHZTME (HOE'TE

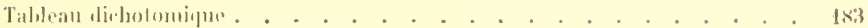

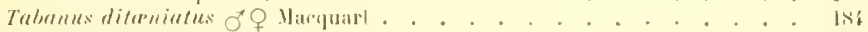

Tulunus fuscipes or livarilo . . . . . . . . . . . . . . . . 187

Thlome diurnus 0 llather . . . . . . . . . . . . . . . 157 
QI IXIE:HI: Q, HOI'1'F:

T.1.tuna liethotomigur

........ 149

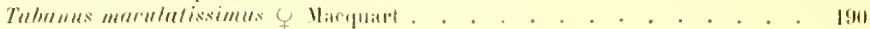

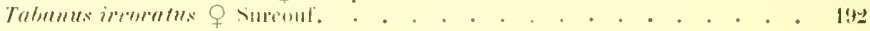

SESAIEMT GRIULIP

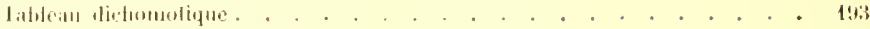

Tubunus fulriauls o lan. . . . . . . . . . . . . . . 144

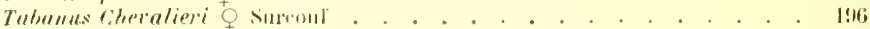

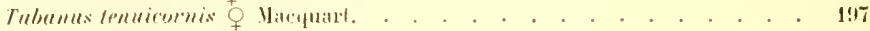

Trabnums sligmn o Wallier. . . . . . . . . . . . . . . . . . 197

Teluanus rapensis $Q$ Wierlensan. . . . . . . . . . . . . . . . 198

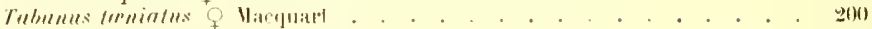

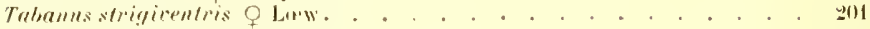

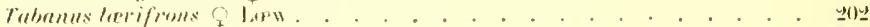

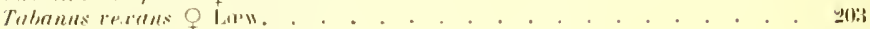

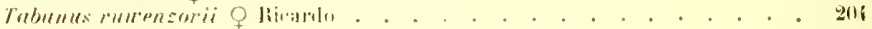

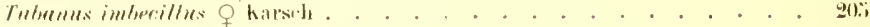

\section{IP'VIDIC:E}

WNZIT:ME GHOP

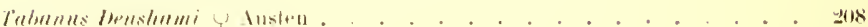

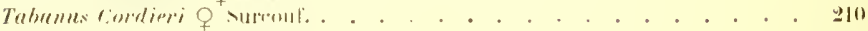

TREIRIEME: GHOI PE

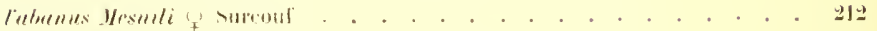

Species incertio sedis

Tulumbs fenestratus o Misllitr. . . . . . . . . . . . . . 913

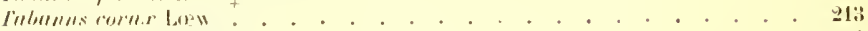

Teluanes begrollei Bignt. . . . . . . . . . . . . . . . . . . 214

Thlunus helues O Wiallier . . . . . . . . . . . . . . . . 214

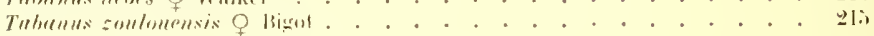

\section{Taons mâles}

P'REMIEK GRUL'PE.

Thlumus fasciatus on Wiedemann . . . . . . . . . . . . . . . 2lti

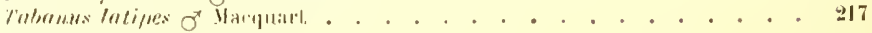


DECXIE'ME GROLPE

\section{TROALIENE GRULP:}

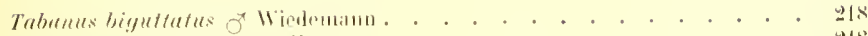

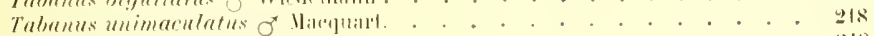

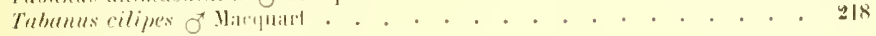

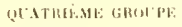

Tabanus raficras of balissot de lieaubois . . . . . . . . . . . 218

CINQITHE MRUCPE

Tubanus pluto of Hilker. . . . . . . . . . . . . . . . 219

SII:U1: (ilsuLPE

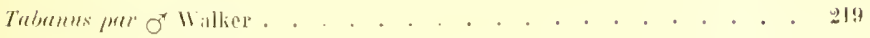

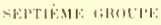

Thbamus Larerani or simonf . . . . . . . . . . . . . . . . . 9l! Tubumus subum! ustus of livarlo. . . . . . . . . . . . . . . . 290

HLITIEME GROLPE

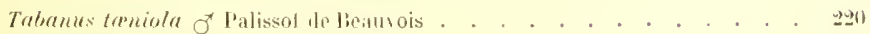

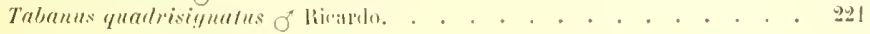

NEUVIĖME GROTPE

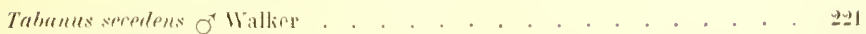

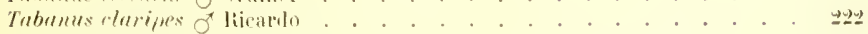

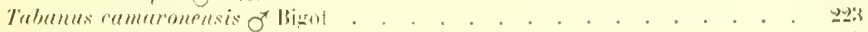

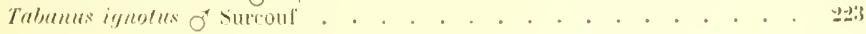

ONZIEME GROLTL:

Tabanus inhambanensis or beroloni . . . . . . . . . . . . . . 92t

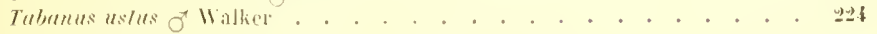

surenul 17 
LOTZLAE GROTPE

Tubanus variabilis or Law

THEIZIEYE GHMLPE

Telualus grafus or harsh

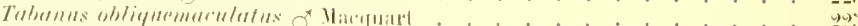

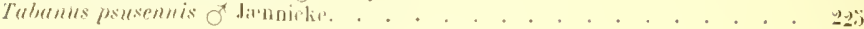

(I'ATORZIEME GROULE

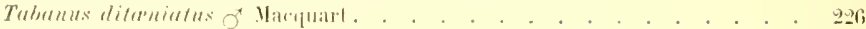

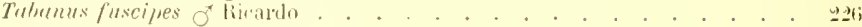

SEI\%II:YE FIROUP:

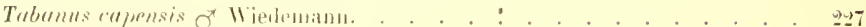

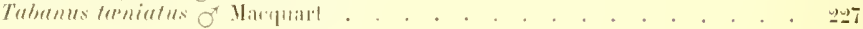

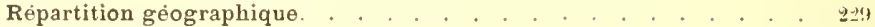

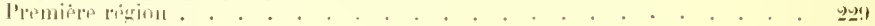

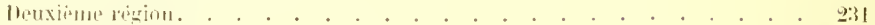

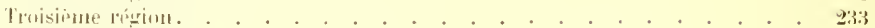

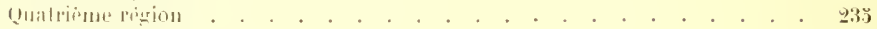

Cimprimertigion . . . . . . . . . . . . . . . . . . 237

Sixime lerint . . . . . . . . . . . . . . . 938

Liste alphabétique des taons . . . . . . . . . . . . . . . @il

Table systématique et bibliographique. . . . . . . . . . . . . @保

Table analytique . . . . . . . . . . . . . . . . . . . . . . 


\section{TABLE IEE FIGTRAS}

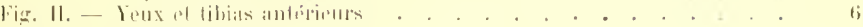

Fier. III. - Vorvilion lo liale. . . . . . . . . . . . . . 7

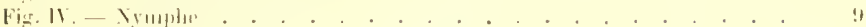

Fin. V. - Vilosion le Tabanus bromius limni . . . . . . . . . . 10

lig. 11. liambes limulates. . . . . . . . . . . 1:3

Fin. 7. - Tabanus latipes V Vitiplual. . . . . . . . . . 17

Fi. 8. - Tabanus Billingtoni 9 Yiskea! . . . . . . . . . . . 301

Fi. 9. - Tabanus marmorosus $Q$ sirroll . . . . . . . . . . . : :

Fig. 10. - Tabanus biguttatus o llierlintimı. . . . . . . . . . 3i

Fig. 12. - Tabanus canus halsh. . . . . . . . . . . is

Fir. 13. - Tabanus combustus o bignt. . . . . . . . . . . . . (ii)

Fi. 14. - Tabanus Laverani firrort" . . . . . . . . . . . . s()

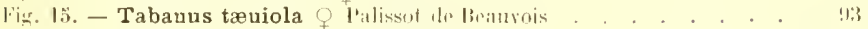

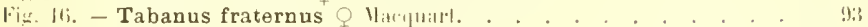

Fis. 17. - Tabanus quadrisignatus 9 licilolo. . . . . . . . . . 9.

Fig. 18. - Tabanus claripes O Rinilto. . . . . . . . . . . . 12y

Fir. 1!. - Tabanus conformis 911 allir. . . . . . . . . . . . 1:31

Fir. 20. - Tabanus disjunctus livallo. . . . . . . . . . . . 1:3א

Fis. 21. - Tabanus Roubaudi finconl. . . . . . . . . . . . 1.ill

Fis. 2.9. - Tabanus gratus L Lipw . . . . . . . . . . . IGX

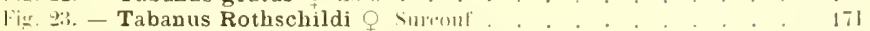

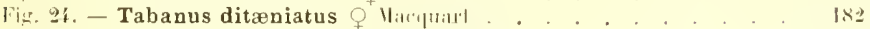

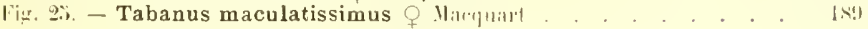

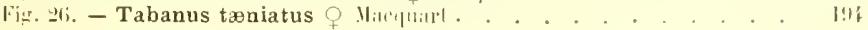




\section{T.IBIE DES C.IRTES}

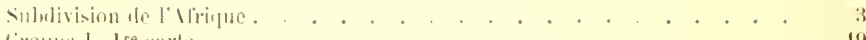

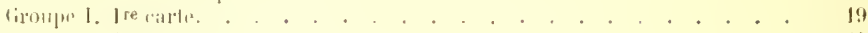

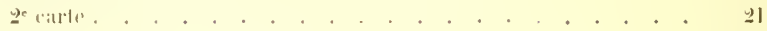

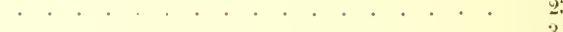

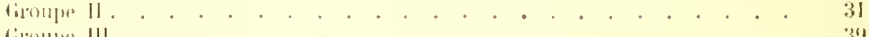

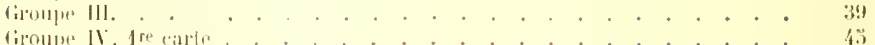

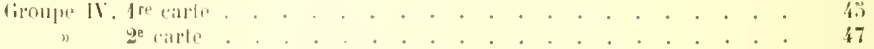

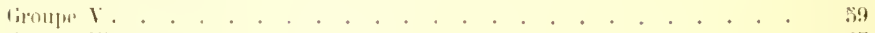

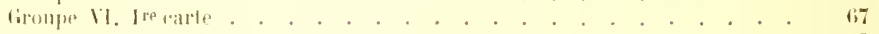

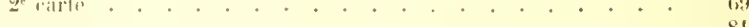

(і)

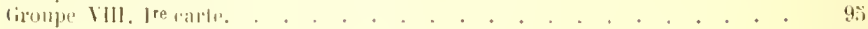

(i)

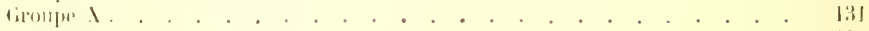

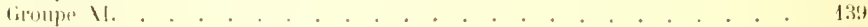

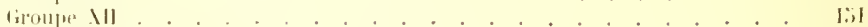

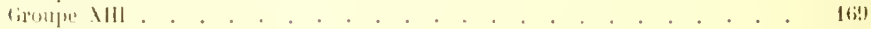

liron

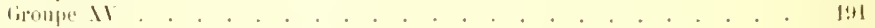

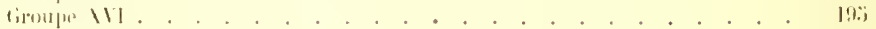

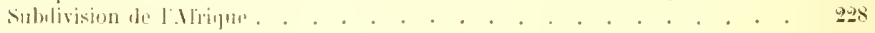



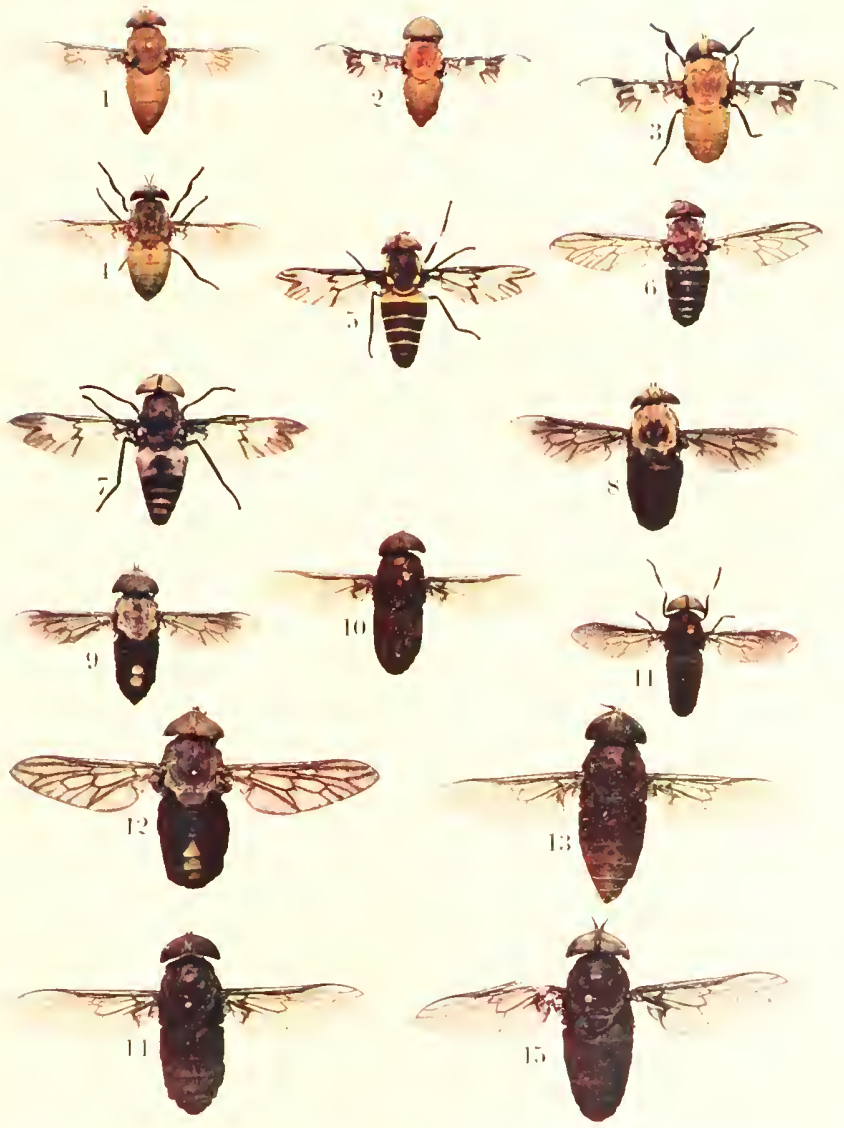

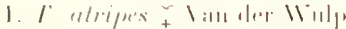

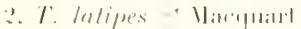

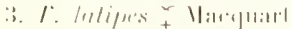

1. I. Pascinlus + lablurian

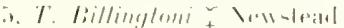

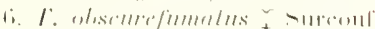

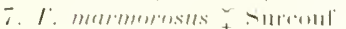

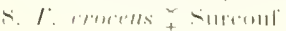

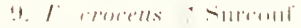

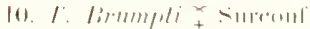

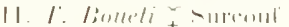

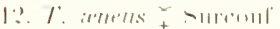

J:3. Ilimlius +

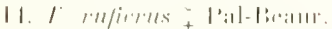

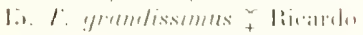




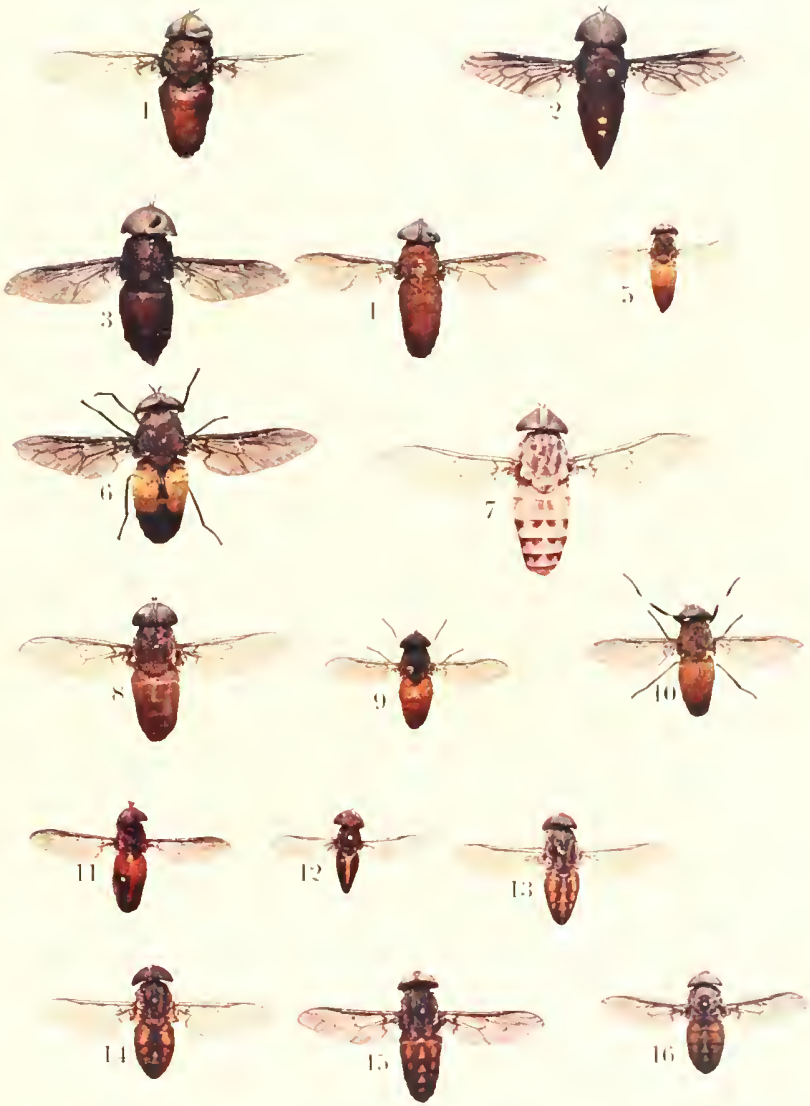

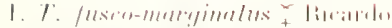

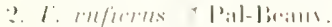

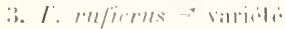

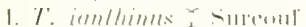

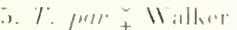

ti. $\%$ flul +11 allar

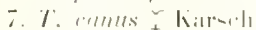

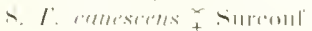

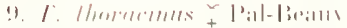

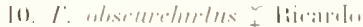

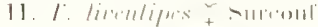

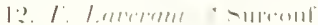

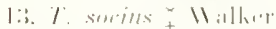

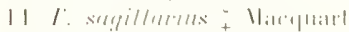

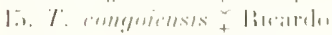

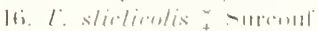




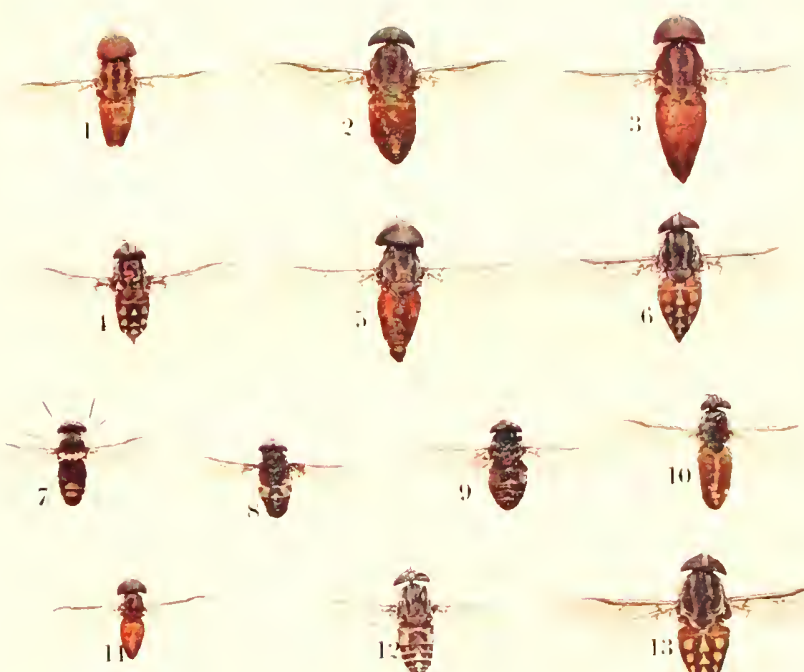

!)
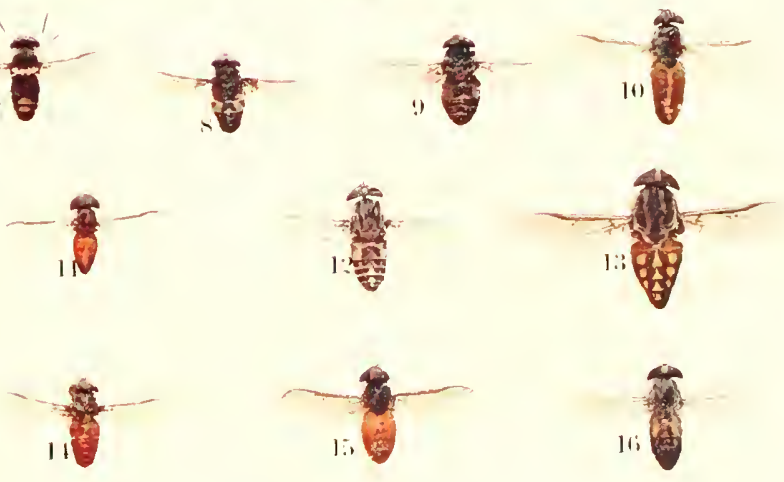

17
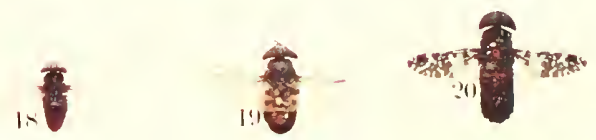

1. I seredens 11 allaed

$\therefore$ I' serestems + II allw

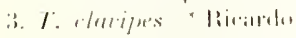

1. $\% 1 / 111 / 114+-111 \%+111$

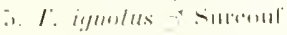

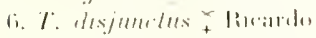

7. Ir myonletes +

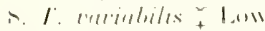

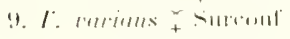

10. I. sulumgustus + liwatho

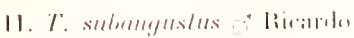

1? $\%$ alrimmus +1 .

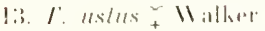

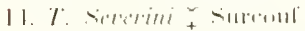

1.. \% ruphirlus + linaluh

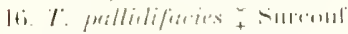

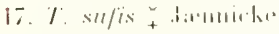

Is. $\%$ e hemblar +

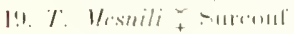

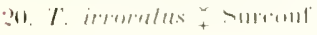







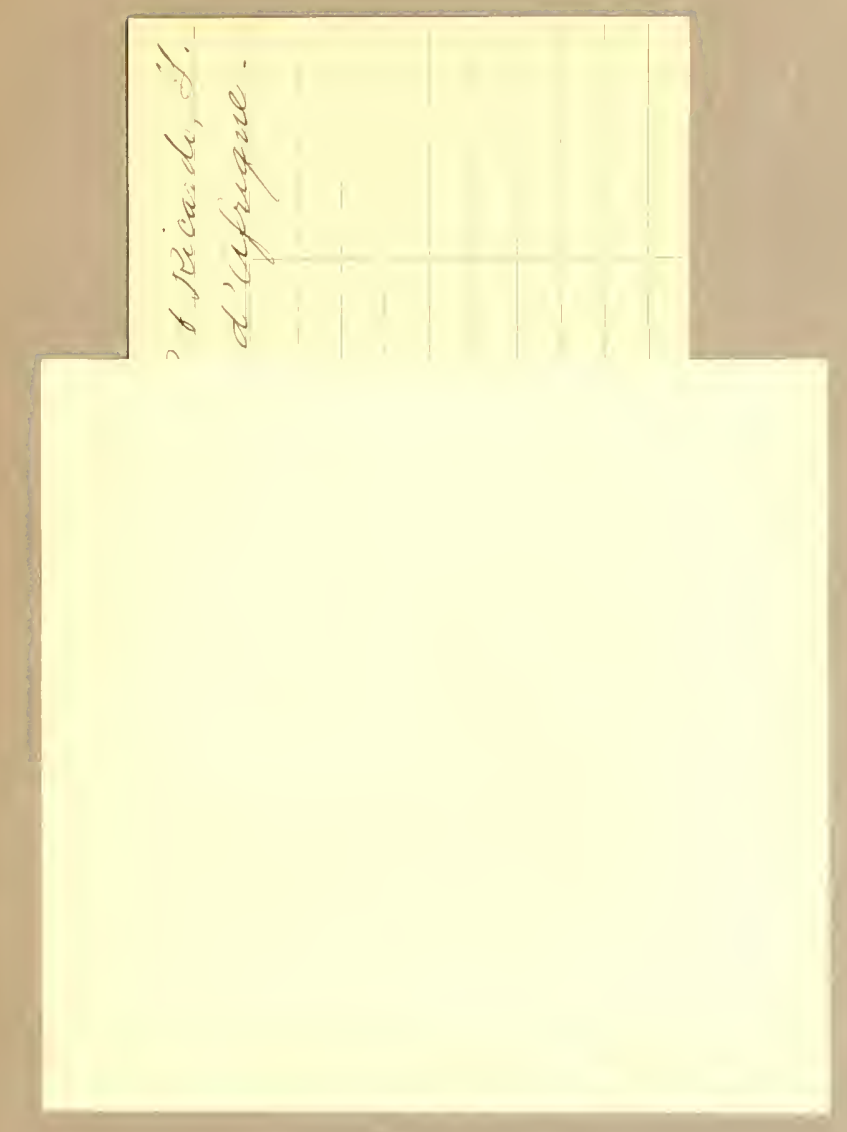




\section{A la même Librairie}

Maladies des Pays chauds, Manuel de Pathologie Exotique, par par Maurice Guibato, Hédecin de réserve de Patrick Manson, traduit de langlais Ja quatrième édition anglaise, entièrement revue et considérablement augmentée, 1 vol. grand in $-8^{\circ}$, de $x v-815$ pages, avec 2.11 figures dans le texte et 7 planches hors texte en couleurs.

Précis de Pathologie Exotique, par E. Jeanselase, Professeur cine agregé à la Faculté de médecine de Paris, Médecin des Hópitaux el E. Rıst, Médecin des Hòpitaux de Paris, Ancien inspecteur général des services sanitaires maritimes et quarantenaires d'Egypte, 1 vol. petit in-80, de la Collection de Précis Médicaux, de xı 809 pages, avec 160 figures dans le texte et 2 planches hors texte en couleurs. Cartonne toile souple.

$12 \mathrm{fr}$.

Cours de Dermatologie exotique, par E. Jeanselme, Profesmédecine de paris, Médecin des Hopitaux. Pecueilli ácé a la Faculté de Lì̀nes, Interne des Hòpilaux, 1 vol. grand in- 80 , de vi-403 pages, avec 5 cartes et 108 figures dans le lexte.

Diagnostic et Séméjologie des Maladies Tropicales, par R. Wurrz, Professeur agrégè, chargé de cours à l'Institut de médecine coloniale de la Faculıé de médecine de Paris el A. Turnoux, Médecin-major de re classe des Troupes coloniales, 1 vol. grand in-80, de xit-5 11 pages, avec 97 figures en noir et en couleurs.

$12 \mathrm{fr}$.

Traité du Paludisme, Par A. Lavenan, Membre de l'Institutel de l'Acagrand in-80, de v1t-622 pages, avec 58 fiecine. Deuxième édition, 1 vol. hors texte en couleurs. . . . . . . . . . . . . . . . . 12 fr.

Trypanosomes et Trypanosomiases, par A. Lavera, Membre mie de médecine et $\mathrm{F}$. Mesnil, Chef de Laboratoire à l'Institut Pasteur, 1 vol. grand in-80, de $\mathrm{x}_{1-4} 18$ pages, avec 61 figures dans le texte et une planche hors texte en couleurs. . . . . . . . . . . . . . . . . . $10 \mathrm{fr}$.

Les Venins, Les Animaux venimeuce et la Sérothérapie antivenimeuse, Les Venins, par A. Calmetre, Membre correspondant de l'Institut et de l'Académiede médecine, Directeur de IInstitut Pasteur de Lille, $t$ vol. in-80 de xv1-396 pages, avec 125 figures dans le texte, rartonne toile anglaise. $12 \mathrm{fr}$.

La Presse Médicale Journal bi-hebdomadaire, paraissant le Mera Presse Medicale, credi et le Samedi. Direction scientihique, L. LaNnouzr, Professeur de Glinique médicale, Doyen de la Faculté de médecine de Paris, Membre de l'Académie de médecine; F. De Lapensonse, Prufesseur de Clinique ophtalmologique à l'llotel-Dieu; H. Roger, Profcsseur de Pathologrie expérimentale à la Faculté de Paris, Médecin de l'hópital de la Charité ; E. Boxxame, Professeur ałréżé, Accoucheur de l'hòpital Lariboisière; M. Lerulle, Professeur agrígé, Médecin de l'hópital Boucicaut, Menbre de l'Académie de médecine; M. Lermorez, Mélecin de l'hòpital Satint-Aatoine; J.-L. l'aune, Professeur agrègé,

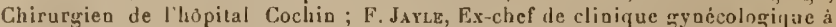
l'hôpital Broca. Secrélnire de la Direction. Rèdaclion: 1'. Desfosses, Secrétaire de la Redaction; J. Demovr, R. Romme, Secritaires.

Prix de l'abonnement annupl: Paris el Departements, 13 fr.: Lnion pustale. 





